ACARORUM CATALOGUS

Petar Beron 



\title{
ACARORUM CATALOGUS VIII
}

\section{Trombidiformes \\ Prostigmata}

\section{Superfamilia Cheyletoidea \\ (Cheyletidae, Psorergatidae, Demodecidae, Harpyrhynchidae, Syringophilidae) Superfamilia Cloacaroidea (Cloacaridae, Epimyodicidae)}

\section{Petar Beron}

\author{
Pensoft
}

National Museum of Natural History, Sofia Bulgarian Academy of Sciences 


\section{ACARORUM CATALOGUS VIII}

\section{Trombidiformes}

Prostigmata

Superfamilia Cheyletoidea

(Cheyletidae, Psorergatidae, Demodecidae,

Harpyrhynchidae, Syringophilidae)

Superfamilia Cloacaroidea

(Cloacaridae, Epimyodicidae)

Petar Beron

First published 2021

ISBN 978-619-248-041-7 (paperback)

ISBN 978-619-248-042-4 (e-book)

Pensoft Series Faunistica No 222

Beron P. 2021. Acarorum Catalogus VIII. Superfamilia Cheyletoidea. (Cheyletidae, Psorergatidae, Demodecidae,Harpyrhynchidae, Syringophilidae) Superfamilia Cloacaroidea (Cloacaridae, Epimyodicidae). Pensoft \& National Museum of Natural History, Sofia, 465 pp.

(c) PENSOFT Publishers

(C) National Museum of Natural History, Sofia

Pensoft Publishers

Prof. Georgi Zlatarski 12, Sofia 1700, Bulgaria

Fax: +359-2-870-42-82

info@pensoft.net

www.pensoft.net

Printed in Bulgaria, June 2021 


\section{Contents}

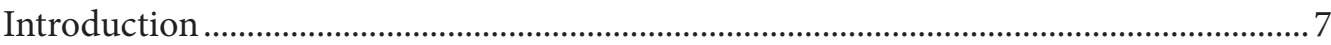

The Mite Superfamily Cheyletoidea.................................................................................. 8

System accepted in this book ..................................................................................... 9

The Mite Family Cheyletidae Leach..................................................................................... 10

Composition of fam. Cheyletidae ............................................................................11

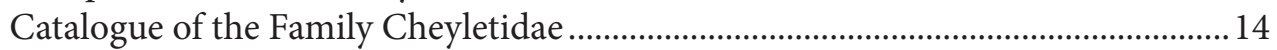

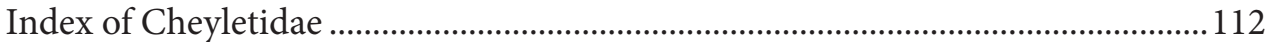

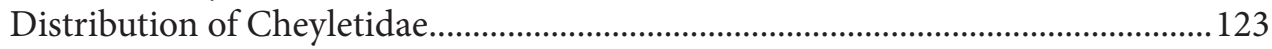

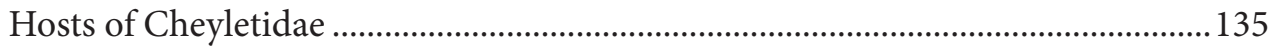

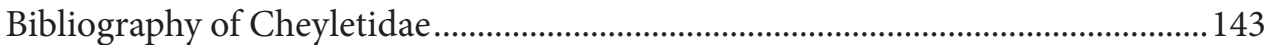

The Mite Family Psorergatidae Dubinin ...........................................................................177

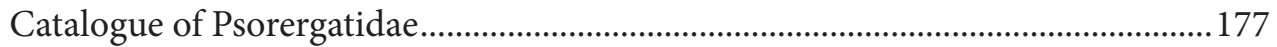

Index of Psorergatidae ............................................................................................190

Distribution of Psorergatidae................................................................................... 192

Hosts of Psorergatidae ..............................................................................................194

Bibliography of Psorergatidae ................................................................................ 198

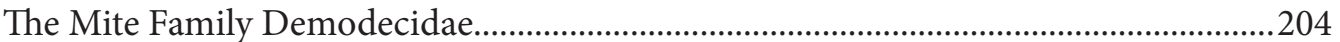

Composition of the family Demodecidae ...............................................................205

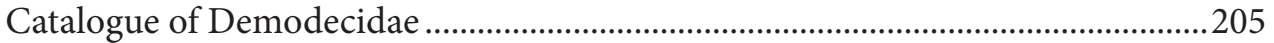

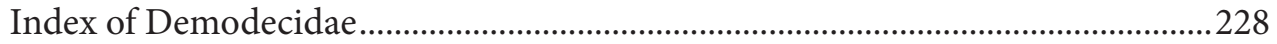

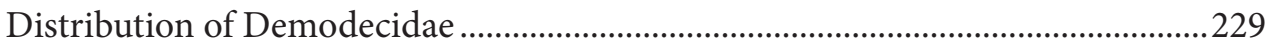

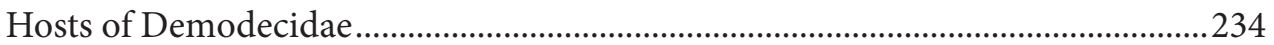

Bibliography of Demodecidae ........................................................................238

The Mite Family Harpirhynchidae Dubinin Nicolet .........................................................267

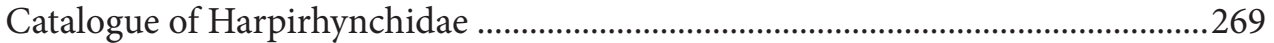

Index of Harpirhynchinae and Harpypalpinae.........................................................296

Distribution of Harpirhynchinae and Harpypalpinae ..............................................299

Hosts of Harpirhynchinae and Harpypalpinae...........................................................302

Bibliography of Harpirhynchinae and Harpypalpinae ..................................................309

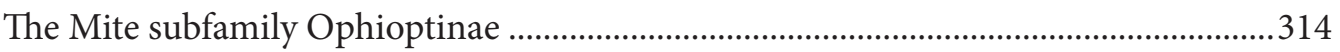

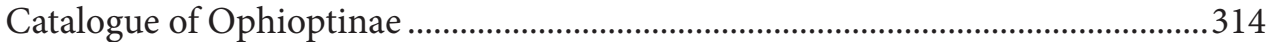




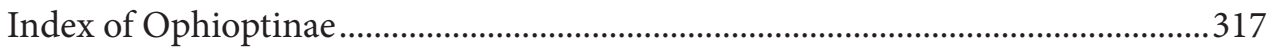

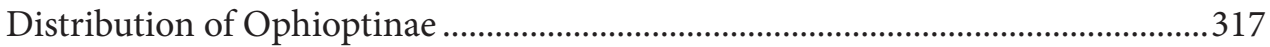

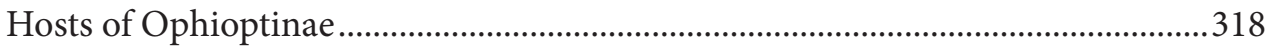

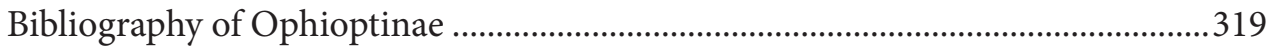

The Mite Family Syringophilidae Lavoipierre .............................................................320

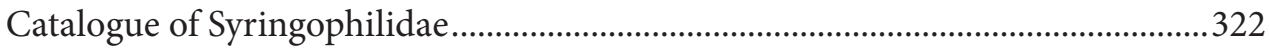

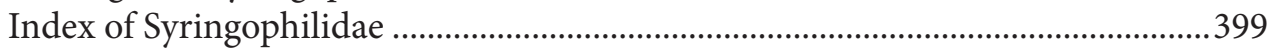

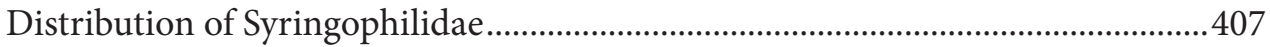

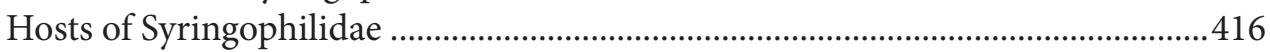

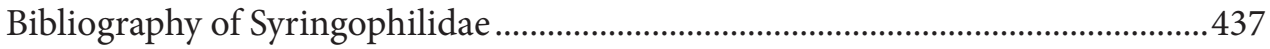

Superfamily Cloacaroidea Bochkov et OConnor........................................................454

The Mite Family Cloacaridae ....................................................................................45

Composition of the family Cloacaridae:...................................................................455

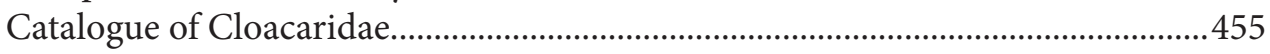

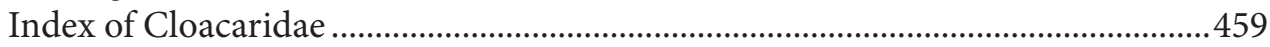

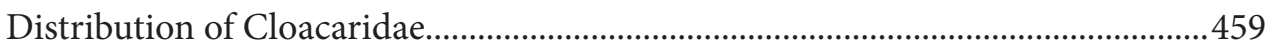

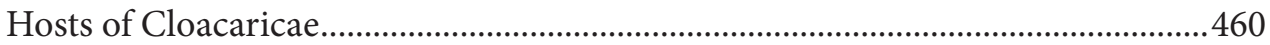

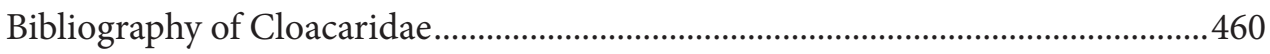

The Mite Family Epimyodicidae......................................................................................462

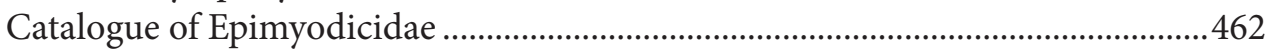

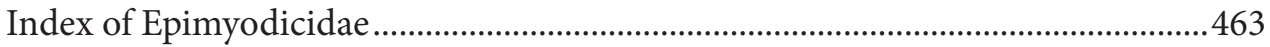

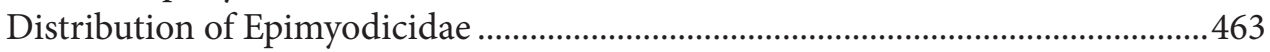

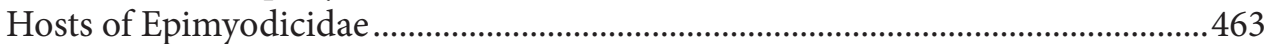

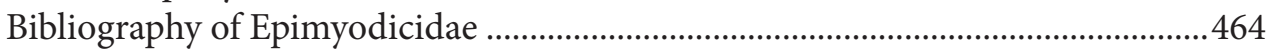

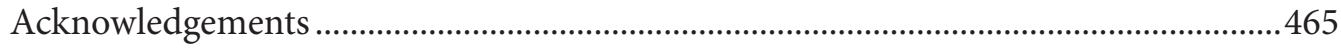




\section{Introduction}

The year 1955 was remarkable in the history of Acarology. Several attempts of classification of various groups have been published almost simultaneously: of the Acaridiae (C.E. Yunker), of the Trombidiform mites (F. Cunliffe) and of Suborder Mesostigmata (J. H. Camin and F.E. Gorirossi). In the same year Dubinin and al. revised many groups of parasites of rodents, Zumpt \& Till wrote their paper on Southafrican mites and many other acarological initiatives took place. Nevertheless, 70 years later the taxonomy of the Acari is still subject of many changes, due to deeper approach and to the new taxa, described from all over the world. From Cheyletidae have been detached several new families, some have been later downgraded to subfamily level (Ophioptinae) and some peculiar new taxa have been described (Cloacaridae, Epimyodicidae and the new superfamily Cloacaroidea). Here the new acquisations are followed with the conviction of the present author that the jugement of the new generation, based on cladistics and DNA analysis, is well founded.

Recently Walter, Lindquist, Smith, Cook \& Krantz (2009) presented a new treatment of what was considered order Acariformes. Formerly divided into three suborders: Prostigmata, Acaridida and Oribatida, in the new system only two entities remain (Trombidiformes and Sarcoptiformes), reminiscent of some very old systems. These entities are ranked as orders, and the Trombidiformes are subdivided into two suborders. Sphaerolichida contains only the superfamilies Lordalycoidea and Sphaerolichoidea, Prostigmata (retaining the rank of suborder) - the other 36 superfamilies. The superfamilies considered in this volume (Cheyletoidea and the newly created superfamily Cloacaroidea Bochkov et OConnor, 2008) share the cohort Raphignathina with Raphignathoidea and Tetranychoidea.

The present volume contains data on 1243 species from 177 genera and seven families. The work was strongly facilitated by the revisions and checklists of different families, written by prominent specialists: Cheyletoidea (Dubinin, 1958, Bochkov, 2002), Cheyletidae (Volgin, 1969, Summers \& Price, 1970, Fain, 1972, Gerson, Fain \& Smiley, 1999, Bochkov \& Fain, 2001), Psorergatidae (Giesen, 1990), Demodecidae (Izdebska \& Rolbiecki, 2020), Harpirhynchidae (Fritsch, 1954; Bochkov \& Literák, 2006; Fain, 1964; Bochkov, Mironov \& Fain, 1999; Bochkov, OConnor \& Klompen, 2015), Syringophilidae (Kethley, 1970; Skoracki, 2011; Skoracki, Zabludovskaya \& Bochkov, 2012; Glowska, Chrzanowski \& Kaszewska, 2015; Zmudzinski \& Skoracki, 2016), and Cloacaridae (Fain, 1968). 


\section{The Mite Superfamily Cheyletoidea}

The superfamily Cheyletoidea is composed of five families. Part of Cheyletidae and all members of Psorergatidae, Demodecidae, Harpirhynchidae and Syringophilidae are obligate parasites, mostly on vertebrates.

Here is the evolution of the concept of Cheyletoidea in the systems of various authors (after Bochkov, 2002, completed)

Cunliffe, 1955 - Cheyletoidea as a new superfamily of Cheyletidae, Myobiidae, Demodecidae, and Heterocheylidae.

Dubinin, 1957 - Cheyletidae, Syringophilidae, Myobiidae, Harpirhynchidae (incl. Ophioptinae)

Baker et al., 1958 - Cheyletidae, Syringophilidae, Myobiidae, Harpirhynchidae, Ophioptidae, Demodecidae, Psorergatidae, Heterocheylidae

Lawrence, 1959 - Cheyletidae, Syringophilidae, Myobiidae, Harpirhynchidae (incl. Psorergates and Ophioptes)

Volgin, 1969 - Cheyletidae, Syringophilidae, Harpirhynchidae (incl. Psorergates), Ophioptidae, Cloacaridae. Myobiidae is raised in a superfamily Myobioidea

Kethley, 1970 - Cheyletidae, Syringophilidae, Myobiidae, Harpirhynchidae, Demodecidae, Psorergatidae, Ophioptidae, Cloacaridae

Smiley, 1970 - Cheyletiellinae becomes a family (Cheyletiellidae)

Fain et al., 1997 - Cheyletiellinae again downgraded to subfamily rank

Bochkov, 1999 - Ophioptidae is downgraded to subfamily of Harpirhynchidae

Bochkov, 2002 - Complete review of the system and phylogeny of Cheyletoidea. Epimyodicinae is raised to family status. Seven families: Cheyletidae, Harpirhynchidae, Syringophilidae, Psorergatidae, Demodicidae, Cloacaridae, Epimyodicidae

Bochkov \& OConnor, 2008 - Epimyodicidae and Cloacaridae form the new superfamily Cloacaroidea Walter et al. (in Krantz \& Walter, 2009) - seven families of Cheyletoidea and two families in Cloacaroidea. This system is followed in the present Catalogue. 


\section{System accepted in this book}

Superfamily Cheyletoidea Cunliffe, 1955

Fam. Cheyletidae Leach, 1815

Chelonotinae Volgin, 1969

Cheyletinae Leach, 1815

Cheyletiellinae Volgin, 1961

Criokerontinae Smiley, 1978

Metacheyletinae Fain, 1972

Ornithocheyletiinae Smiley, 1978

Fam. Psorergatidae Dubinin, 1955

Fam. Harpirhynchidae Dubinin, 1957

Harpirhynchinae Dubinin, 1957

Harpipalpinae Fain, 1972

Ophioptinae Southcott, 1956

Fam. Demodecidae Nicolet, 1855

Fam. Syringophilidae Lavoipierre, 1953

Picobiinae Johnston et Kethley, 1973

Syringophilinae Lavoipierre, 1953

Superfamily Cloacaroidea Bochkov et O'Connor, 2008

Fam. Cloacaridae Camin, Moss, Oliver et Singer, 1967

Cloacarinae Camin, Moss, Oliver et Singer, 1967

Pneumophaginae Fain et Smiley, 1989

Fam. Epimyodicidae Fain, Lukoschus et Rosmalen, 1982 


\title{
The Mite Family Cheyletidae Leach
}

\author{
Cheyletidae Leach, 1815: 399
}

Large family (acc. to Bochkov, 2009b, 380 sp. of 72 genera; acc. to Xia, Klompen \& Childers, 2011, 370 sp. of 73 genera; acc. to the list of Zhang et al., 2011, 75 genera and $438 \mathrm{sp}$.). Contains both predators and permanent parasites of vertebrates. Some are nest or house-dust inhabitants, others live on plants or in insect colonies (phoretic), or are found (Hoffmannita) on Crustacea (Decapoda) and Arachnida (Scorpiones). There are Cheyletids (Cheyletiella parasitivorax, Ch. yasguri) living on domestic animals (rabbits, cats, dogs) and recorded as vectors of mange on Man. According to Bochkov (2004), 280 species of 54 genera $(77.8 \%)$ are predators, 80 sp. of 19 gen. (23.2\%) are parasites of birds and mammals.

The first Cheyletid was described by Schrank (1781, the well known "Acarus" eruditus, type of the genus Cheyletus Latreille, 1796). The family Cheyletidae was described by Leach (1815). Among the many publications until 1969 should be mentionned the revision (and many other papers) of Oudemans (1906), the paper of Baker (1949) on North American Cheyletidae and the analysis of Dubinin (1957). Other acarologists having contributed to the taxonomy of Cheyletidae before 1969 are Heller, Mégnin, Berlese, Michael, Canestrini, Trouessart, Ewing, Zachvatkin, Rohdendorf, Womersley, Cooreman, Ehara, Elbadry, Hara, Sasa, Muma, De Leon, Samšinak, Gerson, Flechtmann, Domrow, Radford, Pelaez, Lawrence, Smiley, Yunker and others.

By the end of the 60es almost simultaneously appeared two world revisions of Cheyletidae. Most remarkable is the monograph of Volgin (1969 in Russian, translated into English in 1987). In his book (and in some earlier publications since 1949) the prominent Russian specialist described 21 valid new genera and restructured the entire system of the family, with new tribes and species groups. Volgin divided these mites in two subfamilies, Cheyletinae and Cheyletiellinae (the parasites of vertebrates). This last subfamily was raised in family rank in the review of Smiley (1970), but was again downgraded in subfamily by Fain et al., 1997). His tribe Chelonotini was raised in subfamily. The other revision (Summers \& Price, 1970) indicates that by this time the Cheyletidae is composed by "close to" 50 genera and 186 species. By now the number of the species is more than double, what is largely the result of the quoted revisions. In the last 50 years many papers appeared on the Cheyletidae of the Philippines (Corpuz-Raros), China-Taiwan (Tseng), Israel and Australia (Gerson), Iran (Saboori, etc.), Pakistan (many authors). Smiley (1978) described the subfamilies Criokerontinae and Ornithocheyletiinae. Other workers who contributed to the study of the taxonomy of Cheyletidae are Thewke, Enns, Klompen, Barilo, Klimov, Mironov, OConnor, Chaudhri, Delfinado, Doğan, Goff, Guilhon, Gupta, Haitlinger, Hassan, Hu, Ide, Kethley, Khaustov, Jeffrey, Kamran, Koç, Ayıldız, Kuznetzov, 
Lin, Lucza, Mathur, Meyer, Nagar, Olivier, Patxot, Putatunda, Kapil, Qayyum, Ramaraju, Rasool, Shiba, Soliman, Tjying, Uchikawa, Vaivanijkul, Wafa, Xia, Yousef, Zaher and others.

A. Fain started publishing papers on Cheyletidae in 1972. Alone or together with Bochkov, Gerson, Lukoschus, Smiley, Nadchatram and other co-authors, he described the subfamily Metacheyletinae, 7 new genera and many new species, mainly parasitic. In the comprehensive papers of Fain, Smiley \& Gerson (1997) and Gerson, Fain \& Smiley (1999) the number of accepted species in the family was stated as "more than 400 " and the genera as "about 75". Fain et al. (1999) compiled a list of all taxa in Cheyletidae. Bochkov \& Fain (1998) left Cheyletidae with 73 valid genera and two genera incertae sedes. Followed the descriptions of Kenyacheylus Fain et Bochkov, 2001, Granulocheyletus Fain et Bochkov, 2002, Picocheyletus Bochkov et OConnor, 2003, Aztecocheyletus Bochkov et Klimov, 2004, Oconnoricheylus Bochkov et Otto, 2010 and Lanceacheyla Xia, Klompen et Childers, 2011.

In this volume the family is considered as composed of six subfamilies, the largest being the Cheyletinae (375 sp.).

\section{Composition of fam. Cheyletidae}

Family Cheyletidae Leach, 1815 - 80 gen., ca. 474 sp. (part of them sp. dubia or inquirendae)[acc. to Zhang et al., 2011 - 438 sp.]

Subfamily Chelonotinae Volgin, 1969 - 1 gen., 1 sp.

Chelonotus Berlese, 1893 - 1 sp.

Subfamily Cheyletiellinae Volgin, 1961 - 4 gen., 33 sp.

Bothrocheyla Volgin, 1964 - 2 sp.

Cheyletiella Canestrini, 1886 - 6 sp.

Eucheyletiella Volgin, 1960 - 7 sp.

Neocheyletiella Baker, 1949 - 18 sp.

Subfamily Cheyletinae Leach, 1815 - 64 gen., 375 sp.

Tribus Cheyletini Leach, 1815 - 23 gen., 189 sp.

Anthribicheyla Thewke, $1980-1 \mathrm{sp}$.

Aztecocheyletus Bochkov et Klimov, 2004 - 1 sp.

Camincheyletus Smiley et Whitaker, $1981-1 \mathrm{sp}$.

Cheletacarus Volgin, 1961 - 5 sp.

Cheletonella Womersley, $1941-7 \mathrm{sp}$.

Cheletomimus Oudemans, 1904 - 68 sp. (many inquir.)

Cheletomimus Oudemans - 19 sp. (incl. 15 inquir.)

Hemicheyletia Volgin, 1969 - $45 \mathrm{sp}$.

Philippicheyla Corpuz-Raros, 1972 - 4 sp.

Cheletophanes Oudemans, $1904-1$ sp. +1 inc. sed.

Cheletophyes Oudemans, $1914-13$ sp. +7 inc. sed. 
Cheyletus Latreille, $1796-48$ sp. ( $35+13$ inc. sed.)

Eucheyletia Baker, 1949 - 14 sp. +1 inc. sed.

Granulocheyletus Fain et Bochkov, $2002-3$ sp.

Kenyacheylus Fain et Bochkov, $2001-1 \mathrm{sp}$.

Ker Muma, 1964 - 7 sp.

Laeliocheyletia Summers et Price, 1970 - 1 sp.

Lanceacheyla Xia, Klompen et Childers, $2011-2$ sp.

Lepidocheyla Volgin, 1963 - 2 sp.

Nidocheyletus Bochkov et O'Connor, 2010 - 1 sp.

Nodele Muma, 1964 - 5 sp.

Paracheyletia Volgin, 1955 - 1 sp.

Paracheyletiella Kuznetzov, 1977 - 1 sp.

Pavlovskycheyla Volgin, 1965 - 3 sp.

Tutacheyla Corpuz - Raros, 1972 - 1 sp.

Zachvatkiniola Volgin, 1969 - 1 sp.

Tribus Acaropsellini Volgin, 1969 - 7 gen., 47 sp.

Acaropsella Volgin, 1969 - 7 sp.

Acaropsellina Summers, $1976-15 \mathrm{sp}$.

Atarsacheylus Thewke, $1980-1 \mathrm{sp}$.

Chelacheles Baker, 1958 - 19 sp.

Neoacaropsis Volgin, 1962 - 1 sp.

Neochelacheles Smiley et Williams, $1972-3$ sp.

Paracaropsis Volgin, 1969 - 1 sp.

Tribus Bakini Volgin, 1969 - 34 gen., 161 sp.

Aegyptocheyla Yousef, 1979 - 1 sp.

Alliea Yunker, 1960 - $2 \mathrm{sp.}$

Bak Yunker, 1961 - 14 sp.

Caudacheles Gerson, 1968 - 3 sp.

Chelacaropsis Baker, $1949-7 \mathrm{sp}$.

Cheletogenes Oudemans, 1905 - 12 sp.

Cheletoides Oudemans, $1904-2 \mathrm{sp}$.

Cheletomorpha Oudemans, $1904-7$ sp.

Cheletopsis Oudemans, $1904-14$ sp.

Cheletosoma Oudemans, 1905 - 1 sp.

Cheyletia Heller, 1884 - 5 sp.

Chiapacheylus De Leon, 1962 - 3 sp.

Columbicheyla Thewke et Enns, 1972 - 3 sp.

Cunliffella Volgin, 1969 - 8 sp.

Dubininiola Volgin, 1969 - 3 sp.

Eucheletopsis Volgin, 1969 - 1 sp.

Eutogenes Baker, 1949 - 14 sp. 
Grallacheles De Leon, 1962 - 3 sp.

Hoffmannita Pelaez, 1962 - 4 sp.

Hylopecheyla Fain, 1972 - 2 sp.

Hypopicheyla Volgin, $1969-2$ sp.

Metacheletoides Fain, $1972-4 \mathrm{sp}$.

Mexecheles De Leon, 1962 - 10 sp.

Microcheyla Volgin, 1966 - 5 sp.

Muricheyla Fain, 1972 - 1 sp.

Neoeucheyla Radford, 1950 - 6 sp.

Oconnoricheylus Bochkov et Otto, $2010-2$ sp.

Oudemansicheyla Volgin, 1969 - 2 sp.

Paramicrocheyla Olivier et Theron, $1989-2$ sp.

Picocheyletus Bochkov et OConnor, 2003 - 1 sp.

Promuricheyla Fain, 1979 - 1 sp.

Prosocheyla Volgin, 1969 - 9 sp.

Samsinakia Volgin, 1965 - $8 \mathrm{sp}$.

Thewkacheyla Ide et Kethley, 1977 - 1 sp.

Subfamily Criokerontinae Smiley, 1978 - 4 gen., 12 sp.

Tribus Niheliini Smiley, $1978-4$ gen., 12 sp.

Criokeron Volgin, 1966 - 2 sp.

Galagocheles Fain, 1979 - 2 sp.

Nihelia Domrow et Baker, 1960 - 3 sp.

Sciurocheyla Volgin, $1969-5$ sp.

Subfamily Metacheyletinae Fain, $1980-1$ genus, 5 sp.

Metacheyletia Fain, 1972 - 5 sp.

Subfamily Ornithocheyletiinae Smiley, $1978-6$ gen., 42 sp.

Tribus Ornithocheyletiini Volgin, $1969-5$ gen., 40 sp.

Apodicheles Fain, 1979 - 4 sp.

Bakericheyla Volgin, 1966 - $5 \mathrm{sp}$.

Apodicheyla Fain, 1979 - 1 sp.

Bakericheyla Volgin, 1966 - 4 sp.

Ornithocheyletia Volgin, $1964-31$ sp.

Tribus Teinocheylini Fain, $1974-1$ genus, 2 sp.

Teinocheylus Fain, $1974-2 \mathrm{sp}$. 


\section{Catalogue of the Family Cheyletidae}

Family Cheyletidae Leach

Cheyletidae Leach, 1815: 399 (Kramer, 1877: 245)

(= Cheyletides Leach, 1815)

Type genus: Cheyletus Latreille, 1796

Subfamily Chelonotinae Volgin

Chelonotini Volgin, 1969: 329

Chelonotinae: Fain, 1979: 408

Type genus: Chelonotus Berlese, 1893

Genus Chelonotus Berlese

Chelonotus Berlese, 1893: 73

Type species: Chelonotus selenorhynchus Berlese, 1893

Chelonotus selenorhynchus Trouessart in Berlese

Chelonotus selenorhynchus Trouessart in Berlese, 1893: 9, 15, 77; Oudemans, 1906: 159;

Baker, 1949: 312; Domrow, 1960: 457 (selenirhynus); 1964: 16; Nadchatram \& Domrow, 1966: 135; Volgin, 1969: 330; Summers \& Price, 1970: 72

Chelonotus oudemansi Baker, 1949: 310 - type: Sulawesi, on Callosciurus tenuis

Chelonotus ewingi Baker, 1949: 311 - type: Sulawesi, Temboan, on Callosciurus prevosti

Type locality: Pulosembeh, Sulawesi (Indonesia)

Distribution: Indonesia (Sulawesi), Malaysia (Malaya, Borneo, Pulau Tioman)

Hosts: Tupaia glis (Scandentia: Tupaiidae), Sundasciurus hippurus, S. [Baginia] lowii, S. [Baginia] tenuis evidens, Lariscus insignis (Rodentia: Sciuridae)

Subfamily Cheyletiellinae Volgin

Cheyletiellinae Volgin, 1961: 255

Type genus: Cheyletiella Canestrini, 1886

Genus Bothrocheyla Volgin

Bothrocheyla Volgin, 1964: 94

Type species: Neoeucheyla (Bothrocheyla) pavlovskyi Volgin, 1964

\section{Bothrocheyla pavlovskyi (Volgin)}

Neoeucheyla (Bothrocheyla) pavlovskyi Volgin, 1964: 94

Neoeucheyla pavlovskyi Volgin: Vysotskaja, 1977: 188

Bothrocheyla pavlovskyi: Volgin, 1969: 262

Neoeucheyla beeri Thewke \& Enns, 1972: 455 
Type locality of Neoeucheyla (Bothrocheyla) pavlovskyi: Murmanskaya and Zakarpatskaya oblasti (Russia, Ukraine)

Distribution: Czech Rep., Russia, Ukraine

Hosts: nests of rodents; on Heteroptera (Aradus lugubris, A. corticalis)

Bothrocheyla typhosa (Summers et Price)

Neoeucheyla typhosa Summers \& Price, 1970: 67

Bothrocheyla typhosa (Summers et Price)

Type locality: Lake Pillsbury, Napa Co., California

Distribution: USA (California)

Host: from soil under pine trees

Genus Cheyletiella Canestrini

Cheyletiella Canestrini, 1886: 170

Type species: Cheyletus parasitivorax Mégnin, 1878

Bicheyletiella Fain, 1972: 48 (synonymized by Fain \& Bochkov, 2001b: 293)

Type species: Bicheyletiella romerolagi Fain, 1972

\section{Cheyletiella blakei Smiley}

Cheyletiella blakei Smiley, 1970: 1072; Keh, 1975: 1; van Bronswijk \& de Kreek, 1976: 315;

Mehl, 1978; Domrow, 1991: 1305; Fain \& Bochkov, 2001c: 90

Type locality: Ithaca, N.Y.

Distribution: Australia (N.S.W., Victoria, Tasmania), France, Norway, Russia, USA (N.Y.)

Hosts: Felis catus (Carnivora: Felidae), Homo sapiens (Primates: Hominidae)

\section{Cheyletiella dengi $\mathrm{Hu}$}

Cheyletiella dengi $\mathrm{Hu}, 1992: 183$

Type locality: Xinxiang (China)

Distribution: China

\section{Cheyletiella parasitivorax (Mégnin)}

Cheyletus parasitivorax Mégnin, 1878: 10; Canestrini, 1879: 40; Mégnin, 1880: 241;

Cheyletiella parasitivorax (Mégnin): Canestrini, 1886: 175; Berlese, 1886: 28; Cooper, 1946: 480; Baker, 1949: 270; Starkoff \& Starkoff, 1950: 96; Barr, 1955: 323; Volgin, 1960: 242; 1969: 360; Alcaino \& Tagle, 1966; Smiley, 1970: 1075; Amaral, 1971: 106; Rak, 1972: 62; 1973: 189; Shepherd \& Edmonds, 1977; Mehl, 1978; Wassel, Whitaker \& Spicka, 1980: 419; Hoffmann et al., 1994: 210; Fain \& Bochkov, 2001: 89; Whitaker et al., 2007: 6; Xia, 2010: 161

[Cheyletiella americana Ewing, 1909b: 77. - Type: Urbana. Illinois, under bark (acc. to Baker, 1949, belongs to Pseudocheylus)] 
Cheletiella parasitivorax: Oudemans, 1906: 212; Hirst, 1917: 132; Womersley, 1941: 59 Ewingella americana Vail \& Augustson, 1943: 419 - Type from Oryctolagus cuniculus, California, USA

Cheyletiella furmani Smiley, 1970: 1074 - Jacksonville, Fla., USA, from Sylvilagus palustris paludicola (Lagomorpha: Leporidae); synonymized by Fain \& Bochkov, 2001c: 88

Cheyletiella katangae Fain, 1972: 48 - Katanga, DR Congo, from Lepus whytei (Lagomorpha: Leporidae)

Cheyletiella dengi Hu \& Hou, 1992: 183 - Type: China, Xinjiang, from Oryctolagus cuniculus (Lagomorpha: Leporidae); synonymized by Fain \& Bochkov, 2001c: 88

Type locality of Cheyletus parasitivorax Mégnin: Probably France

Distribution: Australia, Brazil, Chile, China, Cuba, Danemark, England, France, Greece, Holland, Iran, Italy, Mexico, Nigeria, Norway, Russia, South Africa, New Zealand, USA Hosts: Homo sapiens (Primates: Hominidae), Lepus whytei, Oryctolagus cuniculus, Pronolagus randensis, Romerolagus diazi, Sylvilagus bachmanni, S. floridanus (Lagomorpha: Leporidae), Felis catus (Carnivora: Felidae)

\section{Cheyletiella romerolagi (Fain)}

Bicheyletiella romerolagi Fain, 1972: 49; 1979: 422

Cheyletiella mexicana Uchikawa \& Suzuki, 1979: 21; Hofmann et al., 1994: 211

Cheyletiella romerolagi (Fain): Fain \& Bochkov, 2001b: 293; 2001c: 89

Type locality: Mexico

Distribution: Mexico

Host: Romerolagus diazi (Lagomorpha: Leporidae)

\section{Cheyletiella strandtmanni Smiley}

Cheyletiella strandtmanni Smiley, 1970: 1072; Fain \& Bochkov, 2001c: 89

Type locality: Ma-am Lio

Distribution: Taiwan

Host: Lepus sp. (Lagomorpha: Leporidae)

\section{Cheyletiella yasguri Smiley}

Cheyletiella yasguri Smiley, 1965: 76; Vercammen-Grandjean \& Rak, 1968: 407; Volgin, 1969: 365; Davies \& Kyle, 1969: 136; Bakkers \& Fain, 1972: 25; Rak, 1973: 181; Alcaino \& Calderon, 1976: 40; Mehl, 1978; Fain, Scheepers \& de Groot, 1982: 624; Chin, 1996: 267; Fain \& Bochkov, 2001c: 90

Type locality: New York

Distribution: Chile, England, France, Germany, Holland, Iran, Ireland, Korea, Norway, USA

Host: Canis lupus familiaris (Carnivora: Canidae); phoretic hyperparasite of a louse-fly (Hippobosca longipennis), collected on dog in Iran 
Genus Eucheyletiella Volgin

Eucheyletiella Volgin, 1960: 238

Type species: Cheyletiella ochotonae Volgin, 1960

Eucheyletiella daurica Bochkov et Mironov

Eucheyletiella faini Bochkov \& Mironov, 1999: 148

Type locality: Gorno-Altaysk Province, Chuyskaya steppe, Tashanta Village

Distribution: Kazakhstan, Russia (Tuva, Altai)

Host: Ochotona rufescens (Lagomorpha: Ochotonidae)

Eucheyletiella faini Bochkov et Malikov

Eucheyletiella faini Bochkov \& Malikov, 1997: 43; Bochkov \& Mironov, 1999: 144

Type locality: Kerman Province, near to Shad-Dad Tunnel

Distribution: Iran

Host: Ochotona rufescens (Lagomorpha: Ochotonidae)

Eucheyletiella johnstoni (Smiley)

Cheyletiella johnstoni Smiley, 1965: 77

Eucheyletiella johnstoni (Smiley): Volgin, 1969: 374

Type locality: New Mexico (Santa Fe)

Distribution: USA (New Mexico)

Host: Ochotona princeps (Lagomorpha: Ochotonidae)

\section{Eucheyletiella ochotonae (Volgin)}

Cheyletiella ochotonae Volgin, 1960: 238

Eucheyletiella ochotonae Volgin, 1969: 368; Dubinina \& Bochkov, 1989: 107; Bochkov \& Mironov, 1999: 142

Type locality: Terskey Alatau (Chong Kizilsu), Kirghyzstan

Distribution: Kirghyzstan, Turkmenia

Hosts: Ochotona macrotis, O. roylei, O. rufescens (Lagomorpha: Ochotonidae), Sylvaemus sylvaticus (Rodentia: Muridae)

\section{Eucheyletiella pallasius Bochkov et Mironov}

Eucheyletiella pallasius Bochkov \& Mironov, 1999: 146

Type locality: Tuva, Mugir-Aken

Distribution: Russia (Tuva)

Host: Ochotona pallasi (Lagomorpha: Ochotonidae)

Eucheyletiella pusillinus Bochkov et Mironov

Eucheyletiella pusillinus Bochkov \& Mironov, 1999: 144

Type locality: Orenburg Prov. 
Distribution: Russia

Host: Ochotona pusilla (Lagomorpha: Ochotonidae)

Eucheyletiella takahasii (Sasa et Kono)

Cheyletiella takahashii Sasa \& Kono, 1951: 205

Eucheyletiella takahashii (Sasa et Kono): Volgin, 1969: 372

Eucheyletiella takahasii (Sasa et Kono): Bochkov \& Mironov, 1999: 150

Type locality: Hokkaido

Distribution: Japan (Hokkaido), Russia (Asiatic)

Host: Ochotona hyperborea, O. h. yesoensis (Lagomorpha: Ochotonidae)

Genus Neocheyletiella Baker

Neocheyletiella Baker, 1949: 271

Ornithocheyla Lawrence, 1959: 433

Type species: Neocheyletiella rohwery Baker, 1949

Neocheyletiella aegithali Bochkov et OConnor

Neocheyletiella aegithali Bochkov \& OConnor, 2003: 277; Halliday, Kamran \& Bashir, 2018: 87

Type locality: NW Frontier Prov., Malakand Div., Swat Dist., Yakh Tangai, 2076 m

Distribution: Pakistan

Host: Aegithalos concinnus (Passeriformes: Aegithalidae)

Neocheyletiella alfortensis Guilhon et Euzeby

Neocheyletiella alfortensis Guilhon \& Euzeby, 1984: 497

Type locality: unknown

Distribution: unknown

Host: Leiothrix lutea (Passeriformes: Timaliidae)

Neocheyletiella amandavae Fain

Neocheyletiella amandavae Fain, 1972: 47; 1980: 33

Type locality: Antwerp Zoo

Distribution: Java

Host: Amandava amandava (Passeriformes: Estrildidae)

Neocheyletiella ardeola Bochkov et Ochoa

Neocheyletiella ardeola Bochkov \& Ochoa, 2005: 142

Type locality: Lake Fuhwa

Distribution: Mozambique

Host: Ardeolla ralloides (Pelecaniformes: Ardeidae) 


\section{Neocheyletiella artami Domrow}

Neocheyletiella artami Domrow, 1965: 210; 1970: 273; Summers \& Price, 1970: 77; Smiley,

1978: 236

Type locality: Exeter (Tasmania)

Distribution: Australia (Tasmania, New South Wales)

Host: Artamus cyanopterus (Passeriformes: Artamidae)

\section{Neocheyletiella athene Fain et Bochkov}

Neocheyletiella athene Fain \& Bochkov, 2002a: 159

Type locality: India

Distribution: India

Host: Athene brama (Strigiformes: Strigidae)

\section{Neocheyletiella avicola Fain}

Neocheyletiella avicola Fain, 1972: 47; 1980: 33

Neocheyletiella vestergaardi Smiley, 1978: 236 - Copenhagen, Denmark, caged Erythrura prasina (Passeriformes: Estrildidae)

Type locality: Antwerp Zoo

Distribution: ? (South America, Australia)

Hosts: Ara sp., Agapornis fisheri (Psittaciformes: Psittacidae), Erythrura prasina (Passeriformes: Estrildidae)(N. vestergaardi)

Neocheyletiella lonchurae Fain et Bochkov

Neocheyletiella lonchurae Fain \& Bochkov, 2002a: 159

Type locality: Akanyaru River

Distribution: Rwanda

Host: Lonchura sp. (Passeriformes: Estrildidae)

\section{Neocheyletiella macronycha (Mégnin)}

Cheyletus macronychus Mégnin, 1878: 427; 1880: 243; Poppe, 1888: 237

Cheyletiella macronycha Berlese \& Trouessart, 1889: 136

Cheletiella macronycha: Oudemans, 1906: 212

Neocheyletiella macronycha, Baker, 1949: 272; Fain, 1980: 39; Smiley, 1978: 236

Type locality: Bengal

Distribution: India

Host: birds (Passeriformes)

\section{Neocheyletiella media Fain}

Neocheyletiella media Fain, 1972: 48; 1980: 28

Type locality: host died in Antwerp

Distribution: S. China

Host: Leiothrix lutea (Passeriformes: Timaliidae) 
Neocheyletiella megaphallos (Lawrence)

Ornithocheyla megaphallos Lawrence, 1959: 434; Lindholm, Venter \& Ueckermann, 1998 Neocheyletiella megaphallos (Lawrence): Volgin, 1969: 419; Summers \& Price, 1970: 77

Type locality: Debeete

Distribution: Botswana, South Africa

Hosts: Estrilda erythbronotos (Passeriformes: Estrildidae), Chrysococcyx caprius (Cuculiformes: Cuculidae)

Neocheyletiella microrhyncha (Berlese et Trouessart)

Cheyletiella microrhyncha Berlese \& Trouessart, 1889: 136; Berlese, 1893: 76

Cheletiella microrhyncha: Oudemans, 1906: 212

Neocheyletiella microrhyncha (Berlese \& Trouessart): Baker, 1949: 272; Summers \& Price, 1970: 77; Smiley, 1978: 234; Bochkov \& Galloway, 2001: 2017

Neocheyletiella oudemansi Volgin, 1969: 417 - Belarus, from Delichon urbica and Riparia riparia

Neocheyletiella callawaye Smiley, 1970: 1063; 1978: 236

Type locality: France

Distribution: Belarus, Canada, France, Russia; Rwanda; USA

Hosts: Hirundo rustica, Delichon urbica, Petrochelidon pyrchonota, Cecropis abyssinicus unitatis, Progne subis, Psalidoprocne albiceps, Riparia riparia (Passeriformes: Hirundinidae)

Neocheyletiella nectarinia Bochkov et OConnor

Neocheyletiella nectarinia Bochkov \& OConnor, 2003: 277

Type locality: Romblon Prov., Sibuyan Isl., Magdivang, Tampayan

Distribution: Philippines

Host: Nectarinia jugularis (Passeriformes: Nectariniidae)

Neocheyletiella pittae Fain

Neocheyletiella pittae Fain, 1972: 47; 1980: 33

Type locality: Antwerp Zoo

Distribution: ?

Host: Pitta megarhyncha (Passeriformes: Pittidae)

Neocheyletiella queleae Fain et Bochkov

Neocheyletiella queleae Fain \& Bochkov, 2002 : 155

Type locality: Rubona, Rwanda

Distribution: Rwanda

Hosts: Quelea quelea (Passeriformes: Ploceidae), Streptopelia semitorquata (Columbiformes: Columbidae) 


\section{Neocheyletiella rohweri Baker}

Neocheyletiella rohweri Baker, 1949: 274; Volgin, 1969: 414; Summers \& Price, 1970: 77;

Smiley, 1978: 236

Type locality: Guffey, Colorado

Distribution: USA (Colorado)

Host: Sitta pygmaea melanotus (Passeriformes: Sittidae)

\section{Neocheyletiella siva Fain}

Neocheyletiella siva Fain, 1972: 48; 1980: 37

Type locality: Antwerp Zoo

Distribution: India

Host: Siva [Minia] cyanoptera (Passeriformes: Timaliidae)

Neocheyletiella smallwoodae Baker

Neocheyletiella smallwoodae Baker, 1949: 273; Volgin, 1969: 415; Summers \& Price, 1970:

77; Smiley, 1978: 236

Type locality: Guffei, Colorado

Distribution: USA (Colorado, Delawar)

Host: Leucosticte australis (Passeriformes: Fringillidae, Carduellinae)

\section{Neocheyletiella sp.}

Distribution: Japan, Korea (McClure H. Eliott, N. Ratanaworadhan et al. 1973)

Host: Emberiza cioides (Passeriformes: Emberizidae)

Subfamily Cheyletinae Leach

Cheyletinae Leach, 1815: 399

Type genus: Cheyletus Latreille, 1796

\section{Tribus Cheyletini Leach}

Cheyletini Leach, 1815: 399

Type genus: Cheyletus Latreille, 1796

Genus Anthribicheyla Thewke

Anthribicheyla Thewke, 1980: 335

Type species: Anthribicheyla bocki Thewke, 1980

Anthribicheyla bocki Thewke

Anthribicheyla bocki Thewke, 1980: 337

Type locality: Vera Cruz

Distribution: Mexico

Hosts: Ischnocerus, Ptychoderes (Coleoptera: Anthribidae) 
Genus Aztecocheyletus Bochkov et Klimov Aztecocheyletus Bochkov \& Klimov, 2004: 24

Type species: Nodele conquistador Bochkov et Klimov, 2004

Aztecocheyletus conquistador (Bochkov et Klimov)

Nodele conquistador Bochkov \& Klimov, 2004: 24

Aztecocheyletus conquistador Bochkov \& Klimov, 2004: 23

Type locality: Jalisco, Chamela, Est. biologica

Distribution: Mexico

Host: Azthecanthidium tenochtitlanicum (Hymenoptera: Megachilidae)

Genus Camincheyletus Smiley et Whitaker

Camincheyletus Smiley \& Whitaker, 1981: 111

Type species: Camincheyletus glaucomys Smiley et Whitaker, 1981

Camincheyletus glaucomys Smiley et Whitaker

Camincheyletus glaucomys Smiley \& Whitaker, 1981: 114

Type locality: Oregon

Distribution: USA (Oregon)

Host: Glaucomys sabrinus (nest)(Rodentia: Pteromyidae)

Genus Cheletacarus Volgin

Cheletacarus Volgin, 1961: 248

Type species: Cheletacarus raptor Volgin, 1961

Cheletacarus gryphus Summers et Price

Cheletacarus gryphus Summers \& Price 1970: 36

Type locality: Davis, California

Distribution: USA (California)

Cheletacarus novemdentis Meyer

Cheletacarus novemdentis Meyer, 1988: 9

Type locality: Mountain Zebra Nat. Park

Distribution: South Africa

Cheletacarus ploceus Gupta et Paul

Cheletacarus ploceus Gupta \& Paul, 1985: 6

Type locality: India

Distribution: India 


\section{Cheletacarus raptor Volgin}

Cheletacarus raptor Volgin, 1961: 248; 1969: 169; Lucza, Ripka \& Saly, 1996; Lin et al., 1997; Ripka, Fain \& Bolland, 1999: 366; Xia, 2010: 161; Bochkov \& Abramov, 2016: 336; Khaustov, 2021: 234

Type locality: Kishinev (Moldova)

Distribution: China, Hungary, Moldavia, Russia

Hosts: Diaspidiothis otsreaeformis, D. spurcatus, Parthenolecanium corni (Homoptera: Coccodea)

\section{Cheletacarus rugosus (Womersley)}

Cheletophanes rugosa Womersley, 1941: 62

Cheletonella rugosa: Baker, 1949: 292

Chelacheles rugosus ( Womersley): Volgin, 1961: 253; 1969: 172

Cheletacarus rugosus ( Womersley): Summers \& Price, 1970: 36

Type locality: Brisbane, Queensland

Distribution: Australia (Queensland)

Host: Calymmaderus sp. (Coleoptera: Anobiidae)

Genus Cheletonella Womersley

Cheletonella Womersley, 1941: 60

Type species: Cheletonella vespertilionis Womersley, 1941

Cheletonella daddariensis Aheer et Chaudhri

Cheletonella daddariensis Aheer \& Chaudhri, 1987: 104; Halliday, Kamran \& Bashir, 2018: 86

Type locality: Pakistan

Distribution: Pakistan

Cheletonella emersus Chaudhri, Akbar et Rasool

Cheletonella emersus Chaudhri, Akbar \& Rasool, 1979: 150; Aheer \& Chaudhri, 1987: 103; Halliday, Kamran \& Bashir, 2018: 86

Type locality: Pakistan

Distribution: Pakistan

\section{Cheletonella hoffmannae Smiley}

Cheletonella hoffmannae Smiley, 1996: 241

Type locality: Quincy, Owen Co., Indiana

Distribution: USA (Indiana)

Host: guano of Eptesicus fuscus (Chiroptera: Vespertilionidae) 
Cheletonella iraniensis Salarzehi, Hajizadeh et Ueckermann

Cheletonella iraniensis Salarzehi, Hajizadeh \& Ueckermann, 2019: 189

Type locality: Khomam City

Distribution: Iran

Cheletonella pilosa Tseng

Cheletonella pilosa Tseng, 1977: 240; Xia, 2010: 161

Type locality: Taiwan

Distribution: Taiwan

Cheletonella vespertilionis Womersley

Cheletonella vespertilionis Womersley, 1941: 61; Baker, 1949: 292; Domrow, 1959: 234; 1974: 18; 1991: 1305; Volgin, 1969: 153; 1987: 191; Summers \& Price, 1970: 37; Whitaker, 1973: 1149; Domrow, 1974: 18; Fain \& Nadchatram, 1980: 197; Fain \& Bochkov, 2001; Ezequiel et al., 2001; Ezequiel et al., 2003; Whitaker et al., 2007: 6; Dogan et al., 2011: 782

Cheletonella caucasica Volgin, 1955: 168; 1956: 210; 1969: 154; Rekk, 1976: 68 Algeria, Armenia, Brazil, Georgia, Tajikistan, Ukraine (Cricetulus migratorius, bird's nest) Type locality: Glen Osmond (S. Australia)

Distribution: Australia (N.S.W., South Australia), Brazil, Malaysia, USA (California, Indiana, Pennsylvania, Texas); Armenia, Georgia, Tajikistan, Iran, Belgium, Ukraine Hosts: bat (Chiroptera indet.), Eptesicus fuscus, guano of Myotis lucifugus, M. myotis (Chiroptera: Vespertilionidae), guano of Tadarida punctata (Molossidae), bat guano in Malaysia (Negeri Sembilan), nest of Hylochelidon ariel (Passeriformes: Hirundinidae)

Cheletonella juglandis Xia, Zhu et Ye (sp. inquir.)

Cheletonella juglandis Xia, Zhu \& Ye, 1999: 150

Type locality: Jiangxi, Nanchang

Distribution: China

Genus Cheletomimus Oudemans

Cheletomimus Oudemans, 1904b: 163

Type species: Cheletes berlesei Oudemans, 1904

Subgenus Cheletomimus Oudemans

Cheletomimus Oudemans, 1904b: 163

Type species: Cheletes berlesei Oudemans, 1904

Cheletomimus (Cheletomimus) berlesei (Oudemans)

Cheyletus ornatus Berlese, 1886: 28 (misidentification)

Cheletes berlesei Oudemans, 1904b: 154 
Cheletomimus ornatus (Berlese): Oudemans, 1906: 136;

Cheletomimus berlesei (Oudemans): Baker, 1949: 293; Volgin, 1955: 172; Yunker, 1961: 1032; De Leon, 1962: 134; Summers \& Price, 1970: 9; Rekk, 1976: 68; Patxot \& Goff, 1985: 160

Cheyletomimus berlesei (Oudemans): Evans, 1954: 806; Bochkov, Hakimitabar \& Saboori, 2005: 107; Torre Santana, 2015

Cheletomimus (Ch.) berlesei (Oudemans): Fain, Bochkov \& Corpuz-Raros, 2002: 30

Type locality: Italy

Distribution: Angola, Cuba, Georgia, Greece (ornatus), Great Britain, Iran, Italy, Israel, Mexico, Palestina, Russia, USA (Florida, Hawaiian Is.)

Host: Aspidiotus lataniae (Homoptera: Coccodea), bird's nest

Cheletomimus (Cheletomimus) daltoniensis Corpuz-Raros

Cheletomimus daltoniensis Corpuz-Raros, 1998: 265

Cheletomimus (Cheletomimus) daltoniensis Corpuz-Raros: Fain, Bochkov \& CorpuzRaros, 2002: 33

Type locality: Luzon, Dalton Pass, Santa Fe, Nueva Vizcaya, 1070 m

Distribution: Philippine Is.

\section{Cheletomimus (Cheletomimus) duosetosus Muma}

Cheletomimus duosetosus Muma, 1964: 242; Summers \& Price, 1970: 9; Patxot \& Goff, 1985: 161; Papaioannou-Souliotis, 1994; Feres \& Flechtmann, 1995: 535

Cheletomimus (Cheletomimus) duosetosus Muma: Fain, Bochkov \& Corpuz-Raros, 2002: 33

Type locality: Florida (USA)

Distribution: Brazil, Greece, USA (Florida, Hawaiian Is.)

Cheletomimus (Cheletomimus) ochoai Fain, Bochkov et Corpuz-Raros

Cheletomimus (Cheletomimus) ochoai Fain, Bochkov \& Corpuz-Raros, 2002: 33

Type locality: California, San Francisco

Distribution: USA (California)

Host: on Howea posteriana (calix)

Subgenus Hemicheyletia Volgin

Hemicheyletia Volgin, 1969: 201

Type species: Paracheyletia bakeri Ehara, 1962

Andrecheyla Volgin, 1969: 219

Type species: Cheyletia scutellata De Leon, 1962

Dendrocheyla Volgin, 1969: 205

Type genus: Dendrocheyla bregetovae Volgin, 1969 


\section{Cheletomimus (Hemicheyletia) anarbora (De Leon)}

Paracheyletia anarbora De Leon, 1967: 341

Hemicheyletia anarbora (De Leon): Summers \& Price, 1970: 18; Corpuz-Raros \& Sotto, 1977: 154; Ehara \& Abdul Ghani, 1988: 239; Torre Santana, 2015: 49

Hemicheyletia pusillifolium Lin-Jianzhen, Pen-Wenfu et Chen-Yume, 1994: 141; Lin et al., 2000: 86 - China (Fujian)

Cheletomimus (Hemicheyletia) anarbora: Corpuz-Raros, 1977: 154; Fain, Bochkov \& Corpuz-Raros, 2002: 49

Cheletomimus anarbora: Xia, 2010: 161

Type locality: Trinidad

Distribution: China, Cuba, Malaysia, Philippine Is., Taiwan, Trinidad

\section{Cheletomimus (Hemicheyletia) asiaticus (Volgin)}

Hemicheyletia asiaticus Volgin, 1978: 213

Cheletomimus (Hemicheyletia) asiatica: Fain, Bochkov \& Corpuz-Raros, 2002: 39

Type locality: Sayat Distr., Turkmenistan

Distribution: Turkmenistan (in a burrow of tarantula)

\section{Cheletomimus (Hemicheyletia) athenae (Corpuz-Raros)}

Hemicheyletia athenae Corpuz-Raros, 1988b: 418

Cheletomimus (Hemicheyletia) athenae: Fain, Bochkov \& Corpuz-Raros, 2002: 54

Type locality: Mt. Banahaw at Kinabuhayan, Dolores,

Quezon, Luzon Is., the Philippines

Distribution: Philippine Is.

\section{Cheletomimus (Hemicheyletia) bakeri (Ehara)}

Paracheyletia bakeri Ehara, 1962: 109; Muma, 1964: 245; Gerson, 1967: 360; Kanavel \& Selhime, 1967: 107

Hemicheyletia bakeri (Ehara): Volgin, 1969: 202; Summers \& Price, 1970: 12; Tseng, 1977; Gerson, 1994: 445; Xia et al., 2000

Hemicheyletia tumidus Qayyum \& Chaudhri, 1979: 169. Syn. by Fain, Bochkov \& CorpuzRaros, 2002: 36

Cheletomimus (Hemicheyletia) bakeri (Ehara): Fain, Bochkov \& Corpuz-Raros, 2002: 36;

Bochkov, Hakimitabar \& Saboori, 2005: 107

Cheletomimus bakeri (Ehara): Xia, 2010: 161; Fain, Bochkov \& Corpuz-Raros, 2002: 36;

Bochkov, Hakimitabar \& Saboori, 2005: 107; Halliday, Kamran \& Bashir, 2018: 83

Type locality: Sapporo (Hokaido)

Distribution: Algeria, Australia, Canada, China, DR Congo, Egypt (Hemicheletus b.), Galapagos Isl., Iran, Israel, Japan, Pakistan, Panama, Philippines, Taiwan, USA 
Cheletomimus (Hemicheyletia) blosis (Chaudhri et Akbar)

Hemicheyletia blosis Chaudhri \& Akbar, 1985: 172.

Cheletomimus blosis (Chaudhri et Akbar): Halliday, Kamran \& Bashir, 2018: 83

Type locality: Pakistan

Distribution: Pakistan

\section{Cheletomimus (Hemicheyletia) bregetovae (Volgin)}

Dendrocheyla bregetovae Volgin, 1969: 207

Hemicheyletia bregetovae (Volgin): Diaz Patxot \& Goff, 1985: 161

Cheletomimus (Hemicheyletia) bregetovae: Fain, Bochkov \& Corpuz-Raros, 2002: 54; Koç \& Ayyildiz, 1996:

Hemicheyletia scitula Corpuz-Raros, 1972: 256. Type from Mt. Makling, the Philippines.

Syn. by Fain, Bochkov \& Corpuz-Raros, 2002: 54

Type locality of Dendrocheyla bregetovae: Hosta, Sochi, (Russia)

Distribution: Armenia, Bulgaria, Philippines, Russia, Turkey, Crimea, USA (Hawaian Is. - Oahu)

Host: nests of Apodemus spp. (Rodentia: Muridae)

\section{Cheletomimus (Hemicheyletia) chui (Tseng)}

Hemicheyletia chui Tseng, 1977: 218

Cheletomimus (Hemicheyletia) chui (Tseng): Fain, Bochkov \& Corpuz-Raros, 2002: 39

Cheletomimus chui (Tseng): Xia, 2010: 161

Type locality: Taiwan

Distribution: China, Malaysia, Taiwan

Host: from nest of Muscicapa grandis (Passeriformes: Muscicapidae)

Cheletomimus (Hemicheyletia) congensis (Cunliffe)

Paracheyletia congensis Cunliffe, 1962: 197

Hemicheyletia congensis (Cunliffe): Volgin, 1969: 203; Summers \& Price, 1970: 1

Hemicheyletia congoensis (Cunliffe): Corpuz-Raros, 1998:

Hemicheyletia lacinia Rasool \& Chaudhri, 1979: 2 - Pakistan

Cheletomimus (Hemicheyletia) congensis (Cunliffe): Fain, Bochkov \& Corpuz-Raros, 2002:

38; Bochkov, Hakimitabar \& Saboori, 2005: 107

Cheletomimus congoensis (Cunliffe): Fain, Bochkov \& Corpuz-Raros, 2002: 38; Bochkov,

Hakimitabar \& Saboori, 2005: 107; Halliday, Kamran \& Bashir, 2018: 84

Type locality of Paracheyletia congensis Cunliffe, 1962: Kysenyi (DR Congo)

Distribution: DR Congo, Iran, Pakistan, Philippines

Cheletomimus (Hemicheyletia) cordovensis (De Leon)

Cheyletia cordovensis De Leon, 1962: 219

Hemicheyletia cordovensis (De Leon): Volgin, 1969: 204; Summers \& Price, 1970: 14 
Cheletomimus (Hemicheyletia) cordovensis (De Leon): Fain, Bochkov \& Corpuz-Raros, 2002: 39

Type locality: Cordoba

Distribution: Mexico (Veracruz)

Host: Phoebe psychotrioides (Passeriformes: Tyrannidae)

Cheletomimus (Hemicheyletia) darwinius (Summers et Price)

Hemicheyletia darwinia Summers \& Price, 1970: 16

Cheletomimus (Hemicheyletia) darwinia (Summers et Price): Fain, Bochkov \& CorpuzRaros, 2002: 49

Type locality: Darwin Research Station, Galapagos

Distribution: Ecuador (Galapagos Is.)

Host: From grass near bay shore

Cheletomimus (Hemicheyletia) echis (Chaudhri \& Akbar)

Hemicheyletia echis Chaudhri \& Akbar, 1985: 187

Cheletomimus echis (Chaudhri \& Akbar): Halliday, Kamran \& Bashir, 2018: 84

Type locality: Pakistan

Distribution: Pakistan

Cheletomimus (Hemicheyletia) disparis (Chaudhri \& Akbar)

Hemicheyletia disparis Chaudhri \& Akbar, 1985: 180.

Cheletomimus disparis (Chaudhri \& Akbar): Halliday, Kamran \& Bashir, 2018: 84

Type locality: Pakistan

Distribution: Pakistan

Cheletomimus (Hemicheyletia) glomus (Chaudhri et Akbar)

Hemicheyletia glomus Chaudhri \& Akbar, 1985: 181;

Cheletomimus glomus (Chaudhri et Akbar): Halliday, Kamran \& Bashir, 2018: 84

Type locality: Pakistan

Distribution: Pakistan

Cheletomimus (Hemicheyletia) gracilis Fain, Bochkov et Corpuz-Raros

Cheletomimus (Hemicheyletia) gracilis Fain, Bochkov \& Corpuz-Raros, 2002: 57

Type locality: Hawaii (on Allium cepa from Japan)

Distribution: Peru, the Philippines, USA (Hawaii)

Host: plants

Cheletomimus (Hemicheyletia) granulus (Summers et Price)

Hemicheyletia granula Summers \& Price, 1970: 13

Cheletomimus (Hemicheyletia) granula (Summers et Price): Fain, Bochkov \& CorpuzRaros, 2002: 36 
Type locality: Quetzaltepeque

Distribution: El Salvador

Host: from moss and ant nest

Cheletomimus (Hemicheyletia) greenwoodi Fain, Bochkov et Corpuz-Raros

Cheletomimus (Hemicheyletia) greenwoodi Fain, Bochkov \& Corpuz-Raros, 2002: 54

Type locality: Barstey, Wales, Great Britain

Distribution: Great Britain

Host: from nest of Anthus spinoletta (Passerifpormes: Motacillidae)

Cheletomimus (Hemicheyletia) hastatus (Chaudhri \& Akbar)

Hemicheyletia hastatus Chaudhri \& Akbar, 1985: 183; Afzal, Ali \& Akbar, 2003: 90; Kamran, Khan \& Nazir, 2005: 54

Cheletomimus hastatus (Chaudhri \& Akbar): Halliday, Kamran \& Bashir, 2018: 84

Type locality: Pakistan

Distribution: Pakistan

Cheletomimus (Hemicheyletia) indica (Gupta) (sp. inquir.)

Hemicheyletia indica Gupta, 1991: 234; Fain, Bochkov \& Corpuz-Raros, 2002: 28

Type locality: India

Distribution: India

Cheletomimus (Hemicheyletia) kysenyiensis (Thewke et Enns)

Hemicheyletia kysenyiensis Thewke \& Enns, 1979: 218

Cheletomimus (Hemicheyletia) kysenyiensis (Thewke et Enns): Fain, Bochkov \& CorpuzRaros, 2002: 38

Type locality: Kysenyi (former Zaire)

Distribution: D. R. Congo

Cheletomimus (Hemicheyletia) lanceolata (Corpuz-Raros) (sp. inquir.)

Hemicheyletia lanceolata Corpuz-Raros, 1998: 274; Fain, Bochkov \& Corpuz-Raros, 2002: 28 Type locality: Luzon Is., Pintor, Gamu, Isabela

Distribution: Philippine Is.

Cheletomimus (Hemicheyletia) leytensis (Corpuz-Raros)

Hemicheyletia leytensis Corpuz-Raros, 1988: 419; 1998: 275

Cheletomimus (Hemicheyletia) leytensis (Corpuz-Raros): Fain, Bochkov \& Corpuz-Raros, 2002: 42

Type locality: Babatngon, Leyte

Distribution: Philippines (Leyte, Luzon) 
Cheletomimus (Hemicheyletia) lindquisti (Thewke et Enns)

Hemicheyletia lindquisti Thewke \& Enns 1979: 221

Cheletomimus (Hemicheyletia) lindquisti (Thewke et Enns): Fain, Bochkov \& Corpuz-

Raros, 2002: 57; Bochkov \& Abramov, 2016: 336

Type locality: 2,5 miles SE Kananaskis F. E. Station, Alberta

Distribution: Canada (Alberta), Russia

Cheletomimus (Hemicheyletia) makilingensis (Corpuz-Raros)

Hemicheyletia makilingensis Corpuz-Raros, 1972: 256

Cheletomimus (Hemicheyletia) makilingensis (Corpuz-Raros): Fain, Bochkov \& Corpuz-

Raros, 2002: 39

Type locality: Laguna, Philippines

Distribution: Philippines

Host: Plant host Schizostachium

Cheletomimus (Hemicheyletia) mexicanus (Thewke et Enns)

Hemicheyletia mexicana Thewke \& Enns, 1979: 223

Cheletomimus (Hemicheyletia) mexicanus: Fain, Bochkov \& Corpuz-Raros, 2002: 52

Type locality: three miles north of San Cristobal, Las Casas, Chiapas

Distribution: Mexico (Chiapas)

Cheletomimus (Hemicheyletia) newyorkensis (Delfinado et Khaing-Fields) (spec. inquir.)

Hemicheyletia newyorkensis Delfinado \& Khaing-Fields, 1976: 192; Fain, Bochkov \& Corpuz-Raros, 2002: 28

Type locality: New York, Long Island

Distribution: USA

Cheletomimus (Hemicheyletia) nucella (Chaudhri et Akbar)

Hemicheyletia nucella Chaudhri \& Akbar, 1985: 166; Haq \& Afzal, 2007: 2206

Cheletomimus nucella (Chaudhri et Akbar): Halliday, Kamran \& Bashir, 2018: 84

Type locality: Pakistan

Distribution: Pakistan

Cheletomimus (Hemicheyletia) omissus (Tseng)

Hemicheyletia omissa Tseng, 1977: 222

Cheletomimus (Hemicheyletia) omissa (Tseng): Fain, Bochkov \& Corpuz-Raros, 2002: 60

Cheletomimus omissus (Tseng): Xia, 2010: 161

Type locality: Taiwan

Distribution: China, Taiwan 
Cheletomimus (Hemicheyletia) parcus (Afzal, Ali et Akbar)

Hemicheyletia parcus Afzal, Ali \& Akbar, 2003: 89

Cheletomimus parcus (Afzal, Ali et Akbar): Halliday, Kamran \& Bashir, 2018: 85

Type locality: Pakistan

Distribution: Pakistan

Cheletomimus (Hemicheyletia) parodos (Chaudhri \& Akbar)

Hemicheyletia parodos Chaudhri \& Akbar, 1985: 173.

Cheletomimus parodos (Chaudhri et Akbar): Halliday, Kamran \& Bashir, 2018: 85

Type locality: Pakistan

Distribution: Pakistan

Cheletomimus (Hemicheyletia) recki (Volgin)

Paracheyletia recki Volgin, 1966b: 283; 1969: 183; Fain \& Bochkov, 2001: 51

Cheletomimus (Hemicheyletia) recki (Volgin): Khaustov, 2021: 214

Type locality: Voronezh Reserve

Distribution: Russia

Host: ant hill

Cheletomimus (Hemicheyletia) reticulatus (Jeffrey et Campbell)

Hemicheyletia reticulata Jeffrey \& Campbell, 1975: 103

Cheletomimus (Hemicheyletia) reticulata (Jeffrey et Campbell): Fain, Bochkov \& Corpuz-

Raros, 2002: 42

Type locality: Scotland, Argyll, Island of Gigha

Distribution: Great Britain

Cheletomimus (Hemicheyletia) rostellus (Summers et Price)

Hemicheyletia rostella Summers \& Price, 1970: 17

Cheletomimus (Hemicheyletia) rostellus (Summers et Price): Fain, Bochkov \& Corpuz-

Raros, 2002: 36

Type locality: California (Dorrington, Calaveras Co., etc.)

Distribution: USA (California)

Host: from rotting logs

Cheletomimus (Hemicheyletia) salvus (Chaudhri et Akbar)

Hemicheyletia salvus Chaudhri \& Akbar, 1985: 149

Cheletomimus salvus (Chaudhri et Akbar): Halliday, Kamran \& Bashir, 2018: 85

Type locality: Pakistan

Distribution: Pakistan

Cheletomimus (Hemicheyletia) scutellatus (De Leon)

Cheyletia scutellata De Leon, 1962: 130 
Paracheyletia scutellata (De Leon): Muma, 1964: 246

Andrecheyla scutellata (De Leon): Volgin, 1969: 219

Hemicheyletia scutellata (De Leon): Summers \& Price, 1970: 14

Cheletomimus (Hemicheyletia) scutellatus (De Leon): Fain, Bochkov \& Corpuz-Raros, 2002: 42

Type locality: Florida

Distribution: Mexico (Chiapas), USA (Florida)

Hosts: Swietenia mahagoni (Meliaceae), Xylosma elliptica (Salicaceae)

Cheletomimus (Hemicheyletia) serrulus (Summers et Price)

Hemicheyletia serrula Summers \& Price, 1970: 15

Cheletomimus (Hemicheyletia) serrula (Summers et Price): Fain, Bochkov \& CorpuzRaros, 2002: 48

Type locality: Darwin Res. Station

Distribution: Galapagos Is.

Cheletomimus (Hemicheyletia) tanosis (Kamran, Khan et Nazir)(spec. inquir.) Hemicheyletia tanosis Kamran, Khan \& Nazir, 2005: 52

Cheletomimus tanosis (Kamran, Khan et Nazir): Halliday, Kamran \& Bashir, 2018: 84

Type locality: Chak 98/M.L., 14 km East of Karor, Layyah, Pakistan

Distribution: Pakistan

Host: from pomegranate (Punica granatum)

Cheletomimus (Hemicheyletia) transversa (Corpuz-Raros)

Hemicheyletia transversa Corpuz-Raros, 1972: 257; Corpuz-Raros \& Sotto, 1972: 170

Cheletomimus (Hemicheyletia) transversa (Corpuz-Raros): Fain, Bochkov \& Corpuz-Raros, 2002: 39

Hemicheyletia morii Ehara \& Ghani Ibrahim, 1988: 237 - Kepong, Selangor, Malaysia.

Syn. by Fain, Bochkov \& Corpuz-Raros, 2002: 39

Type locality: Laguna, the Philippines

Distribution: Malaysia, Philippines, Singapore

Host: Plant hosts Centrostemma multiflora and Bambusa sp.

Cheletomimus (Hemicheyletia) trepsis (Chaudhri \& Akbar)

Hemicheyletia trepsis Chaudhri \& Akbar, 1985: 175

Cheletomimus trepsis (Chaudhri \& Akbar): Halliday, Kamran \& Bashir, 2018: 85

Type locality: Pakistan

Distribution: Pakistan

Cheletomimus (Hemicheyletia) tropicus (Shiba)

Cheyletia tropica Shiba, 1976: 169

Hemicheyletia tropica (Shiba): Ehara \& Ghani, 1988: 240 
Cheletomimus (Hemicheyletia) tropica (Shiba): Fain, Bochkov \& Corpuz-Raros, 2002: 48 Type locality: Pasoh Forest Reserve

Distribution: Malaysia

Cheletomimus (Hemicheyletia) uichancoi (Corpuz-Raros)

Hemicheyletia uichancoi Corpuz-Raros, 1972: 258

Cheletomimus (Hemicheyletia) uichancoi (Corpuz-Raros): Fain, Bochkov \& Corpuz-Raros, 2002: 57

Type locality: Laguna, the Philippines

Distribution: Philippines, Vanuatu

Host: Plant host Leucaena leucocephala (Mimosaceae)

Cheletomimus (Hemicheyletia) umbo (Chaudhri et Akbar)

Hemicheyletia umbo Chaudhri \& Akbar, 1985: 185

Cheletomimus umbo (Chaudhri et Akbar): Halliday, Kamran \& Bashir, 2018: 85

Type locality: Pakistan

Distribution: Pakistan

Cheletomimus (Hemicheyletia) vescus (Qayyum et Chaudhri)

Hemicheyletia vescus Qayyum \& Chaudhri, 1979: 167

Cheletomimus (Hemicheyletia) vescus (Qayyum et Chaudhri): Fain, Bochkov \& Corpuz-Raros, 2002: 38; Bochkov, Hakimitabar \& Saboori, 2005: 107

Hemicheyletia laguncula Rasool \& Chaudhri, 1979: 4 - Pakistan. Syn. by Fain, Bochkov \& Corpuz-Raros, 2002: 38

Cheletomimus vescus (Qayyum et Chaudhri): Halliday, Kamran \& Bashir, 2018: 85

Type locality: Pakistan

Distribution: Iran, Pakistan

Cheletomimus (Hemicheyletia) vivus (Chaudhri et Akbar)

Hemicheyletia vivus Chaudhri \& Akbar, 1985: 177

Cheletomimus vivus (Chaudhri et Akbar): Halliday, Kamran \& Bashir, 2018: 85

Type locality: Pakistan

Distribution: Pakistan

Cheletomimus (Hemicheyletia) volgini (Cunliffe)

Paracheyletia volgini Cunliffe, 1963: 197

Dendrocheyla volgini: Volgin, 1969: 210-211

Hemicheyletia volgini (Cunliffe): Summers \& Price, 1970: 14

Cheletomimus (Hemicheyletia) volgini (Cunliffe): Fain, Bochkov \& Corpuz-Raros, 2002: 48 Type locality: Mulunga (D.R. Congo)

Distribution: D. R. Congo, Rwanda 
Cheletomimus (Hemicheyletia) wellsi (Baker)

Cheyletia wellsi Baker, 1949: 300; De Leon, 1962: 132

Paracheyletia wellsi (Baker): Volgin, 1955: 169; Mumma, 1964: 245; Garrett \& Haramoto, 1967: 410

Dendrocheyla wellsi (Baker): Volgin, 1969: 211

Hemicheyletia wellsi (Baker): Summers \& Price, 1970: 15; Flechtmann, 1971: 31; Corpuz Raros \& Sotto, 1977: 157; Tseng, 1977: 224; Gerson, 1994: 445; Feres \& Flechtmann, 1995: 535

Cheletomimus (Hemicheyletia) wellsi: Fain, Bochkov \& Corpuz-Raros, 2002: 45;

Doğan \& Ayyildiz, 2004: 1; Bochkov, Hakimitabar \& Saboori, 2005: 107; Buosi et al., $2006: 7$ Cheletomimus wellsi: Xia, 2010: 161

Type locality: Azores, intercepted at Philadelphia, Pa.

Distribution: Argentina, Australia, Azores, Brazil, Chile, China, Cuba, Iran, Jamaica, Japan, Malaysia, Mexico, Panama, Philippine Is., Puerto Rico, Taiwan (may be wellsinus), Trinidad, Turkey, USA (incl. Hawaii)

Cheletomimus (Hemicheyletia) wellsinus (De Leon)

Paracheyletia wellsina De Leon, 1967: 34

Hemicheyletia wellsina (De Leon): Summers \& Price, 1970: 18; Corpuz-Raros \& Sotto, 1977: 158; Ehara \& Abdul Ghani, 1988

Hemicheyletia arecana Tjying, 1972: 8 - Taiwan. Syn. by Fain, Bochkov \& Corpuz-Raros, 2002: 45

Cheletomimus (Hemicheyletia) wellsina (De Leon): Fain, Bochkov \& Corpuz-Raros, 2002: 45

Cheletomimus (Hemicheyletia) wellsinus (De Leon): Torre Santana, 2015: 50

Cheletomimus wellsina (De Leon): Xia, 2010: 161

Type locality: Trinidad (Caribbean)

Distribution: Algeria, Benin (Dahomey), Cameroon, China, Cuba, Guatemala, Malaysia, Philippine Is., Rwanda, Taiwan, Trinidad, USA (Florida, Texas)

Subgenus Philippicheyla Corpuz-Raros

Philippicheyla Corpuz-Raros, 1972: 265 (genus)

Philippicheyla Corpuz-Raros: Fain \& Bochkov, 2001: 299 (subgenus of Hemicheyletia)

Type species: Philippicheyla filipina Corpuz-Raros, 1972

Cheletomimus (Philippicheyla) ascutatus Fain, Bochkov et Corpuz-Raros Cheletomimus (Philippicheyla) ascutatus Fain, Bochkov \& Corpuz-Raros, 2002: 60 Type locality: Elizabeth La, California

Distribution: USA (California)

Host: from inner tree bark

Cheletomimus (Philippicheyla) crowei Bochkov et Otto Cheletomimus (Philippicheyla) crowei Bochkov \& Otto, 2010: 9 
Type locality: New Zealand

Distribution: New Zealand

Host: from avocado

Cheletomimus (Philippicheyla) notelaeae (Gerson)

Philippicheyla notelaeae Gerson, 1994: 437

Cheletomimus (Philippicheyla) notelaeae (Gerson): Fain, Bochkov \& Corpuz-Raros, 2002: 60

Type locality: Indooroopilly, Queensland

Distribution: Australia (Queensland)

Host: bud scales on branches of Notelaea longifolia (Oleraceae)

Cheletomimus (Philippicheyla) filipina (Corpus-Raroz)

Philippicheyla philipina Corpus-Raroz, 1972: 256

Cheletomimus (Philippicheyla) filipina (Corpus-Raroz): Fain, Bochkov \& Corpuz-Raros, 2002: 60

Type locality: Mt. Makiling, Laguna, the Philippines

Distribution: Philippines

Host: on decomposing bark of a fallen log

Species inquirendae:

Cheletomimus binus Tseng (spec. inquir.)

Cheletomimus binus Tseng, 1973: 76; Salarzehi, 2018:

Type locality: Taiwan

Distribution: Iran, Taiwan

Cheletomimus bisetosus Tseng (spec. inquir.)

Cheletomimus bisetosus Tseng, 1977

Type locality: Taiwan

Distribution: Taiwan

Cheletomimus cambio Aheer, Akbar et Chaudhri (spec. inquir.)

Cheletomimus cambio Aheer, Akbar \& Chaudhri, 1994: 346; Aheer, Akbar \& Chaudhri, 1998: 125; Gerson, Fain \& Smiley, 1999: 50; Fain, Bochkov \& Corpuz-Raros, 2002: 28; Haq \& Afzal, 2007: 2206; Halliday, Kamran \& Bashir, 2018: 84

Type locality: Pakistan

Distribution: Pakistan

Cheletomimus cantor Rasool, Chaudhri et Akbar (spec. inquir.)

Cheletomimus cantor Rasool, Chaudhri \& Akbar, 1980: 28; Chaudhri \& Akbar, 1985: 145; Aheer, Akbar \& Chaudhri, 1998: 125; Gerson, Fain \& Smiley, 1999: 50; Fain, Bochkov \& Corpuz-Raros, 2002: 28; Halliday, Kamran \& Bashir, 2018: 84 
Type locality: Pakistan

Distribution: Pakistan

Cheletomimus citrosinensis Patxot et Goff (spec. inquir.)

Cheletomimus citrosinensis Patxot \& Goff, 1985: 157

Type locality: Oahu I., Mililani

Distribution: USA (Hawaiian Is.)

Cheletomimus domitus Chaudhri \& Akbar

Cheletomimus domitus Chaudhri \& Akbar, 1985: 150; Aheer, Akbar \& Chaudhri, 1998:

126; Halliday, Kamran \& Bashir, 2018: 84

Type locality: Pakistan

Distribution: Pakistan

Cheletomimus fissilis Chaudhri et Akbar

Cheletomimus fissilis Chaudhri \& Akbar, 1985: 155; Aheer, Akbar \& Chaudhri, 1998: 126;

Halliday, Kamran \& Bashir, 2018: 84

Type locality: Pakistan

Distribution: Pakistan

Cheletomimus flecto Aheer, Akbar et Chaudhri (spec. inquir.)

Cheletomimus flecto Aheer, Akbar \& Chaudhri, 1998: 126; Fain, Bochkov \& Corpuz-Raros, 2002: 28; Aheer, Akbar \& Chaudhri, 1998: 126; Halliday, Kamran \& Bashir, 2018: 84 Type locality: Pakistan

Distribution: Pakistan

Cheletomimus heredis Qayyum et Chaudhri (spec. inquir.)

Cheletomimus heredis Qayyum \& Chaudhri, 1979: 9; Halliday, Kamran \& Bashir, 2018: 84 Type locality: Pakistan

Distribution: Pakistan

Cheletomimus larme Aheer, Akbar et Chaudhri (spec. inquir.)

Cheletomimus larme Aheer, Akbar \& Chaudhri, 1994: 348; 1998: 125; Halliday, Kamran \& Bashir, 2018: 84

Cheletomimus larmae Aheer, Akbar \& Chaudhri: Gerson, Fain \& Smiley, 1999: 51; Fain, Bochkov \& Corpuz-Raros, 2002: 28

Type locality: Pakistan

Distribution: Pakistan

Cheletomimus minutus Soliman (spec. inquir.)

Cheletominus [sic, err. typogr.] minutus Soliman, 1975: 99

Type locality: Borg el Arab 
Distribution: Egypt

Cheletomimus sialkotensis Chaudhri et Akbar

Cheletomimus sialkotensis Chaudhri \& Akbar, 1985: 147; Halliday, Kamran \& Bashir, 2018: 85

Type locality: Pakistan

Distribution: Pakistan

Cheletomimus telma Chaudhri et Akbar

Cheletomimus telma Chaudhri \& Akbar, 1985: 149; Aheer, Akbar \& Chaudhri, 1998: 130; Halliday, Kamran \& Bashir, 2018: 85

Type locality: Pakistan

Distribution: Pakistan

Cheletomimus trema Aheer, Akbar et Chaudhri (spec. inquir.)

Cheletomimus trema Aheer, Akbar \& Chaudhri, 1998: 126; Fain, Bochkov \& Corpuz-Raros, 2002: 28; Halliday, Kamran \& Bashir, 2018: 85

Type locality: North West Frontier Province, Dargai

Distribution: Pakistan

Cheletomimus zamia Aheer, Akbar et Chaudhri (spec. inquir.)

Cheletomimus zamia Aheer, Akbar \& Chaudhri, 1994: 348; 1998: 125; Gerson, Fain \& Smiley, 1999: 51; Fain, Bochkov \& Corpuz-Raros, 2002: 28; Halliday, Kamran \& Bashir, 2018: 85

Type locality: Pakistan

Distribution: Pakistan

Genus Cheletophanes Oudemans

Cheletophanes Oudemans, 1904: 162

Type species: Cheyletus montandoni Berlese et Trouessart, 1889

Cheletophanes montandoni (Berlese et Trouessart)

Cheyletus Montandoni Berlese \& Trouessart, 1889: 133

Cheletophanes montandoni (Berlese et Trouessart): Oudemans, 1904b: 162; 1906: 140;

Baker, 1949 : 303; Volgin, 1969: 165; Summers \& Price, 1970: 35

Type locality: Broşteni

Distribution: Romania

Host: Aradus pictus (Heteroptera: Aradidae)

Cheletophanes peregrinus Berlese (inc. sed.)

Cheletophanes peregrinus Berlese, 1921: 194; Baker, 1949: 304 
Type locality: Florence

Distribution: Italy

Host: „in detritis corticis pinorum, ad terram”

Genus Cheletophyes Oudemans

Cheletophyes Oudemans, 1914: 101

Type species: Cheletophyes vitzthumi Oudemans, 1914

Cheletophyes apicola Fain, Lukoschus et Nadchatram

Cheletophyes apicola Fain, Lukoschus \& Nadchatram, 1980: 311; Fain \& Bochkov, 2001: 63

Type locality: Kuala Pilah, Negri Sembilan

Distribution: Malaysia

Host: nest of Xylocopa (Platynopoda) latipes (Hymenoptera: Apidae)

Cheletophyes aurorae Haitlinger

Cheletophyes aurorae Haitlinger, 2000: 86

Type locality: Arusha

Distribution: Tanzania

Host: Xylocopinae (Hymenoptera: Apidae)

Cheletophyes clavipilis Fain, Lukoschus et Nadchatram

Cheletophyes clavipilis Fain, Lukoschus \& Nadchatram, 1980: 310

Type locality: Kuala Pilah, Negri Sembilan

Distribution: Malaysia

Host: nest of Xylocopa (Platynopoda) latipes (Hymenoptera: Apidae)

Cheletophyes decorus Bochkov et Klimov

Cheletophyes decorus Bochkov \& Klimov, 2005: 19

Type locality: Cam Ranh Peninsula, Khanh Hoa Prov. (Vietnam), Trang Prov., Khao

Chong Nature Education Centre (Thailand)

Distribution: Thailand, Vietnam

Host: Xylocopa tranquebarica (Hymenoptera: Apidae)

Cheletophyes eckerti Summers et Price

Cheletophyes eckerti Summers \& Price, 1970: 40; Fain \& Bochkov, 2001: 64

Type locality: Ludhiana (India)

Distribution: Angola, D.R. Congo, India

Host: Xylocopa (Koptortosoma) aestuans (Hymenoptera: Apidae)

Cheletophyes indiacus Smiley et Whitaker

Cheletophyes indiacus Smiley \& Whitaker, 1981: 123; Fain \& Bochkov, 2001: 63 
Cheletophyes xylocopae Ramaraju \& Mohanasundaram, 1999: 121 - Tamil Nadu, Coimbatore, India, from Xylocopa sp. (Hymenoptera: Apidae)

Type locality: India

Distribution: India

Host: Xylocopa sp. (Hymenoptera: Apidae)

Cheletophyes mbomba Klimov, Bochkov et OConnor

Cheletophyes mbomba Klimov, Bochkov \& OConnor, 2006: 56

Type locality: Bas-Uele Prov., Bambesa

Distribution: DR Congo

Host: Xylocopa torrida (Hymenoptera: Apidae)

Cheletophyes occisor Bochkov et Klimov

Cheletophyes occisor Bochkov \& Klimov, 2005: 18

Type locality: Popondetta Distr., Popondetta

Distribution: Papua New Guinea

Host: Xylocopa aruana (Hymenoptera: Apidae)

Cheletophyes panamensis Klompen, Méndez et Lukoschus

Cheletophyes panamensis Klompen, Méndez \& Lukoschus, 1984: 249; Fain \& Bochkov, 2001: 63

Type locality: Capira, Aguacate (Panama)

Distribution: Costa Rica, Panama

Host: Xylocopa (Megaxylocopa) frontalis (Hymenoptera: Apidae)

Cheletophyes torridae Klimov, Bochkov et OConnor

Cheletophyes torridae Klimov, Bochkov \& OConnor, 2006: 55

Type locality: Sangmelima, Fulasi

Distribution: Cameroon

Host: Xylocopa torrida (Hymenoptera: Apidae)

Cheletophyes vespae Fain et Bochkov

Cheletophyes vespae Fain \& Bochkov, 2001: 65

Type locality: KG. Duson, Tampin, N. Sembilan

Distribution: Malaysia (N. Sembilan)

Host: Vespa tropica (Hymenoptera: Vespidae)

Cheletophyes vitzthumi Oudemans

Cheletophyes vitzthumi Oudemans, 1914:101; 1915: 51; Volgin, 1965: 290; 1969: 160; Summers \& Price, 1970: 40; Fain \& Bochkov, 2001: 63 
Type locality: Willowmore, Cape Province

Distribution: South Africa

Host: Xylocopa (Koptorthosoma) caffra (Hymenoptera: Apidae)

Species inquirendae (Fain \& Bochkov, 2001: 61):

Cheletophyes deodikari Putatunda et Kapil

Cheletophyes deodikari Putatunda \& Kapil, 1988: 318

Type locality: Haryana, Hisar

Distribution: India

Host: Xylocopa fenestrata (Hymenoptera: Apidae)

Cheletophyes harnaji Putatunda et Kapil

Cheletophyes harnaji Putatunda \& Kapil, 1988: 320

Type locality: India

Distribution: India

Host: Xylocopa pubescens (Hymenoptera: Apidae)

Cheletophyes newtoni Putatunda et Kapil

Cheletophyes newtoni Putatunda \& Kapil, 1988: 322

Type locality: Haryana, Hisar

Distribution: India

Host: Xylocopa fenestrata (Hymenoptera: Apidae)

Cheletophyes orientalis Putatunda et Kapil

Cheletophyes orientalis Putatunda \& Kapil, 1988: 324

Type locality: Haryana, Hisar

Distribution: India

Host: Xylocopa fenestrata (Hymenoptera: Apidae)

Cheletophyes ruttneri Putatunda et Kapil

Cheletophyes ruttneri Putatunda \& Kapil, 1988: 325

Type locality: Haryana, Hisar

Distribution: India

Host: Xylocopa fenestrata (Hymenoptera: Apidae)

Cheletophyes shendei Putatunda et Kapil

Cheletophyes shendei Putatunda \& Kapil, 1988: 326

Type locality: India

Distribution: India

Host: Xylocopa fenestrata (Hymenoptera: Apidae) 
Genus Cheyletus Latreille

Cheyletus Latreille, 1796: 179

Type species: Acarus eruditus Schrank, 1781

Cheyletus ascutatus Bochkov et OConnor

Cheyletus ascutatus Bochkov \& OConnor, 2003 : 327

Type locality: Leyte Is., 10 km N, 4,5 km E Baybay

Distribution: Philippines (Leyte)

Host: Batomys salomonseni (Rodentia: Muridae)

Cheyletus attiahi Yousef et Issa

Cheyletus attiahi Yousef \& Issa, 1972: 42

Type locality: Egypt

Distribution: Egypt

Cheyletus batomysi Bochkov et OConnor

Cheyletus batomysi Bochkov \& OConnor, 2003: 329

Type locality: Bukidnon Prov., Mindanao Is., Mt. Katangland Range, Camp Phillips, $2250 \mathrm{~m}$

Distribution: Philippines

Host: Batomys salomonseni (Rodentia: Muridae)

Cheyletus bidentatus Fain et Nadchatram

Cheyletus bidentatus Fain \& Nadchatram, 1980: 194

Type locality: Bukit Lanjan Forest Reserve, Selangor, Segamat, Labis Forest Reserve (Johore)

Distribution: Iran, Malaysia (Selangor, Johore)

Host: nest of Hylopetes spadiceus (Rodentia: Sciuridae)

\section{Cheyletus cacahuamilpensis Baker}

Cheyletus cacahuamilpensis Baker, 1949: 282; Volgin, 1949: 114; Costa, 1961: 259; 1967: 109; Summers \& Price, 1970: 26; Fain, 1982: 89; Fain \& Bochkov, 2001; Whitaker et al., 2007: 6; Palyvos et al., 2008: 213; Salarzehi et al., 2018: 465

Eucheyletia mungeri McGregor, 1956: 24 (syn. by Summers \& Price, 1970)

Cheyletus baloghi Volgin, 1969: 116. Type: Crimea (Sevastopol), on Spermophilus [Citellus] pygmaeus (nests)

Type locality: Cave of Cacahuamilpa (Mexico, Guerrero)

Distribution: Algeria, Crimea, Greece, Iran, Israel, Mexico, Peru, USA, South Africa

Hosts: bat guano, Myotis velifer (Chiroptera: Vespertilionidae), Spermophilus [Citellus] pygmaeus (Rodentia: Sciuridae, nests), Microtus guentheri (Rodentia: Arvicolidae) 
Cheyletus carnifex Zachvatkin

Cheyletus carnifex Zachvatkin, 1935: 27; Rohdendorf, 1940: 89; Volgin, 1969: 112; Torre Santana, 2015

Cheyletus aversor Rohdendorf, 1940: 86; Baker, 1949: 283; Volgin, 1955: 163; 1969: 89;

Wharton \& Arlian, 1972: 719; Ždárková, 1979; Shen, 1991; Lin et al., 2000; Xia et al., 2000; Lukáš et al., 2007: 97 - Bulgaria, China, Czech Republic, Russia, Tajikistan, Uganda, Ukraine, USA

Cheyletus beauchampi Baker, 1949: 282 - Portugal, intercepted at Boston, Mass. (syn. by Summers \& Price, 1970)

Cheyletus acarophagus Zacher \& Soliman, 1967: 25

Cheyletus allactaga Fain \& Lukoschus, 1981: 122 - Bulgan-Gol, Mongolia, on Allactaga sibirica (Rodentia: Dipodidae)

Cheyletus zaheri Hassan \& Rakha, 1982: 89

Type locality of Cheyletus carnifex: Moscow

Type locality of Cheyletus aversor: Ordzhonikidze (Caucasus) and Altai

Type locality of Cheyletus acarophagus: Egypt

Type locality of Cheyletus zaheri: Egypt

Distribution: Bulgaria, China, Czech Republic, Iran, Russia, Tajikistan, Uganda, Ukraine Hosts: Allactaga sibirica (Rodentia: Dipodidae)

\section{Cheyletus eruditus (Schrank)}

Acarus eruditus Schrank, 1781: 513

Cheyletus robertsoni Brady, 1875: 302

Cheletes eruditus (Schrank): Oudemans, 1903: 118; 1906: 84

Eutarsus cancriformis Hessling, 1852: 258

Cheyletus ferox Berlese et Trouessart, 1889: 14; Banks, 1906: 134 - "Port-Praslin Nieuw Ireland".

Cheletes ferox (Berlese et Trouessart): Oudemans, 1903a: 83; 1906: 115

Cheyletus eburneus Hardy, 1933: 336

Cheyletus seminivorus, Packard, 1870: 665; Ewing, 1909: 76; 1912: 416

Cheyletus doddi Baker, 1949: 279 - Ithaca, N.Y.

Cheyletus desitus Qayyum \& Chaudhri, 1977: 90

Cheyletus mortelmansi Fain, 1972: 37 - Zoo d'Anvers

Cheyletus eruditus (Schrank): Kramer, 1876: 188; Canestrini, 1886: 171; Haller, 1882: 312;

Oudemans, 1903: 118; Jablonowski, 1918: 4; Hughes, 1948: 102; Baker, 1949: 278;

Starkoff \& Starkoff, 1950: 96; Turk, 1953: 18; Franz, 1954: 367; Haarløv, 1957: 26;

Cwilich \& Hadani, 1965: 54; Volgin, 1969: 78; Shiba, 1969b: 101; 1969a: 159; Shen, 1975; Mumcuoglu, 1976: 365; Kumada et al., 1978: 262; Hallas, 1978: 30; Whitaker et al., 2007: 6; Xia, 2010: 161; Torre Santana, 2015; Halliday, Kamran \& Bashir, 2018: 87 Cheletes strennus Oudemans, 1904: 161; 1906: 8.3

Cheyletus strennus: Baker, 1949: 280 
Type locality: Austria

Distribution: cosmopolitic

Hosts: Coracopsis nigra barkleyi (Psittaciformes: Psittrichasiidae), Didelphis virginiana (Didelphiomorpha: Didelphidae), Pipistrellus abramus (Chiroptera: Vespertilionidae), Peromyscus leucopus (Rodentia: Cricetidae, Neotominae), Menetes berdmorei, Rattus norvegicus (Rodentia: Muridae), Sciurus carolinensis (Rodentia: Sciuridae), Bassariscus astutus (Carnivora: Procyonidae)

\section{Cheyletus funisciuri (Fain)}

Eucheyletia funisciuri Fain, 1972: 38

Cheyletus funisciuri (Fain): Fain \& Bochkov, 2001: 100

Type locality: Rwanda

Distribution: DR Congo, Rwanda

Host: Funisciurus carruthersi (Rodentia: Sciuridae)

Cheyletus gerbillicola Fain et Lukoschus

Cheyletus gerbillicola Fain \& Lukoschus, 1981: 6

Type locality: Askham, South of Kalahari region

Distribution: South Africa

Host: Gerbillurus paeba (Rodentia: Muridae, Gerbillinae)

\section{Cheyletus kivuensis (Fain)}

Eucheyletia kivuensis Fain, 1972: 39

Cheyletus kivuensis (Fain): Fain \& Bochkov, 2001: 100

Type locality: Kivu

Distribution: D.R. Congo

Host: Rodentia indet.

Cheyletus kuznetzovi Bochkov et Khaustov

Cheyletus kuznetzovi Bochkov \& Khaustov, 1999: 116; Dogan, Jalaeian \& Kamali, 2011: 782

Type locality: Dagskiy Reserve (Turkmenistan)

Distribution: Iran, Turkmenistan

Host: from ant hill

\section{Cheyletus legendrei Fain}

Cheyletus legendrei Fain, 1982: 83

Type locality: Tananarive

Distribution: Madagascar

Host: nest of Foudia madagascariensis (Passeriformes: Ploceidae) 


\section{Cheyletus linsdalei Baker}

Cheyletus linsdalei Baker, 1949: 281; Volgin, 1969: 111; Summers \& Price, 1970: 29

Type locality: California, Monterey Co.

Distribution: USA (California)

Host: Citellus beecheyi (Rodentia: Sciuridae)

\section{Cheyletus mafekingensis Fain}

Cheyletus mafekingensis Fain, 1982: 86

Type locality: Mafeking

Distribution: South Africa

Host: Philetarius socius (Passeriformes: Ploceidae)

\section{Cheyletus malaccensis (Oudemans)}

Cheletes malaccensis Oudemans, 1903a: 84; 1906: 88

Cheyletus malaccensis (Oudemans): Baker, 1949: 284; Hughes, 1961: 197; Gerson, 1967: 361; Shiba, 1969a: 161; Flechtmann, 1971: 30; Regev, 1974: 85; Shen, 1975; Nakada, 1975: 151; Kumada et al., 1978: 262; Caceres \& Fain, 1978: 302; Rosa \& Flechtmann, 1979: 196; Ždárková, 1979; Fain \& Nadchatram, 1980: 191; Saleh et al., 1986: 37; Whitaker \& Dietz, 1987: 194; Rostom, 1993 : 29; Mariana et al., 2000: 714; Lukáš et al., 2007: 97; Palyvos et al., 2008: 213; Xia, 2010: 162; Torre Santana, 2015; Halliday, Kamran \& Bashir, 2018: 87

Cheyletus ugandanus Lawrence, 1954: 65 - Karamoja, Uganda, nest of weaver-bird Cheyletus ayyazi Akbar, Aheer \& Ishtiaq, 1993: 293 - Pakistan

Cheyletus polymorphus Volgin, 1949: 584; 1969: 90; Sosnina et al., 1969: 370 - Greece, Bulgaria

Cheyletus caucasicus Zachvatkin, 1949: 288

Cheyletus egypticus Elbadry, 1969: 157 - Egypt

Cheyletus baridos Akbar, Rahi \& Chaudhri, 1988: 5 - Pakistan

Cheyletus wahndoensis Akbar \& Aheer, 1994: 343 - Pakistan

Cheyletus avidus Qayyum \& Chaudhri, 1977: 89 - Pakistan

Cheyletus mianiensis Farooq, Akbar \& Qureshi, 2000: 257 - Pakistan

Cheyletus rafiquiensis Farooq, Akbar \& Qureshi, 2000: 259 - Pakistan

Cheyletus phantosis Akbar \& Aheer, 1994: 342 - Pakistan

Cheletes vorax Oudemans, 1903b: 84; 1906: 122 - Mariana Is., bats (Chiroptera)

Cheyletus vorax (Oud.): Volgin, 1969: 99

Cheyletus rohdendorfi Zachvatkin, 1949: 290; Volgin, 1969: 80; Rekk, 1976: 68 - Baku, Azerbaidjan; Armenia

Cheyletus infensus Akbar, Aheer \& Shaudhri, 1993: 295 - Pakistan

Cheletes fortis Oudemans, 1904: 161; 1906: 96 - on parrot skin, New Guinea 
Cheyletus fortis (Oud.): Baker, 1949: 280; Tanaka, 1953: 73; Shiba, 1966: 142; 1969a: 161; Volgin, 1969: 87; Summers \& Price, 1970: 28; Fain \& Nadchatram, 1980: 191; Patxot \& Goff, 1985: 162; Mariana et al., 2000: 714; Ezequiel et al., 2001: 913

Type locality: Malacca, Straits Settlements

Distribution: Algeria, Australia (Queensland), Azerbaidzan, Bangladesh, Brazil, Bulgaria, Burma, China, Czech Rep., D. R. Congo, Cuba, Greece, India, Iran, Israel, Japan, Malaysia, New Guinea, Pakistan, Philippines, Peru, Russia, Rwanda, Saudi Arabia, Senegal, Tadjikistan, Taiwan, USA (Hawaian is.)

Hosts: Psittinus incertus (Psittaciformes: Psittacidae), Pipistrellus abramus (Chiroptera: Vespertilionidae), Callosciurus sladeni midas, Petinomys setosus (Rodentia: Sciuridae), Thalpomys [Akodon] reinhardti (Rodentia: Arvicolidae, Sigmodontinae); Microtus arvalis (Rodentia: Arvicolidae), nests of birds and rats; for Cheyletus fortis: rodents (Callosciurus, Ratufa, Rattus). According to Fain \& Nadchatram (1980), „accidental contamination“

\section{Cheyletus malayensis Cunliffe}

Cheyletus malayensis Cunliffe, 1962: 201; Patxot \& Goff, 1985: 161; Fain \& Nadchatram, 1980: 192; Mariana et al., 2000: 714; Torre Santana, 2015

Type locality: Rantau Panjang, $5 \mathrm{mi} \mathrm{N}$ of Klang, Selangor, Malaysia

Distribution: Cuba, India, Iran, Malaysia, USA (Hawaian Is. - Oahu)

Hosts: nests of Lonchura malacca (= Munia atricapilla), Munia striata, Pycnonotus goiaver

(Passeriformes: Estrildidae)

Cheyletus misonnei Fain et Lukoschus

Cheyletus misonnei Fain \& Lukoschus, 1981: 1

Type locality: Aminuis

Distribution: Namibia

Host: Tatera sp. (Rodentia: Arvicolidae)

\section{Cheyletus morinus Barilo}

Cheyletus morinus Barilo, 1986: 27

Type locality: Uzbekistan

Distribution: Uzbekistan

\section{Cheyletus nidicolus Fain}

Cheyletus nidicolus Fain, 1972: 37; 1979: 280

Type locality: Butare

Distribution: Rwanda, South Africa

Host: birds' nests

Cheyletus philippinensis Corpuz-Raros

Cheyletus philippinensis Corpuz-Raros, 1988: 328 
Type locality: Philippines

Distribution: Philippines

Hosts: woodpeckers (Picoidea: Picidae), Harpactes ardens (Trogoniformes: Trogonidae)

Cheyletus pluridens Fain et Nadchatram

Cheyletus pluridens Fain \& Nadchatram, 1980: 195

Type locality: Kuantan (Pahang), Ulu Langat Forest Reserve (Selangor)

Distribution: Malaysia (Selangor)

Host: Rhinosciurus laticaudatus (Rodentia: Sciuridae)

Cheyletus pseudomalaccensis Fain

Cheyletus pseudomalaccensis Fain, 1982: 86

Type locality: Potchefstroom, Transvaal

Distribution: South Africa

Host: Plocepasser mahali (Passeriformes: Ploceidae)

Cheyletus punctulatus Fain et Lukoschus

Cheyletus punctulatus Fain \& Lukoschus, 1981: 121

Type locality: $20 \mathrm{~km}$ N. of Oned Draa, Southern Morocco

Distribution: Morocco

Host: Gerbillus pyramidum (Rodentia: Muridae, Gerbillinae)

Cheyletus rarus Shiba

Cheyletus rarus Shiba, 1969: 162

Type locality: Kuwabara-- sho, Matsuyama

Distribution: Japan

Cheyletus rashtiensis Salarzehi, Hajizadeh et Ueckermann

Cheyletus rashtiensis Salarzehi, Hajizadeh \& Ueckermann, 2018: 641; Khaustov, 2021: 228

Type locality: Rasht (Iran)

Distribution: Iran, Russia (Siberia)

Cheyletus rwandae Fain

Cheyletus rwandae Fain, 1972: 38; 1979: 282

Type locality: Butare

Distribution: D. R. Congo, Rwanda

Host: Tachyoryctes rwandae (Rodentia: Rhizomyidae)

Cheyletus schneideri (Oudemans)

Cheyletus schneideri (Oud.): Oudemans, 1902: 15; Turk, 1946: 805; Baker, 1949: 281;

Volgin, 1969: 102 
Cheletes schneideri Oudemans, 1904: 16; 1906: 84

Type locality: San Remo (Italy)

Distribution: England, Italy

\section{Cheyletus strennus (Oudemans)}

Cheletes strennus Oudemans, 1904: 161; 1906: 83

Cheyletus strennus: Baker, 1949: 280

Type locality: unknown

Distribution: unknown

Host: unknown

\section{Cheyletus tanzaniensis (Fain)}

Eucheyletia tanzaniensis Fain, 1972 : 39

Cheyletus tanzaniensis (Fain): Fain \& Bochkov, 2001: 101

Type locality: Tanganyika

Distribution: Tanzania

Hosts: Coleura gallarum (Chiroptera: Emballonuridae); nests of rodents (Dasymys incomtus, Pelomys walambae, Textor sp., Lagonosticta senegallus)

\section{Cheyletus trouessarti Oudemans}

Cheletes trouessarti Oudemans, 1902: 16; 1903: 129; 1906: 88

Cheyletus macrocherus Hardy, 1933: 350

Cheyletus furibundus Rohdendorf, 1940: 85 - Kirzhach, Russia (Vladimirskaya obl.)

Cheyletus davisi Baker, 1949: 283 - Italy, intercepted at Boston, Mass. (syn. by Summers \& Price, 1970)

Cheyletus truculentus Volgin, 1949: 586; 1969: 113 - Karaganda, Kazakhstan

Cheyletus praedabundus Kuzin, in Rohdendorf, 1940: 85 - Rostov on Don, Russia

Cheyletus woodroffei Jeffrey, 1980: 46 - Upton Grey, Hampshire, England, from a bat roost (probably Plecotus auritus)

Cheyletus trouessarti (Oudemans): Oudemans, 1903: 16; Baker, 1949: 280; Volgin, 1969: 109; Shen, 1975; Caceres \& Fain, 1978: 302; Mumcuoglu, 1976: 365; Hallas, 1978: 30; Ždárková, 1979; Haitlinger, 1982: 442; Philips \& Dindal, 1990: 177; Lucza, Ripka \& Saly, 1996; Bochkov, Hakimitabar \& Saboori, 2005: 106; Lukáš et al., 2007: 97; Xia, 2010: 162 Type locality: unknown

Distribution: Bulgaria, China, Czech Rep., Denmark, England, Holland, Hungary, India, Iran, Kazakhstan, Peru, Poland, Russia, Switzerland, USA

\section{Cheyletus trux Rohdendorf}

Cheyletus trux Rohdendorf, 1940: 87; Baker, 1949: 282; Rekk, 1976: 68; Vysotskaja, 1977:

188; Efremova \& Bochkov, 1990: 99; Eliopoulos \& Papadoulis, 2001:

Cheyletus hendersoni Baker, 1949: 279 - Arkansas Cave, USA; "Czechoslovakia” 
Cheyletus tenuipilis Fain, Feldman-Musham \& Mumcuoglu, 1980: 35 - La Louvière (Belgium); Louadi \& Robaux, 1992: 183

Type locality: Ivanovo, Tatarstan

Distribution: Algeria, Belarus, Belgium, “Czechoslovakia”, Georgia, Greece, Israel, Russia, Switzerland, Ukraine, USA (Arkansas, New Mexico, Colorado)

Host: nests, stored grain, house dust

Cheyletus venator Vitzthum

Cheletes venator Vitzthum, 1920: 2

Cheyletus venator (Vitzthum) Volgin, 1969: 119

Type locality: Amani

Distribution: Tanzania

Host: nest of Xylocopa (Afroxylocopa) nigrita (Hymenoptera: Apidae)

Cheyletus vivatus Qayyum et Chaudhri

Cheyletus vivatus Qayyum \& Chaudhri, 1977: 92; Halliday, Kamran \& Bashir, 2018: 87

Cheyletus spatiosus Qayyum \& Chaudhri, 1977: 94

Cheyletus tutela Qayyum \& Chaudhri, 1977: 95

Type locality: Pakistan

Distribution: Pakistan

Cheyletus volgini Fain et Bochkov

Cheyletus volgini Fain \& Bochkov, 2001: 95

Type locality: Russia

Distribution: Russia

Cheyletus zumpti Fain

Cheyletus zumpti Fain, 1972: 38; 1979: 281

Type locality: Garamba Nat. Park

Distribution: D. R. Congo, South Africa, Nigeria, Rwanda

Host: nests of rodents; type from Tatera (Rodentia: Arvicolidae)

Species inquirendae:

Cheyletus acer Oudemans

Cheletes acer Oudemans, 1904b:162; 1906: 112

Cheyletus acer (Oudemans): Baker, 1949: 286; Volgin, 1969: 94

Type locality: Colombia

Distribution: Colombia

Host: Aves (Birdskin)

Cheyletus alacer (Oudemans)

Cheletes alacer Oudemans, 1904b: 162; 1906: 108 
Cheyletus alacer Oudemans: Baker, 1949: 285

Type locality: Colombia

Distribution: Colombia

Host: skin of Pyrrhura cruentata (Psittaciformes: Psittacidae)

Cheyletus audax Oudemans

Cheletes audax Oudemans, 1904: 162; 1906: 99

Cheyletus audax (Oudemans): Baker, 1949: 285; Volgin, 1969: 93

Type locality: New Guinea

Distribution: New Guinea

Host: skin (Aves indet.)

Cheyletus burmiticus Cockerell

Cheyletus burmiticus Cockerell, 1917: 41; Baker, 1949: 287 (inc. sedis)

Type locality: Burma

Distribution: Burma

Host: Burmese amber

Cheyletus clavispinus Banks

Cheyletus clavispinus Banks, 1902: 172; 1906: 746

Type locality: Indianapolis (Indiana)

Distribution: USA (Indiana)

Host: Beneath wings of an Aradus (Heteroptera)

Cheyletus crassus Qayyum et Chaudhri

Cheyletus crassus Qayyum \& Chaudhri, 1977: 87; Chaudhri, Akbar \& Rasool, 1979: 153; Akbar, Rahi \& Chaudhri, 1988: 5; Gerson, Fain \& Smiley, 1999: 55; Farooq, Akbar \&

Qureshi, 2000: 257; Fain \& Bochkov, 2001: 84; Halliday, Kamran \& Bashir, 2018: 86 Type locality: Pakistan

Distribution: Pakistan

Cheyletus ferox Trouessart

Cheyletus ferox Trouessart, 1889: 134; Volgin, 1969: 96

Cheletes ferox, Oudemans, 1906: 115

Type locality: Praslin

Distribution: Seychelle Is.

Host: Coracopsis nigra barklyi (Psittaciformes: Psittacidae)

Cheyletus intrepidus (Oudemans)

Cheletes intrepidus Oudemans, 1903b: 84; 1906: 119

Cheyletus intrepidus, Volgin, 1969: 98

Type locality: Colombia 
Distribution: Colombia

Host: Aves indet.

Cheyletus nigriceps Mola

Cheyletus nigriceps Mola, 1907: 43

Type locality: Europe

Distribution: Europe

Cheyletus parumsetosus Karpelles

Cheyletus parumsetosus Karpelles, 1884: 238

Cheletes parumsetosus: Oudemans, 1906: 81

Type locality: Europe

Distribution: Europe

Cheyletus patagiatus Nordenskiold

Cheyletus patagiatus Nordenskiold, 1900: 37

Type locality: Finland?

Distribution: Finland?

Cheyletus promptus Oudemans

Cheletes promptus Oudemans, 1904b: 161; 1906: 81

Type locality: New Guinea

Distribution: New Guinea

Host: bird's skin

Cheyletus rapax (Oudemans)

Cheletes rapax Oudemans, 1903a: 84; 1906: 103

Cheyletus rapax (Oudemans): Baker, 1949: 284; Volgin, 1969: 85

Type locality: Mariana is.

Distribution: Mariana Is.

Hosts: birds and mammals in det.

Cheyletus saevus (Oudemans)

Cheletes saevus Oudemans, 1904b: 161; 1906: 84

Cheyletus saevus Oudemans: Baker, 1949: 287

Type locality: Netherlands

Distribution: Netherlands

Host: in dust

Genus Eucheyletia Baker

Eucheyletia Baker, 1949: 294

Type species: Eucheyletia bishoppi Baker, 1949 


\section{Eucheyletia bakeri Volgin}

Eucheyletia bakeri Volgin, 1963a: 62; 1969: 149; Fain \& Bochkov, 2001: 49

Type locality: Alaska

Distribution: USA (Alaska)

Host: Bombus sp. (Hymenoptera: Apidae)

\section{Eucheyletia bishoppi Baker}

Eucheyletia bishoppi Baker, 1949: 295; Volgin, 1963: 57; 1969: 142; Summers \& Price, 1970: 32; Fain \& Bochkov, 2001: 49; Xia et al., 2004; Xia, 2010: 162

Eucheyletia asiatica Volgin, 1955: 166; 1969: 144; Vysotzkaja, 1974: 136 - Kazakhstan, Ukraine, in nests of rodents

Eucheyletia oregonensis Smiley \& Whitaker, 1981: 11 - type from Oregon, nest of Glaucomys sabrinus (Sciuridae); Shen, 1991; Xia, 2010: 162

Type locality: California

Distribution: China, Kazakhstan, Ukraine, USA (California, Oregon)

Hosts: nest of Neotoma fuscipes (Rodentia: Arvicolidae), soil samples; from Tamias striatus and nest of Glaucomys sabrinus (Sciuridae); from Sorex pacificus and S. trowbridgei (Soricomorpha: Soricidae)

\section{Eucheyletia bothrophila Volgin}

Eucheyletia bothrophila Volgin, 1963a: 44; 1969: 126; Vysotskaja, 1977: 188; Efremova \& Bochkov, 1990: 102; Fain \& Bochkov, 2001: 49

Type locality: Khustovskiy Distr., Zakarpatskaya obl.

Distribution: Belarus, Ukraine

Host: nests of rodents (Myodes = Clethrionomys glareolus, Microtus arvalis, M. nivalis, Sylvaemus flavicollis, Sciurus vulgaris)

\section{Eucheyletia eoa Volgin}

Eucheyletia eoa Volgin, 1963a: 52; 1969: 134; Fain \& Bochkov, 2001: 49

Type locality: Olginski Region, Imanskiy Region (Primorskiy Krai)

Distribution: Russia (Primorskiy Krai in the Far East)

Host: nests of rodents (Eutamias sibiricus, Apodemus speciosus)

\section{Eucheyletia flabellifera (Michael)}

Cheyletus flabellifer Michael, 1878: 135; Mégnin, 1880: 241

Cheyletus (Cheyletia) flabellifer: Berlese, 1893: 64

Cheyletia flabellifera: Oudemans, 1904a: 162

Cheletia flabellifera, Oudemans, 1906: 127

Eucheyletia flabellifera (Michael): Baker, 1949: 295; Volgin, 1963a: 47; 1969: 124;

Kolebinova, 1967: 75; Shen, 1975; Haitlinger, 1982: 442; Fain \& Bochkov, 2001: 49; Xia, 2010: 162; Salarzehi et al., 2018: 480

Eucheyletia taurica Volgin, 1963a: 48; 1969: 130 - Gurzuf, Crimea 
Type locality: Warwikshire (England)

Distribution: Austria, Belgium, Bulgaria, China, England, Germany, Holland, Iran, Poland, Crimea

Hosts: Myodes [Clethrionomys] glareolus, Microtus arvalis (Rodentia: Arvicolidae), Sylvaemus flavicollis, S. sylvaticus (Muridae), Glis glis (Gliridae), Crocidura russula, C. suaveolens (Soricomorpha: Soricidae), Talpa europaea (Soricomorpha: Talpidae), birds

\section{Eucheyletia hardyi Baker}

Eucheyletia hardyi Baker, 1949: 296; Volgin, 1963: 56; 1969: 141; Summers \& Price, 1970: 33; Mosquera, 1989: 73; Fain \& Bochkov, 2001: 49

Type locality: Harlingen, Texas

Distribution: Argentina, Chile, USA (Texas)

Host: nest of Neotoma micropus (Rodentia: Arvicolidae)

\section{Eucheyletia harpyia (Rohdendorf)}

Cheyletia harpyia Rohdendorf, 1940: 90

Eucheyletia harpyia (Rohdendorf): Baker, 1949: 294; Volgin, 1963a: 59; 1969: 148; Shen, 1975; Fain \& Bochkov, 2001: 49; Xia, 2010: 162

Type locality: Ivanovskaya Oblast (Russia)

Distribution: China, Russia

Eucheyletia nidicola Definado et Khaing-Fields

Eucheyletia nidicola Definado \& Khaing-Fields, 1976: 193

Type locality: New York

Distribution: USA

Eucheyletia omissa Xia, Liang et Zhu

Eucheyletia omissa Xia, Liang \& Zhu, 2004; Xia, 2010: 162

Type locality: China

Distribution: China

\section{Eucheyletia pavlovskyi Volgin}

Eucheyletia pavlovskyi Volgin, 1963a: 54; 1969: 138; Fain \& Bochkov, 2001: 49

Type locality: Karaganda Area

Distribution: Kazahstan

Host: nest of Lagurus lagurus (Rodentia: Arvicolidae)

\section{Eucheyletia sibirica Volgin}

Eucheyletia sibirica Volgin, 1963a: 61; 1969: 151; Efremova \& Bochkov, 1990: 98; Fain \& Bochkov, 2001: 49

Type locality: Asinovskiy Region, Tomskaya obl.

Distribution: Belarus, Russia 


\section{Eucheyletia sinensis Volgin}

Eucheyletia sinensis Volgin, 1963a: 50; 1969: 133; Summers \& Price, 1970: 33; Whitaker,

Baccus, Cleveland \& Gu, 1990; Fain \& Bochkov, 2001: 49; Xia, 2010: 162

Type locality: Yunnan

Distribution: China (Yunnan), Nepal, Taiwan

Hosts: Niviventer fulvescens, Rattus losea [humiliatus] celsus (Rodentia: Muridae)

\section{Eucheyletia variformis Lin}

Eucheyletia variformis Lin, 2000: 187

Type locality: Fujian

Distribution: China

Eucheyletia womersleyi Volgin (inc. sed.)

Eucheyletia womersleyi Volgin, 1963: 59; 1969: 121

Type locality: Australia

Distribution: Australia

Genus Granulocheyletus Fain et Bochkov

Granulocheyletus Fain \& Bochkov, 2002: 37

Type species: Granulocheyletus corpuzrarosae Fain et Bochkov, 2002

Granulocheyletus corpuzrarosae Fain et Bochkov

Granulocheyletus corpuzrarosae Fain \& Bochkov, 2002: 38

Type locality: Weelawadjia Cave, near Eneabba, Western Australia

Distribution: Australia

\section{Granulocheyletus gallowayi Fain}

Granulocheyletus gallowayi Fain, 2003: 100

Type locality: Motunau Isl., New Zealand, from the nest of Eudyptula albosignata (Sphenisciformes: Spheniscidae)

Distribution: New Zealand

\section{Granulocheyletus kureatollensis (Goff)}

Hemicheyletia kureatollensis Goff, 1982: 83

Granulocheyletus kureatollensis (Goff): Fain \& Bochkov, 2002: 40; Fain, 2003: 101

Type locality: Kure Atoll

Distribution: Hawaiian Is.

Genus Kenyacheylus Fain et Bochkov

Kenyacheylus Fain \& Bochkov, 2001: 56

Type species: Kenyacheylus troglodytes Fain et Bochkov, 2001 
Kenyacheylus troglodytes Fain et Bochkov

Kenyacheylus troglodytes Fain \& Bochkov, 2001: 56

Type locality: Kimakia Cave, Kiboko, 140 km SE Nairobi

Distribution: Kenya

Genus Ker Muma

Ker Muma, 1964: 250

Type species: Ker palmatus Muma, 1964

Ker acidalia Aheer, Akbar et Chaudhri

Ker acidalia Aheer, Akbar \& Chaudhri, 1997: 119; Halliday, Kamran \& Bashir, 2018: 87

Type locality: Pakistan

Distribution: Pakistan

Ker afrotropicalis Fain et Bochkov

Ker afrotropicalis Fain \& Bochkov, 2001: 59

Type locality: Musha, Rwanda

Distribution: D.R. Congo, Rwanda

Host: nest of Grammomys sp. (Rodentia: Muridae)

Ker bakeri Zaher et Soliman

Ker bakeri Zaher \& Soliman, 1967: 24; Summers \& Price, 1970: 22; Rosa \& Flechtmann, 1979: 196; Ždárková, 1979; Fain \& Bochkov, 2001: 58; Ezequiel et al., 2001: 913

Type locality: Giza (Egypt)

Distribution: Brazil, Egypt, Malaysia, "Czechoslovakia"

Host: house dust

Ker caeterus Barilo

Ker caeterus Barilo, 1986: 299; Fain \& Bochkov, 2001: 58

Type locality: Uzbekistan

Distribution: Uzbekistan

Ker mercedesae Corpuz-Raros (species dubia)

Ker mercedesae Corpuz-Raros, 1998: 279; Fain \& Bochkov, 2001: 58

Type locality: Leyte Is., Visayas St. Col. Agriculture, Baybay, Leyte

Distribution: Philippines

\section{Ker palmatus Muma}

Ker palmatus Muma, 1964: 226; Volgin, 1969: 226; Summers \& Price, 1970: 22; Patxot \& Goff, 1985: 161; Shen, 1991; Lin et al., 2000; Fain \& Bochkov, 2001: 58; Xia, 2010: 162 Type locality: Florida (Sebring)

Distribution: China, USA (Florida, Hawaian Is - Nihoa) 


\section{Ker pintoriensis Corpuz-Raros}

Ker pintoriensis Corpuz-Raros, 1998: 281; Fain \& Bochkov, 2001: 58

Type locality: Luzon Is., Pintor, Gamu, Isabela

Distribution: Philippines

Genus Laeliocheyletia Summers et Price

Laeliocheyletia Summers \& Price, 1970: 21

Type species: Laeliocheyletia teretis Summers et Price, 1970

Laeliocheyletia teretis Summers et Price

Laeliocheyletia teretis Summers \& Price, 1970: 21

Type locality: Quetzaltepeque

Distribution: El Salvador

Host: Tenebrionidae (Coleoptera)

Genus Lanceacheyla Xia, Klompen et Childers

Lanceacheyla Xia, Klompen \& Childers, 2011: 30

Type species: Lanceachyla whartoni Xia, Klompen et Childers, 2011

Lanceacheyla filipina Corpuz-Raros et Naredo

Lanceacheyla filipina Corpuz-Raros \& Naredo, 2016: 2

Type locality: Luzon Is., Santol Cave, Biak—na-bato Nat. Park, San Miguel, Bulacan Prov.

Distribution: Philippines

Lanceacheyla whartoni Xia, Klompen et Childers

Lanceacheyla whartoni Xia, Klompen \& Childers, 2011: 30

Type locality: Florida, Hardee Co, $10 \mathrm{~km}$ S of Ona, Lilly

Distribution: USA (Florida)

Host: citrus trees

Genus Lepidocheyla Volgin

Lepidocheyla Volgin, 1963: 940

Type species: Lepidocheyla gracilis Volgin, 1963

\section{Lepidocheyla caucasica Volgin}

Lepidocheyla caucasica Volgin, 1978: 940; Fain \& Bochkov, 2001: 55

Hemicheyletia hissariensis Mathur \& Mathur, 1981: 69 (syn of L. gracilis, acc. to Fain \& Bochkov, 2001: 55) - Hissar, India (debris of the wheat straw)

Type locality: Azerbaidjan, Nahichevanskaya obl., Karachuk Village

Distribution: Azerbaidjan, India 


\section{Lepidocheyla gracilis Volgin}

Lepidocheyla gracilis Volgin, 1963: 940; 1969: 221; Lin et al., 1998; Fain \& Bochkov, 2001:

55; Bochkov, Hakimitabar \& Saboori, 2005: 103; Xia, 2010: 162

Type locality: Verkhnaya Terrasa (Tadjikistan), Donezk (Ukraine)

Distribution: China, Iran, Israel, Tadjikistan, Turkmenia, Ukraine

Genus Nidocheyletus Bochkov et OConnor

Nidocheyletus Bochkov \& OConnor, 2010: 60

Type species: Nidocheyletus kennedyae Bochkov et OConnor, 2010

Nidocheyletus kennedyae Bochkov et OConnor

Nidocheyletus kennedyae Bochkov \& OConnor, 2010: 60

Type locality: Michigan

Distribution: USA (Michigan)

Genus Nodele Muma

Nodele Muma, 1964: 252

Neocheletophyes Volgin, 1965: 296

Type species: Nodele calamondin Muma, 1964

Type species of Neocheletophyes Volgin: Cheletophyes philippinensis Baker, 1949

Nodele calamondin Muma

Nodele calamondin Muma, 1964: 252; Volgin, 1969: 162; Summers \& Price, 1970: 41;

Fain \& Bochkov, 2001: 54; Bochkov, Hakimitabar \& Saboori, 2005: 107

Nodele simplex Wafa et Soliman, 1968: 223 - Giza, Egypt - synonymized by Bochkov et al., 2001: 123

Type locality: Florida, Fort Myers

Type locality of Nodele simplex: Giza, Egypt

Distribution: Egypt, Iran, USA (Florida, California)

Nodele coccineae Thewke et Enns

Nodele coccineae Thewke \& Enns, 1968: 216; Summers \& Price, 1970: 42; Fain \& Bochkov, 2001: 54

Type locality: Missouri

Distribution: USA (Missouri)

Host: in galleries of bark beetle, Pseudopityophthorus minutissimus, in scarlet oak

Nodele $\boldsymbol{m u}$ Haines

Nodele mu Haines, 1988: 362; Fain \& Bochkov, 2001: 54

Type locality: ? Bogor, Java

Distribution: Indonesia or Tanzania

Host: Dinoderus minutus (Coleoptera: Bostrychidae) 


\section{Nodele philippinensis (Baker)}

Cheletophyes philippinensis Baker, 1949: 288

Neocheletophyes philippinensis (Baker): Volgin, 1965: 296

Nodele philippinensis (Baker): Muma, 1964: 252; Volgin, 1969: 163; Summers \& Price, 1970: 42; Diaz Patxot \& Goff, 1985: 161; Fain \& Bochkov, 2001: 54

Type locality: Bataan (Philippines)

Distribution: Philippines, USA (Oahu I., Hawaii Is.)

\section{Nodele superba Kuznetzov}

Nodele superba Kuznetzov, 1977: 923; Fain \& Bochkov, 2001: 54

Type locality: Ukraine

Distribution: Ukraine

\section{Genus Paracheyletia Volgin}

Paracheyletia Volgin, 1955: 168

Type species: Cheyletus pyriformis Banks, 1904

\section{Paracheyletia pyriformis (Banks)}

Cheyletus pyriformis Banks, 1904: 17; 1906: 135

Cheyletus longipalpus Ewing, 1909: 54

Cheyletia pyriformis, Garman, 1948: 1; Baker, 1949: 298

Paracheyletia assimilis Volgin, 1955: 168 - England (syn. by Summers \& Price, 1970: 19) Paracheyletia pyriformis, Volgin, 1969: 177; Summers \& Price, 1970: 19; André, 1975: 31; Bochkov, Hakimitabar \& Saboori, 2005: 107; Bochkov \& Abramov, 2016; Khaustov, 2021: 233

Paracheyletia hortensis Volgin, 1969: 181; (acc. to Fain \& Bochkov, 2001, a synonym) Krasnodarskaiy Kray, Slavyanskiy Rayon, Russia

Paracheyletia samsinaki Volgin, 1966b: 281; 1969: 179 (acc. to Fain \& Bochkov, 2001, a synonym) - Prague, Czech Rep., on Scolytus pygmaeus (Coleoptera: Scolytidae)

Type locality: Kirkwood, Mo.

Distribution: Algeria, Belgium, Czech Rep., Germany, Iran, Russia, USA (Montana, Illinois, Massachusetts, New York)

\section{Paracheyletia recki Volgin}

Paracheyletia recki Volgin, 1966b: 283; 1969: 183; Fain \& Bochkov, 2001: 51

Type locality: Voronezh Reserve

Distribution: Russia

Host: ant hill

\section{Genus Paracheyletiella Kuznetzov}

Paracheyletiella Kuznetzov, 1977: 926

Type species: Paracheyletiella volgini Kuznetzov, 1977 


\section{Paracheyletiella volgini Kuznetzov}

Paracheyletiella volgini Kuznetzov, 1977: 926

Type locality: Crimea

Distribution: Crimea

Host: sparrow nest

Genus Pavlovskicheyla Volgin

Pavlovskicheyla Volgin, 1965: 290

Type species: Cheletophyes semenovi Kuzin in Rohdendorf, 1940

Pavlovskicheyla philippicana Corpuz - Raros

Pavlovskicheyla philippicana Corpuz - Raros, 1998: 285; Fain \& Bochkov, 2001 : 58

Type locality: Luzon Is., Sta Rosa, Laguna

Distribution: Peru, Philippine Islands

Pavlovskicheyla platydemae Thewke et Enns

Pavlovskicheyla platydemae Thewke \& Enns, 1975: 671

Type locality: Missouri

Distribution: USA (Missouri)

Host: Platydema sp. (Coleoptera: Tenebrionidae)

\section{Pavlovskicheyla semenovi (Kuzin)}

Cheletophyes semenovi Kuzin in Rohdendorf, 1940: 94; Bakeri, 1949: 290

Pavlovskicheyla semenovi: Volgin, 1965: 292; 1969: 229; Fain \& Bochkov, 2001: 57

Type locality: Andizhanskaya Prov.

Distribution: Uzbekistan

Host: from old seeds of the cotton-plant

Genus Tutacheyla Corpuz - Raros

Tutacheyla Corpuz - Raros, 1972: 266

Indonesicheyla Thewke, 1980: 339

Type species: Tutacheyla robusta Corpuz-Raros, 1972

Type species of Indonesicheyla Thewke: I. buruensis Thewke, 1980

\section{Tutacheyla robusta Corpuz-Raros}

Tutacheyla robusta Corpuz-Raros, 1972: 266

Indonesicheyla buruensis Thewke, 1980: 339 - type: Indonesia (Buru)

Tutacheyla buruensis (Thewke)

Type locality: Philippines

Distribution: Indonesia (Buru), Philippines

Host: pin-hole borer (Coleoptera: Platypodidae) 
Genus Zachvatkiniola Volgin

Zachvatkiniola Volgin, 1969: 156

Type species: Eucheyletia reticulata Cunliffe, 1962

\section{Zachvatkiniola reticulata (Cunliffe)}

Eucheyletia reticulata Cunliffe, 1962: 200; Volgin, 1963: 63; Volgin, 1963: 63; 1969: 157;

Summers \& Price, 1970: 34; Shen, 1975; Nishida (Version 2009)

Zachvatkiniola reticulata (Cunliffe): Volgin, 1969: 157; Bochkov, Hakimitabar \& Saboori, 2005: 106; Xia, 2010: 162

Type locality: Tahiti (Hawaiian Quarantine)

Distribution: China (Guandong), Georgia, Iran, Japan, French Polynesia (Tahiti)

Tribus Acaropsellini Volgin

Acaropseini [sic] Volgin, 1969: 307

Acaropsellini: Bochkov \& Fain, 2003: 24

Type genus: Acaropsella Volgin, 1969

Genus Acaropsella Volgin

Acaropsella Volgin, 1969: 314

Type species: Neoacaropsis rohdendorfi Volgin, 1962

\section{Acaropsella kinshasensis Fain}

Acaropsella kinshasensis Fain, 1972: 39; Fain \& Bochkov, 2001: 68; Xia, 2010: 160

Acaropsella konoi Tseng, 1977: 245

Acaropsella filipina Corpuz-Raros, 1988: 415

Type locality: Kinshasa (Congo)

Distribution: DR Congo, Philippines, Taiwan

\section{Acaropsella kulagini (Rohdendorf)}

Acaropsis kulagini Rohdendorf, 1940: 78; Baker, 1949: 313

Neoacaropsis kulagini: Volgin, 1962: 699

Acaropsella kulagini (Rohdendorf): Volgin, 1969: 319; Summers \& Price, 1970: 63; Fain

\& Bochkov, 2001: 68; Bochkov, Hakimitabar \& Saboori, 2005: 105

Type locality: Blagodarnoe Village, Stavropol District (Russia)

Distribution: Iran, Russia, USA (California)

Acaropsella nobilis Rasool, Chaudhri et Akbar

Acaropsella nobilis Rasool, Chaudhri \& Akbar, 1980: 33; Chaudhri \& Akbar, 1985: 198;

Akbar, Jahan \& Mughal, 2008: 188; Gerson, Fain \& Smiley, 1999: 39; Halliday, Kamran

\& Bashir, 2018: 80

Type locality: Pakistan 
Distribution: Pakistan

\section{Acaropsella rohdendorfi (Volgin)}

Neoacaropsis rohdendorfi Volgin, 1962: 697

Acaropsella rohdendorfi (Volgin): Volgin, 1969: 315; Summers \& Price, 1970: 63; Fain \&

Bochkov, 2001: 68

Type locality: Kishinev, under Lepidosaphes ulni (Homoptera: Coccodea)

Distribution: Moldova

\section{Acaropsella schmidtmanni Price}

Acaropsella schmidtmanni Price, 1972: 47; Fain \& Bochkov, 2001: 68

Type locality: California

Distribution: USA (California)

Acaropsella shaziai Akbar, Jahan et Mughal

Acaropsella shaziai Akbar, Jahan \& Mughal, 2008: 188; Halliday, Kamran \& Bashir, 2018: 80 Type locality: Shekhupura, Punjab

Distribution: Pakistan

\section{Acaropsella volgini (Gerson)}

Neoacaropsis volgini Gerson, 1967: 361

Acaropsis aegyptiaca Wafa \& Soliman, 1968: 221 - Egypt (Giza and Tahreer), nest of birds, debris (synonymized by Fain \& Bochkov, 2001: 67)

Acaropsella aegyptiaca (Wafa et Soliman): Summers \& Price, 1970: 64

Acaropsella volgini (Gerson): Volgin, 1969: 317; Summers \& Price, 1970: 64; Rostom, 1993 :

29 (with “?”); Koç, 1998: 195; Fain \& Bochkov, 2001: 68; Salarzehi, 2018:

Acaropsella walii Akbar, Jahan et Mughal

Acaropsella walii Akbar, Jahan \& Mughal, 2008: 189; Halliday, Kamran \& Bashir, 2018: 80 Type locality: Pakistan

Distribution: Pakistan

\section{Genus Acaropsellina Summers}

Acaropsellina Summers, 1976: 191

Acaropsis Moquin -Tandon, 1863: 314

Type species: Acaropsis sollers Kuzin in Rohdendorf, 1940

Acaropsellina altilis (Haq et Zia)

Acaropsis altilis Haq \& Zia, 2001b: 61; Haq, Khan, Riaz \& Rasool, 2004: 182; Haq \& Afzal, 2007: 2206

Acaropsellina altilis (Haq et Zia): Halliday, Kamran \& Bashir, 2018: 82

Type locality: Pakistan 
Distribution: Pakistan

Acaropsellina anarsia Summers

Acaropsellina anarsia Summers, 1976: 193

Type locality: Colorado

Distribution: USA

Acaropsellina clamo (Qayyum et Chaudhri)

Acaropsis clamo Qayyum et Chaudhri, 1979: 158; Haq, Khan, Riaz \& Rasool, 2004: 182

Acaropsellina clamo (Qayyum \& Chaudhri): Gerson, Fain \& Smiley, 1999: 39; Halliday,

Kamran \& Bashir, 2018: 82

Type locality: Pakistan

Distribution: Pakistan

Acaropsellina culpa (Haq et Zia)

Acaropsis culpa Haq \& Zia, 2001b: 64; Haq, Khan, Riaz \& Rasool, 2004: 182

Acaropsellina culpa (Haq et Zia): Halliday, Kamran \& Bashir, 2018: 82

Type locality: Pakistan

Distribution: Pakistan

\section{Acaropsellina docta (Berlese)}

Cheyletus doctus Berlese, 1886: 33; 1893: 74

Acaropsis docta (Berlese): Oudemans, 1905: 209; Baker, 1949: 314; Volgin, 1969: 309;

Summers \& Price, 1970: 62; Shen, 1975; Rizk et al., 1979; Qayyum \& Chaudhri, 1979c:

2; Farooq, 2000: 41; Haq \& Zia, 2001a: 60; Haq \& Zia, 2001b: 68; Haq, Khan, Riaz \&

Rasool, 2004: 183; Palyvos \& Emmanouel, 2006: 25

Acaropsellina docta (Berlese): Summers, 1976: 194; Lucza, Ripka \& Saly, 1996; Gerson, Fain \&

Smiley, 1999: 39;.Xia, 2010: 161; Salarzehi et al., 2018; Halliday, Kamran \& Bashir, 2018: 82 Type locality: Florence, Italy

Distribution: China, Egypt, Great Britain, Greece, Holland, Hungary, India, Italy, Iran, Iraq

Acaropsellina hirnai (Akbar, Jahan \& Qazi)

Acaropsis hirnai Akbar, Jahan \& Qazi, 2007: 92

Acaropsellina hirnai (Akbar, Jahan \& Qazi): Halliday, Kamran \& Bashir, 2018: 82

Type locality: Pakistan

Distribution: Pakistan

Acaropsellina humilis (Haq et Zia)

Acaropsis humilis Haq \& Zia, 2001b: 72; Haq, Khan, Riaz \& Rasool, 2004: 183

Acaropsellina humilis (Haq et Zia): Halliday, Kamran \& Bashir, 2018: 82

Type locality: Pakistan

Distribution: Pakistan 
Acaropsellina levis (Corpuz-Raros)

Neoacaropsis levis Corpuz-Raros, 1972: 263

Acaropsellina levis (Corpuz-Raros): Fain \& Bochkov, 2001: 68

Type locality: Laguna

Distribution: Philippines

Host: plant (Morus alba)

Acaropsellina nanchangensis (Xia et Zhu)

Acaropsella nanchangensis Xia \& Zhu, 1997: 6; Xia, 2010: 160

Acaropsellina nanchangensis (Xia et Zhu): Fain \& Bochkov, 2001: 68

Type locality: Nanchang

Distribution: China

Acaropsellina opsis (Rasool, Chaudhri et Akbar)

Acaropsis opsis Rasool, Chaudhri \& Akbar, 1980: 34

Acaropsellina opsis (Rasool, Chaudhri \& Akbar): Gerson, Fain \& Smiley, 1999: 40; Halliday,

Kamran \& Bashir, 2018: 81

Type locality: Pakistan

Distribution: Pakistan

Acaropsellina orbis (Qayyum et Chaudhri)

Acaropsis orbis Qayyum \& Chaudhri, 1979: 2

Acaropsellina orbis (Qayyum \& Chaudhri): Gerson, Fain \& Smiley, 1999: 40; Halliday,

Kamran \& Bashir, 2018: 81

Type locality: Pakistan

Distribution: Pakistan

Acaropsellina philippinensis (Corpuz-Raros et Sotto)

Acaropsis philippinensis Corpuz-Raros \& Sotto, 1977: 144

Acaropsellina philippinensis (Corpuz-Raros \& Sotto): Corpuz-Raros, 1988: 415

Type locality: Tanggalian, Piddig, Ilocos Norte

Distribution: Philippine Is.

Acaropsellina platessa (Aheer, Akbar et Chaudhri)

Acaropsis platessa Aheer, Akbar \& Chaudhri, 1991: 336

Acaropsellina platessa (Aheer, Akbar et Chaudhri): Gerson, Fain \& Smiley, 1999: 40;

Halliday, Kamran \& Bashir, 2018: 81

Type locality: Pakistan

Distribution: Pakistan

Acaropsellina porta (Qayyum et Chaudhri)

Acaropsis porta Qayyum \& Chaudhri, 1979: 5 
Acaropsellina porta (Qayyum et Chaudhri): Gerson, Fain \& Smiley, 1999: 40; Halliday, Kamran \& Bashir, 2018: 82

Type locality: Pakistan

Distribution: Pakistan

Acaropsellina sheikhupuraensis (Akbar, Jahan et Qazi)

Acaropsis sheikhupuraensis Akbar, Jahan \& Qazi, 2007: 90

Acaropsellina sheikhupuraensis (Akbar et al.): Halliday, Kamran \& Bashir, 2018: 82

Type locality: Pakistan

Distribution: Pakistan

Acaropsellina sigilla (Haq, Khan, Riaz \& Rasool)

Acaropsis sigilla Haq, Khan, Riaz \& Rasool, 2004: 184

Acaropsellina sigilla (Haq, Khan, Riaz \& Rasool): Halliday, Kamran \& Bashir, 2018: 82

Type locality: Pakistan

Distribution: Pakistan

Acaropsellina shorkotiensis (Akbar, Rahi et Chaudhri)

Acaropsis shorkotiensis Akbar, Rahi \& Chaudhri, 1988: 2; Haq, Khan, Riaz \& Rasool, 2004: 184

Acaropsellina shorkotiensis (Akbar et al.): Gerson, Fain \& Smiley, 1999: 40; Halliday, Kamran \& Bashir, 2018: 82

Type locality: Pakistan

Distribution: Pakistan

Acaropsellina sodalis (Haq \& Zia)

Acaropsis sodalis Haq \& Zia, 2001b: 70

Acaropsellina sodalis (Haq \& Zia): Halliday, Kamran \& Bashir, 2018: 82

Type locality: Pakistan

Distribution: Pakistan

Acaropsellina sollers (Kuzin in Rohdendorf)

Acaropsis callida Kuzin in Rohdendorf, 1940: 78 - Rostov na Don, Russia, in grain

Acaropsis sollers: Kuzin in Rohdendorf, 1940: 78; Baker, 1949: 314; Volgin, 1969: 311;

Summers \& Price, 1970: 61; Rizk et al., 1979; Efremova \& Bochkov, 1990: 102; Ros-

tom, 1993: 29; Athanassiou et al., 2003: 413

Acaropsellina sollers (Kuzin in Rohdendorf): Bochkov, Hakimitabar \& Saboori, 2005: 105; Xia, 2010: 161

Type locality: Leningrad, Russia

Distribution: Belarus, Greece, India, Iran, Iraq, Kazakhstan, Russia, Saudi Arabia, Turkmenistan, Uzbekistan 
Acaropsellina subitus (Akbar, Mushtaq et Akbar)

Acaropsis subitus Akbar, Mushtaq \& Akbar, 1999: 264

Acaropsellina subitus (Akbar, Mushtaq \& Akbar): Halliday, Kamran \& Bashir, 2018: 82

Type locality: Pakistan

Distribution: Pakistan

Acaropsellina summersi (Smiley et Whitaker)

Acaropsis summersi Smiley \& Whitaker, 1981: 121

Acaropsellina summersi (Smiley et Whitaker): Whitaker \& Maser, 1985: 69; Gerson, Fain \& Smiley, 1999: 40

Type locality: Oregon

Distribution: USA

Host: Glaucomys sabrinus (nest)(Rodentia: Sciuridae, Sciurinae)

Acaropsellina tyrophagus (Elbadry)

Acaropsis tyrophagus Elbadry, 1969: 162

Acaropsellina tyrophagus (Elbadry): Gerson, Fain \& Smiley, 1999: 40

Type locality: Egypt

Distribution: Egypt

Acaropsellina unicus (Haq, Khan, Riaz et Rasool)

Acaropsis unicus Haq, Khan, Riaz \& Rasool, 2004: 186

Acaropsellina unictus (Haq, Khan, Riaz et Rasool): Halliday, Kamran \& Bashir, 2018: 82

Type locality: Muzaffarabad, N. Kashmir

Distribution: Pakistan

Acaropsellina venutus (Akbar, Mushtaq et Akbar)

Acaropsis venutus Akbar, Mushtaq \& Akbar, 1999: 163

Acaropsellina venutus (Akbar, Mushtaq et Akbar): Halliday, Kamran \& Bashir, 2018: 82

Type locality: Pakistan

Distribution: Pakistan

Acaropsellina vitrus (Aheer, Akbar et Chaudhri)

Acaropsis vitrus Aheer, Akbar \& Chaudhri 1991: 336; Akbar, Mushtaq \& Akbar, 1999:

263; Farooq, 2000: 41; Haq \& Zia, 2001a: 60; Haq, Khan, Riaz \& Rasool, 2004: 182;

Haq \& Afzal, 2007: 2206.

Acaropsellina vitrus (Aheer, Akbar et Chaudhri): Gerson, Fain \& Smiley, 1999: 40; Halliday,

Kamran \& Bashir, 2018: 82

Type locality: Pakistan

Distribution: Pakistan 
Genus Atarsacheylus Thewke

Atarsacheylus Thewke, 1980: 333

Type species: Atarsacheylus vichii Thewke, 1980

Atarsacheylus vichii Thewke

Atarsacheylus vichii Thewke, 1980: 333

Type locality: one mile north of Vichy, Maries Co., Missouri

Distribution: USA (Missouri)

Genus Chelacheles Baker

Chelacheles Baker, 1958: 235

Type species: Chelacheles strabismus Baker, 1958

Chelachecaropsis Attiah, 1973: 349

Type species: Chelachecaropsis bakeri Attiah, 1973

Chelacheles aigamuxa Bochkov et OConnor

Chelacheles aigamuxa Bochkov \& OConnor, 2004: 559

Type locality: South Africa

Distribution: South Africa

Chelacheles alexandrinus Hassan et Gomaa

Chelacheles alexandrinus Hassan \& Gomaa 1981: 115; Bochkov et al., 1999: 118; Fain \& Bochkov, 2001: 82; Bochkov \& OConnor, 2004: 556

Type locality: Egypt, Barg El-Arab, Northern Coast

Distribution: Crimea, Egypt

Host: litter

Chelacheles apanaskevichi Bochkov et Abramov

Chelacheles apanaskevichi Bochkov \& Abramov, 2016: 337; Khaustov, 2021: 234

Type locality: Tula Province, near Suvorov town

Distribution: Russia

Chelacheles bacchusi Bochkov, Haustov et Kuznetzov

Chelacheles bacchusi Bochkov, Haustov \& Kuznetzov, 1999: 117; Gerson et al., 1999: 7;

Eliopoulos \& Papadoulis, 2001: 31; Bochkov \& OConnor, 2004: 556

Type locality: Sudak

Distribution: Crimea

Chelacheles baiwanganae Corpuz-Raros et Sotto

Chelacheles baiwanganae Corpuz-Raros \& Sotto, 1977: 152; Fain \& Bochkov, 2001c: 82;

Bochkov \& Fain, 2001: 82; Bochkov \& OConnor, 2004: 551 
Type locality: Los Baños, Laguna

Distribution: Philippine Is., Thailand

Host: beetle (Coleoptera: Scolytidae); in Thailand from Sinoxylon crassum (Coleoptera: Bostrichidae)

\section{Chelacheles bakeri (Attiah)}

Chelachecaropsis bakeri Attiah, 1973: 349

Chelacheles bakeri (Attiah): Fain \& Bochkov, 2001b: 299; 2001c: 83

Chelacheles humilis Rasool, Chaudhri \& Akbar, 1980: 30; Fain \& Bochkov, 2001c: 83

Type locality: Cairo

Distribution: Egypt

Host: rice plantation

Chelacheles bipanus Summers et Price

Chelacheles bipanus Summers \& Price, 1970: 79; Shen, 1991; Fain \& Bochkov, 2001c: 83; Xia, 2010: 161

Type locality: American Canyon, Solano Co., California

Distribution: China, USA (California)

Chelacheles humilis Rasool, Chaudhri et Akbar

Chelacheles humilis Rasool, Chaudhri \& Akbar, 1980: 30; Chaudhri \& Akbar, 1985: 194;

Gerson, Fain \& Smiley, 1999: 47; Halliday, Kamran \& Bashir, 2018: 83

Type locality: Pakistan

Distribution: Pakistan

Chelacheles hellenicus Eliopoulos et Papadoulis

Chelacheles hellenicus Eliopoulos \& Papadoulis, 2001: 31; Fain \& Bochkov, 2001c: 83;

Bochkov \& OConnor, 2004: 573

Type locality: Attiki Co., Athens, Votanikos

Distribution: Greece

Chelacheles indra Bochkov et Otto

Chelacheles indra Bochkov \& Otto, 2010: 16

Type locality: India (intercepted on Australian border)

Distribution: India

Host: from Sinoxylon crassum (Coleoptera: Bostrichidae)

Chelacheles klimovi Bochkov et OConnor

Chelacheles klimovi Bochkov \& OConnor, 2004: 559

Type locality: NY, Nassau Co., Tobay Beach

Distribution: USA

Host: wrack from beach 


\section{Chelacheles lanceolatus Tseng}

Chelacheles lanceolatus Tseng, 1977: 259; Lin \& Zhang, 1997: 197; Corpuz-Raros, 1998:

263; Gerson et al., 1999: 47; Fain \& Bochkov, 2001: 82; Bochkov \& OConnor, 2004:

578; Xia, 2010: 161

Type locality: Taiwan

Distribution: Taiwan

Host: Chicken feathers (accidental?)

Chelacheles michalskii Samšinák

Chelacheles michalskii Samšinák, 1962: 183; Volgin, 1969: 305; Liu et al., 1997; Fain \& Bochkov, 2001c: 83; Bochkov, Hakimitabar \& Saboori, 2005: 105; Xia, 2010: 161;

Torre Santana, 2015

Type locality: Prague

Distribution: China, Cuba, Czech Rep., Iran

Host: «In den Gängen von Scolytus multistriatus und S. pygmaeus»

Chelacheles peritremaculatus Thewke

Chelacheles peritremaculatus Thewke, 1974: 33; Hassan \& Gomaa, 1981: 115; Corpuz-Raros, 1998: 263; Bochkov et al., 1999: 117; Gerson et al., 1999: 47; Fain \& Bochkov, 2001c: 82; Eliopoulos \& Papadoulis, 2001: 31; Bochkov \& OConnor, 2004: 565

Type locality: Seven miles of West Plains, Howell Co., Missouri, on oak branches

Distribution: USA (Missouri)

Chelacheles robustus Corpuz-Raros

Chelacheles robustus Corpuz-Raros, 1998: 263; Eliopoulos \& Papadoulis, 2001: 31; Fain \& Bochkov, 2001c: 82; Bochkov \& OConnor, 2004: 570

Type locality: Leyte I., Baybay, Visayas St., Col. of Agriculture Campus

Distribution: Philippine Is. (Leyte)

Host: rice mill

Chelacheles seminole Bochkov et OConnor

Chelacheles seminole Bochkov \& OConnor, 2004: 570

Type locality: Florida, Lee Co., Punta Rassa

Distribution: USA (Florida)

Host: fungi Daldinia concentrica (Xylariales: Xylariaceae)

Chelacheles stigmaeoides (Barilo)

Chelachecaropsis stigmaeoides Barilo, 1989: 137; Gerson et al., 1999: 47

Chelacheles stigmaeoides (Barilo): Fain \& Bochkov, 2001c: 83; Bochkov \& OConnor, 2004: 575

Type locality: Samarkand area

Distribution: Uzbekistan 
Host: soil from ant hill (Formica sp.)

\section{Chelacheles strabismus Baker}

Chelacheles strabismus Baker, 1958: 234; Patxot \& Goff, 1985: 160; Fain \& Bochkov, 2001: 83

Chelacheles algericus Fain \& Bochkov, 2001: 83; syn. by Bochkov \& OConnor, 2004 - terra typica: Algeria

Type locality: Lisbon (Portugal)

Distribution: Algeria, Portugal, USA (Hawaiian Is. - Oahu)

\section{Chelacheles striola Lin}

Chelacheles striola Lin in Lin \& Zhang, 1997: 245; Bochkov \& OConnor, 2004: 567; Xia, 2010: 161

Type locality: Fujian

Distribution: China

Chelacheles temoak Bochkov et OConnor

Chelacheles temoak Bochkov \& OConnor, 2004: 569

Type locality: Nevada, Washoe Co., Patrick

Distribution: USA (Nevada)

Host: Diadasia sphaeralcearum (Hymenoptera: Apidae)

Chelacheles thomasi Bochkov et Otto

Chelacheles thomasi Bochkov \& Otto, 2010: 12

Type locality: Sydney/Rosebury

Distribution: Australia

Host: from oranges

Genus Neoacaropsis Volgin

Neoacaropsis Volgin, 1962: 685

Type species: Neoacaropsis granulatus Volgin, 1962

\section{Neoacaropsis granulatus Volgin}

Neoacaropsis granulatus Volgin, 1962: 685; 1969: 320; Xie et al., 2000; Fain \& Bochkov, 2001: 68; Xia, 2010: 161

Type locality: Sochi (Krasnodarskiy Kray)

Distribution: China, Russia

Genus Neochelacheles Smiley et Williams

Neochelacheles Smiley \& Williams, 1972: 312

Type species: Neochelacheles messersmithi Smiley et Williams, 1972 
Neochelacheles corpuzrarosae Bochkov et OConnor

Neochelacheles corpuzrarosae Bochkov \& OConnor, 2004: 582

Type locality: Negros Oriental, Lake Balinsasayao, 3 km N 14 km W Dumaguete City

Distribution: Philippines

Host: from fungi of the superfamily Polyporaceae (Agarycales)

Neochelacheles mendicus Bochkov et OConnor

Neochelacheles mendicus Bochkov \& OConnor, 2004: 587

Type locality: Luzon Island, Baltalason

Distribution: Philippines

Host: Bysrax tuberculatus (Coleoptera: Tenebrionidae)

Neochelacheles messersmithi Smiley et Williams

Neochelacheles messersmithi Smiley \& Williams, 1972: 312; Fain \& Bochkov, 2001c: 81

Type locality: College Park, Meryland

Distribution: USA (Meryland, Ohio)

Host: Bolitotherus cornutus (Coleoptera: Tenebrionidae, Bolitophaginae)

Genus Paracaropsis Volgin

Paracaropsis Volgin, 1969: 322

Type species: Acaropsis travisi Baker, 1949

\section{Paracaropsis travisi (Baker)}

Acaropsis travisi Baker, 1949: 322

Paracaropsis travisi (Baker): Volgin, 1969: 324; Summers \& Price, 1970: 39

Paracaropsis strofi Samšinák, 1956 (syn. by Summers \& Price, 1970: 39)

Type locality: Newton, Georgia

Distribution: USA (Georgia)

Host: Sceloporus woodi (Sauria: Iguanidae)

Tribe Bakini Volgin

Bakini Volgin, 1969: 299

Type species: Bak sanctaehelenae Yunker, 1961

Genus Aegyptocheyla Yousef

Aegyptocheyla Yousef, 1979: 366

Type species: Aegyptocheyla summersi Yousef, 1979

\section{Aegyptocheyla summersi Yousef}

Aegyptocheyla summersi Yousef, 1979: 366

Type locality: Kalyobia and Giza Governorates

Distribution: Egypt 
Genus Alliea Yunker

Alliea Yunker, 1960: 278

Type species: Alliea laruei Yunker, 1960

\section{Alliea laruei Yunker}

Alliea laruei Yunker, 1960: 278; Volgin, 1969: 233

Type locality: Tampa (Florida)

Distribution: USA (Florida)

Host: Rattus norvegicus (Rodentia: Muridae)

\section{Alliea prasadi Corpuz-Raros}

Alliea prasadi Corpuz-Raros, 1998: 261

Type locality: Luzon Is., UPLB Campus, College, Laguna

Distribution: Philippines

Genus Bak Yunker

Bak Yunker, 1961:1023

Type species: Bak sanctaehelenae Yunker, 1961

Bak deleoni Yunker

Bak deleoni Yunker, 1961: 1028; Volgin, 1969: 302; Summers \& Price, 1970: 80; Patxot \& Goff, 1985: 159; Fain \& Bochkov, 2001: 80

Type locality: Coral Gables (Florida)

Distribution: D.R. Congo, USA (Florida, Hawaiian Is.)

\section{Bak elongatus Patxot et Goff}

Bak elongatus Patxot \& Goff, 1985: 158; Fain \& Bochkov, 2001: 80

Type locality: Oahu I., Honolulu, Waimanalo

Distribution: USA (Hawaiian Is.)

Bak faini Corpuz-Raros

Bak faini Corpuz-Raros, 2000: 323; Fain \& Bochkov, 2001: 80; Xia, 2010: 161

Type locality: Philippines

Distribution: China, Philippines

Bak furcatus Gerson et Fain

Bak furcatus Gerson \& Fain, 1991: 17; Corpuz Raros, 2000; Fain \& Bochkov, 2001c: 80

Type locality: Chantaburi (Thailand)

Distribution: Philippines, Thailand

Host: in debris from a hive of Apis cerana (Hymenoptera: Apidae) 


\section{Bak gersoni Corpuz-Raros}

Bak gersoni Corpuz-Raros, 2000: 326; Fain \& Bochkov, 2001c: 80

Type locality: Philippines

Distribution: Galapagos Is., Philippines

Bak indonesiensis Bochkov et Otto

Bak indonesiensis Bochkov \& Otto, 2010: 17

Type locality: Indonesia (intercepted on Australian border)

Distribution: Indonesia

Host: beetles (Coleoptera indet.)

Bak iranica Pactinat-Saeij, Bagheri et Skvarla

Bak iranica Pactinat-Saeij, Bagheri \& Skvarla, 2017: 848; Salarzehi, 2018

Type locality: Amol, Mazandaran Prov.

Distribution: Iran

\section{Bak ligyscutatus Flechtmann}

Bak ligyscutatus Flechtmann, 1971: 48 (according to Fain \& Bochkov, 2001c: 78, invalid species, published only in a thesis, but actually published regularly)

Type locality: Piracicaba, São Paulo

Distribution: Brazil

Host: decomposing organic material

\section{Bak micidus Summers et Price}

Bak micidus Summers \& Price, 1970: 81; Fain \& Bochkov, 2001c: 79; Xia, 2010: 161

Type locality: Santa Cruz, Old Bella Vista Trail

Distribution: China, Galapagos Is. (Ecuador)

Bak nadchatrami Fain et Bochkov

Bak nadchatrami Fain et Bochkov, 2001c: 80

Type locality: Belakang Pasar, Lama Kepong, Kuala Lumpur

Distribution: Malaysia

Bak ozarkensis Thewke et Enns

Bak ozarkensis Thewke \& Enns, 1974: 43; Fain \& Bochkov, 2001c: 79

Type locality: four miles west of Macks Creek, Camden Co., Missouri

Distribution: USA (Missouri, Pennsylvania)

Bak payatus Corpuz-Raros et Sotto

Bak payatus Corpuz-Raros \& Sotto, 1977: 146; Fain \& Bochkov, 2001c: 79 
Type locality: Laguna

Distribution: Philippine Is.

Bak sanctaehelenae Yunker

Bak sanctaehelenae Yunker, 1961: 1023; Volgin, 1969: 300; Summers \& Price, 1970: 80;

Fain \& Bochkov, 2001c: 79

Type locality: Napa Co., Mt. St. Helena, California

Distribution: USA (California)

Bak truncatus Corpuz-Raros et Sotto

Bak truncatus Corpuz-Raros \& Sotto, 1977: 148; Fain \& Bochkov, 2001c: 79

Type locality: Makiling Bot, Gardens, Mt. Makiling, Laguna

Distribution: Philippine Is.

Bak sp. - India

Genus Caudacheles Gerson

Caudacheles Gerson, 1968: 645

Type species: Caudacheles khayae Gerson, 1968

Caudacheles khayae Gerson

Caudacheles khayae Gerson, 1968: 645; Fain \& Bochkov, 2001: 77; Salarzehi, 2018:

Type locality: Rehovot (Israel)

Distribution: Iran, Israel

Caudacheles lieni Tseng

Caudacheles lieni Tseng, 1977; Fain \& Bochkov, 2001: 77; Bochkov, Hakimitabar \& Saboori, 2005: 104; Xia, 2010: 162

Type locality: Taiwan

Distribution: Iran, Taiwan

Caudacheles trigintaduae Lin et Zhang

Caudacheles trigintaduae Lin \& Zhang, 1997: 136

Type locality: China

Distribution: China

Genus Chelacaropsis Baker

Chelacaropsis Baker, 1949: 315

Type species: Chelacaropsis moorei Baker, 1949

Cheletonata Womersley, 1955 = Cheletacaropsis Baker, 1949 (syn. by Fain \& Bochkov, 2001a: 294) 
Chelacaropsis apus Fain

Chelacaropsis apus Fain, 1972: 40

Type locality: Butare

Distribution: Rwanda

Host: Apus affinis, "dejections"(Apodiformes: Apodidae)

Cheletacaropsis kenyensis Fain et Bochkov

Cheletacaropsis kenyensis Fain \& Bochkov, 2001b: 70

Type locality: Kimakia Cave, Hunter's Lodge, Kiboko, 140 km SE Nairobi

Distribution: Kenya

Cheletacaropsis milesi (Womersley)

Cheletonata milesi Womersley, 1955: 214

Cheletacaropsis milesi (Womersley): Fain \& Bochkov, 2001b: 73

Type locality: Northern Australia

Distribution: Australia

Chelacaropsis moorei Baker

Chelacaropsis moorei Baker, 1949: 315; Volgin, 1969: 326

Type locality: Florida

Distribution: China, USA (Florida)

Host: Glaucomys volans querceti (Rodentia: Pteromyidae)

Chelacaropsis reticulata Soliman

Chelacaropsis reticulata Soliman, 1975: 97

Type locality: Farskour, Domiatta, North of Deltha

Distribution: Egypt

\section{Chelacaropsis rwandana Fain}

Chelacaropsis rwandana Fain, 1972: 40

Type locality: Butare

Distribution: Rwanda

Host: Spermestes cucullatus (Passeriformes: Estrildidae)

Chelacaropsis terrestris Corpuz-Raros et Sotto

Chelacaropsis terrestris Corpuz-Raros \& Sotto, 1977: 150

Type locality: Los Baños, Laguna

Distribution: Philippine Is.

Genus Cheletogenes Oudemans

Cheletogenes Oudemans, 1905: 208 
Type species: Cheyletus ornatus Canestrini et Fanzago, 1876

Cheletogenes aceriai Khan

Cheletogenes aceriai Khan, 1970: 278; Fain \& Bochkov, 2001: 85; Gerson, Fain \& Smiley, 1999: 47; Halliday, Kamran \& Bashir, 2018: 82

Type locality: Pakistan

Distribution: Pakistan

Host: mango tree bugs

Cheletogenes carinatus Aheer, Akbar et Chaudhri

Cheletogenes carinatus Aheer, Akbar \& Chaudhri, 1992: 39; Fain \& Bochkov, 2001: 85

(Species dubia); Gerson, Fain \& Smiley, 1999: 47; Aheer, Akbar \& Chaudhri, 1992:

39; Halliday, Kamran \& Bashir, 2018: 83

Type locality: Pirmahal

Distribution: Pakistan

Host: on Vitis vinifera (plant)

Cheletogenes dissitus Akbar, Rahi et Chaudhri (Species dubia)

Cheletogenes dissitus Akbar, Rahi \& Chaudhri, 1988: 4; Gerson, Fain \& Smiley, 1999: 47;

Fain \& Bochkov, 2001: 85; Halliday, Kamran \& Bashir, 2018: 83

Type locality: Pakistan

Distribution: Pakistan

Cheletogenes iconis Chaudhri et Akbar

Cheletogenes iconis Chaudhri \& Akbar, 1985: 128; Fain \& Bochkov, 2001: 85; Aheer, Akbar

\& Chaudhri, 1992: 36; Halliday, Kamran \& Bashir, 2018: 83

Type locality: $3 \mathrm{~km}$ S Multan

Distribution: Pakistan

Host: on Gossypium hirsutum

Cheletogenes meihuashanense Lin et Liu

Cheletogenes meihuashanense Lin \& Liu, 1994: 220 (223); Lin et al., 2000: 86; Xia, 2010: 161

Type locality: Meihuashan, Fujian

Distribution: China (Fujian)

\section{Cheletogenes monosetosus Tseng}

Cheletogenes monosetosus Tseng, 1977: 229; Ehara \& Abdul Ghani, 1988: 239; Fain \&

Bochkov, 2001: 85; Xia, 2010: 161

Type locality: Taiwan

Distribution: Malaysia, Taiwan

Host: from trees (Gigantochloa rostrata, Bambusa glaucescens) 
Cheletogenes ornatus (Canestrini et Fanzago)

Cheyletus ornatus Canestrini \& Fanzago, 1876: 106; 1877: 147; Canestrini, 1886: 173;

Starkoff \& Starkoff, 1950: 96

Cheyletus saccardianus Berlese, 1886: 32; Oudemans, 1906: 153

Cheyletia ornata, Oudemans, 1904: 154

Cheyletus cocciphilus Banks, 1914: 56. Syn. by Baker, 1949: 305

Cheletogenes ornatus (Canestrini et Fanzago): Oudemans, 1905: 208; 1906: 153; Baker,

1949: 305; Cooreman, 1951: 33; Volgin, 1955: 174; 1969: 296; Gerson, 1967: 359;

Wafa \& Soliman, 1968: 222; Summers \& Price, 1970: 53; Flechtmann, 1971: 30; Shiba,

1976: 173; Nachev \& Trenchev, 1987; Yousef, Zaher \& Kandil, 1979: 228; Ripka, Fain

\& Bolland, 1999: 366; Fain \& Bochkov, 2001: 85; Buosi et al., 2006: 7

Cheyletogenes ornatus (Canestrini et Fanzago): Namaghi, 2010: 135

Type locality: Italy

Distribution: Algeria, Armenia, Australia, Brazil, Chile, China, Cuba, Egypt, Georgia, Hun-

gary, India, Iran, Italy, Israel, Kirghizstan, Malaysia, Mexico, Morocco, Philippines, Porto

Rico, Russia, South Africa, USA (Florida, Louisiana, California, Hawaiian Is.), Venezuela

\section{Cheletogenes petiginis Qayyum et Chaudhri (Species dubia)}

Cheletogenes petiginis Qayyum \& Chaudhri, 1977; Fain \& Bochkov, 2001: 85; Rasool \& Chaudhri, 1979a: 7; Chaudhri \& Akbar, 1985: 134; Akbar, Rahi \& Chaudhri, 1988:

4; Gerson, Fain \& Smiley, 1999: 47; Halliday, Kamran \& Bashir, 2018: 83

Type locality: Pakistan

Distribution: Pakistan

\section{Cheletogenes sagacis Chaudhri et Akbar (Species dubia)}

Cheletogenes sagacis Chaudhri \& Akbar, 1985; Aheer, Akbar \& Chaudhri, 1992: 36; Halliday, Kamran \& Bashir, 2018: 83

Type locality: $2 \mathrm{~km}$ N Haripur

Distribution: Pakistan

Host: on plants

\section{Cheletogenes scaber Qayyum et Chaudhri (Species dubia)}

Cheletogenes scaber Qayyum \& Chaudhri, 1977: 111; Fain \& Bochkov, 2001: 85; Salarzehi, 2018; Halliday, Kamran \& Bashir, 2018: 83

Type locality: Pakistan

Distribution: Iran, Pakistan

\section{Cheletogenes vulgatus Rasool et Chaudhri (Species dubia)}

Cheletogenes vulgatus Rasool \& Chaudhri, 1979: 8; Fain \& Bochkov, 2001: 85; Chaudhri \& Akbar, 1985: 129; Akbar, Rahi \& Chaudhri, 1988: 4; Gerson, Fain \& Smiley, 1999: 47; Halliday, Kamran \& Bashir, 2018: 83 
Type locality: Pakistan

Distribution: Pakistan

\section{Cheletogenes waitei Gerson}

Cheletogenes waitei Gerson, 1994: 441; Fain \& Bochkov, 2001: 85

Type locality: Nambour (Queensland)

Distribution: Australia (Queensland)

Genus Cheletoides Oudemans

Cheletoides Oudemans, 1904: 154

Type species: Syringophilus uncinatus Heller, 1880

\section{Cheletoides chirunduensis Fain}

Cheletoides chirunduensis Fain, 1979: 1023

Type locality: Chirundu

Distribution: Zimbabwe

Host: Numida meleagris (Galliformes: Numididae)

\section{Cheletoides uncinatus Heller}

Syringophilus uncinatus Heller, 1880: 188

Cheyletus uncinatus (Heller): Poppe, 1888: 239

Cheletoides uncinatus (Heller): Oudemans, 1904: 154; 1906: 204; Volgin, 1969: 336

Cheletoides uncinata (Heller): Oudemans, 1906: 204; Baker, 1949: 275; Summers \& Price, 1970: 74

Type locality: Kiel

Distribution: Germany

Host: Pavo cristatus (Galliformes: Phasianidae)

Genus Cheletomorpha Oudemans

Cheletomorpha Oudemans, 1904: 162

Acheletomorpha Volgin, 1969: 195

Type species: Acarus lepidopterorum Shaw, 1794

\section{Cheletomorpha bakeri Lawrence}

Cheletomorpha bakeri Lawrence 1954: 67; Summers \& Price, 1970: 51; Fain \& Bochkov, 2001: 52

Acheletomorpha bakeri (Lawrence): Volgin, 1969: 196

Type locality: Karamoja (Uganda)

Distribution: D.R. Congo, Rwanda, Uganda

Host: in nests of birds and rodents 
Cheletomorpha dolosus Aheer et al.

Cheletomorpha dolosus Aheer et al., 1997: 11; Fain \& Bochkov, 2001: 52; Halliday, Kamran \& Bashir, 2018: 86

Type locality: Faisalabad

Distribution: Pakistan

\section{Cheletomorpha lepidopterorum (Shaw)}

Acarus lepidopterorum Shaw, 1794: 187

Cheyletus venustissimus C.L. Koch, 1839: fasc. 23; Canestrini, 1886: 172; Hull, 1918: 32; Starkoff \& Starkoff, 1950: 96

Cheyletus seminivorus Packard, 1878: 665

Cheletomorpha lepidopterorum (Shaw): Oudemans, 1929: 298; Baker, 1949: 302; Volgin,

1969: 190; Summers \& Price, 1970: 49; Shen, 1975; Rekk, 1976: 68; Caceres \& Fain,

1978: 302; Enghoff, 1988: 49; Peredo et al., 1994: 17; Lucza, Ripka \& Saly, 1996; Fain

\& Bochkov, 2001: 52; Petrova et al., 2004: 91; Bochkov, Hakimitabar \& Saboori, 2005:

105; Xia, 2010: 161; Bochkov \& Abramov, 2016; Halliday, Kamran \& Bashir, 2018: 86 Cheyletus rufus Hardy, in André, 1933: 352

Cheletomorpha venustissima, Oudemans, 1904: 162; 1906: 144; Thor, 1912: 390; Zachvatkin,

1935: 25; Rohdendorf, 1940: 92; Womersley, 1941: 62; Starkoff \& Starkoff, 1950: 96;

Cheletomorpha tatami Hara, 1955: 69; Gerson, 1967: 360; Shiba, 1969a: 164

Cheletophyes knowltoni Beer \& Dailey, 1956: 409

Cheyletus longipes Banks, 1909: 133 - Canada, Ontario, Guelph

Type locality: England

Distribution: Armenia, Australia, Azerbaidzan, Belgium, Brazil, Canada, Chile, China, Cuba, Egypt, Denmark, England, France, Georgia, Germany, Greece, Holland, Hungary, India, Indonesia, Iran, Israel, Italy, Japan, Latvia, Malaysia, Malta, Mexico, New Guinea, Norway, Pakistan, Peru, the Philippines, Portugal, Rwanda, New Guinea, Russia, South Africa, Spain, Switzerland, Taiwan, Turkey, USA

Host: Proxenus sp. (Lepidoptera: Noctuidae) and other moths

Cheletomorpha obrutus Qayyum et Chaudhri - Species inquirenda Cheletomorpha obrutus Qayyum \& Chaudhri, 1977: 75; Halliday, Kamran \& Bashir, 2018: 86 Type locality: Pakistan

Distribution: Pakistan

Cheletomorpha opacus Qayyum et Chaudhri - Species inquirenda

Cheletomorpha opacus Qayyum \& Chaudhri, 1977: 73; Halliday, Kamran \& Bashir, 2018: 86 Type locality: Pakistan

Distribution: Pakistan 
Cheletomorpha orientalis Oudemans - Species inquirenda

Cheletomorpha orientalis Oudemans 1928: 343; Baker, 1949: 303; Volgin, 1969: 195;

Summers \& Price, 1970: 51

Type locality: Java

Distribution: Indonesia (Java)

Host: on leaves of orchid, Palaenopsis sp.

Cheletomorpha tenerum Qayyum et Chaudhri

Cheletomorpha tenerum Qayyum \& Chaudhri, 1977: 71; Fain \& Bochkov, 2001: 52; Halliday, Kamran \& Bashir, 2018: 86

Type locality: Pakistan

Distribution: Pakistan

Genus Cheletopsis Oudemans

Cheletopsis Oudemans, 1904: 163

Type species: Cheyletus nörneri Poppe, 1888

Cheletopsis anax Oudemans

Cheletopsis anax Oudemans, 1904b: 170; 1906: 195; Baker, 1949: 309; Volgin, 1969: 354;

Summers \& Price, 1970: 73; Bochkov et al., 2002: 10

Type locality: France

Distribution: France

Host: "Crocethia alba (= Totanus calidris)", ? Tringa totanus (Charadriiformes: Scolopacidae)

\section{Cheletopsis animosa Oudemans}

Cheletopsis animosa Oudemans, 1904b: 170; 1906: 189; Baker, 1949: 310; Volgin, 1969: 352; Summers \& Price, 1970: 73; Bochkov et al., 2002: 10

Type locality: France

Distribution: France, Eastern Nord America

Hosts: ?Tringa totanus (Charadriiformes: Scolopacidae), Sterna hirundo (Charadriiformes: Laridae, Sterninae)

\section{Cheletopsis basilica Oudemans}

Cheletopsis basilica Oudemans, 1904: 170; 1906: 186; Baker, 1949: 310; Volgin, 1969: 351;

Summers \& Price, 1970: 73; Bochkov et al., 2002: 8

Type locality: France

Distribution: France, Poland; USA (Alaska, Michigan)

Hosts: Calidris melanotus, Tringa totanus (Charadriiformes: Scolopacidae), Charadrius hiaticula (Charadriiformes: Charadriidae), Chlidonius niger (Charadriiformes: Laridae) 
Cheletopsis charadrii Mironov, Bochkov et Chirov

Cheletopsis charadrii Mironov, Bochkov \& Chirov, 1991: 52; Bochkov et al., 2002: 18; Chernichko \& Kivganov, 2013: 84

Type locality: Ottuk Village, near Issyk-Kul Lake (Kirghizstan)

Distribution: Kirghizstan, Ukraine

Hosts: Charadrius alexandrinus, Ch. dubius (Charadriiformes: Charadriidae)

Cheletopsis daberti Kivganov et Bochkov

Cheletopsis daberti Kivganov \& Bochkov, 1994: 39 (also “deberti”, laps. typ.); Bochkov et al., 2002: 15; Chernichko \& Kivganov, 2013: 84

Type locality: Tigulskij Res., Odessa Prov., Ukraine

Distribution: Poland, Ukraine; USA (N. Dakota)

Hosts: Calidris temminckii, Tringa glareola, T. nebularia, Micropalama himantopus (Charadriiformes: Scolopacidae)

Cheletopsis ferrugineae Chernichko et Kivganov

Cheletopsis ferrugineae Chernichko \& Kivganov, 2013: 85

Type locality: Tilihul Lagoon

Distribution: Ukraine

Host: Calidris ferruginea (Charadriiformes: Charadriidae)

Cheletopsis impavida Oudemans

Cheletopsis impavida Oudemans, 1904b: 170; 1906: 175; Baker, 1949: 310; Volgin, 1969: 342; Summers \& Price, 1970: 73; Mironov, Bochkov \& Chirov, 1991: 55; Bochkov et al., 2002: 12; Chernichko \& Kivganos, 2013: 83

Type locality: France

Distribution: France, Poland; Russia (Primorie, Crimea), Kazakhstan, Kirghizstan; USA (Kansas, Michigan); Argentina

Hosts: Tringa totanus, T. stagnatilis (Charadriiformes: Scolopacidae), Calidris minutus, C. pusilla, C. ferruginea, C. temminckii, C. ruficollis, Micropalama himantopus (Charadriiformes: Scolopacidae), Rostratula semicollaris (Charadriiformes: Rostratulidae)

Cheletopsis limnodromi Bochkov, Fain et Dabert

Cheletopsis limnodromi Bochkov, Fain \& Dabert, 2002 : 18

Type locality: Sapelo Isl., McIntosh Co., Georgia, USA

Distribution: USA (Georgia)

Host: Limnodromus griseus (Charadriiformes: Scolopacidae)

Cheletopsis magnanima Oudemans - Species inquirenda

Cheletopsis magnanima Oudemans, 1904: 170; 1906: 193; Volgin, 1969: 350; Summers \& Price, 1970: 73; Mironov et al., 1991: 55; Bochkov et al., 2002: 22 
Type locality: Chile (in museum in Angers, France)

Distribution: Chile

Host: Tringa flavipes (Charadriiformes: Scolopacidae, museum bird from Chile)

Cheletopsis mariae Mironov, Bochkov et Chirov

Cheletopsis mariae Mironov, Bochkov \& Chirov, 1991: 51; Bochkov et al., 2002: 12

Type locality: Ottuk Village, Issyk-Kul Lake, Kirghizstan

Distribution: Poland; Kirghizstan

Host: Actitis hypoleucos (Charadriiformes: Scolopacidae)

\section{Cheletopsis norneri Poppe}

Cheyletus Nörneri Poppe, 1888: 239

Cheletopsis Nörneri (Poppe): Oudemans, 1906: 180

Cheletes noerneri Poppe: Poppe, 1909: 51

Cheletopsis norneri (Poppe): Baker, 1949: 310; Volgin, 1969: 345; Mironov et al., 1991:

55; Bochkov et al., 2002: 8

Type locality: France

Distribution: Bulgaria, France, Germany, Poland, Russia, Ukraine; Kazakhstan, Kirghizstan; Egypt; USA; Paraguay

Host: Tringa totanus (Charadriiformes: Scolopacidae), Sterna hirundo, S. repressa, S. simplex, Gelochelidon nilotica, Chlidonias h. hybrida (Charadriiformes: Laridae)

Cheletopsis prosobonialis Bochkov, Fain et Dabert

Cheletopsis prosobonialis Bochkov, Fain \& Dabert, 2002: 22

Type locality: Tepoto Isl., Tuamotu Archipelago

Distribution: French Polynesia (Tuamotu)

Host: Prosobonia cancellata (Charadriiformes: Scolopacidae)

Cheletopsis rynchops Bochkov, Fain et Dabert

Cheletopsis rynchops Bochkov, Fain \& Dabert, 2002:18

Type locality: South Tica-Buzi River, Distr. Vila Machado, Mozambique

Distribution: Mozambique; USA (N. Carolina)

Hosts: Rynchops flavirostris, R. niger (Charadriiformes: Laridae)

Cheletopsis thalasseus Bochkov et OConnor

Cheletopsis thalasseus Bochkov \& OConnor, 2003: 270

Type locality: Florida, Monroe Co., Dry Tortugas, Garden Key

Distribution: USA (Florida)

Host: Thalasseus [Sterna] sandviciensis (Charadriiformes: Laridae, Sterninae) 
Genus Cheletosoma Oudemans

Cheletosoma Oudemans, 1905: 207

Type species: Cheletosoma tyrannus Oudemans, 1905

Cheletosoma tyrannus Oudemans

Cheletosoma tyrannus Oudemans, 1905: 207; 1906: 168; Baker, 1949:315; Volgin, 1969: 332

Type locality: «Tropical America»

Distribution: «Tropical America»

Host: Aramus scolopaceus (Gruiformes: Aramidae)

Genus Cheyletia Haller

Cheyletia Haller, 1884: 233

Type species: Acarus squamosus De Geer, 1776

Cheyletia americana Volgin

Cheyletia squamosa Baker, 1949: 301

Cheyletia americana Volgin, 1969: 240

Type locality: Birnham wood (Wisconsin)

Distribution: USA

\section{Cheyletia aradiphila Volgin}

Cheyletia aradiphila Volgin, 1966b: 279; 1969: 238; Summers \& Price, 1970: 65

Type locality: Regions of Murmansk, Leningrad, Vologda (Totma) and Irkutsk

Distribution: Russia

Host: Aradus lugubris (Heteroptera: Aradidae)

\section{Cheyletia laureata Haller}

Cheyletia laureata Haller, 1884: 234; Karpelles, 1893: 124; Volgin, 1969: 237; Summers \& Price, 1970: 65 (syn. of Cheyletia squamosa)

Type locality: Germany

Distribution: Germany, Hungary

\section{Cheyletia papillifera Volgin}

Cheyletus squamosus Oudemans, 1897: 128 (not Acarus squamosus De Geer)

Cheyletia papillifera Volgin, 1955: 171; 1969: 240; Ždárková, 1979; Summers \& Price, 1970: 65; Solarz, 1989: 243

Type locality: Utrecht, Arnhem

Distribution: Holland, "Czechoslovakia", Poland

Host: Chloris chloris (Passeriformes: Fringillidae) 
Cheyletia squamosa (De Geer)

Acarus squamosus De Geer, 1778: 116

Cheyletia squamosa (De Geer): Summers \& Price, 1970: 65

Cheyletia laureata Haller, 1884: 223

Type locality: Austria

Distribution: Austria

Genus Chiapacheylus De Leon

Chiapacheylus De Leon, 1962: 135

Type species: Chiapacheylus edentatus De Leon, 1962

Chiapacheylus edentatus De Leon

Chiapacheylus edentata De Leon, 1962: 135; Torre Santana, 2015: 49

Chiapacheylus edentatus De Leon: Volgin, 1969: 278; Summers \& Price, 1970: 52

Type locality: Tuxtla Gutierrez (Chiapas)

Distribution: Cuba, Mexico (Chiapas)

Hosts: Xylosoma elliptica, Citharexylum fructicosum

? Chiapacheylus desertorum Zaher et Soliman

Chiapacheylus desertorum Zaher \& Soliman, 1967: 22

Type locality: El-Fayoum and Bury El-Arab

Distribution: Egypt

? Chiapacheylus macrocorneus Zaher et Soliman

Chiapacheylus macrocorneus Zaher \& Soliman, 1967: 23

Type locality: Giza and Tahrcer Proc.

Distribution: Egypt

Genus Columbicheyla Thewke et Enns

Columbicheyla Thewke \& Enns, 1972: 450

Type species: Columbicheyla macroflabellata Thewke et Enns, 1972

Columbicheyla bicicri Lin et Zhang

Columbicheyla bicicri Lin \& Zhang, 1997: 133; Fain \& Bochkov, 2001: 76; Xia, 2010: 161

Type locality: Fudjian

Distribution: China (Fudjian)

Columbicheyla macroflabellata Thewke et Enns

Columbicheyla macroflabellata Thewke \& Enns, 1972: 450; Lin \& Zhang, 1997; Fain \& Bochkov, 2001: 76; Xia, 2010: 161 
Type locality: Missouri

Distribution: China, Malaysia, Philippine Is., USA (Missouri)

Columbicheyla nindota (Corpuz-Raros)

Eucheyletia nindota Corpuz-Raros, 1988b: 417

Columbicheyla nindota (Corpuz-Raros): Corpuz-Raros, 1998: 266

Type locality: Leyte Is.

Distribution: Philippines

Genus Cunliffella Volgin

Type species: Neoeucheyla (Bothrocheyla) pavlovskyi Volgin, 1964

Cunliffella Volgin, 1969: 255 (as subgenus)

Bothrocheyla Volgin 1964: 93

Type species: Neoeucheyla tuberculicoxa Volgin, 1964

Cunliffella bulgarica (Volgin)

Cheyletia bulgarica Volgin, 1955: 171

Neoeucheyla bulgarica (Volgin): Volgin, 1964: 89; 1969: 252; Summers \& Price, 1970: 69;

Corpus-Raros \& Sotto, 1977: 161

Cunliffella bulgarica (Volgin): Fain \& Bochkov, 2001 : 72; Bochkov, Hakimitabar \& Saboori, 2005: 105

Type locality: Pazardzhik Distr. (Bulgaria)

Distribution: Bulgaria, China, Iran, Philippine Is.

Cunliffella dua (Corpuz-Raros)

Neoeucheyla dua Corpuz-Raros, 1998: 282

Cunliffella dua (Corpuz-Raros): Fain \& Bochkov, 2001 : 72

Type locality: Mt. Makiling, Makiling Bot. Gardens, UPLB Forestry Campus, College, Laguna

Distribution: Philippines

Cunliffella maysa (Corpuz-Raros)

Neoeucheyla maysa Corpuz-Raros, 1998: 284

Cunliffella maysa (Corpuz-Raros): Fain \& Bochkov, 2001 : 72

Type locality: Luzon Is., Putting Lupa, Calamba, Laguna

Distribution: Philippines

\section{Cunliffella mumai (Volgin)}

Neoeucheyla mumai Volgin, 1969: 254; Summers \& Price, 1970: 69

Cunliffella mumai (Volgin): Fain \& Bochkov, 2001 : 72 
Type locality: Sebastian (Florida)

Distribution: U. S. A. (Florida)

Host: from Pinus clausa

Cunliffella ornata (Wafa et Soliman)

Neoeucheyla ornata Wafa \& Soliman, 1968: 228; Summers \& Price, 1970: 69

Cunliffella ornata (Wafa et Soliman): Fain \& Bochkov, $2001: 72$

Type locality: Giza

Distribution: Egypt

\section{Cunliffella panamensis (Baker)}

Eucheyla panamensis Baker, 1949: 291; Patxot \& Goff, 1985: 161

Neoeucheyla (Cunliffella) panamensis (Baker): Volgin, 1969: 260

Cunliffella panamensis: Summers \& Price, 1970: 69; Patxot \& Goff, 1985: 161; Fain \&

Bochkov, 2001: 72

Type locality: Panama

Distribution: Panama, USA (Colorado, California, Hawaii Is., Oahu)

Host: among eggs of termites

\section{Cunliffella variegata (Barilo)}

variegata Barilo, 1985: 45

Cunliffella variegata (Barilo): Fain \& Bochkov, 2001: 72; Salarzehi, 2018:

Type locality: Uzbekistan

Distribution: Iran, Tadjikistan, Uzbekistan

\section{Cunliffella whartoni (Baker)}

Eucheyla whartoni Baker, 1949: 69; Volgin, 1964: 93

Neoeucheyla (Cunliffella) whartoni (Baker) Volgin, 1969: 255

Cunliffella tuberculicoxa Volgin, 1964: 90 - type: Murmansk Distr., Russia

Neoeucheyla (Cunliffella) tuberculicoxa Volgin, 1969: 256

Cunliffella tuberculicoxa (Volgin): Summers \& Price, 1970: 71; Fain \& Bochkov, 2001: 72 (syn.)

Cunliffella whartoni: Summers \& Price, 1970: 70; Khaustov, 2021: 235

Type locality: Birnamwood, Wisconsin

Distribution: Estonia, Kyrgyzstan, Russia (as C. tuberculicoxa), USA (Wisconsin)

Hosts (of Cunliffella tuberculicoxa): Aradus lugubris, A. aterrimus, A. nemtschinovi (Heteroptera: Aradidae); C. whartoni is recorded by Khaustov (2021) from ant hill of Formica rufa (Formicidae).

Genus Dubininiola Volgin

Dubininiola Volgin, 1969: 242

Type species: Dubininiola polylepis Volgin, 1969 
Polycheyletus Vaivanijkul, 1979: 251

Type species: Polycheyletus boonkongae Vaivanijkul, 1979

Dubininiola batangeniae (Corpuz-Raros et Sotto)

Oudemansicheyla batangeniae Corpuz-Raros \& Sotto, 1977: 163

Polycheyletus batangenius (Corpuz-Raros et Sotto): Corpuz-Raros, 1980: 63

Dubininiola batangensis (Corpuz-Raros et Sotto): Fain \& Bochkov, 2001 : 300; 2001 : 77

Type locality: Tanauan, Batangas

Distribution: Philippine Is.

Dubininiola boonkongae (Vaivanijkul)

Polycheyletus boonkongae Vaivanijkul, 1979: 251

Dubininiola boonkongae (Vaivanijkul): Fain \& Bochkov, 2001 : 300; 2001: 77

Type locality: Thailand

Distribution: Thailand

\section{Dubininiola polylepis Volgin}

Dubininiola polylepis Volgin, 1969: 242; Fain \& Bochkov, 2001 : 300; 2001 : 77

Type locality: Deynausskiy Rayon

Distribution: Turkmenistan

Host: rodent's burrow

Genus Eucheletopsis Volgin

Eucheletopsis Volgin, 1969: 356

Type species: Cheyletus major Trouessart, 1893 (Oudemans, 1904)

\section{Eucheletopsis major (Trouessart)}

Cheyletus major Trouessart in Berlese, 1893: 74 (nomen nudum)

Cheletopsis major (Trouessart): Oudemans, 1904b: 163; 1906: 200; Baker, 1949: 309

Eucheletopsis major (Trouessart): Volgin, 1969: 356; Summers \& Price, 1970: 73

Type locality: New Guinea

Distribution: New Guinea

Host: Hemiprocne (= Dendrochelidon) mystacea (Apodiformes: Hemiprocnidae)

Genus Eutogenes Baker

Eutogenes Baker, 1949: 304

Type species: Eutogenes foxi Baker, 1949

Eutogenes bakeri Corpuz-Raros

Eutogenes bakeri Corpuz-Raros, 1998: 268

Type locality: Leyte Is., Visayas St. College of Agriculture, Baybay 
Distribution: the Philippines

\section{Eutogenes bicornis Khaustov}

Eutogenes bicornis Khaustov, 2021: 200

Type locality: Tyumen Province, Tyumen Region, Tyumen, Zatyumenskiy park

Distribution: Russia

\section{Eutogenes citri Gerson}

Eutogenes citri Gerson, 1967: 363; Volgin, 1969: 285; Summers \& Price, 1970: 60

Type locality: Karmon

Distribution: Israel

Eutogenes cornutus Corpuz-Raros

Eutogenes cornutus Corpuz-Raros, 1998: 270

Type locality: Luzon Is., Mt. Makiling, Putting Lupa, Calamba, Laguna, $300 \mathrm{~m}$

Distribution: the Philippines

\section{Eutogenes foxi Baker}

Eutogenes foxi Baker, 1949: 304; Volgin, 1969: 281; Summers \& Price, 1970: 58

Type locality: Brownsville (Texas)

Distribution: USA (Texas, Florida), Puerto Rico

\section{Eutogenes frater Volgin}

Eutogenes frater Volgin, 1958: 460; 1969: 282; Summers \& Price, 1970: 60; Fain \& Bochkov, 2001: 87; Bochkov, Hakimitabar \& Saboori, 2005: 104;

Eutogenes africanus Wafa \& Soliman, 1968: 225 - type: Giza, Burg El Arab and the north of the Delta, associated with scale insects, also collected from the bird nests; synonymized by Fain \& Bochkov, 2001: 87

Type locality: Bulgaria

Distribution: Algeria, Bulgaria, Egypt, Iran, Turkey

Eutogenes makilingiensis Corpuz-Raros

Eutogenes makilingiensis Corpuz-Raros, 1998: 271

Type locality: Mt. Makiling, Luzon Is., Putting Lupa, Calamba, Laguna, $300 \mathrm{~m}$

Distribution: Philippines

Eutogenes narashinoensis Hara et Hanada

Eutogenes narashinoensis Hara \& Hanada, 1960: 25; Volgin, 1969: 286; Summers \& Price, 1970: 60

Type locality: Narashino, Chiba Prefecture, free living

Distribution: Japan 


\section{Eutogenes onoi (Shiba)}

Cheletogenes onoi Shiba, 1976: 171

Eutogenes onoi (Shiba): Ehara \& Abdul Ghani, 1988: 240

Type locality: Malaysia

Distribution: Malaysia

Eutogenes pinicola Thewke et Enns

Eutogenes pinicola Thewke \& Enns, 1972: 453

Type locality: Missouri

Distribution: USA (Missouri)

Eutogenes punctata Zaher et Soliman

Eutogenes punctata Zaher \& Soliman, 1966: 65; Wafa \& Soliman, 1968: 225; Summers \& Price, 1970: 60

Eutogenes punctatus Volgin, 1969: 283

Type locality: Giza

Distribution: Egypt

\section{Eutogenes quadrisetatus (Berlese)}

Cheletogenes quadrisetatus Berlese 1913: 79; Wafa \& Soliman, 1968: 224

Cheletogenes quadrisetosus: Baker, 1949: 305

Eutogenes quadrisetatus (Berlese); Volgin, 1969: 284; Summers \& Price, 1970: 60; Xia, 2010: 161

Type locality: Java

Distribution: China, Indonesia (Java)

Eutogenes reticularis Olivier et Theron

Eutogenes reticularis Olivier \& Theron, 1988: 253

Type locality: Transvaal

Distribution: South Africa

Eutogenes vicinus Summers et Price

Eutogenes vicinus Summers \& Price, 1970: 59; Moser \& Roton, 1971: 1775; Smiley \& Moser, 1975: 406

Type locality: San Luis Obispo, California

Distribution: U.S.A. (California, Louisiana)

Genus Grallacheles De Leon

Grallacheles De Leon, 1962: 135

Type species: Grallacheles bakeri De Leon, 1962 
Grallacheles bakeri De Leon

Grallacheles bakeri De Leon, 1962: 135; Muma, 1964: 248; Volgin, 1969: 275; Summers \& Price, 1970: 49; Corpuz - Raros \& Sotto, 1977: 154; Tseng, 1977; Rosa \& Flechtmann, 1979: 196; Ždárková, 1979; Fain \& Bochkov, 2001 : 78; Xia, 2010: 161; Torre Santana, 2015

Paracheyletia woolfordi Cunliffe, 1962: 200 - type from Japan, intercepted in Hawaii; Tahiti; Nishida (Version 2009)

Type locality: Coral Gables (Florida), Mante (Tamaulipas)

Distribution: Angola, Brazil, D.R. Congo, Cuba, Japan, Malaysia, Mexico, Philippines Is., Surinam, Tahiti, Taiwan, USA (Florida, Hawaii), 'Czechoslovakia'

Grallacheles indicus Podder, Gupta et Saha

Grallacheles indicus Podder, Gupta \& Saha, 2006: 333

Type locality: West Bengal

Distribution: India

Grallacheles nanfengensis Xia, Ye et Zhu

Grallacheles nanfengensis Xia, Ye \& Zhu, 1997: 173; Xia et al., 2000; Xia, 2010: 161

Type locality: Jiangxi

Distribution: China (Jiangxi)

Genus Hoffmannita Pelaez

Hoffmannita Pelaez, 1962: 72

Myrmicocheyla Volgin, 1963: 935

Type species: Hoffmannita mexicana Pelaez, 1962

Hoffmannita clavipes (Volgin)

Myrmicocheyla clavipes Volgin, 1963: 935

Hoffmannita navicula: Lin \& Zhang, 1997

Hoffmannita clavipes (Volgin): Volgin, 1969: 266; Xia, 2010: 161; Khausov, 2021: 235 ( Kurgan and Tyumen Provinces)

Type locality: Ushkovo, Russia (Leningradskaya Prov.), ant hills of Formica rufa (Hymenoptera: Formicidae)

Distribution: China, Czech Rep., Russia

Hosts: Aradus betulae, A. corticalis (Heteroptera: Aradidae), ant hills

Hoffmannita gersoni Fain et Bochkov

Hoffmannita gersoni Fain \& Bochkov, $2001: 73$

Type locality: Curacao

Distribution: Netherlands-Antilles

Host: Coenobita clypeata (Decapoda: Coenobitidae) 


\section{Hoffmannita mexicana Pelaez}

Hoffmannita mexicana Pelaez, 1962: 75; Fain \& Bochkov, 2001 : 73

Type locality: Estado de Oaxaca

Distribution: Mexico

Host: Centruroides flavopictus (Scorpiones: Buthidae)

Hoffmannita rimandoi Corpuz-Raros

Hoffmannita rimandoi Corpuz-Raros, 1972: 262

Type locality: Philippines

Distribution: Philippines

Genus Hylopecheyla Fain

Hylopecheyla Fain, 1972: 41

Type species: Hylopecheyla bunguranensis Fain, 1972

\section{Hylopecheyla bunguranensis Fain}

Hylopecheyla bunguranensis Fain, 1972: 42; 1979: 420; Fain \& Bochkov, 2001: 60

Type locality: Bunguran Is., Natunas

Distribution: Indonesia (Natunas, Java)

Hosts: Hylopetes everetti, H. sagitta (Rodentia: Pteromyidae)

\section{Hylopecheyla malayi Fain et Nadchatram}

Hylopecheyla malayi Fain \& Nadchatram, 1980: 197; Fain \& Bochkov, 2001: 59

Type locality: Jeugka Triangle Feedacheme (Pahang)

Distribution: Malaysia

Host: Tupaia glis (Scandentia: Tupaiidae)

\section{Genus Hypopicheyla Volgin}

Hypopicheyla Volgin, 1969: 242

Type species: Hypopicheyla elongata Volgin, 1969

\section{Hypopicheyla elongata Volgin}

Hypopicheyla elongata Volgin, 1969: 243; Summers \& Price, 1970: 65-66; Doğan \& Ayyildiz, 2004: 1; Bayram \& Cobanoglu, 2006; Salarzehi, 2018

Type locality: Russia (many areas in European and Asiatic Russia); Estonia (Tartu)

Distribution: Estonia, Russia, Iran, Turkey; ?USA (California)

Hosts: Aradus aterrimus, A. crenaticollis, A. lugubris, A. nemtschinovi, etc. (Heteroptera: Aradidae)

\section{Hypopicheyla mirabilis (Volgin)}

Cheyletia mirabilis Volgin, 1955: 170; 1956: 212 
Hypopicheyla mirabilis (Volgin): Volgin, 1969: 245; Summers \& Price, 1970: 65; Corpuz-

Raros, 1998; Bochkov, Hakimitabar \& Saboori, 2005: 104

Type locality: Hiva (Uzbekistan)

Distribution: Iran, Philippines, Uzbekistan

Host: Gonocephalum rusticum (Coleoptera: Tenebrionidae)

Genus Metacheletoides Fain

Metacheletoides Fain, 1972: 40

Type species: Metacheletoides numidae Fain, 1972

Metacheletoides akanyaruensis (Fain)

Cheletoides akanyaruensis Fain, 1972: 40

Metacheletoides akanyaruensis (Fain): Fain, 1979: 1017

Type locality: Akanyaru River

Distribution: Rwanda

Host: Numida meleagris intermedia (Galliformes: Numididae)

\section{Metacheletoides crinifer Fain}

Metacheletoides crinifer Fain, 1979: 1018

Type localites: Butare, Gisagara (Rwanda), Marona (Cameroons), Lama Kara (Togo), Lotanoko (D. R. Congo)

Distribution: Cameroons, D. R. Congo, Rwanda, Togo

Hosts: Crinifer piscator, C. zonurus, Corythaixoides leucogaster (Musophagiformes: $\mathrm{Mu}$ sophagidae)

\section{Metacheletoides gisagarensis Fain}

Metacheletoides gisagarensis Fain, 1979: 1021

Type locality: Butare (Rwanda), Molegbe (D. R. Congo), Lama Kara (Togo)

Distribution: D. R. Congo, Rwanda, Togo

Hosts: Crinifer piscator, C. zonurus (Musophagiformes: Musophagidae)

\section{Metacheletoides numidae Fain}

Metacheletoides numidae Fain, 1972: 41; 1979: 1013

Type locality: n. Akanyaru River (Rwanda)

Distribution: Burundi, Ethiopia, Rwanda

Hosts: Numida meleagris intermedia, N. meleagris meleagris (Galliformes: Numididae)

Genus Mexecheles De Leon

Mexecheles De Leon, 1962: 132

Acarocheyla Volgin, 1965: 293 (syn. by Summers \& Price, 1970: 43)

Type species: Mexecheles cunliffei De Leon, 1962 
Mexecheles aztecorum De Leon

Mexecheles aztecorum De Leon, 1962: 133; Summers \& Price, 1970: 45

Type locality: Oaxaca, Tuxtla Gutierez (Chiapas)

Distribution: Mexico (Oaxaca, Chiapas)

Host: on Quercus conzattii and Cocos nucifera

Mexecheles cunliffei De Leon

Mexecheles cunliffei De Leon, 1962: 132; Volgin, 1969: 185; Summers \& Price, 1970: 44

Type locality: Huajuapan de León, Oaxaca

Distribution: Mexico (Oaxaca)

Host: Ipomea murucoides (Convolvulaceae)

Mexecheles hawaiiensis (Baker)

Cheletophyes hawaiiensis Baker, 1949: 289

Mexecheles hawaiiensis (Baker): Muma, 1964: 248; Volgin, 1969: 198; Summers \& Price, 1970: 44; Flechtmann, 1971: 31; Torre Santana, 2015

Mexecheles intermedius De Leon, 1962: 133; Muma, 1964: 248

Acarocheyla hawaiiensis: Volgin, 1965: 293

Type locality: Kailua, Oahu, Hawaii

Distribution: Brazil, Mexico, Panama, USA (Hawaiian Is.)

Mexecheles impolitus (Smiley et Moser)

Acarocheyla impolita Smiley \& Moser, 1970: 231

Mexecheles impolitus (Smiley et Moser): Summers \& Price, 1970: 48; Fain \& Bochkov, 2001: 55

Type locality: Elizabeth, Louisiana

Distribution: USA (Louisiana)

Host: in boring dust and in galleries of Dendroctonus spp. (Coleoptera: Scolytidae)

Mexecheles marshalli (Baker)

Cheletophyes marshalli Baker, 1949: 290

Mexecheles aztecorum De Leon, 1962: 133; Summers \& Price, 1970: 45

Acarocheyla marshalli: Volgin, 1965: 294

Mexecheles marshalli (Baker): Gerson, Fain \& Smiley, 1999: 70

Type locality: Imboden (Arkansas)

Distribution: Mexico, USA (Arkansas)

Mexecheles panneus Summers et Price

Mexecheles panneus Summers \& Price, 1970: 46

Type locality: Lehman Creek, 8 miles W of Baker, Nevada

Distribution: USA (Nevada) 
Mexecheles shiva Bochkov et Ochoa

Mexecheles shiva Bochkov \& Ochoa, 2005: 137

Type locality: Bombay

Distribution: India

Mexecheles thailandensis Fuangarworn et Lekprayoon

Mexecheles thailandensis Fuangarworn \& Lekprayoon, 2010: 60

Type locality: Sasaloy Subdistrict, Tharua District, Ayutthaya Province

Distribution: Thailand

Host: from tree bark infested by scolytid beetles

\section{Mexecheles virginiensis (Baker)}

Cheyletia virginiensis Baker, 1949: 299

Paracheyletia virginiensis (Baker): Volgin, 1955: 169

Mexecheles virginiensis (Baker): De Leon, 1962: 132; Volgin, 1969: 187; Summers \& Price, 1970: 47; Fain \& Bochkov, 2001: 54; Bochkov \& Abramov, 2016; Diumina \& Abramov, 2018: 213; Khaustov, 2021: 234

Acarocheyla virginiensis (Baker): Smiley \& Moser, 1970: 233

Type locality: Gloucester Co., Virginia (USA)

Distribution: India (Kashmir), U.S.A. (Virginia, Louisiana, Nevada, Utah), Russia Host: from boring dust of Ips calligraphus (Coleoptera: Scolytidae)

Mexecheles votandinii Jeffrey

Mexecheles votandinii Jeffrey, 1976: 668; 1977: 654

Type locality: Girvan, Ayrshire (Scotland)

Distribution: Scotland (U.K.)

Genus Microcheyla Volgin

Microcheyla Volgin, 1966b: 277

Type species: Microcheyla parvula Volgin, 1966

Microcheyla bengalensis Gupta et Paul

Microcheyla bengalensis Gupta \& Paul, 1992: 76

Type locality: West Bengal, Medinipur

Distribution: India

Host: Ploceus philippinus (Passeriformes: Ploceidae)

\section{Microcheyla granifera Kuznetzov}

Microcheyla granifera Kuznetzov, 1977: 925

Microcheyla ozkani Koç \& Ayyldiz, 1995: 226 - Turkey (syn. by Fain \& Bochkov, 2001 : 74)

Type locality: Yalta, Crimea 
Distribution: Turkey, Crimea

Microcheyla parvula Volgin

Microcheyla parvula Volgin, 1966b: 278; 1969: 270; Summers \& Price, 1970: 72; Salarzehi, 2018:

Type locality: Ramit (Tadjikistan)

Distribution: Iran, Tadjikistan

Microcheyla paraparvula Olivier et Theron

Microcheyla paraparvula Olivier \& Theron, 1989b: 49

Type locality: Transvaal

Distribution: South Africa (Transvaal)

Microcheyla squamosa Olivier et Theron

Microcheyla squamosa Olivier \& Theron, 1989b: 54

Type locality: Transvaal

Distribution: South Africa (Transvaal)

Genus Muricheyla Fain

Muricheyla Fain, 1972: 42

Type species: Muricheyla sicista Fain, 1972

Muricheyla sicista Fain

Muricheyla sicista Fain, 1972: 43

Type locality: Caucase

Distribution: Caucase

Host: Sicista subtilis (Rodentia: Zapodidae)

Genus Neoeucheyla Radford

Neoeucheyla Radford, 1950: 82

Type species: Cheyletia (Eucheyla) loricata Berlese, 1913

Neoeucheyla bochkovi Fain

Neoeucheyla bochkovi Fain, 2003: 97

Type locality: Algiers

Distribution: Algeria

Host: from a flower in a garden

Neoeucheyla iranica Fain et Ardeshir

Neoeucheyla iranica Fain \& Ardeshir, 2000: 329; Fain \& Bochkov, 2001 : 72; Bochkov, Hakimitabar \& Saboori, 2005: 105 
Type locality: Iran

Distribution: Iran

Host: in a silo

Neoeucheyla loricata (Berlese)

Cheyletia (Eucheyla) loricata Berlese, 1913: 79; Volgin, 1955: 170

Neoeucheyla loricata (Berlese): Volgin, 1969: 25; Summers \& Price, 1970: 67; Fain \&

Bochkov, 2001 : 72; Bochkov \& Mironov, 2007: 75

Type locality: Palermo («in Agri Panormitani muscis»)

Distribution: Italy (Sicily)

Neoeucheyla minuta Barilo

Neoeucheyla minuta Barilo, 1986: 28; Fain \& Bochkov, 2001 : 72; Bochkov \& Mironov, 2007: 75

Type locality: Uzbekistan

Distribution: Uzbekistan

Neoeucheyla macrocorneus Soliman - Species inquirenda

Neoeucheyla macrocorneus Soliman, 1975: 101

Type locality: Giza

Distribution: Egypt

Neoeucheyla ploceus Gupta et Paul - Species inquirenda

Neoeucheyla ploceus Gupta \& Paul, 1992: 74; Bochkov \& Mironov, 2007: 75

Type locality: West Bengal, Medinpur

Distribution: India

Host: Ploceus philippinus (Passeriformes: Ploceidae)

Genus Oconnoricheylus Bochkov et Otto

Oconnoricheylus Bochkov \& Otto, 2010: 2

Type species: Oconnoricheylus speciosus Bochkov et Otto, 2010

Oconnorichelus chimaera (Bochkov et Ochoa)

Alliea chimaera Bochkov \& Ochoa, 2005: 140

Oconnorichelus chimaera: Bochkov \& Otto, 2010: 3

Type locality: Queensland

Distribution: Australia (Queensland)

Oconnoricheylus speciosus Bochkov et Otto

Oconnoricheylus speciosus Bochkov \& Otto, 2010: 3

Type locality: USA 
Distribution: USA

Host: from oranges

Genus Oudemansicheyla Volgin

Oudemansicheyla Volgin, 1969: 272

Type species: Cheletomimus denmarki Yunker, 1961

Oudemansicheyla coprosomae Thewke et Enns

Oudemansicheyla coprosomae Thewke \& Enns, 1976: 369

Type locality: Cobb L., Nelson Co.

Distribution: New Zealand

Oudemansicheyla denmarki (Yunker)

Cheletomimus denmarki Yunker, 1961: 1032; De Leon, 1962: 134

Oudemansicheyla denmarki (Yunker): Volgin, 1969: 272; Summers \& Price, 1970: 10;

Tseng, 1977: 254; Gerson, 1994: 439; Lin et al., 2000: 86; Xia, 2010: 161

Type locality: Florida (USA)

Distribution: Australia, China (Fujian), Taiwan, USA (Florida, Louisiana)

Genus Paramicrocheyla Olivier et Theron

Paramicrocheyla Olivier \& Theron, 1989:238; Fain \& Bochkov, 2001 :299 (genus incertae sedis)

Type species: Paramicrocheyla spinula Olivier et Theron, 1989

Paramicrocheyla spinula Olivier et Theron

Paramicrocheyla spinula Olivier \& Theron, 1989: 238

Type locality: Transvaal

Distribution: South Africa (Transvaal)

Paramicrocheyla ungulina Olivier et Theron

Paramicrocheyla ungulina Olivier \& Theron, 1989: 242

Type locality: Transvaal

Distribution: South Africa (Transvaal)

Genus Picocheyletus Bochkov et OConnor

Picocheyletus Bochkov \& OConnor, 2003: 268

Type species: Picocheyletus tricholaemae Bochkov et OConnor, 2003

Picocheyletus tricholaemae Bochkov et OConnor

Picocheyletus tricholaemae Bochkov \& OConnor, 2003: 268

Type locality: Ogooue - Maritime, $25.2 \mathrm{~km}$ NW Doussala

Distribution: Gabon 
Host: Tricholaema hirsuta flavipunctata (Piciformes: Capitonidae)

Genus Promuricheyla Fain

Promuricheyla Fain, 1979: 413

Type species: Promuricheyla lukoschusi Fain, 1979

Promuricheyla lukoschusi Fain

Promuricheyla lukoschusi Fain, 1979: 413

Type species: Mt. Katanglad, Mindanao

Distribution: Philippines (Mindanao)

Host: Nannosciurus surrutilus (Rodentia: Sciuridae)

Genus Prosocheyla Volgin

Prosocheyla Volgin, 1969: 288

Type species: Cheletogenes oaklandia Baker, 1949

Subgenus Prosocheyla Volgin

Prosocheyla Volgin, 1969: 288

Type species: Cheletogenes oaklandia Baker, 1949

Prosocheyla (P.) acanthus Smiley et Moser [acanthi ?]

Prosocheyla acanthus Smiley \& Moser, 1970: 229; Fain \& Bochkov, 2001: 85

Prosocheyla acantha: Summers \& Price, 1970: 57

Type locality: Maurepas (Louisiana)

Distribution: USA (Louisiana)

Host: outer bark of loblolly pine

Prosocheyla (P.) oaklandia (Baker)

Cheletogenes oaklandia Baker, 1949: 306

Prosocheyla (Prosocheyla) oaklandia (Baker): Volgin, 1969: 289; Fain \& Bochkov, 2001: 85

Prosocheyla oaklandia (Baker): Summers \& Price, 1970: 55 (many localities in California);

Lucza, Ripka \& Saly, 1996 (oaklandi)

Type locality: Oakland, California

Distribution: Hungary, USA (California)

Prosocheyla (P.) ripkai Fain et Bochkov

Prosocheyla ripkai Fain \& Bochkov, 2001: 85

Type locality: Budapest

Distribution: Hungary

Host: from a tree Populus simonii 
Prosocheyla (P.) sinensis Xia, Liang \& Zhu

Prosocheyla (P.) sinensis Xia, Liang \& Zhu, 2005: 205; Xia, 2010: 161

Type locality: Henan

Distribution: China

Prosocheyla (P.) traubi (Baker)

Cheletogenes traubi Baker, 1949: 307

Prosocheyla traubi (Baker): Volgin, 1969: 291; Summers \& Price, 1970: 56; Lucza, Ripka \& Saly, 1996; Ripka, Fain \& Bolland, 1999: 366; Fain \& Bochkov, 2001: 85;

Type locality: Assam, Stillwell Road, near Ledo, India

Distribution: Burma, Hungary, India

Prosocheyla (P.) villosa Bochkov et Haustov

Prosocheyla villosa Bochkov \& Haustov, 1999: 151; Fain \& Bochkov, 2001: 85

Type locality: Crimea, Aj-Danil'

Distribution: Crimea

Host: Xylocleptes bispinus (Coleoptera: Curculionidae)

Subgenus Reckiana Volgin

Reckiana Volgin, 1969: 291

Type species: Cheletogenes buckneri Baker, 1949

Prosocheyla (Reckiana) buckneri (Baker)

Cheletogenes buckneri Baker, 1949: 308

Prosocheyla (Reckiana) buckneri (Baker): Volgin, 1969: 293; Fain \& Bochkov, 2001: 85

Prosocheyla buckneri (Baker): Summers \& Price, 1970: 56

Type locality: Santa Paula, California

Distribution: USA (California)

\section{Prosocheyla (Reckiana) citrifoliata (Muma)}

Cheletogenes citrifoliata Muma, 1964: 241

Prosocheyla (Reckiana) citrifoliata (Muma): Volgin, 1969: 294; Fain \& Bochkov, 2001: 85;

syn. by Summers \& Price, 1970: 56 with Prosocheyla buckneri

Type locality: Florida

Distribution: USA (Fla)

\section{Prosocheyla (Reckiana) hepburni (Lawrence)}

Cheletogenes hepburni Lawrence, 1954: 70

Prosocheyla (Reckiana) hepburni (Lawrence): Volgin, 1969: 292

Prosocheyla hepburni (Lawrence): Summers \& Price, 1970: 58

Type locality: Hamburg, Peddie Distr., South Africa 
Distribution: South Africa

Host: Lepidosaphes beckii (Homoptera: Coccodea: Diaspididae)

Genus Samsinakia Volgin

Samsinakia Volgin, 1965: 296

Cryptocheyla Fain, 1972: 41

Type species: Cheletophyes theodoridis Samšinak, 1959

Samsinakia carabae Ramaraju et Mohanasundram

Samsinakia carabae Ramaraju \& Mohanasundram, 1999: 125; Fain \& Bochkov, 2001: 76

Type locality: Tamil Nadu, Trichy

Distribution: India (Tamil Nadu)

Host: Carabidae (Coleoptera)

Samsinakia charanasriae Fuangarworn et Lekprayoon

Samsinakia charanasriae Fuangarworn \& Lekprayoon, 2010: 64

Type locality: Buang-Chawak Park, Supan Buri Province

Distribution: Thailand

Host: from soil and leaf-litter

Samsinakia gonocephalum Fain

Samsinakia gonocephalum Fain, 1984: 686; Bochkov \& Mironov, 1998: 265; Fain \& Bochkov, 2001: 76

Type locality: DR Congo

Distribution: D. R. Congo (former Zaire), India

Host: Gonocephalum simplex (Coleoptera: Tenebrionidae)

Samsinakia pagongae Corpuz - Raros et Sotto

? Samsinakia pagongae Corpuz-Raros \& Sotto, 1977: 165; Fain \& Bochkov, 2001: 76

Type locality: Cale, Tanauan, Batangas

Distribution: Philippine Is.

Samsinakia theodoridis (Samšinak)

Cheletophyes theodoridis Samšinák, 1959: 81

Samsinakia theodoridis (Samšinák): Volgin, 1965: 298; 1969: 247; Summers \& Price, 1970:

71; Fain \& Bochkov, 2001: 76

Type locality: Tananarive

Distribution: Madagascar

Host: Selinus abacoides (Coleoptera: Tenebrionidae) 
Samsinakia tilae Bochkov et Klimov

Samsinakia tilae Bochkov \& Klimov, 2005: 15

Type locality: Chiapas, $39 \mathrm{~km}$ on road between Tuxtla Gutierrez and Revolutión Mexicana Distribution: Mexico (Chiapas)

Host: Blastinus sp. (Coleoptera: Tenebrionidae)

Samsinakia trilobitus Bochkov et Mironov

Samsinakia trilobitus Bochkov \& Mironov, 1998: 266; Fain \& Bochkov, 2001: 76; Kazemi \& Paktinat-Saeij. 2021: 107

Type locality: Anamalai Hills, Cinchora

Distribution: India, Iran

Host: beetles (Coleoptera: Tenebrionidae)

\section{Samsinakia volgini (Fain)}

Cryptocheyla volgini Fain, 1972: 41

Samsinakia volgini (Fain): Fain, 1980: 105; Gerson, 1994: 444; Fain \& Bochkov, 2001: 76 Type locality: Butare (Rwanda)

Distribution: Australia, D. R. Congo, Rwanda

Host: nest of Spermestes cucullatus (Passeriformes); on Gonocephalum simplex (Coleoptera: Tenebrionidae)

Genus Thewkachela Ide et Kethley

Thewkachela Ide \& Kethley, 1977: 559

Type species: Thewkachela ratufi Ide et Kethley, 1977

Thewkachela ratufi Ide et Kethley

Thewkachela ratufi Ide \& Kethley, 1977: 559; Fain \& Nadchatram, 1980: 199

Type locality: Sabah

Distribution: Malaysia (Sabah), Thailand

Hosts: Ratufa affinis, R. bicolor (Rodentia: Sciuridae)

Subfamily Criokerontinae Smiley

Criokerontinae Smiley, 1978: 239

Type genus: Criokeron Volgin, 1966

Tribe Niheliini Smiley

Niheliinae Smiley, 1978: 239

Type genus: Nihelia Domrow et Baker, 1960

Criokerontini Smiley, 1978: 239

Type genus: Criokeron Volgin, 1966 


\section{Genus Criokeron Volgin}

Criokeron Volgin, 1966: 293

Type species: Nihelia quinta Domrow et Baker, 1963

Criokeron quintus (Domrow et Baker)

Nihelia quinta Domrow \& Baker, 1963: 230

Criokeron quinta: Fain \& Nadchatram, 1980: 199

Criokeron quintus (Domrow et Baker); Volgin, 1966: 219; 1969: 386; Fain \& Lukoschus, 1985: 264

Type locality: Subang, Selangor

Distribution: Malaysia, Thailand

Hosts: Tupaia belangeri, T. glis (Scandentia: Tupaiidae)

Criokeron thailandicus Fain et Lukoschus

Criokeron thailandicus Fain \& Lukoschus, 1985: 266

Type locality: Nakhon Kachisima, Pak Thong Chai, Ban Sakaerat

Distribution: Thailand

Host: Tupaia glis (Scandentia: Tupaiidae)

Genus Galagocheles Fain

Galagocheles Fain, 1979: 629

Type species: Cheletiella lemuricola Lawrence, 1948

Galagocheles lawrencei Bochkov, Mironov et OConnor

Galagocheles lawrencei Bochkov, Mironov \& OConnor, 2006: 189

Type locality: $3 \mathrm{~km}$ SE Nayaoure (Burkina Faso = Upper Volta)

Distribution: Burkina Faso, DR Congo, Rwanda

Hosts: Galago moholi, G. senegalensis (Primates: Galagonidae)

\section{Galagocheles lemuricola (Lawrence)}

Cheletiella lemuricola Lawrence, 1948: 39

Hemicheyletus lemuricola: Lawrence, 1954: 74; 1961: 114

Hemicheyletus (H.) lemuricola: Volgin, 1969: 378

Nihelia lemuricola (Lawrence): Domrow \& Baker, 1963: 226; Summers \& Price, 1970: 75

Galagocheles lemuricola (Lawrence): Fain, 1979: 630; Bochkov, Mironov \& OConnor, 2006: 185

Type locality: Kwa Zulu-Natal, Cato Ridge, nr. Pietermaritzburg

Distribution: D. R. Congo (former Zaire), Rwanda, South Africa

Hosts: Otolemur garnetti, O. [Galago] crassicaudata monteri, Galago moholi (Primates: Galagonidae) 
Genus Nihelia Domrow et Baker

Hemicheyletus Lawrence, 1954: 74

Nihelia Domrow \& Baker, 1960: 194

Type species: Cheletiella curvidens Lawrence, 1948

Nihelia calcarata Domrow et Baker

Nihelia calcarata Domrow \& Baker, 1960: 194; 1963: 226

Hemicheyletus calcaratus (Domrow et Baker): Volgin, 1969: 382

Hemicheyletus calcarata: Nagar, 1978: 102

Hemicheyletus herpestesi: Nagar \& Saxena, 1977: 35

Type locality: Kanchunaburi, Muang, Thailand

Distribution: India, Thailand

Hosts: Herpestes sp. (Thailand), H. edwardsii (India)(Carnivora: Herpestidae)

Nihelia curvidens (Lawrence)

Cheletiella curvidens Lawrence, 1948: 40

Hemicheyletus curvidens, Lawrence, 1954: 74; 1956: 338; 1961: 114

Hemicheyletus (Hemicheyletus) curvidens: Volgin, 1969: 380

Nihelia curvidens (Lawrence): Domrow \& Baker, 1963: 226; Summers \& Price, 1970: 75

Type locality: Natal

Distribution: Angola, South Africa

Hosts: Galerella [Herpestes] sanguinea, Herpestes [Myonax cauui] punctulatus, H. bocagei

(Carnivora: Herpestidae)

Nihelia cynictis (Fain)

Hemicheyletus cynictis Fain, 1979: 625

Nihelia cynictis (Fain)

Type locality: Bloemhof (Transvaal)

Distribution: South Africa (Transvaal)

Host: Cynictis penicillata (Carnivora: Viverridae)

\section{Genus Sciurocheyla Volgin}

Hemicheyletus (Sciurocheyla) Volgin, 1969: 384

Thryonomycheyla Fain, 1972: 42 (syn. by Bochkov \& OConnor, 2004)

Smileycheles Fain, 1979: 627 (syn. by Bochkov \& OConnor, 2004)

Type species: Nihelia squamosa Domrow et Baker, 1963

Type species of Smileycheles Fain: S. camerounensis Fain, 1979

Type species of Thryonomycheyla Fain: T. congolensis Fain, 1972

Sciurocheyla angolensis (Fain et Bochkov)

Thryonomycheyla angolensis Fain \& Bochkov, 2002: 148 
Sciurocheyla angolensis (Fain et Bochkov):

Type locality: Angola

Distribution: Angola

Host: (?) Thryonomys swinderianus (Rodentia: Thryonomyidae)(contamination ?)

Sciurocheyla camerounensis (Fain)

Smileycheles camerounensis Fain, 1979: 627

Sciurocheyla camerounensis (Fain): Bochkov \& OConnor, 2004: 316

Type locality: Foulassi (Cameroon)

Distribution: Cameroon, Tanzania

Hosts: Heliosciurus undulatus (Rodentia: Sciuridae), Zenkerela insignis (Rodentia: Anomaluridae)(cont.?)

\section{Sciurocheyla congolensis (Fain)}

Thryonomycheyla congolensis Fain, 1972: 42

Sciurocheyla congolensis (Fain): Bochkov \& OConnor, 2004: 309

Type locality: D.R. Congo

Distribution: D.R. Congo

Host: (?) Thryonomys swinderianus (Rodentia: Thryonomyidae)(cont.?)

Sciurocheyla funisciurus Bochkov et OConnor

Sciurocheyla funisciurus Bochkov \& OConnor, 2004: 311

Type locality: Cibitoke Prov., Nat. Park, Ndora Zone, E Kirumura, 2100 m

Distribution: Burundi

Host: Funisciurus pyrrhopus (Rodentia: Sciuridae)

Sciurocheyla squamosa (Domrow et Baker)

Nihelia squamosa Domrow \& Baker, 1963: 227; Summers \& Price, 1970: 75

Hemicheyletus (Sciurocheyla) squamosus (Domrow et Baker): Volgin, 1969: 384

Sciurocheyla squamosus (Domrow et Baker): Fain \& Lukoschus, 1985: 264

Sciurocheyla squamosa (Domrow et Baker): Bochkov \& Oconnor, 2004: 309

Type locality: Nan Prov., Pang Nam NU, Bun Yun Distr., betw. Ban Rong Kwang and Ban

Klang Wiang

Distribution: Thailand

Host: Menetes sp. (Rodentia: Sciuridae)

Subfamily Metacheyletiinae Fain

Metacheyletiinae Fain, 1980: 107

Type genus: Metacheyletia Fain, 1972 


\section{Genus Metacheyletia Fain}

Metacheyletia Fain, 1972: 43

Type species: Metacheyletia obesa Fain, 1972

Metacheyletia amazonae Bochkov et OConnor

Metacheyletia amazonae Bochkov \& OConnor, 2003: 266

Type locality: Guyana

Distribution: Guyana

Host: Amazona amazonica (Psittaciformes: Psittacidae)

Metacheyletia degenerata Fain et Bochkov

Metacheyletia degenerata Fain \& Bochkov, 2003: 119

Type locality: Central Africa (Western Tanzania)

Distribution: Central Africa

Host: Serinus mozambicus (Passeriformes: Fringillidae)

Metacheyletia longisetosa Atyeo, Kethley et Pérez

Metacheyletia longisetosa Atyeo, Kethley \& Pérez, 1984: 125

Type locality: Jalisco, Puerto Vallarta

Distribution: Mexico

Host: Amazona finsehi (Psittaciformes: Psittacidae)

Metacheyletia ngaii Bochkov et Skoracki

Metacheyletia ngaii Bochkov \& Skoracki, 2011: 93

Type locality: Tanganyika

Distribution: Tanzania

Host: Corythaixoides leucogaster (Musophagiformes: Musophagidae)

\section{Metacheyletia obesa Fain}

Metacheyletia obesa Fain, 1972: 43; 1980: 107

Type locality: Antwerp Zoo

Distribution:?

Host: Psittacula sp. (Psittaciformes: Psittaculidae)

Subfamily Ornithocheyletiinae Smiley

Ornithocheyletiinae Smiley, 1978: 228

Type genus: Ornithocheyletia Volgin, 1964

Tribe Ornithocheyletiini Volgin

Ornithocheyletiini Volgin, 1969: 387

Apodichelini Fain, Smiley \& Gerson, 1997: 81 
Neocheyletiellini Fain, Smiley \& Gerson, 1997: 81

Type genus: Ornithocheyletia Volgin, 1964

Genus Apodicheles Fain

Apodicheles Fain, 1979: 253

Type species: Apodicheles cypsiurus Fain, 1979

Apodicheles apus Fain

Apodicheles apus Fain, 1979: 255; Fain \& Bochkov, 2002a: 159

Type locality: Butare

Distribution: Rwanda

Host: Apus caffer (Apodiformes: Apodidae)

Apodicheles collocalia Bochkov et OConnor

Apodicheles collocalia Bochkov \& OConnor, 2003: 273

Type locality: Leyte Is., 4 km S, 1 km E Inopacan

Distribution: Philippines (Leyte)

Host: Collocalia esculenta marginata (Apodiformes: Apodidae)

Apodicheles cypsiurus Fain

Apodicheles cypsiurus Fain, 1979: 253

Type locality: Tzaneen, Transvaal

Distribution: South Africa

Host: Cypsiurus p. parvus (Apodiformes: Apodidae)

\section{Apodicheles heteropalpus (Mégnin) - Species inquirenda}

Cheyletus heteropalpus Mégnin, 1878: 11; 1880: 242

Cheyletiella heteropalpus: Berlese, 1886: fasc. 28, 2

Cheyletiella heteropalpa: Oudemans, 1906: 213

Neocheyletiella heteropalpus (Mégnin): Baker, 1949: 272

Cheyletiella heteropalpa: Starkoff \& Starkoff, 1950: 96

Neocheyletiella heteropalpa: Brown, 1971: 1380

Apodicheles heteropalpus (Mégnin)

Type locality: Probably France

Distribution: France (?), Italy, USA

Host: Columba livia (Columbiformes: Columbidae)

Genus Bakericheyla Volgin

Bakericheyla Volgin, 1966: 297

Type species: Cheyletiella chanayi Berlese et Trouessart, 1889 


\section{Subgenus Apodicheyla Fain}

Apodicheyla Fain, 1979: 255

Type species: Bakericheyla (Apodicheyla) africana Fain, 1979

\section{Bakericheyla (Apodicheyla) africana Fain}

Bakericheyla (Apodicheyla) africana Fain, 1979: 256; Fain \& Bochkov, 2001: 88

Type locality: Tzaneen, Transvaal

Distribution: South Africa

Host: Cypsiurus parvus (Apodiformes: Apodidae)

\section{Subgenus Bakericheyla Volgin}

Bakericheyla Volgin, 1966: 297

Type species: Cheyletiella chanayi Berlese et Trouessart, 1889

\section{Bakericheyla (Bakericheyla) benoiti Fain}

Bakericheyla (Bakericheyla) benoiti Fain, 1980: 135

Type locality: Galim

Distribution: Cameroons

Host: Merops [Melittophagus] bullocki (Coraciiformes: Meropidae) on the skin

Bakericheyla (Bakericheyla) chanayi (Berlese et Trouessart)

Cheyletiella Chanayi Berlese \& Trouessart, 1889: 135; Berlese, 1889: fasc. 56, No 3

Cheletiella chanayi (Berlese et Trouessart): Oudemans, 1906: 213

Neocheyletiella chanayi, Baker, 1949: 274; Dubinin, 1957: 90; Volgin \& Nikolaeva, 1965: 300

Bakericheyla chanayi (Berlese et Trouessart): Furman \& Sousa, 1969: 858; Volgin, 1969:

400; 1987: 492; Smiley, 1970: 1060; Summers \& Price, 1970: 77; Domrow, 1991: 1303;

Fain \& Bochkov, 2001: 88

Neocheyletiella faini Lawrence, 1959: 431 - Mossel Bay, Cape Province, from Cossypha dichroa (Passeriformes: Muscicapidae); synonymized by Fain \& Bochkov, 2001: 88

Bakericheyla faini (Lawrence): Volgin, 1969: 405

Bakericheyla (B.) faini (Lawrence): Fain, 1980: 134 - from Cossypha dichroa, Cape Prov., South Africa

Bakericheyla (Bakericheyla) chanayi (Berlese et Trouessart): Fain, 1980: 133

Bakericheyla chanayi latior Fain, 1972: 46 - on Paroaria gularis

Type locality: Lyon, France

Distribution: Australia (Northern Territory), Holland, South Africa

Hosts: Motacilla alba (Passeriformes: Motacillidae), Poephila acuticauda, P. gouldiae (Passeriformes: Estrildidae), Carduelis carduelis, Fringilla coelebs (Passeriformes: Fringillidae), Paroaria gularis (Passeriformes: Thraupidae), Cossypha dichroa (Passeriformes: Muscicapidae) 
Bakericheyla (Bakericheyla) subquadrata (Lawrence)

Neocheyletiella subquadrata Lawrence, 1959: 427

Bakericheyla subquadrata (Lawrence): Volgin, 1969: 411; Summers \& Price, 1970: 78;

Fain \& Bochkov, 2001: 88

Bakericheyla (B.) subquadrata (Lawrence): Fain, 1980: 134

Type locality: Kanji (Botswana)

Distribution: Botswana, South Africa

Host: Merops [Melittophagus pusillus] (Coraciiformes: Meropidae)

Bakericheyla (Bakericheyla) transvaalica (Lawrence)

Neocheyletiella transvaalica Lawrence, 1959: 430

Bakericheyla transvaalica (Lawrence): Volgin, 1969: 406; Summers \& Price, 1970: 78; Fain \& Bochkov, 2001: 88

Bakericheyla (B.) transvaalica Fain, 1980: 134

Type locality: Mabelikwa (Transvaal)

Distribution: former U. S. S. R., South Africa, Rwanda, Zimbabwe

Hosts: Melittophagus pusillus, M. bullockoides, Merops apiaster, M. supercilliosus persicus, M. nubicoides (Coraciiformes: Meropidae)

Genus Ornithocheyletia Volgin

Ornithocheyletia Volgin, 1964: 28

Type species: Ornithocheyletia dubinini Volgin, 1964

Ornithocheyletia aitkeni Fain

Ornithocheyletia aitkeni Fain, 1972: 46; 1981: 201

Type locality: Guama

Distribution: Brazil

Host: Turdus fumigatus (Passeriformes: Turdidae)

Ornithocheyletia argentinensis Fain

Ornithocheyletia argentinensis Fain, 1972: 45; 1981: 198

Type locality: Antwerp Zoo

Distribution: South America, (Argentina)

Hosts: Forpus passerinus, Nandays nanday (Psittaciformes: Psittacidae)

Ornithocheyletia boeroi Brandetti et Bischoff-de-Alzuet

Ornithocheyletia boeroi Brandetti \& Bischoff-de-Alzuet, 1981: 236

Type locality: Argentina

Distribution: Argentina

Host: Cyanocorax chrysops (Passeriformes: Corvidae) 


\section{Ornithocheyletia canadensis (Banks)}

Cheyletiella canadensis Banks, 1909: 133

Neocheyletiella canadensis (Banks): Baker, 1949: 272; Volgin, 1964: 32

Ornithocheyletia canadensis (Banks), Volgin, 1969: 393; Summers \& Price, 1970: 78

Type locality: Guelph (Ontario)

Distribution: Bulgaria, Canada

Host: Picus viridis (Piciformes: Picidae)

Ornithocheyletia columbigallinae Fain et Bochkov

Ornithocheyletia columbigallinae Fain \& Bochkov, 2002: 148

Type locality: Piracicaba

Distribution: Brazil

Host: Columbigallina sp. (Columbifomes: Columbidae)

\section{Ornithocheyletia dubinini Volgin}

Ornithocheyletia dubinini Volgin, 1964: 28; 1969: 390; Summers \& Price, 1970: 78; Smiley, 1970: 1065; 1977: 229; Fain, 1981: 186; Skoracki, Bochkov \& Sikora, 2004: 87 Ornithocheyletia barri Smiley, 1977: 234 - type: Davis, California, on Sturnus vulgaris Type locality: Kishinev (Moldavia)

Distribution: Holland, Moldavia, Poland; USA (California)

Host: Sturnus vulgaris (Passeriformes: Sturnidae)

\section{Ornithocheyletia eulabes Fain}

Ornithocheyletia eulabes Fain, 1981: 200

Type locality: Antwerp Zoo

Distribution: Asia (Antwerp Zoo)

Host: Eulabes javana (Passerifomes: Eulabetidae)

\section{Ornithocheyletia francolini Fain}

Ornithocheyletia francolini Fain, 1972: 44

Type locality: Waterport, N. Transvaal

Distribution: South Africa

Host: Francolinus natalensis (Galliformes: Phasianidae)

\section{Ornithocheyletia garrulax Fain}

Ornithocheyletia garrulax Fain, 1972: 46; 1981: 202

Type locality: Antwerp Zoo

Distribution: ?

Host: Garrulax lencolophus bicolor (Passeriformes: Timaliidae) 
Ornithocheyletia geopeliae Fain

Ornithocheyletia geopeliae Fain, 1981: 192

Type locality: Antwerp Zoo

Distribution: ?

Host: Geopelia striata (Columbiformes: Columbidae)

Ornithocheyletia gersoni Smiley

Ornithocheyletia gersoni Smiley, 1970: 1065

Type locality: Wooster

Distribution: USA (Ohio)

Host: Dendrocopos pubescens (Piciformes: Picidae)

Ornithocheyletia granatina Fain

Ornithocheyletia granatina Fain, 1972: 46; 1981: 204

Type locality: Antwerp Zoo

Distribution: ?

Host: Granatina ianthinogaster (Passeriformes: Estrildidae)

Ornithocheyletia hallae Smiley

Ornithocheyletia hallae hallae Smiley, 1970: 1068; Fain, 1981: 190;

Ornithocheyletia hallae: Haarløv \& Mørch, 1975: 284; Smiley, 1978: 229; Hallas, 1978: 32;

Rosa \& Flechtmann, 1979: 121; Fain \& Bochkov, 2002: 151; Valim \& Gazeta, 2007:

518: Bilal, 2012: 169

Type locality: Brownsville, Texas

Distribution: Brazil, Denmark, England, South Africa, USA (Texas, California), Turkey, Iran

Hosts: Columba livia, Columbigallina sp. (Columbiformes: Columbidae); phoretic on Pseudolynchia canariensis (Diptera: Hippoboscidae)

Ornithocheyletia lamprocolius Fain

Ornithocheyletia lamprocolius Fain, 1972: 45; 1981: 204

Type locality: Central Africa

Distribution: Central Africa

Host: Lamprocolius chloropterus (Passeriformes: Sturnidae)

Ornithocheyletia lawrenceae Smiley

Ornithocheyletia lawrenceae Smiley, 1970: 1065

Type locality: San Antonio

Distribution: USA (Texas)

Host: Psittacula sp. (Psittaciformes: Psittaculidae) 


\section{Ornithocheyletia leiotrix Fain}

Ornithocheyletia leiotrix Fain, 1972: 45; 1981: 198

Type locality: Antwerp Zoo

Distribution: Eastern Asia

Host: Leiothrix lutea (Passeriformes: Timaliidae)

Ornithocheyletia lepidus Fain

Ornithocheyletia lepidus Fain, 1981: 204

Type locality: Antwerp Zoo

Distribution: ?

Host: Garrulax leucolophus bicolor (Passeriformes: Leiothricidae)

Ornithocheyletia lichmerae Smiley

Ornithocheyletia lichmerae Smiley, 1984: 239 (lapsus)

Ornithocheyletia lichmerae Domrow, 1991: 1313

Type locality: Western Australia

Distribution: Australia (W. A.)

Hosts: Geiciphila indistincta, Grallina cyanoleuca (Passeriformes: Monarchidae)

Ornithocheyletia lonchurae Smiley

Ornithocheyletia lonchurae Smiley, 1984: 240; Domrow, 1991: 1313

Type locality: Western Australia

Distribution: Australia (W. A.)

Host: Donacola castaneothorax (Passeriformes: Estrildidae)

\section{Ornithocheyletia lukoschusi Smiley}

Ornithocheyletia lukoschusi Smiley, 1970: 1069; Fain, 1981: 198

Type locality: Nijmegen

Distribution: Holland

Host: Hirundo rustica (Passeriformes: Hirundinidae)

Ornithocheyletia mironovi Chirov et Bochkov

Ornithocheyletia mironovi Chirov \& Bochkov, 1998: 35

Type locality: Lake Issyk - Kul Region, Ottuk Village

Distribution: Kyrgyzstan

Host: Riparia riparia (Passeriformes: Hirundinidae)

Ornithocheyletia orioli Fain et Bochkov

Ornithocheyletia orioli Fain \& Bochkov, 2002: 151

Type locality: Akanyaru River, Rwanda

Distribution: Rwanda 
Hosts: Oriolus oriolus (Passeriformes: Oriolidae), Lybius sp. (Piciformes: Capitonidae), Indicator indicator (Piciformes: Indicatoridae), Pachyphantes superciliosa (Passeriformes: Ploceidae), Chrysococcyx klaasi (Cuculiformes: Cuculidae)

Ornithocheyletia phylloscopi Bochkov, Mironov et Gorgol

Ornithocheyletia phylloscopi Bochkov, Mironov \& Gorgol, 1995: 74

Type locality: Leningradskaya oblast

Distribution: Russia, Ukraine

Host: Phylloscopus trochilis (Passeriformes: Phylloscopidae)

Ornithocheyletia pinguis (Berlese)

Cheyletiella pinguis Berlese, 1889: 56; 1893: 75

Cheletiella pinguis, Oudemans, 1906: 213; Poppe, 1909: Womersley, 1941: 59

Neocheyletiella pinguis, Baker, 1949: 275

Ornithocheyletia pinguis, Volgin, 1964: 34; 1969: 396; Smiley, 1970: 1065; 1977: 229; Fain, 1981: 186

Type locality: Florence

Distribution: Germany, Italy

Hosts: Columba livia domestica (Columbiformes: Columbidae), Sturnus vulgaris (Passeriformes: Sturnidae), Turdus merula (Passeriformes: Turdidae)

Ornithocheyletia prognae Bochkov et Galloway

Ornithocheyletia prognae Bochkov \& Galloway, 2001: 2017

Type locality: Winnipeg, Manitoba

Distribution: Canada

Host: Progne subis (Passeriformes: Hirundinidae)

Ornithocheyletia psittaci Fain

Ornithocheyletia psittaci poicephali Fain

Ornithocheyletia psittaci poicephali Fain, 1981: 194

Type locality: Western Africa

Distribution: Western Africa

Host: Poicephalus senegalus (Psittaciformes: Psittacidae)

Ornithocheyletia psittaci psittaci Fain

Ornithocheyletia psittaci Fain, 1972: 43

Type locality: Antwerp Zoo

Distribution: Western Africa

Host: Psittacus erythacus (Psittaciformes: Psittacidae) 


\section{Ornithocheyletia smileyi Fain}

Ornithocheyletia smileyi Fain, 1972: 45; 1981: 196

Type locality: Antwerp Zoo

Distribution:?

Host: Myopsitta monachus (Psittaciformes: Psittacidae)

Ornithocheyletia turdi Bochkov et Galloway

Ornithocheyletia turdi Bochkov \& Galloway, 2001: 2015

Type locality: Winnipeg, Manitoba

Distribution: Canada

Host: Turdus migratorius (Passeriformes: Turdidae)

\section{Ornithocheyletia volgini Smiley}

Ornithocheyletia volgini Smiley, 1970: 1069; Fain, 1981: 190

Type locality: Wayne Co., Ohio

Distribution: USA (Ohio)

Host: Petrochelidon pyrrhonota (Passeriformes: Hirundinidae)

\section{Ornithocheyletia wauthyi Fain et Bochkov}

Ornithocheyletia wauthyi Fain \& Bochkov, 2002: 152

Type locality: Rwanda

Distribution: Rwanda

Host: Lamprocolius sp. (Passeriformes: Sturnidae)

\section{Tribe Teinocheylini Fain}

Teinocheylini Fain, 1974: 273

Teinocheylinae Fain

Type genus: Teynocheylus Fain, 1974

\section{Genus Teinocheylus Fain}

Teinocheylus Fain, 1974: 271

Type species: Teinocheylus longissimus Fain, 1974

Teinocheylus gundii Fain, Gerrits et Lukoschus

Teinocheylus gundii Fain, Gerrits \& Lukoschus, 1982: 450

Type locality: Chebikar

Distribution: Tunisia

Hosts: Ctenodactylus gundi, C. vali, Massoutiera mzabi (Rodentia: Ctenodactylidae)

\section{Teinocheylus longissimus Fain}

Teinocheylus longissimus Fain, 1974: 271

Type locality: Berbera, N. Somalia 
Distribution: Somalia

Host: Pectinator spekei (Rodentia: Ctenodactylidae)

\section{Index of Cheyletidae}

(Names in bold are accepted as valid)

acantha, Prosocheyla 96

acanthus, Prosocheyla 96

acanyaruensis, Cheletoides 90

acanyaruensis, Metacheletoides 90

Acarocheyla 90

acarophagus, Cheyletus 42

Acaropsella 59

Acaropsellina 60

Acaropsellini 59

Acaropsis 61

Acarus 77

acer, Cheletes 48

acer, Cheyletus (sp. inquir.) 48

aceriai, Cheletogenes 74

Acheletomorpha $\mathbf{7 6}$

acidalia, Ker 54

aegithali, Neocheyletiella 18

Aegyptocheyla (genus inquir.) 69

aegyptiaca, Acaropsella 60

aegyptiaca, Acaropsis $\mathbf{6 0}$

africana, Bakericheyla 105

africanus, Eutogenes $\mathbf{8 6}$

afrotropicalis, Ker 54

aigamuxa, Chelacheles 65

aitkeni, Ornithocheyletia 106

alacer, Cheyletus (sp. inquir.) 48

alexandrinus, Chelacheles 65

alfortensis, Neocheyletiella 18

algericus, Chelacheles $\mathbf{6 8}$

allactaga, Cheyletus 42

Alliea 70

altilis, Acaropsis 61

altilis, Acaropsellina 61

amandavae, Neocheyletiella 18 amazonae, Metacheyletia 103

americana, Cheyletia 81

americana, Cheyletiella 15

americana, Ewingella 16

anarbora, Cheletomimus 26

anarbora, Hemicheyletia 26

anarbora, Paracheyletia 26

anarsia, Acaropsellina 61

anax, Cheletopsis 78

angolensis, Sciurocheyla 101

angolensis, Thryonomycheyla 101

animosa, Cheletopsis 78

Andrecheyla 25

Anthribicheyla 21

apanaskevichi, Chelacheles 65

apicola, Cheletophyes 38

Apodicheles 104

Apodichelini 103

Apodicheyla 105

apus, Apodicheles 104

apus, Chelacaropsis 73

aradiphila, Cheyletia 81

ardeola, Neocheyletiella 18

arecana, Hemicheyletia 34

argentinensis, Ornithocheyletia 106

artami, Neocheyletiella 19

ascutatus, Cheyletus 41

ascutatus, Cheletomimus (Philippicheyla) 34

asiatica, Eucheyletia 51

asiatica, Hemicheyletia 26

asiaticus, Cheletomimus 26

assimilis, Paracheyletia 57

Atarsacheylus 65

athenae, Cheletomimus 26 
athenae, Hemicheyletia 26

athene, Neocheyletiella 19

attiahi, Cheyletus 41

audax, Cheletes 49

audax, Cheyletus (sp. inquir.) 49

aurorae, Cheletophyes 38

aversor, Cheyletus 42

avicola, Neocheyletiella 19

avidus, Cheyletus 44

ayyazi, Cheyletus $\mathbf{4 4}$

Aztecocheyletus 22

aztecorum, Mexecheles 91

bacchusi, Chelacheles 65

baiwanganae, Chelacheles 65

Bak 70

bakeri, Acheletomorpha 76

bakeri, Chelachecaropsis $\mathbf{6 6}$

bakeri, Chelacheles 66

bakeri, Cheletomimus (Hemicheyletia) 26

bakeri, Cheletomorpha 76

bakeri, Eucheyletia 51

bakeri, Eutogenes 85

bakeri, Grallacheles 88

bakeri, Hemicheyletia 26

bakeri, Ker 54

bakeri, Paracheyletia 26

Bakericheyla 104

Bakini 69

baloghi, Cheyletus $\mathbf{4 1}$

baridos, Cheyletus 44

barri, Ornithocheyletia 107

basilica, Cheletopsis 78

batangeniae, Dubininiola 85

batangeniae, Oudemansicheyla 85

batangenius, Polycheyletus 85

batangensis, Polycheyletus $\mathbf{8 5}$

batomysi, Cheyletus 41

beauchampi, Cheyletus 42

beeri, Neoeucheyla 14

bengalensis, Microcheyla 92

benoiti, Bakericheyla 105

berlesei, Cheletes $\mathbf{2 4}$ berlesei, Cheletomimus 24

berlesei, Cheyletomimus $\mathbf{2 5}$

Bicheyletiella 15

bicicri, Columbicheyla 82

bicornis, Eutogenes 86

bidentatus, Cheyletus 41

binus, Cheletomimus (sp.inquir.) 35

bipanus, Chelacheles 66

bisetosus, Cheletomimus (sp. inquir.) 35

bishoppi, Eucheyletia 51

blakei, Cheyletiella 15

blosis, Hemicheyletia 27

bochkovi, Neoeucheyla 93

bocki, Anthribicheyla 21

boeroi, Ornithocheyletia 106

boonkongae, Dubininiola 85

boonkongae, Polycheyletus 85

Bothrocheyla 14

bothrophila, Eucheyletia 51

bregetovae, Cheletomimus 27

bregetovae, Dendrocheyla 27

bregetovae, Hemicheyletia 27

buckneri, Cheletogenes 97

buckneri, Prosocheyla 97

bulgarica, Cheyletia 83

bulgarica, Cunliffella 83

bulgarica, Neoeucheyla 83

bunguranensis, Hylopecheyla 89

burmiticus, Cheyletus (sp. inquir.) 49

buruensis, Indonesicheyla 58

buruensis, Tutacheyla $\mathbf{5 8}$

cacahuamilpensis, Cheyletus 41

caeterus, Ker 54

calamondin, Nodele 56

calawayae, Neocheyletiella 20

calcarata, Nihelia 101

calcarata, Hemicheyletus 101

calcaratus, Hemicheyletus 101

callawaye, Neocheyletiella 20

callida, Acaropsis 63

cambio, Cheletomimus (sp. inquir.) 35

camerounensis, Smileycheles 102 
camerounensis, Sciurocheyla 102

Camincheyletus 22

canadensis, Cheyletiella 107

canadensis, Neocheyletiella 107

canadensis, Ornithocheyletia 107

cancriformis, Eutarsus 42

cantor, Cheletomimus (sp. inquir.) 35

carabae, Samsinakia 98

carinatus, Cheletogenes 74

carnifex, Cheyletus 42

caucasica, Cheletonella 24

caucasica, Lepidocheila 55

caucasicus, Cheyletus 44

Caudacheles 72

chanayi, Bakericheyla 105

chanayi, Cheletiella 105

chanayi, Cheyletiella 105

chanayi, Neocheyletiella 105

charadrii, Cheletopsis 79

charanasriae, Samsinakia 98

Chelacaropsis 73

Chelachecaropsis 65

Chelacheles 65

Cheletacarus 22

Cheletogenes 73

Cheletoides 76

Cheletomimus 24

Cheletomorpha 76

Cheletonella 23

Cheletophanes 37

Cheletophyes 38

Cheletopsis 78

Cheletosoma 81

Chelonotinae 14

Chelonotini 14

Chelonotus 14

Cheyletia 81

Cheyletidae 14

Cheyletides 14

Cheyletiella 15

Cheyletiellinae 14

Cheyletinae 21
Cheyletini 21

Cheyletus 41

Chiapacheylus 82

chimaera, Alliea 94

chimaera, Oconnoricheylus 94

chirunduensis, Cheletoides 76

chui, Cheletomimus (Hemicheyletia) 27

chui, Hemicheyletia 27

citri, Eutogenes 86

citrifoliata, Cheletogenes 97

citrifoliata, Prosocheyla (Reckiana) 97

citrosinensis, Cheletomimus (sp. inquir.) 36

clamo, Acaropsellina 61

clamo, Acaropsis 61

clavipes, Hoffmannita 88

clavipes, Myrmicocheyla $\mathbf{8 8}$

clavipilis, Cheletophyes 38

clavispinus, Cheyletus (sp. inquir.) 49

coccineae, Nodele 56

cocciphilus, Cheyletus 75

collocalia, Apodicheles 104

Columbicheyla 82

columbigallinae, Ornithocheyletia 107

congolensis, Sciurocheyla 102

congolensis, Thryonomycheyla 102

congensis, Cheletomimus 27

congensis, Hemicheyletia 27

congensis, Paracheyletia 27

congoensis, Cheletomimus 27

congolensis, Sciurocheyla 102

conquistador, Aztecocheyletus 21

conquistador, Nodele 22

coprosomae, Oudemansicheyla 95

cordovensis, Cheletomimus 27

cordovensis, Cheyletia 27

cordovensis, Hemicheyletia 27

cornutus, Eutogenes 86

corpuzrarosae, Neochelacheles 69

corpuzrarosae, Granulocheyletus 53

crassus Cheyletus (sp. inquir.) 49

crinifer, Metacheletoides 90

Criokeron 100 
Criokerontinae 99

Criokerontini 99

crowei, Cheletomimus 34

Cryptocheyla 99

culpa, Acaropsellina 61

culpa, Acaropsis 61

cunliffei, Mexecheles 91

Cunliffella 83

curvidens, Cheletiella 101

curvidens, Hemicheyletus 101

curvidens, Nihelia 101

cynictis, Hemicheyletus 101

cynictis, Nihelia 101

cypsiurus, Apodicheles 104

daberti, Cheletopsis 79

daddariensis, Cheletonella 23

daltoniensis, Cheletomimus 25

darwinius, Cheletomimus 28

darwinia, Hemicheyletia $\mathbf{2 8}$

daurica, Eucheyletiella 17

davisi, Cheyletus 47

decorus, Cheletophyes 38

degenerata, Metacheyletia 103

deleoni, Bak 70

Dendrocheyla 25

dengi, Cheyletiella 16

denmarki, Cheletomimus 95

denmarki, Oudemansicheyla 95

deodikari, Cheletophyes (sp. inquir.) 40

desertorum, Chiapacheylus (sp. inquir.) 82

desitus, Cheyletus 42

disparis, Cheletomimus 28

disparis, Hemicheyletia $\mathbf{2 8}$

dissitus, Cheletogenes 74

docta, Acaropsellina 61

docta, Acaropsis 61

doctus, Cheyletus $\mathbf{6 1}$

doddi, Cheyletus 42

dolosus, Cheletomorpha 77

domitus, Cheletomimus 36

dua, Cunliffella 83

dua, Neoeucheyla 83 dubinini, Ornithocheyletia 107

Dubininiola 84

duosetosus, Cheletomimus 25

eburneus, Cheyletus 42

echis, Cheletomimus 28

echis, Hemicheyletia $\mathbf{2 8}$

eckerti, Cheletophyes 38

edentata, Chiapacheylus 82

edentatus, Chiapacheylus 82

egypticus, Cheyletus 44

elongata, Hypopicheyla 89

elongatus, Bak 70

emersus, Cheletonella 23

eoa, Eucheyletia 51

eruditus, Acarus 42

eruditus, Cheletes 42

eruditus, Cheyletus 42

Eucheletopsis 85

Eucheyletia 50

Eucheyletiella 17

eulabes, Ornithocheyletia 107

Eutarsus 42

Eutogenes 85

ewingi, Chelonotus 14

Ewingella 16

faini, Bak 71

faini, Bakericheyla 105

faini, Eucheyletiella 17

faini, Neocheyletiella 105

ferox, Cheletes 49

ferox, Cheyletus (sp. inquir.) 49

ferrugineae, Cheletopsis 79

filipina, Acaropsella 59

filipina, Cheletomimus 35

filipina, Philippicheyla 35

fissilis, Cheletomimus 36

flabellifer, Cheyletus 51

flabellifera, Cheletia $\mathbf{5 1}$

flabellifera, Cheyletia $\mathbf{5 1}$

flabellifera, Eucheyletia 51

flecto, Cheletomimus (sp. inquir.) 36

fortis, Cheletes $\mathbf{4 4}$ 
fortis, Cheyletus 45

foxi, Eutogenes 86

francolini, Ornithocheyletia 107

frater, Eutogenes 86

funisciuri, Cheyletus 43

funisciuri, Eucheyletia 43

funisciurus, Sciurocheyla 102

furcatus, Bak 70

furibundus, Cheyletus 47

furmani, Cheyletiella $\mathbf{1 6}$

Galagocheles 100

gallowayi, Granulocheyletus 53

garrulax, Ornithocheyletia 107

geopeliae, Ornithocheyletia 108

gerbillicola, Cheyletus 43

gersoni, Bak 71

gersoni, Hoffmannita 88

gersoni, Ornithocheyletia 108

gisagarensis, Metacheletoides 90

glaucomys, Camincheyletus 22

glomus, Cheletomimus 28

lomus, Hemicheyletia 28

gonocephalum, Samsinakia 98

gracilis, Cheletomimus 28

gracilis, Lepidocheyla 56

Grallacheles 87

granatina, Ornithocheyletia 108

granifera, Microcheyla 92

granulatus, Neoacaropsis 69

Granulocheyletus 53

granulus, Cheletomimus 28

granulus, Hemicheyletia $\mathbf{2 8}$

greenwoodi, Cheletomimus 29

gryphus, Cheletacarus 22

gundii, Teinocheylus 111

hallae, Ornithocheyletia 108

hardyi, Eucheyletia 52

harnaji, Cheletophyes (sp. inquir.) 40

harpyia, Cheyletia 52

harpyia, Eucheyletia 52

hastatus, Cheletomimus 29

hastatus, Hemicheyletia 29 hawaiiensis, Cheletophyes 91

hawaiiensis, Mexecheles 91

hellenicus, Chelacheles 66

Hemicheyletia 25

Hemicheyletus 101

hendersoni, Cheyletus 47

hepburni, Cheletogenes 97

hepburni, Prosocheyla 97

heredis, Cheletomimus (sp. inquir.) 36

herpestesi, Hemicheyletus 101

heteropalpa, Cheletiella 104

heteropalpa, Cheyletiella 104

heteropalpus, Apodicheles 104

heteropalpus, Cheyletus 104

heteropalpa, Neocheyletiella 104

heteropalpus, Neocheyletiella 104

hirnai, Acaropsellina 62

hirnai, Acaropsis 62

hissariensis, Hemicheyletia 55

hoffmannae, Cheletonella 23

Hoffmannita 88

hortensis, Paracheyletia 57

humilis, Chelacheles 66

Hylopecheyla 89

Hypopicheyla 89

iconis, Cheletogenes 74

impavida, Cheletopsis 79

impolita, Acarocheyla 91

impolitus, Mexecheles 91

indiacus, Cheletophyes 38

indica, Cheletomimus 29

indica, Hemicheyletia (sp. inquir.) 29

indicus, Grallacheles 88

Indonesicheyla $\mathbf{5 8}$

indonesiensis, Bak 71

indra, Chelacheles 67

infensus, Cheyletus 44

intermedius, Mexecheles 91

intrepidus, Cheletes 49

intrepidus, Cheyletus (sp. inquir.) 49

iranica, Bak 71

iranica, Neoeucheyla 93 
iraniensis, Cheletonella 24

johnstoni, Cheyletiella 17

johnstoni, Eucheyletiella 17

juglandis, Cheletonella (sp. inquir.) 24

katangae, Cheyletiella 16

kennedyae, Nidocheyletus 56

Kenyacheylus 53

kenyensis, Chelacaropsis 73

Ker 54

khayae, Caudacheles 72

kinishasensis, Acaropsella 59

kivuensis, Cheyletus 43

kivuensis, Eucheyletia 43

klimovi, Chelacheles 67

knowltoni, Cheletophyes 77

konoi, Acaropsella 59

kulagini, Acaropsella 59

kulagini, Acaropsis 59

kulagini, Neoacaropsis $\mathbf{5 9}$

kureatollensis, Granulocheyletus 53

kureatollensis, Hemicheyletia 53

kuznetzovi, Cheyletus 43

kysenyiensis, Hemicheyletia 29

kysenyiensis, Cheletomimus 29

lacinia, Hemicheyletia 27

Laeliocheyletia 55

laguncula, Hemicheyletia 33

lamprocolius, Ornithocheyletia 108

Lanceacheyla 55

lanceolata, Cheletomimus(Hemicheyletia) 29

lanceolatus, Chelacheles 67

larmae, Cheletomimus (sp. inquir.) 66

laruei, Alliea 70

latior, Bakericheyla chanayi 105

laureata, Cheyletia 81

lawrenceae, Ornithocheyletia 108

lawrencei, Galagocheles 100

legendrei, Cheyletus 43

leiotrix, Ornithocheyletia 109

lemuricola, Cheletiella 100

lemuricola, Cheyletiella 100

lemuricola, Galagocheles 100 lemuricola, Hemicheyletus $\mathbf{1 0 0}$

lemuricola, Nihelia 100

Lepidocheyla 55

lepidopterorum, Acarus 77

lepidopterorum, Cheletomorpha 77

lepidus, Ornithocheyletia 109

levis, Acaropsellina 62

levis, Neoacaropsis $\mathbf{6 2}$

leytensis, Hemicheyletia 29

leytensis, Cheletomimus 29

lichmerae, Ornithocheyletia 109

lieni, Caudacheles 72

ligyscutatus, Bak 71

limnodromi, Cheletopsis 79

lindquisti, Cheletomimus (Hemicheyletia) 30

linsdalei, Cheyletus 44

lonchurae, Neocheyletiella 19

lonchurae, Ornithocheyletia 109

longipalpus, Cheyletus 57

longipes, Cheyletus 77

longisetosa, Metacheyletia 103

longissimus, Teinocheylus 111

loricata, Cheyletia (Eucheyla) 94

loricata, Neoeucheyla 94

lukoschusi, Ornithocheyletia 109

lukoschusi, Promuricheyla 96

macrocherus, Cheyletus 47

macrocorneus, Chiapacheylus (sp. inquir.) 82

macrocorneus, Neoeucheyla 94

macroflabellata, Columbicheyla 82

macronycha, Cheyletiella 19

macronycha, Neocheyletiella 19

macronychus, Cheyletus 19

mafekingensis, Cheyletus 44

magnanima, Cheletopsis 79

major, Cheletopsis $\mathbf{8 5}$

major, Eucheletopsis 85

major, Cheyletus 85

makilingensis, Cheletomimus (Hemichey-

letia) 30

makilingiensis, Eutogenes 86

malaccensis, Cheletes 44 
malaccensis, Cheyletus 44

malayensis, Cheyletus 45

malayi, Hylopecheyla 89

mariae, Cheletopsis 80

marshalli, Acarocheyla 91

marshalli, Cheletophyes 91

marshalli, Mexecheles 91

maysa, Cunliffella 83

maysa, Neoeucheyla 83

mbomba, Cheletophyes 39

media, Neocheyletiella 19

megaphallos, Neocheyletiella 20

megaphallos, Ornithocheyla 20

meihuashanense, Cheletogenes 74

mendicus, Neochelacheles 69

mercedesae, Ker 54

mericourti, Acaropsis (=Acaropsellina docta?)

Metacheletoides 90

Metacheyletia 103

Metachyletiinae 102

messersmithi, Neochelacheles 69

Mexecheles 90

mexicana, Cheyletiella 16

mexicana, Hemicheyletia 30

mexicana, Hoffmannita 89

mexicanus, Cheletomimus 30

mianiensis, Cheyletus 44

michalskii, Chelacheles 67

micidus, Bak 71

Microcheyla 92

microrhyncha, Cheletiella 20

microrhyncha, Cheyletiella 20

milesi, Chelacaropsis 73

milesi, Cheletonata 73

microrhyncha, Neocheyletiella 20

minuta, Neoeucheyla 94

minutus, Cheletomimus (sp. inquir.) 37

mirabilis, Cheyletia 89

mirabilis, Hypopicheyla 89

mironovi, Ornithocheyletia 109

missonei, Cheyletus 45

monosetosus, Cheletogenes 74 montandoni, Cheletophanes 37

montandoni, Cheyletus 37

moorei, Chelacaropsis 73

morii, Hemicheyletia 32

morinus, Cheyletus 45

mortelmansi, Cheyletus 42

mu, Nodele 57

mumai, Cunliffella 83

mumai, Neoeucheyla 83

mungeri, Eucheyletia 41

Muricheyla 93

Myrmicocheyla $\mathbf{8 8}$

nadchatrami, Bak 72

nanchangensis, Acaropsella 62

nanchangensis, Acaropsellina 62

nanfengensis, Grallacheles 88

narashinoensis, Eutogenes 86

navicula, Hoffmannita $\mathbf{8 8}$

nectarinia, Neocheyletiella 20

Neoacaropsis 68

Neochelacheles 68

Neocheletophyes 56

Neocheyletiella 18

Neocheyletiellini 104

Neoeucheyla 93

newtoni, Cheletophyes (sp. inquir.) 40

newyorkensis, Cheletomimus (Hemichey-

letia) (sp. inquir.) 30

ngaii, Metacheyletia 103

nidicola, Eucheyletia 52

nidicolus, Cheyletus 45

Nidocheyletus 56

nigriceps, Cheyletus (sp. inquir.) 50

Nihelia 101

Niheliinae 99

Niheliini 99

nindota, Columbicheyla 83

nindota, Eucheyletia 83

nobilis, Acaropsella 60

Nodele 56

noerneri, Cheletes 80

nörneri, Cheyletus 80 
notelaeae, Cheletomimus(Philippicheyla) 35 notelaeae, Philippicheyla 35

novemdentis, Cheletacarus 22

numidae, Metacheletoides 90

oaklandia, Cheletogenes 96

oaklandia, Prosocheyla 96

obesa, Metacheyletia 103

obrutus, Cheletomorpha 77

occisor, Cheletophyes 39

ochoai, Cheletomimus 25

ochotonae, Cheyletiella 17

ochotonae, Eucheyletiella 17

Oconnoricheylus 94

omissa, Eucheyletia 52

omissa, Hemicheyletia 30

omissa, Cheletomimus (Hemicheyletia) 30

omissus, Cheletomimus (Hemicheyletia) 30

onoi, Cheletogenes 87

onoi, Eutogenes 87

opacus, Cheletomorpha 77

opsis, Acaropsellina 62

opsis, Acaropsis 62

orbis, Acaropsellina 62

orbis, Acaropsis $\mathbf{6 2}$

oregonensis, Eucheyletia 51

orientalis, Cheletomorpha 78

orientalis, Cheletophyes (sp. inquir.) 40

orioli, Ornithocheyletia 109

ornata, Cheyletia 75

ornata, Cunliffella 84

ornata, Neoeucheyla 84

ornatus, Cheletogenes 75

ornatus, Cheyletogenes 75

ornatus, Cheyletus 75

Ornithocheyla 18

Ornithocheyletia 106

Ornithocheyletiinae 103

Ornithocheyletiini 103

oudemansi, Chelonotus 14

oudemansi, Neocheyletiella 20

Oudemansicheyla 95

ozarkensis, Bak 72 ozkani, Microcheyla 92

pagongae, Samsinakia 98

pallasius, Eucheyletiella 17

palmatus, Ker 54

panamensis, Cheletophyes 39

panamensis, Cunliffella 84

panamensis, Eucheyla $\mathbf{8 4}$

panamensis, Neoeucheyla 84

panneus, Mexecheles 91

papillifera, Cheyletia 81

Paracaropsis 69

Paracheyletia 57

Paracheyletiella 58

Paramicrocheyla (genus inquir.) 95

paraparvula, Microcheyla 93

parasitivorax, Cheletiella 16

parasitivorax, Cheyletiella 15

parasitivorax, Cheyletus 15

parcus, Cheletomimus 31

parcus, Hemicheyletia 31

parumsetosus, Cheyletus 50

parvula, Microcheyla 93

patagiatus, Cheyletus 50

Pavlovskicheyla 58

pavlovskyi, Eucheyletia 52

pavlovskyi, Bothrocheyla 14

pavlovskyi, Neoeucheyla 14

payatus, Bak 72

peregrinus, Cheletophanes 37

peritremaculatus, Chelacheles 67

petiginis, Cheletogenes 75

phantosis, Cheyletus 44

philippicana, Pavlovskicheyla 58

Philippicheyla 34

philippinensis, Acaropsellina 62

philippinensis, Acaropsis 62

philippinensis, Cheletohyes 57

philippinensis, Neocheletophyes 57

philippinensis, Cheyletus 45

philippinensis, Nodele 57

phylloscopi, Ornithocheyletia 110

Picocheyletus 95 
pilosa, Cheletonella 24

pinguis, Cheletiella 110

pinguis, Cheyletiella 110

pinguis, Neocheyletiella 110

pinguis, Ornithocheyletia 110

pinicola, Eutogenes 87

pintoriensis, Ker 55

pittae, Neocheyletiella 20

platessa, Acaropsellina 63

platessa, Acaropsis 63

platydemae, Pavlovskicheyla 58

ploceus, Cheletacarus 22

ploceus, Neoeucheyla 94

pluridens, Cheyletus 46

poicephali, Ornithocheyletia psittaci 110

Polycheyletus $\mathbf{8 4}$

polylepis, Dubininiola 85

polymorphus, Cheyletus 44

porta, Acaropsellina 63

porta, Acaropsis 63

praedabundus, Cheyletus 47

prasadi, Alliea 70

prognae, Ornithocheyletia 110

promptus, Cheletes $\mathbf{5 0}$

promptus, Cheyletus 50

Promuricheyla 96

prosobonialis, Cheletopsis 80

Prosocheyla 96

pseudomalaccensis, Cheyletus 46

psittaci, Ornithocheyletia 110

psittaculae, Ornithocheyletia?

punctata, Eutogenes 87

punctatus, Eutogenes 87

punctulatus, Cheyletus 46

pusillifolium, Hemicheyletia 26

pusillinus, Eucheyletiella 17

pyriformis, Cheyletia $\mathbf{5 7}$

pyriformis, Cheyletus 57

pyriformis, Paracheyletia 57

quadrisetatus, Cheletogenes 87

quadrisetosus, Cheletogenes 87

quadrisetatus, Eutogenes 87 queleae, Neocheyletiella 20

quinta, Nihelia 100

quinta, Criokeron 100

quintus, Criokeron 100

rabiosus, Cheyletus [Kazakhstan]?

rafiquiensis, Cheyletus $\mathbf{4 4}$

rapax, Cheletes $\mathbf{5 0}$

rapax, Cheyletus 50

raptor, Cheletacarus 23

rarus, Cheyletus 46

ratufi, Thewkachela 99

rashtiensis, Cheyletus 46

recki, Paracheyletia 31

Reckiana 97

reticularis, Eutogenes 87

reticulata, Chelacaropsis 73

reticulata, Eucheyletia 59

reticulata, Hemicheyletia 31

reticulata, Zachvatkiniola 59

rimandoi, Hoffmannita 89

ripkai, Prosocheyla 96

robertsoni, Cheyletus $\mathbf{4 2}$

robusta, Tutacheyla 59

robustus, Chelacheles 67

rohdendorfi, Acaropsella 60

rohdendorfi, Cheyletus 44

rohdendorfi, Neoacaropsis $\mathbf{6 0}$

rohweri, Neocheyletiella 21

romerolagi, Bicheyletiella 16

romerolagi, Cheyletiella 16

rostella, Hemicheyletia 31

rostellus, Cheletomimus 31

rufus, Cheyletus 77

rufus, Acaropsis?

rugosa, Cheletonella 23

rugosa, Cheletophanes 23

rugosus, Chelacheles 23

rugosus, Cheletacarus 23

ruttneri, Cheletophyes (sp. inquir.) 40

rwandae, Cheyletus 46

rwandana, Chelacaropsis 73

rynchops, Cheletopsis 80 
saccardianus, Cheyletus 75

saevus, Cheletes $\mathbf{5 0}$

saevus, Cheyletus 50

sagacis, Cheletogenes 75

salvus, Cheletomimus 31

salvus, Hemicheyletia 31

samsinaki, Paracheyletia 57

Samsinakia 98

sanctaehelenae, Bak 72

scaber, Cheletogenes 75

schmidtmanni, Acaropsella 60

schneideri, Cheletes 47

schneideri, Cheyletus 46

scitula, Hemicheyletia 27

Sciurocheyla 101

scutellata, Andrecheyla 32

scutellata, Cheyletia 32

scutellata, Hemicheyletia 32

scutellata, Paracheyletia 32

scutellatus, Cheletomimus 32

selenorhynchus, Chelonotus 14

semenovi, Cheletophyes 58

semenovi, Pavlovskicheyla 58

seminivorus, Cheyletus 42 or 77

seminole, Chelacheles 68

serrulus, Cheletomimus (Hemicheyletia) 32

serrula, Hemicheyletia 32

shaziai, Acaropsella 60

sheikhupuraensis, Acaropsellina 63

sheikhupuraensis, Acaropsis 63

shendei, Cheletophyes (sp. inquir.) 40

shiva, Mexecheles 92

shorkotiensis, Acaropsellina 63

shorkotiensis, Acaropsis 63

sialcotensis, Cheletomimus 37

sibirica, Eucheyletia 52

sicista, Muricheyla 93

sigilla, Acaropsellina 63

sigilla, Acaropsis 63

simplex, Nodele 56

sinensis, Eucheyletia 53

sinensis, Prosocheyla 97 siva, Neocheyletiella 21

smallwoodae, Neocheyletiella 21

Smileycheles 101

smileyi, Ornithocheyletia 111

sodalis, Acaropsellina 63

sodalis, Acaropsis 63

sollers, Acaropsellina 63

sollers, Acaropsis 64

spatiosus, Cheyletus $\mathbf{4 8}$

speciosus, Oconnoricheylus 94

spinula, Paramicrocheyla 95

squamosa, Cheyletia 82

squamosa, Nihelia 102

squamosa, Sciurocheyla 102

squamosa, Microcheyla 93

squamosus, Acarus 102

squamosus, Cheyletus $\mathbf{8 1}$

squamosus, Hemicheyletus 102

squamosus, Sciurocheyla 102

stigmaeoides, Chelachecaropsis $\mathbf{6 8}$

stigmaeoides, Chelacheles 67

strabismus, Chelacheles 68

strandtmanni, Cheyletiella 16

strenuus, Cheletes (= eruditus ?) 42

strenuus, Cheyletus $\mathbf{4 2}$

striola, Chelacheles 68

stroti, Paracaropsis 69

subitus, Acaropsellina 64

subitus, Acaropsis 64

subquadrata, Bakericheyla 106

subquadrata, Neocheyletiella 106

summersi, Acaropsellina 64

summersi, Acaropsis 64

summersi, Aegyptocheyla 69

superba, Nodele 57

takahashii, Cheyletiella 18

takahashii, Eucheyletiella 18

takahasii, Eucheyletiella 18

tanosis, Hemicheyletia 32

tanosis, Cheletomimus 32

tanzaniensis, Eucheyletia 47

tanzaniensis, Cheyletus 47 
tatami, Cheletomorpha 77

taurica, Eucheyletia 51

Teinocheyletinae 111

Teinocheylini 111

Teinocheylus 111

telma, Cheletomimus 37

temoak, Chelacheles 68

tenerum, Cheletomorpha 78

tenuipilis, Cheyletus 48

teretis, Laeliocheyletia 55

terrestris, Chelacaropsis 73

thailandensis, Mexecheles 92

thailandicus, Criokeron 100

thalasseus, Cheletopsis 80

theodoridis, Cheletophyes 98

theodoridis, Samsinakia 98

Thewkachela 99

thomasi, Chelacheles 68

Thryonomycheyla 101

tilae, Samsinakia 99

torridae, Cheletophyes 39

transvaalica, Bakericheyla 106

transvaalica, Neocheyletiella 106

transversa, Cheletomimus (Hemicheyletia) 32

traubi, Cheletogenes 97

traubi, Prosocheyla 97

travisi, Acaropsis 69

travisi, Paracaropsis 69

trema, Cheletomimus (sp. inquir.) 37

trepsis, Cheletomimus 32

trepsis, Hemicheyletia 32

tricholaemae, Picocheyletus 95

trigintaduae, Caudacheles 72

trilobitus, Samsinakia 99

troglodytes, Kenyacheylus 54

tropica, Cheyletia 33

tropica, Hemicheyletia 33

tropicus, Cheletomimus (Hemicheyletia) 33

trouessarti, Cheletes 47

trouessarti, Cheyletus 47

truculentus, Cheyletus 47

truncatus, Bak 72 trux, Cheyletus 47

tuberculicoxa, Cunliffella 84

tuberculicoxa, Neoeucheyla $\mathbf{8 4}$

tumidus, Hemicheyletia 26

turdi, Ornithocheyletia 111

Tutacheyla 58

tutela, Cheyletus 48

typhosa, Bothrocheyla 15

typhosa, Neoeucheyla 15

tyrannus, Cheletosoma 81

tyrophagus, Acaropsellina 64

tyrophagus, Acaropsis 64

ugandanus, Cheyletus 44

uichancoi, Cheletomimus 33

uichancoi, Hemicheyletia 33

umbo, Cheletomimus 33

umbo, Hemicheyletia 33

uncinata, Cheletoides 76

uncinatus, Cheletoides 76

uncinatus, Cheyletus 76

ungulina, Paramicrocheyla 95

unicus, Acaropsellina 64

unicus, Acaropsis 64

variegata, Cunliffella 84

variformis, Eucheyletia 53

venator, Cheletes $\mathbf{4 8}$

venator, Cheyletus 48

venustissima, Cheletomorpha 77

venustissimus, Cheyletus 77

venustus, Acaropsellina 64

venustus, Acaropsis 64

vescus, Cheletomimus 33

vescus, Hemicheyletia 33

vespae, Cheletophyes 39

vespertilionis, Cheletonella 24

vestergaardi, Neocheyletiella 19

vicinus, Eutogenes 87

vichii, Atarsacheylus 65

villosa, Prosocheyla 97

virginiensis, Acarocheyla 92

virginiensis, Cheyletia 92

virginiensis, Paracheyletia 92 
virginiensis, Mexecheles 92

vitrus, Acaropsellina 64

vitrus, Acaropsis 64

vitzthumi, Cheletophyes 39

vivatus, Cheyletus 48

vivus, Cheletomimus 33

vivus, Hemicheyletia 33

volgini, Acaropsella 60

volgini, Cheletomimus 33

volgini, Cheyletus 48

volgini, Cryptocheyla 99

volgini, Dendrocheyla 33

volgini, Hemicheyletia 33

volgini, Neoacaropsis $\mathbf{6 0}$

volgini, Ornithocheyletia 111

volgini, Paracheyletia 33

volgini, Paracheyletiella 58

volgini, Samsinakia 99

votandinii, Mexecheles 92

vulgatus, Cheletogenes 75

vorax, Cheletes 44

vorax, Cheyletus 44

wahndoensis, Cheyletus 44

waitei, Cheletogenes 75 walii, Acaropsella 60

wauthyi, Ornithocheyletia 111

wellsi, Cheletomimus (Hemicheyletia) 34

wellsi, Cheyletia 34

wellsi, Dendrocheyla 34

wellsi, Hemicheyletia 34

wellsi, Paracheyletia 34

wellsina, Hemicheyletia 34

wellsina, Paracheyletia 34

wellsina, Cheletomimus (Hemicheyletia) 34

wellsinus, Cheletomimus (Hemicheyletia) 34

whartoni, Cunliffella 84

whartoni, Eucheyla 84

whartoni, Lanceacheyla 55

whartoni, Neoeucheyla 58

womersleyi, Eucheyletia 53

woodroffei, Cheyletus 47

woolfordi, Paracheyletia $\mathbf{8 8}$

xylocopae, Cheletophyes 39

yasguri, Cheyletiella 16

Zachvatkiniola 59

zaheri, Cheyletus 42

zamia, Cheletomimus (sp. inquir.) 37

zumpti, Cheyletus 48

\section{Distribution of Cheyletidae}

\section{EUROPE}

Armenia (Volgin, 1969) - Cheletogenes ornatus, Cheletomimus (Hemicheyletia) bregetovae, Cheletomorpha lepidopterorum, Cheyletus malaccensis [syn. rohdendorfi]

Austria (Franz, 1954; Willmann, 1951) - Cheyletia squamosa, Cheyletus eruditus

Azerbaidjan (Zachvatkin, 1949; Halilova, 1967; Volgin, 1963, 1969, 1978; Abdullaeva, 1978; Abdullaeva \& Aslanov, 1989; Aslanov \& Mehtieva, 1992) - Cheyletus eruditus, Ch. malaccensis (= polymorphus, rohdendorfi), Ch. carnifex [syn. aversor], Cheletomorpha lepidopterorum, Lepidocheyla caucasica

Belarus (Efremova \& Bochkov, 1990) - Acaropsellina sollers, Cheyletus eruditus, Ch. trux, Eucheyletia bothrophila, E. sibirica, Neocheyletiella microrhyncha (= oudemansi)

Belgium (Dufait, 1968; André, 1975; Fain \& Bochkov, 2001) - Cheyletiella (?) parasitivorax, Eucheyletia flabellifera, Cheletonella vespertilionis, Cheletomorpha lepidopterorum, Paracheyletia pyriformis 
Bulgaria (Volgin, 1955, 1958, 1964a, 1964b, 1969; Zankov, 1955; Kolebinova, 1967; Sosnina et al., 1969; Nachev \& Trenchev, 1987; Beron, 2011; Trencheva \& Trenchev, 2018) - Cheletogenes ornatus, Cheletopsis norneri, Cheyletus carnifex [syn. aversor], Ch. malaccensis (= polymorphus), Eucheyletia flabellifera, Eutogenes frater, Cheletomimus (Hemicheyletia) bregetovae, Neoeucheyla bulgarica, Ornithocheyletia canadensis Croatia (Pagliarini, 1979) - Cheyletus eruditus

Czech Rep. (Samšinak, 1956; Volgin, 1966; Fain \& Bochkov, 2001; Lukáš et al., 2007) Chelacheles michalskii, Cheyletus carnifex (= aversor), Ch. eruditus, Ch. malaccensis, Ch. trouessarti, Paracaropsis strofi, Paracheyletia pyriformis (= samsinaki),

“Czechoslovakia” (Ždárková, 1979) - Acaropsellina docta, Cheletomorpha lepidopterorum, C. hendersoni, Cheyletia papillifera, Cheyletus carnifex (= aversor), C. eruditus, C. malaccensis, C. trouessarti, Grallacheles bakeri, Ker bakeri

Denmark (Haarløv \& Mørch, 1975; Haarløv, 1957; Enghoff, 1988; Hallas, 1978) - Cheletomorpha lepidopterorum, Cheyletiella parasitivorax, Ch. yasguri, Cheyletus eruditus, Ch. trouessarti, Ornithocheyletia hallae

Estonia (Volgin, 1964) - Cunliffella whartoni (= tuberculicoxa)

Finland (Harju, Husman, Merikoski \& Pennanen, 2006) - Mexecheles virginiensis

France (contin.)(Megnin, 1883; Guilhon \& Marchand, 1972, 1974) - Cheletomorpha lepidopterorum, Cheyletiella blakei, Ch. parasitivorax, Ch. yazguri, Neocheyletiella microrhyncha

Georgia (Volgin, 1963; Rekk, 1976; Fain, Bochkov \& Corpuz-Raros, 2002) - Cheletogenes ornatus, Cheletomimus (Ch.) berlesei, Cheletomorpha lepidopterorum, Cheletonella caucasica, Cheyletus eruditus, Ch. malaccensis [syn. rohdendorfi], Ch. trux, Eucheyletia reticulata

Germany (Heller, 1880; Haller, 1882; Poppe, 1909; Rack, 1971; Fain \& Bochkov, 2001) - Cheletoides uncinatus, Cheletomorpha lepidopterorum, Cheletopsis noerneri, Cheyletiella yazguri, Cheyletus eruditus, Eucheyletia flabellifera, Ornithocheyletia pinguis, Paracheyletia pyriformis

Great Britain (Michael, 1878; Hull, 1918; Turk, 1946, 1953; Evans, 1954; Griffiths, 1960; Beesley, 1963; Walton, 1974; Hughes, 1976; Jeffrey, 1976, 1978, 1979, 1980; Fain \& Bochkov, 2001; Fain, Bochkov \& Corpuz-Raros, 2002; Baker \& Craven, 2003) Acaropsellina docta, A. sollers, Cheletogenes ornatus, Cheletomimus (Hemicheyletia) berlesei, Cheletomimus greenwoodi, Cheletomorpha lepidopterorum, Cheyletiella parasitivorax, Ch. yasguri, Cheyletus eruditus, Ch. trouessarti (= Ch. woodroffei), Eucheyletia flabellifera, Ornithocheyletia hallae

Greece (Papaioannou-Souliotis, 1994; Eliopoulos \& Papadoulis, 2001; Athanassiou et al., 2003; Palyvos \& Emmanouel, 2006; Palyvos, Emmanouel \& Saitanis, 2008) - Acaropsellina docta, A. sollers, Chelacheles hellenicus, Cheletogenes berlesei (= ornatus), Cheletomimus duosetosus, Cheletomorpha lepidopterorum, Cheyletiella parasitivorax, Cheyletus aversor, Ch. cacahuamilpaensis, Ch. eruditus, Ch. malaccensis (=Ch. polymorphus), Ch. trouessarti, Ch. trux (= tenuipilis),

Holland (Oudemans, 1903; Smiley, 1970; Bronswijk et al., 1972; Volgin, 1987; Fain \& Bochkov, 2001) - Acaropsellina docta, Bakericheyla chanayi, Cheletomorpha lepidop- 
terorum, Cheyletiella parasitivorax, Ch. yasguri, Cheyletus eruditus, Ch. trouessarti, Eucheyletia flabellifera, Ornithocheyletia lukoschusi

Hungary (Dudich, Kolosváry \& Szalay, 1940; Komlovszky, 1980, 1984; Lucza, Ripka \& Saly, 1996; Ripka, Fain \& Bolland, 1999; Fain \& Bochkov, 2001 ) - Acaropsellina docta, Cheletacarus raptor, Cheletogenes ornatus, Cheyletus aversor, Ch. eruditus, Ch. trouessarti, Prosocheyla ripkai, P. traubi

Ireland (Baker, 1969; Dodd, 1970) - Cheyletiella yasguri

Iceland (Sellnick, 1940) - Cheyletus eruditus

Italy (contin.) (Berlese, 1886, 1889; Oudemans, 1904; Starkoff \& Starkoff, 1950; Volgin, 1987;) - Acaropsellina docta, Apodicheles heteropalpus, Cheletogenes ornatus, Cheletomimus (Ch.) berlesei, Cheyletiella parasitivorax, Cheletomorpha lepidopterorum (=Ch. venustissima), Cheyletus eruditus, Ch. schneideri, Ornithocheyletia pinguis

Sicily (Berlese, 1913) - Neoeucheyla loricata

Latvia (Petrova \& Salmane, 2000; Petrova, Salmane \& Čudare, 2004) - Cheletomorpha lepidopterorum

Malta (Pfliegler et al., 2017) - Cheletomorpha lepidopterorum

Moldavia (Volgin, 1961) - Cheletacarus raptor

Norway (Thor, 1912; Austad, 1968; Dotterud, Korsgaard \& Falk, 1995) - Cheyletiella parasitivorax, Cheletomorpha lepidopterorum (=Ch. venustissima), Cheyletus eruditus

Poland (Willmann, 1949; Wegner \& Przyborowski, 1958; Haitlinger, 1982, 2000; Kivganov \& Bochkov, 1994; Haitlinger \& Jankowska, 2005; Solarz, 1989; Skoracki, Bochkov \& Sikora, 2004) - Acaropsis docta, Cheletophyes aurorae, Cheletopsis daberti, Cheyletia papillifera, Cheyletus eruditus, Ch. trouessarti, Eucheyletia flabellifera, Ornithocheyletia dubinini

Portugal (cont.) (Baker, 1949, 1958, Bochkov \& OConnor, 2004) - Chelacheles strabismus, Cheletomorpha lepidopterorum, Cheyletus eruditus

Madeira (Willmann, 1939) - Cheyletus eruditus

Russia (all, continent.)(Rohdendorf, 1940; Volgin, 1962, 1964, 1966, 1969; Fain, 1972; Hughes, 1976; Bochkov, Mironov \& Gorgol, 1994; Bochkov \& Mironov, 1999; Fain \& Bochkov, 2001; Chernichko \& Kivganov, 2013; Bochkov \& Abramov, 2016; Diumina \& Abramov, 2018; Khaustov et al., 2019; Khaustov, 2021) - Acaropsella kulagini, Acaropsellina sollers, Chelacheles apanaskevichi, Cheletacarus raptor, Cheletogenes ornatus, Cheletomimus berlesei, Ch. lindquisti, Ch, recki, Cheletomorpha lepidopterorum, Cheletopsis impavida, Cheyletia aradiphila, Cheyletiella blakei, Ch. parasitivorax, Cheyletus eruditus, Ch. malaccensis (= polymorphus), Ch. rashtiensis, Ch. trouessarti (= truculentus Volgin), Ch. volgini, Cunliffella tuberculicoxa, Eucheyletia eoa, E. harpyia, E. sibirica, Eucheyletiella daurica, E. pallasius, E. takahasii, Eutogenes frater, Mexecheles virginiensis, Muricheyla sicista, Neoacaropsis granulatus, Neocheyletiella microrhyncha, Ornithocheyletia phylloscopi, Paracheyletia pyriformis

Crimea (Volgin, 1963a; Kuznetsov, 1977; Bochkov, Khaustov \& Kuznetsov, 1999; Fain \& Bochkov, 2018) - Chelacheles alexandrinus, Cheletopsis impavida, Cheyletus cacahua- 
milpensis, Eucheyletia flabellifera (= taurica), Mictrocheyla granigera, Paracheyletia volgini, Prosochela villosa

Slovakia (Ždárková, 1979) - see Czechoslovakia

Spain (cont.) (Mihelcic, 1958; Zapatero Ramos et al., 1982; Cordero del Campillo et al., 1994) - Cheletonella vespertilionis, Cheyletus eruditus

Sweden (Boström et al., 1997) - Cheyletidae (Gotland I.)

Switzerland (Schweizer, 1922, 1951; Schweizer \& Bader, 1963; Mumcuoglu, 1976) - Cheletomorpha lepidopterorum, Cheyletus eruditus, Ch. trouessarti, Eucheyletia flabellifera

Turkey (all) (Özer et al., 1986, 1989; Özkan, Ayyıldız \& Soysal. 1988; Koç \& Ayyıldız, 1995, 1996; Koç, 1998; Fain \& Bochkov, 2001; Dogan \& Ayyıldız, 2002, 2004; Bayram \& Çobanoğlu, 2006; Bilal, 2012; Akpınar, Çobanoğlu \& Öğreten, 2017; Çobanoğlu, Cilbircioğlu \& Zabludovskaya, 2020; Doğan S. et al., 2019; Doğan, Doğan \& Erman, 2019) - Acaropsella volgini, Bothrocheyla pavlovskyi, Cheletomimus (Hemicheyletia) bregetovae, Ch. (H.) wellsi, Cheletomorpha lepidopterorum, Cheyletus eruditus, Ch. malaccensis, Eutogenes frater, Hypopicheyla elongata, Microcheyla granifera (= ozkani), Ornithocheyletia hallae

Ukraine (Volgin, 1969; Vysotskaja [= Vysotzkaja], 1974, 1977; Kuznetsov, 1977; Kivganov \& Bochkov, 1994; Bochkov \& Khaustov, 1999; Bochkov, Khaustov \& Kuznetsov, 1999; Fain \& Bochkov, 2001; Chernichko \& Kivganov, 2013) - Bothrocheyla pavlovskyi, Chelacaropsis moorei, Chelacheles alexandrinus, Ch. bacchusi, Cheletopsis charadrii, Ch.daberti, Ch. ferruginea, Cheyletus cacahuamilpensis [syn. baloghi], Ch. eruditus, Ch. trux, Eucheyletia bishoppi [= asiatica], E. bothrophila, E. taurica, Microcheyla granifera, Nodele superba, Paracheyletiella volgini, Prosocheyla villosa

"Yugoslavia" (Wikerhauser \& Pinter, 1987) - Cheyletiella blakei

\section{AFRICA}

Algeria (Louadi \& Robaux, 1992; Fain \& Bochkov, 2001; Fain, Bochkov \& Corpuz-Raros, 2002; Fain, 2003) - Chelacheles strabismus (= algericus), Cheletogenes ornatus, Cheletomimus (Hemicheyletia) bakeri, Cheletonella vespertilionis, Cheyletus cacahuamilpensis, Ch. malaccensis, Ch. trux (sub tenuipilis), Eutogenes frater, Neoeucheyla bochkovi, Paracheyletia pyriformis

Angola (Fain \& Bochkov, 2001, 2002) - Cheletomimus (Ch.) berlesei, Cheletophyes eckerti, Nihelia curvidens, Sciurocheyla angolensis

Botswana (Lawrence, 1959) - Neocheyletiella megaphallos

Burkina Faso (Bochkov, Mironov \& OConnor, 2006) - Galagocheles lawrencei

Burundi (Fain \& Bochkov, 2001; Bochkov \& OConnor, 2004) - Chelacaropsis apus, Sciurocheyla funisciurus, Bakericheyla benoiti, Cheletophyes torridae, Sciurocheyla [Smileycheles] camerounensis

Cameroon (Fain, Bochkov \& Corpuz-Raros, 2002) - Cheletomimus wellsina 
Congo DR (Cunliffe, 1962, Fain, 1972; Fain \& Bochkov, 2001; Fain, Bochkov \& CorpuzRaros, 2002; Klimov, Bochkov \& OConnor, 2006; Bochkov, Mironov \& OConnor, 2006) - Acaropsella kinshasensis, Bak deleoni, Cheletomimus (Hemicheyletia) bakeri, Ch. (H.) congensis, Cheletomorpha bakeri, Cheletophyes eckerti, Ch. mbomba, Cheyletiella parasitivorax (= katangae), Cheyletus funisciuri, Ch. kivuensis, Galagocheles lawrencei, G. lemuricola, Grallacheles bakeri, Ker afrotropicalis, Samsinakia volgini, Sciurocheyla congolensis

Egypt (El Badry \& Zaher, 1960; Zaher \& El Badry, 1962; Zaher \& Soliman, 1966, 1967; Wafa \& Soliman, 1968; Elbadry, 1969; Rasmi et al., 1972; Yousef \& Issa, 1972; Attiah, 1973; El Tawab \& Yousef, 1975; Soliman, 1975, 1977; Yousef, 1975, 1979; Hassan \& Gomaa, 1982; Yousef, Zaher \& Kandil, 1979; Saleh et al., 1985, 1986; Fain \& Bochkov, 2001; Negm \& Mesbah, 2014; Metwally et al., 2016) - Acaropsella aegyptiaca, Acaropsellina docta, Aegyptocheyla summersi, Chelacaropsis moorei, Ch. reticulata, Chelacheles alexandrinus, Ch. bakeri, Cheletogenes ornatus, Cheletomorpha lepidopterorum, Cheyletus egypticus, Ch. eruditus, Ch. malaccensis (incl. fortis), Eutogenes frater (= africanus), E. punctata, Ker bakeri, Neoeucheyla macrocorneus, N. ornata, Nodele calamondin [=simplex]; Genus incertae sedis: Aegyptocheyla; Sp. inquirenda: Cheletomimus minutus

Gabon (Bochkov \& OConnor, 2003) - Picocheyletus tricholaemae

Kenya (Fain \& Bochkov, 2001) - Kenyacheylus troglodytes, Chelacaropsis kenyensis

Madagascar (Samšinak, 1959; Volgin, 1965; Fain, 1982) - Cheyletus legendrei; Samsinakia theodoridis

Morocco (Cooreman, 1951; Fain \& Lukoschus, 1981) - Cheletogenes ornatus, Cheyletus punctulatus

Mozambique (Rodriges, 1967a, 1967b, 1968; Bochkov \& Ochoa, 2005) - Neocheyletiella ardeola

Nigeria (George et al., 1992) - Cheyletiella parasitivorax

Rwanda (Fain, 1972; Fain \& Bochkov, 2001, 2002a; Fain, Bochkov \& Corpuz-Raros, 2002; Bochkov, Mironov \& OConnor, 2006) - Apodicheles apus, Bakericheyla transvaalica, Chelacaropsis apus, Ch. rwandana, Cheletomimus wellsina, Cheletomorpha bakeri, Ch. lepidopterorum, Cheyletus funisciuri, Ch. tanzaniensis, Galagocheles lawrencei, G. lemuricola, Ker afrotropicalis, Neocheyletiella lonchurae, N. megaphallos, N. microrhyncha, N. queleae,

Sainte-Helene (Summers \& Price, 1970) -

Senegal (Gueye-N'Diaye \& Fain, 1987) - Cheyletus malaccensis

Somalia (Fain, 1974) - Teinocheylus longissimus

South Africa (Lawrence, 1948, 1954; Meyer \& Ryke, 1960; Fain, 1982; Meyer, Loots, van Pletzen, Engelbrecht \& Walker, 1973; Meyer, 1988; Fain \& Lukoschus, 1981; Olivier \& Theron, 1988, 1989a, 1989b; Lindholm, Venter \& Ueckermann, 1998; Bochkov \& OConnor, 2004) - Apodicheles cypsiurus, Bakericheyla africana, B. faini, B. subquadrata, B. transvaalica, Chelacheles aigamuxa, Cheletacarus novemdentis, Cheyletiella 
parasitivorax, Cheyletus cacahuamilpensis, Ch. mafekingensis, Ch. gerbillicola, Ch. pseudomalaccensis, Eutogenes reticularis, Galagocheles lemuricola, Microcheyla paraparvula, M. squamosa, Neocheyletiella megaphallos, Nihelia curvidens, N. cynictis, Paramicrocheyla spinula, P. ungulina, Prosocheyla (Reckiana) hepburni

Tanzania (Vitzthum, 1920; Fain, 1972; Haitlinger, 2000; Fain \& Bochkov, 2001; Bochkov \& OConnor, 2004; Bochkov \& Skoracki, 2011) - Cheletophyes aurorae, Cheyletus venator, Ch. tanzaniensis, Metachyletia ngaii, Sciurocheyla camerounensis

Tunisia (Fain, Gerrits \& Lukoschus, 1982) - Teinocheylus gundii

Uganda (Lawrence, 1954) - Cheletomorpha bakeri, Cheyletus malaccensis (= ugandanus) Zambia (Mwase \& Baker, 2006) - Cheyletus spp.

Zimbabwe (Fain, 1979) - Bakericheyla transvaalica, Cheletoides chirunduensis

\section{ASIA}

Bangladesh (Asmat \& Abdul Kader, 1990) - Cheyletus malaccensis

Burma (Myanmar)(Summer \& Price, 1970) - Cheyletus malaccensis (= fortis), Prosocheyla China (Volgin, 1963a; Shen, 1975, 1984, 1991; Lung-Shut, 1984; Whitaker, Shen, 1975, 1991; Wang, 1989; Baccus, Cleveland \& Gu, 1990; Shen, 1991; Fang Lin, Qian Kaiming \& Gao Cheng, 1991; Hu \& Hou, 1992; Lin, 1997; Lin \& Liu, 1994; Xia, Ye \& Zhu, 1997a, 1997b; Xia \& Zhu, 1997; Lin \& Zhang, 1992, 1997; Lin, Zhang \& Yang, 1997, 1998; Xia, Rong \& Zhimin, 1997; Lin et al., 2000; Zhu et al., 2000; Fain \& Bochkov, 2001; Xia, Liang, Zeng \& Zhu, 2004; Xia, 2010) - Acaropsellina docta, A. nanchangensis, A. sollers, Bak faini, Caudacheles trigintaduae, Chelacaropsis moorei, Ch. bipanus, Cheletacarus raptor, Chelacheles bipanus, Ch. michalskii, Ch. striola, Cheletogenes meihuashanense, Ch. ornatus, Cheletomimus (Hemicheyletia) anarbora (= Hemicheyletia pusillifolium), Ch. bakeri, Ch. chui, Ch. omissus, Ch. wellsi, Ch. wellsinus, Cheletomorpha lepidopterorum, Cheyletiella parasitivorax (= dengi), Cheyletus carnifex, Ch. eruditus, Ch. malaccensis, Ch. trouessarti, Columbicheyla bicicri, C. macroflabellata, Eucheyletia bishoppi, E. flabellifera, E. harpyia, E. omissa, E. sinensis, E. variformis, Eutogenes quadrisetatus, Grallacheles bakeri, G. nanfengensis, Hoffmannita clavipes (= H. navicula), Ker palmatus, Lepidocheyla gracilis, Neoacaropsis granulatus, Oudemansicheyla denmarki, Prosocheyla sinensis, Zachvatkiniola reticulate. Sp. inquirendae: Cheletonella juglandis

India (Baker, 1949; Summers \& Price, 1970; Fain, 1972; Nagar \& Saxena, 1977; Nagar, 1979; Gupta \& Chattopadhyay, 1978; Smiley \& Whitaker, 1981; Mathur \& Mathur, 1981; Gupta \& Paul, 1985, 1992; Putatunda \& Kapil, 1988; Gupta, 1991, 2002; Bochkov \& Mironov, 1998; Ramaraju \& Mohanasundaram, 1999; Fain \& Bochkov, 2001, 2002a; Putatunda, Kumar \& Banerjee, 2004; Bochkov \& Ochoa, 2005; Podder, Gupta \& Saha, 2006; Bochkov \& Otto, 2010) - Chelacaropsis moorei, Chelacheles indra, Cheletophyes deodikari, Ch. eckerti, Ch. harnaji, Ch. haryanaensis, Ch. Indiacus (=xylocopae), Ch. newtoni, Ch. orientalis, Ch. ruttneri, Ch. shendei, Cheyletus eruditus, Grallacheles indicus, Lepidocheyla caucasica [= hissariensis], Mexecheles shiva, M. virginiensis, 
Microcheyla bengalensis, Neocheyletiella athene, N. siva, Nihelia calcarata, Prosocheyla (P.) traubi, Samsinakia carabae, S. gonocephalum, S. trilobitus; Neoeucheyla ploceus (Sp. inquir.)

Andaman and Nicobar Is. (Gupta \& Ghosh, 1980) - Chelacaropsis moorei

Indonesia (Berlese, 1913; Domrow, 1960; 1964: 17; Fain, 1972; Thewke, 1980; Haines, 1988; Fain \& Bochkov, 2001; Bochkov \& Otto, 2010) - Bak indonesiensis, Chelonotus selenirhynchus [= Ch. oudemansi $=$ Ch. ewingi], Cheletophyes apicola, Eutogenes quadrisetatus, Hylopecheyla bunguranensis, Neocheyletiella amandavae, Nodele mu, Tutacheyla buruensis

Iran (Vercammen-Grandjean \& Rak, 1968; Rak, 1972, 1973; Sepasgozarian, 1978; Kamali, 1990; Faraji \& Kamali, 1993; Yazdani \& Ebrahimi, 1993; Bochkov \& Malikov, 1996; Fathipour, Kamali \& Ostovan, 1999; Ardeshir et al., 2000; Fain \& Ardeshir, 2000; Bochkov, Fain \& Ardeshir, 2001; Fain \& Bochkov, 2001; Kamali, Ostovan \& Atamehr, 2001; Merhnejad \& Ueckermann, 2001; Bochkov, Hakimitabar \& Saboori, 2005; Ostovan \& Kamali, 1997; Sahraeian et al., 2006; Ardeshir \& Nematollahi, 2008; Namaghi, 2010; Beyzavi \& Ostovan, 2011; Doğan, Jalaeian \& Kamali, 2011; Modarres, 2012; Pactinat-Saeij, Bagheri \& Skvarla, 2017; Ardeshir, 2017; Salarzehi, Hajizadeh, Hakimitabar \& Ueckermann, 2018; Salarzehi, Hajizadeh \& Ueckermann, 2018, 2019) - Acaropsella kulagini, A. volgini, Acaropsellina docta, A. sollers, Bak iranica, Caudacheles khayae, C. lieni, Chelacheles michalskii, Ch. strabismus, Cheletogenes ornatus, Ch. scaber, Cheletomimus (Ch.) berlesei, Ch. binus, Ch. (Hemicheyletia) bakeri, Ch. (H.) congensis, Ch. (H.) vescus, Ch. (H.) wellsi, Ch. parasitivorax, Ch. yasguri, Cheyletus bidentatus, Ch. cacahuamilpensis, Ch. carnifex $(=C h$. acarophagus = allactaga $=$ zacheri), Ch. eruditus, Cheyletus kuznetzovi, Ch. malayensis, Ch. malaccensis, Ch. rashtiensis, Ch. trouessarti, Cheletomorpha lepidopterorum, Cheletonella iraniensis, C. vespertilionis, Cunliffella bulgarica, C. variegata, Eucheyletiella faini, E. flabellifera, Eutogenes frater, Hypopicheyla elongata, H. mirabilis, Lepidocheyla gracilis, Microcheyla parvula, Neoeucheyla iranica, Nodele calamondin, Paraheyletia pyriformis, Samsinakia trilobites, Zachvatkiniola reticulata

Iraq (Rizk et al., 1979) - Acaropsellina docta, A. sollers

Israel and Palestine (Shulov, 1957; Cwilich \& Hadani, 1965; Costa, 1961: 259; 1967: 109; Gerson, 1967, 1968; Feldman-Musham et al., 1985; Fain \& Bochkov, 2001 Fain, Bochkov \& Corpuz-Raros, 2002) - Acaropsella volgini, Caudacheles khayae, Cheletogenes ornatus, Cheletomimus (Ch.) berlesei, Cheletomimus (Hemicheyletia) bakeri, Cheletomorpha lepidopterorum, Ch. tatami, Cheyletiella sp., Cheyletus cacahuamilpensis, Ch. eruditus, Ch. malaccensis, Ch. trux (= tenuipilis), Eutogenes citri, Lepidocheyla gracilis, Neoacaropsis volgini

Japan (Sasa \& Kono, 1951; Tanaka, 1953; Hara, 1955; Hara \& Hanada, 1960; Ehara, 1962; Shiba, 1969a, 1969b; Summers \& Price, 1970; McClure, Ratanaworadhan et al. 1973; Kumada et al., 1978; Razzaq, Shiraishi, Manabe \& Ohbayashi, 2001) - Chelacaropsis moorei, Cheletomorpha lepidopterorum [Cheletophyes tatami Hara], Cheyletus er- 
uditus, Ch. malaccensis (= fortis), Ch. rarus, Cheletomimus (Hemicheyletia) bakeri, Ch. (H.) wellsi, Eucheyla favosa, Eucheyletiella takahashii, Eutogenes narashinoensis, Neocheyletiella sp., Zachvatkiniola reticulata

Kazakhstan (Kuzin, 1940; Volgin, 1955, 1963; Bochkov \& Mironov, 1999) - Acaropsellina sollers, Cheyletus trouessarti [sub Ch. praedabundus Kuzin], [Cheyletus rabiosus], Eucheyletia asiatica, E. pavlovskyi, Eucheyletiella pallasianus

Korea (Nord and South) (McClure, Eliott, Ratanaworadhan et al. 1973; Shin, 1996; SangHun Kim et al., 2008) - Cheyletiella parasitivorax, Ch. yasguri, Neocheyletiella sp.

Kyrghyzstan (Volgin, 1969; Dubinina \& Bochkov, 1989; Chirov \& Mironov, 1998; Chirov \& Bochkov, 1998) - Cheyletus carnifex, Cunliffella tuberculicoxa, Eucheyletiella ochotonae, Ornithocheyletia mironovi

Malaysia (continent.)(Oudemans, 1903; Nadchatram \& Domrow, 1966; Shiba, 1976; Yunus \& Ho, 1980; Fain \& Nadchatram, 1980; Fain, Lukoschus \& Nadchatram, 1980; Ehara \& Abdul Ghani, 1988; Mariana et al., 2000: 714; Fain \& Bochkov, 2001) - Cheletogenes monosetosus, Ch. ornatus, Cheletomimus (Hemicheyletia) anarbora, Ch. (H.) transversus (= Hemicheyletia morii), Ch. (H.) tropicus, Ch. (H.) wellsi, Ch. (H.) wellsinus, Cheletomorpha lepidopterorum, Cheletophyes apicola, Ch. clavipilis, Ch. vespae, Cheletonella vespertilionis, Chelonotus selenirhynchus, Cheyletus malaccensis (= Ch. fortis), Ch. malayensis, Ch. trouessarti, Criokeron quintus, Eutogenes onoi, Grallacheles bakeri, Hylopecheyla malayi, Ker bakeri

Sarawak \& Sabah (Domrow, 1964; Ide \& Kethley, 1977) - Chelonotus selenirhynchus, Thewkachela ratufi

Mongolia (Fain \& Lukoschus, 1981) - Cheyletus carnifex [syn. allactaga]

Nepal (Summers \& Price, 1970) - Eucheyletia sinensis

"New Guinea” (Summers \& Price, 1970)

Pakistan (Khan, 1970; Qayyum \& Chaudhri, 1977a, 1977b, 1977c, 1979a, 1979b; Rasool \& Chaudhri, 1979; Rasool, Chaudhri \& Akbar, 1980; Chaudhri \& Akbar, 1985; Akbar, Rahi \& Chaudhri, 1988; Aheer, Akbar \& Chaudhri, 1988, 1991, 1992, 1993, 1994, 1998; Baloch, 1991; Akbar, Aheer \& Ishtiaq, 1993; Aheer et al., 1997; Farooq, 2000; Farooq, Akbar \& Qureshi, 2000; Haq \& Zia, 2001a, 2001b; Haq et al., 2004; Fain, Bochkov \& Corpuz-Raros, 2002; Bochkov \& OConnor, 2003; Afzal, Ali \& Akbar, 2003; Kamran, Khan \& Nazir, 2005; Haq \& Afzal, 2007; Akbar, Jahan \& Mughal, 2008; Halliday, Kamran \& Bashir, 2018) - Acaropsella nobilis, A. shaziai, A. walii, Acaropsellina campusensis, A. clamo, A. opsis, A. orbis, A. platessa, A. porta, A. shorkotiensis, A. subitus, A. venustus, A. vitrus, Chelacheles humilis, Cheletogenes aceriai, Ch. carinatus, Ch. dissitus, Ch. iconis, Ch. petigenis, Ch. sagacis, Ch. scaber, Ch. vulgatus, Cheletomimus (Hemicheyletia) bakeri, Ch. (H.) tanosus, Ch. (H.) vescus, Cheletomorpha dolosus, Ch. lepidopterorum, Ch. obrutus, Ch. opacus, Ch. tenerum, Cheletonella daddaciensis, Ch. emersus, Cheyletus crassus, Ch. eruditus, Ch. malaccensis $(=$ ayyazi, $=$ baridos $=$ infensus $=$ phantosis $=$ wahndoensis $)$, Ch. vivatus, Ker 
acidalia, Neocheyletiella aegithali; $\mathbf{S p}$. inquirendae: Cheletomimus cambio, Ch. cantor, Ch. larmae, Ch. telma, Ch. trema, Ch. zamia, Cheletomorpha obrutus

Philippines (Summers \& Price, 1970; Corpuz - Raros, 1972, 1980, 1988a, 1988b, 1998, 2000; Corpuz - Raros \& Sotto, 1977; Malaban \& Corpuz-Raros, 1998; Fain, Bochkov \& Corpuz-Raros, 2002; Bochkov \& OConnor, 2003, 2004; Corpuz-Raros L. A., J.C.B. Naredo. 2016) - Acaropsella filipina, Acaropsellina levis, A. philippinensis, Alliea prasadi, Apodicheles collocalia, Bak faini, B. furcatus, B. gersoni, B. payatus, B. truncatus, Chelacaropsis terrestris, Chelacheles baiwanganae, Ch. robustus, Cheyletus ascutatus, Ch. batomysi, Ch. malaccensis (= fortis), Ch. malayensis, Cheletogenes ornatus, Cheletomimus (Ch.) daltoniensis, Cheletomimus (Hemicheyletia) athenae, Ch. (H.) bakeri, Ch. (H.) leytensis, Ch. (H.) transversa, Ch. (H.) uichancoi, ?Ch. (H.) wellsi, Ch. (H.) wellsinus, Cheletomimus (Philippicheyla) philipina, Cheletomorpha dolosus, Ch. tenerum, Cunliffella duo, C. maysa, Eucheyletia nindota, Eutogenes makilingensis, Hoffmannita rimandoi, Ker mercedesae, K. pintoriensis, Neochelacheles corpuzrarosae, N. mendicus, Pavlovskicheila philippicana, Samsinakia pagongae. Species inquirendae: Hemicheyletia lanceolata

Saudi Arabia (Soliman \& Al-Yousif, 1980; el-Bahrawy \& al-Dakhil, 1993; Rostom, 1993) - Acaropsella ? volgini, Acaropsellina sollers, Cheyletus eruditus, Ch. malaccensis

Singapore (Fain, Bochkov \& Corpuz-Raros, 2002) - Cheletomimus (Hemicheyletia) asiaticus

Sri Lanka (Parameswaran Pillai, 1957) - Cheyletus munroi [var. bituberculatus]

Tadjikistan (Volgin, 1963) - Cheyletus carnifex, Ch. malaccensis (= polymorphus), Lepidocheyla gracilis

Taiwan (Sugimoto, 1942; Summers \& Price, 1970; Tjying I. - S. (= Tseng), 1971, 1972; Tseng, 1973, 1977, 1982, 1984) - Acaropsella kinshasensis (= konoi), Bak micidus, Caudacheles lieni, Chelacheles lanceolatus, Cheletogenes monosetosus, Cheletomorpha lepidopterorum, Cheyletiella strandtmanni, Cheletomimus anarbora, Ch. (H.) bakeri, Ch. chui, Ch. omissus, Ch. (H.) wellsinus, Cheletonella pilosa, Cheyletus eruditus, Ch. malaccensis, Ch. trouessarti, Eucheyletia sinensis, Oudemansicheyla denmarki, Zachvatkiniola reticulata

Thailand (Vaivanijkul, 1979; Uchikawa \& Suzuki, 1980; Fain \& Lukoschus, 1985; Lekprayoon \& Smiley, 1986; Gerson \& Fain, 1991; Bochkov \& OConnor, 2004; Bochkov \& Klimov, 2005; Fuangarworn \& Lekprayoon, 2010) - Bak furcatus, Chelacaropsis moorei, Criokeron quintus, C. thailandicus, Chelacheles baiwanganae, Cheletophyes decorus, Cheyletus eruditus, Dubininiola boonkongae, Mexecheles thailandensis, Samsinakia charanasriae, Sciurocheyla squamosa

Turkmenia (Volgin, 1969, 1978) - Acaropsellina sollers, Dubininiola polylepis, Cheletomimus (Hemicheyletia) asiaticus, Lepidocheyla gracilis

Uzbekistan (Kuzin, 1940; Volgin, 1955; Barilo, 1985, 1986a, 1986b, 1989) - Acaropsellina sollers, Chelacheles [Chelachecaropsis] stigmaeoides, Cheyletus carnifex, Ch. morinus, Hypopicheyla mirabilis, Ker caeterus, Neoeucheyla minuta, Pavlovskicheyla semenovi 
Vietnam (Bochkov \& Klimov, 2005) - Cheletophyes decorus

\section{NORTH AMERICA}

Canada (Banks, 1909; Ayalew \& Vaillancourt, 1976; Thewke \& Enns, 1979; Bochkov \& Galloway, 2001; Fain, Bochkov \& Corpuz-Raros, 2002) - Cheletomimus (Hemicheyletia) bakeri, Ch. (H.) lindquisti, Cheletomorpha lepidopterorum, Cheyletiella yasguri, Mexecheles virginiensis, Neocheyletiella microrhyncha, Ornithocheyletia canadensis, O. prognae, O. turdi

Mexico (Baker, 1949; De Leon, 1962; Pelaez, 1962; Fain, 1972; Thewke \& Enns, 1979; Uchikawa \& Suzuki, 1979; Thewke, 1980; Atyeo, Kethley \& Pérez, 1984; Hoffmann, Cervantes \& Morales-Malacara, 1994; Estébanes - González, 1997; Fain \& Bochkov, 2001; Bochkov \& Klimov, 2004, 2005) - Anthribicheyla bocki, Cheletogenes ornatus, Cheletomimus berlesei, Ch. cordovensis, Ch. scutellatus, Cheyletiella parasitivorax, Cheyletiella [syn. Bicheyletiella] romerolagi [= mexicana], Cheyletus cacahuamilpensis, Ch. eruditus, Chiapacheylus. edentatus, Hoffmannita mexicana; Metacheyletia longisetosa, Mexecheles hawaiiensis, M. marshalli, Nodele (Aztecocheyletus) conquistador, Samsinakia tilae

USA (Banks, 1902, 1904, 1906; Ewing, 1909a, 1909b, 1912; Miller, 1925; Cooper, 1946; Baker, 1949; Yunker, 1960, 1961; De Leon, 1962; Muma, 1964; Vercammen-Grandjean \& Rak, 1968; Summers \& Price, 1970; Smiley \& Moser, 1970, 1975; Hughes, 1976; Smiley, 1970, 1996; Moser \& Roton, 1971; Smiley \& Williams, 1972; Whitaker, 1973; Whitaker \& Wilson, 1974; Thewke \& Enns, 1972, 1974, 1975; Keh, 1973, 1975; Delfinado \& Khaing - Fields, 1976; Thefke, 1974, 1977; Whitaker, Pascal \& Mumford, 1979; Whitaker, Tieben \& Maser, 1980; Smiley \& Whitaker, 1981; Whitaker \& Maser, 1985; Philips \& Dindal, 1990; Cudmore, 1986; Fain \& Bochkov, 2001; Fain, Bochkov \& Corpuz-Raros, 2002; Bochkov \& OConnor, 2003, 2004; Whitaker et al., 2007; Bochkov \& Otto, 2010; Xia, Klompen \& Childers, 2011) - Acaropsella kulagini, Acaropsellina sollers, Alliea laruei, Anthribicheyla bocki, Atarsacheylus vichii, Bak deleoni, B. ozarkensis, B. sanctaehelenae, Bothrocheyla typhosa, Camincheyletus glaucomys, Chelacheles bipanus, Ch. peritremaculatus, Ch. seminole, Ch. strabismus, Ch. temoak, Cheletacarus gryphus, Cheletogenes ornatus, Cheletomimus (Ch.) berlesei, Ch. duosetosus, Cheletomimus (Hemicheyletia) bakeri, Ch.(H.) newyorkensis, Ch. (H.) rostella, Cheletonella vespertilionis, Ch. hoffmannae, Cheletopsis thalasseus, Cheyletiella blakei, Ch. parasitivorax (= furmani = americana), Ch. yasguri, Cheyletus cacahuamilpensis (nr), Ch. carnifex (= aversor), Ch. eruditus, Ch. linsdalei, Ch. malaccensis, Ch. trouessarti, Ch. trux (= hendersoni), Cunliffella whartoni, Eucheyletia bishoppi (= E. oregonensis), E. hardyi, Eucheyletiella johnstoni, Eutogenes pinicola, E. vicinus, Grallacheles bakeri, Ker palmatus, Lanceacheyla whartoni, Mexecheles impolitus, M. panneus, M. virginiensis, Modele calamondin, M. coccineae, Neochelacheles messersmithi, Neocheyletiella roweri, N. smallwoodae, Ornithocheyletia gersoni, O. hallae hallae, O. lawrenceae, Oudemansicheyla denmarki, Paracaropsis travisi, Paracheyletia 
pyriformis (= Cheyletus longipalpus), Pavlovskicheyla platydemae, Prosocheyla (P.) acantha, P. (P.) oaklandia, P. (Reckiana) buckneri

Alaska (Volgin, 1963) - Eucheyletia bakeri

Hawaiian Is. (Baker, 1949; Garrett \& Haramoto, 1967; Haramoto, 1969; Summers \& Price (1970); Goff, 1982, 1983, 1987; Paxot \& Goff, 1985; Fain, Bochkov \& CorpuzRaros, 2002) - Bak deleoni, B. elongatus, Chelacheles strabismus, Cheletogenes ornatus, Cheletomimus (Ch.) berlesei, Ch. (Ch.) duosetosus, Ch. (Hemicheyletia) bakeri, Ch. (H.) bregetovae, Ch. (H.) gracilis, Ch. (H.) granulae, Ch. (H.) kureatollensis, Ch. (H.) wellsi, Cheyletus eruditus, Ch. malaccensis (=Ch. fortis), Ch. malayensis, Cunliffella panamensis, Grallacheles bakeri, Ker palmatus, Mexecheles hawaiiensis, Nodele philippinensis; Incertae sedis: Cheletomimus citrosinensis

\section{SOUTH and CENTRAL AMERICA}

Argentina (Brandetti \& Bischoff-de-Alzuet, 1981; Mosquera, 1989) - Cheletomimus (Hemicheyletia) wellsi, Eucheyletia hardyi, Ornithocheyletia boeroi

Brazil (Flechtmann, 1968, 1971; Reis \& Paschoal, 1968; Arruda et al., 1969; Amaral, 1971; Fain, 1972; Rosa \& Flechtmann, 1979; Whitaker \& Dietz, 1987; Bagio et al., 1988a, 1988b; Feres \& Flechtmann, 1995; Ezequiel et al., 2001, 2003; Buosi et al., 2006; Valim \& Gazeta, 2007) - Bak ligyscutatus, Cheletogenes ornatus, Cheletomimus duosetosus, Ch. wellsi, Cheletomorpha lepidopterorum, Cheletonella vespertilionis (= caucasica), Cheyletiella parasitivorax, Cheyletus eruditus, Ch. malaccensis (= fortis), Eutogenes sp., Ker bakeri, Mexecheles hawaiiensis, Ornithocheyletia aitkeni, O. hallae

Chile (contin.)(Baker, 1949; Alcaino \& Tagle, 1966; Alcaino \& Calderon, 1976; Artigas \& Casanueva, 1983; Casanueva \& Artigas, 1985; Peredo et al., 1994; Fain \& Bochkov, 2001) - Cheletogenes ornatus, Cheletomorpha lepidopterorum, Cheyletiella parasitivorax, Ch. yasguri, Cheletomimus (Hemicheyletia) wellsi, Cheyletus eruditus

Columbia (Oudemans, 1903; Baker, 1949) - Cheyletus eruditus, Ch. intrepidus

Costa Rica - Cheletophyes panamensis

Cuba (de la Cruz, 1971; Cuervo, Dusbábek, de la Cruz \& Abreu, 1983; Socarras, 1987; Torre Santana, 2015) - Chelacheles michalskii, Cheyletiella parasitivorax, Cheletogenes ornatus, Cheletomimus (Ch.) berlesei, Cheletomimus anarbora, Cheletomimus (Hemicheyletia) wellsi, Ch. wellsinus, Cheletomorpha lepidopterorum, Cheyletus carnifex, Ch. eruditus, Ch. malaccensis, Ch. malayensis, Chiapacheylus edentatus, Grallacheles bakeri, Mexecheles hawaiiensis

\section{Ecuador (contin.)}

Galapagos Is. (Summers \& Price, 1970; Fain \& Bochkov, 2001; Fain, Bochkov \& CorpuzRaros, 2002) - Bak gersoni, B. micidus, Cheletomimus (Hemicheyletia) bakeri, Ch. (H.) darwinia, Ch. (H.) serrula

El Salvador (Summers \& Price, 1970) - Cheletomimus (Hemicheyletia) granulus, Laeliocheyletia teretis

Guatemala (Fain, Bochkov \& Corpuz-Raros, 2002) - Cheletomimus wellsina 
Honduras - Mexecheles virginiensis

Jamaica (Corpuz-Raros \& Sotto, 1977) - Cheletomimus (Hemicheyletia) wellsi

Netherlands-Antilles (Fain \& Bochkov, 2001) - Hoffmannita gersoni

Panama (Thewke, 1980; Klompen, Mendez \& Lukoschus, 1984; Fain, Bochkov \& CorpuzRaros, 2002) - Anthribicheyla bocki, Cheletomimus (Hemicheyletia) bakeri, Ch. (H.) wellsinus, Cheletophyes panamensis, Cunliffella panamensis, Mexecheles hawaiiensis

Peru (Caceres \& Fain, 1975, 1978a, 1978b; Fain \& Bochkov, 2001) - Cheletomorpha lepidopterorum, Cheyletus cacahuamilpensis, Ch. malaccensis, Ch. trouessarti, Pavlovskycheyla philippicana

Porto Rico (Banks, 1914)

Suriname (Fain \& Bochkov, 2001) - Grallacheles bakeri

Trinidad (De Leon, 1967) - Cheletomimus anarbora, Ch. wellsina

Venezuela (Fain \& Bochkov, 2001) - Cheletogenes ornatus

\section{OCEANIA}

Australia (continent)(Womersley, 1941, 1942a, 1942b, 1956; Champ, 1965, 1966; Shepherd \& Edmonds, 1977; Smiley, 1984; Koch, 1986; Domrow, 1991; Gerson, 1994; Fain \& Bochkov, 2000; 2002b; Bochkov \& Ochoa, 2005; Bochkov \& Otto, 2010) - Chelacheles thomasi, Cheletacarus rugosus, Cheletogenes ornatus, Ch. waitei, Cheletomimus (Hemicheyletia) bakeri, Cheletomimus (Philippicheyla) notelaeae, Cheletomorpha lepidopterorum, Chelacaropsis [syn. Cheletonata] milesi, Cheletonella vespertilionis, Cheletophanes rugosus, Cheyletiella blakei, Ch. parasitivorax, Ch. yasguri, Cheyletus eruditus, Ch. malaccensis, Eucheyletia womersleyi, Granulocheyletus corpuzrarosae, Mexecheles virginiensis, Neocheyletiella ardeola, N. artami, Oconnoricheylus chimaera, Oudemansicheyla denmarki, Ou. coprosomae, Ornithocheyletia lawrenceae, O. lichmerae, O. lonchurae, Prosocheyla traubi, Samsinakia volgini

Tasmania (Domrow, 1991) - Cheyletiella blakei

Papua New Guinea (Oudemans, 1904; Bochkov \& Klimov, 2005) - Eucheletopsis major, Cheletophyes occisor

French Polynesia (Tahiti)(Cunliffe, 1962; Nishida, Version 2009) - Grallacheles bakeri (= Paracheyletia woolfordi), Zachvatkiniola reticulata

Marianne Is. (Oudemans, 1903a) - Cheyletus malaccensis (= vorax), Ch. rapax

New Guinea (Fain \& Bochkov, 2001) - Cheletomorpha lepidopterorum

New Zealand (Womersley, 1942a; Lamb, 1952; Bull, 1953; Davies \& Kyle, 1969; Thewke \& Enns, 1976; Gerson, 1994; Fain, 2003; Bochkov \& Otto, 2010) - Cheletomimus crowei, Cheyletiella parasitivorax, Granulocheyletus gallowayi, Oudemansicheyla coprosomae Vanuatu (Fain, Bochkov \& Corpuz-Raros, 2002) - Cheletomimus (Hemicheyletia) uichancoi 


\section{Hosts of Cheyletidae}

\section{Arthropoda}

Crustacea

Decapoda

Fam. Coenobitidae

Coenobita clypeata - Hoffmannita gersoni

\section{Arachnida}

\section{Scorpiones}

Fam. Buthidae

Centruroides flavopictus - Hoffmannita mexicana

\section{Insecta}

\section{Coleoptera}

Fam. Scolytidae

Dendroctonus frontalis - Mexecheles impolitus, M. virginiensis, Prosocheyla acanthus

Fam. Tenebrionidae

Blastinus sp. - Samsinakia tilae

Bolitotherus cornutus - Neochelacheles messersmithi

Gonocephalum simplex - Samsinakia gonocephalum, G. volgini

Platydema - Pavlovskicheyla platydemae

Selinus abacoides - Samsinakia theodoridis

\section{Fam. Bostrychidae}

Dinoderus minutus - Nodele mu

Sinoxylon crassum - Chelacheles baiwanganae

Xyloperthoides nidipennis - Chelacheles aigamuxa

\section{Heteroptera}

Fam. Aradidae

A. aterrimus - Cunliffella tuberculicoxa, Hypopicheyla elongata

A. betulae - Hoffmanita clavipes

A. corticalis - Hoffmanita clavipes

A. crenaticollis - Hypopicheyla elongata

A. lugubris - Cheyletia aradiphila, Cunliffella tuberculicoxa, Hypopicheyla elongata

A. nemtschinovi - Cunliffella tuberculicoxa, Hypopicheyla elongata

\section{Hymenoptera}

Fam. Apidae

Diadasia sphaeralcearum - Chelacheles temoak

Xylocopa aruana - Cheletophyes occisor

Xylocopa (Megaxylocopa) frontalis - Cheletophyes panamensis 
Xylocopa caffra - Cheletophyes vitzthumi

X. fenestata-Cheletophyes deodicari, Ch. newtoni, Ch. orientalis, Ch. ruttneri, Ch. shendei

X. pubescens - Cheletophyes harnaji

Xylocopa tranquebarica - Cheletophyes decorus

Xylocopa sp. - Cheletophyes xylocopae

Xylocopinae - Cheletophyes aurorae

\section{Fam. Megachilidae}

Azthecanthidium tenochtitlanicum - Nodele (Aztecocheyletus) conquistador

Fam. Vespidae

\section{Diptera}

Vespa tropica - Cheletophyes vespae

\section{Fam. Hippoboscidae}

Pseudolynchia canariensis - Ornithocheyletia hallae

\section{Vertebrata}

Reptilia

Sauria

Fam. Iguanidae

Sceloporus woodi - Paracaropsis travisi

Aves

\section{Apodiformes}

Fam. Apodidae

Apus affinis - Chelacaropsis apus

A. caffer - Apodicheles apus

Collocalia esculenta - Apodicheles collocalia

Fam. Hemiprocnidae

Charadriiformes

Hemiprocne (= Dendrochelidon) mystacea - Eucheletopsis major

Fam. Charadriidae

Charadrius dubius - Cheletopsis charadrii

Ch. hiaticula - Cheletopsis basilica

Fam. Rostratulidae

Rostratula semicollaris - Cheletopsis impavida

Fam. Scolopacidae

Actitis hypoleucos - Cheletopsis mariae

Calidris ferruginea - Cheletopsis impavida

C. melanotis - Cheletopsis basilica

C. minutus - Cheletopsis impavida

C. pusilla - Cheletopsis impavida

C. ruficollis - Cheletopsis impavida

C. temminckii - Cheletopsis daberti, Ch. impavida 
Limnodromus griseus - Cheletopsis limnodromi

Micropalama himantopus - Cheletopsis daberti, Ch. impavida

Prosobonia cancellata - Cheletopsis prosobonialis

Tringa flavipes - Cheletopsis magnanima

T. glareola - Cheletopsis daberti

T. nebularia - Cheletopsis daberti

T. stagnatilis - Cheletopsis impavida

T. totanus - Cheletopsis basilica, Ch. norneri

Fam. Laridae

Subfam. Sterninae

Thalasseus [Sterna] sandvicensis - Cheletopsis thalasseus

\section{Columbiformes}

\section{Fam. Columbidae}

Columba livia - Ornithochyletia hallae, O. pinguis

Columbigallina sp. - Ornithochyletia columbigallinae, O. hallae

Streptopelia semitorquata - Neocheyletiella quleae

\section{Coraciiformes}

Fam. Meropidae

Melithophagus bullockoides - Bakericheyla (B.) transvaalica

M. pusillus - Bakericheyla transvaalica, B. subquadrata

Merops apiaster - Bakericheyla (B.) transvaalica

M. nubicoides - Bakericheyla (B.) transvaalica

M. supercilliosus persicus - Bakericheyla (B.) transvaalica

Merops [Melithophagus] bullocki - Bakericheyla benoiti

\section{Cuculiformes}

\section{Fam. Cuculidae}

Chrysococcyx caprius - Neocheyletiella (= Ornithocheyla) megaphallos Ch. klaasi - Ornithochyletia orioli

\section{Galliformes}

\section{Fam. Numididae}

Numida meleagris meleagris - Metacheletoides numidae

N. meleagris intermedia - Metacheletoides akanyaruensis, M. numidae

Fam. Phasianidae

Francolinus natalensis - Ornithocheyletia francolini

\section{Gruiformes}

Fam. Aramidae

\section{Musophagiformes}

Aramus scolopaceus - Cheletosoma tyrannus

Fam. Musophagidae

Corythaxoides leucogaster - Metacheyletia ngaii

Crinifer piscator - Metacheletoides gisagarensis, M. crinifer 


\section{Passeriformes}

C. zonurus - Metacheletoides gisagarensis, M. crinifer

Fam. Aegithalidae

Aegithalos concinnus - Neocheyletiella aegithali

Fam. Artamidae

Artamus cyanopterus - Neocheyletiella artami

Fam. Corvidae

Cyanocorax chrysops - Ornithochyletia boeroi

Fam. Estrildidae

Donacola castaneothorax - Ornithochyletia lonchurae

Erythrura prasina - Neocheyletiella vestergaardi

Estrilda erythronotos - Neocheyletiella megaphallos

Granatina ianthinogaster - Ornithochyletia granatina

Lonchura striata - Chelacaropsis moorei

Poephila acuticauda - Bakericheyla chanayi

P. gouldiae - Bakericheyla chanayi

Spermestes cucullatus - Chelacaropsis rwandana

\section{Fam. Fringillidae}

Carduelis carduelis - Bakericheyla chanayi

Chloris chloris - Cheyletia papillifera

Leucosticte australis - Neocheyletiella vestergaardi

Serinus mozambicus - Metacheyletia degenerata

\section{Fam. Hirundinidae}

Cecropis abyssinicus unitatis - Neocheyletiella microrhyncha

Delichon urbica - Neocheyletiella microrhyncha

Hirundo rustica - Neocheyletiella microrhyncha

Petrochelidon pyrchonota - Neocheyletiella microrhyncha

Progne subis - Neocheyletiella microrhyncha

Psalidoprocne albiceps - Neocheyletiella microrhyncha

Riparia riparia - Neocheyletiella microrhyncha

Fam. Monarchidae

Geiciphila indistincta - Ornithocheyletia lichmerae

Grallina cyanoleuca - Ornithocheyletia lichmerae

Fam. Motacillidae

Motacilla alba - Bakericheyla chanayi

Fam. Muscicapidae

Cossypha dichroa - Bakericheyla (B.) faini

Fam. Nectariniidae

Nectarinia jugularis - Neocheyletiella nectarinia

Fam. Oriolidae

Oriolus oriolus - Ornithochyletia orioli 
Fam. Phylloscopidae

Phylloscopus trochilis - Ornithocheyletia phylloscopi

Fam. Pittidae

Pitta megarhyncha - Neocheyletiella pittae

Fam. Ploceidae

Foudia madagascariensis - Cheyletus legendrei

Pachyphantes superciliosa - Ornithochyletia orioli

Philetarius socius - Cheyletus mafekingensis

Plocepasser mahali - Cheyletus pseudomalaccensis

Ploceus philippinus - Microcheyla bengalensis, Neoeucheyla ploceus

Quelea quelea - Neocheyletiella queleae

\section{Fam. Sittidae}

Sitta pygmaea melanotis - Neocheyletiella rohweri

\section{Fam. Sturnidae}

Lamprocolius chloropterus - Ornithochyletia lamprocolius

Lamprocolius sp. - Ornithochyletia wauthyi

Sturnus vulgaris - Ornithocheyletia dubinini, O. pinguis

\section{Fam. Timaliidae}

Leiothrix lutea - Neocheyletiella media, N. alfortensis, Ornithocheyletia leiothris

\section{Fam. Turdidae}

Turdus fumigatus - Ornithocheyletia aitkeni

T. merula - Ornithocheyletia pinguis

Fam. Tyrannidae

\section{Piciformes}

Phoebe psychotrioides - Hemicheyletia cordovensi

\section{Fam. Capitonidae}

Lybius sp. - Ornithochyletia orioli

Tricholaema hirsuta flavipunctata - Picocheyletus tricholaemae

Fam. Indicatoridae

Indicator indicator - Ornithochyletia orioli

Fam. Leiothricidae

Garrulax leuolophus bicolor - Ornithocheyletia garrulax

\section{Fam. Picidae}

Dendrocopos pubescens - Ornithocheyletia gersoni

Picus viridis - Ornithocheyletia canadensis

\section{Pelecaniformes}

\section{Fam. Ardaeidae}

Ardeolla ralloides - Neocheyletiella ardeola 


\section{Psittaciformes}

\section{Fam. Psittacidae}

Amazona finschi - Metacheyletia longisetosa

Ara sp. - Neocheyletiella avicola

Forpus passerinus - Ornithocheyletia argentinensis

Nandays nanday - Ornithocheyletia argentinensis

Psittacus erythacus - Ornithocheyletia psittaci psittaci

Pyrrhura cruentata - Cheyletus alacer

Fam. Psittaculidae

Agapornis fisheri - Neocheyletiella avicola

Fam. Psittrichasidae

\section{Strigiformes}

Coracopsis nigra barkleyi - Cheyletus eruditus (= ferox)

Fam. Strigidae

\section{Trogoniformes}

Athene brama - Neocheyletiella athene

Fam. Trogonidae

\section{Mammalia}

Harpactes ardens - Cheyletus philippinensis

\section{Didelphiomorpha}

Fam. Didelphidae

Didelphis virginiana - Cheyletus eruditus

\section{Soricomorpha}

\section{Fam. Soricidae}

Sorex fumeus - Eucheyletia bishoppi

S. pacificus - Eucheyletia bishoppi

S. trowbridgei - Eucheyletia bishoppi

\section{Scandentia}

Fam. Tupaiidae

Tupaia belangeri - Criokeron quintus

T. glis - Criokeron quintus, C. thailandicus, Hylopecheyla malayi

\section{Chiroptera}

Fam. Emballonuridae

Coleura gallarum - Cheyletus tanzaniensis

Fam. Vespertilionidae

Eptesicus fuscus - Cheletonella vespertilionis

Myotis velifer - Cheyletus cacahuamilpensis (nr)

\section{Rodentia}

Fam. Anomaluridae

(?) Zenkerella insignis - Sciurocheyla camerounensis 


\section{Fam. Ctenodactylidae}

Ctenodactylus gundi - Teynocheylus gundii

C. vali - Teynocheylus gundii

Massoutiera mzabi - Teynocheylus gundii

Pectinator spekei - Teinocheylus longissimus

\section{Fam. Muridae}

Batomys salomonseni - Cheyletus ascutatus, Ch. batomysi

Menetes berdmorei - Cheyletus eruditus

Niviventer fulvescens - Eucheyletia sinensis

Rattus norvegicus - Cheyletus eruditus, Ch. trouessarti

R. humiliatus celsus - Eucheyletia sinensis

R. whiteheadi - Cheletophyes apicola

\section{Subfam. Gerbillinae}

Gerbillurus paeba - Cheyletus gerbillicola

Gerbillus pyramidum - Cheyletus punctulatus

\section{Fam. Arvicolidae}

Microtus agrestis - Cheyletus trouessarti

M. arvalis - Cheyletus malaccensis (= polymorphus)

M. guentheri - Cheyletus cacahuamilpensis

M. pinetorum - Eucheyletia bishoppi

\section{Fam. Cricetidae}

Neotoma cinerea - Eucheyletia bishoppi

$N$. floridana - Cheyletus malaccensis

N. magister - Cheyletus malaccensis

Peromyscus leucopus - Cheyletus eruditus

Thalpomys [Akodon] reinhardti - Cheyletus malaccensis

\section{Fam. Sciuridae}

Callosciurus sladeni midas - Cheyletus malaccensis

Citellus beecheyi - Cheyletus lindsdalei

Funisciurus carruthersi - Cheyletus funisciuri

Glaucomys sabrinus (nest) - Acaropsellina summersi, Camincheyletus glaucomys, Eucheyletia bishoppi (= oregonensis)

Heliosciurus undulatus - Sciurocheyla camerounensis

Hylopetes spadiceus - Hylopecheyla bunguranensis

Lariscus insignis - Chelonotus selenirhynchus

Nannosciurus surrutilus - Promuricheyla lukoschusi

Petinomys setosus - Cheyletus malaccensis

Ratufa affinis - Thewkachela ratufi

R. bicolor - Thewkachela ratufi

Rhinosciurus laticaudatus - Cheyletus pluridens

Spermophilus beldingi-Cheyletus lindsdalei 
Sundasciurus hippurus - Chelonotus selenirhynchus

S. lowii - Chelonotus selenirhynchus

S. tenuis - Chelonotus selenirhynchus

Tamias striatus - Eucheyletia bishoppi

\section{Lagomorpha}

\section{Fam. Leporidae}

Lepus whytei - Cheyletiella parasitivorax (= katangae)

Lepus sp. - Cheyletiella strandtmanni

Oryctolagus cuniculus - Cheyletiella parasitivorax

Romerolagus diazi - Cheyletiella [=Bicheyletiella] romerolagi [= mexicana], Ch. parasitivorax

Sylvilagus bachmanni - Cheyletiella parasitivorax

S. floridanus - Cheyletiella parasitivorax

S. palustris paludicola - Cheyletiella parasitivorax (= furmani)

\section{Fam. Ochotonidae}

Ochotona hyperborea yesoensis - Eucheyletiella takahasii

O. hyperborea - Eucheyletiella takahasii

O. pallasi - Eucheyletiella pallasius

O. princeps - Eucheyletiella johnstoni

O. pusillinus - Eucheyletiella pusillinus

O. roylei - Eucheyletiella ochotonae

O. rufescens - Eucheyletiella faini, E. ochotonae

\section{Carnivora}

Fam. Canidae

Canis familiaris - Cheyletiella yasguri (phoretic)

\section{Fam. Felidae}

Felis catus - Cheyletiella blakei, Ch. parasitivorax

\section{Fam. Herpestidae}

Herpestes bocagei - Nihelia curvidens

H. punctulatus - Nihelia curviden

$H$ sanguineus - Nihelia curvidens

H. edwardsii - Nihelia calcarata

\section{Fam. Mustelidae}

Spilogale putorius - Eucheyletia bishoppi

Fam. Procyonidae

Bassariscus astutus - Cheyletus eruditus, Eucheyletia hardyi

Fam. Viverridae

Cynictis penicillata - Nihelia cynictis

\section{Primates}

\section{Fam. Galagonidae}

Galago senegalensis - Galagocheles lawrencei 


\section{G. moholi - Galagocheles lawrencei, G. lemuricola \\ Otolemur garnetti - Galagocheles lemuricola \\ O. crassicaudata monteri - Galagocheles lemuricola}

\section{Fam. Hominidae}

Homo sapiens - Cheyletiella blakei, Ch. parasitivorax

\section{Bibliography of Cheyletidae}

Afzal M., M.A. Ali, R. Akbar. 2003. A new species of the genus Hemicheyletia (Acarina, Cheyletidae) from Chang Manga Forest. - Biologia (Lahore), 49(1-2): 89-91.

Ahadiyat A., H. Ostovan, A. Saboori. 2004. Mites associated with Scolytus amygdali GuerinMeneville, 1847 in Karaj region. Proceeding of 16th Iranian Plant Protection Congress, University of Tabriz. p. 251.

Aheer G.M., S. Akbar, W. M. Chaudhri. 1991. Two new species of genus Acaropsis (Acarina: Cheyletidae) from Pakistan. - Acarologia, 32: 335-340.

Aheer G.M., S. Akbar, W. M. Chaudhri. 1992. Three new species of genus Cheletogenes Oudemans (Acarina: Cheyletidae) from Pakistan. - Acarologia, 33(1): 35-43.

Aheer G.M., S. Akbar, W. M. Chaudhri. 1994. The genus Cheletomimus (Acarina: Cheyletidae). I. Descriptions of three new species. - Acarologia, 35(4): 345-351.

Aheer G.M., S. Akbar, W. M. Chaudhri. 1997. New species of the genera Cheletomorpha and $\operatorname{Ker}$ (Acarina: Cheyletidae) from Pakistan. - Acarologia, 38: 117-121.

Aheer G.M., S. Akbar, W. M. Chaudhri. 1998. New species of the genus Cheletomimus Oudemans (Cheyletidae: Acarina) and a study of phenetic affinities of the species from Pakistan. - Pakistan J. of Zoology, 30(2): 125-132.

Aheer G.M., W. M. Chaudhri. 1987. A new mite species of genus Cheletonella (Acarina: Cheyletidae) from Pakistan. - Journal of Agricultural Research, Pakistan, 25: 103-109.

Akbar S., G.M. Aheer. 1994. Mite fauna of summer vegetables in Punjab. - Pakistan Journal of Zoology, 26: 339-345.

Akbar S., G.M. Aheer, A. Ishtaq. 1993. New predatory mites from summer vegetables at Gujranwala. - Pakistan Journal of Zoology, 25: 293-297.

Akbar S., S. Jahan, M.S. Mughal. 2008. Two new species of the genus Acaropsella Volgin (Prostigmata: Cheyletidae) from Punjab Pakistan. - Acarologia, 48(3-4): 187-190.

Akbar S., M. Sarwar Rahi, W. M. Chaudhri. 1988. Three new mite species of the family Cheyletidae from Pakistan. - Florida Entomologist, 71(1): 1-7.

Akimov I.A., V.T. Gorgol. 1990. [Predatory and parasitic cheyletid mites]. - Naukova Dumka, Kiev: 1-120 (in Russian).

Akpınar, D., Çobanoğlu, S., Öğreten, A. 2017. Traits of Cheyletidae family and their usage possibilities in biological control. Turkish Journal of Agricultural and Natural Sciences, 4: 9-13. Alcaino H., M.T. Calderon. 1976. Cheyletiella yasguri (Acarina: Cheyletidae) en perros de Chile. - Arch. Med. Vet., 8: 40-42. 
Alexander J. O’D. 1972. Mites and skin diseases. - Clin. Med., 79: 14-19.

Amaral V. do. 1971. Ocorrencia do ácaro Cheyletiella parasitivorax (Mégnin 1878)(Acarina

Cheyletidae), en coelho domestico (Oryctolagus cuniculus), no Brasil. - Biológico, Săo Paulo, 37: 106-107.

André H. 1975. Observations sur les Acariens corticoles de Belgique. - Fondation universitaire luxembourgeoise, Série "Notes de recherche", 5-31.

Ardeshir F., M.R. Nematollahi. 2008. Mite fauna on wheat stored and new record of Cunliffella variegata (Barilo, 1985) in Esfahan Province. Proceeding of 18th Iranian Plant Protection Congress; University of Bu-Ali Sina. p. 274.

Ardeshir F. 2017. Cheyletid mites (Acari: Trombidiformes) in stored grains in Iran. - Persian J. Acarology, 6(1): 11-24.

Ardeshir F., D.S. Georges-Gridelet, P. Grootaert, L. Tirry, G. Wauthy. 2000. Preliminary observations on mites associated with stored grains in Iran. - Belgian Journal of Entomology, 2: 287-293.

Arruda G.P. et al. 1969. Ácaros associados a plantas no nordeste do Brasil. - Res. II Reun. An. Soc. Brasil. Entomol., Recife, 35-36.

Artigas J. N., M. E. Casanueva. 1983. Acaras del polvo de las habitaciones en Chile (Acari). - Gayana (Zool.), 47 : 3-106

Asmat G.S.M., Abdul Kader. 1990. A preliminary survey of mites from the nest of certain birds in the Chittagong University campus. - Bangladesh J. Zool., 18(2): 177-183.

Athanassiou Ch. G., N.G. Kavallieratos, N.E. Palyvos, C. Th. Buchelos. 2003. Three - dimensional distribution and sampling indices of insects and mites in horizontally - stored wheat. - Applied Entomology and Zoology, 38(3): 413-426.

Attiah H.H. 1973. Chelachecaropsis bakeri, a new genus and species associated with stored food mites in Egypt (Acarina, Cheyletidae). In: M. Daniel and B. Rosicky (eds), Proceedings of the $3^{\text {th }}$ International Congress of Acarology, Akademia, Prague, pp. 349-352.

Atyeo W. T., J. B. Kethley, T.M. Pérez. 1984. Paedomorphosis in Metacheyletia (Acari: Cheyletidae), with the description of a new species. - Journal of Medical Entomology, 21: 125-131.

Austad R. 1968. Cheyletiella parasitivorax en dyremidd som årsak til klaende utslett hos menneske. - Tidd. Norsdk. Laegforen., 88: 28-30.

Avidov Z., D. Blumberg, U. Gerson. 1968. Cheletogenes ornatus (Acarina: Cheyletidae), a predator of the chaff scale on citrus in Israel. - Israel J. Entomol., 3: 77-94.

Baggio D., J. Croce, F.M. Castro. 1988a. Ácaros do pó domiciliar de Cubatão, São Paulo. - Rev. Bras. Alerg. Imunopatol., 11(5): 178.

Baggio D., J. Croce, F.M. Zuppi, W. Mello. 1988b. Ácaros do pó domiciliar em Luiz Antônio, São Paulo. - Rev Bras Alerg Imunopatol., 11(5): 179.

Baker A.S., J.C. Craven. 2003. Checklist of the mites (Arachnida: Acari) associated with bats (Mammalia: Chiroptera) in the British Isles. - Syst. \& Applied Acarology Special Publications, 14: 1-20.

Baker E. W. 1949. A review of the mites of the family Cheyletidae in the United States National Museum. - Proceedings of the the United States National Museum, 99: 267-320. 
Baker E. W. 1958. Chelacheles strabismus, a new genus and species of mite from Portugal (Acarina, Cheyletidae). - Proceeding of the Entomological Society of Washinglon, 60: 234-235.

Baker K.P. 1969. Infestation of domestic animals with the mite Cheyletiella parasitivorax. - The Veterinary Record, 88: 561.

Bakkers E. J. M., A. Fain. 1972. Dermatitis in man and in a dog caused by the mite Cheyletiella yasguri Smiley. - Brit. J. Dermatol., 87: 245-247.

Baloch Umar Khan. 1991. Taxonomic studies on Cheyletid mites of stored grain and stored products in Pakistan. - Sindh Agriculture Universituy, Tandojam (Hyderabad), Sindh.

Banks N. 1902. New genera and species of acarians. - Canadian Ent., 34: 171-176.

Banks N. 1904. A treatise on the Acarina, or Mites. - Proc. U.S. Nat. Mus., 28(1382): 1-114.

Banks N. 1907. A preliminary list of the Arachnida of Indiana, with keys to families and genera of spiders. - Rep. Indiana Geol. Surv., 31: 715-747.

Banks N. 1915. The Acarina or Mites. - Report No 8, U.S. Dep. of Agriculture.

Barilo A. B. 1985. A new species of the genus Neoeucheyla Radford (Cheyletidae; Prostigmata) from southern regions of Uzbekistan. - Uzbekskii Biol. Zhur., 1985: 45-47 (in Russian).

Barilo A. B. 1986a. New species of stigmaeid and cheyletid mites (Acariformes, Stigmaeidae, Cheyletidae) from Uzbekistan. - Nauchnye Dokl. vyssh. Shk. Biol. Nauki. 1986: 25-29.

Barilo A. B. 1986b. Ker caeterus sp. n. (Prostigmata, Cheyletidae) from Uzbekistan. - Zool. Journal, 65: 298-300 (in Russian with English summary).

Barilo A. B. 1989. New species of mites of the families Raphignathidae, Stigmaeidae, Cheyletidae from Central Asia. - Zool. Journal, 68(10): 134-138 (in Russian with English summary).

Barker P.S. 1992. Bionomics of Nodele calamondin Muma (Acarina: Cheyletidae) fed on Lepidoglyphus destructor (Schrank)(Acarina: Glycyphagidae) at two constant temperatures. - Canadian J. Zool., 70(12): 2333-2337.

Barr A.R. 1955. A case of "mange" of the domestic rabbit due to Cheyletiella parasitivorax (Mégnin). - J. Parasitol., 41: 323.

Bayram S., S. Cobanoglu. 2006. Astigmata and Prostigmata (Acari) of bulbaceous ornamental plants in Ankara, Turkey. - Acta Phytopathologica et Entomologica Hungarica, 41(3-4): 367-381.

Bedford G.A.H. 1932. A synoptic check-list and host-list of the ectoparasites found on South African Mammalia, Aves, and Reptilia. - $18^{\text {th }}$ Rep. Vet. Res. Un. S. Afr., part 1.

Beer R. E., D. R. Dailey. 1956. Biological and systematic studies of two species of cheyletid mites, with a description of a new species (Acarina: Cheyletidae). - Univ. Kan. Sci. Bull., 38(5): 393-437.

Beesley W. N. 1963. Cheyletiella parasitivorax (Acarina: Trombidioidea) as a parasitic mite in Britain. - Parasitology, 53(1): 651-652.

Berlese A. 1882-1903. Acari, Myriopoda et Scorpiones hucusque in Italia reperta. - Padova. Berlese A. 1885. Sopra alcuni Acari. Lettera del dott. Antonio Berlese al dott. G. Haller in Zurigo. - Boll. Soc. Ent. Ital., 17: 145-148. 
Berlese A. 1913. Acari nuovi. - Manipoli VII-VIII. - Redia, 9: 77-111.

Berlese A. 1921. Centuria quinta di Acari nuovi. - Redia, 14: 143-195.

Berlese A., E. Trouessart. 1889. Diagnoses d'Acariens nouveaux ou peu connus. - Bulletin de la Bibliothèque Scientifique de l'Ouest, $2^{\mathrm{e}}$ année, $2^{\mathrm{e}}$ partie, 9: 1-23.

Beron P. 2011. Checklist and Bibliography of the fauna of Acari (Arachnida) in Bulgaria. Nat. Mus. Natur. Hist. Sofia: 130 p.

Beyzavi G.R., H. Ostovan. 2011. New record of a genus and species of Cheyletidae for Iranian mites fauna. - Plant Prot. J., 3(2): 177-183.

Bjarke T., L. Hellgren, K. Orstadius. 1973. Cheyletiella parasitivorax dermatitis in man. - Acta Dermato-venerol., 53: 217-224.

Bochkov A. V. 2002. Classification and phylogeny of mites of the superfamily Cheyletoidea (Acari: Prostigmata). - Entomologicheskoe Obozrenie [In Russian but also translated into English in Entomol. Rev.]. 81: 488-513.

Bochkov, A.V. 2004a. A new finding of Teinocheylus gundii Fain and al., 1982 (Acari: Cheyletidae) from a ctenodactylid rodent (Rodentia: Ctenodactylidae). - Zoosystematica Rossica, 13(1): 4.

Bochkov, A.V. 2004b. [Mites of the family Cheyletidae (Acari: Prostigmata): phylogeny, distribution, evolution and analysis of host-parasite relationships]. - Parazitologiya, 38(2): 122-138. [In Russian with English summary]

Bochkov A. V. 2008. New observations on phylogeny of cheyletoid mites (Acari: Prostigmata: Cheyletoidea). - Trudy Zool. Instituta, 312(1-2): 54-73.

Bochkov A. V. 2009a. A review of mites of the parvorder Eleutherengona (Acariformes: Prostigmata) - permanent parasites of mammals. - Acarina Supplement No 1: 3-149.

Bochkov A. V. 2009b. Origin and evolution of parasitism in mites of the infraorder Eleutherengona (Acari: Prostigmata). Report II. Superfamily Cheyletoidea. - Parazitologiya (St. Petersburg), 43(2): 97-117.

Bochkov A., V.V. Abramov. 2016. To fauna of the free-living Cheyletidae (Acariformes: Cheyletoidea) of the European part of Russia. - Systematic and Applied Acarology, 21(3): 335-346.

Bochkov A. V., A. Fain. 2001. Phylogeny and system of the Cheyletidae (Acari: Prostigmata) with special reference to their host-parasite associations. - Bull. Inst. Roy. Sci. Nat. Belg., Entomologie, 71: 5-36.

Bochkov A. V., A. Fain, F. Ardeshir. 2001. Redescription of Nodele calamondin Muma, 1964 (Acari: Cheyletidae). - Bull. S. R. B. E./ K. B. V. E., 137: 123-126.

Bochkov A. V., A. Fain, J. Dabert. 2002. A revision of the genus Cheletopsis (Acari Cheyletidae). - Bull. Inst. Roy. Sci. Nat. Belg., Entomologie, 72: 5-26.

Bochkov A. V., T.D. Galloway. 2001. Parasitic cheyletoid mites (Acari: Cheyletoidea) associated with passeriform birds (Aves: Passeriformes) in Canada. - Canadian Journal of Zoology, 79: 2014-2028.

Bochkov A. V., T.D. Galloway. 2004. New species and records of Cheyletoid mites (Acari: Cheyletoidea) from birds in Canada. - J. of Kansas Entom. Society, 77(1): 26-44. 
Bochkov A. V., M. Hakimitabar, A. Saboori. 2005. A review of the Iranian Cheyletidae (Acari: Prostigmata). - Belg. J. Entomology, 7: 99-109.

Bochkov A. V., A. A. Haustov [= Khaustov]. 1999a. Prosocheyla villosa sp. n., a new mite species from Crimea (Prostigmata: Cheyletidae). - Genus (Wroclaw), 10(1): 151-154.

Bochkov A. V., A. A. Khaustov. 1999b. Description Cheyletus kuznetzovi sp. n. (Acariformes, Cheyletidae) from Turkmenia. - Zoologicheskiy Zhurnal, 78: 115-116 (In Russian). Also published in English in Entomological Review, 79(4): 482-483.

Bochkov A. V., A. A. Khaustov, N.N. Kuznetsov. 1999. A new mite species ot the genus Chelacheles (Acariformes, Cheyletidae) from the Crimea. - Zoologicheskiy Zhurnal, 78: 117-120 (In Russian). Also published in English in Entomological Review, 79(4): 484-487. Bochkov A.V., P.B. Klimov. 2004. A new predaceous mite Nodele (Aztecocheyletus) conquistador subgen. n., sp. n. (Acari: Cheyletidae) phoretic on Aztecontidium tenochtitlanicum (Hymenoptera: Megachilidae). - Acarina, 12: 23-27.

Bochkov A. V., P.B. Klimov. 2005. Three new species of predaceous Cheyletidae (Acari: Prostigmata) phoretic on insects. - Acarina, 13(1): 15-22.

Bochkov A. V., V. G. Malikov. 1996. Eucheyletiella faini sp. n. (Acari: Cheyletidae), a new species of parasitic mites trom Ochotona rufescens (Gray) (Lagomorpha, Ochotonidae). - Acarina, Russian Journal of Acarology, 4(1-2): 43-48.

Bochkov A. V., S. V. Mironov. 1997. On a taxonomy of predatory mites of the genus Neoeucheyla Radford, 1950 and related genera (Acari: Cheyletidae). - Acarina, Russian Journal of Acarology, 5(1-3): 73-78.

Bochkov A. V., S. V. Mironov. 1998. Samsinakia trilobitus sp. n., a new cheyletid mite from South India (Acari: Cheyletidae). - Entomologische Mitteilungen aus dem Zoologischen Museum Hamburg, 12(157): 265-268.

Bochkov A. V., S. V. Mironov. 1999. A systematic review of the parasitic mite genus Eucheyletiella Volgin, 1969 (Prostigmata: Cheyletidae). - Mitt. Hamb. zool. Mus. Inst., 96: 141-155.

Bochkov A. V., S. V. Mironov, V. T. Gorgol. 1994. Ornithocheyletia phylloscopi sp. n. (Acariformes: Cheyletidae), new species of parasitic mite from the willow warbler. -Acarina, Russian J. of Acarology, 2(1-2): 73-80.

Bochkov A. V., S. V. Mironov, B. M. OConnor. 2006. Revision of the genus Galagocheles Fain (Acari: Cheyletidae), parasites of galagos (Primates: Galagonidae). - Systematic Parasitology, 63(3): 183-202.

Bochkov A. V., R. Ochoa. 2005. Three new cheyletid species (Acari: Cheyletidae) from the National Museum of Natural History entomological collection, USA. - Acarina, 0 13(2): 137-144.

Bochkov A. V., B.M. OConnor. 2003a. New cheyletid mites (Acari, Cheyletidae) associated with birds. - Acta Parasitol., 48: 265-279.

Bochkov A. V., B.M. OConnor. 2003b. Two new species of the genus Cheyletus Latreille, 1796 (Acari: Cheyletidae) associated with Philippine vertebrates. - Internat. J. Acarol., 29(4): $327-330$. 
Bochkov A. V., B.M. OConnor. 2004a. Systematics of the genus Sciurocheyla (Acari, Cheyletidae), parasites of tropical squirrels (Rodentia, Sciuridae). - J. Med. Entomol., 41(3): 308-320.

Bochkov A. V., B.M. OConnor. 2004b. Phylogeny, taxonomy and biology of mites of the genera Chelacheles and Neochelacheles (Acari: Cheyletidae). - Invertebrate Systematics, 18: 547-592.

Bochkov A. V., B.M. OConnor. 2010. Nidocheyletus kennedyae n. gen., n.sp. (Prostigmata: Cheyletidae) from nests of passerine birds in Michigan, U.S.A. - Int. J. Acarology, 36(1): 59-71.

Bochkov A. V., J. Otto. 2010. Five new species of predaceous cheyletid mites (Acari: Prostigmata: Cheyletidae). - Zootaxa, 2727: 1-20.

Bochkov A.V., E. Sidorchuk. 2016. A New Eocene Free-Living Cheyletid Mite from Baltic Amber. - Acta Palaeontologica Polonica, 61(4): 869-874.

Bochkov A.V., M. Skoracki. 2011. A new species of cheyletid mite Metacheyletia ngaii n. sp. (Acariformes: Cheyletidae) from quills of Corythaxoides leucogaster (Musophagidae) from Tanzania. - Acarology, 51: 93-97

Bochkov A.V., M. Skoracki. 2012. New and poorly known predaceous cheyletids (Acariformes: Cheyletidae) from feather quills.- International Journal of Acarology, 68: 486-496.

Boczek J. 1959. Biologia i Ecologia Sierposza Rozkruszkowca (Cheyletus eruditus)(Schrank, 1781)(Acarina, Cheyletidae). - Prace nauk., I. 0. R., 1: 175-230.,

Boström S. et al., 1997. Characterization of the mite fauna (Acari) in Swedish barn dust. Intern. J. Acarology, 23(2): 127-132.

Brandetti E., A. Bischoff-de-Alzuet, J.E. Ledd. 1981. Morfologia y biologia de Ornithocheyletia boeroi sp.n. ectoparasito hematofago de Cianocorax chrysops (Acarina, Cheyletidae). Revista de la Sociedad Entomologica Argentina, 1980, 39(3-4): 235-242.

Bronswijk J. E. M. H. van, L. H. Jansen, A. J. Ophof. 1972. Invasion of a house by the dog parasite Cheyletiella yasguri (Smiley 1965), a mite causing prurigo in Man. - Dermatologica, 145: 338-343.

Bronswijk J. E. M. H. van, E. J. de Kreek. 1976. Cheyletiella (Acari: Cheyletiellidae) of dog, cat and domesticated rabbit, a review. - Journal of Medical Entomology, 13: 315-327.

Brown N. Sandra. 1971. A survey of the Arthropod parasites of pigeons (Columba livia) in Boston. - J. Parasitol., 57(6): 1379-1380.

Bull P.C. 1953. Parasites of the wild rabbit (Oryctolagus cuniculus (L.) in New Zealand. - N.Z. J. Sci. Tech., Ser.B, 34: 341-372.

Buosi R., R. J. F. Feres, A. R. Oliveira, A. C. Lofego, F. A. Hernandes. 2006. Ácaros Plantícolas (Acari) da "Estação Ecológica de Paulo de Faria”, Estado de São Paulo., Brasil. - Biota Neotropica, 6(1) 1-20.

Caceres I., A. Fain. 1975. Notes sur les Acariens des denrées alimentaires du Perou. - Bull. Ann. Soc. r. belge Ent., 111: 143-150.

Caceres I., A. Fain. 1978. Notes sur la faune acarologique des poussières de maison du Pérou. - Proc. of the 4th Intern. Congr. of Acarology, 1974: 259-261.

Caceres I., A. Fain. 1978. Notes sur la faune acarologique des poussières de maisons du Pérou. - Bull. Ann. Soc. r. belge Ent., 114: 301-303. 
Canestrini G. 1885. Prospetto dell'Acarofauna italiana. Part 2. Famiglia dei Cheyletini. - Atti Red. Inst. Veneto, Ser.6, 3: 1656-1666, pl. XIX, fig.3.

Canestrini G. 1886. Prospetto dell'Acarofauna italiana. Famiglie: Erythraeini, Cheyletini, Bdellini, Eupodini e Analgesini. Atti del reale Ist. Veneto di scienze, lettere ed arti, ser. 6: 159-311.

Canestrini G. 1892. Prospetto dell'acarofauna italiana. Parte V.a. Intorno alla metamorfosi degli Ixodini (nota). Abbozzo del sistema acarologico. Famiglia dei Phytoptini (Phytoptidae). - pp. 544-722. Padova. (Prosperini).

Canestrini G., F. Fanzago. 1876. Nuovi acari italiani (ser.2). - Atti Soc.Veneto-Trentina Sci. Nat. 5 (fasc. 1) : 99-111.

Carroll H. F., J. H. Theis. 1973. Cheyletiella mite dermatitis: a review. - J. Amer. Anim. Host. Assoc., 9: 573-576.

Casanueva M.E., J.N. Artigas. 1985. Distribución geográfica y estacional de los ácaros del polvo de habitación en Chile (Arthropoda: Acari). - Gayana Zoología, 49:3-76.

Chartet L.D., M.S.Mulla, M.Sanchez-Medina.1978. Domestic Acari of Colombia: occurrence and distribution of Acari in house dust. - Acarologia, 19(2): 302-317.

Chaudry W.M., S. Akbar, A. Rasool. 1979. Studies on the Predatory Leaf Inhabiting Mites of Pakistan. Project No. PK-ARS-30. University of Agriculture, Faisalabad, Pakistan, 234 pp.

Chaudry W.M., S. Akbar. 1985. Studies on the Biosystematics and Control of Mites of Field Crops, Vegetables and Fruit Plants in Pakistan. University of Agriculture, Faisalabad, Pakistan, 314 pp.

Chernichko K.I., D.A. Kivganov. 2013. Review of Cheyletid mites of the genus Cheletopsis (Acari: Cheyletidae) from the quills of waders in Southern Ukraine, with description of a new species. - Vestnik zoologii, 47(1): 82-86.

Chirov P. A., A. V. Bochkov. 1998. Ornitocheyletia mironovi sp. n. (Acari: Cheyletidae) a new species of parasitic mites of the bank swallow from Kirghizia. - Acarina, Russian J. of Acarology, 6(1-2): 35-36.

Clark J.D., Hyong-Sun Ah. 1976. Cheyletiella parasitivorax (Megnin) a parasitic mite causing mange in the domestic rabbit. - J. Parasitol., 62(1): 125.

Clarke M. L. 1958. Cheyletiella parasitivorax infestation in the cat. - Vet. Rec., 70: 502.

Cockerell T.D.A. 1917. Arthropods in Burmese amber. - Psyche, 24: 40-45.

Cooper K. W. 1946. The occurrence of the mite Cheyletiella parasitivorax (Mégnin) in North America with notes on its synonymy and "parasitic" habit. - J. Parasitol., 32 (5): 480-482.

Cooreman J. 1946. A propos de Cheletomorpha lepidopterorum (Shaw, 1794) (Acarien, Cheyletidae). - Lambillionea, 46(4-5): 70-71.

Cooreman J. 1951. Sur quelques Acariens vivant parmi les colonies de Coccides au Maroc. Revue de Pathologie végétale et d'Entomologie agricole de France, 30(1): 30-34.

Corpuz-Raros L. A. 1972. Systematic studies of Philippine cheyletid mites. I. Preliminary report of species mainly from Laguna. -The Philippine Entomologist, 2: 247-271.

Corpuz-Raros L. A. 1980. Systematic studies of Philippine cheyletid mites (Acarina). III. Polycheyletus batangenius (Corpuz-Raros), comb. nov. - Kalikasan, Philippine Journal of Biology, 9: 61-68. 
Corpuz-Raros L. A. 1988a. Systematic studies of Philippine cheyletid mites (Acarina, Cheyletidae). IV. The genus Cheyletus Latreille. - Philippine Journal of Science, 117: 327-341.

Corpuz-Raros L. A. 1988b. Systematic studies of Philippine cheyletid mites (Acarina). V. New species and new records, with a note on the synonymy of Tutacheyla Corpuz-Raros. Philippine Journal of Science, 117: 413-427.

Corpuz-Raros L. A. 1998. Twelve new species and one new record of Cheyletidae (Acari) from the Philippines. - International Journal of Acarology, 24: 259-290.

Corpuz-Raros L.A. 2000a. Two new species and a new record of Bak from the Philippines (Acari: Cheyletidae). - Int. J. Acarol., 26(4): 321-328.

Corpuz-Raros L.A. 2000b. Guide to Philippines predatory mites: Family Cheyletidae Leach. - UPLB Museum Publications in Natural History, The Museum of Natural History, University of the Philippines, Los Baños College, Laguna, Philippines, 2: 1-80.

Corpuz-Raros L. A., J.C.B. Naredo. 2016. Lanceacheyla filipina, a new species of cave-inhabiting mite from the Philippines (Acari: Cheyletidae: Cheyletinae). - Philipp. Ent., 30(1): 1-9.

Corpuz-Raros L. A., J. M. Sotto. 1977. Systematic studies of Philippine cheyletid mites (Acarina, Cheyletidae). II. New species and new records. - Kalikasan, Philippine Journal of Biology, 6: 143-170.

Cruz J. de la. 1971. Nuevos reportes de ectoparasitos de los animales de laboratorio de Cuba.

- Revista Cub. Cienc. Vet., 2: 101-102.

Cuervo N., F. Dusbábek, J. de la Cruz, R. Abreu. 1983. Los acaros (Acarina: Pyrogliphidae, Cheyletidae, Saproglyphidae y Glycyphagidae) de los polvos domesticos en Cuba. - Rev. Cub. Med. Trop., 35: 83-103.

Cunliffe F. 1955. A proposed classification of the Trombidiforme mites (Acarina). - Proc. Ent. Soc. Washington, 57: 209-218.

Cunliffe F. 1962. New species of Cheyletidae (Acarina). - Proc. Ent. Soc. Washington, 64(3): 197-202.

Curtis C.F. 2004. Current trends in the treatment of Sarcoptes, Cheyletiella and Otodectes mite infestations in dogs and cats. - Vet. Dermatol., 15(2): 108-114.

Cwilich R., A. Hadani. 1965. Infestation of chicks with mites of the genera Haemolaelaps and Cheyletus. - Refuah Veterinarith, Israel, 22(1): 55-54.

Çobanoğlu S., C. Cilbircioğlu, S. Zabludovskaya. 2020. New record of predatory mite (Prostigmata: Ereynetidae) with some cheyletid mites (Acari: Cheyletidae) from garlic agrosystems in Turkey. - Int. J. Acarol., 46(2): 111-116.

Da Silva Ezequiel O., G.S. Gazeta, M. Amorim, N.M. Serra-Freire. 2001. Evaluation of the acarofauna of the domiciliary ecosystem in Juiz de Fora, State of Minas Gerais, Brazil. - . Memórias do Instituto Oswaldo Cruz, 96(7): 911-916.

Davies J. H. T. 1941. Cat itch: Cheyletiella and Notoedres compared. - Brit. J. Dermatol., 53: 18-24. Davies J. H. T., M.G. Kyle. 1969. Cheyletiella yasguri infestation of a dog. - N.Z. Vet. J., 17: 136. De Geer C. 1778. Mémoires pour servir à l'histoire des Insectes. Vol.7, Stockholm.

De Leon D. 1962. Three new genera and seven new species of Cheyletids (Acarina: Cheyletidae). - The Florida Entomologist, 45(3): 129-137.

De Leon D. 1967. Some Mites of the Caribbean Area. - Lawrence, Kansas: Allen Press, Inc., 60 p. 
Delfinado M. D., A. A. Khaing - Fields. 1976. Terrestrial mites of New York (Acarina). IV. Cheyletidae and Cheyletiellidae. - J. New York Entomological Society, 84(3): 189-196.

Di Palma A., G. Nuzzaci, G. Alberti. 2009. Morphological, ultrastructural and functional adaptations of the mouthparts in Cheyletid mites (Acari: Actinedida: Cheyletidae). Internat. J. of Acarology, 35(6): 521-532.

Diumina A.V., V.V. Abramov. 2018. Redescription of Mexecheles virginianiensis male (Acariformes: Cheyletidae) from the European part of Russia. - Acarina, 26(2): 213-217.

Dodd K. 1970. Cheyletiella yasguri: Widespread infestation in a breeding kennel. - Vet. Rec., 86: 346-347.

Doğan S., N. Ayyildiz. 2004. The first records of two cheyletid mites from Turkey: Chelotomimus (Hemicheyletia) wellsi (Baker, 1949) and Hypopicheyla elongata Volgin, 1969 (Acari: Cheyletidae). - Zootaxa, 583: 1-4.

Doğan S., S. Doğan, O. Erman. 2019. Eutogenes frater Volgin (): a new member of the acarofauna of Turkey. - Türk Tarım ve Doğa Bilimleri Dergisi, 6(4): 685-695.

Doğan S., M. Jalaeian, H. Kamali. 2011. New records of two cheyletid mite species (Acari: Cheyletidae) from Iran. - Turk J. Zool., 35(5): 781-782.

Doğan S., B. Kabasakal, S. Doğan. 2019. A long-legged mite species from Turkey: Cheletomorpha lepidopterorum (Shaw) (Acari: Cheyletidae). - Erzincan Binali Iildirim University, Journal of Science and Technology, 12(1): 136-147

Domrow R. 1960. The genus Chelonotus Berlese (Acarina, Cheyletidae). - Acarologia, 2: $456-460$.

Domrow R. 1964. The ear mite of squirrels. - The Malayan Nature Journal, 18: 16-19.

Domrow R. 1965. Some mite parasites of Australian birds. - Proceeding of the Entomological Society of Washinglon, 90: 190-217.

Domrow R. 1970. The male of Neocheyletiella artami Domrow (Acari: Cheyletidae). - Proc. Linnean Soc. New South Wales, 94(3): 273-276.

Domrow R. 1974. Miscellaneous mites from Australian vertebrates. - Proc. Linnean Soc. New South Wales, 99(1): 15-35.

Domrow R. 1991. Acari Prostigmata (excluding Trombiculidae) Parasitic on Australian Vertebrates: an Annotated Checklist, Keys and Bibliography. - Invertebr. Taxon., 4: 1283-1376.

Domrow R., E. W. Baker. 1960. Malaysian Parasites XLIV. A new genus of mites from a Thai mongoose (Acarina, Cheyletidae). - Studies of the Institute of Medical Research of Malaya, 29: 194-197.

Domrow R., E. W. Baker. 1963. The genus Nihelia (Acarina, Cheyletidae). - Acarologia, 5: 225-231.

Domrow R., D.J.W. Smith. 1956. Acarina from five hundred native mammals from Queensland. - Proc. Linnean Soc. of New South Wales, 29?(3): 201-206.

Driunina A.V., V.V. Abramov. 2018. Redescription of Mexecheles virginianus male (Acariformes: Cheyletidae) from the European part of Russia. - Acarina, 26(2): 213-217.

Dubinin V.B. 1957. [New system of the superclass Chelicerata]. - Bulletin de la Société des Naturalistes de Moscow, Biologie, 62: 25-33. 
Dubinin V.B. 1958. New classification of mites of the super-families Cheyletoidea W. Dub. and Demodicoidea W.Dub. (Acariformes, Trombidiformes). - Parasitol. Sborn. Zool. Inst. Akad. Nauk SSSR Leningrad, 17: 71-136 (in Russian).

[Dubinina E.V., A.V. Bochkov] Дубинина Е. B., А.В. Бочков. 1989. [New data concerning the ectoparasites of the big-eared Ochotona in Kyrghyzstan]. - Entomological Research in Kyrghyzstan, 20: 107-116 (in Russian).

Dudich E., G. Kolosváry, L. Szalay. 1940. Bars vármegye pókszabású (Arachnoidea-) faunájának alapvetése. - Budapest, 71 p.

Dufait R. 1968. Cheyletiella parasitivorax bij honden. - Vlaams Diergeheesk., 37: 162-163.

Edwards A.R. 1952. Support for the view that Cheyletus eruditus Schrank (Acarina, Trombidiformes) has heteromorphic males. - Ent. Mon. Mag., 88: 107.

Edwards A.R. 1958. Cleavage in Cheyletus eruditus (Acarina). - Nature, London (4620), 181: 1409-1410.

Efremova G.A., A.V. Bochkov. 1990. The mites of families Cheyletidae and Cheyletiellidae (Acariformes: Trombidiformes) of the Belorussian fauna. - Vesti AN BSSR, 6: 98-103 (in Belorussian, summ. Engl.).

Ehara S. 1962. Mites of greenhouse plants in Hokkaido, with a new species of Cheyletidae. Annotationes Zoologicae Japonenses, 35: 106-111.

Ehara S., I. Abdul Ghani. 1988. Cheyletid mites associated with plants in the Malay Peninsula, with description of a new species (Acarina: Cheyletidae). - Proceedings of the Japan Academy, 64, ser. B: 237-240.

Elbadry E. 1969. Two new species of cheyletid mites from milled wheat (Acarina: Cheyletidae). - Journal of Stored Products Research, 5: 157-167.

Elbadry E., M. A. Zaher. 1960. First record on the predatory mites of family Cheyletidae in Egypt (Acarina). - Bull. Ent. Soc. Egypte, 44: 287-290.

El-Bahrawy A.A., M.A. al-Dakhil. 1993. Studies on the interrelation between rodents and their ectoparasitic acarines in Riyadh region, Saudi Arabia. - J. Egypt Soc. Parasitol., 23: 675-685.

Eliopoulos P.A., G.T. Papadoulis. 2001. New records of mites (Acari: Cheyletidae) from stored products with description of a new species in Greece. - Int. J. Acarology, 27(1): 29-33.

Enghoff H. 1988. The moth mite Cheletomorpha lepidopterorum (Shaw, 1794) new to Denmark. - Entomol. Medd., 56(1): 49-52.

Estébanes - González M.L. 1997. Acarofauna en nidos de Aves silvestres en Mexico. - Acta zool. Mex.(n.s.), 71: 1-15.

Evans G. O. 1954. Some new and rare species of Acarina. - Proc. Zool. Soc. London, 123: 793-811.

Ewing H. E. 1909a. New North American Acarina. - Trans. Ac. Sci. of St. Louis, 18: 53-77.

Ewing H. E. 1909b. A Systematic and Biological Study of the Acarina of Illinois. - University of Illinois. The University Studies, 3(6): 119 pp.

Ewing H. E. 1912. The life history and habits of Cheyletus seminivorus Packard. - J. econ. Entomol., 5: 415-420. 
Ewing S. A., J. E. Mosier, T. S. Fox. 1967. The occurrence of Cheyletiella spp. on dogs with skin lesions. - J. Am. Vet. Med. Ass., 151: 64-67.

Eyndhoven G. L. van. 1964. Cheletomorpha lepidopterorum (Shaw, 1794) (= Ch. venustissima) (Acari, Cheyletidae) on Lepidoptera. - Beaufortia, 11 (136): 53-60.

Eyndhoven G. L. van. 1970. Further notes on Cheletomorpha lepidopterorum (Acari: Cheyletidae), transported by Lepidoptera. - Entomol. Berichten, 30: 24-25.

Ezequiel O. da Silva, G. Salles Gazêta, M. Amorim, N. Maués Serra-Freire. 2001.

Evaluation of the Acarofauna of the domiciliary ecosystem in Juiz de Fora, State of Minas Gerais, Brazil. - Mem. Inst. Oswaldo Cruz, Rio de Janeiro, 96(7): 911-916.

Ezequiel O. da Silva, G. S. Gazêta, N. M.da Serra Freire. 2003. Dermatological manifestations caused by mites of the Cheyletidae family: a case report. - Anais Bras. Dermatol., 78(1):

Fain A. 1972. Notes sur les acariens des famillies Cheyletidae et Harpyrhynchidae producteurs de gale chez les oiseaux ou les mammifères. - Acta Zoologica et Pathologica Antverpiensia, 56: 37-60.

Fain A. 1974. Teinocheylus longissimus n.g., n.sp. a new fur mite from Pectinator spekei (Cheyletidae: Trombidiformes). - Acarologia, 16: 271-273.

Fain A. 1979a. Cheyletidae (Acari, Prostigmata) parasitic on Afrotropical primates, Carnivora and rodents. - Revue de Zoologie africaine, 93: 621-632.

Fain A. 1979b. New Cheyletidae from Afrotropical swifts (Apodidae). - International Journal of Acarology, 5: 253-258.

Fain A. 1979c. Acariens du genre Cheyletus (Prostigmata: Cheyletidae) récoltés dans la Région afrotropicale. - International Journal of Acarology, 5: 275-284.

Fain A. 1979d. Idiosomal and leg chaetotaxy in the Cheyletidae. - International Journal of Acarology, 5: 305-310.

Fain A. 1979e. Notes on the genera Cheletoides Oudemans and Metacheletoides Fain (Acarina, Cheyletidae) with description of three new species. - Revue de Zoologie africaine, 93: 1011-1025.

Fain A. 1979f. Observations on cheyletid mites parasitic on mammals (Acari, Cheyletidae and Cheyletiellidae). - Acarologia, 21: 408-422.

Fain A. 1980a. Notes on some poorly known species of the genus Neocheyletiella Baker, 1949 (Acari: Cheyletidae) with a key to the genus. - Systematic Parasitology, 2: 25-39.

Fain A. 1980b. Le genre Bakericheyla Volgin (Acari, Cheyletidae) dans la Région Afrotropicale. Description d'une espèce nouvelle. - Revue de Zoologie africaine, 94(1): 133-137.

Fain A. 1980c. Notes on genera Samsinakia Volgin, 1965 and Metacheyletia Fain, 1972 (Acari: Cheyletidae). - International Journal of Acarology, 6: 103-108.

Fain A. 1981. Revision of the genus Ornithocheyletia Volgin, 1964 (Acari: Cheyletidae). Systematic Parasitology, 2: 181-205.

Fain A. 1982. Three new species of Cheyletus from Afrotropical region and Madagascar (Acari, Cheyletidae). - Revue de Zoologie africaine, 96: 79-89.

Fain A. 1984. Samsinakia gonocephalum n. sp., a new cheyletid mite from an Afrotropical beetle Gonocephalum simplex Fab. (Acari, Cheyletidae). - Revue de Zoologie africaine, 98: 684-688. 
Fain A. 2003. Two new species of Cheyletidae (Acari Prostigmata) of the genera Neoeucheyla Radford, 1950 and Granulocheyletus Fain \& Bochkov, 2002. - Bulletin S.R.B.E./K.B.V.E., 139: 97-101.

Fain A., F. Ardeshir. 2000. Notes on the genus Neoeucheyla Radford, 1950 (Acari : Cheyletidae) with description of a new species from Iran. - Int. J. of Acarology, 26(4): 329-334.

Fain A., A. Bochkov. 2001a. A review of the genus Cheyletus Latreille, 1776 (Acari: Cheyletidae). - Bull. Inst. Roy. Sci. Nat. Belg., Entomologie, 71: 83-114.

Fain A., A. Bochkov. 2001b. Observations on the taxonomic status of some cheyletid genera (Acari Cheyletidae). - Belgian Journal of Entomology, 3: 291-301.

Fain A., A. Bochkov. 2001c. A review of some genera of cheyletid mites (Acari: Prostigmata) with descriptions of new species. - Acarina, 9(1): 47-95.

Fain A., A. Bochkov. 2002a. On some new or little known species of parasitic Cheyletidae (Acari : Prostigmata). - Acarologia, 42(2): 145-160.

Fain A., A. Bochkov. 2002b. A new genus and species of Cheyletid mite (Acari : Cheyletidae) from a cave in Western Australia. - Internat. J. Acarol., 28(1): 37-40.

Fain A., A. Bochkov. 2003. A new species of the genus Metacheyletia Fain, 1972 (Acari: Cheyletidae) parasitizing Serinus mozambicus (Passeriformes: Fringillidae) in Central Africa. - Internat. J. Acarol., 29(2): 119-121.

Fain A., A. Bochkov, L.A. Corpuz-Raros. 2002. A revision of the Hemicheyletia generic group (Acari: Cheyletidae). - Bull. Inst. Roy. Sci. Nat. de Belgique, Entomologie, 72: 27-66.

Fain A., B. Feldman-Muhsam, Y. Mumcuoglu. 1980. Cheyletus tenuipilis n.sp. (Acari: Cheyletidae), nouvel acarien des poussières de maisons en Europe occidentale et en Israël. Bulletin et Annales de la Société royale belge d'Entomologie, 116: 35-44.

Fain A., P. Gerrits, F. S. Lukoschus. 1982. Teinocheylus gundii spec. nov. from Ctenodactylus gundi (Acari, Cheyletidae). - Revue de Zoologie africaine, 96: 448-456.

Fain A., F. S. Lukoschus. 1981a. Two new species of the genus Cheyletus Latreille, 1976 (Acari, Cheyletidae) from South African gerbils. - Bulletin de I'lnstitut royal des Sciences naturelles de Belgium, 53: 1-7.

Fain A., F. S. Lukoschus. 1981b. Two new species of Cheyletus Latreille, 1796 (Acari, Cheyletidae, Prostigmata) from gerbils. - Rivista di Parassitologia. 42: 121-125.

Fain A., F. S. Lukoschus. 1985. Description of a new species in the genus Criokeron Volgin, 1966 and of the male of Criokeron quintus (Domrow and Baker) (Acari: Cheyletidae). - Acarologia. 26: 261-268.

Fain A., F. S. Lukoschus, M. Nadchatram. 1980. Two new species of Cheletophyes Oudemans, 1914 (Prostigmata: Cheyletidae) from the nest of a carpenter bee in Malaysia.- International Journal of Acarology, 6: 309-312.

Fain A., M. Nadchatram. 1980. Cheyletid parasites of commensals in Malaysia (Acari: Cheyletidae). - International Journal of Acarology, 6: 191-200.

Fain A., L. Scheepers, W. De Groot. 1982. Dermatite prurigineuse de longue durée chez une femme, produite par l'acarien parasite du chien Cheyletiella yasguri Smiley. - Rev. Méd. Liège, 37(18): 623-624. 
Fain A., R. L. Smiley, U. Gerson. 1997. New observations on the chaetotaxy and solenidiotaxy in the Cheyletidae (Acari: Prostigmata). - Bulletin de I'Institut royal des Sciences naturelles de Belgique, 67: 65-87.

Fang Lin, Qian Kaiming, Gao Cheng. 1991. The report on rabbit cheyletid fur mite and rabbit listrophorid fur mite. - J. Shanghai Agricult. College, 9(2): 126-128.

Favati N., J. Galliano. 1962. Infestione da Cheyletiella parasitivorax (Megnin, 1878) nel cane. - Parassitologia, 4: 69-73.n

Feldman-Muhsam B., Y. Mumcuoglu, T. Osterovich. 1985. A survey of house dust mites (Acari, Pyroglyphidae and Cheyletidae). - J. Med. Entom., 22: 663-669.

Feres R.J.F., C.H.W. Flechtmann. 1995. Mites (Acari) associated with bamboo (Bambusa sp., Poaceae) in a wood area from northwestern Sao Paulo State, Brazil. - Rev. Bras. Zool., 12(3): 533-546.

Flechtmann C. H. W. 1968. Nota sôbre ácaros de produtos armazenados. - Solo, Piracicaba, 40(1): 63-65.

Flechtmann C. H. W. 1971. Alguns Trombidiformes do Brasil e do Paraguai (Acari). Escola Superior de Agricultura «Luiz de Queiroz», Universidade de Sao Paolo, Piracicaba, Brasil, 63 pp.

Foxx T. S., S. A. Ewing. 1969. Morphologic features, behavior and life history of Cheyletiella yasguri. - Am. J. Vet. Res., 302: 269-284.

Franz H. 1954. Ordnung Acarina. - In: H. Franz. Die Nordost-Alpen im Spiegel ihrer Landtierwelt. Universit. Verlag Wagner, Innsbruck: 329-452.

Fuangarworn M., Ch. Lekprayoon. 2010. Two new species of cheyletid mites (Acari: Prostigmata) from Thailand. - Zootaxa, 2494: 59-68.

Furman D. P., O. E. Sousa. 1968. Morphology and biology of a nest producing mite, Bakericheyla chanayi (Acarina: Cheyletidae). - Ann. Ent. Soc. Amer., 62(4): 858-863.

Garman P. 1948. Mite species from apple trees in Connecticut. - Conn. Agric. Exp. Sta. New Haven Bull., 520: 1-27.

Garrett L.E., F.H. Haramoto. 1967. A catalogue of Hawaiian Acarina. - Proc. Hawaii. Ent. Soc., 19, 1966: 381-414.

George J.B.D., S. Otobo, J. Ogunleye, B. Adediminiyi. 1992. Louse and mite infestation in domestic animals in northern Nigeria. - Tropical Animal Health and Production, 24(2): 121-124.

Gerson U. 1967. Some Cheyletid and Pseudocheylid mites from Israel. - Acarologia, 9 (2): 359-369.

Gerson U. 1968. Caudacheles, a new genus in the family Cheyletidae (Acarina: Prostigmata). - Acarologia, 4 (10): 645-649.

Gerson U. 1971. The mites associated with citrus in Israel. - Israel J. Entomol., 6: 5-22.

Gerson U. 1994. The Australian Cheyletidae (Acari: Prostigmata). - Invertebrate Taxonomy, 8: $435-447$.

Gerson U., A. Fain. 1991. A new species of Bak (Acari: Cheyletidae) from Thailand, with a key to species. - Acarologia, 32(1): 17-21.

Gerson U., A. Fain, R. L. Smiley. 1999. Further observations on the Cheyletidae (Acari), with a key to the genera of the Cheyletinae and a list of all known species in the family. - Bull. Inst. r. Sci. Nat. Belg. Ent., 69: 35-86. 
Gerson U., R. L. Smiley. 1990. Acarine Biocontrol Agents: An Illustrated Key and Manual. Chapman and Hall, pp. 174.

Gething M.A. 1973. Cheyletiella infestations in small animals. - Vet. Bull., 43(2): 63-69.

Goff L. M. 1982. A new species of Hemicheyletia (Acari: Cheyletidae) from Kure Atoll, northwestern Hawaiian Islands. - Proceedings of the Hawaiian Entomological Society, 24: 83-86.

Goff L. M. 1983. Mites from Kahoolawe I. soil and litter./ Ornithocheyletia leiothrix Fain and Neocheyletiella media Fain. / Dematophagoides evansi Fain. - Proceedings, Hawaiian Entomological Society, 24(2/3): 157-158.

Goff M. L. 1987. A Catalog of Acari of the Hawaiian Islands. - Research Extension Series 075, 75 pp.

Gorgol V.T., V.I. Petrova. 1989. New data about predation of mite Cheletomorpha lepidopterorum. - Proc. Latvian Acad. Sci., 8, 505: 105-108.

Gorgol V.T., A.V. Yastrebtsov. 1989. Skeleto-muscular system in some cheyletid mites (Trombidiformes: Cheyletidae). - Vestn. Zool., 1989(4): 74-81.

Griffiths D.A. 1960. Some field habitats of mites of stored food products. - Ann. Appl. Biol., 48(1): 134-144. doi:10.1111/j.1744-7348.1960.tb03512.x

Gueye-N’Diaye A., A. Fain. 1987. Note sur les acariens des denrées alimentaires au Sénégal. - Revue Zool. afr., 101: 365-370.

Guilhon J., Marchand. 1974. Une nouvelle espèce d'acarien pellicole en France. - C.r. hebd. Séanc. Acad. Sci. Paris (Sér.D), 278(1): 73-74.

Guilhon J., J. Euzeby. 1984. A new species of Cheyletidae parasite of cage-birds. - Bull. Acad. Vet. de France, 57(4): 495-500.

Gupta S. K. 1991. Studies on predatory prostigmatid mites of northeast India with descriptions of new species and new records from India. - Records of the Zoolological Survey of India, 88: 207-239.

Gupta S.K. 2002. Memoirs of the Zoological Survey of India: Volume 19: Number 2: A Monograph on Plant Inhabiting Predatory Mites of India: Part I: Orders: Prostigmata, Astigmata and Cryptostigmata. 183 pp.

Gupta S.K., S. Chattopadhyay. 1978. Studies on acari associated with bird nests in Bengal, India. - Indian J. Acarol., 3: 77-86.

Gupta S.K., S.K. Ghosh. 1980. Some prostigmatid mites (Acarina) from Andaman and Nicobar Islands. - Record zool. Survey of India, 77(1-4): 189-213.

Gupta S. K., K. Paul. 1985. Some mites associated with birds' nests in West Bengal, with descriptions of eleven new species. - Bull. Zool. Survey of India, 7(1) : 1-23

Gupta S.K., K. Paul. 1987. Some mites associated with birds' nests in West Bengal, with descriptions of eleven new species. - Bulletin of the Zoological Survey of India, 7: 1-23.

Gupta S. K., K. Paul. 1992. Nest associated acarines of India with descriptions of seven new species and notes on other arthropod associates. - Entomon, 17: 71-86.

Haarløv N. 1942. A Morphologic-Systematic-Ecological Investigation of Acarina. - Meddel. Grønland, 128(1): 1-71. 
Haarløv N., J. Mørch. 1975. Interaction between Ornitocheyletia hallae Smiley 1970 (Acarina: Cheyletiellidae) and Micromonospora chalcea (Foulerton 1905) Orskov 1923 (Streptomycetaceae, Actinomycetales) in the skin pigeon. - Acarologia, 17 (2): 284-299.

Hafiz A. 1935. The embryological development of Cheyletus eruditus. - Proc. Roy. Soc. London Ser. B 117: 174-201.

Haghighi R., H. Ostovan. 2011. The first report of Caudacheles khayae (Acari: Cheyletidae) associated with elm bark beetles in Iran. - Plant protection J., 3(1): 79-84.

Haines C. P. 1988. A new species of predatory mite (Acarina: Cheyletidae) associated with bostrichid beetles on dried cassava. - Acarologia, 29(4): 361-375.

Haitlinger R. 1982. Acarina (Myobiidae, Cheyletidae, Pygmephoridae, Trombiculidae, Dermanyssidae) nowe lub rzadkie w faunie Polski. - Wiadomości Parazytol., 28(3-4): 435-444 (in Polish, summ. Engl.).

Haitlinger R. 2000. Cheyletophyes aurorae n.sp. (Acari: Prostigmata: Cheyletidae) from Xylocopinae (Hymenoptera: Anthophoridae) in Tanzania. - Zeszyty naukowe Akademii rolniczej we Wroclawiu, Zootechnika XLVII, 400: 85-89.

Haitlinger R., A. Jankowska. 2005. Arthropods occuring on Rattus norvegicus (Berkenhout, 1769)(Rodentia: Muridae) in Poland. - Zeszyty naukowe Akademii rolniczej we Wroclawiu, 529, Zootechnika LIII: 35-44.

Haitlinger R., G. Walter. 1997. Data relating to the distribution and host-specifity of bat-infesting mites (Acari, Mesostigmata, Prostigmata, Astigmata) in Germany. - Drosera, '97(2): 95-112.

Hajizadeh J., J. Noei, L. Salehi, H. Ostovan. 2011. Cheyletid mites associated with stored rice in Iran, the first record of Chelacheles strabismus from Iran and a key for their identification. - J. Entomol. Soc. Iran, 30(2): 85-88.

Haq I., M. Afzal. 2007. Mites associated with stored grains and their products in Faisalabad district. - African Crop Science Conference Proceedings, 8, 2205-2207.

Haq A., A.K. Khan, A.B. Riaz, A. B. Rasool. 2004. Studies on the taxonomy of two new species of genus Acaropsis Moquin-Tandon (Acarina: Cheyletidae) from Pakistan along with an old species with new host and locality. - Journal of Agricultural Research, 42: 181-190.

Haq A., M.A. Zia. 2001a. Two new species of genus Acaropsis Moquin-Tandon (Acarina: Cheyletidae) from Pakistan. - Journal of Agricultural Research, 39: 59-66.

Haq A., M.A. Zia. 2001b. Taxonomic studies of two new species of genus Acaropsis MoquinTandon (Acarina: Cheyletidae) from Pakistan. Journal of Agricultural Research, 39: 67-75.

Hallas Th. E. 1978. Fortegnelse over danske mider (Acari). - Ent. Meddr., 46: 27-45.

Haller G. 1882. Beitrag zur Kenntniss der Milbenfauna Württembergs. - Jh. Ver. Vaterl. Naturk. Würtemberg, 38: 293-325.

Haller G. 1884. Beschreibung einiger neuen Milben. - Arch. f. Naturgesch., 50(2): 217-235.

Halliday B. 1998. Mites of Australia. A Checklist and Bibliography. - Monographs on Invertebrate Taxonomy. Vol.5. CSIRO: 317 pp.

Halliday B., M. Kamran, M.H. Bashir. 2018. Checklist of the mites of Pakistan. - Zootaxa, 4464: 178 pp. 
Hara J. 1955. A new species of the Cheyletid mite found on tatami, Japanese rice straw matting. - Japan J. Exp. Med., 25: 69-70.

Hara J., M. Hanada. 1960. On a newly recorded mite, Eutogenes narashinoensis new species, from Japan (Acarina: Cheyletidae). - Jap. J. San. Zool., 11: 25-27.

Harju A., T. Husman, R. Merikoski, S. Pennanen. 2006. Exposure of workers to mites in Finnish groceries. - Ann. Agric. Environ. Med., 13: 341-344.

Hart C.B., J.C. Malone. 1958. The occurrence of the rabbit fur mite, Cheyletiella parasitivorax (Mégnin, 1878), on the dog. - Ver. Rec., Lond., 70: 991-993.

Hassan M. F., E. A. Gomaa. 1982. A new species of the genus Chelacheles from Egypt (Acarina: Cheyletidae). - Bull. of the Zoological Society of Egypt, 31: 115-117.

Hassan M. F., M.A. Rakha. 1982. Cheyletid mites inhabiting rat burrows in Egypt, with description of new species Cheyletus zaheri (Actinedida: Cheyletidae). - Bulletin of the Zoological Society of Egypt, 31: 87-90.

Heller A. 1880. Die Schmarotzer mit besonderer Berücksichtigung der für Menschen wichtigen - Die Naturkrafte, 30: 186-188.

Hessling Th. von. 1852. Einige Notizen über den Weichselzopf. - Illustr. Med. Zeit., 1(5): 255-259.

Hirst S. 1914. Preliminary list of the Acari occuring on the brown rat (Mus norvegicus) in Great Britain, with the description of a new species (Haemogamasus oudemansi). - Bull. Ent. Res., 5(2): 119-124.

Hirst S. 1917. On the occurrence of the pseudoparasitic mite Cheyletiella parasitivorax (Mégnin) on the domestic cat. - Ann. Nat. Hist. Ser.8, 20: 132-133.

Hoffmann A., F.A. Cervantes, J.B. Morales-Malacara. 1994. Ectoparasitos del conejo zacatuche (Romerolagus diazi). - Anales Inst. Biol. Univ. Nac. Autón. México, Ser. Zool., 65(1): 209-215.

Hoffmann A., G. López-Campos. 2000. Biodiversidad de los ácaros en México. 1 st Ed., Conabio, México D.F., México, 230 pp.

Htut T. 1994. A case study of bite reactions in Man and domestic dust samples that implicate the house dust mite predator Cheyletus malaccensis Oudemans (Acari: Cheyletidae). Indoor and Built Environment, 3(2): 103-107.

Hu Jian-De. 1992. On the morphology of the genus Cheyletiella and a description of a new species from Xinjiang, China (Acari: Cheyletiellidae). - Acta Zootaxonomica Sinica, 17(2): 183-188.

Hu J. - D., G. Hou. 1992. On the morphology of the genus Cheyletiella and description of a new species from Xinjiang, China (Acari: Cheyletiellidae). - Acta Zootaxonomica Sinica, 17: 183-188 (in Chinese with English Abstract).

Hughes A. M. 1948. The Mites associated with stored food products. London: 1-166.

Hughes A. M. 1957. Silk production in the Cheyletidae (Acarina: Arachnida). - Nature, 180: $815-816$.

Hughes A. M. 1959. Mites, or the Acari. - Univ. of London, The Athlone Press, 225 pp.

Hughes A. M. 1961. The Mites associated with stored food products. London: 1-287.

Hughes T. E. 1958. The respiratory system of the mite Cheyletus eruditus (Schrank, 1781). Proc. Zool. Soc. Lond., 130(2): 231-239. 
Hull J.E. 1918. Terrestrial Acari of the Tyne Province. - Trans. Nat. Hist. Soc. Northumb. (N.S.), 5(1): 13-88.

Humphreys M. 1958. Cheyletiella parasitivorax infestation of the dog. - Vet. Rec., Lond., 442. Ide G. S., J. B. Kethley. 1977. Thewkachela ratufi n.g. n.sp., an unusual new cheyletid mite (Cheyletidae: Acariformes) from giant squirrel, Ratufa, (Sciuridae: Rodentia) in Sabah and Thailand. - Annals of the Entomological Society of America, 70: 559-562.

Jablonowski J. 1918. Ordo Acarina. In: Fauna Regni Hungariae, Budapest: 2-5.

Jeffrey I. G. 1976. An undescribed species of Mexecheles (Acarina: Cheyletidae) from Scottish farm. - Acarologia, 17(4), 1975: 668-670.

Jeffrey I. G. 1978. The female and juvenile stages of Mexecheles votandinii Jeffrey (Acarina: Cheyletidae) with further notes on the male. - Acarologia, 19(4)(1977): 654-657.

Jeffrey I. G. 1980. A new Cheyletus (Acarina: Cheyletidae) from a bat-roost. - Acarologia, 21(1)(1979): 46-54.

Jeffrey I. G., J. B. Campbell 1975. A new species of Hemicheyletia (Acarina: Cheyletidae). Journal of Stored Product Research, 11: 103-105.

Kamali K., H. Ostovan, A. Atamehr. 2001. A catalog of mites \& ticks (Acari) of Iran. Islamic Azad University Scientific Publication Center, 196 pp.

Kamran M., B.S. Khan, Z. Nazir. 2005. A new predatory mite species of the genus Hemicheyletia (Cheyletidae: Acarina) from Punjab, Pakistan. - Pak. J. Agri. Sci., 42(3-4): 52-54.

Kanavel R.F., A.G. Selhime. 1967. Biological studies on Paracheyleta bakeri (Acarina: Cheyletidae). - Fla Ent., 50: 107-113.

Karpelles L. 1884a. Beiträge zur Naturgeschichte der Milben. - Berl. Entomol. Zeitschr., 28: 1 -34.

Karpelles L. 1884b. Neue Milben. - Berl. Entomol. Zeitschr., 28(2): 231-244.

Kazemi Sh., S. Paktinat-Saeij. 2021. First record of Samsinakia trilobitus (Prostigmata, Cheyletidae) from Iran. - Persian Journal of Acarology, 10(1): 107-110.

Keh B. 1973. Dermatitis in man traced to dog infested with Cheyletiella yasguri Smiley (Acari: Cheyletiellidae) - in California. - Calif. Vector Views, 20(10-12): 77-79.

Keh B. 1975. Intense pruritis in man and concurrent infestation of Cheyletiella blakei Smiley on cats in the home in California. - Vect. Views, 22(1): 1-4.

Keh B., R. S. Lane, S. P. Shachter. 1987. Cheyletiella blakei, an ectoparasite of cats, as cause of cryptic arthropod infestations affecting humans. - Western Journal of Medicine, 146: 192-194.

Kethley J. B. 1990. Acarina: Prostigmata (Actinedida). In: Dindal, D. (ed), Soil Biology Guide, John Wiley, New York, pp. 667-756.

Khan A. W. 1970. Some very common and highly destructive mites: Acarina in mango buds in West Pakistan. - Science in India, 7: 276-279.

Khaustov A.A., P. Klimov, V.A. Trach, A.N. Bobylev, V.M. Salavatulin, V.A. Khaustov, A.V. Tolstikov. 2018. Review of mites (Acari) associated with the European spruce bark beetle, Ips typographus (Coleoptera: Curculionidae: Scolytinae) in Asian Russia. - Acarina, 26(1): 3-79.

Khaustov A.A. 2021. Review of free-living predatory cheyletid mites (Acari: Cheyletidae) of Western Siberia, Russia. - Systematic \& Applied Acarology, 16(1): 199-239. 
Kivganov D. A., A. I. Bochkov 1994. A new mite species of the genus Cheletopsis (Acari: Cheyletidae). - Vestnik Zoologii 1994, 2: 39-43.

Klimov P.B. 1997. [Data on the biology and systematics of Paracaropsis travisi (Acari, Cheyletidae), a new mite for Russia]. - Fundamental'nye problemy okhrany okr. Sredy, 1(9-10): 9-11, Vladivostok (in Russian).

Klimov P.B., A.V. Bochkov, B.M. OConnor. 2006. Host specifity and multivariate diagnostics of cryptic species in predacious cheyletid mites of the genus Cheletophyes (Acari: Cheyletidae) associated with large carpenter bees. - Biological Journal of the Linnean Society, 87: 45-58.

Klompen J. S. H., E. Mendez, F. S. Lukoschus. 1984. A new species of the genus Cheletophyes Oudemans, 1914 (Prostigmata: Cheyletidae) from the nest of a carpenter bee in Panama. - Acarologia, 25: 249-251.

Koch C.L. 1839. Deutschlands Crustaceen, Myriapoden und Arachniden. Fasc. 23, Regensburg. Koffman M., P. Viriden. 1949. On the occurrence Cheyletiella parasitivorax in cats and rabbits. - Nord. Vet. Med., 1: 449-454.

Koç K. 1998. A new record of Acaropsella Volgin (Acari: Prostigmata, Cheyletidae) for the fauna of Turkey. - Turkish Journal of Zoology, 22(3): 195-197.

Koç K., N. Ayyıldız. 1995. A new species of Microcheyla from Turkey (Acarina: Cheyletidae). - Genus, 6: 225-228.

Koç K., N. Ayyıldız. 1996. Türkiye faunası için yeni iki keyletid (Acari, Prostigmata, Cheyletidae) türü. - Turkish Journal of Zoology, 20: 215-221.

Kolebinova M. 1967. Faunistic studies on some Acari of the families Myobiidae and Cheyletidae, parasitizing small mammals in Bulgaria. - Bull. Inst. Zool. Mus., 25: 73-77 (In Bulg., summ. Rus., Engl.).

Kral F., J. P. Uscavage. 1960-61. Cheyletiella parasitivorax infestation in a dog. - J. Small Anim. Pract., 1: 277-278.

Kramer P. 1877. Grundzüge zur Systematik der Milben. - Archiv f. Naturgeschichte, 43: 215-247.

Kucerova Z. 2004. Stored product psocids as one of the preys of the of the predatory mite Cheyletus eruditus (Schrank)(Acarina, Cheyletidae). - IOBC/WPRS Bull., 27(9): 191-195.

Kumada N., H. Itoh, Ch. Suto, S. Mizuno, K. Uchikawa. 1978. Pipistrellus abramus (Temminck) as a nuisance in the Tokai district, Japan, and arthropods associated with this bat. - Medical entomology and zoology, 29(3): 261-263.

Kumar P., H. Naqi. 1990. Study of host stage density effect on cannibalism in Acaropsis sollers predatory mites and its role as a biological control agent (Acari, Cheyletidae). - Indian J. Helminthol., 42(1): 21-24.

Kutzer E. 1963. Über eine echte Raubmilbenräude beim Hund, hervorgerufen durch Cheyletiella parasitivorax (Mégnin, 1878). - Wien. Tierarztl. Monatsschr., 50(8): 784-788.

Kuznetzov N. N. 1977. On the fauna of Cheyletid mites (Acariformes, Cheyletidae) of the Crimea. - Revue d'Entomologie de l'URSS, 56(4): 923-928 (in Russian with English summary). 
Kuznetsov N.N., Petrov, V.M. 1984. Predatory Mites of Baltic States. Zinatne, Riga, 143.

Latreille P. 1796. Precis de caractères génériques des insectes, disposés dans un ordre naturel. An. 5 (1797), Paris.

Lawrence R. F. 1948. Some new pilicolus mites from South African mammals. - Parasitology, 39: $39-42$.

Lawrence R. F. 1954. The known African species of Cheyletidae and Pseudocheylidae (Acarina, Prostigmata). - Ann. Natal Mus., 13(1): 65-77.

Lawrence R. F. 1959. New mite parasites of African birds (Myobiidae, Cheyletidae). - Parasitology, 49(3-4): 416-438.

Lawrence R. F. 1961. In F. Zumpt, ed. The Arthropod parasites of vertebrates in Africa south of the Sahara. Publ. South African Inst. Med. Res., 11: 1-457.

Leach W.E. 1815. A tabular view of the external characters of four classes of animals, etc. Trans. Linn. Soc. London, 11: 399.

Lekprayoon C., R. L. Smiley. 1986. Chelacaropsis moorei Baker (Acari: Cheyletidae): redescription of the male and female. - International Journal of Acarology, 12: 69-72.

Lin J., H. Liu. 1994. A new species of Cheletogenes (Acari: Cheyletidae) from Fujian. - Entomotaxonomia, 16: 218-224.

Lin J., W. Pen, Y. Chen. 1994. A new species of the genus Hemicheyletia Volgin from Fujian (Acari: Cheyletidae). - Wuyi Science Journal, 11: 141-143.

Lin Jian-zhen, Yan-xuan Zhang. 1997. Three new species and a new record of Cheyletinae from Fujian (Acari: Cheyletidae). - Wuyi Science Journal, 13: 131-138.

Lin Jian-zhen, Yan-xuan Zhang. 2000. Cheyletoidea. In: Insect fauna of Fujian. Fujian Science \& Technology Press, 184-197 (in Chinese).

Lin J.-Z., Y.-X. Zhang, M. Yang. 1997. Two new records of the Cheyletinae from China (Acari: Cheyletidae). - Acta Zootaxonomica Sinica, 22(4): 367.

Lin J.-Z., Y.-X. Zhang, M. Yang. 1998. Two new records of the Cheyletinae from China (Acari: Cheyletidae). - Acta Zootaxonomica Sinica, 23(3): 336.

Lin J.-Z., Y.-X. Zhang, Yanxuan Zhang, Qiaoyun Liu, Jie Ji. 2000. Checklist of mites from moso bamboo in Fujian, China. - Syst. \& Applied Acarology, Special Publ. 4: 81-92.

Lindholm A.K., G.J. Venter, E.A. Ueckermann. 1998. Persistence of passerine ectoparasites on the diederik cuckoo Chrysococcyx caprius. - J. of Zoology, 244(1): 145-153.

Lomholt S., O. Jersild, H. Boas. 1917. To Tilfaelde af Dyrfnat hos Mennesket (Cheyletiella parasitivorax) - Dansk. dermatologisk Selskabs Mode 2. - Maj. Hospitalstid, 61: 1098-1099.

Louadi K., P. Robaux. 1992. Etude des populations d'Acariens pulvicoles dans l'Est algérien selon les gradients climatiques propres à cette région. - Acarologia, 33(2): 177-191.

Lucza Z., G. Ripka, K. R. Saly. 1996. Data to the Cheyletidae fauna of Hungary. - Folia Entomologica Hungarica, 57: 105-108.

Lukáš J., V. Stejskal, V. Jarošík, E. Zd’árkova. 2007. Differential natural performance of four Cheyletus predatory mite species in Czech grain stores. - J. of Stored Products Research, 43(1): 97-102.

Lung-Shut L. 1984. Stored grain mites in China. Their distribution and effects. In: Griffiths D.A., C.A. Bowman (Eds).VI, 2: 1002-1005. 
Macchioni F. 2003. Observations on the pidgeon Cheyletiellidae Volgin, 1966. - Annali della Facoltà di Medicina veterinaria, 56: 75-82.

Macchioni F., M. Magi, F. Mancianti \& S. Perrucci. 2005. Phoretic association of mites and Mallophaga with the pigeon fly Pseudolynchia canariensis. - Parasite, 12(3): 277-279.

Macchioni F., Magi, M., F. Mancianti. \& S. Perrucci. 2009. Aspectos da associação forética dos ácaros Myialges spp. (Astigmata: Epidermoptidae) Ornithocheyletia hallae Volgin (Prostigmata: Cheyletidae) com a mosca Pseudolynchia canariensis (Macquart) (Diptera: Hippoboscidae). - Neotropical Entomology, 38: 578-81.

Malaban J.M., L.A. Corpuz-Raros. 1998. Mites associated with Apis mellifera Linnaeus and Apis cerana Fabricius in the Southern Tagalog Region, Philippines. - Philippine Entomologist, 12: 123-135.

Marchiondo A.A., T.S. Foxx. 1978. Scanning electron microscopy of the solenidion on genu I of Cheyletiella yasguri and C. parasitivorax. - J. Parasitol., 64: 925-927.

Mariana A., T.M. Ho, M. Sofian - Azirun, A.L. Wong. 2000. House dust mite fauna in the Klang Valley, Malaysia. - Southeast Asian J. Trop. Med. Public Health, 31(4): 712-721.

Mathur S., R. B. Mathur. 1981. Hemicheyletia hissariensis, a new species of cheyletid mite from India. - Acarologia, 7: 69-70.

McClure H. Eliott, N. Ratanaworadhan et al. 1973. Some ectoparasites of the birds of Asia. -219 pp.

McGregor E.A. 1956. The mites of citrus trees in southern California. - Mem. So. Calif. Acad. Sci., 3(3): 1-24.

Mégnin P. 1878. Mémoire sur un nouveau groupe d’Acariens, les Cheylétides parasites. - Journ. de l'Anatomie et de la Physiologie, 14(3): 416-441.

Mégnin P. 1883. Sur le Cheiletus heteropalpus Mégnin parasite auxiliaire des oiseaux et sur sa nidification. - Bull. Soc. Zool. Fr., 8(3) : 157-160.

Mégnin P. 1892. Les acariens parasites. Encyclopédie scientifique des aide-mémoires dirigée par M. Léauté. Paris, 1-182.

Mehl R. 1978. Henvendelser angående skadedyr, ektoparassiter og andre plagsomme dyr i 1972-1976. - Fauna, 31: 250-267.

Merhnejad M.R., E.A. Ueckermann. 2001. Mites (Arthropoda, Acari) associated with Pistachio trees (Anacardiaceae) in Iran (I). - Syst. Appl. Acarol., 6: 1- 12.

Mertins J.W., A. Bochkov. 2014. Key to Species of the Genus Neocheyletiella (Acariformes: Cheyletidae), With Description of a New Species. - J. Med. Entomol., 51(6):1116-1121.

Metwally A.M., · A. A. Abdallah, H. Gamaleldein, H.M. Elbeltagy. 2016. Mites associated with stored products 2016. - Annals of Agric. Sci. Moshtohor, 54(3): 649-658.

Meyer M.K.P. 1973. Trombidiformes Reuter, 1909. p. 6-19. - In: Meyer et al., eds.: Acari of the Ethiopian Region. Entomology Memoir No29: 45 pp.

Meyer Smith M. K. P., E.A. Ueckermann. 1988. South African Acari. Ill. On the mites of the Mountain Zebra National Park. - Koedoe, 31: 1-29. 
Meyer M.K.P., P.A.J. Ryke. 1960. Acarina of the families Anystidae, Pseudocheylidae and Cheyletidae (Prostigmata) found associated with plants in South Africa. - J. ent. Soc. S. Africa, 23: 177-193.

Meyer Smith M. K. P., E. A. Ueckermann. 1988. South African Acari. III. On the mites of the Mountain Zebra National Park. - Koedoe, 31: 1-29.

Michael A. D. 1878. On a species of Acarus of the genus Cheyletus, believed to be new. - J. Roy. Mirc. Soc., 1: 133-138.

Mihelčič F. 1958. Prostigmata Südeuropas (Spanien). - Eos, Madrid, 34(3): 269-290.

Miller A. E. 1925. An introductory study of the Acarina, or mites, of Ohio. - Bull. Ohio Agricult. Experimental station, 386: Cheyletidae - p. 96-97.

Mironov S. V., A. V. Bochkov, P. A. Chirov. 1991. Mites of the genus Cheletopsis (Acariformes, Cheyletidae) from Charadriiformes birds of the Middle Asia. - Kyrg. Resp. Ilim Akadem Kabarlary Khim - Tekhnol Zhana Biol Ilim, 4: 50-56.

Modarres Awal M. 2012. List of agricultural pests and their natural enemies in Iran. Ferdowsi University of Mashhad Press. pp. 759.

Moradi Faradonbeh M., H. Ostovan, B. M. OConnor, M. Gheibi, O. Joharchi, F. Macchioni. 2019. Promyialges italicus sp. nov. (Astigmata: Epidermoptidae) with redescriptions of mites of the families Epidermoptidae and Cheyletidae (Prostigmata) associated with Pseudolynchia canariensis (Diptera: Hippoboscidae) from Iran and Italy. - Persian Journal of Acarology, 8(1): 27-46.

Mola P. 1907. Nuovi acari parasiti. - Zool. Anz., 32(2): 41-44.

Moser J.C., L.M. Roton. 1971. Mites associated with southern pine beetles in Allen Parish, Louisiana. - Can. Entomol., 103: 1775-1798.

Mosquera S. 1989. Eucheyletia hardyi Baker, 1949 y Haemogamasus pontiger (Berlese, 1904), dos acaros nuevos para la fauna Argentina. - Neotropica (La Plata), 34(9), 1988: 105-108.

Moxham J. W., T. T. Goldfinch, A. C. G. Heat. 1968. Cheyletiella parasitivorax infestation of cats associated with skin lesions of man. - N. Z. Vet. J., 16: 50-52.

Muma M. H. 1964. Cheyletidae (Acarina: Trombidiformes) associated with citrus in Florida. - Flo. Entomol., 47: 239-253.

Mumcuoglu Y. 1976. House dust mites in Switzerland I. Distribution and taxonomy. - J. Med. Ent., 13(3): 361-373.

Mwase E.T., A.S. Baker. 2006. An annotated checklist of mites (Arachnida: Acari) of Zambia.

- Zootaxa, 1106: 1-24

Mykytowskcz R. 1957. Parasitic habit of the rabbit mite Cheyletiella parasitivorax Mégnin). C. S. I. R. O. Wildlife Res., 2: 164.

Nachev P.D., G. Trenchev. 1987. The acarofauna associated with some Coccidae in Bulgaria. - Pochvoznanie, Agrokhimiya i Rastitelna Zashtita, 22: 81-86 (in Bulgarian).

Nadchatram M., R. Domrow. 1966. Observations on the Fauna of Pulau Tioman and Pulau Tulai 10. Parasitic Acarina of the Mammals. - Bull. Nat. Museum, Republic of Singapore, 34: 129-140. Nagar S. K. 1978. Identity, synonymy, host and distribution of Hemicheyletus calcarata (Domrow and Baker, 1960) n. comb. (Acarina: Cheyletidae). - Acarologia, 20(1): 102-103. 
Nagar S. K., V. K. Saxena. 1977. A new mite Hemicheylatus herpestesi n. sp. (Prostigmata: Cheyletidae) from the Indian Mongoose Herpestes edwardsii Geoffroy. - Annals of Zoology, 13(1): 35-42.

Nakada E. 1975. Cheyletus malaccensis Oudemans, 1903. Morphological characters of adult and immature stages. - Animal Hygiene (Eisei Dobutsu), 26: 151-165.

Negm M.W., A.E. Mesbah. 2014. Review of the mite family Cheyletidae (Acari: Trombidiformes: Cheyletoidea) of Egypt. - Int. J. Acarol., 40(5): 390-396. doi:10.1080/0164795 4.2014.930511

Nishida G.M. Version 2009. French Polynesia Mite Checklist.

Nordenskiöld E. 1900. Anteckningar om Acarider samlade i hö. - Medd. Soc. Faun. Fenn., 26: 34-38.

Nörner C. 1883. Einiges über Cheyletiden. - Verhandlungen der zoologisch-botanischen Gesellschaft in Wien, 33: 645-648.

Norris J. D. 1958. Observations on the control of mite infestations in stored wheat by Cheyletus spp. (Acarina, Cheyletidae). - Ann. Appl. Biol., 46(3): 411-422.

Olivier P. A. S., P. O. Theron. 1988. A new species of Eutogenes Baker, 1949 (Acari: Cheyletidae) from South Africa. - Phytophylactica. 20: 253-256.

Olivier P. A. S., P. D. Theron. 1989a. A new genus and species of Cheyletidae (Acari: Prostigmata) from South Africa. - Journal of the Entomological Society of Southern Africa. 52: 237-243.

Olivier P. A. S., P. D. Theron. 1989b. The genus Microcheyla Volgin, 1966 (Acari: Prostigmata: Cheyletidae) from South African soils. - Phytophylactica, 21(1): 49-54.

Olsen S., H. Roth. 1947. On the mite Cheyletiella parasitivorax, occuring on cats, as a facultative parasite of man. - J. Parasitol., 33(5): 444-445.

Oudemans A. C. 1897. List of Dutch Acari.IV. - Tijdschr. Ent., 40: 111-135.

Oudemans A. C. 1902. Tijdschrift der Nederlandse Dierkunde Vereenigin., 8: 15-16.

Oudemans A. C. 1903a. Acarologische Aanteekeningen VI. - Entomologische Berichten Nederlands Entomol. Ver., 1(12): 83-88.

Oudemans A. C. 1903b. Notes on Acari. XI. - Tijdschr. Ent., 46: 93-134.

Oudemans A. C. 1903c. Notes sur les Acariens. Memoires de la Societe Zoologique de France, 16: 5-32.

Oudemans A. C. 1904a. Acarologische Aanteekeningen XII. - Entomologische Berichten Nederlands Ent. Ver., 1 (18): 160-164.

Oudemans A. C. 1904b. Acarologische Aanteekeningen XIII. - Entomologische Berichten Nederlands Ent. Ver., 1: 169-174.

Oudemans A. C. 1904c. Acarologische Aanteekeningen XI. - Entomologische Berichten Nederlands Ent. Ver., 1(17): 153-155.

Oudemans A. C. 1905. Acarologische Aanteekeningen XV. - Entomologische Berichten Nederlands Ent. Ver., 1(21): 207-210.

Oudemans A. C. 1906. Révision des Chélétinés. - Mémoires de la Société Zoologique de France, 19: 36-218. 
Oudemans A. C. 1914. Acarologische Aanteekeningen LIV. - Entomologische Berichten Nederlands Entomol. Ver., 4 (78): 101-103.

Oudemans A. C. 1926. Kritisch historisch overzicht der acarologie.

Oudemans A. C. 1928. Acarologische Aanteekeningen. XCIII. - Entomologische Berichten Nederlands Entomol. Ver., 7(162): 343.

Özer M., S. Toros, S. Çobanoğlu, S. Çınarlı. 1986. Beneficial mites of stored products in İzmir province, 1st Turkish National Congress on Biological Control, Abstract book pg. 280-292, Adana, Turkey. (In Turkish)

Özer, M., Toros, S., Çobanoğlu, S., Çınarlı, S., Emekçi, M. 1989. The determination, distribution and habitats of Acarina group harmful on stored grain, flour and flour products and dried fruits in the province of İzmir. - Turkish Journal of Agriculture and Forestry, 13, 1154-1189. (In Turkish)

Özkan M., N. Ayyıldız, Z. Soysal. 1988. The Acari fauna of Turkey. - Doğa Türk Zooloji Dergisi, 12: 75-85. (In Turkish)

Packard A.S. 1869. Guide to the study of insects, and a treatise on those injurious and veneficial to crops: for the use of colleges, farm-schools, and agriculturists. P. 665.

Packard A.S. 1878. A Guide to the Study of Insects. New York: Henry Holt and Co., 715 pp.

Pactinat-Saeij S., M. Bagheri, M. Skvarla. 2017. First record of the genus Bak Yunker (Acariformes: Prostigmata: Cheyletidae) from Iran with description of a new species. - Acarologia (Paris), 57(4): 847-853.

Pagliarini N. 1979. Studies on the mites of stored cereals in Yugoslavia. In: Rodriguez J.G. (Ed). Recent Advances in Acarology. New York, Academic Press. P. 305-309. doi:10.1016/ B978-0-12-592201-2.50043-9

Paktinat-Saeij, M. Bagheri, M. Skvarla. 2017. First record of the genus Bak Yunker (Acariformes: Prostigmata: Cheyletidae) from Iran with description of a new species. - Acarologia, 57(4): 847-863. doi:10.24349/acarologia/20174199

Palyvos N.E., N.G. Emmanouel. 2006. Seasonal abundance and vertical distribution of mites in flat storage containing wheat. - Phytoparasitica, 34(1): 25-36.

Parameswaran Pillai P.R. 1957. Pests of stored fish and prawns. - Bull. Res. Inst. Univ. Kerala, 5C, 3: 1-79.

Patxot J.D., M.L. Goff. 1985. Two new species and new records of Cheyletidae (Acari) in Hawaii with a key to the species. - Intern. J. Acarol., 11: 157-162.

Pelaez D. 1962. Hoffmannita mexicana gen. et sp. nov., Cheyletidae parasito de un alacrán del géner Centruroides (Acarina, Prostigmata). - Anales de la Escuela Nacional de Ciencia Biologicas, 11: 71-78.

Peredo A.A., R.I. Martínez, P.M. Aqueveque, M.E. Casanueva. 1994. Presencia de Cheletomorpha lepidopterorum (Shaw, 1794) en Actinidia chinensis Planch «Kiwi» en Chile y redescripción del macho (Acari: Cheyletidae). - Gayana Zoologia, 58(1): 17-26.

Petrova V., I. Salmane. 2000. Some mite (Acari) species from mass-rearing laboratories of commercial mushrooms and beneficial arthropods in Latvia. - Ekológia (Bratislava), 19: 211-212. 
Petrova V., I. Salmane, Z. Čudare. 2004. The predatory mite (Acari, Parasitiformes: Mesostigmata (Gamasina); Acariformes: Prostigmata) community in strawberry agrocenosis. - Acta Univ. Latviensis, Biology, 676: 87-95.

Pfliegler W.P., A. Schönhofer, W. Niedbała, P. Vella, A. Sciberras, A. Vella. 2017. New records of mites (Acari) and harvestmen (Opiliones) from Malta with a preliminary checklist of maltese Arachnida. - Soil Organisms, 89: 85-110.

Philips J.R., D.L. Dindal. 1990. Invertebrate populations in the nests of a Screech owl (Otus asio) and an American kestrel (Falco sparverius) in Central New York. - Entomological News, 101(3): 170-192.

Pillars A. W. N. 1925. Cheyletiella parasitivorax Mégnin causing lesions in the domestic rabbits - Vet. J. (London), 81: 96-97.

Podder S., S.K. Gupta, G.K. Saha. 2006. Description of a new species of Grallacheles De Leon (Acari: Cheyletidae) from floor dust in India. - Entomon, 31(4): 333-338.

Poppe S.A. 1888. Über parasitische Milben. - Abh. Naturwiss. Verein, Bremen, 10: 205-240.

Poppe S.A. 1909. Nachtrag zur Milben - Fauna der Umgegend Bremens. - Abh. Nat. Ver. Bremen, 19: 47-67.

Price D. W. 1972. A new species of Acaropsella Volgin from the nest of a turkey vulture (Acarina: Cheyletidae). - Proceedings of the Entomological Society of Washington, 74: 45-49.

Principato M. 1989. Observation of a rare anomaly in Cheyletiella parasitivorax (Acarina: Cheyletidae): atrophy of a third right leg. - J. Med. Entomol., 26(4): 378-379.

Pulpán J., P. H. Verner. 1965. Control of Tyroglyphoid mites in stored grain by the predatory mite Cheyletus eruditus (Shrank). - Can J. Zool., 43: 417-432.

Putatunda B. N., R. P. Kapil. 1988. Seven new species of Cheletophyes (Acari: Prostigmata: Cheyletidae) associated with carpenter bees in India. - In: hannaBasavanna, G.P. and Viraktamath, C.A. (eds), Progress in Acarology, Vol. I, pp. 317-327.

Putatunda B. N., R. Kumar, D.P. Banerjee. 2004. Mites parasitic and/or associated with poultry and some mammals in Hisar, Haryana with key to identification. - Indian J. Anim. Res., 38(1): $1-7$.

Qayyum H. A., W. M. Chaudhri. 1977a. Descriptions of new mites of the genus Cheletomorpha Oudemans (Acarina: Cheyletidae) from Pakistan. - Pakistan Journal of Zoology, 9: 71-77.

Qayyum H. A., W. M. Chaudhri. 1977b. Descriptions of six new mite species of the genus Cheyletus Latreille (Acarina: Cheyletidae) from Pakistan. - Pakistan Journal of Zoology, 9: 87-97.

Qayyum H. A., W. M. Chaudhri. 1977c. Descriptions of two new mite species of the genus Cheletogenes Oudemans (Acarina: Cheyletidae) from Pakistan. - Pakistan Journal of Agricultural Sciences, 14: 110-114.

Qayyum H. A., W. M. Chaudhri. 1979a. Description of Cheletomimus heredis, new species (Acarina: Cheyletidae) from Pakistan. - Pakistan Entomologist, 1: 9-12.

Qayyum H. A., W. M. Chaudhri. 1979b. Mites of the genus Hemicheyletia (Acarina: Cheyletidae) described from Pakistan. - Pakistan Journal of Zoology, 11: 167-172.

Rack G. 1971. Cheyletiella yasguri Smiley, 1965 (Acarina, Cheyletiellidae), ein fakultativ menschenpathogener Parasit des Hundes. - Z. Parasitenk, 36: 321-334. 
Radford C.D. 1947. Parasitic mites from snakes and rodents (Acarina: Cheyletidae, Listrophoridae and Laelaptidae). - Proc. Zool. Soc. London, 117: 228-240.

Radford C.D. 1950. Systematic check list of mite genera and type species. - Int. Union Biol. Sci. (ser.C), 1: 1-232.

Rak H. 1972. Cheyletiella parasitivorax (Mégnin) (Acar., Cheyletidae) from cat flea (Ctenocephalides felis felis (Bouché)) in Iran. - Entomologist's Monthly Magazine, 108: 62.

Rak H. 1973. Ectoparasites of dogs and cats in Iran. - Entomologist's Monthly Magasine, 108: 189.

Ramaraju K., M. Mohanasundaram. 1999. Two new cheyletid mites (Acari: Cheyletidae) from Tamil Nadu, India. - International Journal of Acarology, 25(2): 121-127.

Rasool A., W. M. Chaudhri. 1979a. Two new species of the genus Hemicheyletia Volgin (Acarina: Cheyletidae) from Pakistan. - Pakistan Entomologist, 1: 1-6.

Rasool A., W. M. Chaudhri. 1979b. Description of a new species of the genus Cheletogenes Oudemans (Cheyletidae) from Pakistan. - Pakistan Entomologist, 1: 7-10.

Rasool A., W. M. Chaudhri, S. Akbar. 1980. Studies on the mites of the family Cheyletidae from Pakistan. - Pakistan Entomologist, 2: 27-36.

Razzaq A., M. Shiraishi, T. Manabe, N. Ohbayashi. 2001. External features of cheyletid predatory mite Hemicheyletia bakeri (Ehara) found in citrus orchards in Japan. - Pak. J. Biol. Sci., 4(5): 597-601.

Reed C. M. 1961. Cheyletiella parasitivorax infestation of pups. - J. Amer. Vet. Med. Ass., 138: 306-307.

Regev S. 1974. Morphological and genetic evaluation of male polymorphism in Cheyletus malaccensis (Cheyletidae: Acarina). - Acarologia, 16(1): 85-93.

Reis P.R., A.D. Paschoal. 1968. Alguns ácaros de produtos armazenados do Estado de São Paulo. - An. I Reun. An. Soc. Brasil . Entomol., Piracicaba, 63-64.

Rekk G. F. 1976. Catalogue of the Acarofauna of Georgian SSR. - Mezniereba, Tbilisi: 127 pp.

Ripka G., A. Fain, H.R. Bolland. 1999. New data to the knowledge on the corticolous mite fauna in Hungary (Acari: Prostigmata, Astigmata, Oribatida). - Acta Phytopathologica et Entomologica Hungarica, 34(4): 363-371.

Rizk G.N., E. Badry, S.M. Hafez. 1979. The effectiveness of predacious and parasitic mites in controlling Tribolium confusum Duv. - Mesop. J. Agric., 14(2): 167-182.

Rohdendorf B. B. 1940. Keys to the Cheyletidae and Pediculoididae. - Wiss. Ber. Moscow State Univ., 42: 69-98.

Rosa A. E., C. H. W. Flechtmann. 1979a. Ocorréncia de Ornithocheyletia hallae Smiley, 1970 (Acari: Prostigmata: Cheyletiellidae) parasitando Columbigallina sp. (Aves) no Brasil. Ecossistema, 4: 121-122.

Rosa A. E., C. H. W. Flechtmann. 1979b. Mites in house dust from Brazil. - Intl. J. Acar., 5(3): 195-198.

Rostom Z. M.F.1993. Survey of some granivorous and non-granivorous insects and mites in stores in Saudi Arabia. - J. Stored Prod. Res., 29(1): 27-31. 
Salarzehi S., J.Hajizadeh, M.Hakimitabar, E.Ueckermann. 2018. A contribution to the knowledge of cheyletid mites of Iran with redescription of Eucheyletia flabellifera (Michael, 1878)(Prostigmata: Cheyletidae). - Acarologia, 58(2) : 457-470.

Salarzehi S., J.Hajizadeh, E.Ueckermann. 2018. A new species of Cheyletus Latreille (Prostigmata: Cheyletidae) from Iran and a key to the Iranian species. - Acarologia, 58(3): 640-646.

Salarzehi S., J.Hajizadeh, E.Ueckermann. 2019. A new species of Cheletonella Womersley (Prostigmata : Cheyletidae) from Iran and a key to the species. - Acarologia, 59(2): 188-195.

Saleh S.M., M.S. El-Helaly, F.H. El-Gayar. 1985. Survey on stored product mites of Alexandria (Egypt). - Acarologia, 26(1): 87-93.

Saleh S.M., M.S. El-Helaly, F.H. El-Gayar. 1986. Life history of the predatory mite Cheyletus malaccensis (Oudemans). - Acarologia, 27(1): 37-40.

Samšiňák K. 1956. Die Milben (Acari) auf der Fliege Laphria flava L. - Mém. Soc. Zool. tchécoslov., 20: 353-357.

Samšiňák K. 1958. Roztoci ze slnečnicovych semen (Acari). - Českoslov. Parasitol., 5(1): 185-198.

Samšiňák K. 1959. Contribution à l' étude des Acariens de Madagascar. - Mém. Inst. Sci. Madagascar, Sér. A, 13: 81-84.

Samšiňák K. 1962. Chelacheles michalskii n. sp. (Acari, Cheyletidae). - Čas. Čs. Spol. ent. (Acta Soc. ent. Čechoslov.), 59(2): 183-185.

Sang-Hun Kim, Hyung-Kyou Jun, Kun-Ho Song, D. Gram, Duck-Hwan Kim. 2008. Prevalence of fur mites in pet rabbits in South Korea. - Veterinary Dermatology, 19(3): 189-190.

Sasa M., R. Kano. 1951. Cheyletiella takahasii sp. n., a new species of parasitic mite from Ochotona of Hokkaido, Japan (Acarina, Cheyletidae). - Japan. Journ. exper. Medicine, 21: 205-207.

Schaffer M. H., N. F. Baker, P. C. Kennedy. 1958. Parasitism by Cheyletiella parasitivorax. A case report of the infestation in a female dog and its litter. - Cornell Vet., 48(4): 440-447.

Schrank F.P. 1781. Enumeratio Insectorum Austriae indigenorum. Aug. Vindel.

Schuster R. 1997. Landeskundlich bedeutsame Milbenfunde in Kärnten und Osttirol (Arachnida, Acari). - Carinthia II, 187./ 107 Jhg.: 465-470.

Schweizer J. 1922. Beitrag zur Kenntnis der terrestrischen Milbenfauna der Schweiz. - Verhdl. Naturf. Ges., Basel, 33: 23-112.

Schweizer J. 1948. Landmilben aus der Umgebung des Schweizerischen Nationalparks. Ergebn. d. wissensch. Untersuchg. d. SNP, II (N.F.), 20: 1-28.

Schweizer J. 1951. Die Landmilben des Schweizerischen Nationalparks. 2 Teil: Trombidiformes Reuter 1909. - Rés. rech. sci. entreprises au Parc Nat. Suisse, 3 (NF): 119 -172.

Schweizer J., C. Bader. 1963. Die Landmilben der Schweiz (Mittelland, Jura und Alpen): Trombidiformes Reuter. - Denkschr. d. Schweiz. Naturf. Ges., 84(2): 209-378.

Sellnick M. 1940. Die Milbenfauna Islands. - K. Vet. O. Vitterh. Samh. Handl., Ser. B, 6(14): 129 pp.

Sepasgozarian H. 1978. Stored pests of Iran and their control. University of Tehran press, Publication No. 1026. pp. 287. 
Shen Chao-peng [Z.-P.]. 1975. Preliminary notes on Chinese Cheyletida mites and the life history of Cheyletus malaccensis Ouds. (Acarina: Cheyletidae). - Acta Entomologica Sinica, 18(3): 316-324.

Shen Z.-P. 1984. Cheyletidae. In: Jiangxi University (ed.). Agricultural Acarology in China [Zhongguo Nongyue Manlei]. Shanghai Science and Technology Publishing House, Shanghai, 364-378 (in Chinese).

Shen Z.-P. 1991. Cheyletidae mites of stored grain in China. - Grain Storage, 20(4): 21-30.

Shepherd R.C.H., J.W. Edmonds. 1977. Ectoparasites of the wild rabbit Oryctolagus cuniculus (L.) in Victoria: The occurrence of the mites Leporacarus gibbus (Pagenstecher) and Cheyletiella parasitivorax (Megnin) and the louse Haemodipsus ventricosus (Denny). - J. Aust. Entomol. Soc., 16: 237-244

Shiba M. 1969a. On some mites from «Tatami» (Japanese rice straw matting). - Rep. Res. Matsuyama Shinonome Junior College, 4(1): 147-170.

Shiba M. 1969b. Taxonomic Investigations on Free-living Mites in the Subalpine Forest on Shiga Heights IBP Area II. Prostigmata. - Bull. Nat. Sci. Mus., Tokyo, 12(1): 65-115.

Shiba M. 1976. Taxonomic investigations on free-living Prostigmata from the Malay Peninsula. - Nature Life in South East Asia. 7: 83-229.

Shin Sung-Shik. 1996. A case report of Cheyletiella infestations on a Whippet dog in Korea. - The Korean J. of Parasitology, 34(4): 267-271.

Shulov A. 1957. Additions to the fauna of Acarina of Israel (excluding ticks and gall mites). Bull. Res. Council of Israel, Sect. B. Biology and Geology, 6B (3-4): 233-238.

Skoracki M., A.V. Bochkov, B. Sikora. 2004. A new species and new records of the cheyletoid mites (Acari: Cheyletoidea) from passeriform birds in Poland. - Belgian Journal of Entomology, 6: 83-90.

Skvarla M., J.R. Fisher, A.P.G. Dowling. 2014. Redescription of Paracaropsis travisi (Baker, 1949)(Trombidiformes: Cheyletidae), with range expansion, additional host records, and reevaluation of cheyletid chaetotaxy based on the sejugal furrow. - Acarologia, 54(3): 335-345.

Smiley R. L. 1965. Two new species of the genus Cheyletiella (Acarina: Cheyletidae). - Proc. Entomol. Soc. Washington, 67(2): 75-79.

Smiley R. L. 1970. A review of the family Cheyletiellidae (Acarina). - Annals of the Entomological Society of America, 63: 1056-1078.

Smiley R. L. 1978. Further studies on the family Cheyletiellidae (Acarina). - Acarologia, 19: 225-241.

Smiley R. L. 1984. Two new species of Ornithocheyletia (Acari: Cheyletidae) from Australian birds. - International Journal of Acarology, 10: 239-250.

Smiley R. L. 1996. New species of Cheletonella (Acari: Prostigmata: Cheyletidae) and a new key to the species. - Anales Institute Biologico de Universita Autonom. Mexico, Ser. Zool., 67: 239-244.

Smiley R. L., J. C. Moser. 1970. Three cheyletids found with pine bark beetles (Acarina: Cheyletidae). - Proceedings of the Entomological Society of Washington, 72: 229 -236. 
Smiley R. L., J. C. Moser. 1975. Redescription of Eutogenes vicinus Summers and Price, a predatory polymorphic Cheyletid mite with descriptions of males and immature stages (Acarina: Cheyletidae). - Proc. Ent. Soc. Washington, 77(4): 405-418.

Smiley R. L., J. O. Whitaker Jr. 1981. Studies on the idiosomal and leg chaetotaxy of the Cheyletidae (Acari) with descriptions of a new genus and four new species. - International Journal of Acarology, 1: 109-128.

Smiley R. L., G. L. Williams. 1972. A new genus and species of Cheyletidae (Acarina). -Proceedings of the Entomological Society of Washington, 74: 312-315.

Socarrás A.A. 1987. Observaciones preliminares sobre la biología de Cheyletus eruditus (Prostigmata: Cheyletidae) en condiciones de laboratorio. - Reporte de Investigación del Inst. de Ecologia y Sistemática, Ac. Sci. de Cuba, 41: 1-10.

Soliman Z. R. 1975. Three new species of cheyletid mites from Egypt (Acari: Prostigmata) with a key to genera. - Acarologia, 17(1): 95-102.

Soliman Z. R. 1977. Three new species of Cheyletid mite from Egypt with a key to genera (Acarina : Prostigmata). - Dtsch. Ent. Z., N.F., 24, Heft I - III: 207-212.

Stam J. W. E., H. J. W. M. Cremers. 1978. Ornitocheyletia hallae Smiley, 1970 (Acarina: Cheyletidae) as the cause of pruritis in a racing pigeon. - Tijdschr. Diergeneesk., 103(20): 1114-1115.

Starkoff O., I. Starkoff. 1950. Contributo alla conoscenza dell'acarofauna di Roma e dintorni IIa nota: sottordini Sarcoptiformes e Prostigmata. - Rivista di Parassitologia, 11(2): 85-101.

Sugimoto M. 1942. Parasites and parasitic diseases of domestic rabbits. - Sylvia (Journal of the Taihoku Society of Agriculture and Forestry), 5(5): 550-577.

Summers F. M. 1975. Males of three species of Cheyletus (Acarina: Cheyletidae). - Proceedings of the Entomological Society of Washington, 77(4): 446-455.

Summers P. M. 1976. A new genus for several cheyletid mites formerly in Acaropsis (Acarina: Cheyletidae). - Proceedings of the Entomological Society of Washington, 78: 190-194.

Summers F. M., D. W. Price. 1970. Review of the mite family Cheyletidae. - University of California Publications in Entomology, 61: 1-153.

Summers P. M., R. L. Witt. 1971. The gnatosoma of Cheyletus cacahuamilpensis Baker (Acarina: Cheyletidae). - Proc. Entomol. Soc. Washigton, 73 (2): 158-168.

Summers P. M., R. L. Witt. 1973a. Nesting behavior of Cheyletus eruditus (Acarina: Cheyletidae). - Pan-Pac. Ent., 48(4): 261-269.

Summers P. M., R. L. Witt. 1973b. Oviposition and mating tendencies of Cheyletus malaccensis (Acarina: Cheyletidae). - Fla. Entomol., 56(4): 277-285.

Summers P. M., R. Witt, S. Regev. 1972. Evaluation of several characters by which five species of Cheyletus are distinguished (Acarina: Cheyletidae). - Proceedings of the Entomological Society of Washington, 74: 230-252.

Sweatman G.K. 1962. Parasitic mites of non-domesticated animals in New Zealand. - New Zealand Entomologist, 3(1): 15-23.

Tanaka H. 1953. Studies on the seven species of cheyletid mites of Japan. - Japan. J. San. Zool., 4: 72-80. 
Thewke S. E. 1974. A new species of Chelacheles (Acarina: Cheyletidae) from Missouri with a key to the known species. - Entomological News, 85: 33-39.

Thewke S. E. 1977. A Revision of the Mite Family Cheyletidae: Acari: Prostigmata. Univ. of Missuri, Columbia, 1366 p.

Thewke S. E. 1980. Three new genera and species of cheyletid mites (Acarina: Cheyletidae) from the U. S. A., Mexico, Panama and Indonesia. - Journal of the Kansas Entomological Society, 53: 333-342.

Thewke S. E., W. R. Enns. 1968. A new species of predaceous mite (Acarina: Cheyletidae) from galleries of bark beetles in Missouri. - Acarologia, 10: 215-219.

Thewke S. E., W. R. Enns. 1972. A new genus and three new species of cheyletid mites (Acarina: Cheyletidae) from Mis $\neg$ souri and Michigan. - Journal of the Kansas Entomological Society, 45: 450-459.

Thewke S. E., W. R. Enns. 1974. A new species of Bak Yunker (Acarina: Cheyletidae) from Missouri with a revised key to the known species. - Journal of the Kansas Entomological Society, 47: 42-53.

Thewke S. E., W. R. Enns. 1975. A new species of Pavlovskicheyla (Acarina: Cheyletidae) from the elytra of Platydema ruficorne (Coleoptera: Tenebrionidae) from Missouri. - Acarologia, 17(4): 671-682.

Thewke S. E., W. B. Enns. 1976. Oudemansicheyla coprosomae sp. nov. (Acarina: Cheyletidae) from New Zealand. - Journal of the Kansas Entomological Society, 49: 360-362.

Thewke S. E., W. B. Enns. 1979. Three new species of Hemicheyletia Voegin (Acarina: Cheyletidae) with a key to the known world species. - Journal of the Kansas Entomological Society, 52: 218-225.

Thewke S. E., R.E. Wilbur. 1968. A new species of predaceous mite (Acari: Cheyletidae) from galleries of bark beetles Missouri. - Acarologia, 10: 215-219.

Thomsett L.R. 1968. Mite infestations of man contracted from dogs and cats. - Brit. Med. J., 3: 93-95.

Thor S. 1912. Norwegische Cunaxidae und Cheyletidae I. - Zool. Anz., 39(11/12): 389-390.

Torre Santana P.E. de la. 2015. Las especies de la familia Cheyletidae (Acari: Trombidiformes) en Cuba. - Revista Cubana de Ciencias Biológicas, 4(1): 47-55.

Trencheva K.G., G.S. Trenchev. 2018. Three new species of Tenuipalpidae (Acari: Prostigmata) for the fauna of Bulgaria. - Journal of Entomology and Zoology Studies, 6(4): 1794-1798.

Tseng Y. - H. [Tjying I. - S.]. 1971. Cheyletid mites associated with stored products in Taiwan (I) (Acarina: Cheyletidae). - Inspection Research, Bulletin 4, p. 36.

Tseng Y. - H. [Tjying I. - S.]. 1972. A new cheyletid mite Hemicheyletia arecana n. sp. from Taiwan (Acarina: Prostigmata). - Plant Protection Bulletin, 14: 8-10.

Tseng Y. - H. 1973. Two new predatory mites from Taiwan (Acarina: Cheyletidae: Prostigmata). - Plant Protection Bulletin, 15: 76-77.

Tseng Y. - H. 1977. A contribution to the knowledge of Formosan cheyletid mites (Acarina: Prostigmata). - The Proceedings of the National Science Council, 10(2): 213-263.

Turk F.A. 1946. Studies of Acari. V. - Notes on and descriptions of new and little-known British Acari. - Ann. Mag. Nat. Hist. (11) 12: 785-820. 
Turk F.A. 1953. I. - A synonymic catalogue of British Acari: Part I. - Ann. Mag. Nat. Hist., 12th Ser., 61: 1-26.

Uchikawa K., H. Suzuki. 1979. Cheyletiella mexicana sp. nov. (Acarina: Cheyletiellidae) parasitic on Romerolagus diazi (Mammalia, Leporidae). - Tropical Medicine, 21(1): 21-27.

Uchikawa K., H. Suzuki. 1980. Studies on the Parasite Fauna of Thailand 3. Mites associated with Thai mammals. - Tropical Medicine, 22(1): 13-25.

Vail E. L., G. F. Augustson. 1943. A new ectoparasite (Acarina: Cheyletidae) from domestic rabbits. - J. Parasitol. 29: 419-421.

Vaivanijkul P. 1979. Polycheyletus boonkongae n.g., n.sp. from Thailand (Acari: Cheyletidae). - International Journal of Acarology, 5: 251-252.

Valim M.P., G.S. Gazeta. 2007. Phoretic association of the mites Myialges anchora Sergeant \& Trouessart (Acaridida, Epidermoptidae) and Ornithochyletia hallae Smiley (Actinedida, Cheyletiellidae) with Pseudolynchia canariensis (Macquart) (Diptera, Hippoboscidae). - Revista Brasileira de Entomologia, 51(4): 518-519.

Vercammen-Grandjean P. H., H. Rak. 1968. Cheyletiella yasguri Smiley, 1965, un parasite de Canidés aux Etats-Unis et hyperparasite d'Hippoboscidae en Iran (Acarina: Cheyletidae). - Ann. Parasitol. (Paris), 43(3): 405-412.

Vitzthum H. 1920. Acarologische Beobachtungen. II. - Arch. f. Naturgesch., 1918, 84A(6): $1-40$.

Vitzthum H. 1931. Terrestrische Acarinen (unter Ausschluss der Oribatiden und Ixodiden) der Deutschen Limnologischen Sunda-Expedition. - Archiv f. Hydrobiologie, Suppl. 9 (Tropische Binnengewässer II): 59-134.

Volgin V. I. 1949. Materials for the systematics of predaceous mites of the genus Cheyletus Latr. - C.R. Acad. Sci. U.S.S.R., Moskow, n.ser., 64: 583-586.

Volgin V. I. 1955. [The family Cheyletidae Leach - predatory mites. - In: The mites on rodents in the U.S.S.R.]. Moscow - Leningrad: 152-176.

Volgin V. I. 1958. Data on the taxonomy of predatory mites of the family Cheyletidae. I. Genus Eutogenes Baker, 1949. - Entomol. Rev., 37 (2): 460-463 (In Russian, summ. Engl.).

Volgin V. I. 1960. On the taxonomy of predatory mites of the family Cheyletidae. II. Genus Cheyletiella. - Parasitol. Sbornik, 19: 237-248.

Volgin V. I. 1961. On the taxonomy of predatory mites of the family Cheyletidae. III. Genus Cheletacarus Volgin, gen. nov. - Parasitol. Sbornik, 20: 248-256.

Volgin V. I. 1962. - On the taxonomy of predatory mites of the family Cheyletidae. IV. The genus Neoacaropsis Volgin, gen. nov. - Rev. Entomol., 41(3): 685-691 (In Russian).

Volgin V. I. 1963a. On the taxonomy of predatory mites of the family Cheyletidae. V. Genus Eucheyletia Baker, 1949. - Parasitol. Sbornik, 21: 44-68 (in Russian, short summary in English).

Volgin V. I. 1963b. Two new genera of predatory mites of the family Cheyletidae (Trombidiformes). - Entomol. Rev., 42(4): 504-508.

Volgin V. I. 1964a. On the taxonomy of predatory mites of the family Cheyletidae. VI. The genus Ornithocheyletia Volgin. - Gen. n. - Zool. Zh., 43 (1): 28-36. 
Volgin V. I. 1964b. On the taxonomy of predatory mites of the family Cheyletidae. VII. The genus Neoeucheyla Radford, 1950. - Parasitol. Sbornik, 22: 88-97 (in Russian, summ. Engl.).

Volgin V. I. 1965. [On the artificial nature of the genus Cheletophyes Oudm. (Acarina, Cheyletidae)]. - Tr. Zool. Inst., 35: 288-299 (in Russian).

Volgin V. I. 1966a. Morphological peculiarities of the mites of the family Cheyletidae (Acarina, Trombidiformes) and their ontogenetical development. - Rev. Entomol. URSS, 45(1): 217-231.

Volgin V. I. 1966b. A new genus and new species of mites of the family Cheyletidae Leach (Acarina, Trombidiformes). In: [New species of insects in the fauna of the USSR and the bordering countries], Trudi Zool. Instituta, Moscow - Leningrad, 37: 277-285 (in Russian).

Volgin V. I. 1969a. Acarina of the Family Cheyletidae of the World. Akademia Nauk, Leningrad, USSR, in Russian, pp. 432. In English, 1987, Amerind Publishing Co., New Delhi, pp. 532.

Volgin V.I. 1978. New species of predatory cheyletid mites (Acariformes, Trombidiformes). - Entomological Review, 57: 213-217 (in Russian with English summary).

Volgin V.I., I.I. Nikolaeva. 1965. On parasitism of predacious mites of the genus Neocheyletiella Baker, 1949 (Acarina, Cheyletidae) - Tr. Zool. Inst., 35: 300-304 (in Russian).

Vysotzkaja S.O. 1974. Biocoenotic relationshipes between ectoparasites of the common redbacked vole, Clethrionomys glareolus Schreb., and inhabitants of its nests in the Transcarpathian region of the Ukraine. - Parazitologicheskiy sbornik, 26: 114-143.

Wafa A.K., Z.R. Soliman. 1968. Five genera of family Cheyletidae (Acarina) in the U.A.R. with a description of four new species. - Acarologia, 10(2): 220-229.

Wang X.-Z. 1989. Cheyletidae. In: Deng G.- F., Wang H.-F., Xin J.-L., Wang D.-Q., Wu W.-N. \& Wang X.-Z. (eds) An Outline of Acari in China [Zhongguo Piman Gangyao]. Science Press, Beijing, 227-234 (in Chinese).

Wassel M.E., J.O. Whitaker, E.J. Spicka. 1980. The ectoparasites and other associates of the Cottontail Rabbit, Sylvilagus floridanus, in Indiana. - Proc. Indiana Acad. Sci., Zoology, 89: 418-420.

Wegner Z., T. Przyborowski. 1958. Pasozyty zewnetrzne szczurow portu gdynskiego. - Wiad. parazyt., Warszawa, 4: 773-774.

Weitkamp R. A. 1964. Cheyletiella parasitivorax parasitism in dogs. - J. A. V. M. A., 144 (6): 597-599.

Wharton G. W., L. G. Arlian. 1972. Predatory behaviour of the mite Cheyletus aversor. - Ann. Behav., 20(4): 719-723.

Whitaker J. O., Jr., J.T. Baccus, A.G. Cleveland, Yi-M. Gu. 1990. Ectoparasites of some mammals from Southern China. - Bull. Soc. Vector Ecol., 15(1): 53-58.

Whitaker J. O., Ch. Maser. 1985. Mites (excluding Chiggers) of mammals of Oregon. - Great Basin Naturalist, 45(1): 67-76. 
Whitaker J. O., D.D. Pascal, R.M. Mumford. 1979. Ectoparasites of the red squirrel (Tamiasciurus hudsonicus) and the Eastern Chipmunk (Tamias striatus) from Indiana. - J. Med. Entomol., 16(4): 350-351.

Wikerhauser T., L. Pinter. 1987. Parasitic mite Cheyletiella blakei in a cat. First case report in Yugoslavia. - Vet. Arch., 57(2): 63-70.

Willmann C. 1939. Die Arthropodenfauna von Madeira nach den Ergebnissen der Reise von Prof. Dr. O. Lundblad Juli - August 1935. XIV. Terrestrische Acari (exkl. Ixodidae). Arkiv för Zoologi, 31A(10): 1-42.

Willmann C. 1949a. Das Männchen der Milbe, Cheyletiella pinguis Berlese, 1889, aufgefunden. - Ber. Münch. Tierärztl. Wschr., 1949, 5: 56-57.

Willmann C. 1949b. Beiträge zur Kenntnis des Salzgebietes von Ciechocinek. I. Milben aus den Salzwiesen und Salzmooren von Ciechocinek an der Weichsel. - Ver. Mus. f. Natur-Völker-u. Handelskunde in Bremen, A, 1: 106-135.

Womersley H. 1942a. Notes on the Cheyletidae (Acarina, Trombidoidea) of Australia and New Zealand, with descriptions of new species. - Rec. S.A. Museum, 7: 51-64.

Womersley H. 1942b. Miscellaneous additions to the acarine fauna of Australia. - Trans. Royal Soc. of South Australia, 66(1): 85-92.

Womersley H. 1956. A new genus and two new species of Acarina from Northern Australia. - Proc. Lin. Soc. N.S. Wales, 80(3): 214-216.

Woodroffe G. 1956. Some insects and mites associated with bat - roosts with a discussion of the feeding of the Cheyletids (Acarina). - Entomologist's Monthly Mag., 92: 138-142.

Xia Bin. 2010. A review of progress on the systematics and biology of the family Cheyletidae in China, with a checklist of the Chinese cheyletids. P. 158-164. - In: Zhang, Z.-Q., Hong, X.-Y. \& Fan, Q.-H. (eds) Xin Jie-Liu Centenary: Progress in Chinese Acarology. Zoosymposia, 4, 1-345.

Xia Bin, H. Klompen, C. Childers. 2011. A new genus and species of Cheyletidae (Acariformes: Prostigmata) from citrus trees in Florida. - Zootaxa, 2796: 29-36.

Xia B., Gong Z.-Q., Yu L.-P. 2005. The effects of temperatures on development and survivorship of Cheyletus eruditus. - Journal of Nanshang University (Natural Science), 29(3): 286-289.

Xia B., R. Ye, Z. Zhu. 1997a. A new species of the genus Grallacheles (Acari: Cheyletidae) from Jiangxi, China. - Systematic and Applied Acarology, 1: 173-175.

Xia B., R. Ye, Z. Zhu. 1997b. One new species of the genus Acaropsella from China (Acari: Cheyletidae). - Acta Arachnologica Sinica, 6(2): 143-145 (in Chinese, English Summ.).

Xia B., R. Ye, Z. Zhu. 2000. Notes and key to known species of the Cheyletidae (Acari: Prostigmata) from Jiangxi. - Chinese science abstracts, 6(7): 885-886 (in Chinese, English Summ.).

Xia B., Z. - M. Zhu, R. Ye. 1999. A new species of the genus Cheletonella (Acari: Cheyletidae) from China and a key to the species. - Systematic and Applied Acarology, 4: 149-151.

Xia B., G. Liang, L. Zeng, Z. Zhu. 2004. On species of the genus Eucheyletia Baker from China (Acari, Cheyletidae). - Acta Zootaxonomica Sinica, 29(1): 89-92 (in Chinese, English Summ.). 
Xia B., G. Liang, Z. Zhu. 2005. A new species of the genus Prosocheyla Volgin (Acari, Cheyletidae) with a key to the known world species. - Acarologia, 56(2-3): 203-206.

Xia B., Luo D.-M., Zou Z.-W., Xu R., Zhu Z.-M. 2007. Predation of Cheyletus eruditus on Aleuroglyphus ovatus. - Chinese Bull. of Entomology, 44(4): 549-552 (in Chinese, English Summ.).

Xia B., Zhang T., Zou Z.-W. 2007. The Predation of Cheletomorpha lepidopterorum on Tyrophagus putrescentiae. - J. of Nanchang University (Natural Science), 31(6): 579-582 (in Chinese, English Summ.).

Xia B., Zhu Z.-M., Ye R., Wang S.-J., Tu D. 2001. Notes and key to known species of Hemicheyletia (Acari: Cheyletidae) from China. - Chinese Science Abstracts, 7(9): 1168-1169 (in Chinese, English Summ.).

Xie S.-Y., Wen X., Chen K.-S. 2000. Four new records of the stored product mite species in China. - Grain Storage, 29(5): 17-19 (in Chinese, English Summ.).

Yazdani A., J. Ebrahimi. 1993. Predators of scale insects on pistachio tree in Kerman province. - Proc. 11th Plant Protec. Cong. Iran. Guilan, Rasht, p. 210.

Yousef A. E.-T. A. 1979. Aegyptocheyla summersi n. gen., n. sp. (Acarina: Prostigmata: Cheyletidae). - Acarologia, 1978, 20(3): 365-367.

Yousef Abdel-Tawab A., M.A. Zaher, M.M. Kandil. 1980. Comparative morphological studies on the developmental stages of Cheletogenes ornatus (C. \& F.)(Prostigmata - Cheyletidae). - Acarologia, 21(2), 1979: 228-233.

Yousef Abdel-Tawab A., M.A. Zaher, M.M. Kandil. 1982. Effect of prey and temperature on the development and biology of Cheyletus malaccensis Oudemans (Acari: Cheyletidae). - J. Appl. Entomology, 93(1): 39-42.

Yunker C. E. 1960. Alliea laruei, n. gen., n. sp., (Acarina: Cheyletidae) from Rattus norvegicus (Erxleben) in Florida. - Proceedings of the Helmintological Society of Washington, 27: 279-281.

Yunker C. E. 1961. The genera Bak, new genus, and Cheletomimus Oudemans, with descriptions of three new species (Acarina: Cheyletidae). - Canad. Entomol., 93 (2): 1023-1053.

Zachvatkin 1935. A Short Key to the granary mites, pp.1-31. 2d ed. Moskow (in Russian).

Zaher M. A., Z. R. Soliman. 1966. Eutogenes punctata n. sp. (Acarina: Cheyletidae). - Bull. Soc. Entomol. d'Egypte, 49: 65-66.

Zaher M. A., Z. R. Soliman. 1967. The family Cheyletidae in the U. A. R., with a description of four new species (Acarina). - Bulletin de la Société Entomologique d'Egypte, 51: 21-26.

Zaher M. A., A. El -Tawab A. Yousef, M. M. Kandil. 1981. Effect of food on the biology of Cheletogenes ornatus (C. \& F.)(Acari: Prostigmata: Cheyletidae). - Acarologia, 22(4): 361- 366.

Zankov Z.D. (Занков 3. Д.) 1955. [A predator mite] (Cheyletia bulgarica Volg.). - Lozarstvo i Vinarstvo, 1: 34-36 (in Bulgarian).

Zhang Y.-X., Lin J.-Z. 1996. The predatory effect of Cheyletus malaccensis and Lepidoglyphus destructor. - Entomological Journal of East China, 5(1): 65-68 (in Chinese, English Summ.). 
Zhang Y.-X., Lin J.-Z., Hou A.-P. 1997. Studies on the relationship between Cheyletus malaccensis (Acarina: Cheyletidae) and Lepidoglyphus destructor. - J. of the Fujian Academy of Agricultural Sciences, 12(1): 44-47 (in Chinese, English).

Zhu Z.-M., Zhou K., Xia B., Yu L.-P., Tu D. 2000. Effects of temperature on the development period of Cheyletus trouessarti. - J. of Nanchang University (Natural Science), 24(4): 307-309 (in Chinese, English Summ.).

Ždárková E. 1979. Cheyletid fauna associated with stored products in Czechoslovakia. - J. Stored Prod. Res., 15: 11-16.

Ždárková E. 1986. Mass rearing of the predator Cheyletus eruditus (Schrank) (Acarina: Cheyletidae) for biological control of acarid mites infesting stored products. - Crop Protection (Guildford, Surrey), 5(2): 122-124.

Ždárková E., P. Horák. 1997. Bionomics of Eucheyletia taurica Volgin, a species new to Central Europe (Acarina: Cheyletidae). - Acarologia, 38(2): 123-125.

Ždárková E., J. Lukáš, P. Horák. 2003. Compatibility of Cheyletus eruditus (Schrank)(Acari: Cheyletidae) and Cephalonomia tarsalis (Ashmead) (Hymenoptera: Bethylidae) in biological control of stored grain pests. - Plant Protect. Sci., 39(1): 29-34. 


\title{
The Mite Family Psorergatidae Dubinin
}

\author{
Psorergatidae Dubinin, 1955: 150
}

The itch-mites of the family Psorergatidae are tiny inhabitants of the skin of Soricomorpha, Macroscelidea, Scandentia, Rodentia, Lagomorpha, Carnivora, Primates, and Artiodactyla.

The first species of this peculiar mites was described as early as 1883 by J.B. Tyrrell in Canada. For the next 70 years very little attention was given to the tiny intradermal Psorergatid mites. In Europe the example of Michael (1889) who described from England the second Psorergatid species (under the "new" genus Goniomerus), was not followed until the time of Dubinin and Fain. In his paper on rodent parasites (1955) and in his revision of Cheyletoidea (1957) Dubinin described, among other new families, Psorergatidae with only two species.

Meanwhile, several new species have been described from countries outside Europe, mainly from bigger mammals (now in subgenus Psorobia): P. ovis Womersley, 1941 (Australia), P. cercopitheci Zumpt et Till, 1955 (South Africa), P. bos Johnston, 1964 (USA).

The papers of Fain and co-authors from 1959 to 1973 laid down the structure of the family, but it was F. Lukoschus who, between 1967 and 1979, together with his co-authors Beaujean, Driessen, Fain, De Cock, Fauran, Giesen, Maa, Hallmann, Rack, Rosmalen, described 47 (?) of all 77 species in the family. Giesen (1990) made the comprehensive revision of the family. Other modern authors are R. Haitlinger (1979-1997), A. Baker (2005) and others. As Psorergatids seem to be host-specific, many new species are to be discovered.

For the time being the family Psorergatidae consists of 77 species [73, acc. to Zhang et al., 2011], distributed among three genera, as pointed by Fain (1959a, b):

Psorergates Tyrrell, $1883-49$ sp.

Psorergatoides Fain, 1959 - 18 sp.

Psorobia Fain, 1959 - 10 sp.

\section{Catalogue of Psorergatidae}

Family Psorergatidae Dubinin

Psorergatidae Dubinin, 1955: 150

Type genus: Psorergates Tyrrell, 1883

Genus Psorergates Tyrrell

Psorergates Tyrrell, 1883: 337

Type species: Psorergates simplex Tyrrell, 1883

Goniomerus Michael, 1889: 400

Type species: Goniomerus musculinus Michael, 1889 
Psorergates agrestis Lukoschus, Fain et Beaujean

Psorergates (Psorergates) agrestis Lukoschus, Fain \& Beaujean, 1967: 149

Psorergates agrestis, Giesen, 1990: 56

Type locality: Hoensbroek (Limburg, Holland)

Distribution: Holland

Host: Microtus agrestis bailloni (Rodentia: Arvicolidae)

Psorergates apodemi Fain, Lukoschus et Hallmann

Psorergates (Psorergates) apodemi Fain, Lukoschus \& Hallmann, 1966: 261; Sosnina, 1970: 538; Beron, 1973: 182

Psorergates apodemi: Haitlinger, 1980: 700; Giesen, 1990: 56

Type locality: Hatert (Holland)

Distribution: Austria, Bulgaria, Germany, Holland, Poland, Romania, Switzerland, Ukraine, f. Tchecoslovakia

Hosts: Sylvaemus flavicollis, S. sylvaticus, S. microps, S. uralensis (Rodentia: Muridae, Murinae)

Psorergates arvalis Lukoschus, Fain et Beaujean

Psorergates (Psorergates) arvalis Lukoschus, Fain \& Beaujean, 1967: 171

Psorergates arvalis, Giesen, 1990: 56

Type locality: Parque Nacional de Aigües Tortes, Lerida

Distribution: Spain

Host: Microtus arvalis meridianus (Rodentia: Arvicolidae)

Psorergates auricola Lukoschus, Fain et Beaujean

Psorergates auricola Lukoschus, Fain \& Beaujean, 1967: 167

Type locality: Jaca

Distribution: Spain

Host: Microtus [Pitymys] duodecimcostatus flavescens (Rodentia: Arvicolidae)

Psorergates baueri Lukoschus, de Cock et Driessen

Psorergates baueri Lukoschus, de Cock \& Driessen, 1971: 190

Type locality: St. Michael, Salzburg

Distribution: Austria

Host: Neomys fodiens (Soricomorpha: Soricidae)

Psorergates callipidis Lukoschus, Fain et Beaujean

Psorergates (Psorergates) callipidis Lukoschus, Fain \& Beaujean, 1967: 156; Lukoschus, 1967: 203

Type locality: El Puerto, Asturia

Distribution: Spain 
Host: Sylvaemus sylvaticus callipides (Rodentia: Muridae, Murinae)

Psorergates canadensis Kok, Lukoschus et Clulow

Psorergates canadensis Kok, Lukoschus \& Clulow, 1971: 1243

Type locality: Sudbury (Ont.)

Distribution: Canada (Ontario, Manitoba)

Host: Microtus pennsylvanicus (Rodentia: Arvicolidae)

Psorergates cinereus Kok, Lukoschus et Clulow

Psorergates cinereus Kok, Lukoschus \& Clulow, 1971: 1239

Type locality: Sudbury (Ont.)

Distribution: Canada (Ontario, Manitoba)

Host: Sorex cinereus (Soricomorpha: Soricidae)

Psorergates crocidurae Lukoschus

Psorergates (Psorergates) crocidurae Lukoschus, 1968a: 81; Giesen \& Lukoschus, 1983: 410

Psorergates crocidurae Giesen, 1990: 54

Type locality: Nijmegen (Holland)

Distribution: China, Germany, France, Holland, Spain

Host: Crocidura russula russula (Soricomorpha: Soricidae)

Psorergates cryptotis Giesen et Lukoschus

Psorergates cryptotis Giesen \& Lukoschus, 1983: 404

Type locality: Cerro Punta

Distribution: Panama

Host: Cryptotis n. nigrescens (Soricomorpha: Soricidae)

Psorergates deomydis Lukoschus, Fain et Beaujean

Psorergates (Psorergates) deomydis Lukoschus, Fain \& Beaujean, 1967: 159

Psorergates deomydis Giesen, 1990: 56

Type locality: Albert (= Virunga) Nat. Park

Distribution: DR Congo

Host: Deomys ferrugineus chrystyi (Rodentia: Muridae, Deomyinae)

Psorergates desmanae Lukoschus

Psorergates desmanae Lukoschus, 1968b: 125

Type locality: Moulis (Ariège)

Distribution: France

Host: Galemys pyrenaicus (Soricomorpha: Talpidae, Desmaninae) 
Psorergates dissimilis Fain, Lukoschus et Hallmann Psorergates (Psorergates) dissimilis Fain, Lukoschus \& Hallmann, 1966: 267

Psorergates dissimilis, Giesen, 1990: 56; Haitlinger, 1978: 143

Type locality: Hamert, Limburg Prov.

Distribution: Austria, Holland, Poland, Switzerland, Crimea

Hosts: Microtus arvalis, Myodes [Clethrionomys] glareolus (Rodentia: Arvicolidae)

Psorergates doriae Giesen, Lukoschus et Nadchatram

Psorergates doriae Giesen, Lukoschus \& Nadchatram, 1982: 319

Type locality: Kampong Taii, Sarawak

Distribution: Malaysia (Sarawak)

Host: Crocidura [doriae] foetida (Soricomorpha: Soricidae)

Psorergates dremomydis Giesen, Lukoschus et Nadchatram

Psorergates dremomydis Giesen, Lukoschus \& Nadchatram, 1982: 316

Type locality: Gunong (Mt.) Brinchang, 1500 m, Pahang

Distribution: Malaysia (Pahang)

Host: Dremomys rufigenis (Rodentia: Sciuridae, Sciurinae)

Psorergates eliomydis Lukoschus, Fain et Beaujean

Psorergates (Psorergates) eliomydis Lukoschus, Fain \& Beaujean, 1967: 156

Psorergates eliomydis, Giesen, 1990: 56

Type locality: Ntra. Sra. del Pilar (Formentera, Ibiza)

Distribution: Spain (Ibiza)

Host: Eliomys quercinus ophiusae (Rodentia: Gliridae)

Psorergates etruscus de Cock, Lukoschus et Ariani

Psorergates etruscus de Cock, Lukoschus \& Ariani, 1970: 1

Type locality: Naples (Italy)

Distribution: Italy

Host: Suncus etruscus (Soricomorpha: Soricidae)

Psorergates fritzi Giesen, Nutting et Nadchatram

Psorergates fritzi Giesen, Nutting \& Nadchatram, 1989: 133

Type locality: Fraser's Hill, Pahang, $1200 \mathrm{~m}$

Distribution: Malaysia (Pahang), Singapore

Host: Tupaia glis (Scandentia: Tupaiidae)

Psorergates glaucomys Ah, Peckham et Ateyo

Psorergates glaucomys Ah, Peckham \& Ateyo, 1973: 369

Type locality: Savannah, Chatham Co., Georgia 
Distribution: U.S.A. (Georgia)

Host: Glaucomys v. volans (Rodentia: Sciuridae)

Psorergates hispanicus Lukoschus, Fain et Beaujean

Psorergates hispanicus Lukoschus, Fain \& Beaujean, 1967: 163; Giesen, 1990: 56

Type locality: Jaca

Distribution: Spain

Hosts: Mus musculus commissarius, M. spretus (Rodentia: Muridae, Murinae)

\section{Psorergates meati?}

Psorergates meati ? - Giesen, 1990: 56

Type locality: Spain

Distribution: Spain

Host: Sylvaemus sylvaticus callipides (Rodentia: Muridae, Murinae)

Psorergates mexicanus Giesen, Lukoschus, Whitaker et Gettinger

Psorergates mexicanus Giesen, Lukoschus, Whitaker \& Gettinger, 1983: 169

Type locality: Socorro Co. (New Mexico)

Distribution: USA (New Mexico)

Host: Notiosorex crawfordi (Soricomorpha: Soricidae)

Psorergates micromydis Lukoschus, Fain et Beaujean

Psorergates micromydis Lukoschus, Fain \& Beaujean, 1967: 143

Type locality: Hellevoetsluis

Distribution: Holland

Host: Micromys minutus soricinus (Rodentia: Muridae, Murinae)

Psorergates microti Fain, Lukoschus et Hallmann

Psorergates microti Fain, Lukoschus \& Hallmann, 1966: 266

Type locality: Hatert

Distribution: Holland

Hosts: Microtus agrestis, Myodes [Clethrionomys] glareolus (Rodentia: Arvicolidae)

\section{Psorergates muricola Fain}

Psorergates (Psorergates) muricola Fain, 1961: 66; Fain, Lukoschus \& Hallmann, 1966: 259;

Lukoschus, Fain \& Beaujean, 1967: 175; Beron, 1970: 146; 1973: 182

Psorergates muricola: Haitlinger, 1987a: 82; Sosnina, 1970: 539; Giesen, 1990: 56

Type locality: Kawa, Lake Albert, DR Congo

Distribution: Belgium, Bulgaria, DR Congo, France, Holland, Italy, Poland, Russia

Hosts: Sylvaemus flavicollis, S. sylvaticus, Hybomys univittatus, Lophuromys aquilus, Mus musculus, Otomys [irroratus elgonis] tropicalis (Rodentia: Muridae, Murinae) 
Psorergates muscardinus Lukoschus, de Cock et Driessen

Psorergates muscardinus Lukoschus, de Cock \& Driessen, 1971: 185

Type locality: München

Distribution: Germany

Host: Muscardinus avellanarius (Rodentia: Gliridae)

Psorergates musculinus (Michael)

Goniomerus musculinus Michael, 1889: 405; Canestrini, 1894: 812

Psorergates simplex var. musculinus, Neumann, 1893: 21

Psorergates musculinus (Michael): Oudemans, 1906: 78; Hirst, 1919: pl. XII, fig. 52;

Vitzthum, 1929: 54; Turk, 1953; Zumpt \& Till, 1955: 269; Dubinin, 1955: 152; Fain,

Lukoschus \& Hallmann, 1966: 254; Rioux \& Golvan, 1961:

Type locality: England

Distribution: England, Russia

Hosts: Microtus agrestis, Myodes [Clethrionomys] glareolus (Rodentia: Arvicolidae), Sylvaemus sylvaticus (Muridae)

Psorergates neerlandicus Lukoschus, de Cock et Driessen

Psorergates neerlandicus Lukoschus, de Cock \& Driessen, 1971: 192

Type locality: Texel Island, the Netherlands

Distribution: Holland

Host: Microtus oeconomus (Rodentia: Arvicolidae)

Psorergates oeconomi Lukoschus, Fain et Beaujean

Psorergates oeconomi Lukoschus, Fain \& Beaujean, 1967: 139

Type locality: Zuidland, Rockanje (Holland)

Distribution: Holland

Host: Microtus oeconomus arenicola (Rodentia: Arvicolidae)

\section{Psorergates oettlei Till}

Psorergates simplex, Lavoirierre, 1946: 81 (nec Tyrrell, 1883)

Psorergates oettlei Till, 1960: 75

Psorergates (Psorergates) oettlei (Till): Fain, 1961: 61; Fain, Lukoschus \& Hallmann, 1966: 258

Type locality: Johannesburg

Distribution: South Africa

Host: Mastomys [Rattus] natalensis (Rodentia: Muridae, Murinae)

Psorergates olawaensis Haitlinger

Psorergates olawaensis Haitlinger, 1987: 539

Type locality: Wroclaw 
Distribution: Poland

Host: Crocidura suaveolens (Soricomorpha: Soricidae)

Psorergates paraxeri Giesen et Lukoschus

Psorergates paraxeri Giesen \& Lukoschus, 1982: 2

Type locality: Zoutpansberg (22 $58^{\prime}$ S, $29^{\circ} 56^{\prime}$ E), Mahalapye (Botswana)

Distribution: Botswana, South Africa

Host: Paraxerus cepapi (Rodentia: Sciuridae)

Psorergates peromysci Giesen, Lukoschus, Whitaker et Gettinger

Psorergates peromysci Giesen, Lukoschus, Whitaker \& Gettinger, 1983: 164

Type locality: Lively (Ontario), Abercorn (Quebec), Lincoln Co. (Oregon), Oconne Co. (Georgia)

Distribution: Canada (Ontario, Quebec), USA (Oregon, Georgia)

Hosts: Peromyscus leucopus, P. maniculatus (Rodentia: Cricetidae)

Psorergates pinetorum Giesen, Lukoschus, Whitaker et Gettinger

Psorergates pinetorum Giesen, Lukoschus, Whitaker \& Gettinger, 1983: 166

Type locality: Huntingdon Co. (Pennsylvania), Bicknell (Indiana)

Distribution: USA (Pennsylvania, Indiana)

Host: Microtus pinetorum (Rodentia: Arvicolidae)

Psorergates pitymydis Lukoschus, Fain et Beaujean

Psorergates (Psorergates) pitymydis Lukoschus, Fain \& Beaujean, 1967: 151

Psorergates pitymydis, Giesen, 1990: 56

Type locality: Arganda nr. Madrid, Jaca (Huesca)

Distribution: Spain

Host: Microtus [Pitymys] duodecimcostatus flavescens (Rodentia: Arvicolidae)

\section{Psorergates polonicus Haitlinger}

Psorergates polonicus: Haitlinger, 1986: 425

Type locality: Wroclaw - Swojczyce

Distribution: Poland

Host: Microtus [Pitymys] subterraneus (Rodentia: Arvicolidae)

Psorergates quercinus Lukoschus, de Cock et Driessen

Psorergates quercinus Lukoschus, de Cock \& Driessen, 1971: 195

Type locality: Madrid

Distribution: Spain

Host: Eliomys quercinus (Rodentia: Gliridae) 


\section{Psorergates rattus Fain et Goff}

Psorergates rattus Fain \& Goff, 1986: 197; Cierocka \& Izdebska, 2019: 314

Type locality: Oahu I., Nuuanu (Hawaiian Is.)

Distribution: USA (Hawaiian Islands), Poland

Host: Rattus norvegicus (Rodentia: Muridae, Murinae)

Psorergates simplex Tyrrell

Psorergates simplex Tyrrell, 1883: 337; Neumann, 1893: 13; Canestrini, 1894: 809; Oudemans, 1906: 77; Vitzthum, 1929: 54; Dubinin, 1953: 276; Zumpt \& Till, 1955: 269; Dubinin, 1955: 151; Till, 1960: 75; Fain, 1961: 61; Spicka, 1976: 418; Kamali, Ostovan \& Atamehr, 2001; Izdebska \& Fryderyk, 2012: 20

Psorergates (Psorergates) simplex (Tyrrell): Fain, Lukoschus \& Hallmann, 1966: 252

Type locality: Canada

Distribution: Canada, France, Holland, Iran, Russia, USA (Indiana)

Hosts: Mus musculus (Rodentia: Muridae, Murinae); Arvicola terrestris, Microtus arvalis (Arvicolidae)

Psorergates sorici Lukoschus

Psorergates sorici Lukoschus, 1968: 85

Type locality: Nijmegen (Holland)

Distribution: Holland

Host: Sorex araneus araneus (Soricomorpha: Soricidae)

Psorergates squamipes Lukoschus, Louppen et Maa

Psorergates squamipes Lukoschus, Louppen \& Maa, 1974: 51

Type locality: Arisan, $2300 \mathrm{~m}$

Distribution: Taiwan

Host: Anourosorex squamipes (Soricomorpha: Soricidae)

Psorergates talpae Lukoschus

Psorergates (Psorergates) talpae Lukoschus, 1968: 75

Type locality: Nijmegen (Holland)

Distribution: Holland, Spain

Host: Talpa europaea europaea (Soricomorpha: Talpidae, Talpinae)

Psorergates townsendi Giesen, Lukoschus, Whitaker et Gettinger

Psorergates townsendi Giesen, Lukoschus, Whitaker \& Gettinger, 1983: 169

Type locality: Lincoln Co. (Oregon)

Distribution: USA (Oregon)

Host: Microtus townsendi (Rodentia: Arvicolidae) 
Psorergates tupaiae Giesen et Lukoschus

Psorergates tupaiae Giesen \& Lukoschus, 1982: 259

Type locality: Mt. Kenepai (Borneo)

Distribution: Malaysia (Borneo)

Host: Tupaia dorsalis (Scandentia: Tupaiidae)

Psorergates urotrichi Giesen et Lukoschus

Psorergates urotrichi Giesen \& Lukoschus, 1983: 405

Type locality: Hakuba, Nagano

Distribution: Japan

Host: Urotrichus talpoides (Soricomorpha: Talpidae)

Psorergates watsoni Kok, Lukoschus et Clulow

Psorergates watsoni Kok, Lukoschus \& Clulow, 1971: 1243

Type locality: Sudbury (Ont.)

Distribution: Canada (Ontario)

Host: Peromyscus maniculatus (Rodentia: Cricetidae)

Psorergates zibethicalis Lukoschus, Fain et Beaujean

Psorergates (Psorergates) zibethicalis Lukoschus, Fain \& Beaujean, 1967: 134

Psorergates zibethicalis,

Type locality: Prümzurlay in der Eifel, Germany

Distribution: [Germany]

Host: Ondatra zibethica (Rodentia: Arvicolidae)

\section{Psorergates sp.}

Psorergates sp.

Distribution: Canada

Host: Microsorex koyi (Soricomorpha: Soricidae)

\section{Genus Psorergatoides Fain}

Psorergatoides Fain, 1959b: 232 (Subgenus of Psorergates)

Psorergatoides: Giesen, 1990: 22 (Genus)

Type species: Psorergates (Psorergatoides) kerivoulae Fain, 1959

Psorergatoides artibei Lukoschus, Rosmalen et Fain

Psorergatoides artibei Lukoschus, Rosmalen \& Fain, 1973: 70

Type locality: Paramaribo

Distribution: Suriname

Host: Artibeus lituratus fallase (Chiroptera: Phyllostomatidae, Stenodermatinae) 
Psorergatoides australiensis Giesen, Lukoschus et Fain

Psorergatoides australiensis Giesen, Lukoschus \& Fain, 1982: 315; Domrow, 1991: 1336;

Halliday, 1998: 79

Type locality: Geikie Gorge, W. A. (18 $05^{\circ}$ S; $125^{\circ} 43^{\prime}$ E)

Distribution: Australia (Western Australia)

Hosts: Vespadellus [Eptesicus] pumilus, V. [E.] [douglasi] douglasorum, Nyctophilus arnhemensis, N. gouldi, N. walkeri (Chiroptera: Vespertilionidae)

Psorergatoides desmodus Lukoschus, Louppen et Fauran

Psorergatoides desmodus Lukoschus, Louppen \& Fauran, 1979: 311

Type locality: Cayenne

Distribution: French Guyana

Host: Desmodus rotundus (Chiroptera: Phyllostomatidae, Desmodontinae)

\section{Psorergatoides emballonurae Fain}

Psorergates (Psorergatoides) emballonurae Fain, 1959b: 241

Psorergatoides emballonurae Fain

Type locality: Buin (Bougainville Is.)

Distribution: Papua New Guinea (Bougainville)

Host: Mosia [Emballonura] nigrescens (Chiroptera: Emballonuridae)

Psorergatoides glossophagae Lukoschus, Rosmalen et Fain, 1973

Psorergatoides glossophagae Lukoschus, Rosmalen \& Fain, 1973: 63

Type locality: Leonsberg

Distribution: Suriname

Host: Glossophaga soricina (Chiroptera: Phyllostomatidae, Glossophaginae)

Psorergatoides guyanensis Lukoschus, Louppen et Fauran

Psorergatoides guyanensis Lukoschus, Louppen \& Fauran, 1979: 322

Type locality: Rochambeau

Distribution: French Guyana

Host: Rhinophylla pumilio (Chiroptera: Phyllostomatidae)

\section{Psorergatoides hipposideros Fain}

Psorergates (Psorergatoides) hipposideros Fain, 1959b: 237

Psorergatoides hipposideros (Fain): Giesen, 1990: 55

Type locality: Bunia (Ituri); Grotte de Kakontwe

Distribution: DR Congo

Hosts: Hipposideros abae, H. caffer centralis (Chiroptera: Hipposideridae) 
Psorergatoides indicicola Lukoschus, Rosmalen et Fain

Psorergatoides indicicola Lukoschus, Rosmalen \& Fain, 1973: 67

Type locality: Lelydorp

Distribution: Suriname

Hosts: Saccopteryx bilineata, S. canescens (Chiroptera: Emballonuridae)

\section{Psorergatoides kerivoulae (Fain)}

Psorergatoides kerivoulae Fain, 1959a: 61

Psorergates (Psorergatoides) kerivoulae Fain, 1959b: 239

Psorergates kerivoulae Fain: Haitlinger, 1979b: 109

Type locality: Bokuma, Pawa (DR Congo)

Distribution: Belgium, DR Congo, Ivory Coast, Malaysia (Borneo), Poland

Hosts: Kerivoula cuprosa, K. [harrisoni] lanosa, Myotis bocagei, M. muricola, M. myotis, M. mystacinus, Plecotus auritis (Chiroptera: Vespertilionidae)

Psorergatoides laviae (Fain)

Psorergates laviae Fain, 1959a: 64

Psorergates (Psorergatoides) laviae Fain, 1959b: 247

Psorergatoides laviae (Fain): Giesen, 1990: 54

Type locality: Akanyaru River, Gabiro, Kagera Nat. Park

Distribution: Rwanda

Host: Lavia frons (Chiroptera: Megadermatidae)

\section{Psorergatoides lonchorhinae Fain}

Psorergatoides (Psorergatoides) lonchorhinae Fain, 1959b: 243

Psorergatoides lonchorhina Fain, Giesen, 1990: 54

Type locality: San Esteban

Distribution: Venezuela

Hosts: Saccopteryx canescens [canina] (Chiroptera: Emballonuridae), Lonchorhina aurita (Chiroptera: Phyllostomatidae)

Psorergatoides molossi Lukoschus, Rosmalen et Fain

Psorergatoides molossi Lukoschus, Rosmalen \& Fain, 1973: 72

Type locality: Lelydorp

Distribution: Suriname

Host: Molossus molossus (Chiroptera: Molossidae)

\section{Psorergatoides nyctali Baker}

Psorergatoides nyctali Baker, 2005: 67; Heddergott \& Claussen, 2008: 57

Type locality: Dartington Hall, near Totnes, Devon, England

Distribution: England, Germany

Host: Nyctalus noctula (Chiroptera: Vespertilionidae) 


\section{Psorergatoides nycteris (Fain)}

Psorergates (Psorergatoides) nycteris Fain, 1959a: 56

Psorergatoides nycteris (Fain): Giesen, 1990: 54

Type locality: Musha, nr. Akanyaru; Elisabethville (= Lubumbashi)

Distribution: DR Congo, Rwanda

Host: Nycteris macrotis (Chiroptera: Nycteridae)

Psorergatoides peropteryx Lukoschus, Louppen et Fauran

Psorergatoides peropteryx Lukoschus, Louppen \& Fauran, 1979: 316

Type locality: Cayenne

Distribution: French Guyana

Hosts: Cormura brevirostris, Peropteryx macrotis (Chiroptera: Emballonuridae)

\section{Psorergatoides rhinolophi (Fain)}

Psorergates rhinolophi Fain, 1959a: 59

Psorergates (Psorergatoides) rhinolophi Fain, 1959b: 235

Psorergatoides rhinolophi (Fain): Giesen, 1990: 54; Heddergott \& Claussen, 2008: 57

Type locality: Lubudi, Uvira (DR Congo), Kambisa (Angola)

Distribution: Angola, Belgium, Burma, DR Congo, France, Germany, Italy, Spain

Hosts: Rhinolophus aethiops, Rh. affinis, Rh. clivosus zuluensis, Rh. euryale, Rh.ferrumequinum, Rh. fumigatus, Rh. hipposideros, Rh. hildebrandti, Rh. mehelyi (Chiroptera: Rhinolophidae)

Psorergatoides surinamensis Lukoschus, Louppen et Fauran

Psorergatoides surinamensis Lukoschus, Louppen \& Fauran, 1979: 319

Type locality: Wageningen

Distribution: Suriname

Hosts: Lophostoma [Tonatia] carrikeri, Lophostoma brasiliense [T. nicaraguae] (Chiroptera: Phyllostomatidae)

Psorergatoides tadaridae Giesen, Lukoschus et Nadchatram

Psorergatoides tadaridae Giesen, Lukoschus \& Nadchatram, 1982: 325

Type locality: Gombak Forest Reserve, Selangor

Distribution: Malaysia (Selangor)

Host: Mops [Tadarida] mops (Chiroptera: Molossidae)

Genus Psorobia Fain

Psorobia Fain, 1959a: 55

Psorergates (Psorobia): Fain, 1961: 61

Type species: Psorergates ovis Womersley, 1941

Psorobia bos (Johnston)

Psorergates bos Johnston, 1964: 1; Oberem \& Malam, 1983: 63 
Psorobia bos (Johnston): Fain, 19; Giesen \& Yunker, 1985: 37; Baker, Andrews \& Fox, 1996; Andrews, Baker, Fox \& Brizuela, 1997: 183.

Type locality: New Mexico

Distribution: Great Britain, South Africa, USA (New York, New Mexico)

Host: Bos taurus (Artiodactyla: Bovidae)

Psorobia castoris Kok, Lukoschus et Clulow

Psorobia castoris Kok, Lukoschus \& Clulow, 1970: 1419

Type locality: Blezard Valley (Ontario)

Distribution: Canada (Ontario)

Host: Castor canadensis (Rodentia: Castoridae)

Psorobia cercopitheci Zumpt et Till

Psorergates cercopitheci Zumpt \& Till, 1955: 272; Seier, 1985: 236

Psorobia cercopitheci (Zumpt \& Till): Fain, 19...

Type locality: Bedford, Cape Province

Distribution: South Africa

Host: Cercopithecus aethiops pygerythus (Primates: Cercopithecidae)

Psorobia elephantuli Giesen, Spicka et Whitaker

Psorobia elephantuli Giesen, Spicka \& Whitaker, 1985: 147

Type locality: Mezuna, Tunis

Distribution: Tunisia

Host: Elephantulus rozeti (Macroscelidea: Macroscelididae)

Psorobia foinae (Fain et Lukoschus)

Psorergates (Psorobia) foinae Fain \& Lukoschus, 1968: 3

Psorobia foinae (Fain et Lukoschus): Giesen, 1990: 56

Type locality: Torgny, Belgium

Distribution: Belgium

Host: Martes foina (Carnivora: Mustelidae)

Psorobia hystrici (Till)

Psorergates hystrici Till, 1957: 329

Psorobia hystrici (Till): Giesen, 1990: 56

Type locality: Winburg, Orange Free State

Distribution: South Africa

Host: Hystrix africaeaustralis (Rodentia: Hystricidae)

Psorobia lagomorphae Giesen, Spicka et Whitaker

Psorobia sp.: Wassel, Whitaker \& Spicka, 1980: 419 
Psorobia lagomorphae Giesen, Spicka \& Whitaker, 1985: 148

Type locality: Parke Co., 8 mi. S. Rockville, Indiana

Distribution: USA (Indiana)

Host: Sylvilagus floridanus (Lagomorpha: Leporidae)

Psorobia mustelae (Lukoschus)

Psorergates (Psorobia) mustelae Lukoschus, 1969: 111

Psorobia mustelae (Lukoschus): Giesen, 1990: 56

Type locality: Nijmegen (Holland)

Distribution: Holland

Hosts: Mustela erminea, M. nivalis (Carnivora: Mustelidae)

Psorobia ovis (Womersley)

Psorergates ovis Womersley, 1941: 56; Carter, 1941: 193; Davis, 1954: 255; Fiedler \& du

Toit, 1954: 21; Zumpt \& Till, 1955: 270; Whitten \& Elliott, 1956: 19; Sinclair \& Girson,

1970; Sinclair, 1990: 59; Whitten, 1962: 9; Pomroy, 1985: 18

Psorergates (Psorobia) ovis (Womersley): Fain, 1961: 62

Psorobia ovis (Womersley): Fain, 1959a: 56

Type locality: Australia

Distribution: Australia, New Zealand, South Africa, USA

Host: Ovis aries (Artiodactyla: Bovidae)

Psorobia zumpti (Fain)

Psorergates (Psorobia) zumpti Fain, 1965: 295

Psorobia zumpti (Fain): Giesen, 1990: 56

Type locality: Bloemfontein

Distribution: South Africa

Host: Cryptomys hottentotus (Rodentia: Bathyergidae)

N.B.: In the general paper of Giessen (1990) are mentioned also Psorergates alleni and P. ramai.

\section{Index of Psorergatidae}

(Names in bold are accepted as valid)

agrestis, Psorergates 178

alleni, Psorergates

apodemi, Psorergates 178

artibei, Psorergatoides 185

arvalis, Psorergates 178

auricola, Psorergates 178 australiensis, Psorergatoides 186

baueri, Psorergates 178

bos, Psorergates 189

bos, Psorobia 189

callipidis, Psorergates 178

canadensis, Psorergates 179 
castoris, Psorobia 189

cercopitheci, Psorergates 189

cercopitheci, Psorobia 189

cinereus, Psorergates 179

crocidurae, Psorergates 179

cryptotis, Psorergates 179

deomydis, Psorergates 179

desmanae, Psorergates 179

desmodus, Psorergatoides 186

dissimilis, Psorergates 180

doriae, Psorergates 180

dremomydis, Psorergates 180

elephantuli, Psorobia 189

eliomydis, Psorergates 180

emballonurae, Psorergatoides 186

etruscus, Psorergates 180

foinae, Psorergates (Psorobia) 189

foinae, Psorobia 189

fritzi, Psorergates 180

glaucomys, Psorergates 180

glossophagae, Psorergatoides 186

Goniomerus 177

guyanensis, Psorergatoides 186

hipposideros, Psorergatoides 186

hispanicus, Psorergates 181

hystrici, Psorergates 189

hystrici, Psorobia 189

indicicola, Psorergatoides 187

kerivoulae, Psorergatoides 187

lagomorphae, Psorobia 190

laviae, Psorergates 187

laviae, Psorergatoides 187

lonchorhina, Psorergatoides 187

lonchorhinae, Psorergatoides 187

meati, Psorergates 181

mexicanus, Psorergates 181

micromydis, Psorergates 181

microti, Psorergates 181

molossi, Psorergatoides 187

muricola, Psorergates 181

muscardinus, Psorergates 182 musculinus, Goniomerus 182

musculinus, Psorergates 182

mustelae, Psorergates (Psorobia) 190

mustelae, Psorobia 190

neerlandicus, Psorergates 182

nyctali, Psorergatoides 187

nycteris, Psorergatoides 188

oeconomi, Psorergates 182

oettlei, Psorergates 182

olawaensis, Psorergates 182

ovis, Psorergates 190

ovis, Psorobia 190

paraxeri, Psorergates 183

peromysci, Psorergates 183

pinetorum, Psorergates 183

pitymydis, Psorergates 183

polonicus, Psorergates 183

Psorergates 177

Psorergatidae 177

Psorergatoides 185

Psorobia 188

peropteryx, Psorergatoides 188

quercinus, Psorergates 183

ramai, Psorergates

rattus, Psorergates 184

rhinolophi, Psorergates $\mathbf{1 8 8}$

rhinolophi, Psorergatoides 188

simplex, Psorergates 184

sorici, Psorergates 184

squamipes, Psorergates 184

surinamensis, Psorergatoides 188

tadaridae, Psorergatoides 188

talpae, Psorergates 184

townsendi, Psorergates 184

tupaiae, Psorergates 185

urotrichi, Psorergates 185

watsoni, Psorergates 185

zibethicalis, Psorergates 185

zumpti, Psorergates (Psorobia) 190

zumpti, Psorobia 190 


\section{Distribution of Psorergatidae}

\section{EUROPE}

Austria (Lukoschus, de Cock \& Driessen, 1971) - Psorergates apodemi, P. baueri, P. dissimilis

Azerbaidzhan (Gadjiev et al., 1988) - Psorobia ovis

Belgium (Fain, 1959; Fain \& Lukoschus, 1968) - Psorergates muricola, Psorergatoides kerivoulae, P. rhinolophi; Psorobia foinae

Bulgaria (Beron, 1970, 1973) - Psorergates apodemi, P. muricola

Denmark

Groenland (Giesen, 1990) - Psorergates sp.

France (Neumann, 1893; Lukoschus, 1968a, 1968b) - Psorergates desmanae, P. simplex, Psorergatoides rhinolophi

Germany (Lukoschus, Fain \& Beaujean, 1967; Lukoschus, de Cock \& Driessen, 1971; Heddergott \& Claussen, 2008) - Psorergates apodemi, P. muscardinus, P. zibethicalis, Psorergatoides nyctali, $P$. rhinolophi

Great Britain (Michael, 1889; Andrews, Baker, Fox \& Brizuela, 1997; Baker, 2005) - Psorergates musculinus, Psorergatoides nyctali, Psorobia bos

Holland (Fain, Lukoschus \& Hallmann, 1966; Lukoschus, Fain \& Beaujean, 1967; Lukoschus, 1968a, 1969; Lukoschus, de Cock \& Driessen, 1971; Giessen, 1990) - Psorergates agrestis, P. apodemi, P. crocidurae, P. dissimilis, P. micromydis, P. microti, P. neerlandicus, P. oeconomi, P. simplex, P. sorici, P. talpae; Psorobia mustelae

Italy (Piana, 1886; de Cock, Lukoschus \& Ariani, 1970) - Psorergates etruscus; Psorergatoides rhinolophi

Poland (Haitlinger, 1978, 1979b, 1986, 1987, 1997; Izdebska \& Fryderyk, 2012; Cierocka \& Izdebska, 2019) - Psorergates apodemi, P. dissimilis, P. microti, P. muricola, P. musculinus, $P$. olawaensis, $P$. polonicus, $P$. rattus, $P$. simplex, Psorergatoides kerivoulae

Romania (Haitlinger, 1980) - Psorergates apodemi

Russia (all) (Dubinin, 1953, 1955; Sosnina, 1970) - Psorergates apodemi, P. musculinus, P. simplex

Spain (cont.) (Lukoschus, Fain \& Beaujean, 1967; Lukoschus, 1967, 1968a; Lukoschus, de Cock \& Driessen, 1971; Zapatero Ramos et al., 1982) - Psorergates arvalis, P. auricola, P. callipidis, P. crocidurae, P. eliomydis [Ibiza], P. hispanicus, P. meati, P. pitymydis, $P$. quercinus, $P$. talpae; Psorergatoides rhinolophi

Sweden (Giesen, 1990) - Psorergates sp.

Switzerland (Sosnina, 1970) - Psorergates apodemi

Ukraine (Dubinin, 1955; Sosnina, 1970) - Psorergates apodemi, P. dissimilis

AFRICA

Angola (Giesen, 1990) - Psorergatoides rhinolophi 
Botswana (Giesen \& Lukoschus, 1982) - Psorergates paraxeri

Congo DR (formerly Zaire)(Fain, 1959a, 1959b, 1961; Fain, Lukoschus \& Hallmann, 1966; Lukoschus, Fain \& Beaujean, 1967; ) - Psorergates deomydis; Psorergatoides kerivoulae, $P$. nycteris, $P$. rhinolophi

Ivory Coast (Giesen, 1990) - Psorergatoides kerivoulae

Madagascar (Giesen, 1990) - Psorergatidae gen. sp.

Rwanda (Fain, 1959a) - Psorergatoides laviae, P. nycteris

South Africa (Lavoipierre, 1946; Fiedler \& du Toit, 1954; Zumpt \& Till, 1955; Till, 1960; Fain, 1965; Oberem \& Malan, 1983) - Psorergates cepapi, P. oettlei, P. paraxeri; Psorobia bos, $P$. cercopitheci, $P$. hystrici, $P$. ovis, $P$. zumpti

Tunisia (Giesen, Spicka \& Whitaker, 1985) - Psorobia elephantuli

ASIA

Burma (Giesen, 1990) - Psorergatoides rhinolophi

China (Giesen, 1990) - Psorergates crocidurae

Iran (Kamali, Ostovan \& Atamehr, 2001) - Psorergates simplex

Japan (Giesen \& Lukoschus, 1983) - Psorergates urotrichi

Malaysia (cont.) (Beck, 1971; Giesen, Lukoschus \& Nadchatram, 1982; Giesen, Nutting \& Nadchatram, 1989; Giesen \& Lukoschus, 1982) - Psorergates doriae, P. dremomydis, P. fritzi; Psorergatoides tadaridae

Sarawak and Sabah (Giesen \& Lukoschus, 1982) - Psorergates tupaiae; Psorergatoides kerivoulae

Singapore (Giesen, Nutting \& Nadchatram, 1989) - Psorergates fritzi

Taiwan (Lukoschus, Louppen \& Maa, 1974) - Psorergates squamipes

\section{NORTH AMERICA}

Canada (Tyrrell, 1883; Kok, Lukoschus \& Clulow, 1970, 1971; Giesen, Lukoschus, Whitaker \& Gettinger, 1983) - Psorergates canadensis, P. cinereus, P. peromysci, P. simplex, P. watsoni; Psorobia castoris

Mexico (Giesen, 1990) - Psorergates mexicanus

USA (Ah, Peckham \& Ateyo, 1973; Spicka, 1976; Wassel, Whitaker \& Spicka, 1980; Giesen, Lukoschus, Whitaker \& Gettinger, 1983; Giesen, Spicka \& Whitaker, 1985; Giesen \& Yunker, 1985;) - Psorergates glaucomys, P. mexicanus, P. peromysci, P. pinetorum, $P$. simplex, P. townsendi; Psorobia bos, P. lagomorphae, P. ovis

Hawaii Is. (Fain \& Goff, 1986) - Psorergatus rattus

\section{SOUTH and CENTRAL AMERICA}

French Guyana (Lukoschus, Louppen \& Fauran, 1979) - Psorergatoides desmodus, P. guyanensis, $P$. peropteryx

Panama (Giesen \& Lukoschus, 1983) - Psorergates cryptotis 
Suriname (Lukoschus, Rosmalen \& Fain, 1973) - Psorergatoides artibei, P. glossophagae, P. molossi, P. nidicicola, P. surinamensis

Venezuela (Fain, 1959b) - Psorergates sp., Psorergatoides lonchorhinae

\title{
OCEANIA
}

Australia (continent) (Womersley, 1941; Carter, 1941; Toop, 1948; Murray, 1961; Downing \& Mort, 1962; Sutherland, 1965; Giesen, Lukoschus \& Fain, Sinclair \& Girson, 1970; 1982; Sinclair, 1990; Domrow, 1991) - Psorergatoides australiensis; Psorobia ovis New Zealand (Whitten \& Elliott, 1956; Whitten, 1962; Pomroy, 1985) - Psorobia ovis Papua New Guinea (Fain, 1959b) - Psorergatoides emballonurae

\section{Hosts of Psorergatidae}

\author{
Mammalia \\ Soricomorpha \\ Fam. Soricidae \\ Anourosorex squamipes - Psorergates squamipes \\ Crocidura doriae - Psorergates [doriae] foetida \\ C. lasiura - Psorergates crocidurae \\ C. russula russula - Psorergates crocidurae \\ C. suaveolens - Psorergates olawaensis \\ Cryptotis n. nigrescens - Psorergates cryptotis \\ Microsorex koyi - Psorergates sp. \\ Neomys fodiens - Psorergates baueri \\ Notiosorex crawfordi - Psorergates mexicanus \\ Sorex araneus - Psorergates sorici \\ S. cinereus - Psorergates cinereus \\ Suncus etruscus - Psorergates etruscus
}

Fam. Talpidae

Galemys pyrenaicus - Psorergates desmanae

Neurotrichus gibbsi - Psorergates sp.

Talpa europaea europaea - Psorergates talpae

Urotrichus talpoides - Psorergates urotrichi

\section{Afrosoricida}

Fam. Tenrecidae

Macroscelidea

Echinops telfairi - Psorergatidae sp.

Fam. Macroscelididae

Elephantulus rozeti - Psorobia elephantuli 


\section{Scandentia}

Fam. Tupaiidae

Tupaia dorsalis - Psorergates tupaiae

T. glis - Psorergates fritzi

\section{Chiroptera}

Fam. Emballonuridae

Cormura brevirostris - Psorergatoides peropteryx

Mosia [Emballonura] nigrescens - Psorergatoides emballonurae

Peropteryx macrotis - Psorergatoides peropteryx

Saccopteryx canina - Psorergatoides lonchorhinae

S. bilineata - Psorergatoides indicicola

S. canescens - Psorergatoides indicicola

\section{Fam. Hipposideridae}

Hipposideros caffer centralis - Psorergatoides hipposideros

H. abae - Psorergatoides hipposideros

\section{Fam. Megadermatidae}

Lavia frons - Psorergatoides laviae

\section{Fam. Molossidae}

Molossus ater - Psorergatoides molossi

M. molossus - Psorergatoides molossi

Mops [Tadarida] mops - Psorergatoides tadaridae

\section{Fam. Nycteridae}

Nycteris macrotis - Psorergatoides nycteris

\section{Fam. Phyllostomatidae}

Artibeus lituratus fallase - Psorergatoides artibei

Desmodus rotundus - Psorergatoides desmodus

Glossophaga soricina - Psorergatoides glossophagae

Lonchorhina aurita - Psorergatoides lonchorhinae

Rhynophylla pumilio - Psorergatoides gyuanensis

Lophostoma [Tonatia] carrikeri - Psorergatoides surinamensis

Lophostoma brasiliense [Tonatia nicaraguae] - Psorergatoides surinamensis

\section{Fam. Rhinolophidae}

Rhinolophus aethiops - Psorergatoides rhinolophi

Rh. affinis - Psorergatoides rhinolophi

Rh. clivosus zuluensis - Psorergatoides rhinolophi

Rh. euryale - Psorergatoides rhinolophi

Rh. fumigatus - Psorergatoides rhinolophi

$R h$. hildebrandti - Psorergatoides rhinolophi

Rh. hipposideros - Psorergatoides rhinolophi

$R h$. mehelyi-Psorergatoides rhinolophi

\section{Fam. Vespertilionidae}


Kerivoula cuprosa - Psorergatoides kerivoulae

K. [harrisoni bellula] lanosa-Psorergatoides kerivoulae

Myotis bocagei - Psorergatoides kerivoulae

M. muricola - Psorergatoides kerivoulae

M. myotis - Psorergatoides kerivoulae

M. mystacinus - Psorergatoides kerivoulae

Nyctalus noctula - Psorergatoides nyctali

Nyctophilus arnhemensis - Psorergatoides australiensis

N. gouldi - Psorergatoides australiensis

N. walkeri - Psorergatoides australiensis

Plecotus auritis - Psorergatoides kerivoulae

Vespadellus [Eptesicus] pumilus - Psorergatoides australiensis

Vespadellus douglasorum [E. douglasi] - Psorergatoides australiensis

\section{Rodentia}

\section{Fam. Arvicolidae}

Arvicola terrestris - Psorergates simplex

Myodes [Clethrionomys] glareolus - Psorergates microti, P. dissimilis

M. [C.] gapperi atabascae - Psorergates sp.

M. [C.] gapperi proteus - Psorergates sp.

M. [C.] rufocanus - Psorergates sp.

M. [C.] rutilus - Psorergates sp.

Dicrostonyx groenlandicus - Psorergates sp.

Microtus agrestis bailloni - Psorergates agrestis

M. agrestis - Psorergates microti

M. arvalis - Psorergates simplex, $P$. dissimilis

M. arvalis meridianus - Psorergates arvalis

M. [Pitymys] duodecimcostatus flavescens - Psorergates auricola, P. pitymydis

M. nivalis - Psorergates sp.

M. oeconomus - Psorergates neerlandicus

M. oeconomus arenicola - Psorergates oeconomi

M. pennsylvanicus - Psorergates canadensis

M. [Pitymys] pinetorum - Psorergates pinetorum

M. townsendi-Psorergates townsendi

Microtus townsendi - Psorergates townsendi

Ondatra zibethica - Psorergates zibethicalis

Fam. Bathyergidae

Cryptomys hottentotus - Psorobia zumpti

Fam. Castoridae

Castor canadensis - Psorobia castoris

\section{Fam. Cricetidae}

Peromyscus maniculatus - Psorergates watsoni, P. peromysci 
P. leucopus - Psorergates peromysci

Reithrodontomys megalotis - Psorergates sp.

Sigmodon hispidus - Psorergates sp.

Fam. Gliridae (Myoxidae)

Eliomys quercinus - Psorergates pitymydis

E. quercinus ophiusae - Psorergates eliomydis

Muscardinus avellanarius - Psorergates muscardinus

\section{Fam. Heteromyidae}

Heteromys anomalus - Psorergates sp.

\section{Fam. Hystricidae}

Hystrix africaeaustralis - Psorobia hystrici

Fam. Muridae

Deomys ferrugineus chrystyi - Psorergates deomydis

Hybomys univittatus - Psorergates muricola

Lophuromys aquilus - Psorergates muricola

Micromys minutus soricinus - Psorergates micromydis

Mus musculus - Psorergates muricola, P. simplex

M. musculus commissarius - Psorergates hispanicus

M. spretus - Psorergates hispanicus

Otomys [irroratus elgonis] tropicalis - Psorergates muricola

Rattus norvegicus - Psorergates rattus

R. natalensis - Psorergates oettlei

Sylvaemus sylvaticus - Psorergates apodemi, P. muricola

S. sylvaticus callipides - Psorergates callipidis, P. meati

S. flavicollis - Psorergates apodemi, P. muricola

S. microps - Psorergates apodemi

S. uralensis - Psorergates apodemi

\section{Fam. Sciuridae}

Dremomys rufigenis - Psorergates dremomydis

Glaucomys v. volans - Psorergates glaucomys

Paraxerus cepapi - Psorergates paraxeri

\section{Lagomorpha}

Fam. Leporidae

\section{Carnivora}

Sylvilagus floridanus - Psorobia lagomorphae

\section{Fam. Mustelidae}

Martes foina - Psorobia foinae

Mustela erminea - Psorobia mustelae

M. nivalis - Psorobia mustelae

\section{Primates}

Fam. Cercopithecidae 
Cercopithecus aethiops pygerythrus - Psorobia cercopitheci

C. mona mona - Psorobia sp.

Macaca arctoides (USA, captured) - Psorobia sp.

\section{Artiodactyla}

\section{Fam. Bovidae}

Bos taurus - Psorobia bos

Ovis aries - Psorobia ovis

\section{Bibliography of Psorergatidae}

Ah Hyong-Sun, J. C. Peckman, W. T. Atyeo. 1973. Psorergates glaucomys sp. n. (Acari: Psorergatidae), a cystogenous mite from the southern flying squirrel (Glaucomys v. volans), with histopathological notes on a mite-induced dermal cyst. - J. Parasitol., 59(2): 369-374.

Andrews A.S. Baker, M. Fox, C. Brizuela. 1997. A cattle skin condition in Britain associated with the cattle itch mite Psorobia bos. - Veterinary Record, 140: 183.

Baker A. 2005. Psorergatoides nyctali (Prostigmata: Psorergatidae), a new mite species parasitizing the bat Nyctalus noctula (Mammalia: Chiroptera) in the British Isles. - Syst. \& Applied Acarology, 10: 67-74.

Baker A.S., A.H. Andrews, M.T. Fox. 1996. First record of the cattle itch mite, Psorobia bos (Prostigmata: Psorergatidae), in the Palaearctic Region. - Syst. \& Applies Acarology, 1: 213-216.

Beck A. J. 1971. A survey of bat ectoparasites in West Malaysia. - J. of Med. Entomology, 8(2): 147-152.

Bell D. S., W. D. Pounden, B. H. Edgington, O. G. Bentley. 1952. Psorergates ovis - a cause of itchiness in sheep. - J. Amer. Vet. Med. Assoc., 120: 117-120.

Beresford-Jones W. P., M. A. 1965. Occurrence of the mite Psorergates simplex in mice. - Austral. Vet. J., 41: 289-290.

Beron P. 1970. Sur quelques Acariens (Myobiidae, Psorergatidae, Spinturnicidae, Sarcoptidae et Listrophoroidea) de Bulgarie et de l'île de Crète. - Bull. Inst. Zool., Sofia, 32: 143-149.

Beron P. 1973. Catalogue des Acariens parasites et commensaux des Mammifères en Bulgarie. - Bull. Inst. Zool. Mus. Sofia, 37: 167-199.

Canestrini G. 1894a. Prospetto dell'Acarofauna italiana. Parte VI. Famiglia dei Psoroptidi (Psoroptidae) pendice ai Fitoptidi italiani. Varia: Psorergates, Hemisarcoptes, Histiogaster, Psoroptes ovis, Otodectes furonis. Gli Epidermoptini. - Padova, Stabilimento Prosperini.

Canestrini G. 1894b. Varia. Psorergates, Hemisarcoptes, Histiogaster. Appendice ai psoroptidi. - Prospetto dell'Acarofauna Italiana: 809-812.

Carter H. B. 1941. A skin disease of sheep due to an ectoparasitic mite, Psorergates ovis Womersley, 1941. - Austr. Vet. J., 17: 193-201.

Cierocka K., J. Izdebska. 2019. Psorergatidae mite infestation in the brown rat Rattus norvegicus (Rodentia, Muridae): the first record of Psorergates rattus (Acariformes, Prostigmata) in Europe. - Turkish Journal of Zoology, 43: 314 -317. 
Cock De A. W. A. M., Lukoschus F. S., A. P. Ariani. 1970. Psorergates etruscus sp. n. (Acari: Psorergatidae), a new itch mite from Suncus etruscus (Soricidae). - Ann. Ist. Mus. Zool. Univ. Napoli, 19(4): 1-9.

Cook R. 1956. Murinae ear mange: The control of Psorergates simplex infestation. - Brit. Vet. J., $112(1): 22-25$.

Davis J. W. 1954. Studies of the sheep mite, Psorergates ovis. - Amer. J. Vet. Res., 15: 255-257. Domrow R. 1991. Acari Prostigmata (excluding Trombiculidae) parasitic on Australian vertebrates: an annotated checklist, keys and bibliography. - Invertebr. Taxon., 4: 1283-1376.

Downing W., P. Mort. 1962. Experiments in the control of the itch mite, Psorergates ovis, Womersley 1941. Part 1 and 2. - Austr. Vet. J., 38: 77-85 and 269-274.

[Dubinin W. В.] В.Б. Дубинин. 1953. [The parasitofaune of the rodents and its changement in the delta of Volga] Паразитофауна мышевидных грызунов и ее изменения в дельте Волги. - Parazitologicheskiy sbornik, 15: 252-301 (in Russian).

Dubinin W. 1955. Acariens de rongeurs d'U. R. S. S. II. Psorergatidae. - Trav. Acad. Sci. U. R. S. S., Zool., 59: 150-152 (In Russian).

Dubinin W. 1957. New classification of the mites of the superfamilies Cheyletoidea W. Dub. and Demodicoidea W. Dub. (Acariformes, Trombidiformes). - Mag. Parasit., 17: 103-131.

Fain A. 1959a. Les Acariens psoriques parasites des Chauves-souris. III. Le genre Psorergates Tyrrell (Trombidiformes-Psorergatidae). - Bull. et Ann. Soc. R. entomol. Belg., 95(1-4): 54-69.

Fain A. 1959b. Les Acariens psoriques parasites des Chauves-souris. IX. Nouvelles observations sur le genre Psorergates Tyrrell. - Bull. et Ann. Soc. R. entomol. Belg., 95(7-8): 232-248.

Fain A. 1961. Notes sur le genre Psorergates Tyrrell. Description de Psorergates ovis Womersley et d'une espèce nouvelle. - Acarologia, 3(1): 60-71.

Fain A. 1965. Sur un cas de gale chez un rat-taupe (Cryptomys hottentotus) produite par un acarien du genre Psorergates (Psorergatidae: Trombidiformes). - Acarologia, 7: 295-300.

Fain A., M.L. Goff. 1986. Psorergates rattus (Acari: Psorergatidae), a new species of parasitic mite from Rattus norvegicus in Hawaii. - Intern. J. Acarol., 12(2): 107-110.

Fain A., F. Lukoschus. 1968. Psorergates (Psorobia) foinae sp. n., acarien producteur de gale chez la foine en Belgique. - Bull. Inst. r. Sci. nat. Belg., 44 (14): 1-6.

Fain A., F. Lukoschus, P. Hallmann. 1966. Le genre Psorergates chez les Muridés. Description de trois espèces nouvelles (Psorergatidae: Trombidiformes). - Acarologia, 8(2): 251-274.

Fain A., F. S. Lukoschus, G. Rack. 1973. Notes on parasitic mites from some small mammals in Liberia. - Mitt. Hamburg Zool. Mus. Inst., 71: 165-174.

Fiedler O. G. H., R. du Toit. 1954. Australian Itch. A note on its occurence in South Africa. - J. S. Afr. Vet. Med. Assoc., 25(2): 21-22.

Flynn R. J. 1959. Follicular acariasis of mice caused by Psorergates simplex successfully treated with aramite. - Amer. J. Vet. Res., 20: 198-200.

Flynn RJ, B.H. Jaroslow. 1956. Nidification of a mite (Psorergates simplex Tyrrell, 1883: Myobiidae) in the skin of mice. - J. Parasitology, 42: 49-52.

Gerlach A. C. 1857. Krätze und Räude, entom. und klin. bearbeitet. - Berlin, 1(1), plate 8, fig. 44. 
Giesen K. M. T. 1990. A review of the parasitic mite family Psorergatidae (Cheyletoidea: Prostigmata: Acari) with hypotheses on the phylogenetic relationships of species and species group. - Zool. Verhandelingen, 259, 69 pp.

Giesen K. M. T., F. S. Lukoschus. 1982a. A new species of the genus Psorergates Tyrell, 1883 (Acarina: Prostigmata: Psorergatidae) parasitic on tree-shrew Tupaia dorsalis (Mammalia: Scandentia) from Borneo. - Zool. Mededel., 56 (20): 259-266.

Giesen K. M. T., F. S. Lukoschus. 1982b. A new itch mite (Acarina: Prostogmata: Psorergatidae) from the South African bush squirrel Paraxerus cepapi. - Bull. Inst. r. Sci. nat. Belg., 53(18): 1-9.

Giesen K. M. T., F. S. Lukoschus. 1983. Two new species of the genus Psorergates Tyrell, 1883 (Acarina: Prostigmata: Psorergatidae) parasitic on Insectivores, with a key to the known species from Insectivora hosts. - Acarologia, 24 (4): 403-415.

Giesen K. M. T., F. S. Lukoschus, A. Fain. 1982. Parasites of Western Australia. XV. A new species of Psorergatoides (Acarina: Psorergatidae) from Australian bats. - Rec. West. Aust. Mus., 9(4): 315-323.

Giesen K. M. T., F. S. Lukoschus, M. Nadchatram. 1982. Three new itch mites of the family Psorergatidae Dubinin, 1955 (Acarina, Prostigmata) from Malaysian mammals. - Malay. Nat. J., 35: 315-328.

Giesen K. M. T., F. S. Lukoschus, J. O. Whitaker Jr., D. Gettinger. 1983. Four new species of itch mites (Psorergatidae: Prostigmata) from small mammals in North America. - J. Med. Entomol., 20(2): 164-173.

Giesen K. M. T., W. B. Nutting, M. Nadchatram. 1989. A new mite species of the genus Psorergates Tyrrell (Acari: Prostigmata: Psorergatidae) parasitic on the tree shrew Tupaia glis from Malaysia and Singapore, with notes on pathology. - Raffles Bull. of Zool., 37(1-2): $132-141$.

Giesen K. M. T., B. M. O'Connor. (in press.) Phylogenetic and coevolutionary patterns in the genus Psorergates (Acarti: Psorergatidae), with description of a new species from Hodomys alleni (Rodentia: Muridae). - Submitted to Systematic Parasitology.

Giesen K. M. T., E. J. Spicka, J. O. Whitaker Jr. 1985. Two new species of the genus Psorobia, Fain 1959 (Psorergatidae: Prostigmata) from two new host orders, Lagomorpha and Insectivora. - Acarologia, 26(2): 147-153.

Giesen K. M. T., C. E. Yunker. 1985. Psorobia bos Johnston, 1964. The cattle itch mite. Description of the male (Acari: Psorergatidae). - Acarologia, 26(1): 37-41.

Gill D., R. W. Strandtmann. 1977. Ectoparasites of the Collared lemming (Dicrostonyx torquatus) on Bathurst Island, N. W. T., Canada. - J. Med. Entomol., 14(1): 101-106.

Graham N. P. H. 1943. Some observations on the bionomics of the itch mite (Psorergates ovis) of sheep and its control with lime-sulphur dips. - J. Counc. Scient. \& Indust. Res., Australia, 16: 206-214.

Graham N. P. H. 1959. The control of itch mite (Psorergates ovis) in sheep. - The Australian Veterinary Journal, April: 153 
Grigorjeva L.A. 2007. Peculiarities of the skin lesions in small mammals parasitized by Psorergates apodemi and $P$. dissimilis (Cheyletoidea: Psorergatidae). - Parazitologiya (St. Petersburg), 41(3): 235-239.

Haitlinger R. 1978. Psorergates dissimilis Fain, Lukos., Hallm. and Psorergates apodemi Fain, Lukos., Hallm. (Psorergatidae: Acarina) two new mites species for fauna of Poland. Przegląd Zoologiczny, 22(2): 143-146.

Haitlinger R. 1979a. Pasożyty zewnętrzne nietoperzy Dolnego Śląnska V. Trombidiformes, Sarcoptiformes (Acarina). - Wiadomości paraz., 25(1): 105-118.

Haitlinger R. 1979b. Pasożyty zewnętrzne nietoperzy Dolnego Śląnska VI. Acarina, Siphonaptera, Diptera (Nycteribiidae). - Wiadomości paraz., 25(1): 119-140.

Haitlinger R. 1980. Stawonogi (Siphonaptera, Anoplura, Coleoptera, Acarina) zebrane z drobnych ssaków Rumunii. - Wiadomosci Parazytol., 26(6): 679-710.

Haitlinger R. 1982. Acarina (Myobiidae, Cheyletidae, Pygmephoridae, Trombiculidae, Dermanyssidae) nowe lub rzadkie w faunie Polski. - Wiadomości parazitol., 28(3-4): 435-444.

Haitlinger R. 1986. Psorergates polonicus sp. n. (Acari, Prostigmata, Psorergatidae) from Pitymys subterraneus (de Sel. Longch.). - Bull. Ent de Pologne, 56: 425-426.

Haitlinger R. 1987a. Psorergates olawaensis sp.n. (Acari, Prostigmata, Psorergatidae) from Crocidura suaveolens (Pall.). - Bull. Ent. de Pologne, 57: 539-540.

Haitlinger R. 1987b. Trichoecius apodemi Fain, Munting, Lukoschus, 1969 and some mites species (Myocoptidae, Myobiidae, Psorergatidae, Haemogamasidae) new for the fauna of Poland. - Wiadomości Parazytologiczne, 33 (1): 81-83.

Haitlinger R. 1997. Arthropods communities occuring on small mammals from wooded areas of urban agglomeration of Wrocław. - Zeszyty naukowe Akademii rolniczej we Wrocławiu, Zootechnika XLIII, 323: 47-64.

Heddergott M., A. Claussen. 2008. Kommentierte Checklist der Milben der Gattung Psorergatoides Thueringens (Acari). Check - Listen Thueringer Insekten, 16: 57-58.

Izdebska J. N., S. Fryderyk. 2012. New for the fauna of Poland species of Psorergates spp. with the data of occurrence of mites from Psorergatidae family (Acari, Prostigmata) in native mammals. - Annals of Parasitology, 58: 19-22.

Johnston D. E. 1964. Psorergates bos, a new mite parasite of domestic cattle (Acari: Psorergatidae). - Ohio Agric. Exper. Sta. Res. Circ., 129: 3-7.

Kok N. J. J., F. S. Lukoschus, F. V. Clulow. 1970. Psorobia castoris spec. nov. (Acarina: Psorergatidae), a new itch mite from the beaver Castor canadensis. - Can. J. Zool., 48: 1419-1423.

Kok N. J. J., F. S. Lukoschus, F. V. Clulow. 1971. Three new itch mites from Canadian small mammals (Acarina: Psorergatidae). - Can. J. Zool., 49(9): 1239-1248.

Lavoipierre M. M. J. 1946. New records of Acari from Southern Africa and the Belgian Congo. - J. ent. Soc. S. Afr., 9: 79-81.

Lavoipierre M. M. J., W. Crewe. 1955. The occurrence of a mange-mite Psorergates sp. (Acarina), in a West African monkey. - Ann. Trop. Med. \& Parasitol., 49: 351.

Lee K.J., C.M. Lang, H.C. Hughes, R.D. Hartshorn. 1981. Psorergatic mange (Acari: Psorergatidae) of the stumptail macaque (Macaca arctoides). - Lab. Anim. Sci., 31(1): 77-79. 
Lukoschus F. S. 1967. Krätzmilben an spanischen Kleinsäugern (Psorergatidae: Trombidiformes). - Rev. Iber. Parasitol., 27(3-4): 203-224.

Lukoschus F. S. 1968a. Neue Krätzmilben von einheimischen Insektivoren (Psorergatidae: Trombidiformes). - Tijdschr. v. Entomol., 111(3): 75-88.

Lukoschus F. S. 1968b. Psorergates desmanae sp. n., eine neue Krätzmible von Galemys pyrenaicus (Psorergatidae: Trombidiformes). - Bull. Mus. Nat. Hist. Nat., Paris, 40(1): 125-131.

Lukoschus F. S. 1969. Psorergates (Psorobia) mustelae spec. nov. eine neue Krätzmilben von Mustela nivalis L. (Acarina: Psorergatidae). - Zool. Anz., 183 (1-2): 110-118.

Lukoschus F. S., A. W. A. M. De Cock, F. M. Driessen. 1971. Four new species of the genus Psorergates Tyrell from European hosts (Acarina, Psorergatidae). - Tijdschr. Entomol., 114(4): 185-200.

Lukoschus F. S., A. Fain, M. M. J. Beaujean. 1967. Beschreibung neuer Psorergates-Arten (Psorergatidae: Trombidiformes). - Tijdschr. V. Entomol., 110(7): 133-181.

Lukoschus F. S., J. M. W. Louppen, P. Fauran. 1979. Parasitic mites of Surinam, XIV. New observations on the genus Psorergatoides Fain, 1959 (Psorergatidae: Trombidiformes), with the key to the known species. - Intl. J. Acar., 5: 311-324.

Lukoschus F. S., J. M. W. Louppen, T. C. Maa. 1974. Psorergates squamipes, n. sp. (Acari: Psorergatidae), a skin mite from Anourosorex squamipes (Insectivora) in Taiwan. - Pacific Insects, 16: 51-56.

Lukoschus F. S., P. G. Rosmalen, A. Fain. 1973. Parasitic mites of Surinam, XI. Four new species of the genus Psorergatoides Fain, 1959 (Psorergatidae: Trombidiformes). - Tijdschr. Ent., 116: 63-81.

Meleney W. P., N. M. Albuquerque. 1958. A case of experimentally induced Psoroptic Acariasis fatal to a rabbit. - Unpublished data.

Michael A. D. 1889. On some unrecorded parasitic Acari found in Great Britain. - J. Linn. Soc. (Zool.) London, 20: 400-406.

Murray M. D. 1961. The life cycle of Psorergates ovis Womersley, the itch mite of sheep. Austral. J. Agric. Res., 12: 965-973.

Neuman M. G. 1893a. Note preliminaire sur le Psorergates simplex, acarien parasite de la souris. - Compt. rend. Soc. Biol., 330.

Neuman M. G. 1893b. Sur un acarien (Psorergates simplex Tyrrell) de la souris. - Bull. Soc. Hist. Natur. Toulouse., 27: 13-22.

Oberem P.T., F.S. Malan. 1983. First record of the mite, Psorergates bos, (Acari: Sarcoptiformes) on cattle in South Africa. - Proc. Congr. Ent. Soc. Sth. Afr., 4: 63.

Oudemans A.C. 1906. Révision des Chélétinés. - Mém. Soc. Zool. de France, 19: 36-144.

Piana G. P. 1886. Cisti cutanee contenenti acari nei topi. Annuario p. anno scolastico 1885-86. - R. Scuola Sup. di Med. Vet. Milano: 122.

Pomroy W. E. 1985. Mites. Itch mite (Psoregates ovis), sheep scab (Psoroptes ovis). In: Charleston, W.A. G., ed. Ectoparasites of sheep in New Zealand their control. - Palmerston North, The Sheep and Beef Cattle Society of the New Zealand Veterinary Association. Pp. 18-22. Raulston G.L.1972. Psorergatic mites in Patas monkeys.- Lab. Anim. Sci., 22: 107-108. 
Rioux J. A., Y. J. Golvan. 1961. Gale a Psorergates musculinus (Michael, 1889) (Acari: Myobiidae) chez les mulots Apodemus sylvaticus (Linné 1758) de massif de caroux (Herault). - Ann. Parasitol. Hum. pom., 36: 785-787.

Roberts I. H., W. P. Meleney. 1965. Psorergatic Acariasis in Cattle. - J. Amer. Vet. Med. Assoc., 146(1): 17-23.

Scott M. T. 1949. The failure of benzene hexachloride to control itch mite (Psorergates ovis). - Austral. Vet. J., 25: 300-301.

Seier J.V. 1985. Psorergatic acariasis in vervet monkeys. - Laboratory Animals, 19: 236-239.

Sheldon W. S. 1966. Psorergatic mange in the sooty mangabey (Cercocebus torquatus atys) monkey. - Lab. Animal. Care, 16: 276-279.

Sinclair A.N., A.J.F. Girson. 1970. Distribution of the itch mite (Psorergates ovis) on some merino sheep. - Australian Vet. Journal, 46.

Sinclair A. 1990. The epidermal location and possible feeding site of Psorergates ovis, the sheep itch mite. - Aust. Vet. J., 67(2): 59-62.

Skerman K.D., N.P.H. Graham, A.N. Sinclair, M.D. Murray (statement). 1960. Psorergates ovis - the itch mite of sheep. - Australian Veterinary Journal, July: 317

Sosnina E. F. 1970. On mites of the genus Psorergates (Trombidiformes: Psorergatidae) - parasites of Muridae and Cricetidae in the USSR. - Parazitologiya, 4(6): 537-541 (in Russian, summ. Engl.).

Spicka E. J. 1976. First report of Psorergates simplex Tyrrell, 1883 (Acari: Psorergatidae) from wild house mouse, Mus musculus, in the United States. - Proc. Indiana Acad. Science 1975, 85: 418-422.

Till W. M. 1957. Two new parasitic mites (Acarina) from the South African porcupine. Parasitology, 47(3-4): 329-334.

Till W. M. 1960. Psorergates oettlei n. sp., a new mange-causing mites from the multimammate rat (Acarina, Psorergatidae). - Acarologia, 2(1): 75-70.

Toop C. R. 1948. The sheep itch mite (Psorergates ovis). - J. Dept. Agric. W. Aust., 25(4): 343-347.

Turk F.A. 1953. I. - A synonymic catalogue of British Acari: Part I. - Ann. Mag. Nat. Hist., $12^{\text {th }}$ Ser., 61: 1-26.

Tyrrell J.B. 1883. On the occurrence in Canada of two species of parasitic mites. - Proc. Canad. Inst. Toronto N.S., 1: 342-343.

Wassel M.E., J.O. Whitaker, E.J. Spicka. 1980. The ectoparasites and other associates of the Cottontail Rabbit, Sylvilagus floridanus, in Indiana. - Proc. Indiana Acad. Sci., Zoology, 89: 418-420.

Whitten L.K. 1962. Parasitic mites of domestic animals in New Zealand. - New Zealand Entomologist, 3(1): 9-12.

Whitten L. K., D. C. Elliott. 1956. A note on the occurrence of the itch mite, Psorergates ovis Womersley, 1941, on New Zealand sheep. - New Zealand Vet. J., 4: 19.

Womersley H. 1941. Notes on the Cheyletidae (Acarina, Trombidoidea) of Australia and New Zealand with descriptions of new species. - Rec. of the S. Austral. Mus., 7: 51-64.

Zumpt F., W. M. Till. 1955. The mange-causing mites of the genus Psorergates (Acarina: Myobiidae) with description of a new species from a South Africain monkey. - Parasitology, 45: $269-274$. 


\title{
The Mite Family Demodecidae Nicolet
}

\author{
Demodecidae Nicolet, 1855: 383
}

The history of the study of Demodecidae starts with Owen (1843), who described the genus Demodex. Hirst (1919) published a comprehensive review of Demodex - the only known genus and mentioned that already there are more than 230 publications on the group, mostly on veterinary cases (D. canis, etc.). Nutting (1964) added information about 650 more titles, and since there have been hundreds of publications - the literature on Demodecidae is well over 1000 titles. Most of them are of little taxonomical or host - related interest and represent case reports. After Hirst (1918a, 1918b, 1919, 1921, 1923) particularly important were the contributions of Fain $(1959,1960)$ who described the new genera Rhinodex and Stomatodex and 4 of the valid species in the family. Banks (1915) and Dubinin (1958) raised the superfamily Demodicoidea, but this arrangement was not followed. Among the acarologists having contributed to the taxonomy of Demodecidae should be noted C.E. Desch (since 1969), W.B. Nutting (since 1950), V. Bukva (since 1982, described the genera Apodemodex and Soricidex), Lukoschus and co-authors (described the genera Ophthalmodex and Pterodex) and Izdebska and co-authors (since 2000), described the genus Glossicodex and many new species.

According Bochkov, 2009b, this family includes 100 sp., including 86 valid sp. of Demodex. Izdebska \& Rolbiecki (2020) presented a checklist containing 122 valid species of Demodecidae. We have listed here 122 species of Demodecidae, six species are not described according to ICZN and two are nomina nuda. They live in the skin glands of mammals (32 families and 14 orders, fide Bochkov, 2009b). The representatives of Ophthalmodex live in the epithelium of the eyelids, cornea, and lacrimal canals of bats (Hipposideridae, Vespertilionidae, Molossidae, Phylostomatidae) and rodents (Muridae). The Stomatodex prefer the oral cavity and esophagus of bats, rodents and primates. The Rhinodex have been found in the nose cavity of Galagidae (Primates). The small genera Soricidex, Apodemodex and Pterodex inhabit the skin follicles or the epithelium of Soricidae, Muridae and Pteropodidae (fruit bats). Most of the demodecids are monoxenous parasites. Some are important for the animal health, causing demodectic mange, and even to humans (Demodex folliculorum, D. brevis).

Here efforts were made to include in the bibliography most papers containing taxonomic and faunistic information and some of the medical papers (others could be seen in the bibliography of Izdebska \& Rolbiecki, 2020). 


\section{Composition of the family Demodecidae}

Fam. Demodecidae Nicolet, 1855 - 8 genera, 122 species

Apodemodex Bukva, 1996 - $1 \mathrm{sp}$.

Demodex Owen, 1843 - 106 sp.

Glossicodex Izdebska et Rolbiecki, 2016 - 1 sp.

Ophthalmodex Lukoschus et Nutting, 1979 - 7 sp.

Pterodex Lukoschus, Woeltjes, Desch et Nutting, 1980 - 1 sp.

Rhinodex Fain, 1959 - 1 sp.

Soricidex Bukva, 1982 - $1 \mathrm{sp}$.

Stomatodex Fain, 1959 - 4 sp.

\section{Catalogue of Demodecidae}

Fam. Demodecidae Nicolet

Demodicidae Owen, 1843

Demodecidae Nicolet, 1855: 383

Demodicoidea Banks, 1915 (superfamily)

Demodicoidea Dubinin, 1954: 73; 1955: 141; 1957: 124 (superfamily)

Type genus: Demodex Owen, 1843

Genus Apodemodex Bukva

Apodemodex Bukva, 1996: 312

Type species: Apodemodex cornutus Bukva, 1996

Apodemodex cornutus Bukva

Apodemodex cornutus Bukva, 1996: 312; Izdebska \& Rolbiecki, 2020: 10

Type locality: České Budějovice, South Bohemia

Distribution: Czech Rep.

Host: Neomys anomalus (Soricomorpha: Soricidae)

Genus Demodex Owen

Demodex Owen, 1843

Type species: Acarus folliculorum Simon, 1842

Demodex acutipes Bukva et Preisler

Demodex acutipes Bukva \& Preisler, 1988: 69; Izdebska \& Rolbiecki, 2020: 10

Type locality: Czech Rep.

Distribution: Czech Rep., Poland 
Host: Cervus elaphus (Artiodactyla: Cervidae)

\section{Demodex aelleni Fain}

Demodex aelleni Fain, 1960: 81; Izdebska \& Rolbiecki, 2020: 10

Type locality: Geneva

Distribution: Switzerland

Host: Myotis daubentoni (Chiroptera: Vespertilionidae)

\section{Demodex agrarii Bukva}

Demodex agrarii Bukva, 1994: 306; Izdebska \& Cydzik, 2010: 59; Izdebska \& Rolbiecki, 2020: 10

Type locality: Kokšov-Bakša, Eastern Slovakia

Distribution: Poland, Slovakia

Host: Apodemus agrarius (Rodentia: Muridae)

Demodex ailuropodae $\mathrm{Xu}$, Xie, Liu, Zhou et Shi

Demodex ailuropodae Xu, Xie, Liu, Zhou \& Shi, 1986: 163; Izdebska \& Rolbiecki, 2020: 10

Type locality: Shanghai Zool. Garden

Distribution: China

Host: Ailuropoda melanoleuca (Carnivora: Ailuropodidae)

Demodex antechini Nutting et Sweatman

Demodex antechini Nutting \& Sweatman, 1970: 425; Domrow, 1991: 1339; Desch \& Holz, 2006: 81; Izdebska \& Rolbiecki, 2020: 10

Type locality: Canberra

Distribution: Australia

Hosts: Antechinus agilis, A. stuartii (Dasyuromorphia: Dasyuridae, Dasyuridae)

\section{Demodex apodemi Hirst}

Demodex apodemi Hirst, 1918: 145; 1919: 35; Izdebska, 2012: 1101; Izdebska \& Rolbiecki, 2020: 10

Demodex arvicolae var. apodemi Hirst: Dubinin, 1953: 276

Demodex arvicolae apodemi Hirst: Izdebska \& Cydzik, 2010: 59

Type locality: England

Distribution: England, Poland, Russia

Hosts: Apodemus agrarius, Sylvaemus sylvaticus (Rodentia: Muridae). "Specimens on Apodemus agrarius probably belong to the separate species" (Izdebska et al., 2020).

\section{[Demodex araneae Nutting}

Demodex araneae Nutting, 1950: Thesis; Izdebska \& Rolbiecki, 2020: 10

Type locality: laboratory 
Distribution: South America

Host: Ateles sp. (Primates: Atelidae). Note by Izdebska et al., 2020: "Nom. nud.; description not published within the meaning of the ICZN"].

\section{Demodex aries Desch}

Demodex aries Desch, 1986: 367; Bukva, 1990: 82; Domrow, 1991: 1343 (synonymised the species with Demodex ovis Railliet, 1893); Izdebska \& Rolbiecki, 2020: 11

Type locality: Wellington City (New Zealand)

Distribution: Czech Rep., New Zealand

Host: Ovis aries (Artiodactyla: Bovidae)

Demodex artibei Vargas, Bassols, Desch, Quintero et Polaco

Demodex artibei Vargas, Bassols, Desch, Quintero \& Polaco, 1995: 75; Izdebska \& Rolbiecki, 2020: 11

Type locality: Mexico

Distribution: Mexico

Host: Artibeus aztecus (Chiroptera: Phyllostomatidae)

\section{Demodex arvicolae Zschokke}

Demodex arvicolae Zschokke, 1888: 69; Dubinin, 1953: 276; Izdebska \& Rolbiecki, 2020: 11 Type locality: Europe

Distribution: England, Poland, Russia

Hosts: Microtus arvalis (?), M. agrestis, Mus musculus (?)(Rodentia: Arvicolidae)

\section{Demodex aurati Nutting}

Demodex aurati Nutting, 1961: 515; Sarashina \& Sato, 1986: 619; Izdebska \& Rolbiecki, 2020: 11

Type locality: laboratory Golden Hamsters

Distribution: from laboratory animals

Host: Mesocricetus auratus (Rodentia: Cricetidae)

Demodex auricularis Izdebska, Rolbiecki et Fryderyk

Demodex auricularis Izdebska, Rolbiecki \& Fryderyk, 2014: 215; Izdebska \& Rolbiecki, 2020: 11

Type locality: Poland

Distribution: Poland

Host: Sylvaemus sylvaticus (Rodentia: Muridae)

Demodex bandicotae Izdebska, Rolbiecki, Morand et Ribas

Demodex bandicotae Izdebska, Rolbiecki, Morand \& Ribas, 2017; Izdebska \& Rolbiecki, 2020: 11 
Type locality: Laos

Distribution: Laos

Host: Bandicota indica (Rodentia: Muridae)

Demodex bantengi Firda, Nutting et Sweatman

Demodex bantengi Firda, Nutting \& Sweatman, 1987: 227; Izdebska \& Rolbiecki, 2020: 11 Type locality: Indonesia (Bali)

Distribution: Indonesia

Host: Bos javanicus (Artiodactyla: Bovidae)

Demodex bicaudatus Kniest et Lukoschus

Demodex bicaudatus Kniest \& Lukoschus, 1981: 111; Domrow, 1991: 1339; Izdebska \& Rolbiecki, 2020: 11

Type locality: Mitchell Plateau

Distribution: Australia

Host: Macroglossus minimus [lagochilus] (Chiroptera: Pteropodidae)

Demodex bisonianus Kadulski et Izdebska

Demodex bisonianus Kadulski \& Izdebska, 1996: 103; Izdebska, 2006: 171; Izdebska \& Rolbiecki, 2020: 11

Type locality: Prevalence

Distribution: Poland

Host: Bison bonasus (Artiodactyla: Bovidae, Bovinae)

\section{[Demodex bonapartei Nutting}

Demodex bonapartei Nutting, 1950: Thesis; Izdebska \& Rolbiecki, 2020: 11.

Type locality: ?

Distribution: North America (North and East of the Great Lakes)

Host: Mustela erminea cicognanii (Carnivora: Mustelidae). Note by Izdebska \& Rolbiecki, 2020: "Nom. nud.; description not published within the meaning of the ICZN"].

\section{Demodex bovis Stiles}

Demodex folliculorum: Faxon, 1878: 11

Demodex folliculorum var. bovis Stiles, 1892: 289; Railliet, 1893: 638; Cleland, 1913: 591

Demodex bovis Stiles: Canestrini \& Kramer, 1899: 3; Hirst, 1919: 28; 1921: 371; Freire \& Dias, 1947: 247; Desch \& Nutting, 1971; 1973: 500; Esuruoso, 1977: 65; Tashiro et al., 1979: 30; Noorudin \& Rahman, 1985; Matthes \& Bukva, 1993; Candona, Vargas \& Perdomo, 2013; Izdebska \& Rolbiecki, 2020: 12.

Type locality: ?

Distribution: cosmopolitic

Hosts: Bos taurus, Bos taurus indicus, accid. Bison bonasus in farm (Artiodactyla: Bovidae, Bovinae) 


\section{Demodex brevis Akbulatova}

Acarus foliculorum Simon, 1842: 231 (partim, fig. 3, 4)

Demodex folliculorum brevis Akbulatova, 1963: 40

Demodex brevis Akbulatova: Desch \& Nutting, 1972: 171; László \& Zoltán, 1973: 55; Nutting \& Green, 1974: 10; 1976: 307; Hallas, 1978: 30; English \& Nutting, 1981: 362; Andrews, 1988: 97; Tenquist \& Charleston, 2001: 495; Özdemir, 2003; Zeytun \& Karakurt, 2019: 2; Izdebska \& Rolbiecki, 2020: 12

Type locality: Russia

Distribution: cosmopolitan

Host: Homo sapiens (Primates: Hominidae)

Demodex buccalis Bukva, Vítovec et Vlček

Demodex buccalis Bukva, Vítovec \& Vlček, 1985: 158; Izdebska \& Rolbiecki, 2020: 12

Type locality: South Bohemia

Distribution: Czech Rep., Poland

Host: Myodes [Clethrionomys] glareolus (Rodentia: Arvicolidae)

\section{Demodex caballi (Railliet)}

Demodex folliculorum var. equi Railliet, 1895

Demodex equi Railliet, 1895: Gmeiner, 1908

Demodex caballi (Railliet): Izdebska \& Rolbiecki, 2020: 12

Type locality: Europe

Distribution: USA, New Zealand (cosmopolitan?)

Host: Equus caballus (Perissodactyla: Equidae)

\section{Demodex cafferi Nutting et Guilfoy}

Demodex cafferi Nutting \& Guilfoy, 1979: 9; Dräger \& Paine, 1980: 521; Izdebska \& Rolbiecki, 2020: 12

Type locality: South Africa

Distribution: South Africa

Host: Syncerus caffer (Artiodactyla: Bovidae, Bovinae)

\section{Demodex canis Leydig}

Demodex canis Leydig, 1859: 345; Canestrini \& Kramer, 1899: 2; Hirst, 1919: 3; Cooreman, 1946: 2; Starkoff \& Starkoff, 1950: 92; Roberts, 1952: 189; Desch \& Nutting, 1973: 501; Nutting, 1976: 214; Nutting \& Desch, 1978: 140; Desch \& Hillier, 2003: 148; Domrow, 1991: 1340; Izdebska \& Rolbiecki, 2020: 12, many other authors

Demodex folliculorum var. canis: Railliet, 1893: 635

Demodex folliculorum var. caninus Mégnin, 1877: 111

Demodex caninus: Canestrini, 1899: 23; Hirst, 1917: 232

Type locality: Europe

Distribution: cosmopolitic 
Host: Canis lupus familiaris (Carnivora: Canidae)

Demodex caprae Railliet

Demodex folliculorum var. caprae: Railliet, 1893; 1895: 638; Tenquist \& Charleston, 2001: 495

Demodex caprae: Canestrini \& Kramer, 1899: 3; Hirst, 1919: 38; Cooreman, 1946: 2; Dubinin, 1957: 132; Desch \& Nutting, 1973: 501; Lebel \& Nutting, 1973b: 517; Nutting, 1976: 214; Lebel \& Desch, 1979; Tsedev \& Grebenyuk, 1975: 127; Domrow, 1991: 1341; Izdebska \& Rolbiecki, 2020: 12

Type locality: Europe

Distribution: range of host

Host: Capra hircus (Artiodactyla: Bovidae, Caprinae)

Demodex carolliae Desch, Lebel, Nutting et Lukoschus

Demodex carolliae Desch, Lebel, Nutting \& Lukoschus, 1971: 303; Izdebska \& Rolbiecki, 2020: 13

Type locality: Lelydorp (Suriname)

Distribution: Suriname

Host: Carollia perspicillata (Chiroptera: Phyllostomatidae)

Demodex castoris Izdebska, Fryderyk et Rolbiecki

Demodex castoris Izdebska, Fryderyk \& Rolbiecki, 2016: 1; Izdebska \& Rolbiecki, 2020: 13

Type locality: Poland

Distribution: Poland

Host: Castor fiber (Rodentia: Castoridae)

\section{Demodex cati Mégnin}

Demodex folliculorum var. cati Mégnin, 1877: 112; Railliet, 1893: 637

Demodex cati: Canestrini \& Kramer, 1899: 3; Hirst, 1919: 26; Dubinin, 1957: 132; Nutting, 1976: 214; Desch \& Nutting, 1979b: 281; Domrow, 1991: 1341; Yathiraj et al., 1994:

393; Löwenstein, Beck, Bessmann \& Mueller, 2005: 290; Izdebska \& Rolbiecki, 2020: 13

Type locality: Europe

Distribution: range of hosts

Host: Felis catus (Carnivora: Felidae)

Demodex caviae Bacigalupo et Roveda

Demodex caviae Bacigalupo \& Roveda, 1954: 149; Izdebska \& Rolbiecki, 2020: 13

Type locality: laboratory animal

Distribution: Argentina

Host: Cavia porcellus (Rodentia: Caviidae) 
Demodex cervi Prietsch

Demodex cervi Prietsch, 1886; Vanselow, 1910; Kutzer \& Grunberg, 1972: 9; Izdebska \& Rolbiecki, 2020: 13

Type locality: Austria

Distribution: Austria

Host: Cervus elaphus (Artiodactyla: Cervidae)

\section{Demodex chiropteralis Hirst}

Demodex chiropteralis Hirst, 1921: 367; Fain, 1960: 81; Baker \& Craven, 2003: 11; Izdebska \& Rolbiecki, 2020: 13

Type locality: England

Distribution: England, Poland

Host: Plecotus auritus (Chiroptera: Vespertilionidae)

Demodex conicus Izdebska et Rolbiecki

Demodex conicus Izdebska \& Rolbiecki, 1915: 168

Type locality: Poland

Distribution: Poland

Host: Mus musculus (Rodentia: Muridae)

Demodex cornei Izdebska et Rolbiecki

Demodex cornei Izdebska \& Rolbiecki, 2018: 346 (Mason. 1993: 92 - nomen nudum)

Type locality: Poland

Distribution: Poland, India, probably cosmopoliotic

Host: Canis lupus familiaris (Carnivora: Canidae)

Demodex criceti Nutting et Rauch

Demodex criceti Nutting \& Rauch, 1958: 328; Nutting, 1961: 515; Sarashina \& Sato, 1986:

619; Izdebska \& Rolbiecki, 2020: 13

Type locality: laboratory Golden Hamsters, Amherst, Mass.

Distribution: ? [USA]

Host: Mesocricetus auratus (Rodentia: Cricetidae)

Demodex cricetuli Hurley et Desch

Demodex cricetuli Hurley \& Desch, 1994: 529; Izdebska \& Rolbiecki, 2020: 13

Type locality: Massachsetts

Distribution: USA (Massachusetts)

Host: Cricetulus migratorius (Rodentia: Cricetidae)

Demodex cuniculi Pfeiffer

Demodex cuniculi Pfeiffer, 1903; Izdebska \& Rolbiecki, 2020: 14 
Type locality: Europe

Distribution: cosmopolite

Host: Oryctolagus cuniculus (Lagomorpha: Leporidae)

Demodex cyonis Morita, Ohmi, Kiwaki, Ike et Nagata

Demodex cyonis Morita, Ohmi, Kiwaki, Ike \& Nagata, 2018: 323; Izdebska \& Rolbiecki, 2020: 14

Type locality: Japan

Distribution: Japan

Host: Canis lupus familiaris (Carnivora: Canidae)

\section{Demodex dasypodi Desch}

Demodex dasypodi Desch \& Stewart, 2002: 169; Izdebska \& Rolbiecki, 2020: 14

Type locality: Louisiana

Distribution: USA

Host: Dasypus novemcinctus (Cingulata: Dasypodidae)

\section{Demodex desmodi Desch}

Demodex desmodi Desch, 1994: 39; Izdebska \& Rolbiecki, 2020: 14

Type locality: Suriname

Distribution: Suriname

Host: Desmodus rotundus (Chiroptera: Phyllostomatidae, Desmodontinae)

\section{Demodex equi Railliet}

Demodex folliculorum var. equi Railliet, 1893: 639; Bennison, 1943: 36

Demodex equi: Canestrini \& Kramer, 1899: 3; Hirst, 1919; Dubinin, 1957: 132; Kolev, 1958: 299; Domrow, 1991: 1341; Izdebska \& Rolbiecki, 2020: 14

Demodex sp. - Nutting, 1976: 214

Demodex caballi Railliet, 1895; Desch \& Nutting, 1979: 235; Tenquist \& Charleston, 2001: 495. Syn. by Domrow, 1991: 1341

Type locality: Europe

Distribution: cosmopolitic

Host: Equus caballus (Perissodactyla: Equidae)

\section{Demodex erinacei Hirst}

Demodex folliculorum var. erinacei Hirst, 1919: 25; Izdebska \& Rolbiecki, 2020: 14

Type locality: England

Distribution: England

Host: Erinaceus europaeus (Erinaceomorpha: Erinaceidae)

Demodex ermineae Hirst

Demodex ermineae Hirst, 1919: 32 
Type locality: Exeter (England)

Distribution: range of host

Host: Mustela erminea (Carnivora: Mustelidae)

[Demodex felis Silbermayr et al., 2015 - Nomen nudum

Host: Felis catus (Carnivora: Felidae)]

\section{Demodex flagellurus Bukva}

Demodex flagellurus Bukva, 1985: 73; Izdebska, 2000; Izdebska \& Rolbiecki, 2006: 175; 2020: 14

Type locality: České Budějovice, South Bohemia

Distribution: Czech Rep., Poland

Host: Mus musculus (Rodentia: Muridae)

\section{Demodex folliculorum (Simon)}

Acarus folliculorum Simon, 1842: 231 (partim, figs. 1, 2, 8)

Demodex folliculorum (Simon): Haller, 1882: 296 (ex Canis familiaris!); Canestrini \& Kramer, 1899: 2; Poppe, 1909: 66; Starkoff \& Starkoff, 1950: 92; Lamb, 1952: 372;

Desch \& Nutting, 1972: 169; 1978: 422; Xie, Liu, Hsu \& Hseu, 1982: 268; Candotti et al., 1996; Domrow, 1991: 1342; Zeytun \& Karakurt, 2019: 2; Izdebska \& Rolbiecki, 2020: 14 (also many other authors)

Demodex folliculorum longus Akbulatova, 1963: 40

Demodex hominis Leydig, 1859: 345

Type locality: Europe

Distribution: cosmopolitan

Host: Homo sapiens (Primates: Hominidae)

Demodex folliculorum sinensis Xie, Liu, Hsu et Hsu

Demodex folliculorum sinensis Xie, Liu, Hsu \& Hsu, 1982: 267; Izdebska \& Rolbiecki, 2020: 14

Type locality: Shanghai

Distribution: China

Host: Homo sapiens (Primates: Hominidae)

\section{Demodex foveolator Bukva}

Demodex foveolator Bukva, 1984: 45; Izdebska \& Rolbiecki, 2020: 15

Type locality: České Budějovice, South Bohemia

Distribution: Czech Rep.

Host: Crocidura suaveolens (Soricomorpha: Soricidae)

Demodex fusiformis Izdebska et Rolbiecki

Demodex fusiformis Izdebska \& Rolbiecki, 2015: 608; Izdebska \& Rolbiecki, 2020: 15 
Type locality: Poland

Distribution: Poland

Host: Mus musculus (Rodentia: Muridae)

Demodex gapperi Nutting, Emejuaiwe et Tisdel

Demodex gapperi Nutting, Emejuaiwe \& Tisdel, 1971: 660; Nutting, Beekman \& Snyder, 1978: 646; Izdebska \& Rolbiecki, 2020: 15

Type locality: New York State, Massachusetts

Distribution: USA

Host: Myodes [Clethrionomys] gapperi (Rodentia: Arvicolidae)

Demodex gatoi Desch et Stewart

Demodex gatoi Desch \& Stewart, 1999: 167

Type locality: Baton Rouge (USA)

Distribution: USA (Louisiana), Austria, Finland, Poland, Spain

Host: Felis catus [silvestris] (Carnivora: Felidae)

Demodex ghanensis Oppong, Lee et Yasin

Demodex ghanensis Oppong, Lee \& Yasin, 1975: 39; Abu-Samra \& Shuaib, 2014: 77;

Izdebska \& Rolbiecki, 2020: 15

Type locality: Ghana

Distribution: Ghana, Sudan

Host: Bos taurus (Artiodactyla: Bovidae)

\section{Demodex glareoli Hirst}

Demodex arvicolae var. glareoli Hirst, 1919: 35

Demodex glareoli Hirst: Izdebska, Kozina \& Gólcz, 2013: 129; Izdebska \& Rolbiecki, 2020: 15

Type locality: England

Distribution: England

Host: Myodes [Clethrionomys] glareolus britannicus (Rodentia: Arvicolidae)

\section{Demodex gliricolens Hirst}

Demodex gliricolens Hirst, 1921: 368; Izdebska \& Rolbiecki, 2020: 15

Type locality: England (?)

Distribution: England (?)

Host: Arvicola terrestris (Rodentia: Arvicolidae)

Demodex gracilentus Izdebska et Rolbiecki

Demodex gracilentus Izdebska \& Rolbiecki, 2013: 1203; 2020: 15

Type locality: Poland

Distribution: Poland 
Host: Apodemus agrarius (Rodentia: Muridae)

Demodex huttereri Mertens, Lukoschus et Nutting

Demodex huttereri Mertens, Lukoschus \& Nutting, 1983: 489; Izdebska \& Rolbiecki, 2020: 15

Type locality: laboratory animal captured near Fulda, Germany

Distribution: Germany, Poland

Host: Apodemus agrarius (Mammalia: Muridae)

Demodex injai Desch et Hillier

Demodex injai Desch \& Hillier, 2003: 146; Izdebska \& Rolbiecki, 2020: 15

Type locality: Columbus, Ohio

Distribution: USA (Ohio)

Host: Canis lupus familiaris (Carnivora: Canidae)

Demodex intermedius Lukoschus, Mertens, Nutting et Nadchatram

Demodex intermedius Lukoschus, Mertens, Nutting \& Nadchatram, 1984: 234; Izdebska \& Rolbiecki, 2020: 16

Type locality: Bukit Fraser

Distribution: Malaysia

Host: Tupaia glis (Scandentia: Tupaiidae)

Demodex kutzeri Bukva

Demodex kutzeri Bukva, 1987: 175; Kadulski, 1996a: 204; 1996b: 353; Desch et al., 2010: 585; Izdebska \& Rolbiecki, 2020: 16

Demodex cervi sensu Kutzer \& Grünberg, 1972, part.; nec D. cervi Vanselow, 1910

Type locality: Wattens/Tirol

Distribution: Austria, USA

Hosts: Alces alces, Capreolus capreolus, Cervus elaphus, C. elaphus nelsoni, C. nippon pseudaxis, Dama dama, Odocoileus h. hemionus, O. virginianus (Artiodactyla: Cervidae)

Demodex lacrimalis Lukoschus et Jongman

Demodex lacrimalis Lukoschus \& Jongman, 1974: 274; Izdebska \& Rolbiecki, 2020: 16

Type locality: Nijmegen

Distribution: Holland, Italy, Poland

Host: Sylvaemus sylvaticus (Rodentia: Muridae, Murinae)

\section{Demodex leucogasteri Hughes et Nutting}

Demodex leucogasteri Hughes \& Nutting, 1981: 182; Izdebska \& Rolbiecki, 2020: 16

Type locality: Washington State, Tennessee

Distribution: USA (Washington State, Tennessee)

Host: Onychomys leucogaster (Rodentia: Arvicolidae, Neotominae) 


\section{Demodex longior Hirst}

Demodex longior Hirst, 1918: 145; 1919: 25; Izdebska \& Rolbiecki, 2020: 16

Type locality: England

Distribution: England

Host: Sylvaemus sylvaticus (Rodentia: Muridae)

Demodex longissimus Desch, Nutting et Lukoschus

Demodex longissimus Desch, Nutting \& Lukoschus, 1972: 35; Izdebska \& Rolbiecki, 2020: 16

Type locality: Brokopondo, Brownsweg, Lelydorp, Onverwacht, Zandery

Distribution: Suriname

Host: Carollia perspicillata (Chiroptera: Phyllostomatidae)

Demodex lutrae Izdebska et Rolbiecki

Demodex lutrae Izdebska \& Rolbiecki, 2014: 784; Izdebska \& Rolbiecki, 2020: 17

Type locality: Poland

Distribution: Poland

Host: Lutra lutra (Carnivora: Mustelidae)

Demodex macaci Karjala, Desch et Starost

Demodex macaci Karjala, Desch \& Starost, 2005: 948; Izdebska \& Rolbiecki, 2020: 17

Type locality: Laboratory colony

Distribution: laboratory colony

Host: Macaca mulatta (Primates: Cercopithecidae)

Demodex macroglossi Desch

Demodex macroglossi Desch, 1981: 41; Domrow, 1991: 1342; Izdebska \& Rolbiecki, 2020: 17

Type locality: Western Australia

Distribution: Australia (W.A.)

Host: Macroglossus minimus (Chiroptera: Pteropodidae)

Demodex marculus Izdebska et Rolbiecki

Demodex marculus Izdebska \& Rolbiecki, 2015: 607, 2020: 17

Type locality: Poland

Distribution: Poland

Host: Mus musculus (Rodentia: Muridae)

Demodex marsupiali Nutting, Lukoschus et Desch

Demodex marsupiali Nutting, Lukoschus \& Desch, 1980: 84; Izdebska \& Rolbiecki, 2020: 17

Type locality: Lelydorp, Paramaribo, Coronie

Distribution: Suriname

Host: Didelphis marsupialis (Didelphimorphia: Didelphidae) 
Demodex melanopteri Lukoschus, Jongman et Nutting

Demodex melanopteri Lukoschus, Jongman \& Nutting, 1972: 54; Izdebska \& Rolbiecki, 2020: 17

Type locality: Meerzorg, Lelydorp

Distribution: Suriname

Host: Eptesicus brasiliensis melanopterus (Chiroptera: Vespertilionidae)

\section{Demodex melesinus Hirst}

Demodex melesinus Hirst, 1921: 372; Izdebska \& Rolbiecki, 2020: 17

Type locality: England

Distribution: England

Host: Meles meles (Carnivora: Mustelidae)

[Demodex merioni (= meriones) - Nomen nudum

Host: Meriones spp. (Rodentia: Muridae)]

Demodex mexicanus Vargas, Bassols, Desch, Quintero et Polaco

Demodex mexicanus Vargas, Bassols, Desch, Quintero \& Polaco, 1995: 76; Izdebska \& Rolbiecki, 2020: 17

Type locality: Mexico

Distribution: Mexico

Host: Plecotus [Corynorhinus] mexicanus (Chiroptera: Vespertilionidae)

Demodex microti Izdebska et Rolbiecki

Demodex microti Izdebska \& Rolbiecki, 2013: 189; Izdebska \& Rolbiecki, 2020: 17

Type locality: Poland

Distribution: Poland

Host: Microtus arvalis (Rodentia: Arvicolidae)

Demodex mollis Izdebska, Rolbiecki, Fryderyk et Mierzyński

Demodex mollis Izdebska, Rolbiecki, Fryderyk \& Mierzyński, 2017; Izdebska \& Rolbiecki, 2020: 18

Type locality: Poland

Distribution: Poland

Host: Sylvaemus flavicollis (Rodentia: Muridae)

Demodex molossi Desch, Nutting et Lukoschus

Demodex molossi Desch, Nutting \& Lukoschus, 1972 : 43; Izdebska \& Rolbiecki, 2020: 18 Type locality: Lelydorp, Paramaribo

Distribution: Suriname

Host: Molossus molossus (Chiroptera: Molossidae) 


\section{Demodex muscardini Hirst}

Demodex muscardini Hirst, 1917: 433; 1919: 30; Bregetova et al., 1955; Izdebska \& Rolbiecki, 2020: 18

Type locality: England

Distribution: Armenia, Great Britain

Host: Muscardinus avellanarius (Rodentia: Gliridae)

\section{Demodex musculi Oudemans}

Demodex folliculorum var. musculi Oudemans, 1897: 269

Demodex musculi Oudemans, 1905: 12; 1909: 318; Hirst, 1917: 233; Hill et al., 1999: 13; Izdebska \& Rolbiecki, 2020: 18

Demodex arvicolae var. musculi: Hirst, 1919: 34; Dubinin, 1953: 276

Demodex arvicolae musculi Oudemans: Izdebska \& Rolbiecki, 2006: 175

Type locality: Europe

Distribution: England, Poland, Russia, Spain

Host: Mus musculus (Rodentia: Muridae)

[Demodex myotidis Di Benedetto]

Demodex myotidis Di Benedetto, 1961 (MA Thesis); Izdebska \& Rolbiecki, 2020: 18

Type species: USA

Distribution: USA

Hosts: Eptesicus fuscus, Myotis l. lucifugus, M. septentrionalis (Chiroptera: Vespertilionidae). Note by Izdebska \& Rolbiecki, 2020: "Nom. nud.; description not published within the meaning of the ICZN"].

\section{Demodex mystacina Desch}

Demodex mystacina Desch, 1989: 221; Izdebska \& Rolbiecki, 2020: 18

Type locality: Omahuta Kauri Sanctuary

Distribution: New Zealand

Host: Mystacina tuberculata (Chiroptera: Mystacinidae)

\section{Demodex nanus Hirst}

Demodex nanus Hirst, 1918: 146; Desch, 1987: 19; 1989: 228; Bukva, 1993; Haitlinger \& Jankowska, 2005: 38; Izdebska \& Rolbiecki, 2020: 18

Type locality: unknown

Distribution: Great Britain, Poland, Russia

Hosts: Rattus norvegicus, $R$. rattus (Rodentia: Muridae, Murinae)

\section{Demodex neomydis Bukva}

Demodex neomydis Bukva, 1995: 299; Izdebska \& Rolbiecki, 2020: 18

Type locality: České Budějovice, South Bohemia

Distribution: Czech Rep. 
Host: Neomys anomalus (Soricomorpha: Soricidae)

Demodex neoopisthosomae Desch, Lukoschus et Nadchatram

Demodex neoopisthosomae Desch, Lukoschus \& Nadchatram, 1986: 14; Izdebska \& Rolbiecki, 2020: 19

Type locality: Malaysia

Distribution: Malaysia

Host: Eonycteris spelaea (Chiroptera: Pteropodidae, Macroglossinae)

\section{Demodex norvegicus Bukva}

Demodex norvegicus Bukva, 1995: 152; Haitlinger \& Jankowska, 2005: 38; Izdebska \& Rolbiecki, 2020: 19

Type locality: České Budějovice, South Bohemia

Distribution: Czech Rep., Poland

Host: Rattus norvegicus (Rodentia: Muridae, Murinae)

\section{Demodex novazelandica Desch}

Demodex novazelandica Desch, 1989: 226; Izdebska \& Rolbiecki, 2020: 19

Type locality: Omahuta Kauri Sanctuary

Distribution: New Zealand

Host: Mystacina tuberculata (Chiroptera: Mystacinidae)

\section{Demodex nycticeii Desch}

Demodex nycticeii Desch, 1996: 187; Izdebska \& Rolbiecki, 2020: 19

Type locality: Florida

Distribution: USA (Florida)

Host: Nycticeius humeralis (Chiroptera: Vespertilionidae)

\section{Demodex odocoilei Desch et Nutting}

Demodex odocoilei Desch \& Nutting, 1974: 785; Jasques et al., 2001: 221; Gentes, Proctor \& Wobeser, 2007: 758; Turner \& Cano, 2008: 572; Izdebska \& Rolbiecki, 2020: 19 Type locality: Dougherty County (Georgia), Eucha (Oklahoma), Stafford County (Virginia) Distribution: Canada, USA (Georgia, Oklahoma, Virginia, Texas)

Host: Odocoileus hemionus columbianus, O. virginianus (Artiodactyla: Cervidae)

\section{Demodex ovis Railliet}

Demodex folliculorum var. ovis Railliet, 1895: 638; Hirst, 1919: 24; Bukva, 1990: 82; Tenquist \& Charleston, 2001: 497

Demodex ovis Railliet: Hallas, 1978: 30; Izdebska \& Rolbiecki, 2020: 19

Type locality: Europe

Distribution: range of host (incl. Australia, New Zealand, Czech Rep., Israel, Poland)

Host: Ovis aries (Artiodactyla: Bovidae) 
Demodex peromysci Lombert, Lukoschus et Whitaker

Demodex peromysci Lombert, Lukoschus \& Whitaker, 1983: 377; Izdebska \& Rolbiecki, 2020: 19

Type locality: Pennsylvania, Huntingdon Co.

Distribution: USA (Pennsylvania)

Host: Peromyscus leucopus (Rodentia: Cricetidae)

Demodex phocidi Desch, Dailey et Tuomi

Demodex phocidi Desch, Dailey \& Tuomi, 2003: 231; Izdebska et al., 2020: 49; Izdebska \& Rolbiecki, 2020: 19

Type locality: Alaska SeaLife Center, Seward

Distribution: USA (Alaska), Poland

Host: Phoca vitulina (Carnivora Pinnipedia: Phocidae)

Demodex phodopi Desch, Davis et Klompen

Demodex phodopi Desch, Davis \& Klompen, 2006: 75; Izdebska \& Rolbiecki, 2020: 19

Type locality: laboratory colony

Distribution: [USA] (actually, Asia)

Host: Phodopus sungorus (Rodentia: Cricetidae)

\section{Demodex phylloides Csokor}

Demodex phylloides Csokor, 1879: 133; Canestrini, 1899: 968; Canestrini \& Kramer, 1899: 3; Hirst, 1919: 27; Lamb, 1952: 372; Dubinin, 1957: 132; Desch \& Nutting, 1973: 500; Rak, 1976: 509; Quintero, 1977: 42; Tenquist \& Charleston, 2001: 497; Desch \& Stewart, 2002: 173; Reeves et al., 2007: 47; Izdebska \& Rolbiecki, 2020: 19

Demodex folliculorum var. suis Railliet, 1895: 637; Ligrain \& Régulato, 1903: 371

Distribution: Algeria, Argentina, Australia, Brazil, Canada, France, Germany, Iran, Madagascar, Mexico, Poland, Russia, New Zealand, Spain, Sweden, USA

Host: Sus scrofa (Artiodactyla: Suidae)

\section{Demodex phyllostomatis Leydig}

Demodex phyllostomatis Leydig, 1859: 345; Hirst, 1919: 39; Fain, 1960: 80; Izdebska \& Rolbiecki, 2020: 20

Type locality: Suriname

Distribution: Suriname

Host: Phyllostoma hastatum (Chiroptera: Phyllostomatidae)

Demodex plecoti Izdebska, Rolbiecki et Mienzynski

Demodex plecoti Izdebska, Rolbiecki \& Mienzynski, 2019: 377.

Type locality: Poland

Distribution: Poland

Host: Plecotus auritus (Chiroptera: Vespertilionidae) 
Demodex ponderosus Izdebska et Rolbiecki

Demodex ponderosus Izdebska \& Rolbiecki, 2014: 740

Type locality: Poland

Distribution: Poland

Host: Rattus norvegicus (Rodentia: Muridae)

Demodex pseudaxisi Shpringol'ts - Shmidt

Demodex pseudaxisi Shpringol'ts - Shmidt, 1937: 133

Type locality: Far East of Russia

Distribution: Russia

Host: Cervus nippon hortulorum (Arthiodactyla: Cervidae)

Demodex ratti (Hahn) Hirst

Demodex ratti Hirst, 1917: 233; 1919: 37; Dubinin, 1953: 276; Bukva, 1995: 149; Haitlinger

\& Jankowska, 2005: 38

Type locality: Europe; redescription from Vranín, South Bochemia

Distribution: Czech Rep., England, Poland, Russia

Host: Rattus norvegicus (Rodentia: Muridae)

Demodex ratticola Bukva

Demodex ratticola Bukva, 1995: 156; Izdebska \& Rolbiecki. 2012b: 71

Type locality: České Budějovice

Distribution: Czech Rep., Poland

Host: Rattus norvegicus (Rodentia: Muridae)

Demodex rosus Bukva, Vítovec et Vlček

Demodex rosus Bukva, Vítovec \& Vlček, 1985: 151

Type locality: Lišov, distr. of České Budějovice

Distribution: Czech Rep.

Host: Sylvaemus flavicollis (Rodentia: Muridae)

Demodex sabani Desch, Lukoschus et Nadchatram

Demodex sabani Desch, Lukoschus \& Nadchatram, 1984: 55

Type locality: Kuala Selangor Road, 35 km from Selangor, Malaysia

Distribution: Malaysia

Hosts: Leopoldamys sabanus, L. edwardsi, Niviventer cremoriventer, N. rapit, Rattus annandalei, R. tiomanicus jalorensis, Sundamys mulleri (Rodentia: Muridae)

Demodex saimiri Lebel et Nutting

Demodex saimiri Lebel \& Nutting, 1973: 719; Izdebska \& Rolbiecki, 2020: 21

Type locality: Zoo 
Distribution: South America

Host: Saimiri sciureus (Primates: Cebidae, Saimiriinae)

[Demodex sciurei Lebel

Demodex sciurei Lebel, 1970: Master's Thesis

Type locality: Zoo

Distribution: South America

Host: Saimiri sciureus (Primates: Cebidae, Saimiriinae). Note by Izdebska et al., 2020:

"Nom. nud.; description not published within the meaning of the ICZN"].

\section{Demodex sciurinus Hirst}

Demodex sciurinus Hirst, 1923: 994; Izdebska \& Rolbiecki, 2020: 3

Type locality: Inverness (Great Britain)

Distribution: Great Britain, Poland

Host: Sciurus vulgaris (Rodentia: Sciuridae, Sciurinae)

Demodex sinocricetuli Desch et Hurley

Demodex sinocricetuli Desch \& Hurley, 1997: 317; Izdebska \& Rolbiecki, 2020: 21

Type locality: [Maryland, Beltsville]

Distribution: [USA (Maryland), laboratory animals]

Host: Cricetulus barabensis (Rodentia: Cricetidae)

Demodex soricinus Hirst

Demodex soricinus Hirst, 1918: 145; 1919: 37; 1921: 368; Dubinin, 1953: 276; Fain, 1960:

81; van Eyndhoven, 1965: 103; Bukva, 1993: 243; Izdebska, 2004: 47; Izdebska \& Rolbiecki, 2020: 21

Type locality: Great Britain

Distribution: Czech Republic, Great Britain, Holland, Poland, Russia

Hosts: Sorex araneus (Soricomorpha: Soricidae), Plecotus auritus (?, probably wrong host record)(Chiroptera: Vespertilionidae)

Demodex spelaea Desch, Lukoschus et Nadchatram

Demodex spelaea Desch, Lukoschus \& Nadchatram, 1986: 20; Desch, 1989: 226; Izdebska \& Rolbiecki, 2020: 21

Type locality: Malaysia

Distribution: Malaysia

Host: Eonycteris spelaea (Chiroptera: Pteropodidae, Macroglossinae)

Demodex sungori Desch, Davis et Klompen

Demodex sungori Desch, Davis \& Klompen, 2006: 77; Izdebska \& Rolbiecki, 2020: 21

Type locality: laboratory colony 
Distribution: [USA](actually, Asia)

Host: Phodopus sungorus (Rodentia: Cricetidae)

[Demodex sylvilagi Maravelas

Demodex sylvilagi Maravelas, 1962: MA Thesis

Type locality: New England

Distribution: USA

Host: Sylvilagus transitionalis (Lagomorpha: Leporidae). Note by Izdebska et al., 2020:

"Nom. nud.; description not published within the meaning of the ICZN"].

\section{Demodex talpae Hirst}

Demodex talpae Hirst, 1921: 370; Izdebska \& Rolbiecki, 2003: 97, 2020: 21

Type locality: Great Britain

Distribution: Great Britain, Poland

Host: Talpa europaea (Soricomorpha: Talpidae)

\section{Demodex tauri Bukva}

Demodex tauri Bukva, 1986: 364; Izdebska \& Rolbiecki, 2020: 21

Type locality: České Budějovice

Distribution: Czech Rep.

Host: Bos taurus (Artiodactyla: Bovidae, Bovinae)

Demodex tigris Shi, Xie et Hsu

Demodex tigris Shi, Xie \& Hsu, 1985: 385; Izdebska \& Rolbiecki, 2020: 22

Type locality: Shanghai Zool. Garden, Shanghai

Distribution: China

Host: Panthera tigris amoyensis (Carnivora: Felidae)

\section{Demodex tortellinioides Desch et $\mathrm{Holz}$}

Demodex tortellinioides Desch \& Holz, 2006: 81; Izdebska \& Rolbiecki, 2020: 22

Type locality: Victoria

Distribution: Australia

Host: Antechinus agilis (Dasyuromorphia: Dasyuridae)

Demodex transitionalis Moravelas

Demodex transitionalis Moravelas, 1962

Type locality: USA

Distribution: USA

Host: Sylvilagus transitionalis (Lagomorpha: Leporidae). Note by Izdebska et al., 2020:

"Nom. nud.; description not published within the meaning of the ICZN"]. 


\section{Demodex uncii Desch}

Demodex uncii Desch, 1993: 63; Izdebska \& Rolbiecki, 2020: 22

Type locality: Zoo

Distribution: Asia (collected in Zoo in USA)

Host: Uncia uncia (Carnivora: Felidae)

\section{Demodex ursi Desch}

Demodex ursi Desch, 1995: 23; Foster, Cames \& Forrester, 1998: 161; Izdebska \& Rolbiecki, 2020: 22

Type locality: Florida

Distribution: USA (Florida)

Host: Ursus americanus (Carnivora: Ursidae)

Demodex vibrissae Izdebska, Rolbiecki et Fryderyk

Demodex vibrissae Izdebska, Rolbiecki \& Fryderyk, 2016; Izdebska \& Rolbiecki, 2020: 22

Type locality: Poland

Distribution: Poland

Host: Mus musculus (Rodentia: Muridae)

Demodex zalophi Dailey et Nutting

Demodex zalophi Dailey \& Nutting, 1980: 424; Izdebska \& Rolbiecki, 2020: 22

Type locality: San Diego (California), Arlington (Texas) and Australia

Distribution: captive sea lions in California, Texas and Australia

Host: Zalophus californianus (Carnivora Pinnipedia: Otariidae)

Genus Glossicodex Izdebska et Rolbiecki

Glossicodex Izdebska \& Rolbiecki, 2016: 135

Type species: Glossicodex musculi Izdebska et Rolbiecki, 2016

Glossicodex musculi Izdebska et Rolbiecki

Glossicodex musculi Izdebska \& Rolbiecki, 2016: 135; Izdebska \& Rolbiecki, 2020: 22

Type locality: Poland

Distribution: Poland

Host: Mus musculus (Rodentia: Muridae)

Genus Ophthalmodex Lukoschus et Nutting

Ophthalmodex Lukoschus \& Nutting, 1979: 299

Type species: Ophthalmodex artibei Lukoschus et Nutting, 1979

Ophthalmodex apodemi Bukva, Nutting et Desch

Ophthalmodex apodemi Bukva, Nutting \& Desch, 1992: 269; Izdebska \& Rolbiecki, 2020: 22 
Type locality: Česke Budejovice, South Bohemia

Distribution: Czech Rep.

Host: Sylvaemus sylvaticus (Rodentia: Muridae, Murinae)

Ophthalmodex artibei Lukoschus et Nutting

Ophthalmodex artibei Lukoschus \& Nutting, 1979: 301; Izdebska \& Rolbiecki, 2020: 22

Type locality: Lelydorp

Distribution: Suriname

Host: Artibeus lituratus (Chiroptera: Phyllostomatidae, Stenodermatinae)

Ophthalmodex australiensis Woeltjes et Lukoschus

Opthalmodex australiensis Woeltjes \& Lukoschus, 1981: 308 (lapsus)

Ophthalmodex australiensis: Domrow, 1991: 1344; Izdebska \& Rolbiecki, 2020: 22

Type locality: Western Australia

Distribution: Australia

Host: Rhinonicteris aurantius (Chiroptera: Hipposideridae)

Ophthalmodex carolliae Lukoschus, Woeltjes, Desch et Nutting

Ophthalmodex carolliae Lukoschus, Woeltjes, Desch \& Nutting, 1980b: 45; Izdebska \& Rolbiecki, 2020: 22

Type locality: Lelydorp, Zanderij, Onverwacht, Brownsweg

Distribution: Suriname

Host: Carollia perspicillata (Chiroptera: Phyllostomatidae, Carolliinae)

Ophthalmodex juniatae Veal, Giesen et Whitaker

Ophthalmodex juniatae Veal, Giesen \& Whitaker, 1984: 348; Izdebska \& Rolbiecki, 2020: 23

Type locality: Entriken, Huntingdon Co, Penn.

Distribution: USA (Pennsylvania)

Host: Myotis lucifugus (Chiroptera: Vespertilionidae, Vespertilioninae)

Ophthalmodex molossi Lukoschus, Woeltjes, Desch et Nutting

Ophthalmodex molossi Lukoschus, Woeltjes, Desch \& Nutting, 1980b: 47; Izdebska \&

Rolbiecki, 2020: 23

Type locality: Paramaribo

Distribution: Suriname

Host: Molossus molossus (Chiroptera: Molossidae)

Ophthalmodex wilsoni Woeltjes et Lukoschus

Ophthalmodex wilsoni Woeltjes \& Lukoschus, 1981: 311; Domrow, 1991: 1344; Izdebska

\& Rolbiecki, 2020: 23

Type locality: Western Australia 
Distribution: Australia

Host: Vespadelus pumilus (Chiroptera: Vespertilionidae, Vespertilioninae)

Genus Pterodex Lukoschus, Woeltjes, Desch et Nutting

Pterodex Lukoschus, Woeltjes, Desch \& Nutting, 1980: 9

Type species: Pterodex carolliae Lukoschus, Woeltjes, Desch et Nutting, 1980

Pterodex carolliae Lukoschus, Woeltjes, Desch et Nutting

Pterodex carolliae Lukoschus, Woeltjes, Desch \& Nutting, 1980a: 10; Izdebska \& Rolbiecki, 2020: 23

Type locality: Lelydorp, Zanderij, Onverwacht, Brownsweg

Distribution: Suriname

Host: Carollia perspicillata (Chiroptera: Phyllostomatidae, Carolliinae)

Genus Rhinodex Fain

Rhinodex Fain, 1959: 264

Type species: Rhinodex baeri Fain, 1959

Rhinodex baeri Fain

Rhinodex baeri Fain, 1959: 264; Izdebska \& Rolbiecki, 2020: 23

Type locality: Bugesera

Distribution: Rwanda

Host: Galago moholi (Primates: Galagonidae)

Genus Soricidex Bukva

Soricidex Bukva, 1982 : 343

Type species: Soricidex dimorphus Bukva, 1982

Soricidex dimorphus Bukva

Soricidex dimorphus Bukva, 1982: 344; Izdebska, 2004a: 47; Izdebska \& Rolbiecki, 2020: 23

Type locality: Česke Budejovice, South Bohemia

Distribution: Czech Rep., Poland

Host: Sorex a. araneus (Soricomorpha: Soricidae, Soricinae)

Soricidex sp.

Soricidex sp.: Bukva, 1996: 315

Locality: Česke Budejovice, South Bohemia

Distribution: Czech Rep.

Host: Neomys anomalus (Soricomorpha: Soricidae) 


\section{Genus Stomatodex Fain}

Stomatodex Fain, 1959: 267

Type species: Stomatodex galagoensis Fain, 1959

\section{Stomatodex cercarteti Desch}

Stomatodex cercarteti Desch, 1991: 239; Domrow, 1992: 1606; Halliday, 1998: 49; Izdebska \& Rolbiecki, 2020: 23

Type locality: New South Wales

Distribution: Australia

Host: Cercartetus nanus (Diprotodontia: Burramyidae)

\section{Stomatodex corneti Fain}

\section{Stomatodex corneti corneti Fain}

Stomatodex corneti Fain, 1960: 84; Baker \& Craven, 2003: 12; Izdebska \& Rolbiecki, 2020: 23 Type locality: Grottes de Han (Belgium)

Distribution: Belgium, Great Britain, Rwanda

Host: Barbastella barbastellus (Chiroptera: Vespertilionidae), ?Nycteris sp. (Nycteridae)

\section{Stomatodex corneti myotis Fain}

Stomatodex corneti myotis Fain, 1960: 86; Izdebska \& Rolbiecki, 2020: 23

Type locality: Grottes de Han (Belgium)

Distribution: Belgium

Hosts: Myotis dasycneme, M. myotis (Chiroptera: Vespertilionidae)

\section{Stomatodex galagoensis Fain}

Stomatodex galagoensis Fain, 1959: 268; Izdebska \& Rolbiecki, 2020: 23

Type locality: Bugesera

Distribution: Rwanda

Host: Galago moholi (Primates: Galagonidae)

\section{Stomatodex rousetti Fain}

Stomatodex rousetti Fain, 1960: 83; Izdebska \& Rolbiecki, 2020: 24

Type locality: Mahyusha Cave, nr. Katana

Distribution: DR Congo

Host: Rousettus aegyptiacus leachi (Chiroptera: Pteropodidae) 


\section{Index of Demodecidae}

(Names in bold are accepted as valid)

acutipes, Demodex 205

aelleni, Demodex 206

agrarii, Demodex 206

ailuropodae, Demodex 206

antechini, Demodex 206

apodemi, Demodex 206

apodemi, Ophthalmodex 224

Apodemodex 205

araneae, Demodex 206

aries, Demodex 207

artibei, Demodex 207

artibei, Ophthalmodex 225

arvicolae, Demodex 207

arvicolae musculi, Demodex 218

aurati, Demodex 207

auricularis, Demodex 207

australiensis, Ophthalmodex 225

baeri, Rhinodex 226

bandicotae, Demodex 207

bantengi, Demodex 208

bicaudatus, Demodex 208

bisonianus, Demodex 208

bonapartei, Demodex 208

bovis, Demodex 208

bovis, Demodex folliculorum var. 208

brevis, Demodex 209

buccalis, Demodex 209

cabali, Demodex 209

cafferi, Demodex 209

caninus, Demodex 210

caninus, Demodex folliculorum var. 208

canis, Demodex 209

canis, Demodex folliculorum var. 208

caprae, Demodex 210

caprae, Demodex folliculorum var. 210

carolliae, Demodex 210

carolliae, Ophthalmodex 225

carolliae, Pterodex 226 castoris, Demodex 210

cati, Demodex 210

cati, Demodex folliculorum var. 210

caviae, Demodex 210

cercarteti, Stomatodex 227

cervi, Demodex 211

chiropteralis, Demodex 211

conicus, Demodex 211

cornei, Demodex 211

corneti corneti, Stomatodex 211

corneti myotis, Stomatodex 211

corniculatus, Demodex 211

cornutus, Apodemodex 205

criceti, Demodex 211

cricetuli, Demodex 211

cuniculi, Demodex 211

cyonis, Demadex 212

dasypodi, Demodex 212

Demodecidae 205

Demodex 205

Demodicidae 205

Demodicoidea 205

desmodi, Demodex 212

dimorphus, Soricidex 226

equi, Demodex 212

equi, Demodex folliculorum var. 212

erinacei, Demodex 212

erinacei, Demodex canis var. 212

ermineae, Demodex 212

felis, Demodex 213

flagellurus, Demodex 213

folliculorum, Acarus 213

folliculorum, Demodex 213

foveolator, Demodex 213

fusiformis, Demodex 213

galagoensis, Stomatodex 227

gapperi, Demodex 214

gatoi, Demodex 214 
ghanensis, Demodex 214

glareoli, Demodex 214

glareoli, Demodex arvicolae var. 214

gliricolens, Demodex 214

Glossicodex 224

gracilentus, Demodex 214

hominis, Demodex 213

huttereri, Demodex 215

injai, Demodex 215

intermedius, Demodex 215

juniatae, Ophthalmodex 225

kutzeri, Demodex 215

lacrimalis, Demodex 215

leucogasteri, Demodex 215

longior, Demodex 216

longissimus, Demodex 216

longus, Demodex folliculorum 213

lutrae, Demodex 216

macaci, Demodex 216

macroglossi, Demodex 216

marculus, Demodes 216

marsupiali, Demodex 216

melanopteri, Demodex 217

melesinus, Demodex 217

merioni, Demodex 217

mexicanus, Demodex 217

microti, Demodex 217

mollis, Demodex 217

molossi, Demodex 217

molossi, Ophthalmodex 225

muscardini, Demodex 218

musculi, Demodex arvicolae $\mathbf{2 1 8}$

musculi, Demodex folliculorum var. 218

musculi, Demodex 218

musculi, Glossicodex 224

myotidis, Demodex 218

mystacina, Demodex 218

nanus, Demodex 218

neomydis, Demodex 218

neoopisthosomae, Demodex 219

norvegicus, Demodex 219

novazelandica, Demodex 219 nycticeii, Demodex 219

odocoilei, Demodex 219

Ophthalmodex 224

ovis, Demodex folliculorum var. 219

ovis, Demodex 220

peromysci, Demodex 220

phocidi, Demodex 220

phodopi, Demodex 220

phylloides, Demodex 220

phyllostomatis, Demodex 220

plecoti, Demodex 220

ponderosus, Demodex 221

pseudaxisi, Demodex 221

Pterodex 226

ratti, Demodex 221

ratticola, Demodex 221

Rhinodex 226

rosus, Demodex 221

rousetti, Stomatodex 228

sabani, Demodex 221

saimiri, Demodex 221

sciurei, Demodex 222

sciurinus, Demodex 222

sinensis, Demodex folliculorum 213

sinocricetuli, Demodex 222

Soricidex 226

soricinus, Demodex 222

spelaea, Demodex 222

Stomatodex 227

suis, Demodex folliculorum var. 220

sungori, Demodex 222

sylvilagi, Demodex 223

talpae, Demodex 223

tauri, Demodex 223

tigris, Demodex 223

tortellinioides, Demodex 223

transitionalis, Demodex 223

uncii, Demodex 224

ursi, Demodex 224

vibrissae, Demodex 224

wilsoni, Ophthalmodex 225

zalophi, Demodex 224 


\section{Distribution of Demodecidae}

\section{EUROPE}

Armenia (Bregetova et al., 1955) - Demodex muscardini

Austria (Kutzer \& Grünberg, 1972; Bukva, 1987) - Demodex cervi, D. kutzeri

Azerbaidzhan (Gadzhiev et al., 1988) - Demodex bovis, D. canis, D. cuniculi, D. folliculorum, D. phylloides

Belgium (Fain, 1960; de Bosschere et al., 2007) - Stomatodex c. corneti, S. corneti myotis Bulgaria (Drenski, 1921; Kolev, 1958; Hristov \& Mihailov, 1988; Nedelchev, Rusev \& Vassileva, 2001; Iliev, Zhelev, Ivanov \& Prelezov, 2019) - Demodex aurati, D. canis, D. cati, D. caviae, D. equi

Czech Rep. (Daniel, Bozdech \& Moucka, 1959; Bukva, 1982, 1984, 1985a, 1985b, 1986, 1993a, 1993b, 1994, 1995a, 1995b, 1996; Bukva, Nutting \& Desch, 1992; Fleischer et al., 1996) - Apodemodex cornutus, Demodex aries, D. buccalis, D. flagellurus, D. folliculorum, D. foveolator, D. neomydis, D. norvegicus, D. ovis, D. ratti, D. ratticola, D. rosus, D. soricinus, D. tauri; Ophthalmodex apodemi, Soricidex dimorphus

Denmark (Hallas, 1978) - Demodex brevis, D. canis, D. equi, D. folliculorum, D. ovis

Finland (Saari et al., 2009) - Demodex gatoi

France (contin.)(Hirst, 1919; 1921) - Demodex bovis, D. equi, D. phylloides

Germany (Haller, 1882; Poppe, 1909; Hirst, 1919; Hoffman \& Hiepe, 1987) - Demodex bovis, D. equi, D. folliculorum, D. phylloides

Great Britain (Hirst, 1917, 1919; 1921, 1923; Brownlee, 1935; Turk, 1953; Baker \& Craven, 2003) - Demodex arvicolae, D. bovis, D. canis, D. cati, D. chiropteralis, D. cuniculi, D. equi, D. erinacei, D. ermineae, D. folliculorum, D. melesinus, D. ovis, D. ratti, D. sciurinus, D. soricinus, Stomatodex corneti

Greece (Himonas, Theodorides \& Alexakis, 1975) - Demodex spp.

Holland (van Eyndhoven, 1965; Lukoschus \& Jongman, 1974) - Demodex lacrimalis, D. soricinus

Hungary (Dudich et al., 1940) - Demodex folliculorum

Ireland (Baker, 1970, 1973) - Demodex canis

Italy (continent.) (Starkoff \& Starkoff, 1950; Lukoschus \& Jongman, 1974; Candotti et al., 1996) - Demodex canis, D. folliculorum, D. lacrimalis

Norway (Mehl, 1979) - Demodex bovis, D. canis, D. cati, D. caprae, D. equi, D. folliculorum Poland (Kadulski, 1978, 1996a, 1996b, 1997; Kadulski \& Izdebska, 1996; Kadulski, Izdebska \& Konczyk, 1996; Fryderyk \& Izdebska, 2001; Izdebska \& Fryderik, 2000, 2002, 2011, 2012a, 2012b; Izdebska \& Rolbiecki, 2002, 2003, 2004, 2006, 2012a, 2012b, 2013a, 2013b, 2014, 2015a, 2015b, 2016, 2018, 2020; Izdebska, 2000a, 2000b, 2001a, 2001b, 2001c, 2001d, 2002, 2004a, 2004b, 2005, 2006a, 2006b, 2006c, 2007, 2008, 2009, 2012; Izdebska, Rolbiecki \& Fryderik, 2011, 2014, 2016; Haitlinger \& Jankowska, 2005; Izdebska \& Jankowski, 2006; Czepita et al., 2007; Izdebska \& Cydzik, 2010; Izdebska 
\& Krawczyk, 2012) - Demodex acutipes, D. agrarii, D. apodemi, D. auricularis, D. musculi, D. bisonianus, D. bovis, D. brevis, D. buccalis, D. canis, D. caprae, D. castoris, D. cati, D. chiropteralis, D. conicus, D. cornei, D. corniculatus, D. equi, D. flagellurus, $D$. folliculorum, D. fusiformis, D. gatoi, D. glareoli, D. gracilentus, D. injai, D. kutzeri, D. lacrimalis, D. longior, D. lutrae, D. marculus, D. marsupiali, D. melesinus, D. microti, D. mollis, D. musculi, D. nanus, D. norvegicus, D. ovis, D. phocidi, D. phylloides, $D$. plecoti, D. ratti, D. ratticola, D. sciurinus, D. soricinus, D. talpae, D. vibrissae, Glossicodex musculi, Soricidex dimorphus

Romania (Stoenescu et al., 1972; May, Putin \& Aciocîrlánoaia, 1975) - Demodex canis

Russia (all, continent.)(Isaychikov, 1928; Chernobayev, 1928; Rozgon, 1930; Shpringol'ts - Shmid, 1937; Dubinin, 1953; Klinski, 1956, 1959; Polyakov, 1956, 1977; Dubinina, 1987; Moskvina, 2017) - Demodex apodemi, D. aries, D. arvicolae, D. bovis, D. brevis, D. caballi, D. canis, D. caprae, D. cati, D. equi, D. folliculorum, D. musculi, D. nanus, D. ovis, D. phylloides, D. pseudaxisi

Slovakia (Bukva, 1994) - Demodex agrarii

Spain (cont.)(Hirst, 1919) - Demodex phylloides

Canary Islands (Ollé et al., 2020) - Demodex musculi

Sweden (Hist, 1919) - Demodex phylloides

Switzerland (Fain, 1960) - Demodex aelleni

Turkey (all)(Özdemir et al., 2003; Karaer et al., 2009; Pekmezci, Pekmezci \& Bolukbas, 2018; Zeytun \& Karakurt, 2019; Sari, Zeytun, Doğan \& Karakurt, 2019) - Demodex brevis, D. canis, D. folliculorum

\section{AFRICA}

Algeria (Legrain \& Régulato, 1903; Hist, 1919) - Demodex phylloides

Angola (Freire \& Dias, 1947) - Demodex bovis

Botswana (Dräger \& Paine, 1980) - Demodex cafferi

Congo DR (Hirst, 1919; Fain, 1960) - Demodex bovis, Stomatodex rousetti

Egypt (Salib, 2018) - Demodex

Ethiopia (Mulugeta, Yacob \& Ashenafi, 2010; Chanie, Tadesse \& Bogale, 2013) - Demodex bovis

Ghana (Oppong, 1970; Oppong, Lee \& Yasin, 1975) - Demodex ghanensis

Libya (Gabaj et al., 1992) - Demodex sp.

Madagascar (Hist, 1919) - Demodex phylloides

Malawi (Griffiths, 1945) - Demodex bovis

Nigeria (Esuruoso, 1977; Slingerberg, Mohammed \& Bida, 1980; George et al., 1992; Ugbomoiko, Ariza \& Heukelbach, 2008) - Demodex canis, D. folliculorum

Rwanda (Fain, 1959, 1960) - Rhinodex baeri, Rh. galagoensis, Stomatodex c. corneti

South Africa (Du Toit \& Fiedler, 1960; Nutting \& Guilfoy, 1979; Wolhuter et al., 2009) Demodex bovis, D. cafferi, D. canis, D. caprae, D. folliculorum, D. phylloides 
Sudan (Abu-Samra, Hago \& Musa, 1981; Abu-Samra, Imbabi \& Mahgoub, 1981; AbuSamra \& Shuaib, 2014) - Demodex canis, D. ghanensis

Zambia (Mwase \& Baker, 2006) - Demodex bovis, D. canis, D. folliculorum, D. phylloides

ASIA

Bangladesh (Noorudin \& Rahman, 1985) - Demodex bovis

China (Zhang et al., 1982; Xie, Liu, Ye \& Hsu, 1982; Shi, Xie \& Hsu, 1985; Liu, Xu, Xie \& Xu, 1988; Yehua Xu, Hexiu Xie, Sulan Liu, Zhongyong Zhou, Xinquan Shi, 1986; Chen, 199?; Fan et al., 1990; Lin, Wang \& Hong, 2000; Liu, 2007; Zhao, Guo \& Wu, 2009; Zhao, Cheng, Hu \& Ma, 2014) - Demodex ailuropodae, D. brevis, D. caprae, D. folliculorum, D. foliculorum sinensis, D. tigris

Cyprus (Cooreman, 1946) - Demodex canis, D. caprae

India (Hirst, 1919; Sreenivasan \& Rizvi, 1946; Venkatesan, Nandy \& Krishnan, 1979; Chakrabarti \& Pardhan, 1985; Yathiraj et al., 1994; Nayak et all., 1997; Putatunda, Kumar \& Banerjee, 2004, Sivajothi et al., 2013) - Demodex canis, D. cati, D. caprae, D. cornei, D. ovis

Indonesia (Sangvaranond, 1979) - Demodex

Iran (Rafyi, Alavi \& Rak, 1967; Rak, 1969, 1973, 1976; Rak \& Rahgozar, 1975a, 1975b) Demodex canis, D. ovis, D. phylloides

Israel and Palestina (Yeruham, Rosen \& Hadani, 1986) - Demodex ovis

Japan (Tashiro et al., 1979; Sarashina \& Sato, 1986; Morita, 2018) - Demodex aurati, D. criceti [cage], D. cyonis, D. bovis

Korea (Chee, Jeong-Hyun et al., 2008) - Demodex canis

Kuwait (Tarello, 2007) - Demodex sp.

Laos (Izdebska, 2017) - Demodex bandicotae

Malaysia (Beck, 1971; Lukoschus, Mertens, Nutting \& Nadchatram, 1984; Desch, Lukoschus \& Nadchatram, 1984, 1986; Dorny et al., 1994) - Demodex caprae, D. intermedius, D. neoopisthosomae, D. sabani, D. spelaea

Mongolia (Tsedev \& Grebenyuk, 1975; Matthes \& Bukva, 1993) - Demodex bovis, D. caprae Saudi Arabia (Tarello, 2007) - Demodex sp.

Thailand (Tarello, 2007) - Demodex sp.

\section{NORTH AMERICA}

Canada (Hist, 1919; Smith, 1960, 1961a, 1961b; Gentes, Proctor \& Wobeser, 2007) - Demodex odocoilei, D. phylloides

Mexico (Hoffmann et al., 1972; Quintero, 1977; Vargas, Bassols, Desch, Quintero \& Polaco, 1995; Rodriguez-Vivas et al., 2003) - Demodex artibei, D. canis, D. folliculorum, D. mexicanus, D. phylloides

USA (Faxon, 1878; Stiles, 1892; Hirst, 1919; Cram, 1925; Baker \& Nutting, 1950; Besch \& Griffiths, 1956; Carpenter, Freeny \& Patton, 1972; Desch \& Nutting, 1974; Nutting, Beekman \& Snyder, 1978; Dailey \& Nutting, 1980; Hughes \& Nutting, 1981; Lombert, 
Lukoschus \& Whitaker, 1983; Veal, Giesen \& Whitaker, 1984; Forrester, Spalding \& Woodling, 1993; Hurley \& Desch, 1994; Desch, 1995, 1996; Waggie \& Marion, 1997; Foster, Cames \& Forrester, 1998; Desch \& Bonner, 1999; Jacques, Jenks, Hildreth, Schauer \& Johnson, 2001; Desch \& Stewart, 2002; Desch \& Hillier, 2003; Bildfell et al., 2004; Jekl, Hauptman, Jeklova \& Knotek, 2006; Reeves et al., 2007; Turner \& Cano, 2008; Prasad, 2009; Desch et al., 2010; Yabsley et al., 2013) - Demodex bovis, D. brevis, D. canis, D. caprae, D. cricetuli, D. dasypodi, D. equi, D. folliculorum, D. gapperi, D. gatoi, D. injai, D. kutzeri, D. leucogasteri, D. nycticeii, D. odocoilei, D. peromysci, D. phylloides, D. ursi, D. zalophi, Ophthalmodex juniatae

\section{SOUTH and CENTRAL AMERICA}

Argentine (Hirst, 1919) - Demodex phylloides

Aruba (Sutmöller, 1957) - Demodex sp.

Brazil (Hirst, 1919; Rocha \& Pardi, 1954; Faccini, Santos \& Bechara, 2004; Santarém, Farias \& Tostes, 2005; Cardoso \& Franco, 2003) - Demodex bovis, D. phylloides

Chile (Crossley, Gorman \& Alcaino, 1970; Cekalović, 1976: 37; Alcaino \& Gorma, 1999) - Demodex canis

Colombia (Cardona, Vargas \& Perdomo, 2013) - Demodex bovis

Cuba (Guerra et al., 2010) - Demodex spp.

Suriname (Leydig, 1859; Fain, 1960; Desch, Lebel, Nutting \& Lukochus, 1971; Desch \& Nutting, 1972; Lukoschus, Jongman \& Nutting, 1972; Desch, Nutting \& Lukoschus, 1972; Lukoschus \& Nutting, 1979; Nutting, Lukoschus \& Desch, 1980; Lukoschus, Woeltjes, Desch \& Nutting, 1980a, 1980b) - Demodex carolliae, D. desmodi, D. longissimus, D. marsupiali, D. melanopteri, D. molossi, D. phyllostomatis, Ophthalmodex artibei, O. carolliae, O. molossi, Pterodex carolliae

Venezuela (Alvarez et al., 2007) - Demodex sp.

South America (Lebel \& Nutting, 1973) - Demodex saimiri

\section{OCEANIA}

Australia (continent)(Carter, 1942; Nutting \& Wooley, 1965; English, 1969; Nutting \& Sweatman, 1970; Nutting \& Green, 1976; Woeltjes \& Lukoschus, 1981; Veal, Giesen \& Whitaker, 1984; Kniest \& Lukoschus, 1981; Desch, 1981, 1991; Holz, 1998; Domrow, 1991, 1992; Vogelnest et al., 2000; Desch \& Holz, 2006) -Demodex antechini, D. bicaudatus, D. bovis, D. brevis, D. canis, D. caprae, D. cati, D. equi, D. folliculorum, D. macroglossi, D. ovis, D. phylloides, D. tortellinioides, D. zalophi, Demodex sp., Ophthalmodex australiensis, O. wilsoni, Stomatodex cercarteti

New Zealand (Thomson, 1922; Nelson, 1956; Sweatman, 1962; Whitten, 1962; Nutting, Kettle, Tenquist \& Whitten, 1975; Andrews, 1982; Desch, 1986, 1989; Tenquist \& Charleston, 2001; Hill, McKenna \& Mirams, 2008) - Demodex aries, D. brevis, D. canis, D. caprae, D. ermineae, D. folliculorum, D. mystacina, D. novazelandica, D. ovis, D. phylloides

Tokelau (Andrews, 1988) - Demodex 


\section{Hosts of Demodecidae}

\section{Mammalia}

Metatheria

Dasiuromorphia

Fam. Dasiuridae

Antechinus agilis - Demodex antechini, D. tortellinioides

A. stuarti-Demodex antechini

\section{Didelphimorphia}

Fam. Didelphidae

Didelphis marsupialis - Demodex marsupiali

\section{Diprotodontia}

\section{Fam. Phascolarctidae}

Phascolarctos cinereus - Demodex sp.

Fam. Burramyidae

Cercartetus nanus - Stomatodex cercarteti

\section{Eutheria}

\section{Soricomorpha}

Fam. Soricidae

Crocidura suaveolens - Demodex foveolator

Neomys anomalus - Apodemodex cornutus, Demodex neomydis, Soricidex sp.

Sorex araneus - Demodex soricinus, Soricidex dimorphus

Fam. Talpidae

\section{Chiroptera}

Talpa europaea - Demodex talpae

Fam. Pteropodidae

Eonycteris spelaea - Demodex neoopistosomae, D. spelaea

Macroglossus minimus - Demodex bicaudatus, D. macroglossi

Rousettus aegyptiacus leachi - Stomatodex rousetti

Fam. Hipposideridae

Rhinonicteris aurantia - Ophthalmodex australiensis

Fam. Phyllostomatidae

Artibeus aztecus - Demodex artibei lituratus - Ophthalmodex artibei

Carollia perspicillata - Demodex carolliae, D. longissimus, Ophthalmodex carolliae, Pterodex carolliae

Desmodus rotundus - Demodex desmodi

Phyllostoma hastatum - Demodex phyllostomati

Fam. Molossidae

Molossus molossus - Demodex molossi, Ophthalmodex molossi 


\section{Fam. Mystacinidae}

Demodex mystacina, D. novazelandica

\section{Fam. Vespertilionidae}

Barbastella barbastellus - Stomatodes corneti corneti

Eptesicus melanopterus - Demodex melanopteri

Myotis daubentoni - Demodex aelleni

M. lucifugus - Ophthalmodex juniatae

M. myotis - Stomatodes corneti myotis

M. dasycneme - Stomatodes corneti myotis

Nycticeius humeralis - Demodex nycticeii

Plecotus auritus - Demodex chiropteralis, D. plecoti, D. soricinus

P. [Corynorhinus] mexicanus - Demodex mexicanus

Vespadelus pumilus - Ophthalmodex wilsoni

\section{Rodentia}

\section{Fam. Arvicolidae}

Arvicola terrestris - Demodex gliricolens

Microtus agrestis - Demodex agrestis

M. arvalis - Demodex arvicolae

Myodes [Clethrionomys] gapperi - Demodex gapperi

M. [C.] glareolus - Demodex buccalis, D. glareoli

M. [Clethrionomys, Evotomys] glareolus britannicus - Demodex glareoli

\section{Fam. Caviidae}

Cavia - Demodex caviae

\section{Fam. Cricetidae}

Cricetulus migratorius - Demodex cricetuli

C. barabensis - Demodex sinocricetuli

Mesocricetus auratus - Demodex aurati, D. criceti

Peromyscus leucopus - Demodex peromysci

Phodopus sungarus - Demodex phodopi, D. sungori

Fam. Gliridae (Myoxidae)

Muscardinus avellanarius - Demodex muscardini

\section{Fam. Muridae}

Apodemus agrarius - Demodex agrarii, D. apodemi, D. gracilentus, D. huttereri Bandicota indica - Demodex bandicotae

Sylvaemus flavicollis - Demodex corniculatus, D. mollis, D. rosus

S. sylvaticus - Demodex apodemi, D. auricularis, D. lacrimalis, D. longior,

Ophthalmodex apodemi

Leopoldamys sabanus - Demodex sabani

L. edwardsi - Demodex sabani

Mus musculus - Demodex conicus, D. flagellurus, D. fusiformis, D. marculus,

D. musculi, D. vibrissae, Glossicodex musculi 
Niviventer cremoriventer - Demodex sabani

N. rapit - Demodex sabani

Rattus rattus - Demodex nanus

$R$. norvegicus - Demodex nanus, D. norvegicus, D. ponderosus, D. ratti, D. ratticola

R. annandalei - Demodex sabani

R. tiomanicus jalorensis - Demodex sabani

Sundamys mulleri - Demodex sabani

Fam. Sciuridae

Cynomys ludovicianus - Demodex

Sciurus vulgaris - Demodex sciurinus

Fam. Castoridae

Castor fiber - Demodex castoris

\section{Lagomorpha}

Fam. Leporidae

\section{Carnivora}

Oryctolagus cuniculus - Demodex oryctolagi

Fam. Ailuropodidae

Ailuropoda melanoleuca - Demodex ailuropoda

Fam. Canidae

Canis lupus familiaris - Demodex canis, D. cornei, D. cyonis, D. injai

\section{Fam. Felidae}

Felis catus - Demodex gatoi, D. cati

Panthera tigris amoyensis - Demodex tigris

Uncia uncia - Demodex uncii

Fam. Mustelidae

Lutra lutra - Demodex lutrae

Meles meles - Demodex melesinus

Mustela nivalis vulgaris - Demodex sp.

M. erminea - Demodex erminea

Fam. Procyonidae

Procyon lotor - Demodex sp.

Fam. Ursidae

Ursus americanus - Demodex ursi

\section{Carnivora Pinnipedia}

Fam. Otariidae

Zalophus californianus - Demodex zalophi

Fam. Phocidae

Phoca vitulina - Demodex phocidi

\section{Primates}

Fam. Galagonidae 
Galago moholi - Rhinodex baeri, Stomatodex galagoensis

Fam. Cebidae

Saimiri sciureus - Demodex saimiri

\section{Fam. Cercopithecidae}

Macaca mulatta - Demodex macaci

\section{Fam. Callithricidae}

Saguinus geoffroyi - Demodex sp.

S. midas - Demodex sp.

Fam. Atelidae

Ateles sp. - Demodex araneae

\section{Fam. Hominidae}

\section{Perissodactyla}

Homo sapiens - Demodex brevis, D. folliculorum, D. f. sinensis

\section{Fam. Equidae}

\section{Artiodactyla}

Equus caballus - Demodex caballi, D. equi

\section{Fam. Bovidae}

Bison bonasus - Demodex bisonianus, D. bovis

Bos javanicus - Demodex bantengi

B. taurus - Demodex bovis, D. ghanensis, D. tauri

Capra hircus - Demodex caprae

Odocoileus hemionus - Demodex kutzeri, D. odocoilei

O. virginianus - Demodex kutzeri, D. odocoilei

Ovis aries - Demodex aries, D. ovis

Syncerus caffer - Demodex cafferi

Taurotragus oryx - Demodex sp. (nr. D. kutzeri)

\section{Fam. Cervidae}

Alces alces - Demodex kutzeri

Capreolus capreolus - Demodex kutzeri

Cervus elaphus - Demodex acutipes, D. kutzeri

C. elaphus nelsoni - Demodex kutzeri

C. nippon pseudaxis - Demodex kutzeri, D. pseudaxisi

Dama dama - Demodex kutzeri

Odocoileus h. hemionus - Demodex kutzeri

O. virginianus - Demodex kutzeri, D. odocoilei

\section{Fam. Suidae}

Sus scrofa - Demodex folliculorum, D. phylloides 


\section{Bibliography of Demodecidae}

Abu-Samra M.T., B.E.D. Hago, B.E. Musa. 1981. Severe canine demodicosis in the Sudan. First reported cases. - J. Small Anim. Pract., 22(3): 149-152.

Abu-Samra M.T., S.E. Imbabi, E.S. Mahgoub. 1981. Mange in domestic animals in the Sudan. - Annals Trop. Med. Parasitol., 75(6): 627-637.

Abu-Samra M.T., Y.A. Shuaib. 2014. Pathology and pathogenesis of bovine skin and meibomian gland demodicosis. - Rev. Elev. Med. Vet. Pays Trop., 67: 77-85.

Abu-Samra M.T., Y.A. Shuaib. 2015. Bovine demodicosis: Leather from the raw material to the finished product. - J. Soc. Leather Tech. Chem., 99: 80-90.

Abu-Samra M.T., Y.A. Shuaib. 2017. Morphometric and morphologic characteristics of Demodex bovis and Demodex ghanensis (Acarina: Trombidiformes: Demodicidae) isolated from cattle in the Sudan. - J. Anim. Vet. Sci., 3: 25-33.

Agostini A. 1939. Question of Demodex folliculorum in dermatology, study apropos of a case of circumscribed pigmentation of face. - Dermosifilografa, 14: 421-448.

Akbulatova L. 1959. [Detection of the tick Demodex folliculorum in pruritic dermatoses of the face]. - Vestnik Dermat. i Venerol., 33(6): 63-65.

Akbulatova L. 1963. Human demodicosis. - Vestnik Dermat. i Venerol., 38(3): 34-42.

Alcaino H., T. Gorma. 1999. Parasitos de los animales domesticos en Chile. - Parasitologia al dia, 23(1-2): 33-41.

Ali M.H., N. Begum, M.G. Azam, B.C. Roy. 2011. Prevalence and pathology of mite infestation in street dogs at Dinajpur municipality area. - J. Bangladesh Agril. Univ., 9: 111-119.

Alvarez L., O.C. Medina, M.-E. Garcia, H. Garcia. 2007. First report of an unclassified Demodex mite causing demodicosis in a Venezuelan dog. - Ann. Trop. Med. Parasitol., 101(6): 529-532.

Andrews J.R. 1982. The prevalence of hair follicle mites in caucasian New Zealanders. - N.Z. Med. J., 95: 451-453.

Andrews J.R.H. 1988. The epidemiology of Demodex (Demodicidae) infestations in Tokelau Islanders.1, pp. 97-101. In „Progress in Acarology”. Eds. G.L. Channabasavanna \& C.A. Viraktamath. New Delhi, Bombay and Calcutta.

Ayers S.Jr., N.P. Anderson. 1932. Demodex folliculorum its role in the etiology of Acne Rosacea. - Arch. Dermat. Syph., 25: 89-96.

Ayers S.Jr., S. Ayers, III. 1961. Demodectic Eruptions (Demodicosis) in the human. - Arch. Dermat., 83: 816-827.

Bacigalupo J., R. J. Roveda. 1954. Demodex caviae n. sp. - Rev. Med. Vet., Buenos Aires, 36: 149-153.

Baker A.S., J.C. Craven. 2003. Checklist of the mites (Arachnida: Acari) associated with bats (Mammalia: Chiroptera) in the British Isles. - Syst. Appl. Acarol., 14: 1-20.

Baker D. W., W. F. Fisher. 1960. The incidence of demodectic mites in the eyelids of various mammalian hosts. - J. Econ. Entomol., 62: 942. 
Baker D. W., W. B. Nutting. 1950. Demodectic mange in New York State sheep. - Cornell Veterinarian, 40: 140-142.

Baker K. P. 1970. Observations on the epidemiology, diagnosis and treatment of Demodicosis in dogs. - Vet. Rec., 86: 90-91.

Baker K. P. 1973. Demodex spp. In the Meibomian glands of Irish cattle. - Vet. Rec., 92: 699-701.

Baker K. P. 1975. Hyperpigmentation of the skin in canine demodicosis. - Vet. Parasitology, 1(2): 193-197.

Ballweber L.R., J.E. Harkness. 2007. Parasites of guinea pigs. In: Flynn's Parasites of Laboratory Animals; Baker, D.G., Ed.; Blackwell Publishing: Ames, IA, USA: 421-449.

Batey R.G., R.C.A. Thompson, D.G. Nickels. 1981. Demodectic mange in a cat. - Australian Vet. Journal, 57: 49.

Besch E.D., H. Griffiths. 1956. Demonstration of Demodex equi (Railliet, 1895) from a horse from Minnesota. - J. Am. Vet. Med. Assoc., 128: 82-83.

Beck A.J. 1971. A survey of bat ectoparasites in West Malaysia. - J. of Med. Entomology, 8(2): 147-152.

Beerman H., J.H. Strokes. 1934. Rosacea complex and Demodex folliculorum. - Arch. Dermat. Syph., 29: 874-884.

Bell D. S., W. D. Pounden, B. H. Edgington, O. G. Bentley. 1952. Psorergates ovis - a cause of itchiness in sheep. - J. Am. Vet. Med. Assoc., 120: 117-120.

Bennison J. C. 1943. Demodicosis of horses with particular reference to equine members of the genus Demodex. - J. Roy. Army Vet. Corps, 14: 34-49, 66-73.

Bibov A.I. 1965. Demodicosis in cattle. - Veterinaria, 42(10): 48-49.

Bildfell R.J., J. W. Mertins, J.A. Mortenson, D.F. Cottam. 2004. Hair-loss syndrome in blacktailed deer of the Pacific Northwest. - J. of Wildlife Diseases, 40(4): 670-681.

Bochkov A. V. 2009a. A review of mites of the parvorder Eleutherengona (Acariformes: Prostigmata) - permanent parasites of mammals. - Acarina Supplement No1: 3-149.

Bochkov A. V. 2009b. Origin and evolution of parasitism in mites of the infraorder Eleutherengona (Acari: Prostigmata). Report II. Superfamily Cheyletoidea. - Parazitologiya (St. Petersburg), 43(2): 97-117

Bornstein S. 2010. Important ectoparasites of alpaca (Vicugna pacos). - Acta Vet. Scand., 52(Suppl. 1): S17.

Borrel A. 1908. Demodex et infections cutanées. - Comp. Rend. Soc. Biol. Paris, 65(36): 596-597.

Borrel A. 1909. Lèpre et Demodex. - Compt. Rend. Acad. Sci., Paris, 148(1): 50-51.

Bowman D.D., Ch. M. Hendrix, D.S. Lindsay, S.C. Barr. 2002. Feline clinical parasitology. Iowa State University Press.

[Bregetova N.G. et al.]. 1955. Kleshchi Gryzunov Fauny SSSR, Izdatel'stvo Ak. Nauk SSSR: Moskva, Leningrad, 1-459.

Brownlee A. 1935. A species of Demodex found in sheep in Britain. - J. comp. Path. Ther., 48: $68-73$. 
Bukva V. 1982. Soricidex dimorphus g. n., sp. n. (Acari: Demodicidae) from the common shrew, Sorex araneus. - Folia Parasitologica (Praha), 29: 343-349.

Bukva V. 1984. Demodex foveolator sp. n. (Acari: Demodicidae), a new epidermis-dwelling parasite of Crocidura suaveolens (Pallas, 1821). - Folia Parasitologica (Praha), 31: 45-52.

Bukva V. 1985. Demodex flagellurus sp. n. (Acari: Demodicidae) from the preputial and clitoral glands of the house mouse, Mus musculus L. - Folia Parasitologica, 32: 73-81.

Bukva V. 1986. Demodex tauri sp. n. (Acari: Demodicidae), a new parasite of cattle. - Folia Parasitologica, 33: 363-369.

Bukva V. 1987. Demodex kutzeri sp. n. (Acari: Demodicidae), an identical parasite of two species of deer, Cervus elaphus and C. nippon pseudaxis. - Folia Parasitologica, 34: 173-181.

Bukva V. 1988. Synhospitaly of Demodex species (Acari: Demodicidae) in the sheep, Ovis aries L. Abstracts of the V-th European Multicolloquium of Parasitology, September 4-9, 1988, Budapest, p. 150.

Bukva V. 1990a. Transmission of Demodex flagellurus (Acari: Demodicidae) in the house mouse, Mus musculus, under laboratory conditions. - Experimental \& Applied Acarology, 10: 53-60.

Bukva V. 1990b. Three species of the hair follicle mites (Acari: Demodicidae) parasitizing the sheep, Ovis aries L. - Folia Parasitologica, 37: 81-91.

Bukva V. 1991. Structural reduction and topological retrieval: problems in taxonomy of Demodecidae. - In: F. Dusbábek \& V. Bukva (Eds) Modern Acarology, Academia, Prague and SPB Academic Publishing bv, The Hague, 1: 293-300.

Bukva V. 1993a. Sexual dimorphism in the hair follicle mites (Acari: Demodicidae): scanning electron microscopy of Soricidex dimorphus. - Folia Parasitologica, 40: 71-79.

Bukva V. 1993b. Redescription of Demodex soricinus (Acari: Demodecidae) from the sebaceous glands of Sorex araneus (Insectivora). - Folia Parasitologica, Prague, 40: 243-248.

Bukva V. 1994. Demodex agrarii sp.n. (Acari: Demodecidae) from cerumen and the sebaceous glands in the ear of the striped field mouse, Apodemus agrarius (Rodentia). - Folia Parasitologica, 41(4): 305-311.

Bukva V. 1995a. Demodex neomydis sp. n. (Acari: Demodicidae) from the hair follicles of the Mediterranean water shrew, Neomys anomalus (Insectivora: Soricidae). - Folia Parasitologica, 42(4): 299-306.

Bukva V. 1995b. Demodex species (Acari: Demodecidae) parasitizing the brown rat, Rattus norvegicus (Rodentia): redescription of Demodex ratti and description of $D$. norvegicus sp. n. and D. ratticola sp.n. - Folia Parasitologica (Česke Budejovice), 41(4): 149-160.

Bukva V. 1996. Apodemodex cornutus gen. n. et sp. n. (Acari: Demodicidae): new genus and new species of the hair follicles mite from the Mediterranean water shrew, Neomys anomalus (Insectivora: Soricidae). - Folia Parasitologica, 43: 312-316.

Bukva V., W. B. Nutting, C. E. Desch. 1992. Description of Ophthalmodex apodemi sp. n. (Acari: Demodecidae) from the ocular area of Apodemus sylvaticus (Rodentia: Muridae) with notes on pathogenicity. - Int. J. Acarol., 18: 269-276. 
Bukva V., J. Preisler. 1988. Observations on the morphology of the hair follicle mites (Acari Demodicidae) from Cervus elaphus L., 1758 including description of Demodex acutipes sp.n. - Folia parasitol., 35: 67-75.

Bukva V., J. Vítovec, P. Mouha, J. Váhala. 1984. Demodicosis of an African buffalo (Syncerus caffer caffer) in captivity. - Zbl. Vet. Med., 31: 627-633.

Bukva V., J. Vítovec, P. Mouha, J. Váhala. 1988. Pathological process induced by Demodex sp. (Acari: Demodicidae) in the skin of eland, Taurotragus oryx (Pallas). - Folia parasitologica (Praha), 35(1): 87-91.

Bukva V., J. Vítovec, V. Schandl. 1985. The first occurrence of bovine demodicosis in Czechslovakia. - Veter. Med. (Praha), 30: 515-520.

Bukva V., J. Vítovec, M. Vlček. 1985. Demodex rosus sp. n. (Acari: Demodicidae) parasiting the upper digestive tract of rodents. - Folia Parasitologica, 32: 151-162.

Candotti P., G. Varisco, G. Barigazzi, E. Foni, A. Ruffo, P. Boni. 1996. Indagine parassitologica sulla popolazione di cinghiale (Sus scrofa) dell'Appennino parmense. - Suppl. alle Ric. di Biologia della Selvaggina, 24 (Unico): 127-130.

Canépa E., A. Da Grana. 1941. La presencia del Demodex folicullorum Owen en los ganglios linfaticos de perros demodecticos. - Rev. Fac. Agron. Vet. Univ. Buenos Aries, 1 (9): 109-114.

Canestrini G. 1899. Prospetto dell' Acarofauna Ital., Part 8.

Canestrini G., P. Kramer. 1899. Demodicidae und Sarcoptidae. In: Tierreich, 7. Liefg. Berlin: 4-150.

Cardona J., M. Vargas, S. Perdomo. 2013. Descripción clínica de la demodicosis bovina (Demodex bovis) en Córdoba, Colombia. - Rev. Inv. Vet. Perú, 24: 125-129.

Cardoso M.J.L., S.R.V.S. Franco. 2003. Demodicosis in golden hamster (Mesocricetus auratus) - Case in Brasil. - Ars. Vet., 19: 126-128.

Carpenter J.W., J.C. Freeny, C.S. Patton. 1972. Occurrence of Demodex Owen 1843 on a whitetailed deer from Oklahoma. - J. Wildl. Dis., 8: 112-114.

Carter H. B. 1942. A note on the occurrence of the follicle mite (Demodex sp.) in Australian sheep. - Austral. Vet. J., 18: 120-124.

Cekalovic T.K. 1976. Catalogo de los Arachnida: Scorpiones, Pseudoscorpiones, Opiliones, Acari, Araneae y Solifugae de la XII Region de Chile, Magallanes incluyendo la Antarctica Chilena (Chile). - Gayana, Instituto de Biologia, Zoologia, 37.

Chanie M., S. Tadesse, B. Bogale. 2013. Prevalence of bovine Demodicosis in Gondar Zuria District, Amhara Region, Northwest Ethiopia. - Glob. Vet., 11: 30-35.

Chapman R.E. 1973. A clinical manifestation in wool of demodectic infestation of sheep. Australian Vet. J., 18: 120-124.

Chee Jeong-Hyun, Kwon Jung-Kee, Ch Ho-Seong, Ch Kyoung-Oh, Lee Yu-Jin, A.M. AbdelAty, Shin Sung-Shilk. 2008. Survey of ectoparasite infestations in stray dogs of Gwang - ju city, Republic of Korea. - Korean Journal of Parasitology, 46(1): 23-27.

Chen G. 199?. Hair follicle mites. 98-102. - In: Chen X. \& Ma E. [Eds]. Researches of acarology in China. - Chongqing Publishing House, Chongqing [undated], 1-170. 
Chernobayev N. 1928. Demodex folliculorum infecting raw material at the tannery of Spassk, Riezan Prov. USSR. - Vestnik Sovrem. Vet., Moskva, (58) 9: 274-275.

Chesney C.J. 1999. Short form of Demodex species mites in the dog: occurrence and measurements. - J. Small Anim. Pract., 40: 58-61.

Chroustová E., J. Willomitzer, V. Mrva. 1974. Occurrence of demodicosis in sheep. Veterinářství, 24: 115-116.

Cierocka K., J.N.Izdebska, L.Rolbiecki. 2019. Demodex foveolator (Acariformes: Demodecidae) from Crocidura suaveolens (Soricomorpha: Soricidae) - the second observation worldwide, and a checklist of the demodecid mites of soricomorphs. - Ann. Parasitol., 65: 329-332.

Conroy J. D., M. C. Healey, A. G. Bane. 1982. New Demodex species infesting a cat: A case report. - J. Amer. Anim. Hosp. Ass., 18: 405-407.

Cooreman J. 1946. Acariens de l'île de Chypre. (Voyage de M.A. Ball, Octobre - Novembre 1932). - Bull. Mus. Hist. Nat. Belg., 22(8): 1-14.

Cram E. B. 1925. Demodectic mange of the goat in the United States. - J. Amer. Vet. Med. Association, 66: 475-480.

Crossley J., T. Gorman, H. Alcaino. 1970. Sarna demodécica del vacuno en Chile. - Bol. Chil. Parasitol., 25: 126-129.

Csokor J. 1879a. Ueber Haarsackmilben und eine neue Varietät derselben bei Schweinen, Demodex phylloides. - Verh. Zool.- Bot. Ges. in Wien, 29: 419-450.

Csokor J. 1879b. Die Haarsackmilbe des Schweines. Demodex phylloides, eine neue Varietät. - Vierteljahrschrift für Veterinärk., 51: 133-185.

Dailey M.D. 1974. Demodex in captive sea lions. - Presented at the $5^{\text {th }}$ annual meeting of the Internat. Assoc. for Aquatic Medecine. May 1, Sea World, Orlando, Florida.

Dailey M.D., W.B. Nutting. 1980. Demodex zalophi, sp. nov., (Acari: Demodicidae) from Zalophus californianus, the California sea lion. - Acarologia, 21(3-4): 423-428.

Daniel M., V. Bozdech, C. Moucka. 1959. Vyskyt trudnika tukoveho (Demodex, folliculorum Owen 1843) u lidi a jeho epidemiologie [The occurrence of Demodex folliculorum (Owen, 1843) in man and its epidemiology]. - Českoslov. Epidemiol. Microbiol. Immunol., 8(1): 52-60.

De Bosschere H., J. Casaer, A. Neukermans, K. Baert, T. Ceulemans, P. Tavernier, S. Roels. 2007. Severe alopecia due to demodicosis in roe deer (Capreolus capreolus) in Belgium. - The Veterinary Journal, 174: 665-668.

Desch C. E. 1981. A new species of demodicid mite (Acari: Prostigmata) from Western Australia parasitic on Macroglossus minimus (Chiroptera: Pteropodidae). - Rec. West. Aust. Mus., 9: 41-47.

Desch C. E. 1986. Demodex aries sp. n., a sebaceous gland inhabitant of the sheep, Ovis aries, and a redescription of the Demodex ovis Hirst, 1919. - New Zealand J. Zool., 13: 367-376.

Desch C. E. 1987. Redescription of Demodex nanus (Acari: Demodicidae) from Rattus norvegicus and $R$. rattus (Rodentia). - J. Med. Entomol., 24: 19-23. 
Desch C. E. 1989. Two new species of Demodex (Acari: Demodicidae) from the New Zealand short-tailed bat, Mystacina tuberculata Gray, 1843 (Chiroptera: Mystacinidae). - New Zealand J. Zool., 16: 221-230.

Desch C. E. 1991. A New Australian species of Stomatodex Fain (Acari: Demodicidae) from the oesophagus of Cercartetus nanus (Desmarest) (Marsupialia). - J. Aust. Ent. Soc., 30: 239-241.

Desch C.E. 1993. A new species of hair follicle mite (Acari: Demodecidae) from the snow leopard, Panthera uncia (Schreber, 1775)(). - Int. J. Acarol., 19: 63-67.

Desch C.E. 1994. A new species of Demodex Owen, 1843 (Acari: Demodecidae) from the Meibomian glands of the Vampire bat Desmodus rotundus (E. Geoffroy, 1810)(Chiroptera: Phyllostomidae: Desmodontinae) from Surinam. - Int. J. Acarology, 20(1): 39-43. [summary]

Desch C.E. 1995. A new species of Demodex (Acari: Demodecidae) from the black bear of North America, Ursus americanus Pallas, 1780 (Ursidae). - Intern. J. of Acarology, 21: 23-26.

Desch C.E. 1996. Demodex nycticeii: A new species of hair follicle mite (Acari: Demodecidae) from the evening bat, Nycticeius humeralis (Chiroptera: Vespertilionidae). - Int. J. of Acarology, 22(3): 187-191.

Desch C.E., J.J. Andrews, A. Laurie, A. Baeten, H. Zach, G. Jenny, D.W. Powers, L.R. Ballwebe. 2010. New Records of Hair Follicle Mites (Demodecidae) from North American Cervidae. - Journal of Wildlife Diseases, 46(2): 585-590.

Desch C. E., S.T. Bonner. 1999. Demodex gatoi: new species of hair follicle mite (Acari: Demodecidae) from the domestic cat (Carnivora: Felidae). - J. med. Entom., 36(2): 182-185.

Desch C. E., M. D. Dailey, P. Tuomi. 2003. Description of a hair follicle mite (Acari: Demodecidae) parasitic in the earless seal family Phocidae (Mammalia: Carnivora) from the harbor seal Phoca vitulina Linnaeus, 1758. - Internat. J. Acarol., 29(3): 231-235.

Desch C. E., S.L. Davis, H. Klompen. 2006. Two new species of Demodex Owen, 1843, the hair follicle mites (Demodecidae) from the Dzungarian hamster, Phodopus sungarus (Pallas, 1773)(Rodentia: Muridae). - Internat. J. of Acarology, 32(1): 75-80.

Desch C. E., A. Hillier. 2003. Demodex injai: a new species of hair follicle mite (Acari: Demodecidae) from the domestic dog (Canidae). - Journal of Medical Entomology, 40(2): 146-149.

Desch C. E., P.H. Holz. 2006. Demodex tortellinioides n.sp. (Acari: Demodecidae) from the agile Antechinus, Antechinoides agilis Dickman, Parnaby, Crowther and King, 1998 (Marsupialia: Dasyuridae) in Australia. - Internat. J. Acarol, 32(1): 81-84.

Desch C. E., R.J. Hurley. 1997. Demodex sinocricetuli: new species of hair follicle mite (Demodecidae) from the Chinese form of striped hamster, Cricetulus barabensis (Rodentia: Muridae). - J. of Med. Entomology, 34(3): 317-320.

Desch C. E., F. S. Lukoschus, M. Nadchatram. 1984. A new demodicid (Acari: Demodicidae) from the Meibomian glands of Southeast Asian rats (Rodentia: Muridae). - Trop. Biomed., 1: 55-62. 
Desch C. E., F. S. Lukoschus, M. Nadchatram. 1986. Two new species of Demodex (Acari: Demodicidae) from the Meibomian glands of the tropical Old World bat, Eonycteris spelaea (Chiroptera). - Int. J. of Acarology, 12(1): 13-25.

Desch C. E., R. R. Lebel, W. B. Nutting, F. S. Lukoschus. 1971. Parasitic mites of Surinam I. Demodex carolliae n. sp. (Acari: Demodicidae) from the bat Carollia perspicillata. - Parasitology, 62: 303-308.

Desch C. E., W. B. Nutting. 1969. Demodex caprae - structural features of use in demodicid systematics. $-44^{\text {th }}$ Annual Meeting of Am. Soc. Parasitologists, Washington, D.C.

Desch C. E., W. B. Nutting. 1972. Demodex folliculorum (Simon) and D. brevis Akbulatova of man: redescription and reevaluation. - J. Parasitol., 58(1): 169-177.

Desch C. E., W. B. Nutting. 1973. Demodicids (Trombidiformes: Demodicidae) of medical and veterinary importance. - Proc. 3-rd Internat. Congr. Acarology, Prague 1971: 499-505.

Desch C. E., W. B. Nutting. 1974. Demodex odocoilei sp. nov. from the white-tailed deer, Odocoileus virginianus. - Can. J. Zool., 52(6): 785-789.

Desch C. E., W. B. Nutting. 1978a. Morphology and functional anatomy of Demodex folliculorum (Simon) of man. - Acarologia, 19(3): 422-462.

Desch C. E., W. B. Nutting. 1978b. Demodex canis redescription and reevaluation. - Cornell Vet., 68: 139-149.

Desch C. E., W. B. Nutting. 1979a. Redescription of Demodex caballi (=Demodex folliculorum var. equi Railliet, 1895) from the horse, Equus caballus. - Acarologia, 20: 235-240.

Desch C. E., W. B. Nutting. 1979b. Demodex cati Hirst 1919: a redescription. - Cornell Veterinarian, 69: 280-285.

Desch C. E., W. B. Nutting, F. S. Lukoschus. 1972. Parasitic mites of Surinam VII. Demodex longissimus n. sp. from Carollia perspicillata and D. molossi n. sp. from Molossus molossus (Demodicidae: Trombidiformes); Meibomian complex inhabitants of neotropical bats (Chiroptera). - Acarologia, 14(1): 35-53.

Desch C. E., J. O’ Dea, W. B. Nutting. 1970. The proctodeum - a new key character for .demodicids (Demodicidae). - Acarologia, 12(3): 522-526.

Desch C. E. Jr., F. S. Lukoschus, M. Nadchatram. 1984. A new demodicid (Acari: Demodicidae) from the Meibomian glands of Southeast Asian rats (Rodentia: Muridae). - Tropical Biomedicine, 1: 55-62.

Desch C. E. Jr., T.B. Stewart. 1999. Demodex gatoi: new species of hair follicle mite (Acari: Demodecidae) from the domestic cat (Carnivora: Felidae). - J. Med. Entomology, 36(2): 167-170.

Desch C. E. Jr., T.B. Stewart. 2002. First description of a hair follicle mite from the host order Xenarthra: Demodex dasypodi n.sp. (Acari: Demodecidae) from the nine-banded armadillo, Dasypus novemcinctus Linnaeus, 1758 (Dasypodidae). - Int. J. of Acarology, 28(2): 169-174.

Di Benedetto S.A. 1961. The biology of Demodex myotidis sp. nov. (Acarina: Demodicidae) from three species of bats. Master's Thesis, University of Massachusetts Amherst, Amherst, MA, USA. 
Domrow R. 1991. Acari Prostigmata (excluding Trombiculidae) Parasitic on Australian Vertebrates: an Annotated Checklist, Keys and Bibliography. - Invertebr. Taxon., 4: 1283-1376.

Domrow R. 1992. Acari Astigmata (excluding Feather Mites) Parasitic on Australian Vertebrates: an Annotated Checklist, Keys and Bibliography. - Invertebr. Taxon., 6: 1452 -1606.

Dorny P., T. Van Wyngaarden, J. Vercruisse, C. Symoens, A. Jalila. 1994. Survey on the importance of mange in the aethiology of skin lesions in goats in Peninsular Malaysia. - Trop. Anim. Hlth. Prod., 26: 81-86.

Dräger N., G. D. Paine. 1980. Demodicosis in African buffalo (Syncerus cafer cafer) in Botswana. - J. Wildl. Dis., 16: 521-524.

[Drenski P.] Дренски П. 1921. [Notes on the parasitic Acarina in Bulgaria. Preliminary contribution] Бележки върху паразитните акари (Acarina) в България (Предварително съобщение). - Spisanie na BAN, Sofia, 23(11): 81-84 (in Bulgarian).

Dubinin V. B. 1957. Acari: Cheyletoidea and Demodicoidea. - Parasitologicheskiy Sbornik Zoologicheskogo Instituta Akademii Nauk SSSR, 17: 71-136 (in Russian).

Dubinin V.B. 1958. New classification of mites of the super-families Cheyletoidea W.Dub. and Demodicoidea W.Dub. (Acariformes, Trombidiformes). - Parasitol. Sborn. Zool. Inst. Akad. Nauk SSSR Leningrad, 17: 71-136 (in Russian).

Dubinina E.V. 1987. Family Demodicidae. : 297-300. In: Soboleva R.G.[Ed.] [Insects and Acari of the Far East of medical and veterinary importance], Nauka, Leningrad: 1-309.

Dudich E., G. Kolosváry, L. Szalay. 1940. Bars vármegye pókszabású (Arachnoidea-) faunájának alapvetése. - Budapest, 71 p.

Duzgun O.Y., S. Aytekin. 2007. Comparison of Demodex folliculorum density in haemodialysis patients with a control group. - J. of the European Academy of Dermatology and Venereology, 21(4): 480-483.

Du Toit R., O. G. H. Fiedler. 1967. Demodicosis in sheep in South Africa. - J. South Afr. Vet. Med. Ass., 38: 281-282.

English F.P. 1969. Demodex: a cause of blepharitis in Australia. - Med. J. of Australia, 1: 1359-1360.

English F.P. 1971a. Demodex folliculorum and oedema of the eyelash. - British J. of Ophthalmology, 55: 742-746.

English F.P. 1971b. Variant of Demodex folliculorum infesting the eyelids. - British J. of Ophthalmology, 55: 747-749.

English F.P., T. Iwamoto, R.W. Darrell, A.G. Devoc. 1970. The vector potential of Demodex folliculorum. - Arch. Ophthal., 84: 83-85.

English F.P., W.R. Nutting. 1981. Demodicosis of ophthalmic concern. - American J. of Ophthalmology, 91: 362-372.

Enigk K. 1949. Zur Kenntnis der Demodexräude des Hundes. - Zentralbl. Bacteriol. 1 Abt. Orig., 153: 76-90.

Erbağci Z., S. Erkiliç. 2000. Basal cell carcinoma and demodecidosis: Is there an etiologic or coincidental relationship? - Turkish J. of Cancer, 30(3): 111-118.

Erbağci Z., O. Ozgöztaşi. 1998. The significance of Demodex folliculorum density in rosacea. - Int. J. Dermatol., 37(6): 421-425. 
Eyndhoven G.L. van. 1965. Note sur quelques Acariens trouvés sur Sorex araneus (Mamm., Insectivora). - Annales de Parasitologie, Paris, 40(1) : 101-103.

Faccini J.L.H., A.C.G. Santos, G.H. Bechara. 2004. Demodicose bovina no Estado da Paraíba. - Pesq. Vet. Bras., 24(3): 149-153.

Fain A. 1959. Deux nouveaux genres d'Acariens vivant dans l'épaisseur des muqueuses nasale et buccale chez un Lémurien (Trombidiformes: Demodicidae). - Bull. Ann. Soc. Roy. Entomol. Belg., 95(9-10): 263-273.

Fain A. 1960. Les Acariens psoriques parasites des chauves-souris XIII. La famille Demodicidae Nicolet. - Acarologia, 2(1): 80-87.

Fan P., Liu Y., Pan Y., Chen K., Liu H., Zhang J., Li K. 1990. Observations on fine structure and pathogenicity of Demodex tigris. - J. Shanghai Agricult. College, 8(1) : 1-8.

Faure C.L. 1923. Étude histologique de la peau dans la gale démodécique du chien. - Compt. Rend. Soc. Biol., Paris, 88(16): 1176-1178.

Faxon W. 1878. On the presence of Demodex folliculorum in the skin of the ox. - Bull. Mus. Comp. Zool., Harvard Coll., Cambrige Mass., 5 (2): 11-18.

Fehr M., S.Koestlinger. 2011. Ectoparasites in small exotic mammals. - Vet. Clin. Exot. Anim., 16: 611-657.

Ferreira D., N. Sastre, I. Ravera, L. Altet, O. Francino, M. Bardagi, L. Ferrer. 2015. Identification of a third feline Demodex species through partial sequencing of the $16 \mathrm{~S}$ rDNA and frequency of Demodex species in 74 cats using a PCR assay. - Vet. Dermatol., 26: 239-246.

Firda K. E., W. B. Nutting, G. K. Sweatman. 1987. Demodex bantengi n. sp. from Bos javanicus (D’Alton) with notes on gross pathology (Acari: Demodicidae). - Int. J. Acarology, 13: 227-231.

Fisher W. F. 1973. Natural transmission of Demodex bovis Stiles in cattle. - J. Parasitol., 59: 223-224.

Fisher W. F. 1981. Recent advances in psoroptic acariasis and demodectic mange of domestic animals and sarcoptic scabies of humans. - Int. J. Dermatol., 20: 585-588.

Fisher W. F., R. W. Miller, A. L. Everett. 1980. Natural transmission of Demodex bovis Stiles to dairy calves. - Veter. Parasitol., 7: 233-242.

Fleischer P., D. Lukešová, M. Skřivánek, B. Hofírek, I. Štursa. 1996. První nálezy demodikózy koz v České Republice. - Vet. Med. (Praha), 41: 289-293.

Forrester D.J., M.G. Spalding, J.B. Woodling. 1993. Demodicosis in black bears (Ursus americanus) from Florida. - J. of Wildlife Diseases, 29(1): 136-138.

Forton F., B. Seys. 1993. Density of Demodex folliculorum in rosacea: a case-control study using standartized skin-surface biopsy. - Br. J. Dermatol., 128(6): 650-659.

Foster G. W., T.A. Cames, D.J. Forrester. 1998. Geographical distribution of Demodex ursi in black bears from Florida. - J. Wildlife Diseases, 34(1): 161-164.

Freire A.A., V.S. Dias. 1947. Elementos para o estudo das dermatoses bovinas de Angola. Demodex bovis (Stiles, 1892). - An. Serv. Vet. Ind. An. Col. Angola, 1: 247-258.

French F. E. 1962. Biology and morphology of Demodex canis. - Iowa state Univ., Thesis, Dissert. Abstrs., 23(9): 3556-3557. 
French F. E. 1963. Two larval stadia of Demodex canis Leydig (Acarina: Trombidiformes). Acarologia, 5(1): 34-38.

French F. E. 1964. Demodex canis in canine tissues. - The Cornell Vet., 54(2): 271-290.

French F., E. S. Raun, D. L. Baker. 1964. Transmission of Demodex canis Leydig to pups. - Iowa State J. Sci., 38: 291-298.

Friedberger 1876. Ueber den Haarsackmilben - Ausschlag der Hunde. - Arch. Wiss. Prakt. Tierheilk. $1876: 22-25$.

Fryderyk S., J.N. Izdebska. Demodex phylloides (Acari, Demodecidae) as a specific parasite of Sus scrofa (Mammalia, Artiodactyla). - Wiadom. Parazytol., 47(4): 797-800.

Fuss F. 1933. La vie parasitaire du Demodex folliculorum hominis. - Ann. Dermatol. Syphilol., 4: 1053-1062.

Fuss F. 1935. Le cycle evolutif du Demodex folliculorum hominis vu par la microphotographie. - Ann. Dermatol. Syphilol., 6 : 326-331.

Fuss F. 1937. Nouvelle contribution à l'etude du Demodex folliculorum hominis; sexe et reproduction. - Ann. Dermatol. Syphilol., 8: 224-229.

Fuss F. 1946. Sur la vie et les moeurs d'un Acarien parasite habituel de la peau humaine : Demodex folliculorum hominis. - Bull. Soc. Nat. Belges Bruxelles, 27(3-4) : 44-47.

Gabaj M.M., W.N. Beesley, M.A. Awan. 1992. A survey of mites on farm animals in Libya. Ann. Trop. Med. Parasitol., 86(5): 537-542.

Gaiger S.H. 1908. Demodex ovis. - J. of Tropical Veterinary Science, 3: 173 - 174.

Gearhart M. S., J. W. Crissman, M. E. Georgi. 1981. Bilateral lower palpebral demodicosis in a dairy cow. - Cornel Vet., 71: 305-310.

Gentes M.L., H. Proctor, G. Wobeser. 2007. Demodicosis in a Mule Deer (Odocoileus hemionus hemionus) from Saskatchewan, Canada. - J. of Wildlife Diseases, 43: 758-761.

George J.B., S. Otobo, J. Ogunleye, B. Adediminiyi. 1992. Louse and mite infestation in domestic animals in northern Nigeria. - Tropical animal health and production, 24(2): 121-124.

Gmeiner F. 1908. Demodex folliculorum des Menschen und der Tiere. - Arch. Dermat. Syph., 92 (3) : 25-96.

Gmeiner F. 1909. Die Acarus - Räude der Tiere. - Zs. Tiermed., Jena, 13: 1-32, 81-108.

Govyrin, V. A., 1955, Demodecosis in dogs. - Sborn. Nauch. Trudov Leningrad. Inst. Usoversh. Vet. Vrach., Moskva and Leningrad, (10): 27-31.

Gowing G. M. 1964. Advanced demodectid dermatitis in 4-day-old Dachshund puppies. Mod. Vet. Pract., 45 (13): 70.

Graubmann H.D., W. Schulz. 1968. Ein Beitrag zur Histopathologie der Demodikose beim Rind. - Arch. Exp. Veter.-Med., 22: 831-836.

Griffiths J.A. 1945. Demodectic mange of domestic animals in Nyassaland. - J. Comp. Path. Therap., 28: 61-66.

Greve J. H., S. M. Gaafar. 1966. Natural transmission of Demodex canis in dogs. - J. Amer. Veter. Med. Assoc., 148: 1043-1045.

Guerra Y., J.D. Mencho, J.G. Rodríguez Diego, E. Marín, J.L. Olivares. 2010. Demodex spp. en perros con demodicosis, en una región de Cuba. - Rev. Salud Anim., 32: 37-42. 
Guiart J. 1902. La frequence du Demodex chez l'homme. - Bull. Soc. Zool. de France, 27: 128-130.

Gurianova M. P., A. E. Dulebov. 1952. Something new on demodicosis in dogs and its treatment. - Veterinariya (Moskva), 29(10): 29-32 (In Russian).

Haitlinger R., A. Jankowska. 2005. Arthropods occuring on Rattus norvegicus (Berkenhout, 1769)(Rodentia: Muridae) in Poland. - Zeszyty naukowe Akademii rolniczej we Wroclawiu, Zootechnika LIII, 529: 35-44.

Hallas Th.E. 1978. Fortegnelse over Danske Mider (Acari). - Ent. Meddr., 46: 27-45.

Haller G. 1882. Beitrag zur Kenntnis der Milbenfauna Württembergs. - Jh. Ver. Vaterl. Naturk. Württemberg, 38: 293-232.

Hamir A.N., D.E. Snyder, C.A. Hanlon, C.E. Rupprecht. 1993. First report of a Demodex sp. in raccoons (Procyon lotor). - J. Wildlife Dis., 29(1): 139-141.

Harvey R.G. 1990. Demodex cuniculi in dwarf rabbits (Oryctolagus cuniculus). - J. Small Anim. Pract., 31(4): 204-207.

Heath R. 1967. Demodectic mange in dogs - a correction. - Australian Vet. J., 43: 196.

Hickey T.E. et al. 1983. Demodectic mange in a Tamarin (Saguinus geoffroyi). - Lab. Anim. Sci., 33:192-193..

Hill F.I., P.B. McKenna, C.H. Mirams. 2008. Demodex spp. Infestation and suspected demodicosis of alpacas (Vicugna pacos) in New Zealand. - New Zealand Vet. Journal, 56(3): 148.

Hill L.R., P.S. Kille, D.A. Weiss, T.M. Craig, L.G. Coghlan. 1999. Demodex musculi in the skin of transgenic mice. - Contemp. Top. Lab. Anim. Sci., 38(6): 13-18.

Hillier A., C.E. Desch. 1997. A new species of Demodex mite in the dog: a case report. - In: Thirteenth Proc., Annual Members Meeting of Amer. Acad. Veterin. Dermatology and Amer. College Vet. Dermatology: 118-119.

Hillier A., C.E. Desch. 2002. Large-bodied Demodex mite infestation in 4 dogs. - Journal of the American Veterinary Medical Association, 220(5): 623-627.

Himonas C. A., J. T. Theodorides, A. E. Alexakis. 1975. Demodectic mites in eyelids of domestic animals of Greece. - J. Parasitol., 61(4): 767.

Hirst S. 1917a. Remarks on certain species of the genus Demodex Owen (the Demodex of man, the horse, dog, rat and mouse). - Ann. Mag. Nat. Hist., 8 (20): 232-235.

Hirst S. 1917b. On three new parasitic Acari. - Ann. Mag. Nat. Hist., 20: 431-434.

Hirst S. 1918. On four new species of the genus Demodex. - Ann. Mag. Nat. Hist., ser. 9, 2(8): $145-146$.

Hirst S. 1919. Studies on Acari. No 1. The genus Demodex, Owen. Frequency of occurrence and effects of parasitism. List of the species and varieties of the genus Demodex with their hosts and known distribution. - Brit. Mus. Nat. Hist., London, 44 p.

Hirst S. 1921a. On some new or little known Acari, mostly parasitic in habit. - Proc. Zool. Soc. London: 357-378.

Hirst S. 1921b. On three new parasitic mites (Leptus, Schöngastia, and Demodex). - Ann. Mag. Nat. Hist., Ninth Ser., 7: 37-39.

Hirst S. 1922. Mites injurious to domestic animals. - British Mus. Nat. Hist., 13: 1-107. 
Hirst S. 1923. On some new or little known Acari. - Proc. Zool. Soc. London, 4: 971-1000.

Hoffmann G. 1989. Grössenunterscheide bei Demodex bovis (Acari). - Angew. Parasitol., 30(2): 141-143.

Hoffmann A., I. B. de Barrera, C. Méndes. 1972. Nuevos hallazgos de acaros en Mexico. - Rev. de la Sociedad Mexicana de Historia Natural, 33: 151-159.

Holz P.H. 1998. Nodules caused by demodex-like mites in the skin of tiger quolls (Dasyurus maculatus). - Australian Vet. J., 76(1): 59-60.

Hoffman A., I.B. de Barrera, C. Mendez. 1972. Nuevos hallazgos de Acaros en Mexico. Rev. Soc. Mexicana de Historia Natural, 33: 151-159.

Hughes S.E., Wm. B. Nutting. 1981. Demodex leucogasteri n.sp. from Onychomys leucogaster - with notes on its biology and host pathogenesis. - Acarologia, 22(2): 181-186.

Hurley R.J., C. E. Desch. 1994. Demodex cricetuli: new species of hair follicle mite (Acari: Demodecidae) from the Armenian hamster, Cricetulus migratorius (Rodentia: Cricetidae). - J. Med. Entomol., 31(4): 529-533.

Iliev P.T., G. Zhelev, A. Ivanov, P. Prelezov. 2019. Demodex cati and feline immunodeficiency virus co-infection in a cat. - Bulg. J. Vet. Med., 22: 237-242.

Imes M. 1935. Cattle scab and methods of control and eradication. - Farmers' Bulletin No 1017, U.S. Department of Agriculture, 29 pp.

Isaichikov I. M. 1928. Acarus (Demodex) folliculorum als ursache einer massenhaften Schidigung der Felle des Rindes. - Prakt. Vet., Moskva (10): 47-54.

Isaichikov I. M. 1929. Present status of the question on control of Demodex folliculorum in domestic animals. - Vestnik Sovrem. Vet., Moskva, (82) 5 (9): 219-221.

Izdebska J.N. 2000. Demodex spp. (Acari, Demodecidae) in Mus musculus from Poland. Wiad. Parazytol., 46: 277-280.

Izdebska J.N. 2001a. European bison arthropod from closed Polish breeding facilities. - Acta Parasitol., 46: 135-137.

Izdebska J.N. 2001b. The occurence of of parasitic arthropods in two groups of European bison in the Białowieża Primeval Forest. - Wiad. Parazytol., 47: 801-804.

Izdebska J.N. 2002. Demodecidae (Acari: Actinedida): the current status and perspectives of research in Poland. In: Ignatowicz S. (Ed.), Postepy polskiej akarologii [Progress of Polish acarology], Ed. SGGW, Warszawa: 215-223.

Izdebska J.N. 2004a. Species of Demodecidae (Acari, Actinedida), new for the fauna of Poland, in common shrew (Sorex araneus L.). - Zoologica Poloniae, 49(1-4): 47-51.

Izdebska J.N. 2004b. Demodex spp. (Acari: Demodecidae) in brown rat (Rodentia: Muridae) in Poland. - Wiad. Parazytol., 50(2): 333-335.

Izdebska J.N. 2004c. Nužeńce ludzkie Demodex brevis i D. folliculorum. In: Arthropoda. A survey of forms and interactions. (Ed. A. Buczek, C. Blaszak. Wydawnictwo Koliber, Lublin: 173-181.

Izdebska J.N. 2005. Demodectic mites (Acari, Actinedida) in carnivorous mammals (Mammalia, Carnivora) of Poland. In: Arthropoda. A survey of forms and interactions. (Ed. A. Buczek, C. Blaszak). Wydawnictwo Koliber, Lublin: 121-125. 
Izdebska J.N. 2006a. Adaptation to parasitism in skin mites from the Demodecidae family (Acari, Prostigmata). In. Arthropods. Epidemiological importance. In: A. Buczek, C. Błaszak (Eds.), Wyd. Kliber, Lublin 2006: 31-36.

Izdebska J.N. 2006b. Skin mites (Acari: Demodecidae, Psoroptidae, and Sarcoptidae) of the European bison, Bison bonasus. - Biological Lett., 43(2): 169-174.

Izdebska J.N. 2006c. Symptomless Skin Mite Infestation of Horses in Northern Poland. In: Gabryś G., S. Ignatowicz (Eds.); Advances in Polish Acarology; SGGW: Warszawa, Poland: 123-127.

Izdebska J.N. 2007a. Roztocze skórne (Acari: Demodecidae, Psoroptidae, Sarcoptidae) żubra na tle akarofauny ssaków kopytnych - problemy specyficzności żywicielskiej i tropicznej. In: W. Olech (ed.) Rola hodowli ex situ w procesie restytucji żubra. Praca zbiorowa, Gołuchów: 22-27.

Izdebska J.N. 2007b. Demodecidae (Acari, Prostigmata) u gryzoni w Polsce. - Wiad. Parazytol., 53(supp.): 143.

Izdebska J.N. 2008. Nužeńcowate (Demodecidae) In: Fauna Polski - charakterystyka i wykaz gatunków (Red. W. Bogdanowicz, E. Chudzicka, I. Pilipuk, E. Skibińska). Museum i Instytut Zoologii PAN, Warszawa, 3: 128-130, 200-201.

Izdebska J.N. 2009. Selected aspects of adaptations to the parasitism of hair follicle mites (Acari: Demodecidae) from hoofed mammals. - European Bison Conservation Newsletter, 2: 80-88.

Izdebska J.N. 2010a. New data of demodecosis hominis - Ethiology, pathogenesis and diagnosis. In: Buczek, A., Blaszak, C. (Eds). Arthropods, Ecological and Pathological Aspects of Parasite-Host Relationships. Akapit, Lublin, Poland, 137-145.

Izdebska J.N. 2010b. Demodex ssp. (Acari, Demodecidae) and demodecosis in dogs: Characteristics, symptoms, occurrence. - Bull. Vet. Inst. Pulawy, 54: 335-338.

Izdebska J.N. 2012. A new Demodecidae species (Acari) from the yellow-necked mouse Apodemus flavicollis (Rodentia, Muridae) - description with data on parasitism. - Parasitol. Res., 98(6):1101- 1104.

Izdebska J.N., K.Cierocka, L. Rolbiecki, P.Kozina, M.Kolodziej-Sobocińska. 2018. Demodex melesinus (Acariformes: Demodecidae) - the forgotten European badger parasite, rediscovered after 100 years. - Acta Parasitologica, 63: 665-668.

Izdebska J.N., K. Cydzik. 2010. Occurrence of Demodex spp. (Acari, Demodecidae) in the striped field mouse Apodemus agrarius (Rodentia, Muridae) in Poland. - Wiad. Parazytol., 56(1): 59-61.

Izdebska J.N., S. Fryderik. 2000. Demodex spp. (Acari, Demodecidae) in wild Artiodactyla in Poland. - Abstr. of 8the European Multicolloquium of Parasitology. Acta Parasitologica, 45(3): 163-164.

Izdebska J.N., S. Fryderyk. 2002. Zmienność morfologiczna Demodex phylloides (Acari, Demodecidae) u dzika (Sus scrofa). In: Stawonogi w medycynie. (A. Buczek, C. Błaszak Eds.), Wyd. Liber Lublin: 25-34.

Izdebska J.N., S. Fryderyk. 2011. Diversity of three species of the genus Demodex (Acari, Demodecidae) parasitizing dogs in Poland. - Pol. J. Environ. Stud., 20: 565-569. 
IzdebskaJ.N., S. Fryderyk. 2012a. New data on the occurrence of Demodex lacrimalis (Acari, Demodecidae) of the wood mouse Apodemus sylvaticus (Rodentia, Muridae). - Annales UMCS, sectio C, Biologia, 67(1): 7-11.

Izdebska J.N., S. Fryderyk. 2012b. Demodex acutipes Bukva \& Preisler, 1988 (Acari, Demodecidae) - a rare parasite of red deer (Cervus elaphus L.). - Annals of Parasitology, 58(1): $161-166$.

Izdebska J.N., S. Fryderyk, L. Rolbiecki. 2016. Demodex castoris sp. nov. (Acari: Demodecidae) parasitizing Castor fiber (Rodentia), and other parasitic arthropods associated with Castor spp. - Dis. Aquat. Org., 118: 1-10.

Izdebska J.N., Z. Jankowski. 2006. Demodex brevis and D. folliculorum (Actinedida, Demodecidae): Specific human parasites. A comparative study of the effectiveness of diagnostic methods involving autopsy. In: Gabryś G., Ignatowicz S., Eds. Advances in Polish Acarology, SGGW: Warszawa, Poland: 128-136.

Izdebska J.N., P. Kozina, S. Fryderyk. 2013. The occurrence of Demodex spp. (Acari: Demodecidae) in bank vole Myodes glareolus (Rodentia, Cricetidae) with data on its topographical preferences. - Ann. Parasitol., 59: 129-133.

Izdebska J.N., P. Kozina, A. Gólcz. 2013. The occurrence of Demodex spp. (Acari, Demodecidae) in bank vole Myodes glareolus (Rodentia, Cricetidae) with data on its topographical preferences. - Ann. Parasitol., 59: 129-133.

Izdebska J.N., M. Krawczyk. 2012. Skin mites of mammals - the occurrence, significance and research prospects in Poland. (In:) Arthropods. The medical and economic importance. A. Buczek, C. Błaszak (eds.). Akapit, Lublin: 123-131.

Izdebska J.N., L. Rolbiecki. 2002. Parasites on the squirrel (Sciurus vulgaris L.) from the region of Gdańsk, Northern Poland. In: Program a zbornik abstraktum. 5. slovenské a české parasitologické dni. 28-31.05.2002: 59.

Izdebska J.N., L. Rolbiecki. 2003. New data on parasites of mole Talpa europaea (Mammalia, Insectivora) in northern Poland. - Wiad. Parazytol., 49: 97-98.

Izdebska J.N., L. Rolbiecki. 2004. The presence of Demodex spp. in correlation with helminth infestation level in the brown rat Rattus norvegicus (Berk.) of the Tri-City urban agglomeration. - In: (Indykiewiecz P., Barczak T., Eds) Urban fauna in Central Europe in the $21^{\text {st }}$ century. LOGO, Bydgoszcz: 581-584.

Izdebska J.N., L. Rolbiecki. 2006. Correlation between the occurrence of mites (Demodex spp.) and nematodes in house mice (Mus musculus Linnaeus, 1758) in the Gdańsk urban agglomeration. - Biological Lett., 43(2): 175-178.

Izdebska J.N., L. Rolbiecki. 2012a. Topical structure and topography of Demodex spp. (Acari Demodecidae), in brown rat Rattus norvegicus (Rodentia, Muridae). (In:) Arthropods. The medical and economic importance. A. Buczek, C. Błaszak (eds.). Akapit, Lublin: 133-141.

Izdebska J.N., L. Rolbiecki. 2012b. Demodectic mites of the brown rat Rattus norvegicus (Berkenhout, 1769) (Rodentia, Muridae) with a new finding of Demodex ratticola Bukva, 1995 (Acari, Demodecidae). - Annals of Parasitology, 58: 71-74. 
Izdebska J.N., L. Rolbiecki. 2012c. Topical structure and topography of Demodex spp. (Acari Demodecidae), in brown rat Rattus norvegicus (Rodentia, Muridae). In: Arthropods. The Medical and Economic Importance; A. Buczek, A. Blaszak (Eds.); Akapit: Lublin, Poland: 133-141.

Izdebska J.N., L. Rolbiecki. 2013a. Demodex microti n. sp. (Acari: Demodecidae) in Microtus arvalis (Pallas)(Rodentia, Cricetidae) with a checklist of the demodectic mites of cricetids. - Syst. Parasitol., 86: 187-196.

Izdebska J.N., L. Rolbiecki. 2013b. A new species of Demodex (Acari, Demodecidae) with data on topical specifity and topography of demodectic mites in the striped field mouse Apodemus agrarius (Rodentia, Muridae). - J. Med. Entomol., 50: 1202-1207.

Izdebska J.N., L. Rolbiecki. 2014a. Demodex lutrae n.sp. (Acari) in European otter Lutra lutra (Carnivora: Mustelidae) with data from other demodecid mites in carnivores. - J. Parasitol., 100: 784-789.

Izdebska J.N., L. Rolbiecki. 2014b. New species of Demodex (Acari: Demodecidae) with data on parasitism and and occurence of other demodecids of Rattus norvegicus (Rodentia: Muridae). - Annales of the Entomological Soc. of America, 107(4): 740

Izdebska J.N., L. Rolbiecki. 2015a. A new species of the genus Demodex Owen, 1843 (Acari: Demodecidae) from the ear canals of the house mouse Mus musculus L. (Acari: Demodecidae). - Syst. Parasitol., 91: 167-173.

Izdebska J.N., L. Rolbiecki. 2015b. Two new species of Demodex (Acari: Demodecidae) with a redescription of Demodex musculi and data on parasitism in Mus musculus (Rodentia: Muridae). - J. Med. Entomol., 52: 604-613.

Izdebska J.N., L. Rolbiecki. 2016. A new genus and species of demodecid mites from the tongue of a house mouse Mus musculus: Description of adult and immature stages with data on parasitism. - Med. Vet. Entomol., 30: 135-143.

Izdebska J.N., L. Rolbiecki. 2018. The status of Demodex cornei: Description of the species and developmental stages, and data on demodecid mites in the domestic dog Canis lupus familiaris. - Med. Vet. Entomol., 32: 346-357.

Izdebska J.N., L. Rolbiecki. 2020. The Biodiversity of Demodecid Mites (Acariformes: Prostigmata), specific parasites of Mammals with a global checklist and a new finding for Demodex sciurinus. - Diversity, 12, 261: 1-38.

Izdebska J.N., L. Rolbiecki, L. Cierocka, I. Pawliczka. 2020. Demodex phocidi (Acariformes: Demodecidae) from Phoca vitulina (Carnivora: Phocidae) - The second observation in the world and a supplement to the species description. - Oceanol. Hydrobiol. St., 49: 49-55.

Izdebska J.N., L. Rolbiecki, S.Fryderyk. 2011. New data on Demodex huttereri Mertens, Lukoschus et Nutting. 1983 and topical specifity and topography of demodectic mites in striped field mouse Apodemus agrarius. - Wiad. Parazytol., 57: 261-264.

Izdebska J.N., L. Rolbiecki, S.Fryderyk. 2014. Demodex auricularis sp. nov. (Acari: Demodecidae) from the ear canal of the European wood mouse Apodemus sylvaticus (Rodentia: Muridae). - Int. J. Acarol., 40: 214-219. 
Izdebska J.N., L. Rolbiecki, S.Fryderyk. 2016. A new species of Demodex (Acari: Demodecidae) from the skin of the vibrissal area of the house mouse Mus musculus (Rodentia: Muridae), with data on parasitism. - Syst. Appl. Acarol., 21: 1031-1039.

Izdebska J.N., L. Rolbiecki, S.Fryderyk, L. Mierzyński. 2017. Adult and immature stages of the new species of the genus Demodex (Acariformes: Demodecidae) with data on parasitism, topography, and topical specifity of demodectic mites in the yellow-necked mouse, Apodemus flavicollis (Rodentia: Muridae). - J. Parasitol., 103: 320-329.

Izdebska J.N., L. Rolbiecki, L. Mierzyński, K. Bidziński. 2018. Demodectid mites (Acariformes, Demodecidae) in brown long-eared bat Plecotus auritus (Chiroptera, Vespertilionidae) - Second record in the world and systematic status of Demodex chiropteralis Hirst, 1921. - Ann. Parasitol., 64: 109-113.

Izdebska J.N., L. Rolbiecki, L. Mierzyński, K. Bidziński. 2019a. Morphological and ontogenetic characteristics of Demodex plecoti sp.nov. (Acariformes: Demodecidae) from the brown long-earned bat Plecotus auritus (Chiroptera: Vespertilionidae), with comments on parasitism. - Syst. and Applied Acarology, 24(3): 377-388.

Izdebska J.N., L. Rolbiecki, S. Morand, A.Ribas. 2017. A new species and new host record of Demodecidae (Acariformes: Prostigmata) associated with the bandicot rat (Rodentia: Muridae) from Lao PDR with data on parasitism and a checklist of the demodecid mites of rodents. - Syst. and Applied Acarology, 22(11): 1910-1923.

Jablonowski J. 1918. Ordo Acarina. - In: Fauna Regni Hungariae, Budapest: 2-5.

Jacques C.N., J.A. Jenks, M.B. Hildreth, R.J. Schauer, D.D. Johnson. 2001. Demodicosis in a white-tailed deer (Odocoileus virginianus) in South Dakota. - Prairie Naturalist, 33: 221-226.

James S. B., B.L. Raphael. 2000. Demodicosis in red-handed tamarins (Saguinus midas). - J. of Zoo and Wildlife Medicine, 31(2): 251-254.

Jańczak D., A. Ruszczak, I. Kaszak, E. Goląb, K. Barszcz. 2017. Clinical aspects of demodicosis in veterinary and humane medicine. - Med. Weter., 73: 265-271.

Jekl V., K. Hauptman, E. Jeklova, Z. Knotek. 2006. Demodicosis in nine prairie dogs (Cynomys ludovicianus). - Vet. Dermatology, 17(4): 280-283.[summary]

Jimenez I.A., M.R. Odom, S.A. Childs-Sanford, A. Lucio-Forster, D.D. Bowman. 2020. Lynxacarus and Demodex infestation in a captive jaguar (Panthera onca) in La Democracia, Belize. - Vet. Rec. Case Rep., 8: e001037.

Kadlec V. 1975. Demodex suis. - Vet. Med., 20: 441-443.

Kadlec V., J. Kubelka. 1974. Demodicosis in sheep. - Veter. Med. (Praha), 19: 249-254 (In Czech).

Kadulski S. 1978. Ectoparasites of the boar Sus scrofa L. in Poland. In: Proc. Of the Fourth Intern. Congress of Parasitology, A Conference of the World Fed. Of Parasitologists, Warszawa, Poland, August 1978: 211-212.

Kadulski S. 1996a. Ectoparasites of Cervidae in north-east Poland. - Acta parasitologica, 41(4): 204-210. 
Kadulski S. 1996b. Further studies on parasitic arthropods of elk Alces alces from Poland. Wiadomosci Parazytol., 42(3): 349-355.

Kadulski S., J.N. Izdebska. 1996. Demodex bisonianus sp. nov. (Acari: Demodicidae) a new parasite of the bison (Bison bonasus L.). - Wiadomosci Parazytol., 42(1): 103-110.

Kadulski S., J.N. Izdebska, M. Konczyk. 1996. Parasitic Arthropods of Bison bonasus from Bialowieza Primeval Forest. - Wiadomosci Parazytol., 42(2): 255-260.

Kadulski S., B. Szczurek. 2004. Ectoparasites on fallow deer, Dama dama (L.) in Pomerania, Poland. - Acta Parasitol., 49: 80-86.

Karaer Z., A. Kurtdede, K. Ural, B. Sari, C.C. Cingi, M.C. Karakurum, A.E. Haydardedeoğlu. 2009. Demodicosis in a golden (Syrian) hamster (Mesocricetus auratus). - Ankara Üniversitesi Veteriner Fakültesi Dergisi, 56: 227-229.

Karjala Z., C. E. Desch, M. F. Starost. 2005. First description of a new species of Demodex (Acari: Demodecidae) from Rhesus monkey. - J. Med. Entomology, 42(6): 948-952.

Kennedy M.J. 2001. Mange in cattle: Demodectic mange. - Agri-Facts, 4: 1-2.

Kenney D. 1969. Demodex sp. isolated from cutaneous lesions in a sub-adult California sea lion. - Presented ar the $6^{\text {th }}$ Annual Conference on Biological Sonar and diving mammals. Feb. 8. Stanford Research Institute, Palo Alto, California.

Kirk H. 1949. Demodectic mange. - Vet. Rec., 61: 394.

Kirkwood A., S. B. Kendall. 1966. Demodectic mange in cattle. - Vet. Rec., 78: 33-34.

Klinski Yu. D. 1956. Demodicosis of sheep. - Byull. Nauchnotekhn. Inform., 1: 16-17 (In Russian).

Klinski Yu. D. 1957. Distribution, diagnostics and pathomorphology of demodicosis of sheep. - Trudy vsesoyuz. Nauchnoissled. Inst. Veter. Sanit. Ektoparasitol., 12: 3-13 (In Russ.).

Klinski Yu. D. 1959. Materials on morphology and biology of Demodex ovis mites. - Trudy vsesoyuz. Nauchnoissled. Inst. Veter. Sanit. Ektoparasitol., 15: 38-45. (In Russ.).

Kniest F. M., F. S. Lukoschus. 1981. Parasites of Western Australia XIII. A new species of demodicid mite from the Meibomian glands of the bat Macroglossus minimus. - Rec. West. Aust. Mus., 9: 111-118.

Kolev G. 1958. Ein Fall von Demodicose beim Pferd in Bulgarien. - Tr. Sci. Inst. Sup. Med. Vet. Sofia, 5: 299-304 (Bulg., summ. Russ., Germ.).

Koutz F. R. 1953. Demodex folliculorum studies: II. Comparison of various diagnostic methods. - Speculum (Ohio State Univ.), 6: 8-9, 23, 26, 44.

Koutz F. R. 1954. Demodex folliculorum studies: III. A survey of clinical cases in dogs. - J. Am. Vet. Med. Assoc., 124: 131-133.

Koutz F. R. 1955a. Demodex folliculorum studies: IV. Treatment methods. - North Am. Vet., 36: 129-131.

Koutz F. R. 1955b. Demodex folliculorum studies: V. Demodectic mange in cattle. - Vet. Med. Chicago, 50: 305-306.

Koutz F. R. 1957a. Demodex folliculorum studies. VI. The internal phase of canine demodectic mange. - J. Amer. Vet. Med. Assoc., 131: 45-48. 
Koutz F. R. 1957b. Demodex folliculorum studies. VII. The evaluation of systematic drugs in the treatment of demodectic mange in dogs. - Speculum, 11(1): 39-42.

Koutz F. R., H.F. Groves, C.M. Gee. 1960. A survey of Demodex canis in the skin of clinically normal dogs. - Vet. Med., 55: 52-53.

Krawiec D.R., S.M. Gaafar. 1974. Scanning electron micrography of Demodex canis Leydig 1859. - Acarologia, 16(4): 671-676.

Krotova M.B. 1957. The character of the pathological; process in bovine demodicosis and its effect on leather quality. - Tr. Vsesoyuz. Nauch.-issled. Inst. Vet. Sanit. Ektoparazitol., 12: 14-27.

Kuscher A. 1940. Raubmilben beim Hund. - Wein Tieraztl. Monatschr., 27(1): 10-16.

Kutzer D., W. Grünberg. 1972. Demodikose bei Rothirsch (Cervus elaphus). - Zeitschr. f. Parasitenkunde, 40: 9-17.

Lagrain, Regulato. 1903. Rareté des gales sarcoptique et démodectique en Algérie. Sur une épidémie de gale démodectique du Porc. - Arch. Parasitol., Paris, 7(3) : 370-372.

Lamb K.P. 1952. A preliminary list of New Zealand Acarina. - Trans. of the Royal Soc. of New Zealand, 79(3-4): 370-375.

Larinov S. V. 1980. On morphology and biology of demodectic mites. - Tr. Vsesoyuz. Nauch.issled. Inst. Veter. Sanit., 1980: 138-147 (In Russian).

Lawrence H. 1921. The pathogenicity of the Demodex (Owen) in the human being. - Med. J. Australia, year 8, 2, 3: 39-40.

Lebel R.R. 1970. Demodex saimiri sp. nov. and Demodex sciurei sp. nov. ectoparasites of the squirrel monkey, Saimiri sciureus, with notes on demodicids from other subhuman primates. Master's Thesis, Univ. of Massachusetts, Amherst, MA, USA.

Lebel R., C. Desch. 1979. Karyotype and anomalous development in Demodex caprae. - In: Proc. $4^{\text {th }}$ Intl. Congress Acarol. E. Pifl ed.: 525-529.

Lebel R. R., W. B. Nutting. 1973a. Demodectic mites of subhuman primates I: Demodex saimiri sp.n. (Acari: Demodicidae) from the squirrel monkey, Saimiri sciureus. - J. Parasitol., 59(4): 719-722.

Lebel R. R., W. B. Nutting. 1973b. Population dynamics of a parasitic mite Demodex caprae (Trombidiformes: Demodicidae). - Proc. 3-rd Int'l Congress Acarology, Prague, 1971: 517-521.

Legrain, Régulato. 1903. Rareté des gales sarcoptique et démodectique en Algérie. Sur une épidémie de gale démodectique du porc. - Arch. Parasit., 7: 370-372.

Leydig F. 1859. Über Haarsackmilben und Krätzmilben. - Archiv für Naturgeschichte, 25: 338-354.

Lin Weiming, Wang Mingshuang, Hong Hua et al. 2000. A Mite of Demodex Parasitized in Chinese Hamster. - Chinese J. of Laboratory Animal Science, 2.

Liu Hui-ming. 2007. Review of human demodicicosis. - Chinese J. of Pest Control, (3): 3.

Löwenstein C., W. Beck, K. Bessmann, R.S. Mueller. 2005. Feline demodicosis caused by concurrent infestation with Demodex cati and an unnamed species of mite. - Vet. Rec., 157(10): 290-292. 
Lombardini G. 1942. Contributo alla conoscenza cella morfologia dei Demodicidae. Chiave analitica del genere "Demodex" Owen. - Redia, 28: 89-102.

Lombert H. A. P. M., F. S. Lukoschus, J. O. Whitaker, Jr. 1983. Demodex peromysci sp. n. (Acari: Prostigmata: Demodicidae) from the Meibomian glands of Peromyscus leucopus (Rodentia: Cricetidae). - J. Med. Ent., 20: 377-382.

Lukoschus F. S., R. H. G. Jongman. 1974. Demodex lacrimalis sp. n. (Demodicidae: Trombidiformes) from the Meibomian glands of European wood mouse Apodemus sylvaticus. - Acarologia, 16(2): 274-281.

Lukoschus F. S., R. H. G. Jongman, W. B. Nutting. 1972. Parasitic mites of Surinam XII. Demodex melanopteri sp. n. (Demodicidae: Trombidiformes) from the Meibomian glands of the neotropical bat Eptesicus melanopterus. - Acarologia, 14(1): 54-58.

Lukoschus F. S., L. J. A. Mertens, W. B. Nutting, W. B. Nadchatram. 1984. Demodex intermedius sp. n. (Acarina: Prostigmata: Demodicidae) from the Meibomian glands of the tree-shrew Tupaia glis (Scandentia: Tupaiidae). - Malay. Nat. J., 36: 489-498.

Lukoschus F. S., W. B. Nutting. 1979. Parasitic mites of Surinam XIII. Ophthalmodex artibei gen. nov., sp. n. (Prostigmata: Demodicidae) from Artibeus lituratus with notes on pathogenesis. - Int. J. Acarol., 5(4): 299-304.

Lukoschus F. S., A. G. Woeltjes, C. E. Desch, W. B. Nutting. 1980a. Parasitic mites of Surinam XX. Pterodex carolliae gen. nov. sp. n. (Demodicidae) from the fruit bat, Carollia perspicillata. - Int. J. Acarol., 6(1): 9-14.

Lukoschus F. S., A. G. Woeltjes, C. E. Desch, W. B. Nutting. 1980b. Parasitic mites of Surinam XXXV. Two new Ophthalmodex spp. (O. carolliae, O. molossi: Demodicidae) from the bats Carollia perspicillata and Molossus molossus. - Int. J. Acarol., 6(1): 45-50.

Maravelas G.K. 1962. Studies of Demodex sylvilagi n. sp. (Acarina: Demodicidae) from the New England cottontail Sylvilagus transitionalis Bangs with a description of Demodex transitionalis n. sp. Master's Thesis, Univ. of Massachusetts Amherst: Amherst, MA, USA.

Mason K.V. 1993. A new species of demodex mite with D. canis causing canine demodicosis: a case report. - In: Ninth Proceedings, Annual Members Meeting of Amer. Acad. Veterin. Dermatology and Amer. College Vet. Dermatology: 92.

Matthes H.-F. 1991. Untersuchungen zur Infection des Hausrindes mit der Haarbalgmilbe Demodex bovis Stiles, 1892 unter Berücksichtigung der Demodicidae anderer Wirtsspezies. - Faculty of Vet. Med., Humboldt Univ., Berlin, 405 pp. (Thesis)

Matthes H. - F. 1996. New aspects of parthenogenesis in cattle demodicosis (Demodicidae). - 93-96. In: Mitchell R., D. J. Horn, G.R. Needham, W.C. Welbourn (Eds). Acarology, 9: Vol. 1, Proceedings, Ohio Biol. Survey, Columbus: I-XX, 1-718.

Matthes H.-F., V. Bukva. 1993. Features of bovine demodecosis (Demodex bovis Stiles, 1892) in Mongolia: preliminary observations. - Folia Parasitologica, 40: 154-155.

May I., M. Putin, V. Aciocîrlánoaia. 1975. Observaţii privind rîia demodectiča la bovine. - Rev. crest. Animal. 1975 (12) : 48-51.

Mégnin J.P. 1877. Mémoire sur le Demodex folliculorum Owen. - Jour. Anat. Physiol. Normales et Pathologiques de l'Homme et des Animaux, 13(2) : 97-122. 
Mehl R. 1979. Checklist of Norwegian ticks and mites (Acari). - Fauna Norvegica, Ser. B, 26: 31-45.

Mertens K. A. J. M., F. S. Lukoschus \& W. B. Nutting. 1983. Demodex huttereri sp. n. (Acarina: Prostigmata: Demodicidae) from the Meibomian glands of Apodemus agrarius (Rodentia: Muridae). - Bonn. Zool. Beitr., 34: 489-498.

Miskjian H.G. 1951. Demodicidosis (Demodex infestation of the scalp). - Arch. Dermat. \& Syph., 63: 282-283.

Moravvej H., M. Dehghan-Mangabadi, M.-R. Abbasian, G. Meshkat-Razavi. 2007. Association of Rosacea with Demodicosis. - Archives of Iranian Medicine, 10(2): 199-203.

Morita T., A.Ohmi, A. Kiwaki, K. Ilke, K. Nagata. 2018. A new stubby species of demodectic mite (Acari: Demodicidae) from the domestic dog (Canidae). - J. of Medical Entomology, 55(2): 323-328.

Moskvina T.V. 2017. Two morphologically distinct forms of Demodex mites found in dogs with canine demodicosis from Vladivostok, Russia. - Acta Vet. Beogr., 67: 92-91.

Mueller R.S., S.V. Bettenany. 1999. An unusual presentation of canine demodicosis caused by a long bodied Demodex mite in a Lakeland Terrier. - Austr. Vet. Pract., 29: 128-130.

Mulugeta Y., H.T. Yacob, H. Ashenafi. 2010. Ectoparasites of small ruminants in three selectedagro-ecological sites of Tigray Region, Ethiopia. - Trop. Anim. Health Prod., 42: 1219-1224.

Murray M. D. 1959. A clinical case of demodectic mange in a sheep. - Austral. Vet. J., 35: 93.

Murray M. D., W.B. Nutting, R.W. Hewetson. 1976. Letter: Demodectic mange of cattle. Aust. Vet. J., 52(1): 49.

Mwase E.T., A.S. Baker. 2006. An annotated checklist of mites (Arachnida: Acari) of Zambia. - Zootaxa, 1106: 1-24.

Nashat M.A., K.R.Luchins, E.R.Riedel, J.N.Izdebska, M.L.Lepherd, N.S.Lipman. 2017. Characterization of Demodex musculi infestation, associated co-morbidities, and its topographical distribution in a moise strain with defective adaptive immunity. - Comp. Med., 67: 315-329.

Nahrung H. F. 1978. Morphology and functional anatomy of Demodex folliculorum (Simon) of man. - Acarologia (Paris), 19(3): 422-462

Nahrung H. F. 1979. Redescription of Demodex caballi (=D. folliculorum var. equi Railliet, 1895) from the horse, Eques caballus. - Acarologia (Paris), 20(2): 235-240.

Nahrung H. F. 1987. Redescription of Demodex nanus (Acari: Demodicidae) from Rattus norvegicus and $R$. rattus (Rodentia). - Journal of Medical Entomology, 24 (1): 19-23.

Nayak D.C., S.B. Tripathy, P.C. Dey, S.K. Ray, D.N. Mohanty, G.S. Parida, S. Biswal, M. Das. 1997. Prevalence of canine demodicosis in Orissa (India). - Veterinary Parasitology, 73 (3-4): 347-352.

Nelson G.A.H. 1956. Some arthropods affecting man and livestock in New Zealand. - N.Z. Veterinary Journal, 4: 11-18.

Nemeséri L., A. Széki. 1961. Demodicosis in cattle. - Acta veter. Acad. Sci. hung., 11: 209-221. 
Nemeséri L., A. Széki. 1962. Die Rinderdemodikose. - Berl. Munch. Tierarztl. Wochenschr., 16: 304-307.

Nemeséri L., A. Széki. 1966a. Demodicosis in sheep. - Acta vet. Acad. Sci. hung., 16(1): 33-64.

Nemeséri L., A. Széki. 1966b. Demodicosis of swine. - Acta vet. Acad. Sci. hung., 16: 251-261.

Nemeséri L., A. Széki. 1966c. Die Schaf-Demodikose. - In: A. Corradetti, Proc. $1^{\text {st }}$ Int. Congr. Parasitol. (Roma 1964), 2: 1017-1018.

Nicholas W.D. 1943. Demodex folliculorum. Its incidence in routine histologic study of the skin. - Arch. Dermat. Syph., Chicago, 47(6): 793-796.

Nicolet H. 1855. Histoire naturelle des Acariens qui se trouvent aux environs de Paris. - Archive du Muséum d'Histoire Naturelle, Paris, 7: 381-482.

Noorudin M., S.M. Rahman. 1985. A note on bovine demodicosis in Bangladesh. - India J. Vet. Med., 5: 139-140.

Norn M.S. 1970. Demodex folliculorum. Incidence and possible pathogenic role in the human eyelid. - Acta Ophthal. Suppl. 108, 85 p.

Nutting W. B. 1950. Studies on the genus Demodex Owen (Acari, Demodicoidea, Demodicidae). - Ph. D. Thesis, Cornell Univ., Ithaca, New York.

Nutting W. B. 1961. Demodex aurati sp. n. and D. criceti, ectoparasites of the golden hamster (Mesocricetus auratus). - Parasitology, 51: 515-522.

Nutting W. B. 1964. Demodicidae - status and prognostics. - Acarologia, 6: 441-454.

Nutting W. B. 1965. Host-parasite relations: Demodicidae. - Acarologia, 7: 301-317.

Nutting W. B. 1971. Demodex canis - a biologist's viewpoint. - Annual Meeting of Am. Anim. Hosp. Assoc., Miami Beach, Florida.

Nutting W. B. 1973. Adaptations in demodicids (Trombidiformes: Demodicidae) for utilization of the mammalian skin complex. - Proc. 3-rd Internat. Congr. Acarology, Prague, 1971: 531-535.

Nutting W. B. 1976a. Pathogenesis associated with hair follicle mites (Acari: Demodicidae). - Acarologia, 17(3): 493-507.

Nutting W. B. 1976b. Hair follicle mites (Acari: Demodicidae) of man. - Int. J. Dermatol., 15(2): 79-98.

Nutting W. B. 1976c. Hair follicle mites (Demodex spp.) of medical and veterinary concern. - Cornell Veterinarian, 66: 214-231.

Nutting W. B. 1980a. Demodicosis (Acari: Demodicidae) in the California sea lion, Zalophus californicus. - Journal of Medical Entomology, 17(4): 344-347.

Nutting W.B. 1980b. Synhospitality and speciation in the Demodicidae (Trombidiformes). Proc. $4^{\text {th }}$ Intl. Congress Acarology, 1974: 267-272.

Nutting W.B. 1985. Prostigmata-Mammalia. Validation of coevolutionary phylogenies. In Coevolution of parasitic arthropods and mammals, K. C. Kim (ed.). John Wiley and Sons, Inc., New York, New York, pp. 569-640.

Nutting W. B., E.E. Beekman, D.P. Snyder. 1978. Demodex gapperi (Acari: Demodicidae) associated with eylid sealing in the red-backed vole, Clethrionomys gapperi. - J. Med. Entomol., 14(6): 646-648. 
Nutting W. B., H. Beerman. 1965. Atypical giant cells in Antechinus stuartii due to demodicid mites. - I. Invest. Dermatol., 45(6): 504-509.

Nutting W. B., C. E. Desch. 1978. Demodex canis. Redescription and reevaluation. - Cornell Vet., 68: 139-149.

Nutting W. B., C. E. Desch. 1979. Relationships between mammalian and demodicid phylogeny. - In: Recent Advances in Acarology, vol. 2, Ed. J. G. Rodriguez, Academic Press, New York, pp. 339-345.

Nutting W. B., S. O. Emejuaiwe, M. O. Tisdel. 1971. Demodex gapperi sp. n. (Acari: Demodicidae) from the red-backed vole, Clethrionomys gapperi. - J. Parasit., 59: 660-665.

Nutting W. B., K.E. Firda, C.E. Desch. 1989. Topology and histopathology of hair follicle mites (Demodicidae) of man. - Progress in Acarology, 1: 113

Nutting W. B., A.C. Green. 1976. Pathogenesis associated with hair follicle mites (Demodex spp.) in Australian aborigines. - British Journal of Dermatology, 94(3): 307-312.

Nutting W. B., F. M. Guilfoy. 1979. Demodex cafferi n. sp. from the African buffalo, Syncerus caffer. - Intl. J. Acar., 5(1): 9-14.

Nutting W. B., P. R. Kettle, J. D. Tenquist, L.K. Whitten. 1975. Hair follicle mites (Demodex spp.) in New Zealand. - N. Z. J. Zool., 2: 219-222.

Nutting W. B., W.F. Krchheimer, R.E. Pfaltzgraff. 1966. Demodicidiasis and leprosy. - Lepr. Rev., 37(4): 209-216.

Nutting W. B., F. S. Lukoschus, C. E. Desch. 1980. Parasitic mites of Surinam XXXVII. Demodex marsupialis sp. n. from Didelphis marsupialis: adaptation to glandular habitat. - Zool. Meded. Rijks Mus. Nat. Hist. Leiden, 56: 83-90.

Nutting W. B., H. Rauch. 1958. Demodex criceti sp. n. (Acari: Demodicidae) with notes on its biology. - J. Parasitol., 44: 328-333.

Nutting W. B., H. Rauch. 1961. The effect of biotin deficiency in Mesocricetus auratus on parasites of the genus Demodex. - J. Parasitol., 7: 319-322.

Nutting W. B., H. Rauch. 1963. Distribution of Demodex aurati in the host (Mesocricetus auratus) skin complex. - J. Parasitol., 49: 323-329.

Nutting W. B., L. Satterfield, G. Cosgrove. 1973. Demodex sp. infesting tongue, esophagus and oral cavity of Onychomys leucogaster, the grasshopper mouse. - J. Parasitol., 59(5): 893-896.

Nutting W. B., G. K. Sweatman. 1970. Demodex antechini (Acari: Demodicidae) parasitic on Antechinus stuartii (Marsupialia). - Parasitology, 60: 425-429.

Nutting W. B., M. O. Tisdel, S. O. Emejuaiwe. 1968. Y-shaped ovum of a demodicid from Clethrionomys gapperi. - J. Parasit., 54: 393-394.

Nutting W. B., P. Wooley. 1965. Pathology in Antechinus stuartii (Marsupialia) due to Demodex sp. - Parasitology, 55: 383-389.

Okyay P., H. Ertabaklar, E. Savk, S. Erfug. 2006. Prevalence of Demodex folliculorum in young adults: relation with sociodemographic/hygienic factors and acne vulgaris. - J. Eur. Acad. Dermatol. Venereol. 20(4): 474-476. 
Ollé J., Ventura J., C. Feliu, P. Foronda, O. Francino, N. Sastre. 2020. First record of the presence of skin mites (Demodex musculi) in wild house mice from the Canary Islands (Spain). - Int. J. Acarol., 46: 1-4.

Özdemir M.H., U. Aksoy, Ç. Akisu, E. Sönmez, M.A. Çakmak. 2003. Investigating Demodex in forensic autopsy cases. - Forensic Science International, 135: 226-231.

Oppong E. N. W. 1970. Aspects of bovine demodicosis, streptothricosis and besnoitiosis in the Accra Plains of Ghana and a study of Demodex ghanensis sp. n. Ph. D. Thesis, Univ. Dublin.

Oppong E. N. W., R. P. Lee, S. A. Yasin. 1975. Demodex ghanensis sp. nov. (Acari, Demodecidae) parasitic on west Africa cattle. - Ghana J. Sci., 15: 39-43.

Oudemans A.C. 1897. List of Dutch acari, seventh part: Acaridiae Latr. 1806, and Phytoptidae Pagenst. 1861, with synonymical remarks en [sic] description of new species etc. - Tijdschr. Entom., 40: 250-269.

Oudemans A.C. 1905. Acarologische Aanteekeningen XIX. - Ent. Ber., 2(25): 4-12.

Oudemans A.C. 1909. Acarologische Aanteekeningen XXIII. - Ent. Ber., 2(47): 319.

Oudemans A.C. 1921. Over de plaats der Demodicidae in het systeem der Acari. - Entomologische Berichten, 5: 1-7.

Owen R. 1843. Lectures on the comparative anatomy and physiology of the invertebrate animals. From notes taken by W.W. Cooper, and revised by Prof. Owen. - Delivered at the Royal College of Surgeons, in 1843. P. 251-252.

Owen D., C. Young. 1973. The occurrence of Demodex aurati and Demodex criceti in the Syrian hamster (Mesocricetus auratus) in the United Kingdom. - Vet. Rec., 17: 282-284.

Palopoli M.F., V.Tra, K. Matoin, P.D. Mac. 2017. Evolution of host range in the follicle mite Demodex kutzeri. - Parasitology, 144: 594-600.

Pekmezci G.Z., D. Pekmezci, C.S. Bolukbas. 2018. Molecular characterization of Demodex canis (Acarina: Demodicidae) in domestic dogs (Canis familiaris). - Kocatepe Vet. J., 11: 1219-1224.

Polyakov D. K. 1956. The problem of economic losses caused by bovine demodicosis. (In Russian). - Bjull. Nauch.-issled. Inst. Veter. Sanit. Ektoparasit, 1, 16.

Polyakov D. K. 1960. Demodect. - mange in cattle in the northwestern zone and the CentralAsian republics. - Trudy Vsesoyuz. Nauch. - Issled. Inst. Vet. San. i Ektoparazitol., Moskva, 16: 10-14.

Polyakov D. K. 1977. [Demodecosis in cattle]. - Arachnozi I Protozoynie bolezni sel'skohozyaystvennih zhivotnih, Moskow, "Kolos": 62-70 (in Russian).

Poppe S.A. 1909. Nachtrag zur Milben - Fauna der Umgegend Bremens. - Abh. Nat. Ver. Bremen, 19: 47-67.

Prasad V. 2009. Presence of Demodex brevis Akbulatova, 1963 (Acari: Demodecidae) in a pathologic slide in Michigan, USA. - International J. of Acarology, 35(1): 77-78.

Prietsch 1886. Demodex folliculorum bei einem Samburhirsch. - Bericht Veterinärwesen Königreich Sachsen (1885): 89. 
Prietsch 1909. Akarusräude bei einem Reh. - Bericht Veterinärwesen Königreich Sachsen (1908): 77.

Principato M. 1995. Demodecose humaine chez des sujets atteints d’alopècie seborrhéique : modifications périodiques dans le cycle biologique des parasites et considérations cliniques sur les causes de la calvité. - Bull. Soc. Fr. De Parasitologie, 12(1), 1994 : 81-91.

Puccini V., G. Colella. 1975. Prima segnalazione per l'Italia sud-orientale di Demodex phylloides. - Acta med. Vet., 21: 65-72.

Putatunda B. N., R. Kumar, D.P. Banerjee. 2004. Mites parasitic and/or associated with poultry and some mammals in Hisar, Haryana with key to identification. - Indian J. Anim. Res., 38(1): 1-7.

Quintero T.C. 1977. Frecuencia de Demodex phylloides en diversas regiones anatómicas de cerdos en el Estado de México. - Veterinaria Mex., 7: 42-44.

Railliet A. 1893. Traité de Zoologie médicale et agricole. I. - Asselin et Houzeau, Paris.

Railliet A. 1895. Traité de Zoologie médicale et agricole. I. - Asselin et Houzeau, Paris. Second Edition, pp.1303.

Rak H. 1969. A report on Demodicosis (Demodex canis) Leydig (1859) in Iran. - J. vet. Fac. Univ. of Tehran, 25(1): 157-166.

Rak H. 1973. Ectoparasites of dogs and cats in Iran. - Entomologist's Monthly Magasine, 108: 189.

Rak H. 1975. Mites of medical and veterinary importance in Iran. - Bull. Soc. Pathol. Exot., 68(5): 507-511.

Rak H., R. Rahgozar. 1975a. Demodectic mange in cattle. - Vet. Rec. 79: 482-483.

Rak H., R. Rahgozar. 1975b. Demodectic mange in the eyelid of domestic ruminants in Iran. - Bull. de la Societe de Pathologie Exotique, 68(6) : 591-593.

Rafyi A., A. Alavi, H. Rak. 1967. Les espèces de mites rencontrées en Iran. - J. Vet. Fac. Univ. Tehran, Iran, 23(1) : 38-46.

Ramsay W.R. 1883. On Demodex phylloides (Csokor), in the skin of Canadian swine. - Proc. Canad. Inst., 1: 275-281.

Rather P.A., I. Hassan. 2014. Human Demodex mite: The versatile mite of dermatological importance. - Indian J. Dermatol., 59: 60-66.

Razowski J. (Ed.) 1997. Checklist of Animals of Poland. IV, part I-XXXI Porifera - Symphyla. Reeves W.K., L.A. Durden, Ch. M. Ritzi, K.R. Beckham, P. E. Super, B.M. OConnor. 2007. Ectoparasites and other ectosymbiotic arthropods of vertebrates in the Great Smoky Mountains National Park, USA. - Zootaxa, 1392: 31-68.

Reid J.F.S., I.M. Lauder. 1966. Demodectic mange in cattle. - Vet. Rec., 79: 482-483.

Retnasabapathy A., D. Lourdusamy. 1974. Demodex aurati and Demodex criceti in the golden hamster (Mesocricetus auratus). - Southeast. Asian J. Trop. Med. Public Health., 5: 460.

Rocha U.F., M.C. Pardi. 1954. Sarna em bovinos do Estado de São Paulo causado por Demodex bovis (Stiles, 1892) sic. Acari : Demodicidae. - Revta Fac. Med. Vet., Sao Paulo, 5 : 27-34.

Rodriguez H. 2000. Prevalencia de Demodex sp. en pacientes con Blefaritis. - Anales de la Facultad de Medicina, Univ. Nac. Mayor de San Marcos, 61(4): 299-304. 
Rodriguez-Vivas R.I., A. Ortega-Pacheco, J.A. Rosado-Aguilar, G.M.E. Bolio. 2003. Factors affecting the prevalence of mange-mite infestations in stray dogs of Yucatán, Mexico. Veterinary Parasitology, 115(1): 61-65.

Rubin G. J. 1957. Demodectic mange. - Chas. Pfizer and Co., Dept. Vet. Med. Rev., 14: 1-5. (Ex French 1964).

Rufli T., Y. Mumcuoglu. 1981. The hair follicle mites Demodex folliculorum and Demodex brevis: Biology and medical importance. A review. - Dermatologica, 162: 1-11.

Saari S.A.M. et al. 2009. Demodex gatoi - associated contagious pruritic dermatosis in cats - A report from six households in Finland. - Acta Vet. Scand., 51: 1-8.

Sakulploy R., A. Sangvaranond. 2010. Canine demodicosis caused by Demodex canis and short opisthosomal Demodex cornei in Shi Tzu dog from Bangkok Metropolitan Thailand. Kasetsart Veterinarians, 20: 27-35.

Salib F.A. 2018. First report of bovine demodicosis in native Egyptian cow. - Biomed. J. Sci. Tech. Re., 11: 8424-8426.

Salem N.Y., H. Abdel-Saeed, H.S.Farag, R.A.Chandour. 2020. Canine demodicosis: Hematological and biochemical alterations. - Vet. World EISSN, 13: 68-72.

Sangvaranond A. 1979. Mange infestation in domestic animals (Acarina: Sarcoptidae, Psoroptidae \& Demodicidae) in Bogor and vicinity, west Java, Indonesia. - Biotrop spec. Publ. $6: 45$.

Santarém V.A., M.R. Farias, R.A. Tostes. 2005. Demodectic mange in fattening pigs in São Paulo, Brazil. - Veterinary Parasitology, 131(1-2): 169-171. [summary]

Sari Y., E. Zeytun, S. Doğan, Y. Karakurt. 2019. Oküler dandrufflu hastalarda Demodex folliculorum ve D. brevis (Acari: Demodicidae) yaygınlıği ve yoğunluğu. - Acarol. Stud., 1: 23-43.

Saridomichelakis M., A. Koutinas, E. Papadogiannakis, M. Papazachariadon, M. Liapi, D. Trakas. 1999. Adult - onset demodikosis in two dogs due to Demodex canis and a shorttailed demodectic mite. - J. Small Animal Pract., 40: 529-532.

Schulz W., G Gräfner, T. Hiepe. 1968. Untersuchungen über die Demodikose in Rinderbeständen. - Mh. Vet.-Med. (Jena), 23: 535-540.

Schwill.1952. Die Demodikose des Rindes, eine wirtschaftlich bedeutungsvolle Krankheit. Dtsch. Tierärztl. Wschr., 59: 325-327.

Shi Xin-quan, Xie He-Xiu \& Hsu Yeh-Hwa. 1985. A new species of the genus Demodex (Acariformes: Demodicidae). - Acta Zootaxonomica Sinica, 10: 385-387.

Špringol'ts - Šmid A.I. 1937. Ectoparasites of some species of far eastern deer. Vestnik Dal'nevostoch. Fil. Akad. Nauk SSSR, 26: 133-140 (in Russian).

Shipstone M. 2000. Generalized demodicosis in dogs, clinical perspective. - Aust. Vet. J., 78(4): 240-242.

Silbermayr K., C.Horvath-Ungerboeck, B. Eigner, A. Joachim, L. Ferrer. 2015. Phylogenetic relationships and new genetic tools for the detection and dsiscrimination of the three feline Demodex mites. - Parasit. Res., 114: 747-752. 
Silbermayr K., A. Joachim, B. Litschauer, L. Panakova, N. Sastre, L. Ferrer, C. Horvath-Ungerboeck. 2013. The first case of Demodex gatoi in Austria, detected with fecal flotation. - Parasitol. Res., 112: 2809-2810.

Silbermayr K., C. Horvath-Ungerboeck, B. Eigner, A. Joachim, L. Ferrer. 2015. Phylogenetic relationships and new genetic tools for the detection and discrimination of the three feline Demodex mites. - Parasitol. Res., 114: 747-752.

Simon G. 1842. Ueber eine in der Kranken und normalen Haarsacken des Menschen lebende Milbe. - Mullers Archiv 1842, 218-237.

Simon G. 1851. Die Hautkrankheiten. 295 p.

Sivajothi S., B. Sudhakara Reddy, K.N. Kumari, V.C. Rayulu. 2013. Morphometry of Demodex canis and Demodex cornei in dogs with demodicosis in India. - Int. J. Vet. Health Sci. Res., 1: 1-4.

Sivajothi S., B. Sudhakara Reddy, V.C. Rayulu. 2015. Demodicosis caused by Demodex canis and Demodex cornei in dogs. - J. Parasitol. Dis., 39: 673-676.

Slingerberg J., A. N. Mohammed, S. A. Bida. 1980. Studies on bovine demodicosis in northern Nigeria. Specification and hast parasite relationships. - Vet. I., 2: 90-94.

Smith J.M.B. 1968. Diseases of hedgehogs. - The Veterinary Bulletin, 38: 425-430.

Smith H. J. 1960. Demodicidosis on large domestic animals. - A review. Health of Animals Division, Canada Department of Agriculture, Ottawa, 56 pp.

Smith H. J. 1961a. Bovine demodicosis. I. Incidence in Ontario. - Can. J. comp. Med., 25: $165-169$.

Smith H. J. 1961b. Bovine demodicosis. II. Clinical manifestations in Ontario. - Can. J. comp. Med., 25: 201-204.

Smith H. J. 1967. Bovine demodicosis-lesions and the resulting damage to hides and leather. - Wiad. Parazyt., 13: 543-546.

Sokolovskii, V. A., 1952, Biological cycle of Demodex canis (Leydig). - Sborn. Trudov Kharkov. - Vet. Inst., Kharkov., 21: 328-346.

Sokolovskii V. A. 1954. Increasing immunity against demodecosis in the dog. Tezisy Dokl. 1. Vsesoyuz. Konf. Probl. Vet. Dermat. Arkhnol. i Entom., Moskva, (22-26 Mar.), pp. 131-133. (in Russian).

Sokolovskli V. A. 1954. Mange (demodecosls) of the dog and basic methods of its control. Sborn. Trudov Khar'kov. Vet. Inst., Kharkov, 32: 310-315.

Spickett S.G. 1961a. A preliminary note on Demodex folliculorum Simon (1842) as possible vector of leprosy. - Leprosy Rev., 32(4): 263-268.

Spickett S.G. 1961b. Studies on Demodex folliculorum Simon (1842) I. Life History. - Parasitology, 51(1-2): 181-192.

Sreenivasan M.K., S. W. H. Rizvi. 1946. Demodectic mange of goats in India. - Indian J. vet. Sci., 15(4): 287-289.

Starkoff O., I. Starkoff. 1950. Contributo alla conoscenza dell'acarofauna di Roma e dintorni II ${ }^{a}$ nota: sottordini Sarcoptiformes e Prostigmata. - Rivista di Parassitologia, 11(2): 85-101.

Stiles C. W. 1892. On Demodex folliculorum var. bovis in American cattle. - Canad. Entomol., 24: 286-290. 
Stoenescu D., V. Clipa, A. Gheorghiteanu, N. Curatu, P. Tanase, C. Toma, D. S. Teoderescu. 1972. Observations concernant l'épizootologie, la clinique et le traitement de la démodécose du chien. - Arch. Vet. Bucur., 9: 113-123.

Stromberg B.E., W.B. Nutting. 1973. Adaptive features of the exoskeleton and "pigment" deposits in Demodex spp. (Demodicidae). - Acarologia, 1972, 14(4): 605-611.

Sutmöller P. 1957. Demodicosis bij geiten en varkens op Aruba (Nederlandse Antillen). Tijdsch. Diergeneesk. Utrecht, 82: 42-47.

Sweatman G.K. 1962. Parasitic mites of non-domestic animals in New Zealand. - N.Z. Entomologist, 3: 15-23.

Tandon N., A.K. Hati, A. Thammayya. 1980. Demodex folliculorum in man. - Rec. Zool. Surv. India, 76: 139-142.

Tater K.C., A.P. Patterson. 2008. Canine and feline demodicosis. - Veterinary Medicine, 103: 8.

Tenquist J. D., W. A. G. Charleston. 2001. A revision of the annotated checklist of ectoparasites of terrestrial mammals in New Zealand. - J. of the Royal Society of New Zealand, 31(3) : 481-542.

Thomson G. M. 1922. p.345 In: The Naturalisation of Plants and Animals in New Zealand. Cambridge Univ. Press, London.

Trouessart E. L. 1892. Considérations génerales sur la classification des Acariens suivies d'un essai de classification nouvelle. - Rev. Sci. Nat. Ouest, 2: 20-54.

Trucco T., H. Ahibe, M. Arribillaga. 2019. Sarna demodécica (Demodex bovis) en terneros en la provincia de Entre Ríos. Descripción de un cuadro clínica. - Vet. Arg., 36: 1-8.

[Tsedev B., R.V. Grebenyuk] Б. Цэдэв, Р.В. Гребенюк. 198 . [Ectoparasites of the sheep and goats and some wild animals in Mongolia. Entomological Research in Kirghizsan. ]. Эктопаразиты мелкого рогатого скота и некоторых диких животных Монголии. - Энтомологические исследования в Киргизии: 126-127.

Turk F. A. 1953. I. - A synonymic catalogue of British Acari: Part I. - Ann. Mag. Nat. Hist., $12^{\text {th }}$ Ser., 61: 1-26.

Turner J.C., J. Cano. 2008. Demodectic Mange in a White -Tailed Deer from Walker County, Texas. - J. of Medical Entomology, 45(3): 572-575.

Tüzdil N. 1957. Das Vorkommen von Demodex in der Zunge einer Maus. - Z. Tropenmed. Parasitol., 8: 274-278.

Ugbomoiko U. S., L. Ariza, J. Heukelbach. 2008. Parasites of importance for human health in Nigerian dogs: high prevalence and limited knowledge of pet owners. - BMC Vet. Rec., 4: 49.

Unsworth K. 1946. Studies on the clinical and parasitological aspects of canine demodectic mange. - J. Comp. Path. Therap., 56(2): 155-179.

Valandro M.A., J.P. da Exaltacao Pascon, M.L. de Arruda Mistieri, T. Gallina. 2016. Demodicose felina por Demodex cati. - Acta Sci. Vet., 44(suppl.1): 1-4.

Vanselow P. 1910. Zur Acarusräude des Rindes und des Hirsches. - Vet. - Med. Diss. Univ. Giessen. Buchdruckerei Kirmse \& Wagner, Gössnitz, 55 pp. 
Vargas M., B. Bassols, C.E. Desch, M.T. Quintero, O.J. Polaco. 1995. Description of two new species of the genus Demodex Owen, 1843 (Acari: Demodecidae). - Int. J. Acarology, 20(2): 75-82.

Veal R. A., K. M. T. Giesen, J. O. Whitaker Jr. 1984. A new species of the genus Ophthalmodex Lukoschus \& Nutting 1979 (Prostigmata: Demodicidae) from Myotis lucifugus (Chiroptera: Vespertilionidae). - Acarologia, 25(4): 347-350.

Veena M., H. Dhanalakshmi, K. Kavitha, P.E.D. Souza, G.C. Puttalaksmamma. 2017. Morphological characterization of Demodex mites and its therapeutic management with neem leaves in canine demodicosis. - J. Entomol. Zool. Stud., 5: 661-664.

Venkatesan R. A., S. C. Nandy, G. Krishnan. 1979. Occurrence and possible significance of demodectic mites, Demodex carpae, in the internal tissues of fetal and adult Indian goats. - J. Am. Leather Chem. Assos., 74: 191-197.

Vestweber J.G., R.K. Ridley, J.C. Nietfeld, M.J. Wilkerson. 1999. Demodecosis in American Bison. - Can. Vet. J., 40: 417-418.

Vogelnest L.J., L. Vogelnest, R.S. Mueller. 2000. An undescribed Demodex sp. and demodicosis in a captive koala (Phascolarctos cinereus). - Journal of Zoo and Wildlife Medicine, 31(1): 100-106.

Waggie K.S., P.L. Marion. 1997. Demodex sp. in California ground squirrel. - J. of Wildlife Diseases, 33(2): 368-370. [summary]

Watson M. 1948. Colloidal iodine in the treatment of demodectic mange. - Vet. Rec., 60: 349. Wetzel R. 1958. Zum Problem der Rinder-Demodikose. - Dtsch. Tierärztebl., 6: 156.

Whitten L. K. 1962. Parasitic mites of domestic animals in New Zealand. - N. Z. Entomologist, 3: 9-12.

Wilkerson J.D., D.M.P.Berger. 2007. Parasites of Gerbils. In: Flynn's Parasites of Laboratory Animals. Baker, D.G., Ed. Blackwell Publishing: Ames, IA USA, 413-420.

Woeltjes A.G. W., F. S. Lukoschus. 1981. Parasites of Western Australia XIV. Two new species of Ophthalmodex Lukoschus \& Nutting (Acarina: Prostigmata: Demodicidae) from the eyes of bats. - Rec. West. Aust. Mus., 9(3): 307-313.

Wolhuter J., R.G. Bengis, B.K. Reilly, P.C. Cross. 2009. Clinical demodicosis in African buffalo (Syncerus caffer) in the Kruger National Park. - J. Wildl. Dis., 45: 502 - .

Xu Yehua, Xie Hexiu, Liu Sulan, Zhou Zhongyong, Shi Xemquan. 1986. A new species of the genus Demodex (Acariformes: Demodicidae). - Acta Zool. Sinica, 32: 163-167.

Xie Hexiu, Liu Su-lan, Hsu-Yehua, Hsu Yin-chi. 1982. Taxonomy of family Demodicidae and a new subspecies. - Acta zootaxonomica sinica, 7: 265-269.

Yabsley M.J., S. E. Clay, S. E. J. Gibbs, M. W. Cunningham, M. G. Austel. 2013. Morphologic and Molecular Characterization of a Demodex (Acari: Demodicidae) Species from WhiteTailed Deer (Odocoileus virginianus). - International Scholarly Research Notices, Vol. 2013, Article ID 342918, https://doi.org/10.5402/2013/342918

Yagdiran Duzgun O., S. Aytekin. 2007. Comparison of Demodex folliculorum density in haemodialisis patients with a control group. - J. of the European Academy of Dermatology and Venereology, 21(4): 480-483. 
Yasine A., B.Kumsa, Y.Hailu, D. Ayana. 2015. Mites of sheep and goats in Oromia Zone of Amhara Region, North Eastern Ethiopia: Species, prevalence and farmers awareness. BMC Vet. Res., 11: 1-6.

Yehua Xu, Hexiu Xie, Sulan Liu, Zhongyong Zhou, Xinquan Shi. 1986. A new species of the genus Demodex (Acariformes: Demodicidae). - Acta Zool. sin., 32: 163-167.

Yeruham I., Sh. Rosen, A. Hadani. 1986. Sheep demodicosis (Demodex ovis Railliet, 1895) in Israel. - Rev. Elev. Méd. Vét. Pays trop., 39: 363-365.

Zeytun E., Y. Karakurt. 2019. Prevalence and load of Demodex folliculorum and Demodex brevis (Acari: Demodicidae) in patients with chronic blepharitis in the province of Erzincan, Turkey. - J. Med. Entomol., 8: 2-9.

Zhao Y.E., J. Cheng, L. Hu, J.X. Ma, 2014. Molecular identification and phylogenetic study of Demodex caprae. - Parasitol. Res., 113: 3601-3508.

Zhao Y.E., N. Guo, L.P.Wu. 2009. The effect of temperature on the viability of Demodex folliculorum and Demodex brevis. - Parasitol. Res., 105: 1623-1628.

Zhao Y.E., Y.Peng, X.I.Wang, L.P.Wu, M.Wang, H.I.Yan, S.X.Xiao. 2011. Facial dermatosis associated with Demodex: A case-control study. - J. Zhejiang Univ. Sci., B12: 1008-1015.

Zhao Y.E., L. P. Wu, L. Hu, Y. Xu, Z. H. Wang, W. Y. Liu. 2012. Sequencing for complete rDNA sequences (18S, ITS1, 5. 8S, ITS2, and 28S rDNA) of Demodex and phylogenetic analysis of Acari based on 18S and 28S rDNA. - Parasitology Research, 21(5): 2109-2114.

Zhu Y.E., L. P. Wu. 2012. Phylogenetic relationships in Demodex mites (Acari: Demodicidae) based on mitochondrial 16S rDNA partial sequences. - Parasitology Research, 111(3): 1113-1121. 


\title{
The Mite Family Harpirhynchidae Dubinin
}

\author{
Harpirhynchidae Dubinin, 1957: 94
}

Two of the three subfamilies of Harpirhynchidae include skin parasites of birds.

Harpypalpinae are living on Passeriform birds, Harpirhynchinae - also on Passeriformes, but also on birds of other orders - Anseriformes, Apodiformes, Charadriiformes, Ciconiiformes, Coliiformes, Columbiformes, Galliformes, Pelecaniformes. Harpirhynchids were shown to be highly host specific, with $69.5 \%$ being monoxenous species and $23 \%$ species are associated with several closely related hosts (Bochkov, Klimov \& Skoracki, 2017).

In his description of fam. Harpirhynchidae Dubinin (1957) included the specialized subfamily Ophioptinae (parasites of snakes) as subfamily of this family. Many subsequent researchers (Fain, Beron, Lizaso, etc.) followed Southcott (1956) who described the family Ophioptidae, but Bochkov (2002), based on cladistic analysis, restored the subfamilial status of the snake parasites.

According to Bochkov (2009b), the family contains 84 sp. of 14 genera. In line with Bochkov \& Literák (2006), the family Harpirhynchidae is considered here to contain three subfamilies with 16 genera and ca. 135 species [93 acc. to Zhang et al., 2011], as follows:

\section{Fam. Harpyrhynchidae Dubinin, 1957 - 16 genera, 135 species}

Subfam. Harpypalpinae Fain, 1972 - 2 genera, 19 species

Harpypalpoides Lombert et Moss, 1983 - 9 species

Harpypalpus Dubinin, 1957 - 10 species

Subfam. Harpirhynchinae Dubinin, 1957 - 12 genera, 98 species

Tribus Harpirhynchini Fain, 1972

Anharpyrhynchus Fain, 1995 - 4 species

Crassacarus Bochkov, OConnor et Klompen, 2015 - 6 species

Fainharpirhynchus Bochkov et Galloway, 2013 - 3 species

Harpyrhynchiella Fain, 1972 - 3 species

Harpyrhynchiella Fain, $1972-1$ species

Cypsharpirhynchus Fain, 1995 - 2 species

Harpyrhynchoides Fain, 1972 - 58 species

Harpirhynchus Mégnin, 1878 - 5 species

Harpirhynchus s. str. Mégnin, 1878 - 4 species

Pseudoharpirhynchus Fain, Bochkov et Mironov, 1999 - 1 species

Neharpyrhynchus Fain, 1995 - 9 species

Ralliharpirhynchus Fain, 1995 - 2 species

Trichorhynchiella Fain, 1995 - 1 species 
Tribus Metharpyrhynchini Fain, 1972

Metharpyrhynchus Fain, 1972 - 4 species

Tribus Perharpyrhynchini Fain, 1972

Perharpyrhynchus Fain, 1972 - 2 species

Harpirhynchinae incertae sedes -1 species

Subfam. Ophioptinae Southcott, 1956 - 2 genera, 18 species

Afrophioptes Fain, 1962 - 2 species

Ophioptes Sambon, 1928 - 16 species

The history of the research on Harpirhynchidae starts with the description of "Sarcoptes" nidulans Nitsch, 1818. The same specied is being described under various names all along $19^{\text {th }}$ century by C.L. Koch (1836), Giebel (1871), Murray (1877), Canestrini (1886), Heim (1892), Railliet (1893), Berlese (1895) and others. In the paper of Berlese \& Trouessart (1889) are described three more species.

In his general paper Mégnin (1877) first uses the name Harpirhynchus and under it these mites find place in the family Cheyletidae and remain there for the long 80 years, until the new classification of Dubinin (1957). He motivates the placement of genus Harpirhynchus, together with his new genus Harpypalpus, in a family of it's own, including also the snake parasites of the new subfamily Ophioptinae. Shortly before Dubinin's revision of the family Fritsch (1954) published his revision of these mites, leaving them (plus eight newly described species) in the genus Harpirhynchus. New hiatus follows and in 1972 and 1995 A. Fain described nine more genera and the subfamily Harpypalpidae, thus laying the foundation of the modern system of Harpirhynchidae. Many species known to belong to this family (without Ophioptinae, treated here separately) are described by Fain or with his participation.

Since 1999 begin a series of contributions of A. Bochkov (partly together with A. Fain, S.V. Mironov, H. Klompen, I. Literák, B. Sikora, M. Skoracki, T.D. Galloway and other coauthors). Based on solid analysis, using cladistic and other modern methods, they greatly completed the knowledge on Harpirhynchidae. Other specialists having contributed to the studies on the family are R. Domrow, M. Jeleva, N. Banks, H.E. Ewing, W. W. Moss, A.C. Oudemans, J.H. Oliver, R.F. Lawrence, H.A.P.M. Lombert, P.-Y. Henry, V. Černy, W. Sixl, E.M. Boyd, F. Dusbábek, J. Martinu, and others.

The recent review of Bochkov \& Literák (2006) summarizes the knowledge on the European Harpirhynchidae (28 species, incl. the four described by Martinu, Dusbábek \& Literák, 2008). According to Moss (1979a), the number of species belonging to this family is theoretically more than 2500 . The Ophioptinae was downgraded from family to subfamily status, but is treated here on it's own, as it comprises specific parasites of snakes. 


\section{Catalogue of Harpirhynchidae}

(Harpirhynchinae and Harpypalpinae)

Family Harpirhynchidae Dubinin

Harpirhynchidae Dubinin, 1957: 94

Type genus: Harpirhynchus Mégnin, 1877

Subfamily Harpypalpinae Fain

Harpypalpinae Fain, 1972: 49

Type genus: Harpypalpus Dubinin, 1957

Genus Harpypalpoides Lombert et Moss

Harpypalpoides Lombert \& Moss, 1983: 165

Type species: Harpypalpoides lukoschusi Lombert et Moss, 1983

\section{Harpypalpoides hirundinis (Fain)}

Harpypalpus hirundinis Fain, 1972: 58

Harpypalpoides hirundinis (Fain): Fain, Bochkov \& Mironov, 1999: 53; Bochkov \&

Klompen, 2014a: 469

Type locality: Kinshasa

Distribution: DR Congo

Host: Psalidoprocne sp. (Passeriformes: Hirundinidae)

Harpypalpoides hirundinoides Bochkov et Klompen

Harpypalpoides hirundinoides Bochkov \& Klompen, 2014a: 468

Type locality: Noord Brabant, Schaijk

Distribution: Holland

Host: Hirundo rustica (Passeriformes: Hirundinidae)

Harpypalpoides johnstoni Bochkov et Klompen

Harpypalpoides johnstoni Bochkov \& Klompen, 2014a: 470

Type locality: Holland, Gelderland, Arnhem

Distribution: Holland, Kazakhstan

Hosts: Fringilla coelebs, F. montifringilla (Passeriformes: Fringillidae)

Harpypalpoides lesickii Skoracki, Bochkov et Sikora

Harpypalpoides lesickii Skoracki, Bochkov \& Sikora, 2004: 84; Bochkov \& Literák, 2006:

140; Bochkov \& Klompen, 2014a: 469

Type locality: Poznań environs, Jeziory village 
Distribution: Kazakhstan, Poland

Hosts: Carduelis cannabina, Coccothraustes coccothraustes (Passeriformes: Fringillidae)

Harpypalpoides lukoschusi Lombert et Moss

Harpypalpoides lukoschusi Lombert \& Moss, 1983: 165; Skoracki, Bochkov \& Sikora, 2004:

85; Bochkov \& Literák, 2006: 140; Bochkov \& Klompen, 2014a: 467

Type locality: Utrecht (Holland)

Distribution: Holland, Poland

Hosts: Turdus merula, T. pilaris (Passeriformes: Turdidae)

Harpypalpoides namibiensis Fain, Bochkov et Mironov

Harpypalpoides namibiensis Fain, Bochkov \& Mironov, 1999: 53; Bochkov \& Klompen, 2014a: 469

Type locality: Karas, Aroab

Distribution: Namibia

Host: Emberiza impetuani (Passeriformes: Emberizidae)

Harpypalpoides regulus Bochkov et Klompen

Harpypalpoides regulus Bochkov \& Klompen, 2014a: 471

Type locality: Noord Brabant, Schaijk

Distribution: Holland

Host: Regulus regulus (Passeriformes: Regulidae)

Harpypalpoides sitta Bochkov et Klompen

Harpypalpoides sitta Bochkov \& Klompen, 2014a: 474

Type locality: California, Monterey Co.

Distribution: USA (Arizona, California)

Host: Sitta pygmaea (Passeriformes: Sittidae)

Harpypalpoides sylvia Bochkov et Klompen

Harpypalpoides sylvia Bochkov \& Klompen, 2014: 472; Bochkov \& Klompen, 2014a: 472

Type locality: Amsterdam

Distribution: Holland

Host: Sylvia atricapilla (Passeriformes: Sylviidae)

Genus Harpypalpus Dubinin

Harpypalpus Dubinin, 1957: 100

Type species: Harpirhynchus longipes Fritsch, 1954 (? syn. of Harpirhynchus holopus Berlese et Trouessart, 1889) 


\section{Harpypalpus dubinini Fain}

Harpypalpus dubinini Fain, 1972: 58; Bochkov \& Klompen, 2014a: 465

Type locality: Antwerp Zoo

Distribution: ? Central Africa

Host: Ploceus [Sitagra] intermedius (Passeriformes: Ploceidae)

\section{Harpypalpus holopus (Berlese et Trouessart)}

Harpirhynchus holopus Berlese \& Trouessart, 1889: 18

Sarcoborus holopus: Oudemans, 1906: 76

Harpypalpus holopus (Berlese et Trouessart): Fain, Bochkov \& Mironov, 1999: 46; Bochkov \& Literák, 2006: 140; Bochkov \& Klompen, 2014a: 462

Type locality: Italy

Distribution: Belgium, Italy

Hosts: Passer domesticus (Passeriformes: Passeridae), ? Luscinia svecica (Passeriformes: Muscicapidae)(? Emberiza citronella - Emberizidae - see Bochkov \& Klompen, 2014a)

\section{Harpypalpus lonchura Bochkov et Klompen}

Harpypalpus lonchura Bochkov \& Klompen, 2014a: 460

Type locality: Western Australia, Mount Hart

Distribution: Australia

Host: Lonchura castaneothorax (Passeriformes: Estrildidae)

\section{Harpypalpus longipes (Fritsch)}

Harpyrhynchus longipes Fritsch, 1954: 183

Harpypalpus longipes (Fritsch): Moss, 1979; Skoracki, Bochkov \& Sikora, 2004: 86; Bochkov \& Klompen, 2014a: 458

Type locality: Germany, Bavaria, Middle Franconia, Kreis Freie Stadt Erlangen

Distribution: Germany, Holland, Poland

Hosts: Cyanistes caeruleus, P. major, Periparus ater (Passeriformes: Paridae), Troglodytes troglodytes (Pass.: Troglodytidae), Nucifraga caryocatactes (Pass.: Corvidae), Carduelis cannabina, Spinus spinus, Pyrrhula pyrrhula, Serinus mozambicus (Pass.: Fringillidae)

\section{Harpypalpus pyrrhula Bochkov et Klompen}

Harpypalpus pyrrhula Bochkov \& Klompen, 2014a: 462

Type locality: Gelderland, Nijmegen

Distribution: Holland

Host: Pyrrhula pyrrhula (Passeriformes: Fringillidae)

\section{Harpypalpus serini Fain}

Harpypalpus serini Fain, 1972: 58; Bochkov \& Klompen, 2014a: 465

Type locality: Antwerp Zoo 
Distribution: Central Africa

Host: Serinus mozambicus (Passeriformes: Fringillidae)

Harpypalpus spermestes Fain, Bochkov et Mironov

Harpypalpus spermestes Fain, Bochkov \& Mironov, 1999: 50

Type locality: Luluabourg (Kananga, Kasai Occidental)

Distribution: DR Congo

Host: Ploceus cucullatus (Passeriformes: Ploceidae)

Harpypalpus sturnus Bochkov et Klompen

Harpypalpus sturnus Bochkov \& Klompen, 2014a: 463

Type locality: Gelderland, Nijmegen

Distribution: Holland

Host: Sturnus vulgaris (Passeriformes: Sturnidae)

Harpypalpus taeniopygia Bochkov et Klompen

Harpypalpus taeniopygia Bochkov \& Klompen, 2014a: 461

Type locality: Western Australia, Brooking Spring

Distribution: Australia

Host: Taeniopygia guttata (Passeriformes: Estrildidae)

Harpypalpus tiarae Fain, Bochkov et Mironov

Harpypalpus tiarae Fain, Bochkov \& Mironov, 1999: 49; Bochkov \& Klompen, 2014a: 465

Type locality: Antwerp Zoo

Distribution: Central Africa

Host: Tiaris canora (Passeriformes: Thraupidae)

Subfamily Harpirhynchinae Dubinin

Harpirhynchinae Dubinin, 1957: 100

Type genus: Harpirhynchus Mégnin, 1877

Tribus Harpyrhynchini Fain

Harpyrhynchini Fain, 1972: 50

Type genus: Harpirhynchus Mégnin, 1877

Genus Anharpyrhynchus Fain

Harpyrhynchus (Anharpyrhynchus) Fain, 1972: 50

Harpirhynchus (Anharpyrhynchus) Fain, 1994: 111

Anharpyrhynchus Fain, 1995: 75

Type species: Harpyrhynchus monstrosus Fritsch, 1954 
Anharpyrhynchus apodus Bochkov et Klompen

Anharpyrhynchus apodus Bochkov \& Klompen, 2014: 320

Type locality: New South Wales, Cordeaux Dam, $34.37^{\circ} \mathrm{S} 150.75^{\circ} \mathrm{E}$

Distribution: Australia

Hosts: Lichmera indistincta, Phylidonyris novaehollandiae (Passeriformes: Meliphagidae)

Anharpyrhynchus elizae Bochkov et Klompen

Anharpyrhynchus elizae Bochkov \& Klompen, 2014b: 317; Bochkov, OConnor \& Klompen, 2015: 112

Type locality: Florida, Columbia Co., O'Leno State Park, $29.92^{\circ} \mathrm{N} 82.58^{\circ} \mathrm{W}$

Distribution: USA

Hosts: Cyanocitta cristata, C. stelleri (Passeriformes: Corvidae), Colaptes auratus (Piciformes: Picidae)

Anharpyrhynchus lukoschusi Bochkov et Klompen

Anharpyrhynchus lukoschusi Bochkov \& Klompen, 2014b: 320

Type locality: Western Australia, Mount Hart, $17.02^{\circ} \mathrm{S} 125.07^{\circ} \mathrm{E}$

Distribution: Australia

Hosts: Manorina flavigula, Certhionyx pectoralis (Passeriformes: Meliphagidae)

\section{Anharpyrhynchus monstrosus (Fritsch)}

Harpyrhynchus monstrosus Fritsch, 1954: 188; Dubinin, 1957: 107; Lawrence, 1959a: 425 Harpyrhynchus (Anharpyrhynchus) monstrosus, Fain, 1972: 55

Harpyrhynchus (Anharpyrhynchus) fritschi Fain, 1972: 55 - type from Xanthoura luxuosa, Anvers Zoo

Harpirhynchus monstrosus Fritsch: Domrow, 1991: 1317

Anharpyrhynchus monstrosus (Fritsch): Fain, 1995: 97; Bochkov \& Literák, 2006: 140

Type locality: Germany

Distribution: Australia, Belgium, Germany

Hosts: Corvus monedula, Garrulus glandarius, Xanthoura yncas luxuosa (= Cyanocorax yncas)(Passeriformes: Corvidae); recorded from Australia on: Gliciphila indistincta, Meliornis novaehollandiae, Myzantha flavigula, Myzomela pectoralis (Passeriformes: Meliphagidae)

Genus Crassacarus Bochkov, OConnor et Klompen

Crassacarus Bochkov, OConnor \& Klompen, 2015: 67

Type species: Crassacarus alexfaini Bochkov, OConnor et Klompen, 2015

Crassacarus alexfaini Bochkov, OConnor et Klompen

Crassacarus alexfaini Bochkov, OConnor \& Klompen, 2015: 67

Type locality: Michigan, Livingston Co., 4 mi S. Howell, $42.5495394^{\circ} \mathrm{N}, 83.9409112^{\circ} \mathrm{W}$ 
Distribution: USA

Hosts: Cardinalis cardinalis (Passeriformes: Cardinalidae), Passerina ciris (Pass.: Cardinalidae), Setophaga petechia (Pass.: Parulidae)

\section{Crasacarus cylindripalpus (Fritsch)}

Harpyrhynchus cylindripalpus: Fritsch, 1954: 183; Dubinin, 1957: 108

Harpirhynchus (Pseudoharpirhynchus) cylindripalpus Fritsch: Fain, Bochkov \& Mironov, 1999: 39

Harpyrhynchoides cylindripalpus (Fritsch): Bochkov \& Literák, 2006: 140

Crassacarus cylindripalpus (Fritsch) - Bochkov, OConnor \& Klompen, 2015: 67

Type locality: Germany

Distribution: Germany

Hosts: Fringilla coelebs (Passeriformes: Fringillidae), Passerina ciris (Pass., Cardinalidae)

Crassacarus fritschi Bochkov, OConnor et Klompen

Crassacarus fritschi Bochkov, OConnor \& Klompen, 2015: 71

Type locality: Michigan, Muskegon Co., Muskegon State game Area, along US route 31, $43.2961989^{\circ} \mathrm{N}, 86.0668945^{\circ} \mathrm{W}$

Distribution: USA

Host: Bombycilla cedrorum (Passeriformes: Bombycillidae)

Crassacarus melanerpes Bochkov, OConnor et Klompen

Crassacarus melanerpes Bochkov, OConnor \& Klompen, 2015: 75

Type locality: Kansas, Johnson Co., Craig, $38.97^{\circ} \mathrm{N} 94.82^{\circ} \mathrm{W}$

Distribution: USA

Host: Melanerpes formicivorus (Piciformes: Picidae)

Crassacarus sialia Bochkov, OConnor et Klompen

Crassacarus sialia Bochkov, OConnor \& Klompen, 2015: 73

Type locality: Wyoming, Campbell Co., Gillette, $19.3 \mathrm{~km} \mathrm{E}$ of, $44.29^{\circ} \mathrm{N} 105.5^{\circ} \mathrm{W}$

Distribution: USA

Host: Sialia currucoides (Passeriformes: Turdidae)

Crassacarus tinae Bochkov, OConnor et Klompen

Crassacarus tinae Bochkov, OConnor \& Klompen, 2015: 70

Type locality: Wyoming, Campbell Co., Gillette, $19.3 \mathrm{~km} \mathrm{E}$ of, $44.29^{\circ} \mathrm{N} 105.5^{\circ} \mathrm{W}$

Distribution: USA

Host: Carduelis tristis (Passeriformes: Fringillidae)

Genus Fainharpirhynchus Bochkov et Galloway

Fainharpirhynchus Bochkov \& Galloway, 2013: 414 
Type species: Fainharpirhynchus contopus Bochkov et Galloway, 2013

Fainharpirhynchus contopus Bochkov et Galloway

Fainharpirhynchus contopus Bochkov \& Galloway, 2013: 418; Bochkov, OConnor \& Klompen, 2015: 83

Type locality: Manitoba, Winnipeg

Distribution: Canada, USA (Michigan)

Hosts: Contopus cooperi, C.virens (Parasitiformes: Tyrannidae)

Fainharpirhynchus legatus Bochkov et Klompen

Fainharpirhynchus legatus Bochkov \& Klompen, 2014: 307

Type locality: Sangre Grande, Cumuto, $1.6 \mathrm{~km}$ E on Cumuto Road, $10.58^{\circ} \mathrm{N}, 61.2^{\circ} \mathrm{W}$

Distribution: Trinidad \& Tobago

Host: Legatus leucophaius (Parasitiformes: Tyrannidae)

Fainharpirhynchus mossi Bochkov et Klompen

Fainharpirhynchus mossi Bochkov \& Klompen, 2014: 311

Type locality: North America

Distribution: North America

Host: Myiarchus crinitus (Parasitiformes: Tyrannidae)

Genus Harpyrhynchiella Fain

Harpyrhynchiella Fain, 1972: 56

Type species: Harpyrhynchus reductus Fritsch, 1954

Cypsharpirhynchus Fain, 1995: 75. Syn. by Bochkov (2015)

Type species: Metharpyrhynchus cypsiuri Fain, 1972

Subgenus Cypsharpirhynchus Fain, 1995

Cypsharpirhynchus Fain, 1995: 75. Syn. by Bochkov (2015)

Type species: Metharpyrhynchus cypsiuri Fain, 1972

Harpyrhynchiella (Cypsharpirhynchus) apus Bochkov

Harpyrhynchiella (Cypsharpirhynchus) apus: Bochkov, 2015: 8

Type locality: Kaliningrad Province, Curonian spit

Distribution: Russia

Host: Apus apus (Apodiformes: Apodidae)

\section{Harpyrhynchiella (Cypsharpirhynchus) cypsiuri (Fain)}

Metharpyrhynchus cypsiuri Fain, 1972: 57

Cypsharpirhynchus cypsiuri (Fain): Fain, 1995: 75

Harpyrhynchiella (Cypsharpirhynchus) cypsiuri (Fain): Bochkov, 2015: 7 
Type locality: Kananga (= Luluabourg), Prov. Kasai, DR Congo; Togo

Distribution: DR Congo, Togo

Hosts: Apus affinis, Cypsiurus parvus (Apodiformes: Apodidae)

Subgenus Harpyrhynchiella Fain

Harpyrhynchiella Fain, 1972: 56

Harpyrhynchiella (Harpyrhynchiella): Bochkov, 2015: 4

Type species: Harpyrhynchus reductus Fritsch, 1954

\section{Harpyrhynchiella (Harpyrhynchiella) reductus (Fritsch)}

Harpyrhynchus reductus Fritsch, 1954: 196; Dubinin, 1957: 108

Harpyrhynchiella reductus (Fritsch): Fain, 1972: 56; 1995: 94; Bochkov \& Literák, 2006: 140

Harpyrhynchiella (Harpyrhynchiella) reductus (Fritsch): Bochkov, 2015: 4

Type locality: Germany

Distribution: Germany, Holland

Host: Apus apus (Apodiformes: Apodidae)

\section{Genus Harpyrhynchoides Fain}

Harpyrhynchus (Harpyrhynchoides) Fain, 1972: 50

Harpyrhynchoides (genus): Fain, Bochkov \& Mironov, 1999: 42

Type species: Harpyrhynchus (Harpyrhynchoides) squamosus Fain, 1972

Pseudoharpirhynchus Fain, Bochkov et Mironov, 1999 is syn. by Bochkov, OConnor \& Klompen, 2015. Type: Harpirhynchus agapornis Fain, 1972

Harpyrhynchoides accipiter Bochkov et OConnor

Harpyrhynchoides accipiter Bochkov \& OConnor, 2013: 503; Bochkov, OConnor \& Klompen, 2015: 35

Type locality: Michigan, Chippewa Co., Whitefish Point

Distribution: USA (Michigan, New York)

Host: Accipiter striatus (Accipitriformes: Accipitridae)

Harpyrhynchoides actitis Bochkov, OConnor et Klompen

Harpyrhynchoides actitis Bochkov, OConnor \& Klompen, 2015: 23

Type locality: British Columbia, Vancouver, $49.16^{\circ} \mathrm{N}, 122.86^{\circ} \mathrm{W}$

Distribution: Canada

Host: Actitis macularius (Charadriiformes: Scolopacidae)

\section{Harpyrhynchoides aegolius Bochkov et OConnor}

Harpyrhynchoides aegolius Bochkov \& OConnor, 2013: 501

Type locality: Michigan, Oakland Co., Blissfield

Distribution: USA (Michigan)

Host: Aegolius acadicus (Strigiformes: Strigidae) 


\section{Harpyrhynchoides agapornis (Fain)}

Harpyrhynchus (Harpyrhynchus) agapornis Fain, 1972: 50; 1995: 77

Harpirhynchus (Pseudoharpirhynchus) agapornis Fain: Fain, Bochkov \& Mironov, 1999: 39

Harpyrhynchoides agapornis (Fain): Bochkov, OConnor \& Klompen, 2015: 66

Type locality: South Africa

Distribution: South Africa

Hosts: Agapornis nigrigenis, A. pullarius, A. roseicollis (Psittaciformes: Psittaculidae)

Harpyrhynchoides alaudinus Bochkov

Harpyrhynchus nidulans: Mégnin, 1877 : 429; Oudemans, 1939 (? misidentification)

Harpyrhynchoides alaudinus Bochkov, 2000b: 91; Bochkov \& Literák, 2006: 140

Type locality: Okskiy Reservation, Moskow Prov.

Distribution: France (?), Russia

Host: Alauda arvensis (Passeriformes: Alaudidae)

\section{Harpyrhynchoides alectoris (Fain)}

Harpyrhynchus (Harpyrhynchoides) alectoris Fain, 1972: 54

Harpirhynchus (Harpyrhynchoides) alectoris, Fain, 1994: 117

Harpyrhynchoides alectoris (Fain): Fain, Bochkov \& Mironov, 1999: 45; Bochkov \& Literák, 2006: 140

Type locality: Antwerp Zoo

Distribution: Spain

Hosts: Alectoris graeca, A. rufa (Galliformes: Phasianidae)

\section{Harpyrhynchoides amazonae (Fain)}

Harpyrhynchus (Harpyrhynchoides) amazonae Fain, 1972: 52; 1994: 113

Harpyrhynchoides amazonae (Fain): Bochkov, OConnor \& Klompen, 2015: 59

Type locality: Antwerp Zoo

Distribution: South America

Host: Amazona aestiva (Psittaciformes: Psittacidae)

\section{Harpyrhynchoides anatum (Fain)}

Harpyrhynchus (Harpyrhynchoides) anatum Fain, 1972: 54; 1994: 113; Bochkov \& Literák, 2006: 140

Type locality: Antwerp Zoo

Distribution: Europe

Host: Anas querquedula (Anseriformes: Anatidae)

Harpyrhynchoides artamus Bochkov et Klompen

Harpyrhynchoides artamus Bochkov \& Klompen, 2015: 24

Type locality: SE Asia 
Distribution: SE Asia

Host: Artamus fuscus (Passeriformes: Artamidae)

\section{Harpyrhynchoides asio (Fain)}

Harpyrhynchus (Harpyrhynchoides) asio Fain, 1972: 52; 1994: 128

Harpyrhynchoides asio (Fain): Bochkov \& Literák, 2006: 140

Type locality: Baelen Neet

Distribution: Belgium

Host: Asio otus otus (Strigiformes: Strigidae)

Harpyrhynchoides athene Bochkov, OConnor et Klompen

Harpyrhynchoides athene Bochkov, OConnor \& Klompen, 2015: 56

Type locality: Texas, Lubbock Co., Lubbock, $33.58^{\circ} \mathrm{N}, 101.86^{\circ} \mathrm{W}$

Distribution: USA

Host: Athene canicularia (Strigiformes: Strigidae)

Harpyrhynchoides botaurus Bochkov et Galloway

Harpyrhynchoides botaurus Bochkov \& Galloway, 2013: 409; Bochkov, OConnor \& Klompen, 2015: 33

Type locality: Manitoba, Winnipeg

Distribution: Canada

Host: Botaurus lentiginosus (Pelecaniformes: Ardeidae)

\section{Harpyrhynchoides brevis (Ewing)}

Harpirhynchus brevis Ewing, 1911: 38; Moss et al., 1968: 391; Fain, 1995: 99; Bochkov \& Literák, 2006: 140

Harpyrhynchoides brevis (Ewing): Bochkov \& Klompen, 2014c

Type locality: ?

Distribution: ?

Hosts: Turdus migratorius (Passeriformes: Turdidae), Coccothraustes vespertina, Loxia curvirostra (Pass.: Fringillidae)

\section{Harpyrhynchoides bubulcus Bochkov, OConnor et Klompen}

Harpyrhynchoides bubulcus Bochkov, OConnor \& Klompen, 2015: 28

Type locality: Florida, Orange Co., Orlando, Lake Adaire, $28.5666208^{\circ} \mathrm{N}, 81.2608791^{\circ} \mathrm{W}$

Distribution: USA

Host: Bubulcus ibis (Pelecaniformes: Ardeidae)

Harpyrhynchoides butorides (Boyd)

Harpyrhynchus butorides Boyd, 1968: 19

Harpirhynchus (Harpyrhynchoides) ?herodius, Fain 1994: 119 
Harpyrhynchoides butorides (Boyd): Bochkov, OConnor \& Klompen, 2015: 31

Type locality: Massachusetts, Hampshire, $42.38^{\circ} \mathrm{N}, 72.52^{\circ} \mathrm{W}$

Distribution: USA

Host: Butorides virescens (Pelecaniformes: Ardeidae)

Harpyrhynchoides calidris Bochkov, OConnor et Klompen

Harpyrhynchoides calidris Bochkov, OConnor \& Klompen, 2015: 17

Type locality: Kansas

Distribution: USA

Host: Calidris minutilla (Charadriiformes: Scolopacidae)

\section{Harpyrhynchoides capellae (Fritsch)}

Harpyrhynchus capellae Fritsch, 1954: 179; Dubinin, 1957: 104

Harpirhynchus (Harpyrhynchoides) capellae, Fain, 1994: 117

Harpyrhynchoides capellae (Fritsch): Fain, Bochkov \& Mironov, 1999: 45; Bochkov \&

Literák, 2006: 140

Type locality: Erlangen (Germany)

Distribution: France, Germany

Hosts: Gallinago gallinago, Lymnocryptes minimus (Charadriiformes: Scolopacidae)

\section{Harpyrhynchoides capitatus (Fain)}

Harpirhynchus (Harpyrhynchoides) capitatus Fain, 1976: 129; 1994: 119

Type locality: Antwerp Zoo

Distribution: ?

Host: Columbigallina talpacoti (Columbiformes: Columbidae)

Harpyrhynchoides charadrius Bochkov, OConnor et Klompen

Harpyrhynchoides charadrius Bochkov, OConnor \& Klompen, 2015: 20

Type locality: Texas, Lubbock Co., $33.6^{\circ} \mathrm{N}, 101.83^{\circ} \mathrm{W}$

Distribution: USA

Host: Charadrius vociferus (Charadriiformes: Charadriidae)

\section{Harpyrhynchoides clamator Bochkov}

Harpyrhynchoides clamator Bochkov, 2014: 480

Type locality: Limpopo, Waterberg District Municipality, Bela-Bela, Little Kariba

Distribution: South Africa

Host: Clamator jacobinus (Cuculiformes: Cuculidae)

Harpyrhynchoides coccyzus Bochkov, OConnor et Klompen

Harpyrhynchoides coccyzus Bochkov, OConnor \& Klompen, 2015: 44 
Type locality: Michigan, Washtenaw Co., Ann Arbor, UM Campus, $42.2793409^{\circ} \mathrm{N}$, $83.7386084^{\circ} \mathrm{W}$

Distribution: USA

Hosts: Coccyzus americanus, C. erythrophthalmus (Cuculiformes: Cuculidae)

\section{Harpyrhynchoides columbae (Fain)}

Harpyrhynchus (Harpyrhynchoides) columbae Fain, 1972: 51; 1994

Harpyrhynchoides columbae (Fain): Bochkov \& Literák, 2006: 140

Type locality: Bouillon

Distribution: Belgium

Host: Columba livia domestica (Columbiformes: Columbidae)

\section{Harpyrhynchoides coturnix (Fain)}

Harpyrhynchus (Harpyrhynchoides) coturnix Fain, 1972: 54

Harpirhynchus (Harpyrhynchoides) coturnix, Fain, 1994: 116

Harpyrhynchoides coturnix (Fain): Bochkov, OConnor \& Klompen, 2015: 34

Type locality: Antwerp Zoo

Distribution: North America (unknown locality)

Host: Coturnix delegorguei (Galliformes: Phasianidae)

\section{Harpyrhynchoides coxatus (Fain)}

Harpyrhynchus (Harpyrhynchoides) coxatus Fain, 1972: 54; 1994: 133

Type locality: Antwerp Zoo

Distribution: ?

Hosts: Columbigallina talpacoti, Columbina cruziana, Scardafella squamata (Columbiformes: Columbidae)

\section{Harpyrhynchoides cristagalli (Berlese et Trouessart)}

Harpirhynchus crista - galli Berlese \& Trouessart, 1889: 121; Lawrence, 1959b: 108

Sarcoborus crista - galli: Oudemans, 1906: 77

Harpyrhynchus crista - galli: Lawrence, 1959a: 418

Harpirhynchus (Harpyrhynchoides) cristagalli, Fain, 1994: 130

Harpyrhynchoides cristagalli: Bochkov, OConnor \& Klompen, 2015: 66

Type locality: South Africa

Distribution: Namibia, South Africa

Hosts: Colius colius, C. striatus (Coliiformes: Coliidae)

\section{Harpyrhynchoides crotophaga Bochkov, OConnor et Klompen}

Harpyrhynchoides crotophaga Bochkov, OConnor \& Klompen, 2015: 61

Type locality: Paramaribo, Wegnaar Zee, $05.82^{\circ} \mathrm{N} 55.17^{\circ} \mathrm{W}$ 
Distribution: Suriname

Host: Crotophaga ani (Cuculiformes: Cuculidae)

Harpyrhynchoides gallowayi Bochkov, OConnor et Klompen

Harpyrhynchoides gallowayi Bochkov, OConnor \& Klompen, 2015: 51

Type locality: Manitoba, Winnipeg

Distribution: Canada

Host: Columba livia (Columbiformes: Columbidae)

Harpyrhynchoides heatherae Bochkov et Galloway

Harpyrhynchoides heatherae Bochkov \& Galloway, 2013: 407

Type locality: Manitoba, Winnipeg, Sommerland

Distribution: Canada

Host: Junco hyemalis (Passeriformes: Emberizidae)

\section{Harpyrhynchoides herodius (Boyd)}

Harpyrhynchus herodius Boyd, 1968: 18

Harpyrhynchus (Harpyrhynchoides) herodius Fain, 1994: 112

Harpyrhynchoides herodius (Boyd): Bochkov \& Literák, 2006: 140; Bochkov, OConnor \& Klompen, 2015: 28

Type locality: Massachusetts, USA

Distribution: Belgium, Holland, USA (Massachusetts)

Hosts: Ardea cinerea, A. herodius (Pelecaniformes: Ardeidae)

Harpyrhynchoides ixobrychus Bochkov, OConnor et Klompen

Harpyrhynchoides ixobrychus Bochkov, OConnor \& Klompen, 2015: 32

Type locality: Michigan, Oakland Co.

Distribution: USA

Host: Ixobrychus exilis (Pelecaniformes: Ardeidae)

\section{Harpyrhynchoides kakatoe (Fain)}

Harpyrhynchus (Harpyrhynchoides) kakatoe Fain, 1972: 52; 1994: 120

Harpirhynchus kakatoe: Domrow, 1991: 1317

Harpyrhynchoides kakatoe (Fain): Bochkov, OConnor \& Klompen, 2015: 64

Type locality: Antwerp Zoo

Distribution: Australia

Host: Kakatoe galerita (Psittaciformes: Cacatuidae)

Harpyrhynchoides lawrencei (Fain)

Harpyrhynchus (Harpyrhynchus) lawrencei Fain, 1972: 51 
Harpirhynchus (Harpyrhynchoides) lawrencei, Fain, 1994: 113

Harpyrhynchoides lawrencei (Fain): Bochkov, OConnor \& Klompen, 2015: 60

Type locality: Antwerp Zoo

Distribution: South America

Host: Aratinga nenday (Psittaciformes: Psittacidae)

Harpyrhynchoides megascops Bochkov, OConnor et Klompen

Harpyrhynchoides megascops Bochkov, OConnor \& Klompen, 2015: 39

Type locality: Michigan, Michigan, Oakland Co., Commerce Twp., Marble Ct., $42.5644286^{\circ} \mathrm{N}, 83.4637356^{\circ} \mathrm{W}$

Distribution: USA

Host: Megascops asio (Strigiformes: Strigidae)

\section{Harpyrhynchoides metropeliae (Fain)}

Harpyrhynchus (Harpyrhynchoides) metropeliae Fain, 1972: 53

Harpyrhynchoides metropeliae (Fain): Bochkov, OConnor \& Klompen, 2015: 47

Type locality: Antwerp Zoo

Distribution: South America (unknown locality)

Host: Metropelia caeciliae (Columbiformes: Columbidae)

\section{Harpyrhynchoides modestus (Fain)}

Harpyrhynchus (Harpyrhynchoides) modestus Fain, 1976: 126; 1994: 134; Bochkov \& Galloway, 2013: 413

Harpyrhynchoides modestus (Fain): Bochkov, OConnor \& Klompen, 2015: 54

Type locality: Antwerp Zoo

Distribution: Canada

Host: Metropelia caeciliae (Columbiformes: Columbidae)

\section{Harpyrhynchoides numidae (Lawrence)}

Harpyrhynchus numidae Lawrence, 1959b: 110

Harpirhynchus (Harpyrhynchoides) numidae, Fain, 1994: 117

Harpyrhynchoides numidae (Fain): Bochkov, OConnor \& Klompen, 2015: 64

Type locality: Africa

Distribution: Africa

Host: Numida meleagris (Galliformes: Numididae)

\section{Harpyrhynchoides oenae (Fain)}

Harpyrhynchus (Harpyrhynchoides) oenae Fain, 1972: 53

Harpyrhynchus (Harpyrhynchoides) lamorali Fain, 1972: 53 - on Oena capensis.

Harpyrhynchus (Harpyrhynchoides) oenae lamorali, Fain, 1994: 125. Syn. by Bochkov et al., 1915 
Type locality: Antwerp Zoo

Distribution: ?

Host: Tympanistria tympanistria (Columbiformes: Columbidae)

Harpyrhynchoides parazumpti Fain, Bochkov et Mironov

Harpyrhynchoides parazumpti Fain, Bochkov \& Mironov, 1999: 43; Bochkov \& Literák, 2006: 140; Bochkov, OConnor \& Klompen, 2015: 54

Type locality: Kishinev (Moldavia)

Distribution: Germany, Moldavia, USA

Hosts: Corvus brachyrhynchos, C. frugilegus, C. monedula (Passeriformes: Corvidae)

\section{Harpyrhynchoides pectinifer (Lawrence)}

Harpyrhynchus pectinifer Lawrence, 1959a: 419

Harpirhynchus (Harpyrhynchoides) pectinifer Lawrence: Fain, 1994: 113

Harpyrhynchoides pectinifer (Lawrence): Bochkov, OConnor \& Klompen, 2015: 60

Type locality: Newington, East Transvaal, South Africa

Distribution: Rwanda, South Africa, USA, Zimbabwe

Hosts: Campethera abingoni, C. cailliauti, Colaptes auratus (Piciformes: Picidae)

Harpyrhynchoides phalaropus Bochkov et Galloway

Harpyrhynchoides phalaropus Bochkov \& Galloway, 2013: 410; Bochkov, OConnor \& Klompen, 2015: 28

Type locality: Manitoba, Winnipeg

Distribution: Canada

Host: Phalaropus lobatus (Charadriiformes: Scolopacidae)

Harpyrhynchoides pluvialis Bochkov, OConnor et Klompen

Harpyrhynchoides pluvialis Bochkov, OConnor \& Klompen, 2015: 25

Type locality: Ohio, Wayne Co., Wooster, $40.83^{\circ} \mathrm{N}, 81.88^{\circ} \mathrm{W}$

Distribution: USA

Host: Pluvialis dominica (Charadriiformes: Charadriidae)

\section{Harpyrhynchoides psittaci (Fain)}

Harpyrhynchus (Harpyrhynchoides) psittaci Fain, 1972: 55; 1994: 129

Harpyrhynchoides psittaci (Fain): Bochkov, OConnor \& Klompen, 2015: 66

Type locality: Antwerp Zoo

Distribution: ?

Hosts: Poicephalus senegalus, Psittacus erithacus (Psittaciformes: Psittacidae)

\section{Harpyrhynchoides psittaculae (Fain)}

Harpyrhynchus (Harpyrhynchoides) psittaculae Fain, 1972: 52; 1994: 126 
Type locality: Antwerp Zoo

Distribution: ?

Hosts: Psittacula alexandri, P. cyanocephala (Psittaciformes: Psittaculidae)

Harpyrhynchoides puffinus Mertins in Bochkov, OConnor et Klompen

Harpyrhynchoides puffinus Mertins in Bochkov, OConnor \& Klompen, 2015: 35

Type locality: Florida, Atlantic Ocean adjacent to east cost of Martin Co.

Distribution: USA

Host: Puffinus gravis (Procellariiformes: Procellariidae)

Harpyrhynchoides rosellacinus (Lawrence)

Harpyrhynchus rosellacinus Lawrence, 1959: 238

Harpyrhynchus (Harpyrhynchoides) rosellacinus, Fain, 1994: 139

Harpyrhynchoides rosellacinus (Lawrence):

Type locality: Sydney

Distribution: Australia (incl. Tasmania)

Hosts: Glossopsitta concinna, Lathamus discolor, Platycercus eximius, Trichoglossus chlorolepidotus, T. moluccanus (Psittaciformes: Psittaculidae)

Harpyrhynchoides rubeculinus (Černy et Sixl)

Harpyrhynchus rubeculinus Černy \& Sixl, 1971: 388

Harpyrhynchus (Harpyrhynchoides) rubeculinus (Černy et Sixl): Fain, 1994: 138

Harpyrhynchoides rubeculinus (Černy et Sixl): Fain, Bochkov \& Mironov, 1999: 45;

Bochkov \& Literák, 2006: 140; 2008: 227; Bochkov \& OConnor, 2013: 505; Bochkov, OConnor \& Klompen, 2015: 51

Type locality: Brunnsee (Austria)

Distribution: Austria, Brazil, Czech Republic, France, USA

Hosts: Erithacus rubecula (Passeriformes: Muscicapidae, Saxicolinae), Catharus ustulatus, Turdus amaurochalinus (Pass.: Turdidae), Pheucticus ludovicianus (Pass.: Cardinalidae)

Harpyrhynchoides setophaga Bochkov et Klompen

Harpyrhynchoides setophaga Bochkov \& Klompen, 2014c: 406; Bochkov, OConnor \& Klompen, 2015: 50

Type locality: Winnipeg, Manitoba

Distribution: Canada

Host: Setophaga ruticilla (Passeriformes: Parulidae)

Harpyrhynchoides spizella Bochkov et Klompen

Harpyrhynchoides spizella Bochkov \& Klompen, 2014a: 415

Type locality: California 
Distribution: USA

Host: Spizella passerina (Passeriformes: Passerellidae)

\section{Harpyrhynchoides squamosus (Fain)}

Harpyrhynchus (Harpyrhynchoides) squamosus Fain, 1972: 51; 1976: 126

Harpyrhynchoides squamosus (Fain): Bochkov, OConnor \& Klompen, 2015: 65

Type locality: Antwerp Zoo

Distribution:?

Host: Psittacula cyanocephala (Psittaciformes: Psittaculidae)

\section{Harpyrhynchoides tracheatus (Fritsch)}

Harpyrhynchus tracheatus Fritsch, 1954: 180; Dubinin, 1957: 103

Harpirhynchus (Harpyrhynchoides) tracheatus, Fain, 1994: 120

Harpyrhynchoides tracheatus (Fritsch); Bochkov \& Literák, 2006: 140; Bochkov \& Galloway,

2013: 412; Bochkov \& OConnor, 2013: 505; Bochkov, OConnor \& Klompen, 2015: 36

Type locality: Germany

Distribution: Canada, Germany, Luxemburg, USA

Hosts: Buteo buteo, B. jamaicensis, B. lineatus (Accipitriformes: Accipitridae)

\section{Harpyrhynchoides tyto (Fain)}

Harpyrhynchus (Harpyrhynchoides) tyto Fain, 1972: 51; 1994: 112

Harpyrhynchoides tyto (Fain): Bochkov \& Literák, 2006: 140

Type locality: Crupet, Namur

Distribution: Belgium

Host: Tyto alba (Strigiformes: Tytonidae)

\section{Harpyrhynchoides vercammeni (Lawrence)}

Harpyrhynchus vercammeni Lawrence, 1959a: 423

Harpyrhynchus (Harpyrhynchoides) vercammeni Lawrence: Fain, 1994: 138

Harpyrhynchoides vercammeni (Lawrence): Bochkov, OConnor \& Klompen, 2015: 63

Type locality: Luvungi, Ruzizi, Kivu

Distribution: DR Congo

Host: Centropus toulou grilli (Cuculiformes: Cuculidae)

Harpyrhynchoides vulgaris Bochkov et Galloway

Harpyrhynchoides vulgaris Bochkov \& Galloway, 2004: 27; Bochkov \& Klompen 2014b: 409; Bochkov, OConnor \& Klompen, 2015: 50

Type locality: Manitoba

Distribution: Canada

Hosts: Haemorhous [Carpodacus] purpureus (Passeriformes: Fringillidae), Setophaga ruticilla (Passeriformes: Parulidae) 
Harpyrhynchoides xanthocephalus Bochkov et Klompen

Harpyrhynchoides xanthocephalus Bochkov \& Klompen, 2014a: 212; Bochkov, OConnor

\& Klompen, 2015: 51

Type locality: Nebraska: Cherry Co., Valentine

Distribution: USA

Host: Xanthocephalus xanthocephalus (Passeriformes: Icteridae)

Harpyrhynchoides zenaida Bochkov, OConnor et Klompen

Harpyrhynchoides zenaida Bochkov, OConnor \& Klompen, 2015: 43

Type locality: Michigan, Washtenaw Co., Ann Arbor, UM Campus, $42.2793409^{\circ} \mathrm{N}$,

$83.7386084^{\circ} \mathrm{W}$

Distribution: USA

Host: Zenaida macroura (Columbiformes: Columbidae)

Harpyrhynchoides zumpti (Fain)

Harpyrhynchus (Harpyrhynchoides) zumpti Fain, 1972: 54; 1994: 136; Bochkov \& Galloway, 2001: 2017

Harpyrhynchus (Harpyrhynchoides) diuca Fain, 1972: 53. Type from Antwerp Zoo, on Diuca diuca (Passeriformes: Thraupidae)

Harpyrhynchoides zumpti (Fain): Bochkov \& Galloway, 2001, 2004; Bochkov, OConnor \& Klompen, 2015: 48

Harpyrhynchoides kirgizorum Fain, Bochkov \& Mironov, 1999: 42. Type loc.: Bishkek, Kirghizia, on Emberiza calandra. Syn. By Bochkov \& Klompen, 2014c: 410

Type locality: Bloemenhof, Mafeking

Distribution: Canada, Kirghizia, South Africa

Hosts: Eremopteryx leucotis (Passeriformes: Alaudidae), Diuca diuca (Passeriformes: Thraupidae), Quiscalus quiscula (Passeriformes: Icteridae), Emberiza calandra, Junco hyemalis (Passeriformes: Emberizidae)

Genus Harpirhynchus Mégnin

Harpirhynchus Mégnin, 1878: 8 (written also Harpyrhinchus and Harpyrynchus)

Type species: Sarcoptes nidulans Nitzsch, 1818

Harpicephalus Canestrini, 1886: 170

Type species: Sarcoptes nidulans Nitzsch, 1818

Pseudoharpirhynchus Fain, Bochkov \& Mironov, 1999: 39 (subgenus)

Type species: Harpirhynchus agapornis Fain, 1972

Harpirhynchus dusbabeki Bochkov et Literák

Harpyrhinchus nidulans Nitzsch, 1818: Henry et al., 2004: 34 (misidentification), Literák et al., 2005: 26 (misidentification)

Harpirhynchus dusbabeki Bochkov \& Literák, 2006: 138 
Type locality: Pusté Úl’any (Slovakia)

Distribution: Austria, Croatia, Czech Rep., France, Poland, Slovakia

Host: Panurus biarmicus (Passeriformes: Panuridae)

Harpirhynchus galeridae Fain, Bochkov et Mironov

Harpirhynchus (Harpirhynchus) galeridae Fain, Bochkov \& Mironov, 1999: 39; Bochkov \& Literák, 2006: 140

Type locality: Azerbaijan

Distribution: Azerbaijan

Host: Galerida cristata (Passeriformes: Alaudidae)

Harpirhynchus longipilus Banks (sp. inquir.)

Harpirhynchus longipilus Banks, 1905a: 20; Fain, 1995: 99

Sarcopterus longipilis: Banks, 1905b: 135

Type locality: USA

Distribution: USA

Host: Loxia sp. (Passeriformes: Fringillidae)

\section{Harpirhynchus nidulans (Nitzsch)}

Sarcoptes nidulans Nitzsch, 1818, in Ersch-Gruber, Allg. Encyclop. Wissensch., 1: 250; synonymy in Oudemans, 1906: 75 - 76; 1936: 1132 - 1134

Sarcoptes palumbinus C.L. Koch, 1836: 5, 12; Lucas, 1840: 487; synonymy in Oudemans, 1936: $1134-1135$

Sarcopterus nidulans: Giebel, 1871: 29; Murray, 1877: 314; Berlese, 1895: 5

Harpirhynchus nidulans Mégnin, 1877: 13; Literák et al., 2005: 26; Bochkov \& Literák, 2006: 140

Harpicephalus nidulans: Canestrini, 1886: 170

? Harpirhynchus megnini Heim, 1892: 132

Sarcopterinus nidulans: Railliet, 1893: 697; Berlese, 1913: 130

Sarcoborus nidulans: Oudemans, 1906: 75

Harpyrhynchus nidulans (Nitzsch): Turk, 1953: 18; Jeleva, 1970: 63

Type locality: Germany

Distribution: Bulgaria, Czech Rep., England, Germany, Italy, Slovakia, Switzerland

Hosts: Coccothraustes coccothraustes, Chloris chloris, Fringilla coelebs (Passeriformes: Fringillidae), Alauda arvensis, Galerida cristata (Passeriformes: Alaudidae), Locustella lanceolata (Passeriformes: Locustellidae), Panurus biarmicus (Passeriformes: Panuridae), Corvus corone (Passeriformes: Corvidae), Columba palumbus (Columbiformes: Columbidae)

Harpirhynchus quasimodo Bochkov et Mertins

Harpirhynchus quasimodo Bochkov \& Mertins, 2010: 83

Type locality: Florida 
Distribution: USA (Florida)

Host: Molothrus ater (Passeriformes: Icteridae)

Harpirhynchus sp. 1

Distribution: Turkey

Host: Perdix (Galliformes: Phasianidae)

Harpirhynchus sp. 2

Distribution: SE Asia (McClure H. Eliott, N. Ratanaworadhan et al., 1973)

Host: Eurystomus orientalis (Coraciiformes: Coraciidae)

Genus Neharpyrhynchus Fain

Harpirhynchus (Neharpyrhynchus) Fain, 1972: 50

Neharpyrhynchus Fain, 1995: 78

Type species: Harpirhynchus plumaris Fritsch, 1954

Neharpyrhynchus agelaius Bochkov, OConnor et Klompen

Neharpyrhynchus agelaius Bochkov, OConnor \& Klompen, 2015: 102

Type locality: Michigan, Oakland Co.

Distribution: USA

Host: Agelaius phoeniceus (Passeriformes: Icteridae)

Neharpyrhynchus baile Bochkov et Literák

Neharpyrhynchus baile: Bochkov \& Literák, 2007: 35; Martinu et al. 2008: 207; Bochkov, OConnor \& Klompen, 2015: 98

Type locality: Brazil

Distribution: Brazil, USA

Hosts: Turdus leucomelas (Passeriformes: Turdidae), Dumetella carolinensis (Passeriformes: Mimidae)

Neharpyrhynchus bochkovi Martinu, Dusbábek et Literák

Neharpyrhynchus bochkovi Martinu, Dusbábek \& Literák, 2008: 198

Type locality: Jinacovice

Distribution: Czech Rep.

Host: Turdus merula (Passeriformes: Turdidae)

Neharpyrhynchus bombycilla Bochkov, OConnor et Klompen

Neharpyrhynchus bombycilla Bochkov, OConnor \& Klompen, 2015: 96

Type locality: Michigan, Muskegon Co., Muskegon State game Area, US route 31, $43.2961989^{\circ} \mathrm{N}, 86.0668945^{\circ} \mathrm{W}$

Distribution: USA 
Host: Bombycilla cedrorum (Passeriformes: Bombycillidae)

Neharpyrhynchus campylorhynchus Bochkov, OConnor et Klompen

Neharpyrhynchus campylorhynchus Bochkov, OConnor \& Klompen, 2015: 87

Type locality: USA

Distribution: USA

Host: Campylorhynchus brunneicapillus (Passeriformes: Troglodytidae)

Neharpyrhynchus chlorospingus Bochkov et Literák

Neharpyrhynchus chlorospingus Bochkov \& Literák, 2011: 20; Bochkov, OConnor \& Klompen, 2015: 108

Type locality: Cerro de la Mueste, $9^{\circ} 34^{\circ} \mathrm{N}, 83^{\circ} 45^{\circ} \mathrm{W}$

Distribution: Costa Rica

Host: Chlorospingus pileatus (Passeriformes, Passerellidae)

Neharpyrhynchus domrowi Bochkov et Klompen

Neharpyrhynchus domrowi Bochkov \& Klompen, 2015: 25

Type locality: New South Walles, Loddon Falls, 34.28 S, 150.9 E

Distribution: Australia, Papua New Guinea

Hosts: Acanthorhynchus tenuirostris, Ptiloprora perstriata, Myzomela rodenbergii (Passeriformes: Meliphagidae)

Neharpyrhynchus hippolae Bochkov

Neharpyrhynchus hippolae Bochkov, 2000a: 534; Bochkov \& Literák, 2006: 140; Martinu, Dusbábek \& Literák, 2008: 200

Type locality: Oskui, Novgorodskaya Oblast, Russia

Distribution: Czech Rep., Russia

Host: Hippolais icterina (Passeriformes: Sylviidae)

Neharpyrhynchus icterus Bochkov et OConnor

Neharpyrhynchus icterus Bochkov \& OConnor, 2013: 506; Bochkov, OConnor \& Klompen, 2015: 101

Type locality: California

Distribution: USA

Host: Icterus galbula (Passeriformes: Icteridae)

Neharpyrhynchus loxia Bochkov et Galloway

Neharpyrhynchus loxia Bochkov \& Galloway, 2013: 413; Bochkov, OConnor \& Klompen, 2015: 108

Type locality: Manitoba, Winnipeg

Distribution: Canada 
Host: Loxia curvirostra (Passeriformes: Fringillidae)

Neharpyrhynchus mironovi Bochkov et Literák

Neharpyrhynchus mironovi Bochkov \& Literák, 2011: 23; Bochkov, OConnor \& Klompen, 2015: 104

Type locality: Minas Gerais, Belo Horizonte, Nova Lima

Distribution: Brazil

Host: Dacnis cayana (Passeriformes, Thraupidae)

Neharpyrhynchus novoplumaris (Moss, Oliver et Nelson)

Harpyrhynchus novoplumaris Moss, Oliver \& Nelson, 1968: 377

Neharpyrhynchus novoplumaris: Fain, 1995: 85 (syn. of N. plumaris)

Neharpyrhynchus novoplumaris: Bochkov, 2000a: 537; Bochkov \& OConnor, 2013: 508

(separate species); Bochkov, OConnor \& Klompen, 2015: 108

Type locality: Hopland Field Station, Mendocino Co., California

Distribution: USA (California, Arizona, Kansas, Michigan, Missouri, Maryland, Nebraska, Wyoming, New Mexico), Mexico

Hosts: Certhia familiaris (Certhiidae), Baeolophus [Parus] bicolor (Paridae), Kieneria [Pipilo, Melozone] fusca, Amphispiza bilineata, Spizella passerina ....(Passerellidae), Campylorhynchus brunneicapillus (Troglodytidae), Cardinalis cardinalis (Cardinalidae), Junco hyemalis, J. phaeonotus (Emberizidae)(all Passeriformes)

Neharpyrhynchus oenanthe Bochkov

Neharpyrhynchus oenanthe Bochkov, 2014: 483

Type locality: Akanyaru

Distribution: Rwanda

Host: Oenanthe oenanthe (Passeriformes: Muscicapidae)

Neharpyrhynchus pari Martinu, Dusbábek \& Literák

Neharpyrhynchus pari Martinu, Dusbábek \& Literák, 2008: 201

Type locality: Czech Rep., Brno, Sobesice

Distribution: Czech Rep., USA ?

Hosts: Baeolophus bicolor, Cyanistes caeruleus, Parus major, Periparus ater, Poecile montanus, P. palustris (Passeriformes: Paridae)

Neharpyrhynchus picidarum Bochkov, OConnor et Klompen

Neharpyrhynchus picidarum Bochkov, OConnor \& Klompen, 2015: 88

Type locality: Michigan, Oakland Co., Pontiac, $42.6110959^{\circ} \mathrm{N}, 83.2877398^{\circ} \mathrm{W}$

Distribution: USA

Hosts: Colaptes auratus, Melanerpes formicivorus, M. uropygidialis, Picoides pubescens (Piciformes: Picidae) 


\section{Neharpyrhynchus pilirostris (Berlese et Trouessart)}

Harpirhynchus pilirostris Berlese \& Trouessart, 1889: 137 - 138; Dubinin, 1957: 104;

Lawrence, 1959b: 108

Sarcoborus pilirostris: Oudemans, 1906: 76

Sarcopterus pilirostris (Berlese et Trouessart) Berlese, 1894: fasc. 73, tav. 5, figs.1-5; nec Sarcopterus nidulans Nitzsch, 1818

Harpyrhynchus ovalis Fritsch, 1957: 193; Lawrence, 1959a: 424 (deutonymph)

Harpirhynchus (Neharpyrhynchus) Fain, 1972: 50

Neharpyrhynchus pilirostris (Berlese et Trouessart): Fain, 1995: 81; Bochkov, 2000a: 534;

Bochkov \& Literák, 2006: 140; Martinu, Dusbábek \& Literák, 2008: 203; Bochkov \&

Galloway, 2013: 414; Bochkov, OConnor \& Klompen, 2015: 101

Type locality: Blanc (Indre, France)

Distribution: Canada, Czech Rep., France, Germany, South Africa, USA

Hosts: Passer domesticus (Passeriformes: Passeridae), ? Aegithalos caudatus (Passeriformes:

Aegithalidae)

\section{Neharpyrhynchus plumaris (Fritsch)}

Harpirhynchus plumaris Fritsch, 1954: 193

Harpyrhynchus plumaris: Dubinin, 1957: 108

Harpyrhynchus ovalis Fritsch, 1954: 192; Lawrence, 1959b: 108

? Harpirhynchus novoplumaris Moss et al., 1968: 377 (Fain, 1995)

Neharpyrhynchus novoplumaris, Fain, 1995: 85

Neharpyrhynchus plumaris (Fritsch): Bochkov, 2000a: 536; Martinu, Dusbábek \& Literák, 2008: 203; Bochkov, OConnor \& Klompen, 2015: 111

Type locality: Germany

Distribution: Czech Rep., Germany, Russia

Hosts: Fringilla coelebs, Chloris chloris, Carduelis cannabina (Passeriformes: Fringillidae), Muscicapa striata (Pass.: Muscicapidae), Parus ater, P. palustris (Passeriformes: Paridae), ? Aythya ferina (Anseriformes: Anatidae)

\section{Neharpyrhynchus quiscalus Bochkov, OConnor et Klompen}

Neharpyrhynchus quiscalus Bochkov, OConnor \& Klompen, 2015: 98

Type locality: Michigan, Mecosta Co., 1.3 mi E Big Rapids, $43.698604^{\circ} \mathrm{N}$, $85.4576146^{\circ} \mathrm{W}$

Distribution: USA

Host: Quiscalus quiscula (Passeriformes: Icteridae)

Neharpyrhynchus schoenobaenus Martinu, Dusbábek et Literák

Neharpyrhynchus schoenobaenus Martinu, Dusbábek \& Literák, 2008: 203

Type locality: Czech Rep., Sedlec 
Distribution: Czech Rep.

Host: Acrocephalus schoenobaenus (Passeriformes: Acrocephalidae)

Neharpyrhynchus spinus Martinu, Dusbábek et Literák

Neharpyrhynchus spinus Martinu, Dusbábek \& Literák, 2008: 204

Type locality: Česke Budejovice

Distribution: Czech Rep.

Host: Spinus spinus (Passeriformes: Fringillidae)

Neharpyrhynchus aff. spinus Martinu, Dusbábek et Literák

Type locality: Czech Rep.

Distribution: Paraguay (Literák, Bochkov \& Franco, 2014)

Host: Setophaga pitiayumi (Passeriformes: Parulidae)

Neharpyrhynchus spizella Bochkov, OConnor et Klompen

Neharpyrhynchus spizella Bochkov, OConnor \& Klompen, 2015: 105

Type locality: California, Mendocino Co., Hopland Field Station, $39^{\circ} \mathrm{N} 123.08^{\circ} \mathrm{W}$

Distribution: USA (California, Wyoming)

Host: Spizella passerina (Passeriformes: Passerellidae)

Neharpyrhynchus squamiferus (Fain)

Harpyrhynchus (Neharpyrhynchus) squamiferus Fain, 1972: 55

Neharpyrhynchus squamiferus (Fain): Fain, 1995: 80

Neharpyrhynchus squamifer (Fain): Bochkov, 2000a: 534

Type locality: India

Distribution: India

Host: Temenuchus pagodarum (Passeriformes: Sturnidae)

Neharpyrhynchus tangara Bochkov et Literák

Neharpyrhynchus tangara Bochkov \& Literák, 2011: 20; Literák, Bochkov \& Franco, 2014;

Bochkov, OConnor \& Klompen, 2015: 104

Type locality: Minas Gerais, Belo Horizonte, Nova Lima, Água Limpa

$20^{\circ} 13^{`} \mathrm{~S}, 43^{\circ} 56^{\mathrm{C}} \mathrm{W}$ (Brazil)

Distribution: Brazil, Paraguay, Peru

Hosts: Paroaria capitata, P. capitata, Tangara cayana, Thraupis episcopus (Passeriformes: Thraupidae)

\section{Neharpyrhynchus trochilinus (Fain)}

Harpirhynchus (Neharpyrhynchus) trochilinus Fain, 1972: 55 
Neharpyrhynchus trochilinus (Fain): Fain, 1995: 80; Bochkov, 2000a: 534; Bochkov \& Literák, 2011: 28; Literák et al., 2012: 109; Literák, Bochkov \& Franco, 2014: 184; Bochkov, OConnor \& Klompen, 2015: 94

Type locality: Antwerp Zoo

Distribution: Brazil, Costa Rica, Paraguay, Peru

Hosts: Amazilia lactea, Chlorostilbon lucidus, Chrysolampis moschitus, Eugenes fulgens, Panterpe insignis (Trochiliformes: Trochilidae)

Neharpyrhynchus turdus Bochkov, OConnor et Klompen

Neharpyrhynchus turdus Bochkov, OConnor \& Klompen, 2015: 92

Type locality: Michigan, Washtenaw Co., $4.4 \mathrm{mi}$ ESE Saline, $42.1561503^{\circ} \mathrm{N}, 83.7180519^{\circ} \mathrm{W}$

Distribution: USA

Host: Turdus migratorius (Passeriformes: Turdidae)

Neharpyrhynchus vireo Bochkov, OConnor et Klompen

Neharpyrhynchus vireo Bochkov, OConnor \& Klompen, 2015: 104

Type locality: Florida, Leon Co., $30.49^{\circ} \mathrm{N}, 84.21^{\circ} \mathrm{W}$

Distribution: USA

Host: Vireo olivaceus (Passeriformes: Vireonidae)

Genus Ralliharpirhynchus Fain

Ralliharpirhynchus Fain, 1995: 74

Type species: Metharpyrhynchus porphyrio Fain, 1972

Ralliharpirhynchus limnocarax (Fain)

Metharpyrhynchus limnocorax Fain, 1972: 56

Ralliharpirhynchus limnocorax: Fain, 1995: 74

Type locality: Kinshasa

Distribution: DR Congo

Host: Limnocorax flaviceps (Ralliformes: Rallidae)

Ralliharpirhynchus porphyrio (Fain)

Metharpyrhynchus porphyrio Fain, 1972: 56

Ralliharpirhynchus porphyrio, Fain, 1995: 74

Type locality: Antwerp Zoo

Distribution: ?

Host: Porphyrio porphyrio (Ralliformes: Rallidae)

Genus Trichorhynchiella Fain

Trichorhynchiella Fain, 1995: 75

Type species: Trichorhynchiella paddae Fain, 1995 
Trichorhynchiella myiarchus Bochkov et Klompen

Trichorhynchiella myiarchus Bochkov \& Klompen, 2014b: 304; Bochkov, OConnor \& Klompen, 2015: 113

Type locality: unknown

Distribution: North America

Host: Myiarchus crinitus (Passeriformes: Tyrannidae)

Trichorhynchiella paddae Fain

Trichorhynchiella paddae Fain, 1995: 99

Type locality: Antwerp Zoo

Distribution: ?

Host: Padda oryzivora (Passeriformes: Estrildidae)

Trichorhynchiella phleocryptes Bochkov et OConnor

Trichorhynchiella phleocryptes Bochkov \& OConnor, 2014a: 324; Bochkov, OConnor \& Klompen, 2015: 117

Type locality: Argentina

Distribution: Argentina

Host: Phleocryptes melanops (Passeriformes: Furnariidae)

Tribus Metharpyrhynchini Fain

Metharpyrhynchini Fain, 1972: 56

Type genus: Metharpyrhynchus Fain, 1972

Genus Metharpyrhynchus Fain

Metharpyrhynchus Fain, 1972: 56

Type species: Metharpyrhynchus macrophallus Fain, 1972

Metharpyrhynchus jynx Fain

Metharpyrhynchus jynx Fain, 1972: 57

Type locality: Akanyaru

Distribution: Rwanda

Host: Jynx ruficollis (Piciformes: Picidae)

Metharpyrhynchus macrophallus Fain

Metharpyrhynchus macrophallus Fain, 1972: 56

Type locality: Antwerp Zoo

Distribution: ?

Host: Padda oryzivora (Passeriformes: Estrildidae)

Metharpyrhynchus mossi Fain

Metharpyrhynchus mossi Fain, 1995: 87 
Type locality: Akanyaru, Eastern region of Rwanda

Distribution: Rwanda

Host: Prinia subflava graueri (Passeriformes: Cisticolidae)

Metharpyrhynchus namibiensis Fain

Metharpyrhynchus namibiensis Fain, 1995: 87

Type locality: Arab

Distribution: Namibia

Host: Philetairus socius (Passeriformes: Ploceidae)

Tribus Perharpyrhynchini Fain

Tribus Perharpyrhynchini Fain, 1972: 57

Type genus: Perharpyrhynchus Fain, 1972

Genus Perharpyrhynchus Fain

Perharpyrhynchus Fain, 1972: 57

Type species: Perharpyrhynchus jacana Fain, 1972

Perharpyrhynchus caprimulgus Bochkov et Klompen

Perharpyrhynchus caprimulgus Bochkov \& Klompen, 2014: 313

Type locality: Mozambique

Distribution: Mozambique

Host: Caprimulgus fossii (Caprimulgiformes: Caprimulgidae)

Perharpyrhynchus charadrius Bochkov, OConnor et Klompen

Perharpyrhynchus charadrius Bochkov, OConnor \& Klompen, 2015: 80

Type locality: Michigan, Washtenaw Co., Ann Arbor, UM campus, $42.2793409^{\circ} \mathrm{N}$, $83.7386084^{\circ} \mathrm{W}$

Distribution: USA

Host: Charadrius vociferus (Charadriiformes: Charadriidae)

Perharpyrhynchus elseyornis Bochkov et Klompen

Perharpyrhynchus elseyornis Bochkov \& Klompen, 2014: 314

Type locality: Western Australia, Brooking Springs, $18.12^{\circ} \mathrm{S}, 125.58^{\circ} \mathrm{E}$

Distribution: Australia

Host: Elseyornis melanops (Charadriiformes: Charadriidae)

\section{Perharpyrhynchus jacana Fain}

Perharpyrhynchus jacana Fain, 1972: 57

Type locality: Antwerp Zoo

Distribution: South America 
Host: Jacana spinosa intermedia (Charadriiformes: Jacanidae)

Perharpyrhynchus recurvirostra Fain

Perharpyrhynchus recurvirostra Fain, 1976: 130

Type locality: Antwerp Zoo

Distribution: ?

Host: Recurvirostra avocetta (Charadriiformes: Scolopacidae)

\section{Index of Harpirhynchinae and Harpypalpinae}

(Names in bold are accepted as valid)

accipiter, Harpyrhynchoides 276

actitis, Harpyrhynchoides 276

aegolius, Harpyrhynchoides 276

agapornis, Harpyrhynchoides 277

agapornis, Harpirhynchus 277

agapornis, Harpyrhynchus 277

agelaius, Neharpyrhynchus 288

alaudinus, Harpyrhynchoides 277

alectoris, Harpyrhynchoides 277

alectoris, Harpirhynchus 277

alectoris, Harpyrhynchus 277

alexfaini, Crassacarus 273

amazonae, Harpyrhynchoides 277

amazonae, Harpyrhynchus 277

anatum, Harpyrhynchoides 277

anatum, Harpyrhynchus 277

Anharpyrhynchus 272

apodus, Anharpyrhynchus 273

apus, Harpyrhynchiella 275

artamus, Harpyrhynchoides 277

asio, Harpyrhynchoides 278

asio, Harpyrhynchus $\mathbf{2 7 8}$

athene, Harpyrhynchoides 278

baile, Neharpyrhynchus 288

bochkovi, Neharpyrhynchus 288

bombycilla, Neharpyrhynchus 288

botaurus, Harpyrhynchoides 278

butorides, Harpirhynchus 278 brevis, Harpyrhynchoides 276

brevis, Harpirhynchus (incertae sedis) 278

bubulcus, Harpyrhynchoides 278

butorides, Harpirhynchus $\mathbf{2 7 8}$

calidris, Harpyrhynchoides 279

campylorhynchus, Neharpyrhynchus 289

capellae, Harpyrhynchoides 279

capellae, Harpirhynchus $\mathbf{2 7 9}$

capellae, Harpyrhynchus 279

capitatus, Harpyrhynchoides 279

capitatus, Harpyrhynchus 279

caprimulgus, Perharpyrhynchus 295

charadrius, Harpyrhynchoides 279

charadrius, Perharpyrhynchus 295

chlorospingus, Neharpyrhynchus 289

clamator, Harpyrhynchoides 279

coccyzus, Harpyrhynchoides 279

columbae, Harpyrhynchoides 280

columbae, Harpyrhynchus $\mathbf{2 8 0}$

contopus, Fainharpyrhynchus 275

coturnix, Harpyrhynchoides 280

coturnix, Harpirhynchus $\mathbf{2 8 0}$

coturnix, Harpyrhynchus $\mathbf{2 8 0}$

coxatus, Harpyrhynchus $\mathbf{2 8 0}$

Crassacarus 273

cristagalli, Harpyrhynchoides 280

crista-galli, Harpyrhynchus $\mathbf{2 8 0}$

crista-galli, Sarcoborus $\mathbf{2 8 0}$ 
crotophaga, Harpyrhynchoides 280 cylindripalpus, Crassacarus 274

cylindripalpus, Harpirhynchus 274 cylindripalpus, Harpyrhynchus 274 Cypsharpirhynchus 275 cypsiuri, Cypsharpirhynchus 275 cypsiuri, Harpyrhynchiella 275 cypsiuri, Metharpyrhynchus 275 diuca, Harpyrhynchoides diuca, Harpyrhinchus 286 domrowi, Neharpyrhynchus 289 dubinini, Harpypalpus 271 dusbabeki, Harpirhynchus 286 elizae, Anharpyrhynchus 273 elzeyornis, Perharpyrhynchus 295 Fainharpirhynchus 274 fritschi, (Anharpyrhynchus) 273 fritschi, Crassacarus 274 galeridae, Harpirhynchus 287 gallowayi, Harpyrhynchoides 281 Harpicephalus $\mathbf{2 8 6}$

Harpirhynchus 286

Harpirhynchidae 269

Harpirhynchinae 272

Harpyrhynchini 272

Harpypalpinae 269

Harpypalpoides 269

Harpypalpus 270

Harpyrhynchiella 275

Harpyrhynchoides 276 heatherae, Harpyrhynchoides 281 herodius, Harpyrhynchoides 281 herodius, Harpirhynchus herodius, Harpyrhynchus $\mathbf{2 8 1}$ hippolae, Neharpyrhynchus 289 hirundinis, Harpypalpus 269 hirundinis, Harpypalpoides 269 hirundinoides, Harpypalpoides 269 holopus, Harpypalpus 271 holopus, Harpirhynchus 271 holopus, Sarcoborus 271 icterus, Neharpyrhynchus 289 ixobrychus, Harpyrhynchoides 281 jacana, Perharpyrhynchus 295 johnstoni, Harpypalpoides 269 jynx, Metharpyrhynchus 294 kakatoe, Harpyrhynchoides 281 kakatoe, Harpyrhynchus 281 kirgizorum, Harpyrhynchoides $\mathbf{2 8 5}$ lamorali, Harpyrhynchus oenae $\mathbf{2 8 2}$ lamorali, Harpyrhynchus $\mathbf{2 8 2}$ lawrencei, Harpyrhynchoides 281 lawrencei, Harpirhynchus $\mathbf{2 8 2}$ lawrencei, Harpyrhynchus $\mathbf{2 8 1}$ legatus, Fainharpirhynchus 275 leptoptilus, Harpyrhynchoides leptoptilus, Harpyrhynchus lesickii, Harpypalpoides 269 limnocorax, Methaharpyrhynchus 293 limnocorax, Ralliharpirhynchus 293 lonchura, Harpypalpus 271 longipes, Harpypalpus 271 longipes, Harpyrhynchus 271 longipilus, Harpirhynchus (sp. inquir.) 287 longipilis, Sarcopterus $\mathbf{2 8 7}$ loxia, Neharpyrhynchus 289 lukoschusi, Anharpyrhynchus 273 lukoschusi, Harpypalpoides 270 macrophallus, Metharpyrhynchus 284 megnini, Harpirhynchus 287 megascops, Harpyrhynchoides 282 melanerpes, Crassacarus 274 Metharpyrhynchini 294 Metharpyrhynchus 294 metropeliae, Harpyrhynchoides 282 metropeliae, Harpyrhynchus $\mathbf{2 8 2}$ mironovi, Neharpyrhynchus 290 modestus, Harpyrhynchoides 282 modestus, Harpyrhynchus 282 monstrosus, Anharpyrhynchus 273 monstrosus, Harpyrhynchus 273 mossi, Fainharpirhynchus 275 
mossi, Metharpyrhynchus 294 myiarchus, Trichorhynchiella 294 namibiensis, Harpypalpoides 270 namibiensis, Metharpyrhynchus 295 Neharpyrhynchus 288 nidulans, Harpicephalus 287 nidulans, Harpirhynchus 287 nidulans, Harpyrhynchus 287 nidulans, Sarcoborus 287 nidulans, Sarcopterinus $\mathbf{2 8 7}$ nidulans, Sarcoptes $\mathbf{2 8 7}$ novoplumaris, Harpirhynchus 290 novoplumaris, Neharpyrhynchus 290 numidae, Harpyrhynchoides 282 numidae, Harpirhynchus $\mathbf{2 8 2}$ numidae, Harpyrhynchus $\mathbf{2 8 2}$ oenae, Harpyrhynchoides 282 oenae, Harpyrhynchus $\mathbf{2 8 2}$ oenanthe, Neharpyrhynchus 290 ovalis, Harpyrhynchus 291 paddae, Trichorhynchiella 294 palumbinus, Sarcoptes $\mathbf{2 8 7}$ parazumpti, Harpyrhynchoides 283 pari, Neharpyrhynchus 290 pectinifer, Harpyrhynchoides 283 pectinifer, Harpirhynchus $\mathbf{2 8 3}$ pectinifer, Harpyrhynchus $\mathbf{2 8 3}$ Perharpyrhynchini 295 Perharpyrhynchus 295 phalaropus, Harpyrhynchoides 283 phleocryptes, Trichorhynchiella 294 picidarum, Neharpyrhynchus 290 pilirostris, Harpirhynchus 291 pilirostris, Neharpyrhynchus 291 pilirostris, Sarcoborus 291 pilirostris, Sarcopterus 291 plumaris, Harpirhynchus 291 plumaris, Neharpyrhynchus 291 pluvialis, Harpyrhynchoides 283 porphyrio, Metharpyrhynchus 293 porphyrio, Ralliharpirhynchus 293
Pseudoharpirhynchus 276 psittaci, Harpyrhynchoides 283 psittaci, Harpyrhynchus 283 psittaculae, Harpyrhynchoides 283 psittaculae, Harpyrhynchus 283 puffinus, Harpyrhynchoides 284 pyrrhula, Harpypalpus 271 quasimodo, Harpirhynchus 287 quiscalus, Neharpyrhynchus 291 Ralliharpirhynchus 293 recurvirostra, Perharpyrhynchus 296 reductus, Harpyrhynchiella 276 reductus, Harpyrhynchus 276 regulus, Harpypalpoides 270 rosellacinus, Harpyrhynchoides 284 rosellacinus, Harpyrhynchus $\mathbf{2 8 4}$ rubeculinus, Harpyrhynchoides 284 rubeculinus, Harpyrhynchus 284 Sarcoborus $\mathbf{2 8 0}$

Sarcopterinus $\mathbf{2 8 7}$

schoenobaenus, Neharpyrhynchus 291 serini, Harpypalpus 271 setophaga, Harpyrhynchoides 284 sialia, Crassacarus 274 sitta, Harpypalpoides 270 spermestes, Harpypalpus 272 spinus, Neharpyrhynchus 292 spizella, Harpyrhynchoides 284 spizella, Neharpyrhynchus 292 squamifer, Neharpyrhynchus 292 squamiferus, Neharpyrhynchus 292 squamiferus, Harpirhynchus 292 squamosus, Harpyrhynchoides 285 squamosus, Harpyrhinchus 285 sturnus, Harpypalpus 272 sylvia, Harpypalpoides 270 tabescentium, Harpyrhynchus (Berthold, 1845) - unknown taeniopygia, Harpypalpus 272 tangara, Neharpyrhynchus 292 tiarae, Harpypalpus 272 
tinae, Crassacarus 274

tracheatus, Harpyrhynchoides 285

tracheatus, Harpirhynchus 285

tracheatus, Harpyrhynchus 285

Trichorhynchiella 293

trochilinus, Harpyrhynchus 292

trochilinus, Neharpyrhynchus 292

turdus, Neharpyrhynchus 293

tyto, Harpyrhynchoides 285 tyto, Harpyrhynchus 285

vercammeni, Harpyrhynchoides 285

vercammeni, Harpyrhynchus $\mathbf{2 8 5}$

vireo, Neharpyrhynchus 293

vulgaris, Harpyrhynchoides 285

xanthocephalus, Harpyrhynchoides 286

zenaida, Harpyrhynchoides 286

zumpti, Harpyrhynchoides 286

zumpti, Harpyrhynchus $\mathbf{2 8 6}$

\section{Distribution of Harpirhynchinae and Harpypalpinae}

\section{EUROPE}

Austria (Černy \& Sixl, 1971; Bochkov \& Literák, 2006) - Harpirhynchus dusbabeki, Harpyrhynchoides rubeculinus

Azerbaidjan (Fain, Bochkov \& Mironov, 1999) - Harpirhynchus galeridae

Belgium (Fain, 1972; Fain, Bochkov \& Mironov, 1999) - Anharpyrhynchus monstrosus, Harpypalpus holopus, Harpyrhynchoides asio, H. columbae, H. herodius, H. tyto

Bulgaria (Jeleva, 1970) - Harpirhynchus nidulans

Croatia (Bochkov \& Literák, 2006) - Harpirhynchus dusbabeki

Czech Rep. (Literák et al., 2005; Bochkov \& Literák, 2006, 2008; Martinu, Dusbábek \& Literák, 2008) - Harpyrhynchoides rubeculinus, Harpirhynchus dusbabeki, H. nidulans, Neharpyrhynchus bochkovi, N. hippolae, N. pari, N. pilirostris, N. plumaris, N. schoenobaenus, N. spinus

France (contin.)(Berlese \& Trouessart, 1889; Fain, Bochkov \& Mironov, 1999; Bochkov \& Literák, 2006) - Harpyrhynchoides alaudinus, H. capellae, H. rubeculinus, Harpirhynchus dusbabeki, Neharpyrhynchus pilirostris

Germany (Fritsch, 1954; Bochkov, OConnor \& Klompen, 2015) - Anharpyrhynchus monstrosus, Harpyrhinchiella reductus, Harpyrhinchoides capellae, H. cylindripalpis, H. parazumpti, H. tracheatus, Harpirynchus nidulans, Neharpyrhynchus pilirostris, N. plumaris

Great Britain (Turk, 1953) - Harpirhynchus nidulans

Holland (the Netherlands)(Lombert \& Moss, 1983; Bochkov \& Klompen, 2014a) - Harpypalpoides hirundinoides, $H$. johnstoni, H. lukoschusi, H. regulus, H. sylvia, Harpypalpus longipes, $H$. pyrrhula, $H$. sturnus, Harpirhynchiella reductus, Harpyrinchoides herodius

Italy (contin.)(Canestrini, 1886; Berlese \& Trouessart, 1889) - Harpirhynchus nidulans, Harpypalpus holopus

Luxemburg (Fain, 1994) - Harpyrhynchoides tracheatus

Moldavia (Fain, Bochkov \& Mironov, 1999) - Harpyrhynchoides parazumpti 
Poland (Skoracki, Bochkov \& Sikora, 2004; Bochkov \& Literák, 2006) - Harpypalpoides lesickii, H. lukoschusi, Harpypalpus longipes, Harpirhynchus dusbabeki

Russia (all, continent.)(Bochkov, 2000a, 2000b, 2015) - Harpyrhynchiella apus, Harpyrhynchoides alaudinus, Neharpyrhynchus hippolae, N. plumaris

Slovakia (Literák et al., 2005; Bochkov \& Literák, 2006) - Harpirhynchus dusbabeki, H. nidulans

Spain (cont.)(Fain, Bochkov \& Mironov, 1999) - Harpyrhynchoides alectoris

Switzerland (Fain, 1995) - Harpirhynchus nidulans

Turkey (Aksin \& Erdoğmuş, 2005) - Harpirhynchus sp.

\section{AFRICA}

Congo DR (Fain, 1972; Fain, Bochkov \& Mironov, 1999) - Harpyrhinchiella [Cypsharpirhynchus] cypsiuri, Harpypalpoides hirundinis, Harpypalpus spermestes, Harpyrhynchoides vercammeni, Ralliharpirhynchus limnocorax,

Mozambique (Bochkov \& Klompen, 2014) - Perharpyrhynchus caprimulgus

Namibia (Fain, 1995; Fain, Bochkov \& Mironov, 1999) - Metharpyrhynchus namibiensis, Harpypalpoides namibiensis, Harpyrhinchoides cristagalli

Rwanda (Fain, 1994, 1995; Bochkov, 2015) - Harpyrhynchoides pectinifer, Metharpyrhynchus jynx, M. mossi, Neharpyrhynchus oenanthe

South Africa (Lawrence, 1959; Fain, 1972; Bochkov, 2015) - Harpyrhynchoides clamator, H. cristagalli, H. pectinifer, H. pilirostris, H. zumpti, Harpirhynchus agapornis

Togo (Fain, 1972) - Cypsharpirhynchus cypsiuri

Zimbabwe (Fain, 1994) - Harpyrhynchoides pectinifer

Central Africa (Fain, Bochkov \& Mironov, 1999) - Harpypalpus tiarae

Africa (Lawrence, 1959b) - Harpyrhynchoides numidae

ASIA

India (Fain, 1972) - Neharpyrhynchus squamiferus

Kazakhstan (Bochkov \& Klompen, 2014a) - Harpypalpoides johnstoni, H. lesickii

Kirghizstan (Fain, Bochkov \& Mironov, 1999) - Harpyrhynchoides kirgizorum

Malaysia (Berlese \& Trouessart, 1889) - Harpypalpus sp. pr. holopus

SE Asia (Bochkov \& Klompen, 2015) - Harpyrhynchoides artamus

\section{NORTH AMERICA}

Canada (Bochkov \& Galloway, 2001, 2004; 2013; Bochkov \& Klompen, 2014; Bochkov, OConnor \& Klompen, 2015) - Fainharpyrhynchus contopus, Harpyrhynchoides actitis, H. botaurus, H. gallowayi, H. heatherae, H. modestus, H. phalaropus, H. setophaga, H. tracheatus, H. vulgaris, H. zumpti, Neharpyrhynchus loxia, N. pilirostris

Mexico (Bochkov, OConnor \& Klompen, 2015) - Junco phaeonotus

USA (Banks, 1905; Chaddock, 1941; Boyd, 1968; Moss, Oliver \& Nelson, 1968; Moss, Mitchell \& Johnston, 1970; Bochkov \& Mertins, 2010; Spelding et al., 2010; Bochkov 
\& OConnor, 2013; Bochkov \& Klompen, 2014a, b; Bochkov, OConnor \& Klompen, 2015) - Anharpyrhynchus elizae, Crassacarus alexfaini, C. fritschi, C. melanerpes, C. sialia, C. tinae, Fainharpirhynchus contopus, Harpypalpoides sitta, Harpyrhynchoides accipiter, H. aegolius, H. athene, H. bubulcus, H. butorides, H. calidris, H. charadrius, H. coccyzus, H. herodius, H. ixobrychus, H. megascops, H. parazumpti, H. pectinifer, $H$. pluvialis, puffinus, $H$. rubeculinus, $H$. spizella, $H$. tracheatus, $H$. xanthocephalus, H. zenaida, Harpirhynchus quasimodo, H. longipilis, Neharpyrhynchus agelaius, $N$. baile, N. bombycilla, N. campylorhynchus, N. icterus, N. novoplumaris, ? N. pari, N. quiscalus, N. picidarum, N. pilirostris, N. spizella, N. turdus, N. vireo, Perharpyrhynchus charadrius

North America (Bochkov \& Klompen, 2014b) - Trichorhynchiella myiarchus

\section{SOUTH and CENTRAL AMERICA}

Argentina (Bochkov \& OConnor, 2014) - Trichorhynchiella phleocryptes

Brazil (Bochkov \& Literák, 2007, 2011; Bochkov, OConnor \& Klompen, 2015) - Neharpyrhynchus baile, N. mironovi, N. rubeculinus, N. tangara, N. trochilinus

Costa Rica (Bochkov \& Literák, 2011) - Neharpyrhynchus chlorospingus, N. trochilinus

Paraguay (Literák, Bochkov \& Franco, 2014) - Neharpyrhynchus tangara, N. trochilinus, N. aff. spinus

Peru (Literák et al., 2012) - Neharpyrhynchus tangara, N. trochilinus

Suriname (Bochkov, OConnor \& Klompen, 2015) - Harpyrhynchoides crotophaga

Trinidad and Tobago (Bochkov \& Klompen, 2014) - Fainharpirhynchus legatus

South America (Fain, 1972) - Harpyrhynchoides amazonae, H. metropeliae, Neharpirhynchus trochilinus, Perharpyrhynchus jacana

\section{OCEANIA}

Australia (continent)(Fain, 1972; Moss, 1979a; Filippich \& Domrow, 1985; Domrow, 1991; Bochkov \& Klompen, 2014a, 2015) - Anharpyrhynchus apodus, A. lukoschusi, A. monstrosus, Harpypalpus lonchura, $H$. taeniopygia, Harpyrhynchoides kakatoe, $H$. rosellacinus, Neharpyrhynchus domrowi, Perharpyrhynchus elseyornis

Tasmania - Harpyrhynchoides rosellacinus

Papua New Guinea (Bochkov \& Klompen, 2015) - Neharpyrhynchus domrowi

Unknown distribution (Zoo birds): Harpypalpus dubinini, H. serini, Harpyrhinchoides amazonae, $H$. anatum, $H$. capitatus, $H$. coturnix, $H$. coxatus, $H$. diuca, H. lawrencei, H. leptopilus, $H$. metropeliae, $H$. modestus, $H$. oenae oenae, $H$. oenae, $H$. lamorali, $H$. psittaci, H. psittaculae, H. squamosus, H. trochilinus, Ralliharpirhynchus porphyrio, Trichorhinchiella paddae, Metharpyrhynchus macrophallus, Perharpyrhynchus recurvirostra, P. jacana 


\section{Hosts of Harpirhynchinae and Harpypalpinae}

Aves

Accipitriformes

Fam. Accipitridae

Accipiter striatus - Harpyrhynchoides accipiter

Buteo buteo - Harpyrhynchoides tracheatus

B. jamaicensis - Harpyrhynchoides tracheatus

B. lineatus - Harpyrhynchoides tracheatus

\section{Anseriformes}

\section{Fam. Anatidae}

Anas querquedula - Harpyrhynchoides anatum

Aythya ferina - Neharpyrhynchus plumaris (doubtful - see Bochkov \& Literák, 2006)

\section{Apodiformes}

Fam. Apodidae

Apus affinis - Harpyrhynchiella [Cypsharpirhynchus] cypsiuri

A. apus - Harpyrhynchiella reductus

Cypsiurus parvus - Harpyrhynchiella [Cypsharpirhynchus] cypsiuri

Fam. Trochilidae

Amazilia lactea - Neharpyrhynchus trochilinus

A. chionogaster - Neharpyrhynchus trochilinus

Chlorostilbon lucidus - Neharpyrhynchus trochilinus

Chrysolampis moschitus - Neharpyrhynchus trochilinus

Eugenes fulgens - Neharpyrhynchus trochilinus

Panterpe insignis - Neharpyrhynchus trochilinus

\section{Caprimulgiformes}

Fam. Caprimulgidae

Caprimulgus fossii - Perharpyrhynchus caprimulgus

\section{Charadriiformes}

Fam. Charadriidae

Charadrius vociferus - Harpyrhynchoides charadrius, Perharpyrhynchus charadrius

Elseyornis melanops - Perharpyrhynchus elseyornis

Pluvialis dominica - Harpyrhynchoides pluvialis

Fam. Jacanidae

Jacana spinosa intermedia - Perharpyrhynchus jacana

Fam. Scolopacidae

Actitis macularius - Harpyrhynchoides actitis

Calidris minutilla - Harpyrhynchoides calidris 
Gallinago gallinago - Harpyrhynchoides capellae Lymnocryptes minimus - Harpyrhynchoides capellae

Phalaropus lobatus - Harpyrhynchoides phalaropus

Recurvirostra avocetta - Perharpyrhynchus recurvirostra

\section{Ciconiiformes}

\section{Fam. Ciconiidae}

Coliiformes

Leptoptilus crumeniferus - Harpyrhynchoides leptoptilus

Fam. Coliidae

Colius colius - Harpyrhynchoides cristagalli

C. striatus - Harpyrhynchoides cristagalli

\section{Columbiformes}

\section{Fam. Columbidae}

Columba livia domestica - Harpyrhynchoides columbae

C. livia - Harpyrhynchoides gallowayi, H. modestus

C. palumbus - Harpirhynchus nidulans (= palumbinus)

Columbigallina talpacoti - Harpyrhynchoides capitatus, H. coxatus

Columbina cruziana - Harpyrhynchoides coxatus

Metropelia caeciliae - Harpyrhynchoides metropeliae, H. modestus

Oena capensis - Harpyrhynchoides oenae

Scardafella squamata - Harpyrhynchoides coxatus

Tympanistria tympanistria - Harpyrhynchoides oenae [lamorali]

Zenaida macroura - Harpyrhynchoides zenaida

\section{Cuculiformes}

Fam. Cuculidae

Clamator jacobinus - Harpyrhynchoides clamator

Coccyzus americanus - Harpyrhynchoides coccyzus

C. erythrophthalmus - Harpyrhynchoides coccyzus

Galliformes

Crotophaga cardinalis - Harpyrhynchoides crotophaga

Fam. Phasianidae

Alectoris graeca - Harpyrhynchoides alectoris

A. rufa - Harpyrhynchoides alectoris

Coturnix delegorguei - Harpyrhynchoides coturnix

Fam. Numididae

\section{Charadriiformes}

Numida meleagris - Harpyrhynchoides numidae

\section{Fam. Charadriidae}

Charadrius vociferus - Perharpyrhynchus charadrius

Elseyornis melanops - Perharpyrhynchus elseyornis

Fam. Scolopacidae

Phalaropus lobatus - Harpyrhynchoides phalaropus 


\section{Passeriformes}

Fam. Acrocephalidae

Acrocephalus schoenobaenus - Neharpyrhynchus schoenobaenus

Fam. Aegithalidae

Aegithalos caudatus - Neharpyrhynchus pilirostris (?)

Fam. Alaudidae

Alauda arvensis - Harpyrhynchoides alaudinus, Harpirhynchus nidulans

Galerida cristata - Harpirhynchus galeridae, H. nidulans

Eremopterix leucotis - Harpyrhynchoides zumpti

Fam. Artamidae

Artamus fuscus - Harpyrhynchoides artamus

\section{Fam. Bombycillidae}

Bombycilla cedrorum - Crassacarus fritschi, Neharpyrhynchus bombycilla

Fam. Cardinalidae

Cardinalis cardinalis - Crassacarus alexfaini, Neharpyrhynchus novoplumaris

Passerina ciris - Crassacarus cylindripalpus

Pheucticus ludovicianus - Harpyrhynchoides rubeculinus

Fam. Certhiidae

Certhia familiaris - Neharpyrhynchus novoplumaris

Fam. Cisticolidae

Prinia subflava graueri - Metharpyrhynchus mossi

Fam. Corvidae

Corvus brachyrhinchos - Harpyrhynchoides parazumpti

C. corone - Harpirhynchus nidulans

C. frugilegus - Harpyrhynchoides parazumpti

C. monedula - Anharpyrhynchus monstrosus, Harpyrhynchoides parazumpti

Cyanocitta cristata - Anharpyrhynchus elizae

C. stelleri-Anharpyrhynchus elizae

Garrulus glandarius - Anharpyrhynchus monstrosus

Nucifraga caryocatactes - Harpypalpus longipes

Xanthoura yncas luxuosa - Anharpyrhynchus monstrosus

Fam. Emberizidae

Emberiza calandra - Harpyrhynchoides kirgizorum

E. cioides - Harpirhynchus sp.

E. impetuani - Harpypalpoides namibiensis

Junco hyemalis - Harpyrhynchoides heatherae, H. zumpti, Neharpyrhyncus novoplumaris

J. phaeonotus - Neharpyrhyncus novoplumaris

\section{Fam. Estrildidae}

Lonchura castaneothorax - Harpypalpus lonchura

Padda oryzivora - Metharpyrhynchus macrophallus, Trichorhynchiella paddae Taeniopygia guttata - Harpypalpus taeniopygia 


\section{Fam. Fringillidae}

Amphispiza bilineata - Neharpyrhynchus novoplumaris

Chloris chloris - Harpirhynchus nidulans, Neharpyrhynchus plumaris

Carduelis cannabina - Harpypalpoides lesickii, Harpypalpus longipes, Neharpyrhynchus plumaris

C. tristis - Crassacarus tinae

Coccothraustes coccothraustes - Harpirhynchus nidulans, Harpypalpoides lesickii

C. vespertina - Harpyrhynchoides brevis

Fringilla coelebs - Harpypalpoides johnstoni, Harpirhynchus cylindripalpus, H. nidulans, Neharpyrhynchus plumaris

F. montifringilla - Harpypalpoides johnstoni

Loxia curvirostra - Harpyrhynchoides brevis, Neharpyrhynchus loxia

Loxia sp. - "Harpirhynchus longipilus"

Pyrrhula pyrrhula - Harpypalpus pyrrhula

Serinus mozambicus - Harpypalpus serini

Spinus spinus - Harpypalpus longipes, Neharpyrhynchus spinus

\section{Fam. Furnariidae}

Phleocryptes melanops - Trichorhynchiella phleocryptes

\section{Fam. Hirundinidae}

Hirundo rustica - Harpypalpoides hirundinoides

Psalidoprocne sp. - Harpypalpoides hirundinis

\section{Fam. Icteridae}

Agelaius phoeniceus - Neharpyrhynchus agelaius

Icterus galbula - Neharpyrhynchus icterus

Molothrus ater - Harpirhynchus quasimodo

Quiscalus quiscula - Neharpyrhynchus quiscula

Xanthocephalus xanthocephalus - Harpypalpoides xanthocephalus

\section{Fam. Locustellidae}

Locustella lanceolata - Harpirhynchus nidulans

\section{Fam. Meliphagidae}

Acanthorhynchus tenuirostris - Neharpyrhynchus domrowi

Certhionyx pectoralis - Anharpyrhynchus lukoschusi

Gliciphila indistincta - Anharpyrhynchus monstrosus

Lichmera indistincta - Anharpyrhynchus apodus

Manorina flavigula - Anharpyrhynchus lukoschusi

Meliornis novaehollandiae - Anharpyrhynchus monstrosus

Myzantha flavigula - Anharpyrhynchus monstrosus

Myzomela pectoralis - Anharpyrhynchus monstrosus

M. rosenbergii - Neharpyrhynchus domrowi

Phylidonyris novaehollandiae - Anharpyrhynchus apodus 
Ptiloprora perstriata - Neharpyrhynchus domrowi

Fam. Mimidae

Dumetella carolinensis - Neharpyrhynchus baile

Fam. Muscicapidae

Erithacus rubecula - Harpyrhynchoides rubeculinus

Luscinia svecica - Harpypalpus holopus

Muscicapa striata - Neharpyrhynchus plumaris

Oenanthe oenanthe - Neharpyrhynchus oenanthe

\section{Fam. Panuridae}

Panurus biarmicus - Harpirhynchus nidulans

\section{Fam. Paridae}

Baeolophus bicolor - Neharpyrhynchus pari [novoplumaris?]

Cyanistes caeruleus - Harpypalpus longipes, Neharpyrhynchus pari

P. major - Harpypalpus longipes, Neharpyrhynchus pari

Periparus ater - Neharpyrhynchus pari, [N. plumaris]

Poecile atricapillus - Harpypalpus longipes

P. montanus - Neharpyrhynchus pari

P. palustris - Neharpyrhynchus pari, [N. plumaris]

\section{Fam. Parulidae}

Setophaga petechia - Crassacarus alexfaini

S. pitiayumi - Neharpyrhynchus aff. spinus

S. ruticilla - Harpyrhynchoides setophaga, H. vulgaris

\section{Fam. Passerellidae}

Amphispiza bilineata - Neharpyrhynchus novoplumaris

Chlorospingus pileatus - Neharpyrhynchus chlorospingus

Kieneria [Pipilo, Melozone] fusca - Neharpyrhynchus novoplumaris

Passerina ciris - Crassacarus alexfaini

Spizella passerina - Neharpyrhynchus novoplumaris, N. spizella

\section{Fam. Passeridae}

Passer domesticus - Harpypalpus holopus, Neharpyrhynchus piliristris

Fam. Ploceidae

Ploceus [Sitagra] intermedius - Harpypalpus dubinini

Ploceus [Spermestes] cucullatus - Harpypalpus spermestes

Fam. Regulidae

Regulus regulus - Harpypalpoides regulus

\section{Fam. Sittidae}

Sitta pygmaea - Harpypalpoides sitta

\section{Fam. Sturnidae}

Temenuchus pagodarum - Neharpyrhynchus squamiferus

\section{Fam. Sylviidae}

Hippolais icterina - Neharpyrhynchus hippolae

Sylvia atricapilla - Harpypalpoides sylvia 
Fam. Thraupidae

Dacnis cayana - Neharpyrhynchus mironovi

Diuca diuca - Harpyrhynchoides diuca, H. zumpti

Paroaria capitata - Neharpyrhynchus tangara

P. coronata - Neharpyrhynchus tangara

Tangara cayana - Neharpyrhynchus tangara

Thraupis episcopus - Neharpyrhynchus tangara

Tiaris canora - Harpypalpus tiarae

Fam. Timaliidae

Panurus biarmicus - Harpirhynchus dusbabeki

Fam. Troglodytidae

Campylorhynchus brunneicapillus - Neharpyrhynchus campylochirus, $N$. novoplumaris

Troglodytes troglodytes - Harpypalpus longipes

Fam. Turdidae

Catharus ustulatus - Harpyrhynchoides rubeculinus

Sialia currucoides - Crassacarus sialia

Turdus leucomelas - Neharpyrhynchus baile

T. merula - Harpypalpoides lukoschusi, Neharpyrhynchus bochkovi

T. migratorius - Neharpyrhynchus turdus

T. pilaris - Harpypalpoides lukoschusi

T. amaurochalinus - Harpypalpoides rubeculinus

Fam. Tyrannidae

Contopus cooperi - Fainharpirhynchus contopus

C. virens - Fainharpirhynchus contopus

Legatus leucophaius - Fainharpirhynchus legatus

Myiarchus crinitus - Fainharpirhynchus mossi, Trichorhynchiella myiarchus

Fam. Vireonidae

Vireo olivaceus - Neharpyrhynchus vireo

\section{Pelecaniformes}

Fam. Ardeidae

Ardea cinerea - Harpyrhynchoides herodius

A. herodius - Harpyrhynchoides herodius

Botaurus lentiginosus - Harpyrhynchoides botaurus

Bubulcus ibex - Harpyrhynchoides bubulcus

Butorides virescens - Harpyrhynchoides butorides

Ixobrychus exilis - Harpyrhynchoides ixobrychus

\section{Piciformes}

Fam. Picidae

Campethera abingoni - Harpyrhynchoides pectinifer

C. cailliauti - Harpyrhynchoides pectinifer 
Colaptes auratus - Anharpyrhynchus elizae, Harpyrhynchoides pectinifer, Neharpyrhynchus picidarum

Jynx ruficollis - Metharpyrhynchus jynx

Melanerpes formicivorus - Crassacarus melanerpes, Neharpyrhynchus picidarum

M. uropygydialis - Neharpyrhynchus picidarum

Picoides pubescens - Neharpyrhynchus picidarum

\section{Procellariiformes}

Fam. Procellariidae

\section{Psittaciformes}

Puffinus gravis - Harpyrhynchoides gravis

\section{Fam. Cacatuidae}

Kakatoe galerita - Harpyrhynchoides kakatoe

\section{Fam. Psittacidae}

Amazona aestiva - Harpyrhynchoides amazonae

Nandays nanday - Harpyrhynchoides lawrencei

Poicephalus senegalus - Harpyrhynchoides psittaci

Psittacus erithacus - Harpyrhynchoides psittaci

\section{Fam. Psittaculidae}

Agapornis roseicollis - Harpyrhynchoides agapornis

A. pullarius - Harpyrhynchoides agapornis

A. nigrigenis - Harpyrhynchoides agapornis

Glossopsitta concinna - Harpyrhynchoides rosellacinus

Lathamus discolor - Harpyrhynchoides rosellacinus

Platycercus eximius - Harpyrhynchoides rosellacinus

Psittacula alexandri - Harpyrhynchoides psittaculae

P. cyanocephala - Harpyrhynchoides psittaculae

Trichoglossus chlorolepidotus - Harpyrhynchoides rosellacinus

T. moluccanus - Harpyrhynchoides rosellacinus

\section{Ralliformes}

Fam. Rallidae

Limnocorax flaviceps - Ralliharpirhynchus limnocorax

Porphyrio porphyrio - Ralliharpirhynchus porphyrio

\section{Strigiformes}

Fam. Tytonidae

Centropus toulou grillii - Harpyrhynchoides vercammeni

Tyto alba - Harpyrhynchoides tyto

\section{Fam. Strigidae}

Aegolius acadicus - Harpyrhynchoides aegolius

Asio otus otus - Harpyrhynchoides asio

Athene canicularia - Harpyrhynchoides athene

Megascops asio - Harpyrhynchoides megascops 


\section{Bibliography of Harpirhynchinae and Harpypalpinae}

Aksin N., Z. Erdoğmuş. 2005. Pseudolichus solutocurtus Dubinin, 1956 (Acarina, Pterolichoidea) and Harpyrhynchus sp. (Acarina, Harpyrhinchidae Dubinin, 1957) species recorded for the first time on wild partridge in Turkey. - Turk J. Vet. Anim. Sci., 29: 1149-1156.

Banks N. 1905a. A Treatise of the Acarina, or mites. - Proc. Ent. Soc. Washington, 28: 1-109. Banks N. 1905b. Description of some new mites. - Proc. Ent. Soc. Washington, 8: 133-142. Banks N. 1907. Catalogue of the Acarina, or Mites, of the United States. - Proc. U.S. National Museum, 32 (1553): 595-625.

Berlese A. 1884. Acari, Myriapoda et Scorpiones hucusque in Italia reperta. - 73(5-6).

Berlese A. 1913. Acarotheca italica. Fasciculi Ius et Iius. Praemisso Acarorum systemate

Berlese A., E. Trouessart. 1889. Diagnoses d'Acariens nouveaux ou peu connus. - Bull. Bibl. Sci. de l'Ouest, 2 (2e partie)(9): 1-23 (copy).

Berthold 1845. Lehrb. Zool., p. 349

Bochkov A. V. 2000a. A first record of the mite genus Neharpyrhynchus (Acari: Harpirhynchidae) in Russia. - Parazitologiya, 34(6): 534-538 (in Russian, summ. Engl.).

Bochkov A.V. 2000b. A new Harpirhynchid mite Harpyrhynchoides alaudinus sp.n. (Acari: Harpirhynchidae) from Alauda arvensis (Passeriformes: Alaudidae) from Russia. - Acarina, 8(2): 91-93.

Bochkov A.V. 2009a. A review of mites of the parvorder Eleutherengona (Acariformes: Prostigmata) - permanent parasites of mammals. - Acarina Supplement No1: 3-149.

Bochkov A.V. 2009b. Origin and evolution of parasitism in mites of the infraorder Eleutherengona (Acari: Prostigmata). Report II. Superfamily Cheyletoidea. - Parazitologiya (St. Petersburg), 43(2): 97-117.

Bochkov A.V. 2014a. Two new species of the subfamily Harpirhynchinae (Acari: Harpirhynchidae) from African birds. - Annales Zoologici, 64(3): 479-483.

Bochkov A.V. 2014b. Mites of the genus Harpyrhynchiella Fain, 1972 (Acariformes: Harpirhynchidae), an example of an unusual mating strategy. - Int. J. of Acarology, DOI: 10.1080/01647954.2014.988644

Bochkov A. V., T.D. Galloway. 2001. Parasitic cheyletoid mites (Acari: Cheyletoidea) associated with passeriform birds (Aves: Passeriformes) in Canada. - Canadian Journal of Zoology, 79: 2014-2028.

Bochkov A. V., T.D. Galloway. 2004. New species and records of Cheyletoid mites (Acari: Cheyletoidea) from birds in Canada. - J. of Kansas Entom. Society, 77(1): 26-44.

Bochkov A. V., T.D. Galloway. 2013. New records and new species of mites of the subfamily Harpirhynchinae (Acariformes: Harpirhynchidae) infesting birds in Manitoba, Canada. - Acta Parasitologica, 58(4): 405-419. .

Bochkov A. V., P.B. Klimov, M. Skoracki. 2017. Morphological phylogenetic conflict in the parasitic mite family Harpirhynchidae (Acariformes: Cheyletoidea) correlates with host associations. - Zool. Anzeiger, 271: 33-48. 
Bochkov A. V., H. Klompen. 2014a. A review of the subfamily Harpypalpinae Fain, 1972 (Acariformes: Harpirhynchidae) — parasites of passerine birds. - Zootaxa, 3857(4): 451- 477.

Bochkov A. V., H. Klompen. 2014b. New Harpirhynchinae Dubinin (Acariformes: Harpirhynchidae) - intracutaneous and feather - base parasites of birds. - Zootaxa, 3860(4): 301-324.

Bochkov A. V., H. Klompen. 2014c. A review of the zumpti species group of the genus Harpyrhynchoides (Acariformes: Harpirhynchidae) - ectoparasites of passerines. - Zootaxa, 3884(5): 401-418.

Bochkov A.V., I. Literák. 2006. A review of the European Harpirhynchidae (Acari: Prostigmata) with the description of a new species. - Acta parasitologica, 51(2): 136-142.

Bochkov A.V., I. Literák. 2007. Neharpyrhynchus baile n. sp. (Prostigmata: Harpirhynchidae) parasitizing Turdus leucomelas Viellot (Aves: Turdidae) from Brazil. - Int. J. of Acarology, 33(1): 35-39.

Bochkov A.V., I. Literák. 2008. Description of the life stages of Harpyrhynchoides rubeculinus (Černy and Sixl, 1971)(Prostigmata: Harpirhynchidae). - Internat. J. of Acarology, 34(3): 227-236.

Bochkov A.V., I. Literák. 2011. Mites of the genus Neharpyrhynchus Fain (Acariformes, Harpirhynchidae) from Neotropical birds. - ZooKeys, 89: 15-31.

Bochkov A. V., J.W. Mertins. 2010. Harpyrhinchus quasimodo n.sp. (Acariformes: Harpirhynchidae), a new species parasitizing Molothrus ater (Passeriformes: Icteridae) in Florida, USA. - Internat. J. Acarology, 36(1): 83-87.

Bochkov A. V., S.V. Mironov. 1999. Phylogeny and host-parasite relationships of the mite family Harpirhynchidae (Acari, Prostigmata). - Acarina, 7 (2): 69-87.

Bochkov A. V., B.M. OConnor. 2013. Mites of the Subfamily Harpirhynchinae (Acariformes: Harpirhynchidae) from North American Birds. - J. of Medical Entomology, 50(3): 501-509.

Bochkov A. V., B.M. OConnor. 2014. Mites of the genus Trichorhynchiella (Acariformes: Harpirhynchidae): a new exemple of ovoviviparity in acariform mites. - J. of Medical Entomology, 51(2): 323-330.

Bochkov A. V., B.M. OConnor, H. Klompen. 2015. A review of the mite subfamily Harpirhynchinae (Acariformes: Harpirhynchidae) - parasites of New World birds (Aves: Neognathae). - Zootaxa, 4023: 1-130.

Boyd E. M. 1968. Two new species of Harpirhynchus from Herons in North America (Acarina, Trombidiformes Harpyrhynchidae). - Proceedings of the Helmintological Society of Washington, 35: 18-24.

Chaddock T.T. 1941. Rare mites found on Wisconsin blackbird. - Wisconsin Conservation, 6: $33-34$.

Černy V. \& W. Sixl. 1971. Harpirhynchus rubeculinus spec. nov. eine neue Milbenart aus der Steiermark (Arachnida, Acari, Trombidiformes). - Mitteilungen Naturwissenschaften Ver. Steiermark, 100: 388-390.

Domrow R. 1991. Acari Prostigmata (excluding Trombiculidae) parasitic on Australian vertebrates: an annotated checklist, keys and bibliography. - Invertebr. Taxon., 4: 1283-1376. 
Dubinin V.B. 1957. Acari: Cheyletoidea and Demodicoidea. - Parasitologicheskiy Sbornik Zoologicheskogo Instituta Akademii Nauk SSSR, 17: 71-136. (in Russian).

Ewing H.E. 1911. New predaceous and parasitic mites. - Psyche, 18: 37-43.

Fain A. 1972. Notes sur les Acariens des familles Cheyletidae et Harpyrhynchidae producteurs de gale chez les Oiseaux ou les Mammifères. - Acta Zool. Path. Antwerp., 56: 37-60.

Fain A. 1976. Notes sur les Harpyrhynchidae. Description de quatre espèces nouvelles. (Acarina: Prostigmata). - Acarologia, 18(1): 124-132.

Fain A. 1994. New observations on the Harpyrhynchidae Dubinin, 1957 (Acari: Prostigmata). I. The subgenus Harpirhynchus (Harpyrhynchoides) Fain, 1972. - Bull. Inst. r. Sci. Nat. de Belgique, Entomologie, 64: 109-144.

Fain A. 1995. New observations on the Harpyrhynchidae Dubinin, 1957 (Acari: Prostigmata). II. On some new or little-known taxa in the Harpirhynchinae. - Bull. Inst. r. Sci. Nat. de Belgique, Entomologie, 65: 73-100.

Fain A., A.V. Bochkov, S.V. Mironov. 1999. A contribution to the systematics of the mite family Harpirhynchidae (Acari: Cheyletoidea). - Acarologia, 40(1): 37-54.

Filippich L. J., R. Domrow. 1985. Harpirhynchid mites in a scaly-breasted lorikeet Trichoglossus chlorolepidotus. - Journal of Wildlife Diseases, 21: 451-453.

Fritsch W. 1954. Die Milbengattung Harpyrhynchus Mégnin, 1878. - Zool. Anz., 152: 177-198. Heim F. 1892. Harpirhynchus megnini n. sp. - Ann. Soc. ent. France, Bull. Séan., 61: 132-133. Henry P.-Y., B. Poulin, F. Rousset, F. Renaud, F. Thomas. 2004. Infestation by the mite Harpirhynchus nidulans in the bearded tit Panurus biarmicus. - Bird Study, 51: 34-40.

Hering 1845. Eine neue Krätzmilbe. - Thierärtzl. Ztg., 11(29): 115.

Jeleva M. 1970. On the skin reaction of the host to Harpyrhynchus nidulans (Acari, Cheyletoidea: Harpyrhynchidae). - Ann. Univ. Sofia, Fac. Biol., Geol., Geogr., 63: 63-67.

Koch C.L. 1836. DCMA 9 - Deutschlands Crustaceen, Myriapoden und Arachniden 9: 1-24.

Lawrence R. F. 1959a. New mites parasites of African birds (Myobiidae, Cheyletidae). - Parasitology, 49(3-4): 416-438

Lawrence R. F. 1959b. Acariens (Harpyrhynchidae, Listrophoridae) nouveaux ou peu connus parasites d'oiseaux ou de mammifères. - Acarologia, 1(1): 106-118.

Lawrence R. F. 1959c. New mites parasite (Harpyrhynchus) from the Roselle Parakeet (Trombidiformes, Acari). - Proceedings of the Linnean Society of New South Wales, 84: 238-241.

Literák I., A.V. Bochkov, J. Cárdenas-Callirgos, M. Čapek. 2012. The first records of mites of the genus Neharpyrhynchus (Acariformes: Harpirhynchidae) from birds in Peru. - Neotropical Helminthology, 6: 109-114.

Literák I., A.V. Bochkov, F. Dusbábek, J. Martinu, P. Podzemny, O. Sychra. 2009. Málo známi cizopasníci - rostoči čeledi Harpirhynchidae. - Ziva, 3: 126-128. [in Czech].

Literák I., A.V. Bochkov, S. Franco. 2014. The first record of mites of ther genus Neharpyrhynchus (Acariformes: Harpirhynchidae) from birds in Paraguay. - Neotropical Helminthology, 8(1): 183-188.

Literák I., J. Chytil, A. Trnka, A. Fain, V. Tukač. 2005. Subalar cutaneous cysts with Harpirhynchus nidulans in bearded tits and hawfinches in Central Europe. - Avian Pathology, 34(1): 26-28. 
Lombert H. A. P. M., W. Wayne Moss. 1983. Description and Developmental Cycle of Harpypalpoides lukoschusi gen. et sp. nov. (Acari: Harpyrhynchidae: Harpypalpinae) from the Eurasian Blackbirds, Turdus merula (Aves: Passeriformes: Turdidae). - Proc. Acad. Nat. Sci. Phil., 135: 163-176.

McClure H. Eliott, N. Ratanaworadhan et al. 1973. Some ectoparasites of the birds of Asia. -219 pp.

Martinu J., F. Dusbábek, I. Literák. 2008. A review of mites of the genus Neharpyrhynchus Fain (Acari: Harpirhynchidae) - ectoparasites of wild birds, including description of four new species. - Int. J. of Acarology, 34(2): 197-209.

Mégnin J. P. 1877. Une tumeur cutanée recueillie sur une Alouette. - Ann. Soc. ent. France, 7: 159

Mégnin P. 1878. Mémoire sur les Cheylétides parasites. - Journ. de l’Anatomie et de la Physiologie, 14 (3): 416-441.

Morley L. C., J. E. Shillinger. 1937. Parasitic Tumors in Wild Birds. - Journal of Veterinary Medical Association, 91: 94-97.

Moss W. W. 1979a. Patterns of host-specificity and co-evolution in the Harpyrhynchidae (Acari). - Recent Advances in Acarology, 2: 379-384.

Moss W. W. 1979b. Numerical taxonomic studies of the mite family Harpyrhynchidae (Acari: Cheyletoidea): classification and of the subfamily Harpypalpinae. In manuscript.

Moss W. W., C. J. Mitchell, D.E. Johnston. 1970. New North American host and distribution records for the mite genus Dermanyssus (Acari: Mesostigmata: Dermanyssidae). - J. Med. Entomol., 7(5): 589-593.

Moss W. W., J. H. Oliver Jr., B. C. Nelson. 1968. Karyotypes and developmental stages of Harpyrhynchus novoplumaris n. sp. (Acari: Cheyletoidea: Harpyrhynchidae), a parasite of North American birds (Aves). - Journal of Parasitology, 54: 377-392.

Moss W. W., J. F. Wojcik. 1978. Numerical taxonomic studies of the mite family Harpyrhynchidae (Acari: Cheyletoidea): the higher taxa. - Ann. Ent. Soc. Am., 71: 247-252.

Murray A.1877. Economic Entomology. Aptera. - South Kensington Museum Science Handbook No 1. Chapman and Hall, London, 433 pp.

Nitzsch C.L. 1818. Acarina. - In "Ersch \& Gruber". Allgemeine Encyclopedic Wissenschaft I: 250-251.

Oudemans A.C. 1906. Révision des Chélétinés. - Mém. Soc. Zool. de France, 19: 36-144.

Oudemans A.C. 1936.: 133-135.

Oudemans A.C. 1939. Über Phthiriasis und über ihren Erzeuger, Harpyrhynchus tabescentium (Berthold 1845). - Zeitschrift f. Parasitenkunde, 11: 145-198.

Railliet A. 1893. Traité de zoologie médicale et agricole. - Part I. Asselin et Houzeau, Paris.

Skoracki M., A.V. Bochkov, B. Sikora. 2004. A new species and new records of the cheyletoid mites (Acari: Cheyletoidea) from passeriform birds in Poland. - Belgian Journal of Entomology, 6: 83-90.

Spelding M.G., J.W. Merlins, M.J.Reetz, K. L. Keacher, M.L. Avery, E.C. Greiner. 2010. 
Mite-Filled Cyst on a Brown-headed Cowbird (Molothrus ater) in Florida, USA. - J. of Wildlife Diseases, 46(4): 1279-1283.

Turk F.A. 1953. I. - A synonymic catalogue of British Acari: Part I. - Ann. Mag. Nat. Hist., $12^{\text {th }}$ Ser., 61: 1-26.0

Ward P., M. Lavoipierre, C. Rajamanickam. 1965. A lanceolate warbler Locustella lanceolata bearing large tumors caused by a harpyrhynchid mite (Harpyrhynchus). - Ibis, 107: 543-544. 


\title{
The Mite subfamily Ophioptinae
}

Mites living all their live inside the scales of snakes of the families Colubridae, Elapidae and Viperidae. Their study started by Sambon (1928), followed by Ewing (1933), Allred (1958), and Lisazo (1983), again concerning South American or Cuban snakes. Radford (1947) recorded the group from Asia and Southcott (1956), from Australia, describing these mites as a new family. Fain $(1962,1964,1965)$ described the new genus Afrophioptes from African snakes, nine new species and revised the group, still as a family. Beron (1972) recorded from Bulgaria Ophioptes for the first time in Europe (confirmed by Fain, who prospected thousands of preserved snakes from Europe, without detecting any Ophioptid). It is clear that these mites prefer the warmer climate. Revising Cheyletoidea Bochkov (2002) followed Dubinin (1957), ascribing to them subfamily status in Harpirhynchidae. Still only 18 species in two genera are known.

\section{Catalogue of Ophioptinae}

Subfamily Ophioptinae Southcott (Dubinin)

Ophioptidae Southcott, 1956: 147

Ophioptinae (subf. of Harpirhynchidae): Dubinin, 1957: 98; Bochkov, 2002

Type genus: Ophioptes Sambon, 1928

Genus Afrophioptes Fain

Afrophioptes Fain, 1962: 157

Type species: Afrophioptes whartoni Fain, 1962

Afrophioptes rhodesiensis Fain

Afrophioptes rhodesiensis Fain, 1962: 158

Type locality: Abercorn (now Mbala)

Distribution: "Rhodesia" (Zambia)

Host: "Cerastes" t. tritaeniatus (Serpentes: Viperidae)

\author{
Afrophioptes whartoni Fain \\ Afrophioptes whartoni Fain, 1962: 158; 1964: 48; 1965: 112 \\ Type locality: Musosa, Katanga \\ Distribution: Angola, DR Congo \\ Host: Psammophis s. sibilans (Serpentes: Colubridae)
}

Genus Ophioptes Sambon

Ophioptes Sambon, 1928: 138

Type species: Ophioptes parkeri Sambon, 1928 
Ophioptes beshkovi Beron

Ophioptes beshkovi Beron, 1972: 689

Type locality: Frolosh Village, Kyustendil Distr.

Distribution: Bulgaria

Host: Platyceps najadum (=Coluber dahli) (Serpentes: Colubridae)

Ophioptes boaedoni Fain

Ophioptes boaedoni Fain, 1962: 156; 1964: 35

Type locality: Teturi, Kivu

Distribution: DR Congo

Host: Boaedon fuliginosus (Serpentes: Colubridae)

Ophioptes brevipilis Lizaso

Ophioptes brevipilis Lizaso, 1983: 380

Type locality: Brazil

Distribution: Brazil

Host: Chironius flavolineatus (Serpentes: Colubridae)

Ophioptes coluber Radford

Ophioptes coluber Radford, 1947: 229; Fain, 1964: 43

Type locality: Imphal, Manipur State

Distribution: India

Host: Coelognathus [Coluber] radiatus (Serpentes: Colubridae)

Ophioptes congolensis Fain

Ophioptes congolensis Fain, 1962: 155; 1964: 37

Type locality: Upemba, Mpala

Distribution: DR Congo

Host: Philothamnus hoplogaster (Serpentes: Colubridae)

Ophioptes dromicus Allred

Ophioptes dromicus Allred, 1958b: 107; Fain, 1964: 33

Type locality: Prov. Oriente, Cuba

Distribution: Cuba

Host: Dromicus andreae orientalis (Serpentes: Colubridae)

Ophioptes ekans Mendoza-Roldan et Barros-Battesti

Ophioptes ekans Mendoza-Roldan \& Barros-Battesti, 2017: 2

Type locality: Campo Limpo Paulista

Distribution: Brazil

Host: Crotalus durissus terrificus (Serpentes: Viperidae) 
Ophioptes longipilis Lizaso

Ophioptes longipilis Lizaso, 1983: 378

Type locality: Brazil

Distribution: Brazil

Host: Oxyrhopus trigeminus (Serpentes: Colubridae)

Ophioptes lycodontis Fain

Ophioptes lycodontis Fain, 1964: 38

Type locality: Java

Distribution: Indonesia (Java)

Host: Lycodon subcinctus (Serpentes: Colubridae)

Ophioptes machadoi Fain

Ophioptes machadoi Fain, 1965: 110

Type locality: Mwaoka (45 km S Dundo), Dundo

Distribution: Angola

Host: Dispholidus typus punctatus (Serpentes: Colubridae)

Ophioptes najae Fain

Ophioptes najae Fain, 1962: 156; 1964: 41; 1965: 109

Type locality: Kai Bumba, Mayumbe (DR Congo)

Distribution: Angola, DR Congo

Host: Naja melanoleuca (Serpentes: Elapidae)

Ophioptes parkeri Sambon

Ophioptes parkeri Sambon, 1928: 141; Fain, 1964: 21

Ophioptes oudemansi Sambon, 1928: 141

Type locality: Buenavista, Bolivia

Distribution: Argentina, Bolivia, Brazil, Paraguay

Hosts: Erythrolaprus aesculapii, Leimadophis poecilogyrus, L. poecilogyrus pictostriatus, Liophis anomalus, Clelia rustica (Serpentes: Colubridae)

\section{Ophioptes samboni Southcott}

Ophioptes samboni Southcott, 1956: 142; Fain, 1964: 47

Type locality: Queensland

Distribution: Australia (Queensland)

Host: Rhynchoelaps fasciolatus (Serpentes: Elapidae)

Ophioptes schoutedeni Fain

Ophioptes schoutedeni Fain, 1962: 154; 1964: 35

Type locality: Abercorn (now Mbala) 
Distribution: "Rhodésie" (Zambia)

Host: Boaedon lineatus (Serpentes: Colubridae)

Ophioptes southcotti Fain

Ophioptes southcotti Fain, 1962: 157; 1964: 47

Type locality: Thailand

Distribution: Thailand

Host: Macropisthodon rhodomelas (Serpentes: Colubridae)

Ophioptes tropicalis Ewing

Ophioptes tropicalis Ewing, 1933: 53; Allred, 1958a: 287; Fain, 1964: 47

Type locality: Zoo in Washington D.C.

Distribution: Guiana

Host: Erpetodryas carinatus (Serpentes: Colubridae)

\section{Index of Ophioptinae}

(Names in bold are accepted as valid)

Afrophioptes 314

beshkovi, Ophioptes 315

boaedoni, Ophioptes 315

coluber, Ophioptes 315

congolensis, Ophioptes 315

dromicus, Ophioptes 315

ekans, Ophioptes 315

longipilis, Ophioptes 316

lycodontis, Ophioptes 316

machadoi, Ophioptes 316

najae, Ophioptes 316
Ophioptes 314

Ophioptidae 314

Ophioptinae 314

oudemansi, Ophioptes 316

parkeri, Ophioptes 316

rhodesiensis, Afrphioptes 314

samboni, Ophioptes 316

schoutedeni, Ophioptes 316

southcotti, Ophioptes 317

tropicalis, Ophioptes 317

whartoni, Afrophioptes 314

\section{Distribution of Ophioptinae}

\section{EUROPE}

Bulgaria (Beron, 1974) - Ophioptes beshkovi

\section{AFRICA}

Angola (Fain, 1965) - Afrophioptes whartoni, Ophioptes machadoi, O. najae 
Congo (DR)(Fain, 1962) - Afrophioptes whartoni, Ophioptes boaedoni, O. congolensis, O. najae

Zambia (Fain, 1962) - Afrophioptes rhodesiensis, Ophioptes schoutedeni

ASIA

India (Radford, 1947) - Ophioptes coluber

Indonesia (Fain, 1964) - Ophioptes lycodontis

Thailand (Fain, 1962) - Ophioptes southcotti

CENTRAL and SOUTH AMERICA

Argentina (Sambon, 1928) - Ophioptes parkeri

Bolivia (Sambon, 1928, Fain, 1964) - Ophioptes parkeri

Brazil (Fain, 1964, Lizaso, 1983; Mendoza-Roldan et al., 2017) - Ophioptes brevipilis, O. ekans, O. longipilis, O. parkeri

Cuba (Allred, 1958b; Fain, 1964) - Ophioptes dromicus

Guyana (Ewing, 1933) - Ophioptes tropicalis

Paraguay (Sambon, 1928, Fain, 1964) - Ophioptes parkeri

OCEANIA

Australia (Southcott, 1956) - Ophioptes samboni

\section{Hosts of Ophioptinae}

\section{Serpentes}

\section{Fam. Colubridae}

Boaedon fuliginosus - Ophioptes boaedoni

B. lineatus - Ophioptes schoutedeni

Chironius flavolineatus - Ophioptes brevipilis

Clelia rustica - Ophioptes parkeri

Platyceps najadum (=Coluber dahli) - Ophioptes beshkovi

Coelognathus [Coluber] radiatus - Ophioptes coluber

Erpetodryas carinatus - Ophioptes tropicalis

Erythrolaprus aesculapii - Ophioptes parkeri

Leimadophis poecilogyrus - Ophioptes parkeri

L. poecilogyrus pictostriatus - Ophioptes parkeri

Liophis anomalus - Ophioptes parkeri

Lycodon subcinctus - Ophioptes lycodontis

Macropisthodon rhodomelas - Ophioptes southcotti

Oxyrhopus trigeminus - Ophioptes longipilis

Philothamnus hoplogaster - Ophioptes congolensis 
Psammophis s. sibilans - Afrophioptes whartoni

\section{Fam. Elapidae}

Naja melanoleuca - Ophioptes najae

Rhyncoelaps fasciolatus - Ophioptes samboni

Fam. Viperidae

"Cerastes" t. tritaeniatus - Afrophioptes rhodesiensis

Crotalus durissus terrificus - Ophioptes ekans

\section{Bibliography of Ophioptinae}

Allred D. 1958a. Redescription of Ophioptes tropicalis Ewing, 1933, (Acarina, Ophioptidae). - Proc. Ent. Soc. Wash. 60 (6): 287-288.

Allred D. 1958b. A new species of pit mite (Acarina: Ophioptidae) infesting snakes. - Herpetologica, 14: 107-110.

Beron P. 1974. Deuxième contribution à létude des Acariens parasites des Reptiles: Ophioptes beshkovi sp.n. (Ophioptidae) et Hemilaelaps piger (Berl.) (Ixodorhynchidae) de Bulgarie. - Comptes rendus de l'Ac. Bulg. Sci., 27(5): 689-692.

Bochkov A.V. 2002. Classification and phylogeny of mites of the superfamily Cheyletoidea (Acari: Prostigmata). - Entomologicheskoe Obozrenie [In Russian but also translated into English in Entomol. Rev.]. 81: 488-513.

Ewing H. E. 1933. A new pit-producing mite from the scales of a South-American snake. - J. Parasit. 20 (1): 53-56.

Fain A. 1962. Diagnoses d'Acariens nouveaux. - Rev. Zool. Bot. Afr., 66 (1-2): 154-162.

Fain A. 1964. Les Ophioptidae Acariens parasites des écailles des serpents (Trombidiformes). - Bull. Inst. r. Sci. nat. Belgique, 40(15): 1-57.

Fain A. 1965. Ophioptidae de l'Angola (Acarina: Trombidiformes). - Services Culturais Comp. de Diamantes de Angola, Lisboa, 77: 107-114.

Lizaso N.M. 1983. Acaros ectoparasitas de serpentes. Descricao de Ophioptes longipilis sp.n. e Ophioptes brevipilis sp.n. (Trombidiformes, Ophioptidae). - Memorias Inst. Butantan, 44-45, 1980-1981: 377-378.

Mendoza-Roldan J.A., Barros-Battesti D.M., Bassini-Silva R., Jacinavicius F.C., Nieri-Bastos F.A., Franco F.L. and Marcili A. 2017. A New Species of Pit Mite (Trombidiformes: Harpirhynchidae) from the South American Rattlesnake (Viperidae): Morphological and Molecular Analysis. - Entomology, Ornithology \& Herpetology: Current Research, 6(3): $8 \mathrm{pp}$.

Radford Ch. D. 1947. Parasitic mites from snakes and rodents (Acarina: Cheyletidae, Listrophoridae and Laelaptidae). - Proc. Zool. Soc. London, 117: 228-240.

Sambon L. W. 1928. Ophioptes parkeri. A new species and genus of Cheyletid inhabiting the scales of reptiles. - Ann. Trop. Med. \& Parasit., 22(1): 137-142.

Southcott R.V. 1956. Notes on the Acarine genus Ophioptes, with a description of a new Australian species. - Trans. Roy. Soc. South Australia, 79: 142-147. 


\section{The Mite Family Syringophilidae Lavoipierre}

Syringophilidae Lavoipierre, 1953

The quill mites (Syringophilidae) were considered part of Cheyletidae until Lavoipierre (1953) and Dubinin (1957) made of them separate family. The number of the known genera and species in this family literally exploded after the revision of Kethley (1970) who added to the two genera known by this time (Picobia Haller, 1878 and Syringophilus Heller, 1880) another 15 genera. In 1973 he described another one - Colinophilus. Before him Fritsch (1958) revised the genus Syringophilus and some other authors (Oudemans, Poppe, Banks, Berlese, Hancock, Hirst, Lawrence, Womersley, Clark) added to the knowledge of the family, but mostly in general acarological papers. Johnston \& Kethley (1973) separated the Picobia in a new subfamily Picobiinae.

The bulk of modern information on Syringophilidae comes from the many important papers of the Polish specialist Skoracky and his co-authors (OConnor, Bochkov, Sikora, Glowska, Fain, Mironov, Hebda, Wauthy, Magowsky, Dabert, Spicer, Hendricks, Møller, Tryjanowski, Hromada and others). They described 17 more genera and many species from the entire world. According to Bochkov (2009b), the family includes 167 sp. of 36 genera. In his monograph on Palearctic Syringophilidae Skoracky (2011) analysed all 118 Palearctic species, describing 6 new genera, 25 new species and many new combinations. Chirov \& Kravtsova (1995) described the new genus Mironovia, Kivganov in Kivganov \& Sharafat (1995) - the genus Kethleyana, Philips \& Norton (1978) - the genus Bubophilus. New information is credited also to Russians (Bochkov, Mironov and others, after 2000), Americans (Casto, 1973-1980, OConnor), Chinese (Liu Bai-li) and other acarologists. Now (end December 2020) the family includes more then 420 described species of 63 genera and many more (at least 5000) should be expected, as many species of birds are not examined for Syringophilidae.

The quill mites, as their name shows, are obligatory and permanent ectoparasites living inside the different types of quills. They feed on soft-tissue fluids of their hosts.

So far quill mites have been recorded on more than 440 species of birds, belonging to 109 bird families (including 60 of Passeriformes) and 28 orders (Accipitriformes, Anseriformes, Apodiformes, Bucerotiformes, Ciconiiformes, Charadriiformes, Coliiformes, Columbiformes, Coraciiformes, Cuculiformes, Falconiformes, Galbuliformes, Galliformes, Gruiformes, Musophagiformes, Otidiformes, Passeriformes, Pelecaniformes, Phoenicopteriformes, Piciformes, Procellariiformes, Psittaciformes, Pteroclidiformes, Strigiformes, Suliformes, Tinamiformes, Trogoniformes, Upupiformes), but many other birds may harbour new genera and species of these rather specific mites.

The system of Syringophilidae is accepted here as follows:

Family Syringophilidae Lavoipierre, 1953 - 63 genera, 415 sp.

Subfamily Syringophilinae Lavoipierre, 1953 - 51 gen., 320 sp. 
Apodisyringiana Skoracki, 2005 - 3 sp.

Apodisyringophilus Skoracki et OConnor, 2010 - 1 sp.

Ascetomylla Kethley, 1970 - 2 sp.

Aulobia Kethley, 1970 - 12 sp.

Aulonastus Kethley, 1970 - 17 sp.

Betasyringophiloidus Skoracki, $2011-6$ sp.

Blaszakia Skoracki et Sikora, 2008 - 2 sp.

Bochkovia Skoracki et OConnor, 2010 - 1 sp.

Bubophilus Philips et Norton, 1978 - 4 sp.

Charadriphilus Bochkov et Chistyakov, 2001 (incl. Charadriaulobia Bochkov, Fain et Skoracki, 2004) - 5 sp.

Chenophila Kethley, 1970 - 5 sp.

Colinophilus Kethley, 1973 - 1 sp.

Colisyringophilus Skoracki, Unsoeld et Ozminski, 2013 - 1 sp.

Corvitorotroglus Skoracki et Bochkov, 2010 - 1 sp.

Creagonycha Kethley, 1970 - 3 sp.

Crotophagisyringophilus Skoracki, 2008 - $1 \mathrm{sp}$.

Cuculisyringohilus Skoracki, 2008 - 2 sp.

Fritschisyringophilus Bochkov, Fain et Skoracki, 2004 - 1 sp.

Galliphilopsis Skoracki et Sikora, 2004- 5 sp.

Ixobrychiphilus Skoracki, Zmudzinski et Solarczyk, 2017 - 1 sp.

Kalamotrypetes Casto, $1980-2$ sp.

Kethleyana Kivganov, in Kivganov \& Sharafat, 1995 - 1 sp.

Krantziaulonastus Skoracki, 2011 - 5 sp.

Megasyringophilus Fain, Bochkov et Mironov, 2000 - 11 sp.

Meitingsunes Glowska et Skoracki, 2010 - 8 sp.

Mironovia Chirov et Kravtsova, 1995 - 5 sp.

Neoaulobia Fain, Bochkov et Mironov, $2000-8$ sp.

Neoaulonastus Skoracki, 2004 (= Dissonus Skoracki, 1999, praeoccup.) - 18 sp.

Neobubophilus Skoracki et Unsoeld, 2016 - 2 sp.

Neoperisterophila Skoracki, 2005 - 1 sp.

Neosyringophilopsis Skoracki et Sikora, 2005 - 8 sp.

Niglarobia Kethley, 1970 - 7 sp.

Paraniglarobia Skoracki, $2011-2 \mathrm{sp}$.

Peristerophila Kethley, 1970 (= Castosyringophilus Bochkov et Perez, 2002) - 13 sp.

Phalarophilus Skoracki, Bochkov et OConnor, 2011 - 1 sp.

Philoxanthornea Kethley, 1970 - 3 sp.

Picineoaulonastus Skoracky, Klimovičová, Mucha et Hromada, 2014 - 1 sp.

Picisyringophilus Skoracki et OConnor, 2010 - $1 \mathrm{sp}$.

Procellariisyringophilus Schmidt et Skoracki, 2007 (= Syringonomus Kethley, 1970, praeoccup.) - 1 sp. 
Psittaciphilus Fain, Bochkov et Mironov, 2000 - 4 sp.

Pteroclidisyringophilus Skoracki, Unsoeld et Skorupski, 2011 - 2 sp.

Selenonycha Kethley, 1970 - 3 sp.

Stibarokris Kethley, 1970 - 11 sp.

Syringophiloidus Kethley, 1970 - 48 sp.

Syringophilopsis Kethley, 1970 - 50 sp.

Syringophilus Heller, 1880 - 2 sp.

Terratosyringophilus Bochkov et Perez, 2002 - 5 sp.

Tinamiphylopsis Skoracki et Sikora, 2004 - 2 sp.

Torotrogla Kethley, 1970 - 20 sp.

Trypetoptila Kethley, 1970 - $1 \mathrm{sp}$.

Subfamily Picobiinae Johnston et Kethley, 1973 - 12 gen., 95 sp.

Calamincola Casto, 1978 [= Cuculiphilus Casto, 1977, praeocc.] - $1 \mathrm{sp}$.

Charadriineopicobia Skoracki, Spicer et OConnor, 2014 - $4 \mathrm{sp}$.

Columbiphilus Kivganov et Sharafat, 1995 - 4 sp.

Gunabopicobia Skoracki et Hromada, 2013 - 7 sp.

Lawrencipicobia Skoracki et Hromada, 2013 - 3 sp.

Neopicobia Skoracki, 2011 - 12 sp.

Picobia Haller, 1878 - 42 sp.

Phipicobia Glowska et Schmid, 2014a - 1 sp.

Pipicobia Glowska et Schmid, 2014b - 4 sp.

Pseudopicobia Scoracki, Scibek et Sikora, 2012 - 3 sp.

Rafapicobia Skoracki, 2011 - 13 sp.

Tanopicobia Skoracki, Hromada, Jerzak et Sikora, 2020 - 1 sp.

\section{Catalogue of Syringophilidae}

Family Syringophilidae Lavoipierre

Syringophilidae Lavoipierre, 1953; Dubinin, 1957: 93

Type genus: Syringophilus Heller, 1880

Subfamily Syringophilinae Lavoipierre

Syringophilinae Lavoipierre, 1953

Type genus: Syringophilus Heller, 1880

Genus Apodisyringiana Skoracki

Apodisyringiana Skoracki, 2005: 337

Type species: Apodisyringiana haszprunari Skoracki, 2005 


\section{Apodisyringiana haszprunari Skoracki}

Apodisyringiana haszprunari Skoracki, 2005: 338

Type locality: Sumatra

Distribution: Indonesia (Sumatra)

Host: Hemiprocne comata (Apodiformes: Hemiprocnidae)

\section{Apodisyringiana mystaceae Skoracki}

Apodisyringiana mystaceae Skoracki, 2005: 341

Type locality: Papua New Guinea, New Britain Isl.

Distribution: Papua New Guinea

Hosts: Hemiprocne mystacea mystacea, H. m. aeroplanes (Apodiformes: Hemiprocnidae)

Apodisyringiana hirundapi Skoracki, Kaszewska et Kavetska

Apodisyringiana hirundapi Skoracki, Kaszewska \& Kavetska, 2015: 584

Type locality: Yokohama

Distribution: Japan

Host: Hirundapus caudacutus (Apodiformes: Apodidae)

Genus Apodisyringophilus Skoracki et OConnor

Apodisyringophilus Skoracki \& OConnor, 2010: 5

Type species: Apodisyringophilus collocalius Skoracki et OConnor, 2010

Apodisyringophilus collocalius Skoracki et OConnor

Apodisyringophilus collocalius Skoracki \& OConnor, 2010: 5

Type locality: Philippines, Leyte Isl., Inopacan

Distribution: Philippines

Host: Collocalia esculenta (Apodiformes: Apodidae)

Genus Ascetomylla Kethley

Ascetomylla Kethley, 1970: 24

Type species: Ascetomylla gallinula Kethley, 1970

\section{Ascetomylla gallinula Kethley}

Ascetomylla gallinula Kethley, 1970: 25

Type locality: Johannesburg, Transvaal

Distribution: South Africa

Host: Gallinula chloropus (Gruiformes: Rallidae)

Ascetomylla porzanae (Bochkov et Galloway)

Syringophilopsis porzanae Bochkov \& Galloway, 2001: 31

Ascetomylla porzanae (Bochkov et Galloway): Skoracki, Flannery \& Spicer, 2008: 299 
Type locality: Manitoba: Delta Marsh

Distribution: Canada

Host: Porzana carolina (Gruiformes: Rallidae)

Genus Aulobia Kethley

Aulobia Kethley, 1970: 41

Type species: Syringophilus dendroicae Clark, 1964

Aulobia anthreptes Skoracki et Glowska

Aulobia anthreptes Skoracki \& Glowska, 2008: 184; Skoracki, Hendricks \& Spicer, 2010: 40

Type locality: unknown

Distribution: Southeast Asia (distribution of the host)

Host: Anthreptes malacensis (Passeriformes: Nectariniidae)

Aulobia afroanthreptes Skoracki et Zmudzinski

Aulobia afroanthreptes Skoracki \& Zmudzinski, 2018: 2

Type locality: Tanzania

Distribution: Tanzania

Hosts: Anthreptes longuemarei, A. neglectus (Passeriformes: Nectariniidae)

Aulobia cardueli Skoracki, Hendricks et Spicer

Aulobia cardueli Skoracki, Hendricks \& Spicer, 2010: 36; Skoracki \& Bochkov, 2010: 65;

Skoracki, 2011: 104

Type locality: Texas, Johnson Co., Garen Ranch

Distribution: Germany, Kazakhstan, Poland, Russia, Slovakia, USA (Texas)

Hosts: Cardinalis cardinalis (Passeriformes: Cardinalidae), Acanthis flammea, Carduelis carduelis, C. flavirostris, Serinus citrinella, Spinus psaltria, S. spinus (Passeriformes: Fringillidae)

Aulobia cisticolae Skoracki et Sikora

Aulobia cisticolae Skoracki \& Sikora, 2003: 2; Skoracki et al., 2009: 132; Klimovičova et al., 2014a

Type locality: Kodegbe (Togo)

Distribution: Cameroon, DR Congo, Kenya, Rwanda, Togo

Hosts: Cisticola cantans, C. chubbi, C. erythrops, C. hunteri, C. lateralis (Passeriformes: Cisticolidae)

\section{Aulobia dendroicae (Clark)}

Syringophilus dendroicae Clark, 1964: 86

Aulobia dendroicus (Clark): Kethley, 1970: 41; Skoracki, Hendricks \& Spicer, 2010: 33

Aulobia dendroicae (Clark): Skoracki, 2011: 99 
Type locality: Maryland

Distribution: USA (Maryland, Florida, Georgia, California, Texas)

Hosts: Setophaga [Dendroica] nigrescens, S. coronata (Passeriformes: Parulidae)

Aulobia erythroptera Skoracki et Dabert

Aulobia erythroptera Skoracki \& Dabert, 2001b: 208

Type locality: Kamina (Togo)

Distribution: Togo

Host: Prinia [Heliolais] erythroptera (Passeriformes: Cisticolidae)

Aulobia leucostictus Skoracki

Aulobia leucostictus Skoracki, 2011: 110

Type locality: Europe

Distribution: Europe

Host: Leucosticte arctoa (Passeriformes: Fringillidae)

Aulobia nectariniae Skoracki et Glowska

Aulobia nectariniae Skoracki \& Glowska, 2008: 179

Type locality: New Guinea

Distribution: New Guinea

Hosts: Cinnyris jugularis, Leptocoma sericea (Passeriformes: Nectariniidae)

Aulobia stachyris (Bochkov, Mironov et Skoracki)

Syringophilopsis stachyris Bochkov, Mironov \& Skoracki, 2001: 237

Aulobia stachyris (Bochkov, Mironov et Skoracki): Skoracki, 2011: 107

Type locality: Thirkhed-hunga

Distribution: Nepal

Host: Cyanoderma [Stachyris] pyrrhops (Passeriformes: Timaliidae)

Aulobia sylviae Bochkov et Mironov

Aulobia sylviae Bochkov \& Mironov, 199[8]9: 9; Skoracki, 2011: 99

Type locality: Rybachiy village, Kurshskaya Spit, Kaliningrad Province

Distribution: Croatia, France, Jordan, Kazakhstan, Poland, Russia

Hosts: Sylvia atricapilla, S. curruca, S. nisoria (Passeriformes: Sylviidae)

Aulobia sylviettae (Fain, Bochkov et Mironov)

Syringophilopsis sylviettae Fain, Bochkov \& Mironov, 2000: 57

Syringophilopsis sylvietta: Skoracki \& Dabert, 2002: 138

Aulobia sylviettae Fain, Bochkov et Mironov): Skoracky, Hendricks \& Spicer, 2010: 39;

Skoracki, 2011: 99 Skoracki, 2011: 99; Klimovičova et al., 2014a

Type locality: Rwanda 
Distribution: Kenya, Rwanda

Host: Sylvietta whytii johnstoni (Passeriformes: Macrosphenidae)

Aulobia virens Skoracki et Dabert

Aulobia virens Skoracki \& Dabert, 2001b: 211; Skoracki, 2011: 99

Type locality: Adina (Togo)

Distribution: Togo

Host: Sylvietta virens flaviventris (Passeriformes: Macrosphenidae)

Genus Aulonastus Kethley

Aulonastus Kethley, 1970: 47

Type species: Aulonastus pipili Kethley, 1970

\section{Aulonastus albus Skoracki}

Aulonastus albus Skoracki, 2002: 304; 2011: 136; Klimovičová \& Hromada, 2014: 33

Type locality: Zachodniopomorskie, near Swinoujscie

Distribution: Poland, Slovakia

Hosts: Motacilla alba, M. flava (Passeriformes: Motacillidae)

\section{Aulonastus anthus Skoracki}

Aulonastus anthus Skoracki, 2011: 150

Type locality: Wielkopolska, Katy

Distribution: Poland, Slovakia

Hosts: Anthus cervinus, A. trivialis (Passeriformes: Motacillidae)

\section{Aulonastus emberizicus Skoracki, Hendricks et Spicer}

Aulonastus emberizicus Skoracki, Hendricks \& Spicer, 2010c: 132; Skoracki, 2011: 136

Type locality: Jaff Davis Co., Texas

Distribution: USA (California, Texas)

Hosts: Ammodramus savannarum, Passerculus sandwichensis, Zonotrichia atricapilla

(Passeriformes: Emberizidae)

Aulonastus euphagus Skoracki, Hendricks et Spicer

Aulonastus euphagus Skoracki, Hendricks \& Spicer, 2010c: 134; Skoracki, 2011: 136

Type locality: Mono Co., California

Distribution: USA (California)

Host: Euphagus cyanocephalus (Passeriformes: Icteridae)

Aulonastus fringillus Skoracki

Aulonastus fringillus Skoracki, 2011: 152

Type locality: Wielkopolska, Katy 
Distribution: Germany, Poland

Host: Fringilla coelebs (Passeriformes: Fringillidae)

Aulonastus henicorhina Sikora et Skoracki

Aulonastus henicorhina Sikora \& Skoracki, 2012: 60

Type locality: Ecuador, near Quito

Distribution: Ecuador

Host: Henicorhina leucosticta (Passeriformes: Troglodytidae)

\section{Aulonastus lanius Skoracki}

Aulonastus lanius Skoracki, 2011: 146

Type locality: Europe

Distribution: Europe

Host: Lanius senator (Passeriformes: Laniidae)

Aulonastus loxius Skoracki

Aulonastus loxius Skoracki, 2011: 148

Type locality: Zachodniopomorskie, Darlowko Wschodnie, Bukowo - Kopan

Distribution: Poland

Host: Loxia curvirostra (Passeriformes: Fringillidae)

\section{Aulonastus lusciniae Skoracki}

Aulonastus lusciniae Skoracki, 2002: 301; 2011: 136

Type locality: Slovakia

Distribution: Slovakia

Host: Luscinia luscinia (Passeriformes: Muscicapidae)

Aulonastus nectariniphilus Skoracki et Zmudzinski

Aulonastus nectariniphilus Skoracki \& Zmudzinski, 2018: 1

Type locality: Tanzania

Distribution: Tanzania

Host: Anthreptes reichenowi (Passeriformes: Nectariniidae)

Aulonastus paridus Sikora, Unsöld et Skoracki

Aulonastus paridus Sikora, Unsöld \& Skoracki, 2016: 151

Type locality: $80 \mathrm{mi}$. S. Arusha

Distribution: Tanzania

Hosts: Melaniparus albiventris, M. fringillinus (Passeriformes: Paridae)

Aulonastus pipili Kethley

Aulonastus pipili Kethley, 1970: 48; Skoracki, 2011: 136 
Type locality: Lake Placid, Highlands Co., Florida

Distribution: USA (Florida)

Host: Pipilo erythrophthalmus (Passeriformes: Passerellidae)

Aulonastus pirangus Skoracki, Hendricks et Spicer

Aulonastus pirangus Skoracki, Hendricks \& Spicer, 2010c: 138; Skoracki, 2011: 136

Type locality: Sierra Co., California

Distribution: USA (California)

Host: Piranga ludoviciana (Passeriformes: Cardinalidae)

Aulonastus prunellae Bochkov et Mironov

Aulonastus prunellae Bochkov \& Mironov, 1999: 38; Skoracki, 2011: 140

Type locality: Novgorod Prov., Chudovo Distr., Oskuy Vill.

Distribution: Russia

Host: Prunella modularis (Passeriformes: Prunellidae)

Aulonastus pteroptochos Sikora, Kaszewska et Skoracki

Aulonastus pteroptochos Sikora, Kaszewska \& Skoracki, 2014: 422

Type locality: Chile, Valdivia

Distribution: Chile

Host: Pteroptochos tarnii (Passeriformes: Rhinocriptidae)

Aulonastus similis Broda, Dabert et Glowska

Aulonastus similis Broda, Dabert \& Glowska, 2016: 716; Skoracki, 2017:180

Type locality: Los Tuxtlas Tropical Biological Station(UNAM), $18^{\circ} 350700 \mathrm{~N}, 95^{\circ} 403100 \mathrm{~W}$, Veracruz

Distribution: Mexico

Hosts: Myiozetetes similis (Passeriformes: Tyrannidae), Habia fuscicauda, H. rubica (Pass., Cardinalidae)

Aulonastus sturnellus Skoracki, Hendricks et Spicer

Aulonastus sturnellus Skoracki, Hendricks \& Spicer, 2010c: 141; Skoracki, 2011: 136

Type locality: Johnson Co., Texas

Distribution: USA (Texas)

Host: Sturnella magna (Passeriformes: Icteridae)

Genus Betasyringophiloidus Skoracki

Betasyringophiloidus Skoracki, 2011: 86

Type species: Betasyringophiloidus phoenicurus Skoracki, 2011 
Betasyringophiloidus emberizae Glowska, Broda et Dabert Betasyringophiloidus emberizae Glowska, Broda \& Dabert, 2019: 4 Type locality: Novolitovsk, Partizanskiy District, Primorskiy Kray Distribution: Russia

Host: Emberiza tristrami (Passeriformes: Emberizidae)

Betasyringophiloidus motacillae (Bochkov et Mironov)

Syringophiloidus motacillae Bochkov \& Mironov, 1998: 14; Bochkov \& Galloway, 2001: 2025; Skoracki, Flannery \& Spicer, 2009: 72

Betasyringophiloidus motacillae (Bochkov et Mironov): Skoracki, 2011: 92; Klimovičová \& Hromada, 2014: 33

Type locality: Rybachiy village, Kurshskaya Spit, Kaliningrad Province

Distribution: Canada, Poland, Russia, Slovakia, USA (California)

Host: Motacilla flava (Passeriformes: Motacillidae)

\section{Betasyringophiloidus phoenicurus Skoracki}

Betasyringophiloidus phoenicurus Skoracki, 2011: 87; Pidhorna et al., 2020: 215

Type locality: Zachodniopomorskie, Weltyn (Poland)

Distribution: Poland, Ukraine

Host: Phoenicurus ochruros (Passeriformes: Muscicapidae)

\section{Betasyringophiloidus saxicolus Skoracki}

Betasyringophiloidus saxicolus Skoracki, 2011: 90; Pidhorna et al., 2020: 215

Type locality: Zachodniopomorskie, Weltyn (Poland)

Distribution: Poland, Ukraine

Host: Saxicola rubetra (Passeriformes: Muscicapidae)

\section{Betasyringophiloidus schoeniclus (Skoracki)}

Syringophiloidus schoeniclus Skoracki, 2002: 309

Syringophiloidus schoeniclusi: Skoracki, 2004: 296; Skoracki \& Bochkov, 2010: 65 Betasyringophiloidus schoeniclus (Skoracki): Skoracki, 2011: 96; Skoracki \& Mironov, 2013;

Glowska, Broda \& Dabert, 2019: 6

Type locality: Slovenská Kajňa (Slovakia)

Distribution: Kazakhstan, Mongolia, Poland, Russia, Slovakia

Hosts: Emberiza rutila, E. schoeniclus, E. tristrami (Passeriformes: Emberizidae)

Betasyringophiloidus seiuri (Clark, 1964)

Syringophilus seiuri Clark, 1964: 81

Syringophiloidus seiurus (Clark): Kethley, 1970: 50; Skoracki \& Dabert, 2002: 138

Syringophiloidus seiuri: Bochkov \& Galloway, 2001: 2025; Skoracki, 2011: 38 
Betasyringophiloidus seiuri (Clark, 1964): Skoracki et al., 2016: 458; Skoracki, Spicer \& OConnor, 2016: 458; Glowska, Broda \& Dabert, 2019; Grossi \& Proctor, 2020: 82; Skoracki, 2017: 180

Type locality: Maryland (USA)

Distribution: Canada (Manitoba), DR Congo, Mexico, Togo, USA (Maryland, Florida, S. Carolina, Massachusets, Texas), Kenya

Hosts: Geothlypis philadelphia, Helmitheros vermivorus, Oreothlypis peregrina, O. ruficapilla, Parkesia noveboracensis, Seiurus aurocapilla, Setophaga palmarum, S. pensylvanica (Passeriformes: Parulidae), Melospiza georgiana, M. lincolnii, M. melodia (Passeriformes: Passerellidae), Ploceus bagdafecht (= Othyphantes stuhlmanni), Ploceus [Pachyphantes] superciliosus, Ploceus xanthops, Quelea erythrops (Passeriformes: Ploceidae), Icterus pustulatus (Passeriformes: Icteridae)

Genus Blaszakia Skoracki et Sikora

Blaszakia Skoracki \& Sikora, 2008a: 328

Type species: Galliphilopsis francolinus Skoracki et Sikora, 2004

Blaszakia rossae Skoracki et Sikora

Blaszakia rossae Skoracki \& Sikora, 2008a: 330

Type locality: Katanga, Kongolo

Distribution: DR Congo

Host: Musophaga rossae (Musophagiformes: Musophagidae)

Blaszakia tauracos Skoracki et Sikora

Blaszakia tauracos Skoracki \& Sikora, 2008a: 328

Type locality: Uluguru (Tanzania)

Distribution: Guinea, Tanzania

Hosts: Tauraco livingstoni, T. schalowi (Musophagiformes: Musophagidae)

Genus Bochkovia Skoracky et OConnor

Bochkovia Skoracky \& OConnor, 2010: 6

Type species: Bochkovia phalaropi Skoracky et OConnor, 2010

Bochkovia phalaropi Skoracky et OConnor

Bochkovia phalaropi Skoracky \& OConnor, 2010: 8

Type locality: San Diego Co., San Diego

Distribution: USA

Host: Phalaropus fulicarius (Charadriiformes: Scolopacidae)

Genus Bubophilus Philips et Norton

Bubophilus Philips \& Norton, 1978: 900 
Type species: Bubophilus ascalaphus Philips et Norton, 1978

\section{Bubophilus aluconis Nattress et Skoracki}

Bubophilus aluconis Nattress et Skoracki, 2009: 49; Skoracki, 2011: 321; Skoracki et al., 2012: 37; Skoracki, Unsoeld, Marciniak \& Sikora, 2016: 821

Type locality: England

Distribution: England, Poland, Tanzania

Hosts: Asio otus, Strix aluco, S. woodfordii (Strigiformes: Strigidae)

\section{Bubophilus ascalaphus Philips et Norton}

Bubophilus ascalaphus Philips \& Norton, 1978: 900; Skoracki \& Dabert, 2002: 146; Skoracki, 2011: 319; Skoracki, Unsoeld, Marciniak \& Sikora, 2016: 821

Type locality: Central New York (USA)

Distribution: Cameroon, Nepal, South Africa, USA

Hosts: Bubo africanus, B ascalaphus, B. bubo, B. virginianus (Strigiformes: Strigidae), Tyto alba affinis (Strigiformes: Tytonidae)

Bubophilus asiobius Skoracki et Bochkov

Bubophilus asiobius Skoracki \& Bochkov, 2002: 142; Skoracki, 2011: 319; Skoracki, Unsoeld, Marciniak \& Sikora, 2016: 821

Type locality: Darłówko Wschodnie (Poland)

Distribution: Germany, Poland

Host: Asio otus (Strigiformes: Strigidae)

Bubophilus tytonis Skoracki et Unsold

Bubophilus tytonis Skoracki \& Unsold, 2002: 817

Type locality: Cameroon, Wasa

Distribution: Cameroon

Host: Tyto alba affinis (Strigiformes: Tytonidae)

Genus Charadriphilus Bochkov et Chistyakov

Charadriphilus Bochkov \& Chistyakov, 2001: 295

Type species: Charadriphilus lyudmilae Bochkov et Chistyakov, 2001

Charadriaulobia Bochkov, Fain \& Skoracki, 2004: 136

Type species: Charadriaulobia vanelli Bochkov, Fain et Skoracki, 2004

Charadriphilus gallinago (Bochkov et Mironov)

Aulobia gallinago Bochkov et Mironov, 1998: 8

Charadriaulobia gallinago: Bochkov, Fain \& Skoracki, Fain \& Skoracki, 2004: 138

Charadriphilus gallinago (Bochkov et Mironov): Skoracki, Dabert \& Schmäschke, 2006: 61; Skoracki, 2011: 259 
Type locality: St. Malo (France)

Distribution: France, Poland

Host: Gallinago gallinago (Charadriiformes: Scolopacidae)

Charadriphilus lyudmilae Bochkov et Chistyakov

Charadriphilus lyudmilae Bochkov \& Chistyakov, 2001: 297; Skoracki, 2011: 256

Type locality: Pochi Village, Tosno Distr., Leningrad Distr.

Distribution: Russia

Host: Scolopax rusticola (Charadriiformes: Scolopacidae)

Charadriphilus paraguaiae (Skoracki et Sikora)

Aulobia paraguaiae Skoracki \& Sikora, 2002: 5

Charadriphilus paraguaiae (Skoracki et Sikora): Skoracki, 2011: 256

Type locality: El Bolson - Rio Negro

Distribution: Argentina

Host: Gallinago paraguaiae (Charadriiformes: Scolopacidae)

Charadriphilus ralli Skoracki et Bochkov

Charadriphilus ralli Skoracki \& Bochkov, 2010: 57; Skoracki, 2011: 260; Skoracki et al., 2014: 227

Type locality: Ongtustik Qazaqstan, Vysokoe village, Vysochanskiy Pond (Kazakhstan)

Distribution: Chile, Kazakhstan, Kirghizia, Paraguay, USA

Hosts: Fulica americana, Gallinula melanops, Laterallus melanophaius, Porzana parva, Rallus aquaticus (Gruiformes: Rallidae)

Charadriphilus vanelli (Bochkov, Fain et Skoracki)

Charadriaulobia vanelli Bochkov, Fain \& Skoracki, 2004: 136

Charadriphilus vanelli (Bochkov, Fain et Skoracki): Skoracki, 2011: 256

Type locality: Brazil (Antverp Zoo)

Distribution: Brazil

Host: Vanellus chilensis (Charadriiformes: Charadriidae)

Genus Chenophila Kethley

Chenophila Kethley, 1970: 37

Type species: Chenophila branta Kethley, 1970

Chenophila branta Kethley

Chenophila branta Kethley, 1970: 38; Skoracki, 2011: 267

Type locality: Kensington Park, Oakland Co., Michigan 
Distribution: USA (Michigan)

Host: Branta canadensis (Anseriformes: Anatidae)

Chenophila kanduli Bochkov

Chenophila kanduli Bochkov, in Bochkov \& Mironov, 1999: 7; Skoracki, 2011: 267

Type locality: Rybachiy village, Kurshskaya Spit, Kaliningrad Province

Distribution: Russia, USA

Hosts: Anas clypeata, A. querquedula (Anseriformes: Anatidae)

Chenophila marmaronetta Zmudzinski et Unsoeld

Chenophila marmaronetta Zmudzinski \& Unsoeld, 2017: 477

Type locality: Turkey, Erciş

Distribution: Turkey

Host: Marmaronetta angustirostris (Anseriformes: Anatidae)

Chenophila nanseni Skoracki et Zawierucha

Chenophila nanseni Skoracki \& Zawierucha, 2016: 123

Type locality: Svalbard, Nissenfjella

Distribution: Svalbard

Host: Branta leucopsis (Anseriformes: Anatidae)

Chenophila platyrhynchos Tymcio, Kavetska, Czyz et Skoracki

Chenophila platyrhynchos Tymcio, Kavetska, Czyz \& Skoracki, 2013: 660

Type locality: Szczecin

Distribution: Poland

Host: Anas platyrhynchos (Anseriformes: Anatidae)

Genus Colinophilus Kethley

Colinophilus Kethley, 1973: 2

Type species: Colinophilus wilsoni Kethley, 1970

Colinophilus wilsoni Kethley

Colinophilus wilsoni Kethley, 1973: 2

Type locality: Tall Timbers Res. Station, Leon Co., Florida

Distribution: USA (Florida, Kentucky)

Hosts: Calipepla squamata, Colinus virginianus (Galliformes: Odontophoridae)

Genus Colisyringophilus Skoracki, Unsoeld et Ozminski

Colisyringophilus Skoracki, Unsoeld \& Ozminski, 2013: 214

Type species: Colisyringophilus tanzanicus Skoracki, Unsoeld et Ozminski, 2013 
Colisyringophilus tanzanicus Skoracki, Unsoeld et Ozminski

Colisyringophilus tanzanicus Skoracki, Unsoeld \& Ozminski, 2013: 214

Type locality: Tanzania, Soga, Kibaha District

Distribution: Tanzania

Hosts: Colius striatus, Urocolius macrourus (Coliiformes: Coliidae)

Genus Corvitorotroglus Skoracki et Bochkov

Corvitorotroglus Skoracki \& Bochkov, 2010: 54

Type species: Corvitorotroglus alpha Skoracki et Bochkov, 2010

Corvitorotroglus alpha Skoracki et Bochkov

Corvitorotroglus alpha Skoracki \& Bochkov, 2010: 54

Type locality: Ongtustik Qazaqstan, Chockpak Ornithological Station

Distribution: Kazakhstan

Host: Corvus frugilegus (Passeriformes: Corvidae)

Genus Creagonycha Kethley

Creagonycha Kethley, 1970: 34

Type species: Creagonycha lara Kethley, 1970

Creagonycha lara Kethley

Creagonycha lara Kethley, 1970: 36; Skoracki, 2011: 271

Type locality: Coffee Bluff, Chatham Co., Georgia

Distribution: USA (Georgia)

Host: Larus delawarensis (Charadriiformes: Laridae)

Creagonycha sternae Kivganov

Creagonycha sternae Kivganov, in Kivganov \& Sharafat, 1995: 86; Skoracki, 2011: 274

Type locality: Odessa Prov., Tiligulski liman

Distribution: Ukraine

Host: Sternula albifrons (Charadriiformes: Laridae, Sterninae)

\section{Creagonycha totani (Oudemans)}

Syringophilus totani Oudemans, 1904: 171; 1906: 57

Creagonycha totana (Oudemans): Kethley, 1970: 37; Bochkov \& Mironov, 1999: 10;

Glowska, Chrzanowski \& Kaszewska, 2015: 15

Creagonycha totani (Oudemans): Skoracki et al., 2006; Skoracki, 2011: 271

Type locality: Arcachon, France

Distribution: Canada, France, Kazakhstan, Poland, Togo

Hosts: Calidris alba, C. himantopus, C. minuta, C. temminckii, Tringa glareola, T. totanus (Charadriiformes: Scolopacidae) 


\section{Genus Crotophagisyringophilus Skoracki}

Crotophagisyringophilus Skoracki, 2008a: 76

Type species: Crotosyringophilus io Skoracki, 2008

Crotophagisyringophilus io Skoracki

Crotophagisyringophilus io Skoracki, 2008a: 76; Hromada et al., 2016: 532; Skoracki, 2017:

Type locality: Prov. of Formosa (Argentina)

Distribution: Argentina, Bolivia, Mexico

Hosts: Crotophaga ani, C. sulcirostris (Cuculiformes: Cuculidae, Crotophaginae)

Genus Cuculisyringophilus Skoracki

Cuculisyringophilus Skoracki, 2008b: 155

Type species: Cuculisyringophilus crotophaginus Skoracki, 2008

Cuculisyringophilus chirovi (Bochkov et Mironov)

Niglarobia chirovi Bochkov \& Mironov, 1999: 11

Cuculisyringophilus chirovi (Bochkov et Mironov): Skoracki, 2011: 324; Hromada et al., 2016: 531

Type locality: Ottuk village, Issyk-Kul Lake (Kyrghyzstan)

Distribution: Indonesia, Kyrghyzstan, Papua New Guinea, Tanzania

Hosts: Centropus goliath, Cercococcyx montanus, Cuculus canorus, C. solitarius, Pachycoccyx audeberti, Scythrops novaehollandiae (Cuculiformes: Cuculidae)

\section{Cuculisyringophilus crotophaginus Skoracki}

Cuculisyringophilus crotophaginus Skoracki, 2008b: 156; Hromada et al., 2016: 532

Type locality: Cambyrila (Paraguay)

Distribution: Colombia, Mexico, Paraguay

Hosts: Crotophaga sulcirostris, Guira guira (Cuculiformes: Cuculidae, Crotophaginae)

Genus Fritschisyringophilus Bochkov, Fain et Skoracki

Fritschisyringophilus Bochkov, Fain \& Skoracki, 2004: 139

Type species: Fritschisyringophilus lonchurae Bochkov, Fain et Skoracki, 2004

Fritschisyringophilus lonchurae Bochkov, Fain et Skoracki

Fritschisyringophilus lonchurae Bochkov, Fain \& Skoracki, 2004: 139

Type locality: India (Antwerp Zoo)

Distribution: India

Host: Lonchura punctulata (Passeriformes: Estrildidae) 
Genus Galliphilopsis Skoracki et Sikora

Galliphilopsis Skoracki \& Sikora, 2004: 380

Type species: Galliphilopsis francolinus Skoracki et Sikora, 2004

Galliphilopsis bochkovi Skoracki et Sikora

Galliphilopsis bochkovi Skoracki \& Sikora, 2004: 382; Skoracki, 2011: 310

Type locality: Himalaya

Distribution: Himalaya (no other data)

Host: Tragopan sp. (Galliformes: Phasianidae). Note by Glowska, Chrzanowski \& Kaszewska, 2015: 16: "The specimens recorded on Alectoris barbara and determined as G. bochkovi belong to G. szeptyckii (Skoracki \& Sikora 2011)”.

Galliphilopsis colinus Skoracki et Sikora

Galliphilopsis colinus Skoracki \& Sikora, 2011: 20

Type locality: Zanderij (Suriname)

Distribution: Colombia, Suriname

Hosts: Colinus cristatus, C. cristatus leucopogon (Galliformes: Odontophoridae)

Galliphilopsis francolinus Skoracki et Sikora

Galliphilopsis francolinus Skoracki \& Sikora, 2004: 380; Skoracki, 2011: 310

Type locality: South Africa

Distribution: South Africa

Host: Francolinus levaillantoides (Galliformes: Phasianidae)

Galliphilopsis lophurus Skoracki et Sikora

Galliphilopsis lophurus Skoracki \& Sikora, 2004: 383; Skoracki, 2011: 310

Type locality: unknown

Distribution: unknown; host distribution in the Himalaya

Host: Lophura leucomelanos (Galliformes: Phasianidae)

Galliphilopsis szeptyckii Skoracki et Sikora

Galliphilopsis szeptyckii Skoracki \& Sikora, 2011:20; Glowska, Chrzanowski \& Kaszewska, 2015: 16

Type locality: Adelaide (Australia)

Distribution: Australia, Indonesia, Italy (Sicily), Japan

Hosts: Alectoris barbara, Coturnix chinensis, C. coturnix, C. pectoralis (Galliformes: Phasianidae)

Genus Ixobrychiphilus Skoracki, Zmudzinski et Solarczyk

Ixobrychiphilus Skoracki, Zmudzinski \& Solarczyk, 2017: 270

Type species: Ixobrychiphilus wallacei Skoracki, Zmudzinski et Solarczyk, 2017 
Ixobrychiphilus wallacei Skoracki, Zmudzinski et Solarczyk, 2017

Ixobrychiphilus wallacei Skoracki, Zmudzinski \& Solarczyk, 2017: 270

Type locality: China, Middle Course of Yangtze River

Distribution: China

Host: Ixobrychus cinnamomeus (Pelecaniformes: Ardeidae)

Genus Kalamotrypetes Casto

Kalamotrypetes Casto, 1980: 233

Type species: Kalamotrypetes Casto, 1980

Kalamotrypetes colinastes Casto

Kalamotrypetes colinastes Casto, 1980: 233

Type locality: Texas, La Salle Co.

Distribution: USA

Hosts: Callipepla squamata, Colinus virginianus (Galliformes: Odontophoridae)

Kalamotrypetes cracidus Skoracki et Sikora

Kalamotrypetes cracidus Skoracki et Sikora, 2011: 16

Type locality: Guyana

Distribution: Guyana, Paraguay

Hosts: Ortalis canicollis, Penelope sp. (Galliformes: Cracidae)

Genus Kethleyana Kivganov

Kethleyana Kivganov, in Kivganov \& Sharafat, 1995: 84

Type species: Kethleyana gelochelodoni Kivganov, 1995

Kethleyana gelochelodoni Kivganov

Kethleyana gelochelodoni Kivganov, in Kivganov \& Sharafat, 1995: 86; Skoracki, 2011: 275

Type locality: Odessa Prov., Tiligulski liman

Distribution: Ukraine

Host: Gelochelidon nilotica (Charadriiformes: Laridae)

Genus Krantziaulonastus Skoracki

Krantziaulonastus Skoracki, 2011: 128

Type species: Krantziaulonastus Skoracki, 2011

Krantziaulonastus buczekae (Skoracki)

Aulonastus buczekae Skoracki, 2002: 300

Krantziaulonastus buczekae (Skoracki): Skoracki, 2011: 129

Type locality: Pomorskie, Kuznica-Hel

Distribution: Poland 
Host: Sturnus vulgaris (Passeriformes: Sturnidae)

Krantziaulonastus dubinini Skoracki et Sikora

Krantziaulonastus dubinini Skoracki \& Sikora, 2014: 197

Type locality: Russia, Tula District, Suvorov

Distribution: Russia

Host: Passer domesticus (Passeriformes: Passeridae)

Krantziaulonastus galbulicus (Skoracki)

Aulonastus galbulicus Skoracki, 2008: 48

Krantziaulonastus galbulicus (Skoracki): Skoracki, 2011: 128

Type locality: South America

Distribution: South America

Host: Jacamaralcyon tridactyla (Galbuliformes: Galbulidae)

Krantziaulonastus yoyomi Glowska et Skoracki

Krantziaulonastus yoyomi Glowska \& Skoracki, 2011: 64

Type locality: unknown

Distribution: unknown

Host: Dinemellia dinemelli (Passeriformes: Ploceidae)

Genus Megasyringophilus Fain, Bochkov et Mironov

Megasyringophilus Fain, Bochkov \& Mironov, 2000: 35.

Type species: Megasyringophilus kethleyi Fain, Bochkov et Mironov, 2000

Megasyringophilus aquilus Skoracki, Lontkowski et Stawarczyk

Megasyringophilus aquilus Skoracki, Lontkowski \& Stawarczyk, 2010: 1204; Zmudzinski et al., 2015: 292

Type locality: South Africa

Distribution: Kazakhstan, France, Poland, South Africa, Spain

Hosts: Accipiter nisus, Aquila rapax, Clanga pomarina, Hieraaetus pennatus (Accipitriformes: Accipitridae)

Megasyringophilus cacatua Glowska et Laniecka

Megasyringophilus cacatua Glowska \& Laniecka, 2013: 386

Type locality: Australia, Mount Hart

Distribution: Australia

Host: Cacatua galerita (Psittaciformes: Cacatuidae) 
Megasyringophilus cyanocephala Fain, Bochkov et Mironov

Megasyringophilus cyanocephala Fain, Bochkov \& Mironov, 2000: 40; Bochkov \& Fain, 2003: 3; Skoracki, 2005: 128

Type locality: India (Zoo of Antwerp)

Distribution: India

Hosts: Psittacula cyanocephala, P. eupatria, P. krameri (Psittaciformes: Psittaculidae)

Megasyringophilus dalmas Skoracki et Unsoeld

Megasyringophilus dalmas Skoracki \& Unsoeld, 2016: 822

Type locality: Venezuela

Distribution: Venezuela

Host: Megascops choliba (Strigiformes: Strigidae)

Megasyringophilus dubinini Bochkov et Mironov

Megasyringophilus dubinini Bochkov \& Fain, 2003: 3

Type locality: Sulawesi

Distribution: Indonesia

Host: Trichoglossus ornatus (Psittaciformes: Psittaculidae)

Megasyringophilus eos Skoracki

Megasyringophilus eos Skoracki, 2005b: 132

Type locality: Ceram I.

Distribution: Indonesia (Ceram)

Host: Eos bornea (Psittaciformes: Psittaculidae)

Megasyringophilus geoffroyus Skoracki

Megasyringophilus geoffroyus Skoracki, 2005b: 132

Type locality: New Guinea

Distribution: New Guinea

Host: Geoffroyus geoffroyi (Psittaciformes: Psittaculidae)

Megasyringophilus kethleyi Fain, Bochkov et Mironov

Megasyringophilus kethleyi Fain, Bochkov \& Mironov, 2000: 35; Bochkov \& Fain, 2003

Type locality: Brazil (Zoo of Antwerp)

Distribution: Brazil

Hosts: Aratinga jandaya, A. pertinax, Brotogeris versicolurus (Psittaciformes: Psittacidae)

Megasyringophilus platycercus Bochkov et Fain

Megasyringophilus platycercus Bochkov \& Fain, 2003: 4

Type locality: Australia

Distribution: Australia 
Host: Platycercus eximius (Psittaciformes: Psittaculidae)

Megasyringophilus rhynchopsittae Bochkov et Perez

Megasyringophilus rhynchopsittae Bochkov \& Perez, 2002: 157

Type locality: Mesa de las Guacamayas, Mpio. Janos

Distribution: Mexico

Host: Rhynchopsitta pachyrhyncha (Psittaciformes: Psittacidae)

Megasyringophilus trichoglossus Fain, Bochkov et Mironov

Megasyringophilus trichoglossus Fain, Bochkov \& Mironov, 2000: 37; Skoracki, 2005b: 132

Type locality: New Guinea (Zoo of Antwerp)

Distribution: Australia, Indonesia (Timor), New Guinea

Hosts: Trichoglossus chlorolepidotus, T. euteles (Psittaciformes: Psittaculidae)

Genus Meitingsunes Glowska et Skoracki

Meitingsunes Glowska \& Skoracki, 2010: 61

Type species: Meitingsunes aldwelles Glowska et Skoracki, 2010

Meitingsunes aldwelles Glowska et Skoracki

Meitingsunes aldwelles Glowska \& Skoracki, 2010: 61; Skoracki, 2011: 301; Kaszewska,

Skoracki \& Kavetska, 2016: 480

Type locality: Choco

Distribution: Colombia

Host: Geotrygon frenata (Columbiformes: Columbidae)

Meitingsunes caprimulgus Skoracki, Kaszewska, Unsoeld et Skorupski

Meitingsunes caprimulgus Skoracki, Kaszewska, Unsoeld \& Skorupski, 2015: 128

Type locality: Colombia

Distribution: Colombia

Host: Chordeiles minor (Caprimulgiformes: Caprimulgidae)

Meitingsunes chalcophaps Kaszewska, Skoracki et Kavetska

Meitingsunes chalcophaps Kaszewska, Skoracki \& Kavetska, 2016: 480

Type locality: West Timor

Distribution: Indonesia

Host: Chalcophaps indica (Columbiformes: Columbidae)

\section{Meitingsunes columbicus Skoracki}

Meitingsunes columbicus Skoracki, 2011: 302; Kaszewska, Skoracki \& Kavetska, 2016: 480

Type locality: Wielkopolska, Poznan

Distribution: Cameroon, Kazakhstan, Poland, Russia 
Hosts: Columba livia, C. oenas, C. palumbus, Treron waalia (Columbiformes: Columbidae)

Meitingsunes ptilinopus Kaszewska, Skoracki et Kavetska

Meitingsunes ptilinopus Kaszewska, Skoracki \& Kavetska, 2020: 2

Type locality: Australia

Distribution: Australia and New Guinea

Hosts: Ptilinopus magnificus, P. rivoli (Columbiformes: Columbidae)

Meitingsunes turacoensis Kaszewska, Skoracki et Kavetska

Meitingsunes turacoensis Kaszewska, Skoracki \& Kavetska, 2016: 483

Type locality: Sulawesi, Manado

Distribution: Indonesia (Sulawesi, West Timor)

Hosts: Turacoena manadensis, T. modesta (Columbiformes: Columbidae)

Meitingsunes tympanistria (Skoracki et Dabert)

Peristerophila tympanistria Skoracki \& Dabert, 2002: 141

Meitingsunes tympanistria (Skoracki et Dabert): Glowska \& Skoracki, 2010: 61; Skoracki,

2011: 301; Kaszewska, Skoracki \& Kavetska, 2016: 480

Type locality: Missahohe

Distribution: Togo

Host: Turtur tympanistria (Columbiformes: Columbidae)

Meitingsunes zenaidurae (Clark)

Syringophilus zenadurae Clark, 1964: 83

Peristerophila zenadoura (Clark): Kethley, 1970: 56; Bochkov \& Mironov, 1998: 15; Skoracki \& Glowska, 2008: 159

Peristerophila zenadourae (Clark): Skoracki \& Sikora, 2002: 8; Skoracki \& Bochkov, 2010: 66

Meitingsunes zenadourae (Clark): Glowska \& Skoracki, 2010: 61; Skoracki, 2011: 301

Type locality: Maryland

Distribution: Argentina, N. Africa, Kazakhstan, USA (Maryland)

Hosts: Columba livia, Zenaida auriculata, Z. asiatica, Z. macroura (Columbiformes: Columbidae)

Genus Mironovia Chirov et Kravtsova

Mironovia Chirov \& Kravtsova, 1995: 370

Type species: Mironovia phasiani Chirov et Kravtsova, 1995

Mironovia coturnae Bochkov, Fain et Skoracki

Mironovia coturnae Bochkov, Fain \& Skoracki, 2004: 142; Skoracki, 2011

Type locality: Belgium

Distribution: Belgium, Poland 
Host: Coturnix coturnix (Galliformes: Phasianidae)

Mironovia lagopus Bochkov et Skirnisson

Mironovia lagopus Bochkov \& Skirnisson, 2011: 715; Skoracki \& Sikora, 2011: 13

Type locality: Iceland, Lake Mývatn

Distribution: Iceland, Norway

Hosts: Lagopus lagopus, L. muta (Galliformes: Phasianidae)

Mironovia pavodaptes (Casto)

Kalamotrypetes pavodaptes Casto, 1980: 237

Mironovia pavodaptes (Casto): Skoracki \& Sikora, 2011: 19

Type locality: Texas, Ft. Hood Reservation, Bell Co.

Distribution: USA

Host: Meleagris gallopavo [pavodaptes] (Galliformes: Phasianidae)

Mironovia phasiani Chirov et Kravtsova

Mironovia phasiani Chirov \& Kravtsova, 1995: 370; Skoracki, 2011: 315

Type locality: Tokmak, Kyrghyzstan

Distribution: Kyrghyzstan

Host: Phasianus colchicus (Galliformes: Phasianidae)

Mironovia rouloul Skoracki et Sikora

Mironovia rouloul Skoracki \& Sikora, 2004 : 385; Skoracki, 2011

Type locality: Sumatra

Distribution: Indonesia (Sumatra)

Host: Rollulus rouloul (Galliformes: Phasianidae)

Genus Neoaulobia Fain, Bochkov et Mironov

Neoaulobia Fain, Bochkov \& Mironov, 2000: 40

Type species: Neoaulobia aratingae Fain, Bochkov et Mironov, 2000

Neoaulobia agapornis Fain, Bochkov et Mironov

Neoaulobia agapornis Fain, Bochkov \& Mironov, 2000: 44; Bochkov \& Fain, 2003: 151; Skoracki, 2005: 128

Neoaulobia agaporni: Skoracki \& Dabert, 2002: 138

Type locality: Zambia

Distribution: Ethiopia, Namibia, Tanzania, Zambia

Hosts: Agapornis fisheri, A. nigrigenis, A. personatus, A. roseicollis, A. taranta (Psittaciformes: Psittaculidae) 
Neoaulobia aratingae Fain, Bochkov et Mironov

Neoaulobia aratingae Fain, Bochkov \& Mironov, 2000: 40; Skoracki, 2005b: 128

Type locality: Brazil (Antwerp Zoo)

Distribution: Brazil

Host: Aratinga jandaya (Psittaciformes: Psittacidae)

Neoaulobia cacatua Marciniak, Skoracki et Hromada

Neoaulobia cacatua Marciniak, Skoracki \& Hromada, 2019: 192

Type locality: Australia, Victoria State, Melbourne

Distribution: Australia, Papua New Guinea

Hosts: Calyptorhynchus funereus, Probosciger aterrimus (Psittaciformes: Cacatuidae)

Neoaulobia krafti Skoracki

Neoaulobia krafti Skoracki, 2005b: 129

Type locality: Australia, New Holland

Distribution: Australia

Host: Cacatua tenuirostris (Psittaciformes: Cacatuidae)

Neoaulobia mexicana Bochkov et Perez

Neoaulobia mexicana Bochkov \& Perez, 2002: 149; Bochkov \& Fain, 2003: 3; Skoracki, 2005b: 128

Type locality: Huacana, Piaxtla, Sinaloa

Distribution: Brazil, Mexico

Hosts: Aratinga canicularis, A. pertinax (Psittaciformes: Psittacidae)

Neoaulobia mironovi Bochkov et Perez

Neoaulobia mironovi Bochkov \& Perez, 2002: 146; Skoracki, 2005b: 129

Type locality: Piaxtla, Sinaloa

Distribution: Mexico

Host: Amazona finschi (Psittaciformes: Psittacidae)

Neoaulobia psittaculae Fain, Bochkov et Mironov

Neoaulobia psittaculae Fain, Bochkov \& Mironov, 2000: 44; Skoracki, 2005b: 129

Type locality: India (Zoo of Antwerp)

Distribution: India

Host: Psittacula cyanocephala (Psittaciformes: Psittaculidae)

Neoaulobia puylaerti (Skoracki et Dabert)

Aulobia puylaerti Skoracki \& Dabert, 1999: 523

Neoaulobia puylaerti (Skoracki et Dabert): Skoracki \& Dabert, 2002: 138; Skoracki, 2005b:

129; Glowska et al., 2015: 21 
Type locality: Namound joga (Togo)

Distribution: Indonesia (Java), Philippines (Luzon), Togo

Hosts: Loriculus philippensis, L. pusillus, (Psittaciformes: Psittaculidae), Poicephalus senegalus (Psittaciformes: Psittacidae)

Genus Neoaulonastus Skoracki

Dissonus Skoracki, 1999a: 155 (praeoccup.)

Neoaulonastus Skoracki, 2004: 445

Type species: Dissonus scirpaceus Skoracki, 1999

Neoaulonastus aegithalos Skoracki

Neoaulonastus aegithalos Skoracki, 2011: 122

Type locality: Opolskie, Turawa Lake

Distribution: Poland

Host: Aegithalos caudatus (Passeriformes: Aegithalidae)

Neoaulonastus apalis Klimovičova, Mikula, Kahure et Hromada

Neoaulonastus apalis Klimovičova, Mikula, Kahure \& Hromada, 2014: 573

Type locality: Kenya

Distribution: Kenya

Host: Apalis porphyrolaema (Passeriformes: Cisticolidae)

Neoaulonastus bisetatus (Fritsch)

Syringophilus bisetatus Fritsch, 1958: 238

Dissonus scirpaceus Skoracki, 1999a: 157; Skoracki \& Hebda, 2004: 5

Neoaulonastus scirpaceus (Skoracki): Skoracki, 2004: 445; Skoracki \& Bochkov, 2010: 65

Neoaulonastus bisetatus (Fritsch, 1958): Skoracki, 2011: 113; Klimovičová \& Hromada, 2014: 33; Pidhorna et al., 2020: 215

Type locality: Germany

Type locality of Dissonus scirpaceus Skoracki: Mierzeja Wiślana (Poland)

Distribution: Germany, Jordan, Kazakhstan, Kenya, Poland, Russia, Slovakia, Ukraine Hosts: Acrocephalus arundinaceus, A. baeticatus, A. dumetorum, A. paludicola, A. palustris, A. scirpaceus (Passeriformes: Acrocephalidae), Sylvia atricapilla, S. communis, S. curruca (Passeriformes: Sylviidae)

Neoaulonastus caligatus Skoracki

Neoaulonastus caligatus Skoracki, 2011: 120

Type locality: Europe

Distribution: Europe

Host: Iduna caligata (Passeriformes: Sylviidae) 
Neoaulonastus chrysocolaptes Skoracky et OConnor

Neoaulonastus chrysocolaptes Skoracky \& OConnor, 2010: 22; Skoracki, 2011: 127; Glowska et al., 2015: 21

Type locality: Luzon Is., Cagayan Prov., Penablanca Munic., betw. Bagio and Iland Rivers Distribution: Philippines

Host: Chrysocolaptes lucidus (Piciformes: Picidae)

Neoaulonastus cinnyris Klimovičova, Smolak, Njiroge et Hromada

Neoaulonastus cinnyris Klimovičova, Smolak, Njiroge \& Hromada, 2014: 256

Type locality: Tanzania, Magamba

Distribution: Tanzania

Host: Cinnyris mediocris (Passeriformes: Nectariniidae)

Neoaulonastus granatina Skoracki, Hromada et Unsoeld

Neoaulonastus granatina Skoracki, Hromada \& Unsoeld, 2013: 372

Type locality: Tanzania, Simangiro

Distribution: Tanzania

Host: Granatina ianthinogaster (Passeriformes: Estrildidae)

Neoaulonastus grewlingi Glowska

Neoaulonastus grewlingi Glowska, 2013: 20

Type locality: Australia

Distribution: Australia

Host: Falcunculus frontatus (Passeriformes: Falcunculidae)

\section{Neoaulonastus lonchurus (Skoracki)}

Krantziaulonastus lonchurus Skoracki, 2011: 133

Neoaulonastus lonchurus (Skoracki): Skoracki, Hromada, Prevuznakova \& Wamiti, 2019: 1806

Type locality: China

Distribution: China, Kenya, Uganda

Hosts: Estrilda astrild, E. atricapilla, E. nonnula, E. erythronotus, E. rhodopyga, E. troglodytes, Lonchura punctulata (Passeriformes: Estrildidae)

Neoaulonastus malaconotus Klimovičova, Skoracki, Njoroge et Hromada

Neoaulonastus malaconotus Klimovičova, Skoracki, Njoroge \& Hromada, 2016: 187

Type locality: Liberia, Grassfield (78300N; 88350W, altitude 165 m.a.s.l.).

Distribution: Kenya, Liberia, Somalia

Hosts: Laniarius aethiopicus, Nilaus afer, Telophorus nigrifrons (Passeriformes: Malaconotidae) 


\section{Neoaulonastus oryzivorus (Skoracki)}

Krantziaulonastus oryzivorus Skoracki, 2011: 131

Neoaulonastus oryzivorus (Skoracki): Skoracki et al., 2019; Skoracki, Hromada, Prevuznakova \& Wamiti, 2019: 1804

Type locality: Japan

Distribution: Japan, Kenya, Uganda

Hosts: Estrilda astrild, E. atricapilla, Lonchura oryzivora (Passeriformes: Estrildidae)

Neoaulonastus picidus Skoracki

Neoaulonastus picidus Skoracki, 2011: 124

Type locality: Bardejov (Slovakia)

Distribution: Germany, Poland, Slovakia

Hosts: Dendrocopos leucotos, D. major, Picus canus (Piciformes: Picidae)

Neoaulonastus prionops Klimovičova, Skoracki et Hromada

Neoaulonastus prionops Klimovičova, Skoracki \& Hromada, 2016: 579

Type locality: Meru Forest, $1676 \mathrm{~m}$

Distribution: Kenya

Host: Prionops sulcifrons (Passeriformes: Vangidae)

Neoaulonastus quelea Skoracki, Hromada et Unsoeld

Neoaulonastus quelea Skoracki, Hromada \& Unsoeld, 2013: 372

Type locality: Tanzania, Arusha, Usa-River

Distribution: Tanzania

Host: Quelea quelea (Passeriformes: Ploceidae)

Neoaulonastus remizus Skoracki

Neoaulonastus remizus Skoracki, 2011: 117

Type locality: Opolskie, Turawa Lake

Distribution: Poland

Host: Remiz pendulinus (Passeriformes: Remizidae)

Neoaulonastus riparius Skoracki

Neoaulonastus riparius Skoracki, 2011: 126

Type locality: Wielkopolska, Katy

Distribution: Poland

Host: Riparia riparia (Passeriformes: Hirundinidae)

Neoaulonastus sidorchukae Zmudzinski, Skoracki et Hromada

Neoaulonastus sidorchukae Zmudzinski, Skoracki \& Hromada, 2019: 84

Type locality: Sri Lanka 
Distribution: Sri Lanka

Host: Leptocoma zeylonica (Passeriformes: Nectariniidae)

Neoaulonastus tanzanicus Skoracki, Hromada et Unsoeld

Neoaulonastus tanzanicus Skoracki, Hromada \& Unsoeld, 2013: 369; Skoracki et al., 2016: 1544

Type locality: Tanzania

Distribution: Madagascar, Tanzania

Hosts: Euplectes axillaris, Ploceus nelicourvi (Passeriformes: Ploceidae)

Neoaulonastus zosterops Skoracki, Antczak et Riegert

Neoaulonastus zosterops Skoracki, Antczak \& Riegert, 2009: 129; Skoracki \& Mironov, 2013: 129

Type locality: Cameroon

Distribution: Cameroon, Russia (Primorskiy Krai)

Hosts: Zosterops erythropleurus, Z. senegalensis (Passeriformes: Zosteropidae)

Genus Neobubophilus Skoracki et Unsoeld

Neobubophilus Skoracki \& Unsoeld, 2016: 816

Type species: Neobubophilus atheneus Skoracki et Unsoeld, 2016

Neobubophilus atheneus Skoracki et Unsoeld

Neobubophilus atheneus Skoracki \& Unsoeld, 2016: 816

Type locality: India

Distribution: India

Hosts: Athene brama, A. noctua (Strigiformes: Strigidae)

Neobubophilus cunicularius Skoracki et Unsoeld

Neobubophilus cunicularius Skoracki \& Unsoeld, 2016: 817

Type locality: San Pedro Dept., New Germania Distr.

Distribution: Paraguay

Host: Athene cunicularia (Strigiformes: Strigidae)

Genus Neoperisterophila Skoracki

Neoperisterophila Skoracki, 2005c: 155

Type species: Neoperisterophila regiusi Skoracki, 2005

\section{Neoperisterophila regiusi Skoracki}

Neoperisterophila regiusi Skoracki, 2005c: 156

Type locality: New Guinea (no more data, presumed to come from Papua New Guinea)

Distribution: New Guinea

Host: Cicinnurus regius (Passeriformes: Paradisaeidae) 
Genus Neosyringophilopsis Skoracki et Sikora

Neosyringophilopsis Skoracki \& Sikora, 2005: 22

Type species: Neosyringophilopsis acanthizus Skoracki et Sikora, 2005

Neosyringophilopsis acanthizus Skoracki et Sikora

Neosyringophilopsis acanthizus Skoracki \& Sikora, 2005: 23; Skoracki, 2011: 206

Type locality: Australia, New Holland

Distribution: Australia

Host: Acanthiza inornata (Passeriformes: Acanthizidae)

Neosyringophilopsis aegithali (Bochkov, Mironov et Skoracki)

Syringophilopsis aegythali Bochkov, Mironov \& Skoracki, 2001: 235; Skoracki, 2004c: 57;

Skoracki \& Hebda, 2004: 5

Neosyringophilopsis aegythali Skoracki \& Sikora, 2005: 26

Neosyringophilopsis aegithali: Nattress \& Skoracki, 2009: 53; Skoracki, 2011: 215;

Klimovičová \& Hromada, 2014: 34

Type locality: Oskuy Village, Chudovo Distr., Novgorod Prov., Russia

Distribution: England, Poland, Russia, Slovakia

Host: Aegithalos caudatus (Passeriformes: Aegithalidae)

Neosyringophilopsis garrulaxi (Bochkov, Mironov et Skoracki)

Syringophilopsis garrulaxi Bochkov, Mironov \& Skoracki, 2001: 236

Neosyringophilopsis garrulaxi (Bochkov, Mironov \& Skoracki): Skoracki \& Sikora, 2005:

26; Skoracki, 2011: 206

Type locality: Kolopani, S. Mustang

Distribution: Nepal

Host: Trochalopteron affine [Garrulax affinis] (Passeriformes: Leiotrichidae)

Neosyringophilopsis locustellus (Skoracki)

Syringophilopsis locustellus Skoracki, 2004: 51

Neosyringophilopsis locustellus: Skoracki \& Sikora, 2005: 26; Skoracki, 2011: 218

Type locality: Zachodniopomorskie, Swinoujstie

Distribution: Poland

Hosts: Locustella luscinioides, L. naevia (Passeriformes: Locustellidae)

\section{Neosyringophilopsis lonchurus Skoracki}

Neosyringophilopsis lonchurae Skoracki, 2008: 45 (also as "lonchurus")

Neosyringophilopsis lonchurus: Skoracki, 2011: 206; Skoracki et al., 2019: 1806

Type locality: Africa

Distribution: Kenya, Uganda 
Hosts: Estrilda astrild, E. atricapilla, E. nonnula, E. erythronotus, E. rhodopyga, E. troglodytes, Spermestes cucullata [Lonchurus cucullatus] (Passeriformes: Estrildidae)

Neosyringophilopsis lybidus Skoracki, Klimovičová, Muchai et Hromada

Neosyringophilopsis lybidus Skoracki, Klimovičová, Muchai et Hromada, 2014: 180

Type locality: Kenya, Ewaso Nigro River, W.Naro Moru

Distribution: Kenya

Host: Pogoniulus bilineatus (Piciformes: Lybiidae)

Neosyringophilopsis phylloscopi Bochkov, Mironov et Skoracki

Syringophilopsis phylloscopi Bochkov, Mironov \& Skoracki, 2001: 234; Skoracki, 2004c: 57

Neosyringophilopsis phylloscopi: Skoracki \& Sikora, 2005: 26

Type locality: St. Malo (France)

Distribution: France, Poland, Slovakia

Hosts: Phylloscopus collybita, Ph. trochilus (Passeriformes: Phylloscopidae)

\section{Neosyringophilopsis troglodytis (Fritsch)}

Syringophilus troglodytis Fritsch, 1958: 238; Kethley, 1970: 65; Skoracki, 1999: 184 Syringophilopsis troglodytis: Bochkov \& Mironov, 1998: 5; Bochkov \& Galloway, 2001: 2023; Skoracki, 2004c: 57

Neosyringophilopsis troglodytis Skoracki \& Sikora, 2005: 26; Nattress \& Skoracki, 2007: 143; Skoracki, 2011: 213; Skoracki, Spicer \& OConnor, 2016: 460

Type locality: Germany

Distribution: Canada, England, Germany, Poland

Hosts: Troglodytes aedon, T. troglodytes (Passeriformes: Troglodytidae)

Genus Niglarobia Kethley

Niglarobia Kethley, 1970: 43

Type species: Niglarobia ereuneti Kethley, 1970

Niglarobia cursoriae Skoracki, Dabert et Schmäschke

Niglarobia cursoriae Skoracki, Dabert \& Schmäschke, 2006: 55; Skoracki, 2011: 288

Type locality: Ahlenhorst Distr.

Distribution: Namibia

Host: Cursorius temminckii (Charadriiformes: Glareolidae)

Niglarobia ereuneti Kethley

Niglarobia ereuneti Kethley, 1970: 44; Skoracki, 2011: 288

Type locality: Sapelo Island, Georgia

Distribution: USA (Georgia)

Host: Calidris [Ereunetes] pusilla (Charadriiformes: Scolopacidae) 
Niglarobia helleri (Oudemans)

Syringophilus helleri Oudemans, 1904: 190; 1906: 62; Dubinin, 1957: 94; Fritsch, 1958: 229

Niglarobia helleri (Oudemans): Kethley, 1970: 47; Bochkov \& Mironov, 1998: 12; Skoracki \& Sikora, 2002: 7; Skoracki, Dabert \& Schmäschke, 2006: 56; Skoracki, 2011: 288

Type locality: France

Distribution: Argentina, France, Poland, USA

Hosts: Arenaria interpres, Tringa flavipes, T. ochropus, T. totanus, T. stagnatilis (Charadriiformes: Scolopacidae)

Niglarobia lapponica Zmudzinski et Unsoeld

Niglarobia lapponica Zmudzinski \& Unsoeld, 2019: 367

Type locality: Germany

Distribution: Germany

Host: Limosa lapponica (Charadriiformes: Scolopacidae)

Niglarobia rhinoptili Fain, Bochkov et Mironov

Niglarobia rhinoptili Fain, Bochkov \& Mironov, 2000: 61; Skoracki \& Dabert, 2002: 138;

Skoracki, 2011: 288

Type locality: Bloemhof

Distribution: South Africa

Host: Smutsornis [Rhinoptilus] africanus (Charadriiformes: Glareolidae)

Niglarobia trouessarti (Oudemans)

Syringophilus trouessarti Oudemans, 1904: 190; 1906: 67; Dubinin, 1957: 94

Niglarobia trouessarti (Oudemans): Kethley, 1970: 47; Skoracki, 2011: 288

Type locality: South America ("Amérique chaude")

Distribution: South America

Host: Aramus guarauma [scolopaceus] (Gruiformes: Aramidae)

Niglarobia vanelli Zmudzinski et Skoracki

Niglarobia vanelli Zmudzinski \& Skoracki, 2018: 704

Type locality: Germany

Distribution: Germany, Russia, Tanzania

Hosts: Vanellus albiceps, V. coconatus, V. crassirostris, V. gregarius, V. lugubris, V. senegallus, V. spinosus, V. vanellus (Charadriiformes: Charadriidae)

Genus Paraniglarobia Skoracki

Paraniglarobia Skoracki, 2011: 290

Type species: Paraniglarobia Skoracki, 2011

Paraniglarobia calidridis (Bochkov et Mironov)

Niglarobia calidridis Bochkov \& Mironov, 1999: 12 
Paraniglarobia calidridis (Bochkov et Mironov): Skoracki, 2011: 291

Type locality: Sary-Su river, Kzyl Orda Prov.

Distribution: Kazakhstan

Host: Calidris minuta (Charadriiformes: Scolopacidae)

Paraniglarobia skorackii (Bochkov et Galloway)

Niglarobia skorackii Bochkov \& Galloway, 2004: 40

Paraniglarobia skorackii (Bochkov et Galloway): Skoracki, 2011: 291

Type locality: Manitoba: Whitewater Lake

Distribution: Canada

Host: Tringa melanoleuca (Charadriiformes: Scolopacidae)

Genus Peristerophila Kethley

Peristerophila Kethley, 1970: 53

Type species: Syringophilus columbae Hirst, 1920

Castosyringophilus Bochkov \& Perez, 2002: 151 (syn. by Skoracki, Hromada, Kaszewska \& Sikora, 2020)

Type species: Peristerophila mucuya Casto, 1980

Peristerophila accipitridicus Skoracki, Lontkowski et Stawarczyk

Peristerophila accipitridicus Skoracki, Lontkowski \& Stawarczyk, 2010: 1207; Skoracki \& Bochkov, 2010: 66; Skoracki, 2011: 297; Zmudzinski et al., 2015: 292; Skoracki et al., 2016: 1545

Type locality: bird imported from Africa in Wroclaw Zoo

Distribution: Africa, France, Germany, Kazakhstan, Madagascar

Hosts: Accipiter francesiae, A. nisus, Buteo lagopus, Circaetus gallicus, Terathopius ecaudatus (Accipitriformes: Accipitridae)

\section{Peristerophila claravis (Skoracki et Glowska)}

Castosyringophilus claravis Skoracki \& Glowska, 2008b: 155

Peristerophila claravis (Skoracki et Glowska): Skoracki et al., 2020: 1803; Kaszewska et al., 2020: 358

Type locality: Sierra de Chiquitos

Distribution: Bolivia, Colombia, Ethiopia, Panama, Paraguay, Sudan, Tanzania

Hosts: Claravis pretiosa, Oenas capensis (Columbiformes: Columbidae)

\section{Peristerophila columbae (Hirst)}

Syringophilus columbae Hirst, 1920: 121; 1922: fig. 41; Dubinin, 1957: 94; Brown, 1971: 1380 Peristerophila columba (Hirst): Kethley, 1970: 56; Casto, 1976; Bochkov \& Mironov, 1998: 14

Peristerophila columbae (Hirst): Nattress \& Skoracki, 2009: 53; Skoracki, 2011: 294; Kaszewska et al., 2020: 355 
Type locality: Tennessee (USA)

Distribution: Angola, Argentina, Canada, China, D.R. Congo, England, Germany, Greece, Hungary, India, Iran, Japan, Jordan, Kenya, Nepal, N. Macedonia, Panama, Poland, Portugal, South Africa, Suriname, Tanzania, Turkey, USA (Tennessee, Kansas, etc.) Hosts: Columba arguatrix, C. guinea, C. leuconota, C. livia, C. oenas, C. palumbus, C. trocaz, Geotrygon chiriquensis, Patagioenas speciosa, Streptopelia capicola, S. decaocto, S. decipiens, S. orientalis, S. semitorquata, S. transquebarica, S. turtur, Zenaida auriculata (Columbiformes: Columbidae); Buteo jamaicensis (Accipitriiformes: Accipitridae)

Peristerophila coraciidus Skoracki, Hromada et Sikora

Peristerophila coraciidus Skoracki, Hromada \& Sikora, 2020: 175

Type locality: Papua New Guinea

Distribution: Papua New Guinea

Host: Eurystomus orientalis (Passeriformes: Coraciidae)

Peristerophila falcophila Skoracki, Hromada, Kaszewska, Kavetska et Unsoeld Peristerophila falcophila Skoracki, Hromada, Kaszewska, Kavetska \& Unsoeld, 2018: 744 Type locality: Germany

Distribution: Germany, Kenya, Nepal, Romania, Colombia

Hosts: Falco cherrug, F. peregrinus, F. sparverius (Falconiformes: Falconidae)

Peristerophila forpi Bochkov et Perez

Castosyringophilus forpi Bochkov \& Perez, 2002: 152; Skoracki, 2005: 128

Peristerophila forpi (Bochkov et Perez): Skoracki et al., 2020: 1803

Type locality: Ruiz, Nayarit

Distribution: Mexico

Host: Forpus cyanopygius (Psittaciformes: Psittacidae)

Peristerophila geopelis Kaszewska, Skoracki, Kosicki et Hromada

Peristerophila geopelis Kaszewska, Skoracki, Kosicki \& Hromada, 2020: 353

Type locality: Java, Trinil

Distribution: Indonesia (Java, Sumatra, Sulawesi), Australia

Hosts: Geopelia cuneata, G. placida, G. striata, Ocyphaps lophotes

(Columbiformes: Columbidae)

Peristerophila lature Kaszewska, Kavetska et Skoracki

Peristerophila lature Kaszewska, Kavetska \& Skoracki, 2014: 296; Kaszewska et al., 2020: 358 Type locality: Sulawesi (Indonesia)

Distribution: Indonesia (Sulawesi, Java, Sumatra), Mariana Is., Papua New Guinea Hosts: Ducula luctuosa, D. spilorrhoa, Ptilinopus jambu, P. melanospilus, P. porphyreus, $P$. regina (Columbiformes: Columbidae: Treroninae) 
Peristerophila leucomela Kaszewska, Skoracki, Kosicki et Hromada

Peristerophila leucomela Kaszewska, Skoracki, Kosicki \& Hromada, 2020: 355

Type locality: Australia

Distribution: Australia

Host: Columba leucomela (Columbiformes: Columbidae)

Peristerophila meropis (Skoracki, Hromada et Sikora)

Castosyringophilus meropis Skoracki, Hromada \& Sikora, 2017: 24

Peristerophila meropis (Skoracki, Hromada et Sikora): Skoracki, Hromada, Kaszewska \& Sikora, 2020: 1803

Type locality: Sevilla (Spain)

Distribution: Bosnia \& Herzegovina, France (Corsica), Gibraltar, Greece, Italy (Sardinia), Kenya, N. Macedonia, Morocco, Pakistan, Romania (N. Dobroudja), Russia, Tanzania, Turkey

Hosts: Merops apiaster (Coraciiformes: Meropidae), Todiramphus chloris (Cor.: Alcedinidae)

\section{Peristerophila mucuya Casto}

Peristerophila mucuya Casto, 1980: 1; Skoracki, Hromada, Kaszewska \& Sikora, 2020: 1803; Kaszewska et al., 2020: 359

Castosyringophilus mucuya (Casto): Bochkov \& Perez, 2002: 6; Skoracki, 2005b: 128; Skoracki \& Glowska, 2008b: 158

Type locality: Texas, La Sella

Distribution: Argentina, Australia, Brazil, Colombia, Indonesia (Sumbava Is.), Jordan, Monaco, Paraguay, Peru, Suriname, Trinidad \& Tobago, USA (Texas)

Hosts: Bolborhynchus aymara, Brotogeris versicolurus (Psittaciformes: Psittacidae), Trichoglossus haematodus (Psittaciformes: Psittaculidae), Columbina minuta, C. passerina, C. squammata, C. talpacoti, Geophaps plumifera, Metropelia ceciliae, M. melanoptera, Scardafella squamata, Streptopelia decaocto, Petrophassa plumifera (Columbiformes: Columbidae)

Peristerophila nestoriae Marciniak, Skoracki et Hromada

Peristerophila nestoriae Marciniak, Skoracki \& Hromada, 2019: 2

Type locality: New Zealand

Distribution: New Zealand

Host: Nestor meridionalis (Psittaciformes: Strigopidae)

Peristerophila upupi Klimovičova, Mikula, Kahure et Hromada

Peristerophila upupi Klimovičova, Mikula, Kahure \& Hromada, 2014: 575

Type locality: Nyahururu

Distribution: Kenya 
Host: Upupa epops (Upupiformes: Upupidae)

Genus Phalarophilus Skoracki, Bochkov et OConnor

Phalarophilus Skoracki, Bochkov \& OConnor, 2011: 325

Type species: Phalarophilus fulicarius Skoracki, Bochkov et OConnor, 2011

Phalarophilus fulicarius Skoracki, Bochkov et OConnor

Phalarophilus fulicarius Skoracki, Bochkov \& OConnor, 2011: 328

Type locality: San Diego Co., San Diego

Distribution: USA

Host: Phalaropus fulicarius (Charadriiformes: Scolopacidae)

Genus Philoxanthornia Kethley

Philoxanthornia Kethley, 1970: 50

Philoxanthornea (Zool. Record, misspelling)

Type species: Philoxanthornia anoa Kethley, 1970

\section{Philoxanthornia anoa Kethley}

Philoxanthornia anoa Kethley, 1970: 53; Skoracki, 2011: 282

Type locality: Sand Island

Distribution: Midway Atoll (USA)

Host: Anous tenuirostris (Charadriiformes: Laridae, Sterninae)

\section{Philoxanthornia clarki Kivganov}

Philoxanthornea clarki Kivganov, in Kivganov \& Sharafat, 1995: 87; Skoracki, 2011: 282

Type locality: Odessa Prov., Tiligulski liman

Distribution: Russia, Ukraine

Hosts: Sterna hirundo, Sternula albifrons (Charadriiformes: Laridae, Sterninae), Larus canus (Charadriiformes: Laridae)

\section{Philoxanthornia dubinini Bochkov et Mironov}

Philoxanthornea dubinini Bochkov \& Mironov, 1999: 14; Skoracki, 2011: 285

Type locality: Sorbulak Lake, near Alma-Ata

Distribution: Egypt, Kazakhstan

Hosts: Chlidonias leucopterus, Gelochelidon nilotica (Charadriiformes: Laridae)

Genus Picineoaulonastus Skoracky, Klimovičová, Mucha et Hromada

Picineoaulonastus Skoracky, Klimovičová, Mucha \& Hromada, 2014: 180

Type species: Picineoaulonastus pogoniulus Skoracky, Klimovičová, Mucha et Hromada, 2014 
Picineoaulonastus pogoniulus Skoracky, Klimovičová, Muchai et Hromada Picineoaulonastus pogoniulus Skoracky, Klimovičová, Muchai \& Hromada, 2014: 180 Type locality: Kenya

Distribution: Ethiopia, Kenya, Tanzania

Hosts: Pogoniulus bilineatus, P. pusillus (Piciformes: Lybiidae)

Genus Picisyringophilus Skoracky et OConnor

Picisyringophilus Skoracky \& OConnor, 2010: 12

Type species: Picisyringophilus kratos Skoracky et OConnor, 2010

Picisyringophilus kratos Skoracky et OConnor

Picisyringophilus kratos Skoracky \& OConnor, 2010: 15

Type locality: Michigan, Washtenaw Co., 2mi NW Dexter

Distribution: USA (Michigan)

Host: Picoides pubescens (Piciformes: Picidae)

Genus Procellariisyringophilus Schmid et Skoracki

Syringonomus Kethley, 1970: 56 (praeoccup.)

Procellariisyringophilus Schmid \& Skoracki, 2007: 545

Type species: Syringonomus bulwerius Kethley, 1970

\section{Procellariisyringophilus bulwerius (Kethley)}

Syringonomus bulwerius Kethley, 1970: 59

Procellariisyringophilus bulwerius (Kethley): Schmid \& Skoracki, 2007: 545

Type locality: Nihoa Island, Hawaiian Is.

Distribution: Greece, Indonesia, USA (Hawaiian Is.)

Hosts: Ardenna pacifica, Bulwerius bulwerius, Calonectris diomedea (Procellariiformes: Procellariidae)

Genus Psittaciphilus Fain, Bochkov et Mironov

Psittaciphilus Fain, Bochkov \& Mironov, 2000: 44

Type species: Psittaciphilus amazonae Fain, Bochkov et Mironov, 2000

Psittaciphilus amazonae Fain, Bochkov et Mironov

Psittaciphilus amazonae Fain, Bochkov \& Mironov, 2000: 47; Bochkov \& Fain, 2003;

Skoracki, 2005b: 128

Type locality: Colombia

Distribution: Brazil, Colombia

Hosts: Amazona aestiva, A. amazonica (Psittaciformes: Psittacidae) 
Psittaciphilus fritschi Fain, Bochkov et Mironov

Psittaciphilus fritschi Fain, Bochkov \& Mironov, 2000: 47; Skoracki, 2005b: 128

Type locality: Zoo of Antwerp

Distribution:?

Host: parrot (Psittaciformes)

Psittaciphilus montanus Kaszewska et Skoracki

Psittaciphilus montanus Kaszewska \& Skoracki, 2018: 1

Type locality: Trinidad I., Aripo Mt.

Distribution: Brazil, Panama, Trinidad \& Tobago

Host: Geotrygon montanus (Columbiiformes: Columbidae)

Psittaciphilus patagioenas Kaszewska et Skoracki

Psittaciphilus patagioenas Kaszewska \& Skoracki, 2018: 3

Type locality: Near San Juan River, Chocó Department Lama Hermosa

Distribution: Colombia

Hosts: Patagioenas fasciata, P. speciosa (Columbiiformes: Columbidae)

Genus Pteroclidisyringophilus Skoracki

Pteroclidisyringophilus Skoracki, 2011: 31

Type species: Charadriiphilus re Skoracky et OConnor, 2010

Pteroclidisyringophilus otididus Skoracky, Unsoeld et Skorupski

Pteroclidisyringophilus otididus Skoracky, Unsoeld \& Skorupski, 2014: 349

Type locality: South Tunisia

Distribution: Tunisia

Host: Chalamydotis undulata (Otidiformes: Otididae)

Pteroclidisyringophilus re (Skoracky et OConnor)

Charadriiphilus re Skoracky \& OConnor, 2010: 18

Pteroclidisyringophilus re (Skoracky et OConnor): Skoracki, 2011: 264

Type locality: Red Sea Governate, Wadi el Metuli

Distribution: Egypt

Host: Pterocles senegallus (Pterocliformes: Pteroclidae)

Genus Selenonycha Kethley

Selenonycha Kethley, 1970: 31

Type species: Selenonycha baltoda Kethley, 1970

Selenonycha baltoda Kethley

Selenonycha baltoda Kethley, 1970: 34; Bochkov \& Mironov, 1998: 11 
Type locality: Cameron Parish, Louisiana

Distribution: USA

Hosts: Charadrius placidus, Ch. w. wilsonia, Chlidonius niger (Charadriiformes: Charadriidae)

Selenonycha charadriiformicus Skoracki

Selenonycha charadriiformicus Skoracki, 2011: 276

Type locality: Lake Manzala area (Egypt)

Distribution: Egypt, Germany, Poland, Russia

Hosts: Chroicocephalus genei, Ch. ridibundus (Charadriiformes: Laridae), Charadrius placidus (Charadriiformes: Charadriidae), Philomachus pugnax (Charadriiformes: Scolopacidae)

Selenonycha insperata Zmudzinski et Skoracki

Selenonycha insperata Zmudzinski \& Skoracki, 2017: 594

Type locality: Misiones Prov., Laguna San Jose de Posadas

Distribution: Argentina

Host: Phimosus infuscatus (Pelecaniformes: Threskiornithidae)

Genus Stibarokris Kethley

Stibarokris Kethley, 1970: 62

Type species: Stibarokris phalacrus Kethley, 1970

Ciconichenophilus Skoracky \& OConnor, 2010: 12

Type species: Ciconichenophilus phoeniconaias Skoracky et OConnor, 2010

Stibarokris annae Glowska

Stibarokris annae Glowska, 2015a: 74

Type locality: Uruguay

Distribution: Uruguay

Host: Ciconia maguari (Ciconiiformes: Ciconiidae)

Stibarokris brevisetosus Skoracki et Zmudzinski

Stibarokris brevisetosus Skoracki \& Zmudzinski [in Skoracki et al., 2016], 2016: 1319

Type locality: Turkey, Ekmekci

Distribution: Turkey

Host: Plegadis falcinellus (Pelecaniformes: Threskiornithidae)

Stibarokris dastychi Glowska et Skoracky

Stibarokris dastychi Glowska \& Skoracky, 2011: 64

Type locality: Germany

Distribution: Germany 
Host: Phalacrocorax carbo (Suliformes: Phalacrocoracidae)

Stibarokris geronticus Skoracki et Zmudzinski

Stibarokris geronticus Skoracki \& Zmudzinski [in Skoracki et al., 2016], 2016: 1313

Type locality: South Africa

Distribution: South Africa

Host: Geronticus calurus (Pelecaniformes: Threskiornithidae)

Stibarokris hydrobates Zmudzinski, Solarczyk et Skoradski

Stibarokris hydrobates Zmudzinski, Solarczyk \& Skoradski, 2016: 432

Type locality: Malta, Filfla

Distribution: Malta

Host: Hydrobates pelagicus (Procellariiformes: Hydrobatidae)

Stibarokris langei Bochkov et Mironov

Stibarokris langei Bochkov \& Mironov, 1999: 35; Glowska \& Skoracky, 2011: 68

Type locality: Novgorod Prov., Chudovo Distr., Oskuy Vill.

Distribution: Russia

Host: Ciconia alba (Ciconiiformes: Ciconiidae)

Stibarokris mariasi Glowska

Stibarokris mariasi Glowska, 2015b: 616

Type locality: Johnston Atoll

Distribution: Johnston Atoll

Host: Puffinus pacificus (Procellariiformes: Procellariidae)

Stibarokris phalacrus Kethley

Stibarokris phalacrus Kethley, 1970: 62; Glowska \& Skoracky, 2011: 67

Type locality: Pigeon Key, Monroe Co., Florida

Distribution: USA (Florida)

Host: Phalacrocorax auritus (Suliformes: Phalacrocoracidae)

Stibarokris phoeniconaias (Skoracky et OConnor)

Ciconichenophilus phoeniconaias Skoracky \& OConnor, 2010: 13

Stibarokris phoeniconaias Skoracky \& OConnor, 2010: 25; Glowska \& Skoracky, 2011:

66; Zmudzinski, Solarczyk \& Skoradski, 2016: 434; Skoracki, Zmudzinski, Unsoeld \& Sikora, 2016: 9

Type locality: Botswana

Distribution: Botswana, Turkey, South America

Hosts: Phoeniconaias minor, Phoenicopterus ruber, Ph. chilensis (Phoenicopteriformes: Phoenicopteridae) 
Stibarokris plataleus Skoracki et Zmudzinski

Stibarokris plataleus Skoracki \& Zmudzinski, [in Skoracki et al., 2016]. 2016: 1316

Type locality: Neusiedl See

Distribution: Austria

Host: Platalea leucorodia (Pelecaniformes: Threskiornithidae)

Stibarokris theristicus Skoracki, Zmudzinski et Unsoeld

Stibarokris theristicus Skoracki, Zmudzinski \& Unsoeld [in Skoracki et al., 2016]. 2016: 1313

Type locality: Marajo Island, Pacoval

Distribution: Brazil

Host: Theristicus caudatus (Pelecaniformes: Threskiornithidae)

Genus Syringophiloidus Kethley

Syringophiloidus Kethley, 1970: 48

Type species: Syringophilus minor Berlese, 1887

Syringophiloidus agelaius Bochkov, Skoracki, Hendricks et Spicer

Syringophiloidus agelaius Bochkov, Skoracki, Hendricks \& Spicer, 2011: 209

Type locality: Cibola National Wildlife Refuge (Arizona)

Distribution: USA (Arizona)

Host: Agelaius phoeniceus (Passeriformes: Icteridae)

Syringophiloidus amazilia Skoracki

Syringophiloidus amazilia Skoracki, 2017: 181

Type locality: Veracrux, Los Tuxlas

Distribution: Mexico

Host: Amazilia candida (Passeriformes: Trochilidae)

Syringophiloidus apus Skoracki, Kaszewska et Kavetska

Syringophiloidus apus Skoracki, Kaszewska \& Kavetska, 2015: 584

Type locality: Chile

Distribution: Chile

Host: Apus melba (Apodiformes: Apodidae)

\section{Syringophiloidus artamus Skoracki}

Syringophiloidus artamus Skoracki, 2004: 288; 2011: 36

Type locality: Sumatra

Distribution: Indonesia (Sumatra)

Hosts: Artamus fuscus, A. leucorhynchus (Passeriformes: Artamidae) 
Syringophiloidus auduboni Skoracki, Spicer et OConnor

Syringophiloidus auduboni Skoracki, Spicer \& OConnor, 2016: 462

Type locality: California, Riverside Co.

Distribution: USA

Host: Spizella breweri (Passeriformes: Passerellidae)

Syringophiloidus bombycillae Skoracki

Syringophiloidus bombycillae Skoracki, 2002: 305; 2004: 296; 2011: 36; Skoracki, Spicer \& OConnor, 2016: 468

Type locality: Bardejov (Slovakia)

Distribution: Poland, Slovakia, USA

Hosts: Bombycilla cedrorum, B. garrulus (Passeriformes: Bombycillidae)

Syringophiloidus bucerotidus Skoracki

Syringophiloidus bucerotidus Skoracki, 2017: 39

Type locality: D.R.Congo

Distribution: Cameroon, D.R. Congo, Tanzania

Hosts: Horizocerus hartlaubi, H. albocristatus, Lophoceros pallidirostris, Tockus flavirostris, T. fasciatus, T. deckeni (Bucerotiformes: Bucerotidae)

Syringophiloidus carolae Skoracki, Flannery et Spicer

Syringophiloidus carolae Skoracki et al., 2009: 66; Skoracki, 2011; Skoracki, Spicer \& OConnor, 2016: 464

Type locality: Texas, Hood Co., Jones Ranch

Distribution: USA (Texas)

Hosts: Cardinalis cardinalis, Passerina caerulea (Passeriformes: Cardinalidae), Melanerpes formicivorus (Piciformes: Picidae)

Syringophiloidus carpodaci Bochkov et Apanaskevich

Syringophiloidus carpodaci Bochkov \& Apanaskevich, 2001: 323; Skoracki, 2011: 50

Type locality: Southwestern Altai, Zyryanovskii Distr., Shumovsk Village (Kazakhstan)

Distribution: Kazakhstan, Poland, Russia

Host: Carpodacus erythrinus (Passeriformes: Fringillidae)

Syringophiloidus cincli Skoracki

Syringophiloidus cincli Skoracki, 2017: 184

Type locality: Puebla, Scapa, 3 Mi NE Huauchinango

Distribution: Mexico

Host: Cinclus mexicanus (Passeriformes: Cinclidae) 


\section{Syringophiloidus coccothraustes Skoracki}

Syringophiloidus coccothraustes Skoracki, 2011: 82

Type locality: Wielkopolskie, Katy near Poznan

Distribution: Germany, Poland

Host: Coccothraustes coccothraustes (Passeriformes: Fringillidae)

Syringophiloidus cypsiuri Fain, Bochkov et Mironov

Syringophiloidus cypsiuri Fain, Bochkov \& Mironov, 2000: 51; Skoracki, 2011: 36

Type locality: Kasai Prov., DR Congo

Distribution: DR Congo

Host: Cypsiurus parvus (Apodiformes: Apodidae)

Syringophiloidus daberti Bochkov, Fain et Skoracki

Syringophiloidus daberti Bochkov, Fain \& Skoracki, 2004: 144; Bochkov, Skoracki, Hendricks \& Spicer, 2011: 210; Skoracki et al., 2011; Skoracki, Spicer \& OConnor, 2016: 465 Type locality: Mexico (Anwerp Zoo)

Distribution: Mexico, USA (Texas)

Host: Passerina ciris (Passeriformes: Cardinalidae)

\section{Syringophiloidus delichonum Bochkov}

Syringophiloidus delichonum Bochkov, 2001: 151; Skoracki, 2004: 297; 2011: 69; Skoracki, Spicer \& OConnor, 2016: 465

Type locality: Kaliningradskaya Oblast, Kurshskaya Kosa, Ribachiy Vill. (Russia)

Distribution: Poland, Russia, USA

Hosts: Delichon urbicum, Hirundo rustica (Passeriformes: Hirundinidae)

Syringophiloidus dendrocittae Fain, Bochkov et Mironov

Syringophiloidus dendrocitta Fain, Bochkov \& Mironov, 2000: 55; Skoracki, 2011: 36

Type locality: East Asia (Zoo of Antwerp)

Distribution: East Asia

Hosts: Dendrocitta vagabunda (= rufa rufa)(Passeriformes: Corvidae, Sturnus vulgaris (Passeriformes: Sturnidae), [Cyanocitta stelleri (Passeriformes: Corvidae)]

Syringophiloidus erythrurus Skoracki

Syringophiloidus erythrurus Skoracki, 2004: 293

Type locality: Tenerife

Distribution: Spain (Tenerife)

Hosts: Erythrura trichroa, Lonchura sp. (Passeriformes: Estrildidae)

Syringophiloidus estrildus Skoracki, Hromada, Prevuznakova et Wamiti Syringophiloidus estrildus Skoracki, Hromada, Prevuznakova \& Wamiti, 2019: 1802 Type locality: Masindi 
Distribution: Uganda

Host: Estrilda rhodopyga (Passeriformes: Estrildidae)

Syringophiloidus furthi Glowska et Schmidt

Syringophiloidus Skoracki \& Schmidt, 2014: 61

Type locality: Ogooue Maritime Prov., Moukalaba-Doudou National Park, NW corner of N'dogo Lagoon

Distribution: Gabon

Host: Terpsiphone rufiventer (Passeriformes: Monarchidae)

\section{Syringophiloidus glandarii (Fritsch)}

Syringophilus minor glandarii Fritsch, 1958: 235

Syringophiloidus glandarii (Fritsch): Bochkov \& Mironov, 1998: 14; Skoracki, 2011: 57;

Skoracki, Spicer \& OConnor, 2016: 465

Type locality: Germany

Distribution: Germany, Japan, Kazakhstan, Russia, USA

Hosts: Corvus brachyrhynchos, C. frugilegus, C. monedula, Cyanocitta stelleri, Garrulus glandarius, Pica pica (Passeriformes: Corvidae). For other hosts see the notes in Glowska, Chrzanowski \& Kaszewska, 2015: 32.

Syringophiloidus graculae Fain, Bochkov et Mironov

Syringophiloidus graculae Fain, Bochkov \& Mironov, 2000: 51

Type locality: SE Asia

Distribution: SE Asia

Host: Gracula religiosa (Passeriformes: Sturnidae)

Syringophiloidus hirundinis Skoracki, Moller et Tryjanowski

Syringophiloidus hirundinis Skoracki, Skoracki, Moller \& Tryjanowski, 2003: 17; Pidhorna, 2020: 216

Type locality: Milano (Italy)

Distribution: Denmark, Italy, Poland, Spain, Ukraine

Host: Hirundo rustica (Passeriformes: Hirundinidae)

Syringophiloidus jackowiaki Bochkov, Skoracki, Hendricks et Spicer Syringophiloidus jackowiaki Bochkov, Skoracki, Hendricks \& Spicer, 2011: 208

Type locality: Texas, Trinity Co., Davy Crocket Nat. Forest

Distribution: USA (Texas)

Host: Poecile carolinensis (Passeriformes: Paridae)

Syringophiloidus klimovi Skoracki et Bochkov

Syringophiloidus klimovi Skoracki \& Bochkov, 2010: 61

Type locality: Ongtustik, Qazaqstan 
Distribution: Germany, Kazakhstan

Host: Chloris chloris (Passeriformes: Fringillidae)

Syringophiloidus microcerculus Sikora et Skoracki

Syringophiloidus microcerculus Sikora \& Skoracki, 2012: 58

Type locality: Para, Peixe-Boi

Distribution: Brazil

Host: Microcerculus marginatus (Passeriformes: Troglodytidae)

Syringophiloidus minor (Berlese)

Syringophilus bipectinatus minor Berlese, 1887: 10

Syringophilus minor minor (Berlese): Fritsch, 1958: 235

Syringophiloidus minor (Berlese): Kethley, 1970; Bochkov \& Mironov, 1998; Bochkov \& Galloway, 2001; Skoracki \& Dabert, 2002; Glowska et al., 2007; Skoracki, 2011: 39;

Skoracki, Spicer \& OConnor, 2016: 467

Type locality: Europe

Distribution: Bosnia and Herzegovina, Canada, China, Congo, Croatia, England, Germany, Jordan, Kirghizia, Poland, Russia, Slovakia, USA

Hosts: Passer domesticus, P. hispaniolensis, P. montanus (Passeriformes: Passeridae), Serinus capistratus (Passeriformes: Fringillidae)

Syringophiloidus molothrus Skoracki, Flannery et Spicer

Syringophiloidus molothrus Skoracki, Flannery \& Spicer, 2009: 66; Skoracki, Spicer \& OConnor, 2016: 466

Type locality: Texas, Hood Co.

Distribution: USA (Texas, Michigan)

Host: Icterus galbula, Molothrus ater (Passeriformes: Icteridae)

\section{Syringophiloidus montanus Skoracki}

Syringophiloidus montanus Skoracki, 2002: 310

Type locality: Bardejov

Distribution: Slovakia

Host: Passer montanus (Passeriformes: Passeriformes)

Syringophiloidus nectariniae Skoracki et Zmudzinski

Syringophiloidus nectariniae Skoracki \& Zmudzinski, 2018: 1

Type locality: Tanzania

Distribution: Tanzania

Host: Hedydipna collaris (Passeriformes: Nectariniidae)

Syringophiloidus nkaii Klimovičova, Skoracki, Njoroge et Hromada Syringophiloidus nkaii Klimovičova, Skoracki, Njoroge \& Hromada, 2016: 188 
Type locality: Kenya, Archers Post (altitude 1,200 m.a.s.1.).

Distribution: Kenya

Host: Nilaus afer (Passeriformes: Malaconotidae)

Syringophiloidus oriolus Skoracki

Syringophiloidus oriolus Skoracki, 2004: 283

Type locality: Western Europe

Distribution: China, Western Europe

Hosts: Oriolus chinensis, O. oriolus (Passeriformes: Oriolidae)

\section{Syringophiloidus parapresentalis Skoracki}

Syringophiloidus parapresentalis Skoracki, 2011: 63; Skoracki, Spicer \& OConnor, 2016: 466; Skoracki, Mironov, Hernandes et Valim, 2016: 5

Type locality: Wielkopolskie, Jeziory, near Poznan

Distribution: Brazil, Germany, Jordan, Kazakhstan, Poland, Russia, Slovakia, USA (California)

Hosts: Turdus atrogularis, T. iliacus, T. merula, T. migratorius, T. pilaris, T. rufiventris (Passeriformes: Turdidae)

\section{Syringophiloidus petronicus Skoracki}

Syringophiloidus petronicus Skoracki, 2011: 45

Type locality: Italy

Distribution: Italy

Host: Petronia petronia (Passeriformes: Passeridae)

\section{Syringophiloidus philomelosus Skoracki}

Syringophiloidus philomelosus Skoracki, 2011: 43

Type locality: Al-Dhleil near Amman

Distribution: Jordan

Host: Turdus philomelos (Passeriformes: Turdidae)

Syringophiloidus picidus Skoracki, Klimičova, Muchai et Hromada

Syringophiloidus picidus Skoracki, Klimičova, Muchai \& Hromada, 2014: 184

Type locality: Mutanda (Kenya)

Distribution: Germany, Kenya, Tanzania, Uganda

Host: Dendropicus fuscescens (Piciformes: Picidae)

Syringophiloidus plocei Glowska, Broda et Dabert

Syringophiloidus plocei Glowska, Broda \& Dabert, 2016: 563

Type locality: Ogooue Maritime Province, Gamba Complex of Protected Areas, near mouth of Nyanga River

Distribution: Gabon 
Hosts: Ploceus aurantius, P. cucullatus, P. nigerrimus (Passeriformes: Ploceidae)

Syringophiloidus presentalis Chirov et Kravtsova

Syringophiloidus presentalis Chirov \& Kravtsova, 1995: 376; Skoracki, 2011: 60; Skoracki, Spicer \& OConnor, 2016: 466

Type locality: Bishkek (Kirghizia)

Distribution: France, Kirghizia, Poland, Slovakia, USA

Host: Sturnus vulgaris (Passeriformes: Sturnidae)

Syringophiloidus pseudonigritae Glowska, Dragun-Damian et Dabert

Syringophiloidus pseudonigritae Glowska, Dragun-Damian \& Dabert, 2012b: 65

Type locality: Tanzania

Distribution: Tanzania

Host: Pseudonigrita arnaudi (Passeriformes: Ploceidae)

Syringophiloidus quetzali Skoracki, Mironov et Unsoeld

Syringophiloidus quetzali Skoracki, Mironov \& Unsoeld, 2013: 292

Type locality: Guatemala

Distribution: Ecuador, Guatemala

Hosts: Pharomachrus antisianus, Ph. mocinno (Trogoniformes: Trogonidae)

Syringophiloidus serini Bochkov, Fain et Skoracki

Syringophiloidus serini Bochkov, Fain \& Skoracki, 2004: 144; Skoracki, 2011: 38

Type locality: Central Africa (Antwerp Zoo)

Distribution: Central Africa

Host: Serinus mozambicus (Passeriformes: Fringillidae)

Syringophiloidus sialius Skoracki, Flannery et Spicer

Syringophiloidus sialius Skoracki, Flannery \& Spicer, 2009: 67; Bochkov, Skoracki, Hendricks \& Spicer, 2011: 211; Skoracki, 2011: 38

Type locality: Mendocino Co., Hopland

Distribution: USA (California)

Hosts: Sialia mexicana (Passeriformes: Turdidae), Spizella breweri (Passeriformes: Passerellidae)

\section{Syringophiloidus sporophila Skoracki}

Syringophiloidus sporophila Skoracki, 2017: 185

Type locality: Veracruz, Los Tuxlas

Distribution: Mexico

Host: Sporophila torqueola (Passeriformes: Thraupidae) 


\section{Syringophiloidus stawarczyki Skoracki}

Syringophiloidus stawarczyki Skoracki, 2004: 291; 2011: 38; Skoracki, Mironov, Hernands

\& Valim, 2016: 5

Type locality: Brazil

Distribution: Brazil

Hosts: Euphonia cyanocephala (Passeriformes: Emberizidae), Dacnis cayana, Tachyphonus rufus (Pass., Thraupidae)

Syringophiloidus tarnii Skoracki et Sikora

Syringophiloidus tarnii Skoracki \& Sikora, 2002: 2 (and tarni, p.1); Skoracki, 2011: 38

Type locality: El Bolson, Rio Negro (Argentina)

Distribution: Argentina

Host: Pteroptochos tarnii (Passeriformes: Rhinocryptidae)

Syringophiloidus teledromas Sikora, Kaszewska et Skoracki

Syringophiloidus teledromas Sikora, Kaszewska \& Skoracki, 2014: 420

Type locality: Argentina - RioNegro, Cipolletti, Neuquén

Distribution: Argentina

Host: Teledromas fuscus (Passeriformes: Rhinocryptidae)

Syringophiloidus thryothorus Skoracki, Flannery et Spicer

Syringophiloidus thryothorus Skoracki, Flannery \& Spicer, 2009: 69; Skoracki, 2011:

Type locality: Texas, Hood Co., Jones Ranch

Distribution: USA (Texas)

Host: Thryothorus ludovicianus (Passeriformes: Troglodytidae)

Syringophiloidus weiszii Skoracki, Hromada et Tryjanowski

Syringophiloidus weiszii Skoracki, Hromada \& Tryjanowski, 2001: 30; Skoracki, 2011: 79

Type locality: Bardejov

Distribution: Germany, Slovakia, USA

Hosts: Lanius excubitor, L. ludovicianus (Passeriformes: Laniidae)

Syringophiloidus xanthocephalus Bochkov, Skoracki, Hendricks et Spicer

Syringophiloidus xanthocephalus Bochkov, Skoracki, Hendricks \& Spicer, 2011: 208

Type locality: Cibola National Wildlife Refuge (Arizona)

Distribution: USA (Arizona)

Host: Xantocephalus xanthocephalus (Passeriformes: Icteridae)

Syringophiloidus zonotrichia Bochkov, Skoracki, Hendricks et Spicer

Syringophiloidus motacillae of Bochkov \& Galloway (2001: 2025) and Skoracki et al. (2009:

72) (incorrect determinations) 
Syringophiloidus zonotrichia Bochkov, Skoracki, Hendricks \& Spicer, 2011: 203

Type locality: Garen Ranch, Johnson Co. Texas,

Distribution: Canada, USA (Texas)

Host: Zonotrichia albicolis (Passeriformes: Emberizidae)

\section{Syringophiloidus sp.}

Host: Campylorhynchus brunneicapillum (Paseriformes: Troglodytidae)

Genus Syringophilopsis Kethley

Syringophilopsis Kethley, 1970: 18

Type species: Syringophilus elongatus Ewing, 1911

\section{Syringophilopsis acrocephali Skoracki}

Syringophilopsis acrocephali Skoracki, 1999a: 160; 2011: 179; Klimovičová \& Hromada, 2014: 34; Pidhorna, 2020: 216

Type locality: Mierzeja Wiślana (Poland)

Distribution: Egypt, Poland, Russia, Slovakia, Ukraine

Hosts: Acrocephalus palustris, A. schoenobaenus, A. scirpaceus (Passeriformes: Acrocephalidae)

Syringophilopsis albicollisi Skoracki et Dabert

Syringophilopsis albicollisi Skoracki \& Dabert, 2000: 59; Skoracki, 2011: 155

Type locality: Odjolo

Distribution: Togo

Host: Merops albicollis (Coraciiformes: Meropidae)

Syringophilopsis bartrami Skoracki, Spicer et OConnor

Syringophilopsis bartrami Skoracki, Spicer \& OConnor, 2016: 477

Type locality: Michigan, Wayne Co., Plymouth

Distribution: USA (Michigan)

Host: Spizella passerina (Passeriformes: Passerellidae)

Syringophilopsis blaszaki Skoracki et Dabert

Syringophilopsis blaszaki Skoracki \& Dabert, 1999: 89; Skoracki, 2011: 195; Klimovičová \& Hromada, 2014: 34; Pidhorna, 2020: 216

Type locality: 6 km NW from Darlowko Wschodnie (Poland)

Distribution: Poland, Russia, Slovakia, Ukraine

Hosts: Anthus pratensis, P. trivialis (Passeriformes: Motacillidae)

Syringophilopsis bochkovi Skoracki, Mironov et Bermúdez

Syringophilopsis bochkovi Skoracki, Mironov \& Bermúdez, 2019: 231 
Type locality: Panama, Darien Prov., Parque Nacional Darien, Rancho Frio

Distribution: Panama

Hosts: Corapipo altera, Manacus vitellinus (Passeriformes: Pipridae)

Syringophilopsis bonariensis Skoracki, Mironov, Hernandes et Valim

Syringophilopsis bonariensis Skoracki et al., 2016: 4

Type locality: Minas Gerais, Nova Lima, Agua Limpa, 20¹3’05.07”S 435’00.2”W

Distribution: Brazil

Host: Molothrus bonariensis (Passeriformes: Icteridae)

Syringophilopsis borini Bochkov et Mironov

Syringophilopsis borini Bochkov \& Mironov, 1999: 43; Skoracki, 2004; 2011: 201

Type locality: Novgorod Prov., Chudovo Distr., Oskuy Vill.

Distribution: Poland, Russia, Turkey

Host: Sylvia borin (Passeriformes: Sylviidae)

\section{Syringophilopsis catesbyi Skoracki, Spicer et OConnor}

Syringophilopsis catesbyi Skoracki, Spicer \& OConnor, 2016: 470; Skoracki, 2017: 186

Type locality: Florida, Leon Co.

Distribution: Mexico, USA (Florida)

Hosts: Vireo hypochryseus, V. olivaceus (Passeriformes: Vireonidae)

Syringophilopsis certhiae Skoracki, Hendricks et Spicer

Syringophilopsis certhiae Skoracki, Hendricks \& Spicer, 2011: 6

Type locality: California, Monterey

Distribution: USA

Host: Certhia americana (Passeriformes: Certhiidae)

Syringophilopsis corvinae Skoracki et Sykora

Syringophilopsis corvinae Skoracki \& Sykora, 2003: 5; Skoracki, 2011: 155

Type locality: Galim

Distribution: Cameroon

Host: Corvinella corvina (Passeriformes: Laniidae)

Syringophilopsis davidi Glowska et Laniecka

Syringophilopsis davidi Glowska \& Laniecka, 2012: 385

Type locality: Hurghada

Distribution: Egypt

Host: Calandrella brachydactyla (Passeriformes: Alaudidae) 
Syringophilopsis dendroicae Bochkov et Galloway

Syringophilopsis dendroicae Bochkov \& Galloway, 2001: 2023; Skoracki, Flannery \& Spicer, 2008: 299; Skoracki, 2011: 155; Skoracki, Spicer \& OConnor, 2016: 479

Type locality: Winnipeg, Manitoba, Canada

Distribution: Canada, USA

Hosts: Protonotaria citrea, Setophaga [Dendroica] coronata, S. caerulescens, S. petechia, S. [Dendroica] graciae, Cardellina [Wilsonia] pusilla (Passeriformes: Parulidae)

Syringophilopsis dicruri Skoracki, Hromada et Wamiti

Syringophilopsis dicruri Skoracki, Hromada \& Wamiti, 2011: 35

Type locality: Tsavo West National Park, Komboyo Campside

Distribution: Kenya

Host: Dicrurus adsimilis (Passeriformes: Dicruridae)

\section{Syringophilopsis elongatus (Ewing)}

Syringophilus elongatus Ewing, 1911: 39

Syringophilus icteridae Clark, 1964: 89 - Laurel, Maryland, from Agelaius phoenicius, Euphagus carolinus and Quiscalus quiscula (syn. by Kethley, 1970)

Syringophilopsis elongatus (Ewing): Kethley, 1970: 21; Bochkov \& Galloway, 2001: 2023; Skoracki, Flannery \& Spicer, 2008: 299; Skoracki, 2011: 155; Skoracki, Hendricks \& Spicer, 2011: 19; Skoracki, Spicer \& OConnor, 2016: 473

Type locality: Winnipeg, Manitoba

Distribution: Canada, USA (California, Maryland, Georgia, Nebraska, Arizona)

Hosts: Agelaius phoeniceus, Euphagus carolinus, E. cyanocephalus, Quiscalus mexicanus, Q. quiscula (Passeriformes: Icteridae)

Syringophilopsis emberizae Fain, Bochkov et Mironov

Syringophilopsis emberizae Fain, Bochkov \& Mironov, 2000: 57; Skoracki, 2011: 156

Type locality: Rwanda (Zoo of Antwerp)

Distribution: Rwanda

Host: Sicalis [Emberiza] luteola (Passeriformes: Thraupidae)

Syringophilopsis empidonax Skoracki, Flannery \& Spicer

Syringophilopsis empidonax Skoracki, Flannery \& Spicer, 2008: 294; Skoracki, 2011: 156

Type locality: Texas, Johnson Co., Garen Ranch

Distribution: USA (Texas)

Hosts: Empidonax hammondii, E. wrightii (Passeriformes: Tyrannidae)

Syringophilopsis faini Bochkov et Apanaskevich

Syringophilopsis faini Bochkov \& Apanaskevich, 2001: 321

Type locality: South - western Altai, Zyryanovskii Distr., Shumovsk Village 
Distribution: Kazakhstan

Host: Sylvia curruca (Passeriformes: Sylviidae)

\section{Syringophilopsis fringillae (Fritsch)}

Syringophilus fringillae Fritsch, 1958: 230; Skoracki \& Bochkov, 2010: 65; Skoracki, 2011: 185; Klimovičová \& Hromada, 2014; Pidhorna, 2020: 217

Syringophilopsis fringilla (Fritsch): Kethley, 1970: 21; Bochkov \& Mironov, 1998: 5; Skoracki \& Dabert, 1999: 89; 2000: 59; Nattress \& Skoracki, 2009; Skoracki, 2004c: 54; Klimovičová \& Hromada, 2014: 35

Type locality: Germany

Distribution: England, Germany, Kazakhstan, [Morocco], Poland, Russia, Slovakia, Ukraine

Hosts: [Carduelis carduelis], Fringilla coelebs (Passeriformes: Fringillidae), [Passer montanus (Passeridae), Hirundo rustica (Hirundinidae)]. According to Skoracki (2011: 185), S. fringillae is monoxenic parasite of Fringilla coelebs. The records from other birds concern different other species. Zmudzinski \& Unsoeld (2019) have recorded it from Fringilla montifringilla.

Syringophilopsis garrulus Skoracki et Dabert

Syringophilopsis garrulus Skoracki \& Dabert, 2002: 137; Skoracki, 2011: 163

Type locality: Africa

Distribution: Africa, Algeria, Germany

Host: Garrulus glandarius (Passeriformes: Corvidae)

\section{Syringophilopsis hirundus Skoracki}

Syringophilopsis hirundus Skoracki, 2004c: 60; Nattress \& Skoracki, 2007: 143; Skoracki \& Bochkov, 2010: 66; Skoracki, 2011: 193

Type locality: Świnoujscie (Poland)

Distribution: England, Kazakhstan, Poland

Host: Hirundo rustica (Passeriformes: Hirundinidae)

\section{Syringophilopsis hunanensis Liu Bai-li}

Syringophilopsis hunanensis Liu Bai-li, 1988: 277; Skoracki, 2011: 201 (sp. inquirenda

Type locality: Hunan

Distribution: China (Hunan)

Host: Passer domesticus (Passeriformes: Passeridae)

\section{Syringophilopsis icteri Bochkov et Galloway}

Syringophilopsis icteri Bochkov \& Galloway, 2001: 2019; Skoracki, 2011: 156; 2017: 186;

Skoracki, Hendricks \& Spicer, 2011: 17; Skoracki, Spicer \& OConnor, 2016: 473

Type locality: Winnipeg, Manitoba 
Distribution: Canada, Mexico, USA

Hosts: Icterus bullocki, I. galbula, I. pustulatus, I. spurius (Passeriformes: Icteridae)

Syringophilopsis idunae Skoracki

Syringophilopsis idunae Skoracki, 2011: 174

Type locality: Europe

Distribution: Europe

Host: Iduna caligata (Passeriformes: Acrocephalidae)

\section{Syringophilopsis kazmierski Skoracki}

Syringophilopsis kazmierski Skoracki, 2004c: 46; Skoracki, 2011: 166; Klimovičová \& Hromada, 2014: 35; Pidhorna, 2020: 217

Type locality: Darlówko Wsch. - Kopán (Poland)

Distribution: Germany, Jordan, Poland, Romania, Slovakia, Ukraine

Hosts: Ficedula hypoleuca, F. parva, Phoenicurus phoenicurus (Passeriformes: Muscicapidae)

Syringophilopsis kirgizorum Bochkov, Mironov et Kravtsova

Syringophilopsis kirgizorum Bochkov, Mironov \& Kravtsova, 2000: 354; Skoracki, 2004: 51; Glowska, Skoracki \& Khourly, 2007: 67; Nattress \& Skoracki, 2007: 143; Skoracki, Antczak, Riegert, Fainová \& Mikeš, 2009: 128; Skoracki, 2011: 182

Type locality: Bishkek City (Kyrghyzstan)

Distribution: Cameroon, England, Germany, Jordan, Kyrghyzstan, Poland, Russia

Hosts: Carduelis canabina, C. carduelis, Chloris chloris, Linurgus olivaceus, Rhodospiza obsoleta (Passeriformes: Fringillidae)

Syringophilopsis kristini Skoracki, Tryjanowski et Hromada

Syringophilopsis kristini Skoracki, Tryjanowski \& Hromada, 2002: 11; Skoracki, 2011: 198

Type locality: Kurov

Distribution: Slovakia

Host: Lanius minor (Passeriformes: Laniidae)

\section{Syringophilopsis lagonostictus Skoracki et Dabert}

Syringophilopsis lagonostictus Skoracki \& Dabert, 2002: 138; Skoracki, 2011: 157

Type locality: $6 \mathrm{~km}$ S Mango

Distribution: Togo

Hosts: Lagonosticta rufopicta, L. senegala (Passeriformes: Estrildidae)

Syringophilopsis melittophagi Skoracki et Dabert

Syringophilopsis melittophagi Skoracki \& Dabert, 2001b: 108; Skoracki, 2011: 157

Syringophilopsis melittophagii Skoracki \& Dabert, 2002: 138 
Type locality: $6 \mathrm{~km}$ S Mango

Distribution: Togo

Host: Merops (= Melittophagus) bullocki (Coraciiformes: Meropidae)

Syringophilopsis mimidus Sikora, Fajfer et Skoracki

Syringophilopsis mimidus Sikora, Fajfer \& Skoracki, 2012: 30; Skoracki, Spicer \& OConnor, 2016: 473

Type locality: Central America

Distribution: Central America, USA (Pennsylvania, Florida)

Host: Dumetella carolinensis, Margarops fuscatus (Passeriformes: Mimidae)

Syringophilopsis muscicapus Skoracki

Syringophilopsis muscicapus Skoracki, 2011: 161

Type locality: Japan

Distribution: Japan

Host: Muscicapa dauurica (Passeriformes: Muscicapidae)

Syringophilopsis nitens Skoracki et Dabert

Syringophilopsis nitens Skoracki \& Dabert, 2001b: 107; Skoracki \& Sikora, 2003: 9; Skoracki et al., 2011

Type locality: Atakpame (Togo)

Distribution: Kenya, Togo

Hosts: Hyphanturgus nigricollis brachypterus, Malimbus nitens, Ploceus nigricollis brachypterus, P. vitellinus, Quelea erythrops (Passeriformes: Ploceidae)

\section{Syringophilopsis nucifragus Skoracki}

Syringophilopsis nucifragus Skoracki, 2011: 188

Type locality: Europe

Distribution: Europe

Host: Nucifraga caryocatactes (Passeriformes: Corvidae)

Syringophilopsis pari Skoracki et Mironov

Syringophilopsis pari Skoracki \& Mironov, 2013: 557

Type locality: Primorsky Krai

Distribution: Russia (Far East)

Host: Poecile palustris (Passeriformes: Paridae)

\section{Syringophilopsis passericus Skoracki}

Syringophilopsis passericus Skoracki, 2011: 176

Type locality: Bishkek (Kirghizstan)

Distribution: Kirghizstan 
Host: Passer domesticus (Passeriformes: Passeridae)

\section{Syringophilopsis passerinae (Clark)}

Syringophilus passerinae Clark, 1964: 91; Skoracki, Flannery \& Spicer, 2008: 292; Skoracki, 2011: 157; Skoracki, Hendricks \& Spicer, 2011: 17; Skoracki, Spicer \& OConnor, 2016: 474

Syringophilopsis passerina (Clark): Kethley, 1970: 23; Bochkov \& Mironov, 1999: 7

Type locality: Beltsville, Maryland

Distribution: USA (Maryland, Florida, Texas)

Hosts: Passerina ciris, P. cyanea (Passeriformes: Cardinalidae)

Syringophilopsis philemonis Glowska et Laniecka

Syringophilopsis philemonis Glowska \& Laniecka, 2013: 386

Type locality: Australia, Beagle Bay

Distribution: Australia

Host: Philemon citreogularis (Passeriformes: Meliphagidae)

Syringophilopsis polioptilus Skoracki, Flannery \& Spicer

Syringophilopsis polioptilus Skoracki, Flannery \& Spicer, 2008: 292; Skoracki, 2011: 157

Type locality: Texas, Johnson Co., Garen Ranch

Distribution: USA (Texas)

Host: Polioptila caerulea (Passeriformes: Polioptilidae)

\section{Syringophilopsis rusticus Skoracki}

Syringophilopsis rusticus Skoracki, 2004: 49; Pidhorna, 2020: 218

Type locality: Olszyn (Poland)

Distribution: Poland, Ukraine

Hosts: Hirundo rustica, Riparia riparia (Passeriformes: Hirundinidae)

Syringophilopsis sialiae Skoracki, Flannery et Spicer

Syringophilopsis sialiae Skoracki, Flannery \& Spicer, 2008: 298; Skoracki, 2011: 157

Type locality: Texas, Jeff Davis Co., Fort Davis

Distribution: USA (Texas)

Host: Sialia mexicana (Passeriformes: Turdidae)

\section{Syringophilopsis sittae Skoracki, Hendricks et Spicer}

Syringophilopsis sittae Skoracki, Hendricks \& Spicer, 2011: 9; Skoracki \& Mironov, 2013: Type locality: USA (California), Mendocino Co., UC Hopland

Distribution: Russia, USA

Hosts: Sitta carolinensis, S. europaea (Passeriformes: Sittidae) 


\section{Syringophilopsis spinolettus Skoracki}

Syringophilopsis spinolettus Skoracki, 2004c: 57; Skoracki \& Bochkov, 2010: 66; Skoracki, 2011: 190

Type locality: Darlówko-Wsch. - Kopań (Poland)

Distribution: Kazakhstan, Poland, Slovakia

Host: Anthus spinoletta (Passeriformes: Motacillidae)

Syringophilopsis sturnellus Skoracki, Hendricks et Spicer

Syringophilopsis sturnellus Skoracki, Hendricks \& Spicer, 2011: 13

Type locality: Arizona, Mohave Co.

Distribution: USA (Arizona)

Host: Sturnella neglecta (Passeriformes: Icteridae)

Syringophilopsis sturni Chirov et Kravtsova

Syringophilopsis sturni Chirov \& Kravtsova, 1995: 374; Bochkov \& Mironov, 1998: 5;

Skoracki, 2004: 51; 2011: 171

Type locality: Telek, Chuy Valley, Kyrghyzstan

Distribution: Kazakhstan, Kyrghyzstan, Poland, Crimea

Host: Sturnus vulgaris (Passeriformes: Sturnidae)

Syringophilopsis szeffleri Glowska

Syringophilopsis szeffleri Glowska, 2014: 142

Type locality: Peru, El Caucho Biol. Station

Distribution: Peru

Host: Lophotriccus pileatus (Passeriformes: Tyrannidae)

Syringophilopsis trogoni Skoracki, Mironov et Unsoeld

Syringophilopsis trogoni Skoracki, Mironov et Unsoeld, 2013: 295

Type locality: Jalisco, Chamela Biol. Station of UNAM (Mexico)

Distribution: Costa Rica, Mexico

Hosts: Trogon citreolus, T. melanocephalus (Trogoniformes: Trogonidae)

\section{Syringophilopsis turdi (Fritsch)}

Syringophilus turdi Fritsch, 1958: 233

Syringophilopsis turdi (Fritsch): Bochkov \& Mironov, 1998: 6; Skoracki, 2011: 158; Skoracki, Spicer \& OConnor, 2016: 480

Syringophilopsis turdus: Kethley, 1970: 24; Skoracki \& Sikora, 2002: 7; Skoracki, 2004: 51;

Skoracki, Flannery \& Spicer, 2008: 298; Skoracki \& Bochkov, 2010: 65;

Type locality: Germany

Syringophilus hylocichlae Clark, 1964: 87 
Syringophilopsis hylocichlae (Clark): Kethley, 1970: 21; Skoracki, 2011: 156 (syn. by Skoracki, Spicer \& OConnor, 2016: 480)

Syringophilopsis hylocichla (Clark): Kethley, 1970: 21; Skoracki \& Dabert, 2000: 59; Glowska et al., 2015: 37

Type locality: Beltsville, Maryland, USA

Distribution: Argentina, Germany, Kazakhstan, Poland, Russia, Slovakia, USA (California)

Hosts: Catharus (= Hylocichla) fuscescens (sub S. hylocichlae), Turdus albicollis, T. atrogularis, T. iliacus, T. migratorius, T. philomelos, T. pilaris (Passeriformes: Turdidae)

Syringophilopsis tyranni Bochkov et Galloway

Syringophilopsis tyranni Bochkov \& Galloway, 2004: 35; Skoracki, Flannery \& Spicer, 2008: 298; Skoracki, 2011: 161; Sikora, Kaszewska \& Skoracki, 2014; Skoracki, Spicer \& OConnor, 2016: 474; Skoracki, 2017: 188

Type locality: Winnipeg, Manitoba

Distribution: Brazil, Canada, Mexico, USA (California, Texas, Florida), Peru

Hosts: Myiarchus cinerascens, M. crinitus, Myiopagis subplacens, Satrapa icterophrys, Tyrannus tyrannus, T. dominicensis, T. forficatus, T. verticalis (Passeriformes: Tyrannidae)

Syringophilopsis veselovsky Skoracki, Antczak et Riegert

Syringophilopsis veselovsky Skoracki, Antczak \& Riegert, 2009: 125; Skoracki, 2011: 157

Type locality: Cameroon

Distribution: Cameroon, Kenya

Hosts: Chlorocichla flaviventris, Eurillas latirostris, Pycnonotus barbatus (Passeriformes: Pycnonotidae)

Syringophilopsis wilsoni Skoracki, Spicer et OConnor

Syringophilopsis wilsoni Skoracki, Spicer \& OConnor, 2016: 474

Type locality: California, Mono Co.

Distribution: USA (California)

Host: Pheucticus melanocephalus (Passeriformes: Cardinalidae)

Syringophilopsis yosefi Skoracki, Tryjanowski et Hromada

Syringophilopsis yosefi Skoracki, Tryjanowski \& Hromada, 2002: 14; Skoracki, 2011: 157; Glowska, Chrzanowski \& Kaszewska, 2015: 41

Type locality: Yagoua

Distribution: Cameroon

Host: Lanius sp. (Passeriformes: Laniidae)

Genus Syringophilus Heller

Syringophilus Heller, 1880: 186

Type species: Syringophilus bipectinatus Heller, 1880 
Syringophilus bipectinatus Heller

Syringophilus bipectinatus Heller, 1880: 186; Oudemans, 1906: 47-57; Banks, 1907: 597;

Baker \& Wharton, 1952: 229 - 231; Lavoipierre, 1953: 7; Dubinin, 1957: 94; Fritsch, 1958: 229 - 230; Clark, 1964: 77; Jones, 1968: 28; Kethley, 1970: 18; Gritsenko, 1973:

515; Bochkov \& Mironov, 1998: 5; Skoracki \& Sikora, 2003: 9; 2004: 385; Glowska, Chrzanowski \& Kaszewska, 2015: 41

Type locality: Germany

Distribution: Australia, Belarus, Cameroon, China, Germany, France (Corsica), Kyrghyzstan, Poland, Russia, South Africa (Zululand), Ukraine, USA (Kansas, Colorado)

Hosts: Alectoris rufa, Gallus gallus domesticus, (Galliformes: Phasianidae), Ptilopachus petrosus (Odontophoridae), [Corvus brachyrhynchos (Passeriformes: Corvidae)]

\section{Syringophilus numidae Bochkov}

Syringophilus numidae Bochkov, 2000: 103; Skoracki, 2011: 309

Type locality: Transvaal

Distribution: South Africa

Host: Numida meleagris (Galliformes: Numididae)

Genus Terratosyringophilus Bochkov et Perez

Terratosyringophilus Bochkov \& Perez, 2002: 154

Type species: Peristerophila longisoma Casto, 1979

Terratosyringophilus geotrygonus Skoracki et Glowska

Terratosyringophilus geotrygonus Skoracki \& Glowska, 2008b: 152

Type locality: Cumbre de Valencia

Distribution: Venezuela

Host: Geotrygon linearis (Columbiformes: Columbidae)

\section{Terratosyringophilus longisoma (Casto)}

Peristerophila longisoma Casto, 1979: 225

Terratosyringophilus longisoma (Casto): Bochkov \& Fain, 2003: 154; Glowska, Chrzanowski \& Kaszewska, 2015: 42

Type locality: Texas, Presidio Co.

Distribution: USA (Texas)

Hosts: Zenaida asiatica, Z. macroura (Columbiformes: Columbidae)

Terratosyringophilus loricinus Bochkov et Fain

Terratosyringophilus loricinus Bochkov \& Fain, 2003: 6

Type locality: Halmahera Is.

Distribution: Indonesia

Hosts: Lorius garrulus, Trichoglossus haematodus (Psittaciformes: Psittaculidae) 
Terratosyringophilus pioni Bochkov et Perez

Terratosyringophilus pioni Bochkov \& Perez, 2002: 155

Type locality: Near Cd. del Maiz, S.L.P.

Distribution: Mexico

Host: Pionus senilis (Psittaciformes: Psittacidae)

Terratosyringophilus reichholfi Skoracki et Sikora

Terratosyringophilus reichholfi Skoracki \& Sikora, 2008b: 196; Glowska, Chrzanowski \& Kaszewska, 2015: 42

Type locality: Astrolabe

Distribution: Papua New Guinea

Host: Lorius lory (Psittaciformes: Psittaculidae)

Genus Tinamiphilopsis Skoracki et Sikora

Tinamiphylopsis Skoracki \& Sikora, 2004a: 348

Type species: Tinamiphylopsis elegans Skoracki et Sikora, 2004

Tinamiphilopsis ariconte Skoracki, Sikora et Ozminski

Tinamiphylopsis ariconte Skoracki, Sikora \& Ozminski, 2012: 110; Glowska, Chrzanowski \& Kaszewska, 2015: 42

Type locality: Paraguay

Distribution: Brazil, Paraguay

Hosts: Nothura boraquira, N. minor, Rhynchotus rufescens (Tinamiformes: Tinamidae)

Tinamiphilopsis elegans Skoracki et Sikora

Tinamiphylopsis elegans Skoracki \& Sikora, 2004a: 350

Type locality: Camarones

Distribution: Argentina

Host: Eudromia elegans (Tinamiformes: Tinamidae)

Genus Torotrogla Kethley

Torotrogla Kethley, 1970: 28

Type species: Torotrogla mima Kethley, 1970

Torotrogla aphelocoma Bochkov, Flannery et Spicer

Torotrogla aphelocoma Bochkov, Flannery \& Spicer, 2009: 184; Skoracki, 2011: 227

Type locality: Jeff Davis Co., Fort Davis

Distribution: USA (Texas)

Host: Aphelocoma californica (Passeriformes: Corvidae)

Torotrogla calcarius Skoracki

Torotrogla calcarius Skoracki, 2004d: 310; 2011: 247 
Type locality: Lapland, Finland

Distribution: Finland

Host: Calcarius lapponicus (Passeriformes: Emberizidae)

Torotrogla cardinalis Bochkov, Flannery et Spicer

Torotrogla cardinalis Bochkov, Flannery \& Spicer, 2009: 189; Skoracki, 2011: 227

Type locality: Texas, Hood Co., Jones Ranch

Distribution: USA

Host: Cardinalis cardinalis (Passeriformes: Cardinalidae)

Torotrogla cardueli Bochkov et Mironov

Torotrogla cardueli Bochkov \& Mironov, 1999: 41; Skoracki, 2004d: 314; 2011: 242; Glowska, Chrzanowski \& Kaszewska, 2015: 43

Type locality: Novgorod Prov., Chudovo Distr., Oskuy Vill. (Russia)

Distribution: Finland, Germany, Poland, Russia, Slovakia

Hosts: Linaria cannabina, Carduelis carduelis, Loxia curvirostra, L. leucoptera, L. pytyopsittacus, Serinus canaria, Spinus spinus (Passeriformes: Fringillidae)

Torotrogla coccothraustes Bochkov, Flannery et Spicer

Torotrogla coccothraustes Bochkov, Flannery \& Spicer, 2009: 191; Skoracki, 2011: 228

Type locality: California, Madera Co., Sierra National Forest

Distribution: USA (California)

Host: Coccothraustes vespertinus (Passeriformes: Fringillidae)

Torotrogla cyanocitta Bochkov, Flannery et Spicer

Torotrogla cyanocitta Bochkov, Flannery \& Spicer, 2009: 187; Skoracki, 2011: 227

Type locality: Lake Co., Lake Pillsbury; paratypes: Sierra Co., Bear Trap Meadow

Distribution: USA (California)

Host: Cyanocitta stelleri (Passeriformes: Corvidae)

Torotrogla emberizae Glowska, Laniecka et Romanowska

Torotrogla emberizae Glowska, Laniecka \& Romanowska, 2018: 791

Type locality: Japan

Distribution: Japan

Host: Emberiza fucata (Passeriformes: Emberizidae)

\section{Torotrogla gaudi Bochkov et Mironov}

Torotrogla gaudi Bochkov \& Mironov, 1999: 10; Skoracki \& Skoracka, 1999: 185; Skoracki, 2004d: 313; 2011: 239

Type locality: Rybachiy village, Kurshskaya Spit, Kaliningrad Province

Distribution: Poland, Russia, Slovakia

Hosts: Fringilla coelebs, F. montifringilla, Pyrrhula pyrrhula (Passeriformes: Fringillidae) 
Torotrogla lullulae Skoracki, Hromada et Kuczynski

Torotrogla lullulae Skoracki, Hromada \& Kuczynski, 2001: 87; Skoracki, 2011: 244

Type locality: Zlate (Slovakia)

Distribution: Russia, Slovakia

Hosts: Eremophila alpestris, Lullula arborea (Passeriformes: Alaudidae)

\section{Torotrogla lusciniae Skoracki}

Torotrogla lusciniae Skoracki, 2004d: 310; 2011: 236

Type locality: Italy

Distribution: Italy

Hosts: Luscinia megarhynchos, L. svecica (Passeriformes: Muscicapidae)

Torotrogla merulae Skoracki, Dabert et Ehrnsberger

Torotrogla merulae Skoracki, Dabert \& Ehrnsberger, 2000: 192; Skoracki, 2004d: 313;

Glowska, Skoracki \& Khourly, 2007: 67; Skoracki, 2011: 228; Skoracki, Spicer \& OConnor, 2016: 483

Type locality: Kuznica - Hel (Poland)

Distribution: Germany, Jordan, Poland, Romania, Russia, Slovakia, USA

Hosts: Turdus merula, T. migratorius, T. philomelos, T. torquatus, T. viscivorus (Passeriformes: Turdidae)

\section{Torotrogla mima Kethley}

Torotrogla mima Kethley, 1970: 31; Bochkov, Flannery \& Spicer, 2009: 184; Skoracki, 2011:

228; Sikora et al., 2011; Skoracki, Fajfer, Kavetska \& Sikora, 2012

Type locality: Odum, Wayne Co., Georgia (USA)

Distribution: Argentina, USA (Georgia)

Hosts: Mimus patagonicus, M. polyglottos, M. triurus (Passeriformes: Mimidae)

\section{Torotrogla modularis Nattress et Skoracki}

Torotrogla modularis Nattress \& Skoracki, 2007: 140; Skoracki, 2011: 234; Klimovičová \& Hromada, 2014: 35

Type locality: West Yorkshire (England)

Distribution: England, Mongolia, Poland, Slovakia

Hosts: Anthus pratensis, Prunella modularis (Passeriformes: Prunellidae)

Torotrogla paenae Glowska, Laniecka, Schmidt et Romanowska Torotrogla paenae Glowska, Laniecka, Schmidt \& Romanowska, 2018: 865

Type locality: Omaheke, Aminuis

Distribution: Namibia

Host: Cercotrichas paena (Passeriformes: Muscicapidae) 
Torotrogla piranga Bochkov, Flannery et Spicer

Torotrogla piranga Bochkov, Flannery \& Spicer, 2009: 192; Skoracki, 2011: 228

Type locality: California, Sierra Co., Bear Trap Meadow

Distribution: USA (California)

Host: Piranga ludoviciana (Passeriformes: Cardinalidae)

Torotrogla pycnonotus Skoracki

Torotrogla pycnonotus Skoracki, 2004d: 304; 2011: 250

Type locality: Al-Dhleil near Amman

Distribution: Jordan

Host: Pycnonotus xanthopygos (Passeriformes: Pycnonotidae)

Torotrogla rubeculi Skoracki

Torotrogla rubeculi Skoracki, 2004d: 304; 2011: 231; Klimovičová \& Hromada, 2014: 35

Type locality: Zachodniopomorskie, Darlowko Wschodnie, Bukowo Kopań (Poland)

Distribution: Poland, Russia, Slovakia

Host: Erithacus rubecula (Passeriformes: Muscicapidae)

Torotrogla synallaxis Skoracki, Mironov, Hernandes et Valim

Torotrogla synallaxis Skoracki, Mironov, Hernandes \& Valim, 2016: 252

Type locality: Minas Gerais, Nova Lima, APP do Condominio, Miguelso

Distribution: Brazil

Host: Synallaxis spixi (Passeriformes: Furnariidae)

\section{Torotrogla villosa (Hancock)}

Picobia villosa Hancock, 1895: 382; 1898: 886

Syringophilus villosus (Hancock): Oudemans, 1906: 72

Torotrogla villosa (Hancock): Kethley, 1970: 30; Bochkov, Flannery \& Spicer, 2009: 184;

Skoracki, 2011: 228

Type locality: Arizona

Distribution: USA (Arizona)

Host: Phainopepla nitens (Passeriformes: Ptilogonatidae)

Torotrogla volgini Skoracki et Mironov

Torotrogla volgini Skoracki \& Mironov, 2013: 560

Type locality: Primorskiy Krai, Partizanskiy Distr., Novolitovsk

Distribution: Russia

Host: Emberiza elegans (Passeriformes: Emberizidae)

Genus Trypetoptila Kethley

Trypetoptila Kethley, 1970: 25 
Type species: Trypetoptila casmerodia Kethley, 1970

Trypetoptila casmerodia Kethley

Trypetoptila casmerodia Kethley, 1970: 28

Type locality: McIntosh Co., Georgia

Distribution: USA (Georgia)

Host: Ardea alba egretta (Pelecaniformes: Ardeidae)

Subfamily Picobiinae Johnston et Kethley

Picobiinae Johnston \& Kethley, 1973: 529

Type genus: Picobia Haller, 1878

Lobatinae Casto, 1977: 169

Cuculiphilinae [as nom. nov.](praeoccupied)

Type genus: Cuculiphilus Casto, 1977

Genus Calamincola Casto

Cuculiphilus Casto, 1977: 170 (praeocc. from Uchida, 1926)

Calamincola Casto, 1978: 158

Type species: Cuculiphilus lobatus Casto, 1977

Calamincola lobatus (Casto)

Cuculiphilus lobatus Casto, 1977: 170

Calamincola lobatus (Casto): Casto, 1978: 158; Hromada et al., 2016: 532; Skoracki, 2017: 188

Type locality: South Texas

Distribution: Colombia, Mexico, USA (Texas)

Host: Crotophaga sulcirostris (Cuculiformes: Cuculidae)

Genus Charadriineopicobia Skoracki, Spicer et OConnor

Charadriineopicobia Skoracki, Spicer \& OConnor, 2014: 102

Type species: Charadriineopicobia calidris Skoracki, Spicer et OConnor, 2014

Charadriineopicobia apricaria Skoracki, Sikora et Spicer

Charadriineopicobia apricaria Skoracki, Sikora \& Spicer, 2016: 12

Type locality: St. Malo

Distribution: France

Host: Pluvialis apricaria (Charadriiformes: Charadriidae)

Charadriineopicobia calidris Skoracki, Spicer et OConnor

Charadriineopicobia calidris Skoracki, Spicer \& OConnor, 2014: 103; Skoracki, Sikora \& Spicer, 2016: 18

Type locality: Texas, Calhoun Co., Matagorda Island 
Distribution: USA

Host: Calidris alba (Charadriiformes: Scolopacidae)

Charadriineopicobia leucophaeus (Skoracki, Hendericks et Spicer)

Picobia leucophaeus Skoracki, Hendericks \& Spicer, 2010: 730

Charadriineopicobia leucophaeus (Skoracki, Hendericks et Spicer): Skoracki, Spicer \& OConnor, 2014: 104

Type locality: Texas, Calhoun Co.

Distribution: USA

Host: Leucophaeus atricilla (Charadriiformes: Laridae)

Charadriineopicobia ristowi Zmudzinski et Unsoeld

Charadriineopicobia ristowi Zmudzinski \& Unsoeld, 2019: 373

Type locality: Germany

Distribution: Germany

Host: Limosa lapponica (Charadriiformes: Scolopacidae)

Genus Columbiphilus Kivganov et Sharafat

Columbiphilus Kivganov \& Sharafat, 1995: 82

Type species: Columbiphilus khushakhani Kivganov et Sharafat, 1995

Columbiphilus alectoris (Fain, Bochkov et Mironov)

Picobia alectoris Fain, Bochkov \& Mironov, 2000: 65; Skoracki, Bochkov \& Wauthy, 2004: 164 Picobia alectori: Skoracki \& Dabert, 2002: 138

Columbiphilus alectoris (Fain, Bochkov et Mironov): Skoracki, 2011: 374; Skoracki \&

Sikora, 2011; Skoracki \& Hromada, 2013; Skoracki, Sikora \& Spicer, 2016: 25

Type locality: France (see the notes in Skoracki, Sikora \& Spicer, 2016).

Distribution: France, Rwanda

Hosts: Alectoris rufa, Alectoris sp. (Galliformes: Phasianidae)

Columbiphilus khushalkhani Kivganov et Sharafat

Columbiphilus khushalkhani Kivganov \& Sharafat, 1995: 83; Skoracki, 2011:374; Skoracki, Sikora \& Spicer, 2016: 19

Picobia khushalkhani (Kivganov et Sharafat): Bochkov \& Mironov, 1999: 16; Skoracki, Bochkov \& Wauthy, 2004: 172

Picobia polonica Skoracki, Magowski \& Dabert, 2001: 154 - Type loc. Wielkopolska, Zasutowo, Distr. Poland, India (syn. by Skoracki, Sikora \& Spicer, 2016)

Columbiphilus polonica (Skoracki, Magowski et Dabert): Skoracki, 2011: 375; Skoracki \& Sikora, 2011

Type locality of C. khushalkhani: Kabul (Afghanistan) 
Distribution: Afghanistan, Belgium, Germany, India, Japan, Poland, USA, Central, Eastern and South Africa, USA

Hosts: Columba livia (Columbiformes: Columbidae - accidental host), Coturnix coturnix, Gallus gallus domesticus, G. sonneratii, Francolinus clappertoni (Galliformes: Phasianidae), Acryllium vulturinum, Numida meleagris (Galliformes: Numididae)

Columbiphilus odontophoridus Skoracki et Sikora

Columbiphilus odontophoridus Skoracki \& Sikora, 2011: 28

Type locality: Bogota (Colombia)

Distribution: Colombia, Suriname

Hosts: Colinus cristatus, C. leucopogon (Galliformes: Odontophoridae)

Columbiphilus pteroclesi (Skoracki et OConnor)

Picobia pteroclesi Skoracki \& OConnor, 2010: 27

Columbiphilus pteroclesi (Skoracki et OConnor): Skoracki, 2011: 379; Skoracki, Sikora \& Spicer, 2016: 26

Type locality: Egypt, Red Sea Governate, Wadi el Metuli

Distribution: Egypt

Hosts: Pterocles coronatus, P. senegallus (Pterocliformes: Pteroclidae)

Genus Gunabopicobia Skoracki et Hromada

Gunabopicobia Skoracki \& Hromada, 2013: 196

Type species: Picobia zumpti Lawrence, 1959

Gunabopicobia claravis Kaszewska, Skoracki et Hromada

Gunabopicobia claravis Kaszewska, Skoracki \& Hromada, 2018: 292

Type locality: Cauca Province, Mercaderes

Distribution: Colombia

Host: Claravis pretiosa (Columbiformes: Columbidae)

Gunabopicobia geotrygoni Kaszewska, Skoracki et Hromada

Gunabopicobia geotrygoni Kaszewska, Skoracki \& Hromada, 2018: 292

Type locality: Venezuela, Silla de Caracas

Distribution: Colombia, Martinique, Paraguay, Venezuela

Hosts: Geotrygon chrysiei, G. frenata, G. linearis, G. montana (Columbiformes: Columbidae)

Gunabopicobia lathami Kaszewska, Skoracki et Hromada

Gunabopicobia lathami Kaszewska, Skoracki \& Hromada, 2018: 290

Type locality: Australia

Distribution: Australia, Indonesia, Papua New Guinea 
Hosts: Leucosarcia melanoleuca, Caloenas nicobarica (Columbiformes: Columbidae)

Gunabopicobia leptotila Kaszewska, Skoracki et Hromada

Gunabopicobia leptotila Kaszewska, Skoracki \& Hromada, 2018: 291

Type locality: Formosa Province, Tapiciolé

Distribution: Argentina

Host: Leptotila verreauxi (Columbiformes: Columbidae)

Gunabopicobia masalaje Kaszewska, Kavetska et Skoracki

Gunabopicobia masalaje Kaszewska, Kavetska \& Skoracki, 2014: 294; Skoracki, Sikora \& Spicer, 2016: 26

Type locality: Papua New Guinea

Distribution: Indonesia (Sulawesi), Papua New Guinea

Hosts: Ducula luctuosa, D. pistrinaria, D. rosacea, D. rufigaster, D. spilorrhoa, Ptilinopus iozonus (Columbiformes: Columbidae: Treroninae)

Gunabopicobia metropelia Kaszewska, Skoracki et Hromada

Gunabopicobia metropelia Kaszewska, Skoracki \& Hromada, 2018: 295

Type locality: Neuquén Province, Patagonia

Distribution: Argentina

Hosts: Metropelia melanoptera (Columbiformes: Columbidae)

Gunabopicobia zumpti (Lawrence)

Syringophilus zumpti Lawrence, 1959: 425

Picobia zumpti: Kethley, 1970: 65; Skoracki \& Dabert, 2002: 144; Skoracki, Bochkov \& Wauthy, 2004: 171; Bochkov, Williams \& Proctor, 2005: 122

Neopicobia zumpti: Skoracki, 2011: 384

Gunabopicobia zumpti: Skoracki \& Hromada, 2011: 197; Skoracki, Sikora \& Spicer, 2016:

27; Kaszewska, Skoracki \& Hromada, 2018: 294

Type locality: South Africa

Distribution: South Africa, USA

Hosts: Columba livia, Streptopelia capicola, S. senegalensis, Zenaida macroura (Columbiformes: Columbidae)

Genus Lawrencipicobia Skoracki et Hromada

Lawrencipicobia Skoracki \& Hromada, 2013: 199

Type species: Picobia poicephali Skoracki et Dabert, 2002

Lawrencipicobia calyptorhyncha Marciniak, Skoracki et Hromada

Lawrencipicobia calyptorhyncha Marciniak, Skoracki \& Hromada, 2019: 194

Type locality: Australia 
Distribution: Australia

Host: Calyptorhyncha lathami (Psittaciformes: Cacatuidae)

Lawrencipicobia poicephali (Skoracki et Dabert)

Picobia poicephali Skoracki \& Dabert, 2002: 143, Skoracki, Bochkov \& Wauthy, 2004: 171;

Skoracki, 2005: 128; 2011: 329

Lawrencipicobia poicephali (Skoracki et Dabert): Skoracki \& Hromada, 2014: 199; Skoracki,

Sikora \& Spicer, 2016: 29; Skoracki, Sikora \& Hromada, 2019: 1610

Type locality: Galim

Distribution: Cameroon

Host: Poicephalus senegalus versteri (Psittaciformes: Psittacidae)

Lawrencipicobia sulphurea Marciniak, Skoracki et Hromada

Lawrencipicobia sulphurea Marciniak, Skoracki \& Hromada, 2019: 196

Type locality: West Timor, East Nusa Tenggara Province, Kupang Regency

Fatulaeu District, Camplong I (Tjamplong)

Distribution: Indonesia (West Timor)

Host: Cacacua sulphurea (Psittaciformes: Cacatuidae)

Genus Neopicobia Skoracki

Neopicobia Skoracki, 2011: 383

Type species: Syringophilus anthi Fritsch, 1958

\section{Neopicobia anthi (Fritsch)}

Syringophilus anthi Fritsch, 1958: 240

Picobia anthi: Fain, Bochkov \& Mironov, 2000: 65; Skoracki, Bochkov \& Wauthy, 2004: 172

Neopicobia anthi (Fritsch): Skoracki, 2011: 384

Type locality: "Schlesien"

Distribution: Germany, Poland

Hosts: Anthus cervinus, A. trivialis (Passeriformes: Motacillidae)

Neopicobia cardinalis (Skoracki, Hendricks et Spicer)

Picobia cardinalis Skoracki, Hendricks \& Spicer, 2010: 733

Neopicobia cardinalis (Skoracki, Hendricks et Spicer): Skoracki, 2011: 384

Type locality: Texas, Johnson Co.

Distribution: USA (Texas)

Host: Cardinalis cardinalis (Passeriformes: Cardinalidae)

Neopicobia carpodacus (Skoracki, Hendricks et Spicer)

Picobia carpodacus Skoracki, Hendricks \& Spicer, 2010: 737 
Neopicobia carpodacus (Skoracki, Hendricks et Spicer): Skoracki, 2011: 384; Skoracki, Sikora \& Spicer, 2016: 35

Type locality: California, Monterey County

Distribution: USA (California)

Host: Haemorhous [Carpodacus] purpureus (Passeriformes: Fringillidae)

Neopicobia ea Skoracki et Unsöeld

Neopicobia ea Skoracki \& Unsoeld, in: Skoracki, Unsoeld, Kaszewska \& Kavetska, 2014: 78 Type locality: Venezuela, Rio Caura

Distribution: Brazil, Peru, Venezuela

Hosts: Celeus elegans, C. flavus, C. torquatus (Piciformes: Picidae)

Neopicobia epthianura (Skoracki, Glowska et Sikora)

Picobia epthianura Skoracki, Glowska \& Sikora, 2008: 62

Neopicobia epthianura (Skoracki, Glowska et Sikora): Skoracki, 2011: 384

Type locality: Australia

Distribution: Australia

Host: Epthianura aurifrons (Passeriformes: Meliphagidae)

Neopicobia freya Skoracki et Unsoeld

Neopicobia freya Skoracki \& Unsoeld, in: Skoracki, Unsoeld, Kaszewska \& Kavetska, 2014:

74; Klimovičova et al., 2014a: 6

Type locality: Paraguay

Distribution: Kenya, Panama, Paraguay, Tanzania

Hosts: Dryocopus galeatus, Dendropicos goerthae, D. griseocephalus, Colaptes [Piculus] rubiginosus (Piciformes: Picidae)

Neopicobia hepburni Glowska et Laniecka

Neopicobia hepburni Glowska \& Laniecka, 1914: 636; Skoracki, Sikora \& Spicer, 2016: 37

Type locality: Peru

Distribution: Ecuador, Paraguay, Peru, Guyana

Hosts: Picumnus exilis, P. sclateri, P. olivaceus, P. temminckii (Piciformes: Picidae)

Neopicobia herbicolae Glowska, Laniecka et Milensky

Neopicobia herbicolae Glowska, Laniecka \& Milensky, 1915: 636

Type locality: Guyana

Distribution: Guyana

Host: Emberizoides herbicola (Passeriformes: Thraupidae) 
Neopicobia ictericus (Skoracki et Glowska)

Picobia ictericus Skoracki \& Glowska, in: Skoracki, Glowska, Lontkowski \& Stawarczyk, 2010: 143

Neopicobia ictericus (Skoracki et Glowska): Skoracki, Sikora \& Spicer, 2016: 33

Type locality: Brazil

Distribution: Brazil

Hosts: Cacicus chrysopterus, Pseudoleistes guirahuro (Passeriformes: Icteridae)

Neopicobia modularis (Skoracki et Magowski)

Picobia modularis Skoracki \& Magowski, 2001: 114; Skoracki, Bochkov \& Wauthy, 2004: 171

Neopicobia modularis (Skoracki et Magowski): Skoracki, 2011: 386; Klimovičová \& Hromada, 2014: 36

Type locality: Kuznica - Hel (Poland)

Distribution: Mongolia, Poland, Slovakia

Hosts: Prunella atrogularis, P. modularis (Passeriformes: Prunellidae), Pyrrhula pyrrhula (Pass., Fringillidae)

Neopicobia pari Skoracki, Sikora et Spicer

Neopicobia pari Skoracki, Sikora \& Spicer, 2016: 33

Type locality: China, Fuijan Prov.

Distribution: China, Finland, Japan, N. Macedonia,

Hosts: Parus major, Periparus venustulus, Poecile varius (Passeriformes: Paridae)

Neopicobia troglodytes (Skoracki, Hendricks et Spicer)

Picobia troglodytes Skoracki, Hendricks \& Spicer, 2010: 731

Neopicobia troglodytes (Skoracki et al.): Skoracki, 2011: 384

Type locality: California, Monterey Co.

Distribution: USA (California)

Hosts: Thryomanes bewickii, Troglodytes aedon (Passeriformes: Troglodytidae), Cardellina pusilla (Pass.: Parulidae) 
Genus Phipicobia Glowska et Schmidt Phipicobia Glowska \& Schmidt, 2014a: 194

Type species: Phipicobia pygiptilae Glowska et Schmidt, 2004

Phipicobia pygiptilae Glowska et Schmidt

Phipicobia pygiptilae Glowska \& Schmidt, 2004: 19

Type locality: Guyana

Distribution: Guyana

Host: Pygiptila stellaris (Passeriformes: Thamnophilidae)

Genus Picobia Haller

Picobia Haller, 1878: 81 - 91

Type species: Picobia heeri Haller, 1878

Picobia bernieriarum Skoracki, OConnor et Goodman

Picobia bernieriarum Skoracki, OConnor \& Goodman, 2016: 1541

Type locality: Tulear Prov., Fivondronana de Tolagnaro, Forêt de Marosohy along

Enakara-Antseva trail, $15.5 \mathrm{~km}$ WNW Ranomafana $\left(24^{\circ} 34^{`} \mathrm{~S}, 46^{\circ} 48^{`} \mathrm{E}\right)$

Distribution: Madagascar

Hosts: Bernieria madagascariensis, Xanthomixis zosterops (Passeriformes: Bernieridae)

Picobia biarmicus Skoracki, Bochkov et Wauthy

Picobia biarmicus Skoracki, Bochkov \& Wauthy, 2004: 165; Skoracki, 2011: 328

Type locality: Miedzyodrze

Distribution: Poland

Host: Panurus biarmicus (Passeriformes: Panuridae)

Picobia caudati Skoracki et Hebda

Picobia caudati Skoracki \& Hebda, 2004: 2; Zmudzinski \& Unsoeld, 2016: 355

Type locality: Popielow near Opole (Poland)

Distribution: Germany, Poland

Host: Aegithalos caudatus (Passeriformes: Aegithalidae)

Picobia cetti Skoracki

Picobia cetti Skoracki, 2011: 357

Type locality: Aras

Distribution: Turkey

Host: Cettia cetti (Passeriformes: Cettidae) 
Picobia chloris Bochkov, Mironov et Kravtsova

Picobia chloris Bochkov, Mironov \& Kravtsova, 2000: 352; Skoracki, Bochkov \& Wauthy, 2004: 172; Skoracki, 2011: 328

Type locality: Bishkek City

Distribution: Kyrghyzstan

Host: Chloris chloris (Passeriformes: Fringillidae)

Picobia cichladusa Skoracki, Solarczyk et Sikora

Picobia cichladusa Skoracki, Solarczyk \& Sikora, 2012: 124; Skoracki \& Hromada, 2013: 201

Type locality: Soga

Distribution: Tanzania

Host: Cichladusa arcuata (Passeriformes: Muscicapidae)

Picobia cissa Skoracki, Bochkov et Wauthy

Picobia cissa Skoracki, Bochkov \& Wauthy, 2004: 168; Skoracki, 2011: 328; Skoracki, Sikora \& Spicer, 2016: 52

Type locality: North Indochina

Distribution: North Indochina

Hosts: Cissa chinensis, Urocissa erythrorhyncha (Passeriformes: Corvidae)

Picobia corcoracus Skoracki, Glowska et Sikora

Picobia corcoracus Skoracki, Glowska \& Sikora, 2008: 64; Skoracki, 2011: 328

Type locality: Australia

Distribution: Australia

Host: Corcorax melanorhamphos (Passeriformes: Corcoracidae)

Picobia currucae Skoracki et Magowski

Picobia currucae Skoracki \& Magowski, 2001: 116; Glowska, Skoracky \& Khourly, 2007:

64; Skoracki, 2011: 348

Type locality: Poland, Kuznica-Hel

Distribution: Jordan, Poland, Turkey

Hosts: Sylvia curruca, S. hortensis, S. nisoria (Passeriformes: Sylviidae)

Picobia dinemellia Glowska et Skoracki

Picobia dinemellia Glowska \& Skoracki, 2011: 64; Skoracki \& Hromada, 2013: 201

Type locality: Tanzania

Distribution: Tanzania

Host: Dinemellia dinemelli (Passeriformes: Ploceidae) 


\section{Picobia dryobatis (Fritsch)}

Syringophilus dryobatis Fritsch, 1958: 242

Picobia dryobatis (Fritsch): Kethley, 1970: 65; Skoracki, Bochkov \& Wauthy, 2004: 159;

Nattress \& Skoracki, 2009: 53; Skoracki, Hendricks \& Spicer, 2010: 739; Skoracki, 2011: 334; Klimovičova et al., 2014a: 5; Glowska, Chrzanowski \& Kaszewska, 2015:

50; Skoracki, Sikora \& Spicer, 2016: 62

Picobia dryobates: Bochkov \& Mironov, 1998: 15

Type locality: Germany; neotype from Poland (Darlowko Wschodnie, Bukovo - Kopan)

Distribution: Brazil, Germany, England, Ecuador, Ethiopia, Kenya, Moldavia, Nepal, Paraguay, Poland, Slovakia, Suriname, Tanzania, Trinidad and Tobago, USA, Venezuela, Zambia

Hosts: Campethera nubica, Dendrocopos borealis, D. darjellensis, D. leucotos, D. macei, D. major, D. minor hortorum, D. namaquus, D. stierlingi, D. syriacus, Dendropicos fuscescens, D. goerthae, D. namaquus, D. stierlingi, Dryocopus pileatus, Jynx torquilla, Melanerpes carolinus, M. erythrocephalus, Picoides mixtus, P. pubescens, P. scalaris, P. tridactylus, Veneliornis cassini, V. dignus, V. fumigatus, V. kirkii, V. maculifrons, V. passerines, V. sanguineus, V. spilogaster (Piciformes: Picidae); [Sturnus vulgaris (Passeriformes: Sturnidae)]. Note by Glowska, Chrzanowski \& Kaszewska (2015): "Specimens from Sturnus vulgaris determined as P. dryobatis (Bochkov \& Mironov 1998) belong to Picobia sturni (Skoracki et al. 2004d)".

Picobia dziabaszewskii Glowska, Dragun-Damian et Dabert

Picobia dziabaszewskii Glowska, Dragun-Damian \& Dabert, 2012: 59; Klimovičova et al., 2014a: 5

Type locality: China

Distribution: China, Kenya, Namibia, Tanzania, Zambia

Hosts: Trochalopteron [Garrulax] formosus, Turdoides jardineii, T. leucopygia, T. rubiginosa (Passeriformes: Leiothrichidae)

Picobia echo Skoracki, Solarczyk et Sikora

Picobia echo Skoracki, Solarczyk \& Sikora, 2012: 126; Skoracki \& Hromada, 2013: 202

Type locality: South Kivu, Bukavu

Distribution: DR Congo, Indonesia, Nepal

Host: Cossypha heuglini, Copsychus malabricus, C. saularis (Passeriformes: Muscicapidae)

\section{Picobia eremophila Skoracki}

Picobia eremophila Skoracki, 2011: 363; Skoracki \& Hromada, 2013: 202

Type locality: Egypt

Distribution: Egypt

Host: Eremophila bilopha (Passeriformes: Alaudidae) 


\section{Picobia galerida Skoracki}

Picobia galerida Skoracki, 2011: 367

Type locality: Zachodniopomorskie, near Swinoujscie

Distribution: Poland

Host: Galerida cristata (Passeriformes: Alaudidae)

\section{Picobia heeri Haller}

Picobia Heeri Haller, 1878: 81-91; Oudemans, 1906: 73; Dubinin, 1957: 94; Kethley, 1970: 64; Skoracki, Bochkov \& Wauthy, 2004: 158; Skoracki, 2011: 329; Skoracki et al., 2014: 71; Skoracki, Sikora \& Spicer, 2016: 62

Syringophilus heeri: Poppe, 1888: 238

Picobia helleri [sic]: Bochkov \& Mironov, 1999: 15

Type locality: Zurich (Switzerland)

Distribution: Germany, India, Indonesia (Sumatra), Japan, Nepal, Poland, Switzerland, Thailand

Hosts: Picus awokera, P. canus, P. chlorolophus, P. erythropygius, P. flavinucha, P. punicus, P. squamatus, P. viridis (Piciformes: Picidae)

Picobia illadopsae Skoracki et Hromada

Picobia illadopsae Skoracki \& Hromada, 2013: 202

Type locality: Kakamega, Buyangu, Udo's campsite, $0^{\circ} 21^{\prime} 11^{\prime \prime N}$, 3451'50”E, 1598 m a.s.l. Distribution: Kenya

Host: Illadopsis rufipennis (Passeriformes: Pellorneidae)

Picobia indonesiana Skoracki et Glowska

Picobia indonesiana Skoracki \& Glowska, 2008: 281; Skoracki, 2011: 328

Type locality: Indonesia

Distribution: Indonesia

Hosts: Aplonis panayensis, Enodes erythrophris, Mino dumontii (Passeriformes: Sturnidae)

Picobia lamprotornis Klimovičova, Skoracki, Wamiti et Hromada

Picobia lamprotornis Klimovičova, Skoracki, Wamiti \& Hromada, 2014: 395; Klimovičova et al., 2014a: 2

Type locality: Soysambu Conservancy

Distribution: Kenya

Hosts: Lamprotornis superbus (Passeriformes: Sturnidae), Ploceus ocularis (Passeriformes: Ploceidae)

Picobia lemi Skoracki, Glowska et Sikora

Picobia lemi Skoracki, Glowska \& Sikora, 2008: 60; Skoracki, 2011: 328

Type locality: New Guinea 
Distribution: Papua New Guinea

Hosts: Manucodia ater, M. chalybatus (Passeriformes: Paradisaeidae)

Picobia lonchura Skoracki, Sikora et Spicer

Picobia lonchura Skoracki, Sikora \& Spicer, 2016: 53

Picobia lonchurae: Skoracki, Hromada, Prevuznakova \& Wamiti, 2019: 1804, 2019: 1805

Type locality: Java

Distribution: Indonesia, Uganda

Hosts: Estrilda atricapilla, Lonchura leucogastra (Passeriformes: Estrildidae)

Picobia magellani Skoracki, Sikora et Spicer

Picobia magellani Skoracki, Sikora \& Spicer, 2016: 43

Type locality: Bogota

Distribution: Colombia

Host: Scytalopus magellanicus (Passeriformes: Rhinocriptidae)

Picobia makoli Skoracki, Sikora et Spicer

Picobia makoli Skoracki, Sikora \& Spicer, 2016: 45

Type locality: Leticia

Distribution: Colombia

Host: Xiphocolaptes promeropirhynchus (Passeriformes: Furnariidae)

Picobia mentalis Skoracki et Unsoeld

Picobia mentalis Skoracki \& Unsoeld, in: Skoracki, Unsoeld, Kaszewska \& Kavetska, 2014: 71

Type locality: Malacca

Distribution: Indonesia (Java), Malaysia (Malacca)

Host: Picus mentalis (Piciformes: Picidae)

Picobia meru Klimovičova, Skoracki et Hromada

Picobia meru Klimovičova, Skoracki \& Hromada, 2016: 582

Type locality: Arabuko Sokoke Forest; Ngaye Forest, Meru, 1220 m

Distribution: Kenya

Host: Prionops sulcifrons (Passeriformes: Vangidae)

Picobia myiopagi Glowska et Milensky

Picobia myiopagi Glowska \& Milensky, 2014: 378

Type locality: Guyana

Distribution: Guyana, Peru

Hosts: Elaenia ruficeps, Myiopagis gaimardii, M. subplacens (Passeriformes: Tyrannidae) 
Picobia myrmecocichla Skoracki, Solarczyk et Sikora

Picobia myrmecocichla Skoracki, Solarczyk \& Sikora, 2012: 132; Skoracki \& Hromada, 2013: 205

Type locality: Tanzania

Distribution: Tanzania

Host: Myrmecocichla arnotti (Passeriformes: Muscicapidae)

Picobia neomixis Skoracki, OConnor et Goodman

Picobia neomixis Skoracki, OConnor \& Goodman, 2016: 1538

Type locality: Tulear Prov., Toliara Province, Fivondronana de

Tolagnaro, $1.5 \mathrm{~km}$ NW Manafiafy ( $\left.24^{\circ} 46^{\prime} \mathrm{S}, 47^{\circ} 11^{\prime} \mathrm{E}\right)$

Distribution: Madagascar

Host: Neomixis tenella (Passeriformes: Cisticolidae)

Picobia ochoi Glowska et Milensky

Picobia ochoi Glowska \& Milensky, 2014: 374

Type locality: Guyana, Cuyuni-Mazarini, Paruima

Distribution: Guyana

Host: Tolmomyias sulphurescens (Passeriformes: Tyrannidae)

Picobia oritis Skoracki, Antczak et Riegert

Picobia oritis Skoracki, Antczak \& Riegert, 2009: 133; Skoracki, 2011: 328; Skoracki \& Hromada, 2013: 205; Klimovičova et al., 2014a; Skoracki, Sikora \& Spicer, 2016: 52 Type locality: Cameroon, North-Western Province, Bamenda Highlands,

Mendongbuo, $06^{\circ} 05^{\prime} \mathrm{N}, 10^{\circ} 18^{\prime} \mathrm{E}, 2200 \mathrm{~m}$ a.s.l.

Distribution: Angola, Botswana, Cameroon, Kenya, Somalia, South Africa, Uganda, Zambia

Hosts: Cinnyris erythrocercus, C. oustaleti, C. talatala, C. venustus, Cyanomitra olivacea, C. oritis (Passeriformes: Nectariniidae)

Picobia paludicola Skoracki et Kiljan

Picobia paludicola Skoracki \& Kiljan, 2002: 2; Skoracki, Bochkov \& Wauthy, 2004: 171;

Skoracki, 2011: 328

Type locality: Swinoujscie

Distribution: Poland

Host: Acrocephalus paludicola (Passeriformes: Acrocephalidae)

Picobia passeri Skoracki et Sikora

Picobia passeri Skoracki \& Sikora, 2014: 195

Type locality: Russia, Tula District, Suvorov

Distribution: Russia

Host: Passer domesticus (Passeriformes: Passeridae) 
Picobia phoeniculi Fain, Bochkov et Mironov

Picobia phoeniculi Fain, Bochkov \& Mironov, 2000: 69; Skoracki \& Dabert, 2002: 138;

Skoracki, Bochkov \& Wauthy, 2004: 168; Skoracki \& Hromada, 2013: 206; Skoracki,

Sikora \& Spicer, 2016: 62

Type locality: Rwanda

Distribution: N. Macedonia, Kazakhstan, Pakistan, Rwanda, Tanzania

Hosts: Phoeniculus purpureus ruwenzoriae (Upupiformes: Phoeniculidae), Upupa epops africana, U. epops epops (Upupiformes: Upupidae)

Picobia phoenicuri Skoracki et Hromada

Picobia phoenicuri Skoracki \& Hromada, 2013: 208

Type locality: Tunisia

Distribution: Tunisia

Host: Phoenicurus moussieri (Passeriformes: Muscicapidae)

Picobia ploceus Klimovičova, Skoracki, Wamiti et Hromada

Picobia ploceus Klimovičova, Skoracki, Wamiti \& Hromada, 2014: 395

Type locality: Kenya

Distribution: Kenya

Host: Ploceus ocularis (Passeriformes: Ploceidae)

Picobia psaltriparus Skoracki, Hendricks et Spicer

Picobia psaltriparus Skoracki, Hendricks \& Spicer, 2010: 738

Type locality: Texas, Jeff Davis Co.

Distribution: USA

Host: Psaltriparus minimus (Passeriformes: Aegithalidae)

Picobia pycnonoti Glowska, Skoracki et Khourly

Picobia pycnonoti Glowska, Skoracki \& Khourly, 2007: 64; Skoracki, 2011:346; Klimovičova et al., 2014a: 6; Skoracki, Sikora \& Spicer, 2016: 48

Type locality: Al-Dhleil (Jordan)

Distribution: Jordan, Kenya, Philippines

Hosts: Pycnonotus barbatus, P. goiavier, P. xanthopygus (Passeriformes: Pycnonotidae)

Picobia riparius Skoracki

Picobia riparius Skoracki, 2011: 360

Type locality: Lubuskie, Zielona Gora

Distribution: Poland

Host: Riparia riparia (Passeriformes: Hirundinidae) 


\section{Picobia sayornis Glowska}

Picobia sayornis Glowska, 2014: 140

Type locality: Peru, Tumbes, El Caucho Biol. Station

Distribution: Peru

Host: Sayornis nigricans (Passeriformes: Tyrannidae)

Picobia schmidti Glowska et Milensky

Picobia schmidti Glowska \& Milensky, 2014: 378

Type locality: Peru, Tumbes, El Caucho Biol. Station

Distribution: Guyana, Peru

Hosts: Lophotriccus pilatus, Hemitriccus margaritaceiventer (Passeriformes: Tyrannidae)

Picobia sturni Skoracki, Bochkov et Wauthy

Picobia sturni Skoracki, Bochkov \& Wauthy, 2004: 159; Bochkov \& Mironov, 1998; Skoracki, 2011b; Skoracki, Sikora \& Spicer, 2016: 61

Type locality: Poland, Bukowo - Kopan

Distribution: Japan, Moldavia, Morocco, Poland, Slovakia

Hosts: Sturnus cineraceus, S. unicolor, S. vulgaris (Passeriformes: Sturnidae)

Picobia troglodytidus Sikora et Skoracki

Picobia troglodytidus Sikora \& Skoracki, 2012: 62

Type locality: Costa Rica, Cartago

Distribution: Costa Rica

Host: Henicorhina leucophrys (Passeriformes: Troglodytidae)

\section{Picobia sp.}

Argentina, Skoracki \& Sikora, 2002: 8

Host: Aphrastura spinicauda (Passeriformes: Furnariidae)

Genus Pipicobia Glowska et Schmidt

Pipicobia Glowska \& Schmidt, 2014b: 58

Type species: Picobia locustella Skoracki, Bochkov et Wauthy, 2004

Pipicobia glossopsitta (Skoracki, Glowska et Sikora)

Picobia glossopsitta Skoracki, Glowska \& Sikora, 2008: 67

Neopicobia glossopsitta: Skoracki, 2011: 384

Pipicobia glossopsitta: Glowska \& Schmidt, 2014: 59

Type locality: Australia

Distribution: Australia

Host: Glossopsitta porphyrocephala (Psittaciformes: Psittaculidae) 
Pipicobia locustella (Skoracki, Bochkov et Wauthy)

Picobia locustella Skoracki, Bochkov \& Wauthy, 2004: 164

Neopicobia locustella Scoracki, 2011: 389

Pipicobia locustella (Skoracki, Bochkov et Wauthy): Glowska \& Schmidt, 2014: 58

Type locality: Poland, Swinoujscie

Distribution: Poland

Hosts: Locustella luscinioides, L. naevia (Passeriformes: Locustelliidae)

Pipicobia pyrrholaemus (Skoracki et Glowska)

Picobia pyrrholaemus Skoracki \& Glowska, 2008: 284

Neopicobia pyrrholaemus Scoracki, 2011: 384

Pipicobia pyrrholaemus (Skoracki et Glowska): Glowska \& Schmidt, 2014: 59

Type locality: Australia

Distribution: Australia

Host: Pyrrholaemus sagittatus (Passeriformes: Acanthizidae)

Pipicobia terpsiphoni Glowska et Schmidt

Pipicobia terpsiphoni Glowska \& Schmidt, 2014: 59; Skoracki et al., 2016: 1544

Type locality: Ogooue Maritime Province (Moukalaba-Doudou National Park, Gabon)

Distribution: Gabon, Madagascar

Hosts: Terpsiphone mutata, T. rufiventer (Passeriformes: Monarchidae)

Genus Pseudopicobia Skoracki, Scibek et Sikora

Pseudopicobia Skoracki, Scibek \& Sikora, 2012: 230

Type species: Pseudopicobia nonnula Skoracki, Scibek et Sikora, 2012

Pseudopicobia hapaloptila Skoracki, Scibek et Sikora

Pseudopicobia hapaloptila Skoracki, Scibek \& Sikora, 2012: 234

Type locality: Riobamba

Distribution: Ecuador

Host: Hapaloptila castanea (Galbuliformes: Bucconidae)

Pseudopicobia malacoptila Skoracki, Scibek et Sikora

Pseudopicobia malacoptila Skoracki, Scibek \& Sikora, 2012: 233

Type locality: Antioquia, Mutatá

Distribution: Colombia

Host: Malacoptila panamensis (Galbuliformes: Bucconidae)

Pseudopicobia nonnula Skoracki, Scibek et Sikora

Pseudopicobia nonnula Skoracki, Scibek \& Sikora, 2012: 230 
Type locality: Bogota

Distribution: Colombia

Host: Nonnula frontalis (Galbuliformes: Bucconidae)

Genus Rafapicobia Skoracki

Rafapicobia Skoracki, 2011: 391

Type species: Rafapicobia zirnitra Skoracki, 2011

Rafapicobia automoli Glowska, Laniecka et Milensky

Rafapicobia automoli Glowska, Laniecka \& Milensky, 2015: 489

Type locality: Guyana

Distribution: Guyana

Hosts: Automolus ochralaemus, A. rufipileatus (Passeriformes: Furnariidae)

Rafapicobia brachypteraci Skoracki, Hromada et Sikora

Rafapicobia brachypteraci Skoracki, Hromada \& Sikora, 2020: 171

Type locality: Madagascar

Distribution: Madagascar

Host: Atelornis crossleyi (Coraciiformes: Brachypteracidae)

Rafapicobia brotogeris (Fain, Bochkov et Mironov)

Picobia brotogeris Fain, Bochkov \& Mironov, 2000: 62; Skoracki, Bochkov \& Wauthy, 2004: 163; Skoracki, 2005: 128; 2011: 328

Picobia brotogeri: Skoracki \& Sikora, 2002: 1

Rafapicobia brotogeris (Fain, Bochkov et Mironov): Skoracki, Sikora \& Spicer, 2016: 73

Type locality: Upper Amazonia (Zoo of Antwerp)

Distribution: Brazil

Host: Brotogeris jugularis cyanopterus (Psittaciformes: Psittacidae)

Rafapicobia copsychus Skoracki, OConnor et Goodman

Rafapicobia copsychus Skoracki, OConnor \& Goodman, 2016: 1536

Type locality: Tulear Province, Fivondronana de Tolagnaro, Forêt de Marovony, 19 km

NE Manantenina ( $\left.24^{\circ} 06^{\prime} \mathrm{S}, 47^{\circ} 22^{\prime} \mathrm{E}\right)$

Distribution: Madagascar

Host: Copsychus albospecularis (Passeriformes: Turdidae)

Rafapicobia dendrocolaptesi Skoracki et Solarczyk

Rafapicobia dendrocolaptesi Skoracki \& Solarczyk, 2012: 60

Type locality: Paraguay, Apa-Bergland

Distribution: Argentina, Paraguay

Hosts: Dendrocolaptes platyrostris, D. picumnus (Passeriformes: Furnariidae) 
Rafapicobia lepidocolaptesi Skoracki et Solarczyk

Rafapicobia lepidocolaptesi Skoracki \& Solarczyk, 2012: 62; Skoracki, Sikora \& Spicer, 2016: 78

Type locality: Ecuador, Guale

Distribution: Colombia, Ecuador, Venezuela

Hosts: Lepidocolaptes affinis, L. souleyetii (Passeriformes: Furnariidae)

Rafapicobia melzeri Skoracki, Unsoeld, Skorupski et Kavetska

Rafapicobia melzeri Skoracki, Unsoeld, Skorupski \& Kavetska, 2014: 228; Skoracki, Sikora \& Spicer, 2016: 79

Type locality: Germany, Bayern, Ismaninger Lake

Distribution: Chile, France, Germany, Japan, Kirghizia

Hosts: Pardirallus sanguinolentus, Porzana fusca, P. parva, P. porzana, Rallus aquaticus (Gruiformes: Rallidae)

Rafapicobia milenskyi Glowska et Schmidt

Rafapicobia milenskyi Glowska \& Schmidt, 2014: 198

Type locality: Upper Takutu-Upper Essequibo, Lower Rewa River

Distribution: Guyana

Host: Conopophaga aurita (Passeriformes: Conopophagidae)

Rafapicobia momoti Skoracki, Hromada et Sikora

Rafapicobia momoti Skoracki, Hromada \& Sikora, 2020: 171

Type locality: Brazil

Distribution: Brazil

Host: Baryphthengus ruficapillus (Coraciiformes: Momotidae)

Rafapicobia olszanowskii Skoracki, Zmudzinski et Sikora

Rafapicobia olszanowskii Skoracki, Zmudzinski \& Sikora, 2020: 449

Type locality: Ecuador

Distribution: Ecuador

Host: Semnornis ramphastinus (Piciformes: Semnornithidae)

Rafapicobia ramphastos (Fain, Bochkov and Mironov)

Picobia ramphastos Fain, Bochkov \& Mironov, 2000: 33; Skoracki, Bochkov \& Wauthy, 2004: 163

Rafapicobia ramphastos (Fain, Bochkov and Mironov): Skoracki, Sikora \& Spicer, 2016: 73

Type locality: Guatemala (Zoo of Antwerp)

Distribution: Brazil, Guatemala

Hosts: Ramphastos sulfuratus, R. vietellinus (Piciformes: Ramphastidae) 
Rafapicobia thamnophili Glowska et Schmidt

Rafapicobia thamnophili Glowska \& Schmidt, 2014: 196

Type locality: Guyana, Cuyuni Mazarini, Paruima, ca. 19 km N, at Mt. Holitipu

Distribution: Guyana

Hosts: Hypocnemis cantator, Myrmeciza ferruginea, Myrmoborus leucophrys, Myrmotherula longipennis, Thamnophilus insignis (Passeriformes: Thamnophilidae)

Rafapicobia toxostoma Sikora, Fajfer et Skoracki

Rafapicobia toxostoma Sikora, Fajfer \& Skoracki, 2012: 30

Type locality: USA

Distribution: USA

Host: Toxostoma curvirostre (Passeriformes: Mimidae)

\section{Rafapicobia zirnitra Skoracki}

Rafapicobia zirnitra Skoracki, 2011: 392; Skoracki, Sikora \& Spicer, 2016: 77

Type locality: Zachodniopomorskie, near Swinoujscie

Distribution: China, Japan, Poland

Hosts: Ficedula hypoleuca, Luscinia akahige, L. cyanura, L. sibilans, Phoenicurus auroreus, Saxicola rubetra (Passeriformes: Muscicapidae)

Genus Tanopicobia Skoracki, Sikora, Jerzak et Hromada

Tanopicobia Skoracki, Sikora, Jerzak \& Hromada, 2020: 4

Type species: Tanopicobia trachyphoni Skoracki, Sikora, Jerzak et Hromada, 2020

Tanopicobia trachyphoni Skoracki, Sikora, Jerzak et Hromada

Tanopicobia trachyphoni Skoracki, Sikora, Jerzak \& Hromada, 2020: 4

Type locality: Arusha

Distribution: Tanzania

Host: Trachyphonus erythrocephalus (Piciformes: Lybiidae)

\section{Index of Syringophilidae}

(Names in bold are accepted as valid)

acanthizus, Neosyringophilopsis 348

accipitridicus, Peristerophila 351

acrocephali, Syringophilopsis 367

aegithali, Neosyringophilopsis 348

aegithali, Syringophilopsis $\mathbf{3 4 8}$

aegithalos, Neoaulonastus 344 afroanthreptes, Aulobia 324

agapornis, Neoaulobia 342

agelaius, Syringophiloidus 359

albicollisi, Syringophilopsis 367

albus, Aulonastus 326

aldwelles, Meitingsunes 340 
alectoris, Columbiphilus 382

alectoris, Picobia 382

alpha, Corvitorotroglus 334

aluconis, Bubophilus 331

amazilia, Syringophiloidus 359

amazonae, Psittaciphilus 355

annae, Stibarokris 357

anoa, Philoxanthornia 354

anthi, Neopicobia 385

anthi, Picobia 385

anthi, Syringophilus 385

anthreptes, Aulobia 324

anthus, Aulonastus 326

apalis, Neoaulonastus 344

aphelocoma, Torotrogla 377

Apodisyringiana 322

Apodisyringophilus 323

apricaria, Charadriineopicobia 381

apus, Syringophiloidus 359

aquilus, Megasyringophilus 338

aratingae, Neoaulobia 343

ariconte, Tinamiphilopsis 377

artamus, Syringophiloidus 359

ascalaphus, Bubophilus 331

Ascetomylla 323

asiobius, Bubophilus 331

atheneus, Neobubophilus 347

auduboni, Syringophiloidus 360

Aulobia 324

Aulonastus 326

automoli, Rafapicobia 397

baltoda, Selenonycha 356

Betasyringophiloidus 328

bartrami, Syringophilopsis 367

bernieriarum, Picobia 388

biarmicus, Picobia 388

bisetatus, Neoaulonastus 344

bisetatus, Syringophilus 344

bipectinatus, Syringophilus 376

blaszaki, Syringophilopsis 367

Blaszakia 330

bochkovi, Galliphilopsis 336 bochkovi, Syringophilopsis 367

Bochkovia 330

bombycillae, Syringophiloidus 360

bonariensis, Syringophilopsis 368

borini, Syringophilopsis 368

brachypteraci, Rafapicobia 397

branta, Chenophila 332

brevisetosus, Stibarocris 357

brotogeri, Picobia 397

brotogeris, Rafapicobia 397

brotogeris, Picobia 397

Bubophilus 330

bucerotidus, Syringophiloidus 360

bulwerius, Procellariisyringophilus 355

bulwerius, Syringonomus 355

buszekae, Aulonastus 337

buszekae, Krantziaulonastus 337

cacatua, Megasyringophilus 338

cacatua, Neoaulobia 343

Calamincola 381

calcarius, Torotrogla 377

calidridis, Niglarobia 350

calidridis, Paraniglarobia 350

calidris, Charadriineopicobia 381

caligatus, Neoaulonastus 344

calyptorhyncha, Lawrencipicobia 384

caprimulgus, Meitingsunes 340

cardinalis, Neopicobia 385

cardinalis, Picobia 385

cardinalis, Torotrogla 378

cardueli, Aulobia 324

cardueli, Torotrogla 378

carolae, Syringophiloidus 360

carpodaci, Syringophiloidus 360

carpodacus, Neopicobia 385

carpodacus, Picobia 385

casmerodia, Trypetoptila 381

Castosyringophilus 351

catesbyi, Syringophilopsis 368

caudati, Picobia 388

certhiae, Syringophilopsis 368

cetti, Picobia 388 
chalcophaps, Meitingsunes 340

Charadriaulobia 331

charadriiformicus, Selenonycha 357

Charadriineopicobia 381

Charadriphilus 331

Chenophila 332

chirovi, Cuculisyringophilus 335

chirovi, Niglarobia 335

chloris, Picobia 389

chrysocolaptes, Neoaulonastus 345

cichladusa, Picobia 389

Ciconichenophilus 357

cincli, Syringophiloidus 360

cinnyris, Neoaulonastus 345

cissa, Picobia 389

cisticolae, Aulobia 324

claravis, Castosyringophilus 351

claravis, Gunabopicobia 383

claravis, Peristerophila 351

clarki, Philoxanthornea 354

clarki, Philoxanthornia 354

coccothraustes, Syringophiloidus 361

coccothraustes, Torotrogla 378

colinastes, Kalamotrypetes 337

Colinophilus 333

colinus, Galliphilopsis 336

Colisyringophilus 333

collocalius, Apodisyringophilus 323

columba, Peristerophila 351

columbae, Peristerophila 351

columbae, Syringophilus 351

columbicus, Meitingsunes 340

Columbiphilus 382

copsychus, Rafapicobia 397

coraciidus, Peristerophila 352

corcoracus, Picobia 389

corvinae, Syringophilopsis 368

Corvitorotroglus 334

coturnae, Mironovia 341

cracidus, Kalamotrypetes 337

Creagonycha 334

crotophaginus, Cuculisyringophilus 335
Crotophagisyringophilus 335

Cuculiphilinae 381

Cuculiphilus 381

Cuculisyringophilus 335

cunicularius, Neobubophilus 347

currucae, Picobia 389

cursoriae, Niglarobia 349

cyanocephala, Megasyringophilus 339

cyanocitta, Torotrogla 378

cypsiuri, Syringophiloidus 361

daberti, Syringophiloidus 361

dalmas, Megasyringophilus 339

dastychi, Stibarokris 357

davidi, Syringophilopsis 368

delichonum, Syringophiloidus 361

dendrocittae, Syringophiloidus 361

dendrocolaptesi, Rafapicobia 397

dendroicae, Aulobia 324

dendroicae, Syringophilus 324

dendroicae, Syringophilopsis 369

dendroicus, Aulobia 324

dicruri, Syringophilopsis 369

dinemellia, Picobia 389

Dissonus 344

dryobates, Picobia 390

dryobatis, Picobia 390

dryobatis, Syringophilus 390

dubinini, Krantziaulonastus 338

dubinini, Megasyringophilus 339

dubinini, Philoxanthornea 354

dubinini, Philoxanthornia 354

dziabaszewskii, Picobia 390

ea, Neopicobia 386

echo, Picobia 390

elegans, Tinamiphilopsis 377

elongatus, Syringophilopsis 369

elongatus, Syringophilus 369

emberizae, Syringophilopsis 369

emberizae, Betasyringophilus 329

emberizae, Torotrogla 378

emberizicus, Aulonastus 326

empidonax, Syringophilopsis 369 
eos, Megasyringophilus 339

epthianura, Neopicobia 386

epthianura, Picobia 386

eremophila, Picobia 390

ereuneti, Niglarobia 349

erythroptera, Aulobia 325

erythrurus, Syringophiloidus 361

estrildus, Syringophiloidus 361

euphagus, Aulonastus 326

faini, Syringophilopsis 369

falcophila, Peristerophila 352

forpi, Castosyringophilus 352

forpi, Peristerophila 352

francolinus, Galliphilopsis 336

freya, Neopicobia 386

fringilla, Syringophilopsis $\mathbf{3 7 0}$

fringillae, Syringophilopsis 370

fringillae, Syringophilus $\mathbf{3 7 0}$

fringillus, Aulonastus 326

fritschi, Psittaciphilus 356

Fritschisyringophilus 335

fulicarius, Phalarophilus 354

furthi, Syringophiloidus 362

galbulicus, Aulonastus 338

galbulicus, Krantziaulonastus 338

galerida, Picobia 391

Galliphilopsis 336

gallinago, Aulobia 331

gallinago, Charadriaulobia 331

gallinago, Charadriphilus 331

gallinula, Ascetomylla 323

garrulaxi, Neosyringophilopsis 348

garrulaxi, Syringophilopsis 348

garrulus, Syringophilopsis 370

gaudi, Torotrogla 378

gelochelidoni, Kethleyana 337

geoffroyus, Megasyringophilus 339

geopelis, Peristerophila 352

geotrygoni, Gunabopicobia 383

geotrygonus, Terratosyringophilus 376

geronticus, Stibarokris 358

glandarii, Syringophiloidus 362 glandarii, Syringophilus 362

glandarii, Syringophilus minor 362

glossopsitta, Neopicobia 395

glossopsitta, Picobia 395

glossopsitta, Pipicobia 395

graculae, Syringophiloidus 362

granatina, Neoaulonastus 345

grewlingi, Neoaulonastus 345

Gunabopicobia 383

hapaloptila, Pseudopicobia 396

haszprunari, Apodisyringiana 323

heeri, Picobia 391

heeri, Syringophilus 391

helleri, Niglarobia 350

helleri, Syringophilus 350

helleri, Picobia 391

henicorhina, Aulonastus 327

hepburni, Neopicobia 386

herbicolae, Neopicobia 386

hirundapi, Apodisyringiana 323

hirundinis, Syringophiloidus 362

hirundus, Syringophilopsis 370

hunanensis, Syringophilopsis 370

hydrobates, Stibarokris 358

hylocichla, Syringophilopsis 375

hylocichlae, Picobia

hylocichlae, Syringophilopsis 375

hylocichlae, Syringophilus 374

icteri, Syringophilopsis 370

ictericus, Neopicobia 386

ictericus, Picobia 387

icteridae, Syringophilus 369

idunae, Syringophilopsis 371

illadopsae, Picobia 391

indonesiana, Picobia 391

insperata, Selenonycha 397

io, Crotophagisyringophilus 335

Ixobrychiphilus 336

jackowiaki, Syringophiloidus 362

Kalamotrypetes 337

kanduli, Chenophila 333

kazmierski, Syringophilopsis 371 
Kethleyana 337

kethleyi, Megasyringophilus 339

khushalkhani, Columbiphilus 382

khushalkhani, Picobia 382

kirgizorum, Syringophilopsis 371

klimovi, Syringophiloidus 362

krafti, Neoaulobia 343

Krantziaulonastus 337

kratos, Picisyringophilus 355

kristini, Syringophilopsis 371

lagonostictus, Syringophilopsis 371

lagopus, Mironovia 342

lamprotornis, Picobia 391

langei, Stibarokris 358

lanius, Aulonastus 327

lapponica, Niglarobia 350

lara, Creagonycha 334

lathami, Gunabopicobia 383

lature, Peristerophila 352

Lawrencipicobia 384

lemi, Picobia 392

lepidocolaptesi, Rafapicobia 398

leucomela, Peristerophila 353

leptotila, Gunabopicobia 384

leucophaeus, Charadriineopicobia 382

leucophaeus, Picobia $\mathbf{3 8 2}$

leucostictus, Aulobia 325

Lobatinae 381

lobatus, Calamincola 381

lobatus, Cuculiphilus 381

locustella, Neopicobia 396

locustella, Picobia 396

locustella, Pipicobia 396

locustellus, Neosyringophilopsis 348

locustellus, Syringophilopsis 348

lonchura, Picobia 392

lonchurae, Picobia 392

lonchurae, Fritschisyringophilus 335

lonchurae, Neosyringophilopsis 348

lonchurus, Krantziaulonastus 345

lonchurus, Neosyringophilopsis 348

lonchurus, Neoaulonastus 345 longisoma, Peristerophila 376

longisoma, Terratosyringophilus 376

lophurus, Galliphilopsis 336

loricinus, Terratosyringophilus 376

loxius, Aulonastus 327

lullulae, Torotrogla 379

lusciniae, Aulonastus 327

lusciniae, Torotrogla 379

lybidus, Neosyringophilopsis 349

lyudmilae, Charadriphilus 332

magellani, Picobia 392

makoli, Picobia 392

malaconotus, Neoaulonastus 345

malacoptila, Pseudopicobia 396

mariasi, Stibarokris 358

marmaronetta, Chenophila 333

masalaje, Gunabopicobia 384

Megasyringophilus 338

Meitingsunes 340

melittophagi, Syringophilopsis 371

melzeri, Rafapicobia 398

mentalis, Picobia 392

meropis, Castosyringophilus 353

meropis, Peristerophila 353

meru, Picobia 392

merulae, Torotrogla 379

metropelia, Gunabopicobia 384

mexicana, Neoaulobia 343

microcerculus, Syringophiloidus 363

milenskyi, Rafapicobia 398

mima, Torotrogla 379

mimidus, Syringophilopsis 372

minor, Syringophiloidus 363

minor, Syringophilus 363

mironovi, Neoaulobia 343

Mironovia 341

modularis, Neopicobia 387

modularis, Picobia 387

modularis, Torotrogla 379

molothrus, Syringophiloidus 363

momoti, Rafapicobia 398

montanus, Psittaciphilus 356 
montanus, Syringophiloidus 363

motacillae, Betasyringophiloidus 329

motacillae, Syringophiloidus 329

mucuya, Castosyringophilus 353

mucuya, Peristerophila 353

muscicapus, Syringophilopsis 372

myiopagi, Picobia 392

myrmecocichla, Picobia 393

mystaceae, Apodisyringiana 323

nanseni, Chenophila 333

nectariniae, Aulobia 325

nectariniae, Syringophiloidus 363

nectariniphilus, Aulonastus 327

Neoaulobia 342

Neoaulonastus 344

Neobubophilus 347

neomixis, Picobia 393

Neoperisterophila 347

Neopicobia 385

Neosyringophilopsis 348

nestoriae, Peristerophila 353

Niglarobia 349

nitens, Syringophilopsis 372

nkaii, Syringophiloidus 363

nonnula, Pseudopicobia 396

numidae, Syringophilus 376

nucifragus, Syringophilopsis 372

ochoi, Picobia 393

odontophoridus, Columbiphilus 383

olszanowskii, Rafapicobia 398

oritis, Picobia 393

oriolus, Syringophiloidus 364

oryzivorus, Neoaulonastus 346

oryzivorus, Krantziaulonastus 346

otididus, Pteroclidisyringophilus 356

paenae, Torotrogla 379

paludicola, Picobia 393

paraguaiae, Aulobia 332

paraguaiae, Charadriphilus 332

Paraniglarobia 350

parapresentalis, Syringophiloidus 364

pari, Neopicobia 387 pari, Syringophilopsis 372

paridus, Aulonastus 327

passeri, Picobia 393

passericus, Syringophilopsis 372

passerina, Syringophilopsis 373

passerinae, Syringophilopsis 373

passerinae, Syringophilus 373

patagioenas, Psittaciphilus 356

pavodaptes, Kalamotrypetes 342

pavodaptes, Mironovia 342

Peristerophila 351

petronicus, Syringophiloidus 364

Stibarokris 358

Phalarophilus 354

phalaropi, Bochkovia 330

phasiani, Mironovia 342

philemonis, Syringophilopsis 373

philomelosus, Syringophiloidus 364

Philoxanthornea 354 (misspeling)

Philoxanthornia 354

Phipicobia 388

phoeniconaias, Ciconichenophilus 358

phoeniconaias, Stibarokris 358

phoeniculi, Picobia 394

phoenicuri, Picobia 394

phoenicurus, Betasyringophilus 329

phylloscopi, Neosyringophilopsis 349

phylloscopi, Syringophilopsis 349

picidus, Neoaulonastus 346

picidus, Syringophiloidus 364

Picineoaulonastus 354

Picisyringophilus 355

Picobia 388

Picobiinae 381

pioni, Terratosyringophilus 377

Pipicobia 395

pipili, Aulonastus 327

piranga, Torotrogla 380

pirangus, Aulonastus 328

plataleus, Stibarokris

platyrhynchos, Chenophila 333

passerina, Syringophilopsis 373 
passerinae, Syringophilopsis 373 passerinae, Syringophilus 373 platycercus, Megasyringophilus 339 platyrhynchos, Chenophila 333 plocei, Syringophiloidus 364 ploceus, Picobia 394 pogoniulus, Picineoaulonastus 355 poicephali, Lawrencipicobia 385 poicephali, Picobia 385 polioptilus, Syringophilopsis 373 polonica, Columbiphilus 382 polonica, Picobia 382 porzanae, Ascetomylla 323 porzanae, Syringophilopsis 323 presentalis, Syringophiloidus 365 prionops, Neoaulonastus 346 Procelariisyringophilus 355 prunellae, Aulonastus 328 psaltriparus, Picobia 394 pseudonigritae, Syringophiloidus 365 Pseudopicobia 396

Psittaciphilus 355 psittaculae, Neoaulobia 343 pteroclesi, Columbiphilus 383 pteroclesi, Picobia 383 Pteroclidisyringophilus 356 pteroptochos, Aulonastus 328 ptilinopus, Meitingsunes 341 puylaerti, Aulobia 343 puylaerti, Neoaulobia 343 pycnonoti, Picobia 394 pycnonotus, Torotrogla 380 pygiptilae, Phypicobia 387 pyrrholaemus, Picobia 396 pyrrholaemus, Pipicobia 396 pyrrholaemus, Neopicobia 396 quelea, Neoaulonastus 346 quetzali, Syringophiloidus 365 Rafapicobia 397 ralli, Charadriphilus 332 ramphastos, Rafapicobia 398 ramphastos, Picobia 398 re, Charadriiphilus 356

re, Pteroclidisyringophilus 356

regiusi, Neoperisterophila 347 reichholfi, Terratosyringophilus 377 remizus, Neoaulonastus 346 rhinoptili, Niglarobia 350 rhynchopsittae, Megasyringophilus 340 riparius, Neoaulonastus 346 riparius, Picobia 394 ristowi, Charadriineopicobia 382 rossae, Blaszakia 330 rouloul, Mironovia 342 rubeculi, Torotrogla 380 rusticus, Syringophilopsis 373 saxicolus, Betasyringophilus 329 sayornis, Picobia 395 schmidti, Picobia 395 schoeniclus, Betasyringophiloidus 329 schoeniclus, Syringophiloidus 329 schoeniclusi, Syringophiloidus 329 scirpaceus, Dissonus 344 scirpaceus, Neoaulonastus 344 seiuri, Betasyringophiloidus 329 seiuri, Syringophilus 329 seiuri, Syringophiloidus 329 seiurus, Syringophiloidus 329 Selenonycha 356 serini, Syringophiloidus 365 sialiae, Syringophilopsis 373 sialius, Syringophiloidus 365 sidorchukae, Neoaulonastus 346 similis, Aulonastus 328 sittae, Syringophilopsis 373 skorackii, Niglarobia 350 skorackii, Paraniglarobia 351 spinolettus, Syringophilopsis 374 sporophila, Syringophiloidus 365 stachyris, Aulobia 325 stachyris, Syringophilopsis 325 stawarczyki, Syringophiloidus 366 sternae, Creagonycha 334 Stibarokris 357 
sturnellus, Aulonastus 328

sturnellus, Syringophilopsis 374

sturni, Syringophilopsis 374

sturni, Picobia 395

sulphurea, Lawrencipicobia 385

sylviae, Aulobia 325

sylviettae, Aulobia 325

sylvietta, Syringophilopsis 325

sylviettae, Syringophilopsis 325

synallaxis, Torotrogla 380

Syringonomus 355

Syringophilidae 322

Syringophilinae 322

Syringophiloidus 359

Syringophilopsis 367

Syringophilus 375

szeffleri, Syringophilopsis 374

szeptyckii, Galliphilopsis 336

Tanopicobia 399

tanzanicus, Colisyringophilus 334

tanzanicus, Neoaulonastus 347

tarni, Syringophiloidus 366

tarnii, Syringophiloidus 366

tauracos, Blaszakia 330

teledromas, Syringophiloidus 366

terpsiphoni, Pipicobia 396

Terratosyringophilus 376

thamnophili, Rafapicobia 399

theristicus, Stibarokris 359

thryothorus, Syringophiloidus 366

Tinamiphilopsis 377

Torotrogla 377

totani, Creagonycha 334

totani, Syringophilus 334

toxostoma, Rafapicobia 399

trachyphoni, Tanopicobia 399

trichoglossus, Megasyringophilus 340

troglodytes, Neopicobia 387

troglodytes, Picobia $\mathbf{3 8 7}$

troglodytidus, Picobia 395

troglodytis, Neosyringophilopsis 349

troglodytis, Syringophilopsis 349 troglodytis, Syringophilus 349

trogoni, Syringophilopsis 374

trouessarti, Niglarobia 350

trouessarti, Syringophilus 350

Trypetoptila 380

turacoensis, Meitingsunes 341

turdi, Syringophilopsis 374

turdi, Syringophilus 374

turdus, Syringophilopsis 374

tympanistria, Meitingsunes 341

tympanistria, Peristerophila 341

tyrrani, Syringophilopsis 375

tytonus, Bubophilus 331

upupi, Peristerophila 353

vanelli, Charadriaulobia 332

vanelli, Charadriphilus 332

vanelli, Niglarobia 350

veselovsky, Syringophilopsis 375

villosa, Picobia 380

villosa, Torotrogla 380

villosus, Syringophilus $\mathbf{3 8 0}$

virens, Aulobia 326

volgini, Torotrogla 380

wallacei, Ixobrychiphilus 337

weiszii, Syringophiloidus 366

wilsoni, Colinophilus 333

wilsoni, Syringophilopsis 375

xanthocephalus, Syringophiloidus 366

yosefi, Syringophilopsis 375

yoyomi, Krantziaulonastus 338

zenadoura, Peristerophila 341

zenaidurae, Meitingsunes 341

zenaidurae, Peristerophila 341

zenadourae, Syringophilus 341

zirnitra, Rafapicobia 399

zonotrichia, Syringophiloidus 366

zosterops, Neoaulonastus 347

zumpti, Gunabopicobia 384

zumpti, Neopicobia 384

zumpti, Picobia 384

zumpti, Syringophilus 384 


\section{Distribution of Syringophilidae}

\section{EUROPE}

Austria (Skoracki, Zmudzinski, Unsoeld \& Sikora, 2016) - Stibarokris plataleus

Azerbaidzhan (Amirhanov, 1970, 1973) - Syringophiloidus minor, Syringophilus bipectinatus

Belarus (Bochkov, 2000; Skoracki, 2011) - Syringophilus bipectinatus

Belgium (Bochkov, Fain \& Skoracki, 2004; Skoracki, Sikora \& Spicer, 2016) - Columbiphilus khushalkhani, Mironovia coturnae

Bosnia and Herzegovina (Skoracki, 2011; Skoracki, Sikora \& Spicer, 2016; Skoracki, Hromada \& Sikora, 2017) - Peristerophila meropis, Picobia dryobatis, Syringophiloidus minor

Croatia (Skoracki, 2011) - Aulobia sylviae, Syringophiloidus minor

Denmark (Skoracki, 2011) - Syringophiloidus hirundinis

Finland (Skoracki, 2004d; 2011) - Torotrogla calcarius, T. cardueli

France (Oudemans, 1904, 1906; Bochkov \& Mironov, 199[8]9; Bochkov, Mironov \& Skoracki, 2001; Skoracki, 2011; Skoracki \& Sikora, 2011; Skoracki et al., 2014; Zmudzinski et al., 2015; Skoracki, Sikora \& Spicer, 2016; Skoracki, Spicer \& OConnor, 2016) - Aulobia sylviae, Charadriineopicobia apricaria, Charadriphilus gallinago, Columbiphilus alectoris, Creagonycha totani, Megasyringophilus aquilus, Neosyringophilopsis phylloscopi, Niglarobia helleri, Peristerophila accipitridicus, Rafapicobia melzeri, Syringophiloidus minor, S. presentalis

Corsica (Skoracki, 2011; Skoracki, Hromada \& Sikora, 2017) - Peristerophila meropis, Syringophilus bipectinatus

Germany (Heller, 1880; Fritsch, 1958; Skoracki, 2011; Skoracki et al., 2014; Zmudzinski et al., 2015; Skoracki et al., 2018; Zmudzinski \& Unsoeld, 2019; Kaszewska et al., 2020) Aulobia cardui, Aulonastus fragilis, Bubophilus asiobius, Charadriineopicobia ristowi, Columbiphilus khushalkhani, Neoaulonastus bisetatus, N. picidus, Neosyringophilopsis trogloditis, Neopicobia anthi, Niglarobia lapponica, N. vanelli, Picobia caudati, P. dryobatis, $P$. heeri, Peristerophila accipitridicus, P. columbae, P. falcophila, Rafapicobia melzeri, Selenonycha charadriiformes, Stibarokris dastychi, Syringophiloidus coccothraustes, S. glandarii, S. klimovi, S. minor, S. parapresentalis, S. picidus, S. weiszii, Syringophilopsis fringillae, S. garrulus, S. kazmierski, S. kirgizorum, S. troglodytis, S. turdi, Syringophilus bipectinatus, Torotrogla cardueli, T. merulae

Gibraltar (Skoracki, Hromada \& Sikora, 2017) - Peristerophila meropis

Great Britain (Kethley, 1970; Nattress \& Skoracki, 2007, 2009; Kaszewska et al., 2020) Bubophilus aluconis, Neosyringophilopsis aegithali, N. troglodytis, Picobia dryobatis, Peristerophila columbae, Syringophiloidus minor, Syringophilopsis fringillae, S. hirundus, S. kirgizorum, Torotrogla modularis 
Greece (Skoracki, Hromada \& Sikora, 2017; Kaszewska et al., 2020) - Peristerophila columbae, C. meropis, Procellariisyringophilus bulwerius

Hungary (Kaszewska et al., 2020) - Peristerophila columbae

Iceland (Bochkov \& Skirnisson, 2011) - Mironovia lagopus

Italy (contin.)(Skoracki, Møller \& Tryjanowski, 2003; Skoracki, 2004, 2011) - Syringophiloidus hirundinis, S. petronicus, Torotrogla lusciniae

Sardinia (Skoracki, Hromada \& Sikora, 2017) - Peristerophila meropis

Sicily (Skoracki \& Sikora, 2011) - Galliphilopsis szeptyckii [recorded as «bochkovi»]

Malta (Zmudzinski, Solarczyk \& Skoracki, 2016) - Stibarokris hydrobates

Moldavia (Bochkov \& Mironov, 1999) - Picobia dryobatis, P. sturni

Monaco (Kaszewska et al., 2020) - Peristerophila mucuya

North Macedonia (Skoracki, Sikora \& Spicer, 2016: 62; Skoracki, Hromada \& Sikora, 2017; Kaszewska et al., 2020) - Peristerophila columbae, Picobia phoeniculi, Peristerophila meropis

Norway (Bochkov \& Skirnisson, 2011; Skoracki \& Sikora, 2011) - Mironovia lagopus

Svalbard (Skoracki \& Zawierucha, 2016) - Chenophila nanseni

Poland (Skoracki, 1999a, 1999b, 2002, 2004a, c, d, 2011; Skoracki \& Skoracka, 1999; Skoracki \& Dabert, 1999; Skoracki \& Magowski, 2001; Skoracki \& Bochkov, 2002; Skoracki \& Kiljan, 2002; Skoracki \& Hebda, 2004; Skoracki, Bochkov \& Sikora, 2004; Skoracki, Bochkov \& Wauthy, 2004; Skoracki, Dabert \& Schmäschke, 2006; Skoracki, Michalik \& Sikora, 2010; Skoracki et al., 2012; Skoracki, Sikora \& Spicer, 2016) Aulobia cardueli, A. sylviae, Aulonastus albus, Betasyringophyloidus phoenicurus, $B$. saxicolus, B. schoeniclus, Bubophilus aluconis, B. asiobius, Charadriphilus gallinago, Chenophila platyrhynchos, Columbiphilus Columbiphilus khushalkhani (= polonica), Cregonycha totani, Krantziaulonastus buczekae, Meitingsunes columbicus, Mironovia coturnae, Neoaulonastus [Dissonus] scirpaceus, Neopicobia anthi, Neosyringophilopsis aegithali, N. locustellus, N. phylloscopi, N. troglodytis, Niglarobia helleri, Neopicobia modularis, Peristerophila columbae, Philoxanthornea clarki, Picobia biarmicus, P. caudati, $P$. currucae, $P$. dryobatis, $P$. galerida, $P$. heeri, $P$. paludicola, $P$. riparius, $P$. sturni, Pipicobia locustella, Selenonycha charadriiformes, Stibarokris langei, Syringophiloidus bombycillae, S. carpodaci, S. coccothraustes, S. delichonum, S. hirundinis, S. minor, S. parapresentalis, S. presentalis, Syringophilopsis acrocephali, S. blaszaki, S. borini, S. fringillae, S. hirundus, S. kazmierski, S. kirgizorum, S. rusticus, S. spinolettus, S. sturni, S. troglodytis, S. turdi, Syringophilus bipectinatus, Torotrogla cardueli, T. gaudi, T. merulae, T. modularis, T. rubeculi

Portugal (cont.)

Madeira (Kaszewska et al., 2020) - Peristerophila columbae

Romania (Skoracki, 2011; Skoracki, Hromada \& Sikora, 2017; Skoracki, 2018 ) - Peristerophila meropis, Peristerophila falcophila, Syringophilopsis kazmierski, S. kristini, Torotrogla merulae 
Russia (all, continent.)(Dubinina, 1987; Bochkov, 2000, 2001; Bochkov \& Apanaskevich, 2001; Bochkov \& Chystiakov, 2001; Bochkov \& Mironov, 1998, 1999; Bochkov, Mironov \& Skoracki, 2001; Bochkov \& Chistyakov, 2001; Skoracki, 2011; Skoracki \& Mironov, 2013; Skoracki \& Sikora, 2014; Skoracki, Hromada \& Sikora, 2017) - Aulobia cardueli, A. galinago, A. sylviae, Aulonastus prunellae, Betasyringophiloidus motacillae, B. emberizae, B. schoeniclus, Peristerophila meropis, Charadriphilus lyudmilae, Chenophila canduli, Krantziaulonastus dubinini, Melingsunes columbicus, Neoaulonastus zosterops, Neosyringophilopsis aegithali, Niglarobia vanelli, Philoxanthornea clarki, Picobia passeri, Selenonycha charadriiformicus, Stibarokris langei, Syringophiloidus carpodaci, S. delichonum, S. glandarii, S. minor, S. presentatus, Syringophilopsis blascaki, S. borini, S. fringillae, S. kirgizorum, S. pari, S. sittae, S. sturmi, S. troglodytis, S. turdus, Syringophilus bipectinatus, S. parapresentalis, Torotrogla cardueli, T. gaudi, T. lullulae, T. merulae, T. rubeculi, T. volgini

Slovakia (Skoracki, Hromada \& Kuczynski, 2001; Skoracki, 2002; Skoracki, Tryjanowski \& Hromada, 2002; Skoracki, 2011; Klimovičová \& Hromada, 2014) - Aulobia cardueli, Aulonastus albus, Betasyringophiloidus motacillae, B. schoeniclus, Neoaulonastus bisetatus, N. picidus, Neosyringophylopsis aegithali, N. phylloscopi, Niglarobia helleri, Picobia dryobatis, P. sturni, Syringophiloidus bombycillae, S. minor, S. montanus, S. parapresentalis, S. presentalis, Syringophilopsis acrocephali, S. blaszaki, S. fringillae, S. kazmierski, S. kristini, S. spinolettus, Torotrogla cardueli, T. gaudi, T. lullulae, T. merulae, T. modularis, T. rubeculi

Spain (cont.) (Skoracki, 2011; Zmudzinski et al., 2015; Skoracki, Hromada \& Sikora, 2017) - Peristerophila meropis, Megasyringophilus aquilus, Syringophiloidus hirundinis Canary Isl. (Skoracki, 2004) - Syringophiloidus erythrurus

Switzerland (Haller, 1878) - Picobia heeri

Turkey (entire)(Bochkov \& Mironov, 1998; Skoracki, 2011; Skoracki, Zmudzinski, Unsoeld \& Sikora, 2016; Skoracki, Hromada \& Sikora, 2017; Zmudzinski, Solarczyk \& Skoradski, 2016) - Peristerophila meropis, Chenophila marmaronetta, Peristerophila columbae, Picobia cetti, P. currucae, Stibarokris brevisetosus, S. phoeniconaias

Ukraine (Kivganov \& Sharafat, 1995; Bochkov, 2000; Skoracki, 2011; Pidhorna et al., 2020) - Betasyringophiloidus phoenicurus, B. saxicolus, Creagonycha sternae, Kethleyana gelochelidoni, Neoaulonastus bisetatus, Philoxanthornea clarki, Syringophiloidus hirundinis, Syringophilopsis acrocephali, S. blaszaki, S. fringillae, S. hirundus, S. kazmierski, S. rusticus, S. sturni, Syringophilus bipectinatus, Torotrogla merulae, T. rubeculi

West Europe (Skoracki, 2004) - Syringophiloidus oriolus

Europe (Skoracki, 2011) - Aulobia leucosticte, Aulonastus caligatus, Syringophilopsis S. idunae, S. nucifragus

\section{AFRICA}

Angola (Klimovičova et al., 2014c; Kaszewska et al., 2020) - Picobia oritis 
Botswana (Skoracky \& OConnor, 2010; Klimovičova et al., 2014c) - Picobia oritis, Stibarokris phoeniconaias

Cameroon (Skoracki \& Dabert, 2002; Skoracki, Tryjanowski \& Hromada, 2002; Skoracki \& Sikora, 2003; Skoracki, Antczak, Riegert, Fainová \& Mikeš, 2009; Skoracki \& Hromada, 2013; Skoracki, Unsoeld, Marciniak \& Sikora, 2016; Skoracki, Sikora, Marciniak \& Zmudzinski, 2016) - Aulobia cisticolae, Bubophilus ascalaphus, B. tytonus, Lawrencipicobia poicephali, Meitingsunes columbicus, Neoaulonastus zosterops, Picobia oritis, Syringophiloidus bucerotidus, Syringophilopsis kirgisorum, S. veselovsky, S. yosefi, Syringophilus bipectinatus

Congo DR (Fain, Bochkov \& Mironov, 2000; Skoracki \& Sikora, 2008; Skoracki et al., 2009; Skoracki, Solarczyk \& Sikora, 2012; Skoracki, Sikora, Marciniak \& Zmudzinski, 2016; Kaszewska et al., 2020) - Aulobia cisticolae, Blaszakia schalowi, Peristerophila columbae, Picobia echo, Syringophiloidus bucerotidus, S. cypsiuri, S. minor, S. seiuri

Egypt (Skoracki \& OConnor, 2010; Skoracki et al., 2006; Skoracki, 2011; Glowska \& Laniecka, 2012) - Columbiphilus pteroclesi, Philoxanthornea dubinini, Picobia eremophila, Pteroclidisyringophus re, Selenonycha charadriiformes, Syringophilopsis davidi Ethiopia (Bochkov \& Fain, 2003; Skoracki, 2014; Skoracki, Hromada, Zmudzinski, Unsoeld \& Sikora, 2018; Kaszewska et al., 2020) - Aulonastus nectariniphilus, Neoaulobia agapornis, Peristerophila claravis, Picineoaulonastus pogoniulus, Picobia dryobatis

Gabon (Glowska \& Schmidt, 2014; Glowska, Broda, Gebhard \& Dabert, 2016) - Pipicobia terpsiphoni, Syringophiloidus furthi, S. plocei

Guinea (Skoracki \& Sikora, 2008) - Blaszakia tauracos

Kenya (Skoracki, Hromada \& Wamiti, 2011; Skoracki \& Hromada, 2013; Klimovičova et al., 2014a, 2016; Skoracki et al., 2014; Skoracki, Hromada \& Sikora, 2017; Skoracki et al., 2018; Skoracki, Hromada, Pervuznakova \& Wamiti, 2019; Kaszewska et al., 2020) - Aulobia cisticolae, A. sylviettae, Betasyringophiloidus seiuri, Peristerophila columbae, P. meropis, Neoaulonastus apalis, N. bisetatus, N. lonchurus, N. malaconotus, $N$. oryzivorus, $N$. prionops, Neopicobia freya, Neosyringophilopsis lybidus, Peristerophila columbae, P. falcophila, P. upupi, Picineoaulonastus pogoniulus, Picobia dryobatis, $P$. dziabaszewskii, $P$. hedydipnae, $P$. illanopsae, P. lamprotornis, $P$. meru, $P$. ploceus, $P$. pycnonoti, Syrigophiloidus picidus, Syringophilopsis dicruri, S. veselovsky

Liberia (Klimovičova et al., 2014) - Neoaulonastus malaconotus

Madagascar (Skoracki, OConnor, Goodman \& Marciniak-Musial, 2016; Skoracki, Hromada \& Sikora, 2020) - Neoaulonastus tanzanicus, Peristerophila accipitridicus, Picobia bernieriarum, P. neomixis, Pipicobia terpsiphoni, Rafapicobia brachypteraci, R. copsychus

Morocco (Kethley, 1970; Skoracki, Sikora \& Spicer, 2016; Skoracki, Hromada \& Sikora, 2017) - Peristerophila meropis, Picobia sturni, Syringophilopsis fringillae

Namibia (Bochkov \& Fain, 2003; Skoracki, Dabert \& Schmaschke, 2006; Klimovičova et al., 2014; Glowska, Romanowska, Schmidt \& Dabert, 2018) - Neoaulobia agapornis, Niglarobia cursoriae, Picobia dziabaszewskii, Torotrogla paenae 
Rwanda (Fain, Bochkov \& Mironov, 2000; Skoracki \& Sikora, 2003) - Aulobia cisticolae, Columbiphilus alectoris, Syringophilopsis emberizae, S. sylviettae

Somalia (Klimovičova et al., 2014c) - Neoaulonastus malaconotus, Picobia oritis

South Africa (Lawrence, 1959; Kethley, 1970; Skoracki \& Dabert, 2002; Skoracki, Bochkov \& Wauthy, 2004; Skoracki, Zmudzinski, Unsoeld \& Sikora, 2016; Skoracki, Sikora \& Spicer, 2016; Kaszewska et al., 2020) - Bubophilus ascalaphus, Columbiphilus khushalkhani, Neopicobia zumpti, Galliphilopsis francolinus, Peristerophila columbae, Picobia oritis, Stibarokris geronticus, Syringophilus bipectinatus

Sudan (Kaszewska et al., 2020) - Peristerophila claravis

Tanzania (Bochkov \& Fain, 2003; Skoracki \& Sikora, 2008; Glowska et al., 2012; Skoracki, Solarczyk \& Sikora, 2012; Skoracki, Hromada \& Unsoeld, 2013; Skoracki \& Hromada, 2013; Klimovičova et al., 2014a; Skoracki et al., 2014; Skoracki, Unsoeld, Marciniak \& Sikora, 2016; Skoracki, Sikora, Marciniak \& Zmudzinski, 2016; Hromada, Klimovičova, Unsöld \& Skoracki, 2016; Skoracki, Hromada \& Sikora, 2017; Skoracki et al., 2018; Skoracki, Hromada, Zmudzinski, Unsoeld \& Sikora, 2018; Skoracki, Sikora, Jerzak \& Hromada, 2020; Kaszewska et al., 2020) - Aulobia afroanthreptes, A. nectariniae, Aulonastus nectariniphilus, A. paridus, Blaszakia tauracos, Bubophilus aluconis, Peristerophila meropis, Colisyringophilus tanzanicus, Cuculisyringophilus chirovi, Neoaulobia agapornis, Neoaulonastus cinnyris, N. granatina, N. quelea, N. tanzanicus, Neopicobia freya, Niglarobia vanelli, Peristerophila claravis, P. columbae, Picineoaulonastus pogoniulus, Picobia cichladusa, $P$. dinemellia, $P$. dryobatis, P. dziabaszewskii, P. myrmecocichla, P. phoeniculi, Syringophiloidus bucerotidus, S. pseudonigritae, S. picidus, Syringophilus nectarinae, Tanopicobia trachyphoni

Togo (Skoracki \& Dabert, 1999, 2000, 2001b; Skoracki \& Sikora, 2003; Glowska \& Skoracki, 2010) - Aulobia cisticolae, A. erythroptera, A. virens, Creagonycha totani, Meitingsunes [Peristerophila] tympanistria, Neoaulobia puylaerti, Syringophiloidus seiuri, Syringophilopsis albicollisi, S. lagonostictus, S. melittophagi, S. nitens

Tunisia (Skoracki, 2011; Skoracki \& Hromada, 2013; Skoracki, Unsoeld \& Skorupski, 2014) - Picobia phoenicuri, Pteroclidisyringophilus otididus

Uganda (Skoracki, 2014; Klimovičova et al., 2014c; Skoracki, Hromada, Pervuznakova \& Wamiti, 2019) - Neoaulonastus lonchurae, N. oryzivorus, Picobia lonchurae, P. oritis, Syrigophiloidus estrildus, S. picidus

Zambia (Fain, 2000; Klimovičova et al., 2014c) - Neoaulobia agapornis, Picobia dryobatis, P. dziabaszewskii, P. oritis

Central Africa (Skoracki \& Dabert, 2000; Skoracki, Sikora \& Spicer, 2016) - Columbiphilus khushalkhani, Syringophilopsis albicollisi

Eastern Africa (Skoracki, Sikora \& Spicer, 2016) - Columbiphilus khushalkhani

Continental Africa (Skoracki, 2008) - Neosyringophilopsis lonchurus

ASIA

Afghanistan (Kivganov \& Sharafat, 1995) - Columbiphilus khushalkhani 
China (Liu Bai-li, 1988; Sun - undated; Skoracki, 2011; Glowska, Gragun-Damian \& Dabert, 2012; Skoracki, Sikora \& Spicer, 2016; Skoracki, Zmudzinski \& Solarczyk, 2017; Kaszewska et al., 2020) - Ixobrychiphilus wallacei, Krantziaulonastus lonchurus, Rafapicobia zirnitra, Syringophiloidus minor, S. oriolus, Peristerophila columbae, Picobia dziabaszewskii

India (Bochkov \& Mironov, 1998; Fain, Bochkov \& Mironov, 2000; Bochkov, Fain \& Skoracki, 2004; Skoracki \& Sikora, 2011; Skoracki, Unsoeld, Marciniak \& Sikora, 2016; Skoracki, Sikora \& Spicer, 2016) - Columbiphilus khushalkhani (= polonica), Fritschisyringophilus lonchurae, Neobubophilus atheneus, Peristerophila columbae, Picobia dryobatis

Indonesia (Skoracki, 2005; Skoracki \& Glowska, 2008c; Skoracki \& Sikora, 2011; Kaszewska, Kavetska \& Skoracki, 2014; Skoracki et al., 2014; Kaszewska, Skoracki \& Kavetska, 2016; Hromada, Klimovičova, Unsöld \& Skoracki, 2016; Kaszewska, Skoracki \& Hromada, 2018; Marciniak, Skoracki \& Hromada, 2019; Kaszewska et al., 2020) Apodisyringiana haszprunari [Sumatra], Cuculisyringophilus chirovi, Galliphilopsis szeptyckii, Gunabopicobia lathami, G. masalaje, Lawrencipicobia sulphurea, Meitingsunes chalcophaps, M. turacoenas, Peristerophila geopelis, P. lature, Picobia echo, P. heeri, P. mentalis, Procellariisyringophilus bulwerius

Iran (Bochkov \& Mironov, 1998; Skoracki, 2011; Skoracki, Sikora \& Spicer, 2016) - Peristerophila columbae, Picobia dryobatis

Japan (Skoracki, 2004; 2011; Skoracki \& Sikora, 2011; Skoracki et al., 2014; Skoracki, Kaszewska \& Kavetska, 2015; Skoracki, Sikora \& Spicer, 2016; Glowska, Laniecka \& Romanowska, 2018; Kaszewskaet al., 2020) - Apodisyringiana hirundapi, Columbiphilus khushalkhani, Galliphilopsis szeptyckii, Krantziaulonastus oryzivorus, Peristerophila columbae, Picobia dryobatis P. heeri, P. sturni, Rafapicobia zirnitra, Syringophiloidus glandarii, Syringophilopsis muscicapus, Torotrogla emberizae

Jordan (Glowska, Skoracki \& Khourly, 2007; Skoracki, 2011) - Aulobia sylviae, Peristerophila columbae, P. mucuya, Picobia currucae, P. pycnonoti, Syringophiloidus minor, S. parapresentalis, S. philomelosus, S. presentalis, Syringophilopsis kazmierski, S. kirgizorum, Torotrogla merulae, T. pycnonotus

Kazakhstan (Bochkov \& Mironov, 1999; Bochkov \& Apanaskevich, 2001; Skoracki \& Bochkov, 2010; Skoracky, 2011; Skoracki, Sikora \& Spicer, 2016) - Aulobia cardueli, A. sylviae, Betasyringophyloidus phoenicurus, Charadriphilus ralli, Corvitorotroglus alpha, Creagonycha totani, Megasyringophilopsis aquilus, Meitingsunes columbicus, Neoaulonastus scirpaceus, Niglarobia calidridis, Paraniglarobia calidridis, Peristerophila accipitridicus, Picobia phoeniculi, Philoxanthornea dubinini, Syringophyloidus carpodaci, S. glandarii, S. klimovi, S. parapresentalis, S. presentalis, S. schoeniclusi, Syringophilopsis faini, S. fringillae, S. hirundus, S. spinolettus, S. sturni, S. turdi

Kyrghyzstan (Kirghizia) (Chirov \& Kravtsova, 1995; Bochkov \& Mironov, 1998; Bochkov, Mironov \& Kravtsova, 2000; Bochkov, 2000; Skoracki, 2011; Skoracki et al., 2014, 2016) - Picobia chloris, Charadriphilus ralli, Creagonycha totana, Cuculisyringophilus 
chirovi, Mironovia phasiani, Picobia dyobatis, Rafapicobia melzeri, Syringophiloidus minor, S. presentalis, S. parapresentalis, Syringophilopsis kirgizorum, S. passericus, S. sturni, Syringophilus bipectinatus

Malaysia (Skoracki \& Unsoeld, 2014) - Picobia mentalis

Mongolia (Skoracky, 2011) - Betasyringophyloidus phoenicurus, Neopicobia modularis, Torotrogla modularis

Nepal (Bochkov, Mironov \& Skoracki, 2001; Skoracki, 2011; Skoracki et al., 2014; Skoracki, Unsoeld, Marciniak \& Sikora, 2016; Skoracki, Sikora \& Spicer, 2016; Skoracki et al., 2018; Kaszewska et al., 2020 ) - Aulobia [Syringophilopsis] stachyris, Bubophilus ascalaphus, Neosyringophilopsis garrulaxi, Peristerophila columbae, P. falcophila, Picobia dryobatis, P. echo, P. heeri

Pakistan (Skoracki, Sikora \& Spicer, 2016; Skoracki, Hromada \& Sikora, 2017) - Peristerophila meropis, Picobia phoeniculi

Philippines (Skoracky \& OConnor, 2010; Skoracki, Sikora \& Spicer, 2016) - Apodisyringophilus collocalius, Neoaulonastus chrysocolaptes, Picobia pycnonoti

Sri Lanka (Zmudzinski et al., 2019) - Neoaulonastus sidorchukae

Thailand (Skoracki et al., 2014) - Picobia heeri

S.E. Asia (Skoracki \& Glowska, 2008; Fain, Bochkov \& Mironov, 2000) - Aulobia anthreptes, Syringophiloidus graculae

East Asia (Fain, Bochkov \& Mironov, 2000) - Syringophiloidus dendrocitta

\section{NORTH AMERICA}

Canada (Bochkov \& Galloway, 2001, 2004; Skoracki, Flannery \& Spicer, 2008, 2009; Zmudzinski et al., 2015; Kaszewska et al., 2020) - Ascetomylla [Syringophilopsis] porzanae, Betasyringophyloidus motacillae, Megasyringophilus aquilus, Neosyringophilopsis troglodytis, Paraniglarobia skorackii, Peristerophila columbae, Syringophiloidus minor, S. seiuri, S. zonotrichia, Syringophilopsis dendroicae, S. elongatus, S. icteri, S. tyranni Mexico (Casto, 1977; Bochkov \& Perez, 2002; Bochkov, Fain \& Skoracki, 2004; Skoracki, 2008, 2017; Broda, Dabert \& Glowska, 2016) - Aulonastus similis, Betasyringophiloidus seiuri, Calamincola lobatus, Crotophagisyringophilus io, Cuculisyringophilus crotophaginus, Megasyringophilus kethleyi, Neoaulobia mironovi, Peristerophila forpi, Syringophiloidus amazilia, S. daberti, Syringophilopsis trogoni, S. tyranni, Terratosyringophilus pioni

USA (Hankock, 1895, 1898; Banks, 1907; Ewing, 1911; Hirst, 1920; Rebrassier \& Martin, 1932; Hwang, 1959; Brown, 1971; Casto, 1976, 1977, 1978, 1980a, 1980b; Clark, 1964; Kethley, 1970; Philips \& Norton, 1978; Bochkov, Williams \& Proctor, 2005; Skoracki, Dabert \& Schmäschke, 2006; Skoracki, Flannery \& Spicer, 2008; Bochkov, Flannery \& Spicer, 2009; Skoracki, Hendricks \& Spicer, 2010a, b, c; 2011; Glowska \& Skoracki, 2010; Skoracky \& OConnor, 2010; Bochkov, Skoracki, Hendricks \& Spicer, 2011; Skoracki, Bochkov OConnor, 2011; Skoracki, Fajfer \& Sikora, 2012; Skoracki, Spicer \& OConnor, 2014, 2016; Skoracki, Sikora \& Spicer, 2016) - Aulobia cardueli, 
A. dendroicae, A. erythroptera, Aulonastus emberizicus, A. euphagus, A. pirangus, $A$. sturnellus, Betasyringophyloidus motacillae, B. seiuri, Bochkovia phalaropi, Bubophilus ascalaphus, Calamincola lobatus, Peristerophila mucuya, Charadriineopicobia leucophaeus, Charadriphilus ralli, Chenophila branta, Ch. canduli, Columbiphilus khushalkhani, Creagonycha lara, Kalamotrypetes colinastes, Meitingsunes [Peristerophila] zenaidurae, Mironovia [Kalamotrypetes] pavodaptes, Neopicobia cardinalis, N. carpodacus, N. zumpti, Niglarobia ereuneti, N. helleri, Peristerophila columbae, P. mucuya, Phalarophilus fulicarius, Philoxanthornea anoa, Picisyringophilus kratos, Picobia dryobatis, P. psaltriparus, Rafapicobia toxostoma, Selenonycha baltoda, Syringophiloidus agelaius, S. bombycillae, S. carolae, S. daberti, S. delichonum, S. glandarii, S. jackowiaki, S. minor, S. molothrus, S. parapresentalis, S. presentalis, S. sialius, S. thryothorus, $S$. weiszii, S. xanthocephalus, S. zonotrichia, Syringophilopsis bartrami, S. catesbyi, S. certhiae, S. elongatus [= icteridae], S. empidonax, S. hylocichlae, S. icteri, S. mimidus, S. passerinae, S. polioptilus, S. sialiae, S. sittae, S. sturnellus, S. tyranni, S. wilsoni, Syringophilus bipectinatus, Terratosyringophilus longimana, Torotrogla aphelocoma, $T$. cardinalis, T. coccothraustes, T. cyanocitta, T. merulae, T. mima, T. piranga, T. villosa Hawaiian Is. (Kethley, 1970; Schmid \& Skoracki, 2007) - Procellariisyringophilus bulwerius

\section{SOUTH and CENTRAL AMERICA}

Argentina (Skoracki \& Sikora, 2002, 2004a, 2008; Skoracki, 2008a; Skoracki \& Glowska, 2008b; Sikora et al., 2011; Skoracki \& Solarczyk, 2012; Sikora, Kaszewska \& Skoracki, 2014; Kaszewska, Skoracki \& Hromada, 2018) - Charadriphilus paraguaiae, Crotophagisyringophilus io, Gunabopicobia leptotila, G. metriopelia, Niglarobia helleri, Meitingsunes [Peristerophila] zenaidurae, Peristerophila mucuya, Picobia sp., Rafapicobia dendrocolaptesi, Syringophiloidus tarni, S. teledromas, Syringophilopsis turdi, Tinamiphilopsis elegans, Torotrogla mima

Bolivia (Skoracki, 2008a; Skoracki \& Glowska, 2008) - Peristerophila claravis, Crotophagisyringophilus io

Brazil (Fain, Bochkov \& Mironov, 2000; Bochkov \& Fain, 2003; Bochkov, Fain \& Skoracki, 2004; Pires \& Daemon, 2007; Skoracki \& Glowska, 2008b; Skoracki et al., 2010; Sikora et al., 2012; Skoracki, Sikora \& Ozminski, 2012; Skoracki et al., 2014; Skoracki et al., 2016; Kaszewska \& Skoracki, 2018; Kaszewska et al., 2020) - Charadriaulobia vanelli, Megasyringophilus kethleyi, Neoaulobia aratingae, Peristerophila mucuya, Picobia dryobatis, P. ictericus, Rafapicobia brotogeris, R. ramphastos, Psittaciphilus montanus, Stibarokris theristicus, Syringophiloidus microcerculus, Syringophilopsis bonariensis, S. tyranni, Tinamiphilopsis ariconte, Torotrogla synallaxis

Chile (Sikora, Kaszewska \& Skoracki, 2014; Scoracki et al., 2014; Skoracki, Kaszewska \& Kavetska, 2015) - Aulonastus pteroptochos, Rafapicobia melzeri, Syringophiloidus apus

Colombia (Fain, Bochkov \& Mironov, 2000; Skoracki, 2008; Glowska \& Skoracki, 2010; Skoracki \& Sikora, 2011; Skoracki, Scibek \& Sikora, 2012; Skoracki \& Solarczyk, 2012; Skoracki, Kaszewska, Unsoeld \& Skorupski, 2015; Hromada, Klimovičova, Unsöld \& 
Skoracki, 2016; Kaszewska, Skoracki \& Hromada, 2018; Kaszewska \& Skoracki, 2018; Skoracki et al., 2018; Kaszewska et al., 2020) - Calamincola lobatus, Columbiphilus odontophoridus, Cuculisyringophilus crotophaginus, Galliphilopsis colinus, Gunabopicobia claravis, G. geotrygoni, Meitingsunes aldwelles, M. caprimulgus, Peristerophila claravis, P. falconis, P. mucuya, Picobia magellani, P. makoli, Pseudopicobia malacoptila, P. nonnula, Psittaciphilus amasonae, P. patagioenas, Rafapicobia lepidocolaptesi

Costa Rica (Sikora et al., 2012; 2013) - Henicorhina leucosticta, Syringophilopsis trogoni

Ecuador (Sikora et al., 2012; Skoracki \& Solarczyk, 2012; Skoracki, Scibek \& Sikora, 2012; Skoracki, Mironov \& Unsoeld, 2013; Glowska \& Laniecka, 2014; Skoracki, Zmudzinski \& Sikora, 2020) - Aulonastus henicorhina, Picobia dryobatis, Picumnus sclateri, Pseudopicobia hapaloptila, Rafapicobia lepidocolaptesi, R. olszanowskii, Syringophiloidus quetzali

Guatemala (Skoracki, Bochkov \& Wauthy, 2004; Skoracki, Mironov \& Unsoeld, 2013) Picobia ramphastos, Syringophiloidus quetzali

Guyana (Skoracki \& Sikora, 2011; Glowska \& Milensky, 2014; Glowska, Laniecka \& Milensky, 2014) - Kalamotrypetes cracidus, Neopicobia herbicolae, Picobia myiopagi, P. ochoi, P. schmidti, Rafapicobia automoli

Martinique (Kaszewska, Skoracki \& Hromada, 2018) - Gunabopicobia geotrygoni

Panama (Skoracki et al., 2014; Kaszewska \& Skoracki, 2018; Skoracki, Mironov \& Bermúdez, 2019; Kaszewska et al., 2020) - Neopicobia freya, Peristerophila claravis, $P$. columbae, Psittaciphilus montanus, Syringophilopsis bochkovi

Paraguay (Skoracki, 2008b; Skoracki \& Sikora, 2011; Skoracki, Sikora \& Ozminski, 2012; Skoracki \& Solarczyk, 2012; Skoracki et al., 2014; Skoracki, Sikora \& Spicer, 2016; Skoracki, Unsoeld, Marciniak \& Sikora, 2016; Kaszewska, Skoracki \& Hromada, 2018; Kaszewska et al., 2020) - Charadriphilus ralli, Cuculisyringophilus crotophaginus, Gunabopicobia geotrygoni, Kalamotrypetes cracidus, Neobubophilus cunicularius, Neopicobia freya, N. hepburni, Peristerophila claravis, P. mucuya, Picobia dryobatis, Rafapicobia dendrocolaptesi, Tinamiphilopsis ariconte

Peru (Glowska \& Milensky, 2014; Glowska, 2014; Skoracki et al., 2014; Glowska \& Laniecka, 2014; Kaszewska et al., 2020) - Neopicobia ea, Peristerophila mucuya, Picobia myiopagi, P. sayornis, P. schmidti, Picumnus olivaceus, Syringophilopsis szeffleri, S. tyranni

Suriname (Skoracki \& Sikora, 2011, Skoracki et al., 2014; Kaszewska \& Skoracki, 2018; Kaszewska et al., 2020) - Columbiphilus odontophoridus, Galliphilopsis colinus, Peristerophila columbae, P. mucuya, Picobia dryobatis, Psittaciphilus patagioenas

Trinidad \& Tobago (Kaszewska \& Skoracki, 2018; Skoracki et al., 2014; Kaszewska et al., 2020) - Peristerophila mucuya, Picobia dryobatis, Psittaciphilus montanus

Uruguay (Glowska, 2015a) - Stibarokris annae

Venezuela (Skoracki \& Glowska, 2008; Skoracki \& Solarczyk, 2012; Skoracki et al., 2014; Skoracki, Unsoeld, Marciniak \& Sikora, 2016; Kaszewska, Skoracki \& Hromada, 
2018) - Gunabopicobia geotrygoni, Megasyringophilus dalmas, Neopicobia ea, Picobia dryobatis, Rafapicobia lepidocolaptesi, Terratosyringophilus geotrygonus

South America (Oudemans, 1904; Skoracki, 2008; Zmudzinski, Solarczyk \& Skoradski, 2016) - Krantziaulonastus galbulicus, Niglarobia trouessarti, Stibarokris phoeniconaias

\section{OCEANIA}

Australia (Domrow, 1991; Skoracki, 2005b; Skoracki, Glowska \& Sikora, 2008, 2011; Skoracki \& Glowska, 2008c; Glowska \& Laniecka, 2013; Glowska, 2013; Kaszewska, Skoracki \& Hromada, 2018; Marciniak, Skoracki \& Hromada, 2019; Kaszewska et al., 2020) - Galliphilopsis szeptyckii, Gunabopicobia lathami, Lawrencipicobia calyptorhyncha, Megasyringophilus cacatua, Meitingsunes ptilinopus, Neoaulobia cacatua, N. krafti, Neopicobia epthianura, N. glossopsitta, Neoaulonastus grewlingi, Peristerophila geopelis, P. leucomela, Picobia corcoracus, Syringophilopsis philemonis, Syringophilus bipectinatus

Johnston Atoll (Glowska 2015) - Stibarokris mariasi

Mariana Islands (Kaszewska, Kavetska \& Skoracki, 2014) - Peristerophila lature Midway Atoll (Kethley, 1970) - Philoxanthornea anoa

New Zealand (Marciniak, Skoracki \& Hromada, 2019) - Peristerophila nestoriae

Papua New Guinea (Skoracki, 2005c; Skoracki \& Sikora, 2008b; Skoracki, Glowska \& Sikora, 2008; Kaszewska, Kavetska \& Skoracki, 2014; Hromada, Klimovičova, Unsöld \& Skoracki, 2016; Marciniak, Skoracki \& Hromada, 2019; Kaszewska, Skoracki \& Hromada, 2018; Skoracki, Hromada \& Sikora, 2020) - Apodisyringiana mystaceae [New Britain], Aulobia nectariniae, Cuculisyringophilus chirovi, Gunabopicobia lathami, G. masalaje, Lawrencipicobia sulphurea, Meitingsunes ptilinopus, Neoaulobia cacatua, Neoperisterophila regiusi, Peristerophila coraciidus, P. lature, Picobia lemi, Terratosyringophilus reichholfi

Locality unknown (Skoracki \& Dabert, 2002) - Syringophilopsis garrulus

\section{Hosts of Syringophilidae}

Aves

Accipitriformes

Fam. Accipitridae

Accipiter francesiae - Peristerophila accipitridicus

A. nisus - Megasyringophilopsis aquilus, Peristerophila accipitridicus

A. rapax - Megasyringophilus aquilus

Buteo jamaicensis - Peristerophila columbae

B. lagopus - Peristerophila accipitridicus

Circaetus gallicus - Peristerophila accipitridicus

Clanga pomarina - Megasyringophilus aquilus 
Hieraaetus pennatus - Megasyringophilus aquilus

Terathopius ecaudatus - Peristerophila accipitridicus

Anseriformes

Fam. Anatidae

Anas clypeata - Chenophila canduli

A. platyrhynchos - Chenophila platyrhynchos

A. querquedula - Chenophila canduli

Branta canadensis - Chenophila branta

B. leucopsis - Chenophila nanseni

Marmaronetta angustirostris - Chenophila marmaronetta

Apodiformes

Fam. Apodidae

Apus melba - Syringophiloidus apus

Collocalia esculenta - Apodisyringophilus collocalius

Cypsiurus parvus - Syringophiloidus cypsiuri

Hirundapus caudacutus - Apodisyringiana hirundapi

Fam. Hemiprocnidae

Hemiprocne comata - Apodisyringiana haszprunari

H. mystacea mystacea - Aposyringiana mystaceae

Fam. Trochilidae

Amazilia candida - Syringophiloidus amazilia

\section{Bucerotiformes}

Fam. Bucerotidae

Horizocerus hartlaubi - Syringophiloidus bucerotidus

H. albocristatus - Syringophiloidus bucerotidus

Lophoceros pallidirostris - Syringophiloidus bucerotidus

Tockus flavirostris - Syringophiloidus bucerotidus

T. fasciatus - Syringophiloidus bucerotidus

T. deckeni - Syringophiloidus bucerotidus

Caprimulgiformes

Fam. Caprimulgidae

Charadriiformes

Chordeiles minor - Meitingsunes caprimulgus

Fam. Charadriidae

Charadrius w. wilsonia - Selenonycha baltoda

Ch. placidus - Selenonycha charadriiformicus

Pluvialis apricaria - Charadriineopicobia apricaria

P. dominica - Selenonycha baltoda

Vanellus albiceps - Niglarobia vanelli

V. chilensis - Charadriphiaulobia vanelli

V. coconatus - Niglarobia vanelli 
V. crassirostris - Niglarobia vanelli

V. gregarius - Niglarobia vanelli

V. lugubris - Niglarobia vanelli

V. senegallus - Niglarobia vanelli

V. spinosus - Niglarobia vanelli

V. vanellus - Niglarobia vanelli

\section{Fam. Glareolidae}

Cursorius temminckii - Niglarobia cursoriae

Smutsornis [Rhinoptilus] africanus - Niglarobia rhinoptili

\section{Fam. Laridae}

Chroicocephalus genei - Selenonycha charadriiformicus

Ch. ridibundus - Selenonycha charadriiformicus

Larus californicus - Selenonycha baltoda

L. canus - Philoxanthornia clarki

L. delawarensis - Creagonycha lara

Leucophaeus atricilla - Charadriineopicobia leucophaeus, Selenonycha baltoda

Subfam. Sterninae

Anous tenuirostris - Philoxanthornia anoa

Chlidonias leucopterus - Philoxanthornia dubinini

Ch. niger - Selenonycha baltoda

Gelochelidon nilotica - Kethleyana gelochelidoni, Philoxanthornia dubinini

Sterna hirundo - Philoxanthornia clarki

Sternula albifrons - Philoxanthornia clarki, Creagonycha sternae

\section{Fam. Scolopacidae}

Arenaria interpres - Niglarobia helleri

Calidris alba - Charadriineopicobia calidris, Creagonycha totani

C. himantopus - Creagonycha totani

C. minuta - Creagonycha totana, Paraniglarobia calidridis

C. pugnax - Selenonycha charadriiformicus

C. pusilla - Niglarobia ereuneti

C. temminckii - Creagonycha totani

Galinago gallinago - Aulobia galinago, Charadriphilus galinago

G. paraguaiae - Charadriphilus paraguaiae

Limosa lapponica - Niglarobia lapponica, Charadriineopicobia risbowi

Phalaropus fulicarius - Bochkovia phalaropi, Phalarophilus fulicarius

Philomachus pugnax - Selenonycha charadriformicus

Scolopax rusticola - Charadriphilus lyudmilae

Tringa flavipes - Niglarobia helleri

T. glareola - Creagonycha totani

T. melanoleuca - Niglarobia skorackii 
T. ochropus - Niglarobia helleri

T. stagnatilis - Niglarobia helleri

T. totanus - Creagonycha totani, Niglarobia helleri

\section{Ciconiiformes}

\section{Fam. Ciconiidae}

Ciconia alba - Stibarokris langei

C. maguari - Stibarokris annae

\section{Coliiformes}

Fam. Coliidae

Colius striatus - Colisyringophilus tanzanicus

Urocolius macrourus - Colisyringophilus tanzanicus

\section{Columbiformes}

\section{Fam. Columbidae}

Chalcophaps indica - Meitingsunes chalcophaps

Chordeiles minor - Meitingsunes caprimulgus

Claravis pretiosa - Peristerophila claravis, Gunabopicobia claravis

Columba arguatrix - Peristerophila columbae

C. guinea - Peristerophila columbae

C. leucomela - Peristerophila leucomela

C. leuconota - Peristerophila columbae

C. livia - Columbiphilus khushalkhani, Meitingsunes columbicus, M. zenaidurae, Gunabopicobia zumpti, Peristerophila columbae

C. oenas - Meitingsunes [Peristerophila] zenadourae, Peristerophila columbae

C. palumbus - Meitingsunes columbicus, Peristerophila columbae

C. trocaz - Peristerophila columbae

Columbina minuta - Peristerophila mucuya

C. passerina - Peristerophila mucuya

C. squammata - Peristerophila mucuya

C. talpacoti - Peristerophila mucuya

Ducula luctuosa - Gunabopicobia masalaje, Peristerophila lature

D. pistrinaria - Gunabopicobia masalaje

D. rosacea - Gunabopicobia masalaje

D. rufigaster - Gunabopicobia masalaje

D. spilorrhoa - Gunabopicobia masalaje, Peristerophila lature

Geopelia cuneata - Peristerophila geopelis

G. placida - Peristerophila geopelis

G. striata - Peristerophila geopelis

Geophaps plumifera - Peristerophila mucuya

Geotrygon chiriquensis - Peristerophila columbae

G. chrysie - Gunabopicobia geotrygoni

G. frenata-Gunabopicobia geotrygoni, Meitingsunes aldwelles 
G. linearis - Terratosyringophilus geotrygonus, Gunabopicobia geotrygoni

G. montana - Gunabopicobia geotrygoni, Psittaciphilus montanus

Leptotila verreauxi - Gunabopicobia leptotila

Leucosarcia melanoleuca - Gunapicobia lathami

Metriopelia ceciliae - Peristerophila mucuya

M. melanoptera - Peristerophila mucuya, Gunabopicobia metropelia

Ocyphaps lophotes - Peristerophila geopelis

Patagioenas fasciata - Psittaciphilus patagioenas

P. speciosa - Psittaciphilus patagioenas, Peristerophila columbae

Petrophassa plumifera - Peristerophila mucuya

Ptilinopus iozonus - Gunabopicobia masalaje

P. jambu - Peristerophila lature

P. magnificus - Meitingsunes ptilinopus

P. melanospilus - Peristerophila lature

P. porphyreus - Peristerophila lature

P. regina - Peristerophila lature

P. rivoli - Meitingsunes ptilinopus

Stigmatopelia senegalensis - Gunabopicobia zumpti

Streptopelia capicola - Gunabopicobia zumpti, Peristerophila columbae

S. decaocto - Peristerophila columbae

S. decipiens - Peristerophila columbae

S. orientalis - Peristerophila columbae

S. semitorquata - Peristerophila columbae

S. tranquebarica - Peristerophila columbae

S. turtur-Peristerophila columbae

Treron waalia - Meitingsunes columbicolus

Turacoena manadensis - Meitingsunes turacoenas

T. modesta - Meitingsunes turacoenas

Turtur tympanistria - Meitingsunes [Peristerophila] tympanistria

Zenaida auriculata - Meitingsunes [Peristerophila] zenadourae, Peristerophila columbae

Z. asiatica - Meitingsunes [Peristerophila] zenadourae, Terratosyringophilus longisoma

Z. macroura - Gunabopicobia zumpti, Meitingsunes [Peristerophila] zenadourae,

Terratosyringophilus longisoma?

\section{Coraciiformes}

Fam. Alcedinidae

Todiramphus chloris - Peristerophila meropis

Fam. Brachypteracidae

Atelornis crossleyi - Rafapicobia brachypteraci 
Fam. Meropidae

Melittophagus bullocki - Syringophilopsis melittophagi

Merops albicollis - Syringophilopsis albicollisi

Fam. Momotidae

Cuculiformes

Baryphthengus ruficapillus - Rafapicobia momoti

Fam. Cuculidae

Centropus goliath - Cuculisyringophilus chirovi

Cercococcyx montanus - Cuculisyringophilus chirovi

Cuculus canorus - Cuculisyringophilus chirovi

Crotophaga ani - Crotophagisyringophilus io

C. solitarius - Cuculisyringophilus chirovi

C. sulcirostris - Cuculiphilus lobatus, Crotophagisyringophilus sulcirostris,

Cuculisyringophilus crotophaginus

Guira guira - Cuculisyringophilus crotophaginus

Pachycoccyx audeberti - Cuculisyringophilus chirovi

Scythrops novaehollandiae - Cuculisyringophilus chirovi

\section{Falconiformes}

Fam. Falconidae

Falco cherrug - Peristerophila falcophila

F. peregrinus - Peristerophila falcophila

Galbuliformes

F. sparverius - Peristerophila falcophila

Fam. Bucconidae

Hapaloptila castanea - Pseudopicobia hapaloptila

Malacoptila panamensis - Pseudopicobia malacoptila

Nonnula frontalis - Pseudopicobia nonnula

Fam. Galbulidae

\section{Galliformes}

Jacamaralcyon tridactyla - Krantziaulonastus galbulicus

Fam. Cracidae

Ortalis canicollis - Kalamotrypetes cracidus

Penelope sp. - Kalamotrypetes cracidus

\section{Fam. Odontophoridae}

Calipepla squamata - Colinophilus wilsoni, Kalamotrypetes colinastes

Colinus cristatus - Columbiphilus odontophoridus, Galliphilopsis colinus

C. leucopogon - Columbiphilus odontophoridus, Galliphilopsis colinus

C. virginianus - Colinophilus wilsoni, Kalamotrypetes colinastes

Fam. Phasianidae

Alectoris barbara - Gallophilopsis szeptickii [ publ. as «bochkovi»]

A. rufa-Columbiphilus alectoris, Syringophilus bipectinatus 
[Alectoris sp]. - Columbiphilus alectoris, Picobia alectori

Coturnix chinensis - Galliphilopsis szeptyckii

C. coturnix - Columbiphilus khushalkhani, Galliphilopsis szeptyckii, Mironovia coturnae

C. pectoralis - Galliphilopsis szeptyckii

Francolinus levaillantoides - Gallophilopsis francolinus

F. clappertoni-Columbiphilus khushalkhani

Gallus gallus domestica - Columbiphilus khushalkhani (=polonica), Syringophilus bipectinatus

G. sonneratii - Columbiphilus khushalkhani (=polonica)

Lagopus lagopus - Mironovia lagopus

L. muta - Mironovia lagopus

Lophura leucomelanos - Gallophilopsis lophurus

Phasianus colchicus - Mironovia phasiani

Ptilopachus petrosus - Syringophilus bipectinatus

Rollulus rouloul - Mironovia rouloul

Tragopan sp. - Gallophilopsis bochkovi

Meleagris gallopavo [pavodaptes] - Mironovia [Kalamotrypetes] pavodaptes

\section{Fam. Numididae}

Acryllium vulturinum - Columbiphilus khushalkhani

Numida meleagris -Syringophilus numidae

Numida meleagris mitratus - Columbiphilus khushalkhani

\section{Gruiformes}

Fam. Aramidae

Aramus guarauna (= scolopaceus) - Niglarobia trouessarti

\section{Fam. Rallidae}

Fulica americana - Charadriphilus ralli

Gallinula chloropus - Ascetomylla gallinula

G. melanops - Charadriphilus ralli

Laterallus melanophaius - Charadriphilus ralli

Pardirallus sanguinolentus - Rafapicobia melzeri

Rallus aquaticus - Charadriphilus ralli, Rafapicobia melzeri

Porzana carolina - Ascetomylla porzanae

P. parva - Charadriphilus ralli, Rafapicobia melzeri

P. porzana-Rafapicobia melzeri

\section{Musophagiformes}

\section{Fam. Musophagidae}

Musophaga rossae - Blaszakia rossae

Tauraco livingstoni - Blaszakia tauracos

T. schalowi - Blaszakia tauracos

\section{Otidiformes}


Fam. Otididae

\section{Passeriformes}

Chlamydotis undulata - Pteroclidisyringophilus otididus

Fam. Acanthizidae

Acanthiza inornata - Neosyringophilopsis acanthizus

Pyrrholaemus sagitatus - Neopicobia pyrrholaemus

Fam. Acrocephalidae

Acrocephalus arundinaceus - Neoaulonastus bisetatus

A. baeticatus - Neoaulonastus bisetatus

A. dumetorum - Neoaulonastus bisetatus (= scirpaceus)

A. paludicola - Neoaulonastus bisetatus, Picobia paludicola

A. palustris - Neoaulonastus bisetatus

A. schoenobaenus - Syringophilopsis acrocephali

A. scirpaceus - Neoaulonastus bisetatus (= scirpaceus), Syringophilopsis acrocephali

Iduna caligata - Neoaulonastus caligatus, Syringophilopsis idunae

Fam. Aegithalidae

Aegithalos caudatus - Neoaulonastes aegithalos, Neosyringophilopsis aegythali,

Picobia caudati

Psaltriparus minimus - Picobia psaltriparus

Fam. Alaudidae

Calandrella brachydactyla - Syringophilopsis davidi

Eremophila alpestris - Torotrogla lullulae

Galerida cristata - Picobia galerida

Lullula arborea - Torotrogla lullulae

Fam. Artamidae

Artamis fuscus - Syringophiloidus artamis

A. leucorhynchus - Syringophiloidus artamis

Fam. Bernieridae

Bernieria madagascariensis - Picobia bernieriarum

Xanthomixis zosterops - Picobia bernieriarum

Fam. Bombycillidae

Bombycilla cedrorum - Syringophiloidus bombycillae

B. garrulus - Syringophiloidus bombycillae

Fam. Calcariidae

Calcarius lapponicus - Torotrogla calcarius

Fam. Cardinalidae

Cardinalis cardinalis - Neopicobia cardinalis, Syringophiloidus carolae, Torotrogla cardinalis

Habia fuscicauda - Aulonastus similis

H. rubica - Aulonastus similis 
Passerina ciris - Syringophiloidus daberti, Syringophilopsis passerinae . cyanea - Syringophilopsis passerinae, Syringophiloidus carolae

Piranga ludoviciana - Aulonastus pirangus, Torotrogla piranga

\section{Fam. Certhiidae}

Certhia americana - Syringophilopsis certhiae

\section{Fam. Cettiidae}

Cettia cetti - Picobia cetti

Fam. Cinclidae

Cinclus mexicanus - Syringophiloidus cincli

\section{Fam. Cisticolidae}

Apalis porphyrolaema - Neoaulonastus apalis

Cisticola cantans - Aulobia cisticolae

C. chubbi - Aulobia cisticolae

C. erythrops - Aulobia cisticolae

C. hunteri - Aulobia cisticolae

C. lateralis - Aulobia cisticolae

Neomixis tenella - Picobia neomixis

Prinia [Heliolais] erythroptera - Aulobia erythroptera

\section{Fam. Conopophagidae}

Conopophaga aurita - Rafapicobia milenskyi

\section{Fam. Corcoracidae}

Corcorax melanorhamphos - Picobia corcoracus

\section{Fam. Corvidae}

Aphelocoma californica - Torotrogla aphelocoma

Cissa chinensis - Picobia cissa

Corvus brachyrhynchos - Syringophilus bipectinatus, Syringophiloidus glandarii

C. frugilegus - Corvitorotroglus alpha, Syringophiloidus glandarii

C. monedula - Syringophiloidus glandarii

Cyanocitta stelleri - Syringophiloidus glandarii [also published as S. presentalis], Torotrogla cyanocitta

Dendrocitta vagabunda [rufa rufa] - Syringophiloidus dendrocittae

Garrulus glandarius - Syringophiloidus glandarii, S. presentalis, Syringophilopsis garrulus

Nucifraga caryocatactes - Syringophilopsis nucifragus

Pica pica - Syringophiloidus glandarii

Urocissa erythrorhyncha - Picobia cissa

\section{Fam. Dicruridae}

Syringophilopsis dicruri - Dicrurus adsimilis

\section{Fam. Emberizidae}

Ammodramus savanarum - Aulonastus emberizicus

Emberiza elegans - Torotrogla volgini 
E. rutila - Betasyringophiloidus schoeniclus

E. schoeniclus - Betasyringophiloidus schoeniclus

E. tristrami - Betasyringophiloidus schoeniclus

Passerculus sandwichensis - Aulonastus emberizicus

Tachyphonus rufus - Syringophiloidus stawarczyki

\section{Fam. Estrildidae}

Erythrura trichroa - Syringophiloidus erythrurus

Estrilda astrild - Neoaulonastus lonchurus, N. oryzivorus

E. atricapilla - Neoaulonastus lonchurus, N. oryzivorus, Picobia lonchurae

E. nonnula - Neoaulonastus lonchurus

E. erythronotus - Neoaulonastus lonchurus

E. rhodopyga - Neoaulonastus lonchurus, Syringophiloidus estrildus

E. troglodytes - Neoaulonastus lonchurus

Granatina ianthinogaster - Neoaulonastes granatina

Lagonosticta rufopicta - Syringophilopsis lagonostictus

L. senegala - Syringophilopsis lagonostictus

Lonchura leucogastra - Picobia lonchura

L. oryzivora - Neoaulonastus oryzivorus

L. punctulata - Fritschsyringophilus lonchurae, Neoaulonastus lonchurus

Lonchura sp. - Syringophiloidus erythrurus

Spermestes cucullatus [Lonchura cucullata] - Neosyringophilopsis lonchurus

\section{Fam. Falcunculidae}

Falcunculus frontatus - Neoaulonastus grewlingi

\section{Fam. Fringillidae}

Acanthis flammea - Aulobia cardueli

Carduelis cannabina - Syringophilopsis kirgizorum, Torotrogla cardueli

C. cardinalis - Aulobia cardueli

C. carduelis - Aulobia cardueli, Syringophilopsis kirgizorum, Torotrogla cardueli

C. citrinella - Aulobia cardueli

C. flavirostris - Aulobia cardueli

Carpodacus erythrinus - Syringophiloidus carpodaci

Chloris chloris - Picobia chloris, Syringophilopsis kirgizorum, Syringophiloidus klimovi

Coccothraustes coccothraustes - Syringophiloidus coccothraustes

C. vespertinus - Torotrogla coccothraustes

Euphonia cyanocephala - Syringophiloidus stawarczyki

Fringilla coelebs - Syringophilopsis fringillae, Torotrogla gaudi, Aulonastus fringillus

F. montifringilla - Syringophilopsis fringillae, Torotrogla gaudi

Haemorhous purpureus - Neopicobia carpodacus 
Leucosticte arctoa - Aulobia leucostictus

Linurgus olivaceus - Syringophilopsis kirgizorum

Loxia curvirostra - Torotrogla cardueli, Aulonastus loxius

L. leucoptera - Torotrogla cardueli

L. pytiopsittacus - Torotrogla cardueli

Pyrrhula pyrrhula - Neopicobia modularis, Torotrogla gaudi

Rhodospiza obsoleta - Syringophilopsis kirgizorum

Serinus canaria - Torotrogla cardueli

S. capistratus - Syringophiloidus minor

S. mozambicus - Syringophiloidus serini

Spinus psaltria - Aulobia cardueli

S. spinus - Aulobia cardueli, Torotrogla cardueli

\section{Fam. Furnariidae}

Aphrastura spinicauda - Picobia sp.

Automolus ochralaemus - Rafapicobia automoli

A. rufipileatus - Rafapicobia automoli

Dendrocolaptes platyrostris - Rafapicobia dendrocolaptesi

D. picumnus - Rafapicobia dendrocolaptesi

Lepidocolaptes affinis - Rafapicobia lepidocolaptesi

L. souleyetii - Rafapicobia lepidocolaptesi

Synallaxis spixi - Torotrogla synallaxis

Xiphocolaptes promeropirhynchus - Picobia makoli

\section{Fam. Hirundinidae}

Delichon urbicum - Syringophiloidus delichonum, S. glandarii

Hirundo rustica - Syringophiloidus delichonum, S. hirundinis, Syringophilopsis

fringillae, S. rusticus

Riparia riparia - Picobia riparius, Syringophilopsis rusticus

\section{Fam. Icteridae}

Agelaius phoeniceus - Syringophiloidus agelaius, Syringophilopsis elongatus [= S. icteridae]

Cacicus chrysopterus - Picobia ictericus

Euphagus carolinus - Syringophilopsis elongatus

E. cyanocephalus - Aulonastus euphagus, Syringophilopsis elongatus

Icterus bullockii - Syringophilopsis icteri

I. galbula - Syringophiloidus molothrus, Syringophilopsis icteri

I. pustulatus - Betasyringophiloidus seiuri, Syringophilopsis icteri

I. spurius - Syringophilopsis icteri

Molothrus ater - Syringophiloidus molothrus

M. bonariensis - Syringophilopsis bonariensis

Pheucticus melanocephalus - Syringophilopsis wilsoni

Pseudoleistes guirahuro - Picobia ictericus 
Quiscalus mexicanus - Syringophilopsis elongatus

Q. quiscula - Syringophilopsis elongatus

Sturnella magna - Aulonastus sturnellus

S. neglecta - Syringophilopsis sturnellus

Xanthocephalus xanthocephalus - Syringophiloidus xanthocephalus

\section{Fam. Laniidae}

Corvinella corvina - Syringophillopsis corvinae

Lanius excubitor - Syringophiloidus weiszii

L. ludovicianus - Syringophiloidus weiszii

L. minor - Syringophilopsis kristini

L. senator - Aulonastus lanius

Lanius sp. - Syringophilopsis yosefi

\section{Fam. Leiothrichidae}

Trochalopteron formosum [Garrulax formosus] - Picobia dziabaszewskii

Trochalopteron affine [Garrulax affinis] - Neosyringophilopsis garrulaxi

Turdoides jardineii - Picobia dziabaszewskii

T. leucopygia - Picobia dziabaszewskii

T. rubiginosa - Picobia dziabaszewskii

Fam. Locustellidae

Locustella luscinioides - Neosyringophilopsis locustellus, Neopicobia locustella

L. naevia - Neosyringophilopsis locustellus, Neopicobia locustella

\section{Fam. Macrosphenidae}

Sylvietta virens flaviventris - Aulobia virens

S. whytii johnstoni - Aulobia sylviettae

\section{Fam. Malaconotidae}

Laniarius aethiopicus - Neoaulonastus malaconotus

Nilaus afer- Neoaulonastus malaconotus

Telophorus nigrifrons- Neoaulonastus malaconotus

\section{Fam. Meliphagidae}

Epthianura aurifrons - Neopicobia epthianura

Philemon citreogularis - Syringophilopsis philemonis

\section{Fam. Mimidae}

Dumetella carolinensis - Syringophilopsis mimidus

Margarops fuscatus - Syringophilopsis mimidus

Mimus patagonicus - Torotrogla mima

M. polyglottos - Torotrogla mima

M. triurus - Torotrogla mima

Toxostoma curvirostre - Rafapicobia toxostoma

\section{Fam. Monarchidae}

Terpsiphone mutata - Pipicobia terpsiphone

T. rufiventer - Pipicobia terpsiphone, Syringophiloidus furthi 


\section{Fam. Motacillidae}

Anthus cervinus - Aulonastes anthus, Neopicobia anthi

A. pratensis - Syringophilopsis blaszaki, Torotrogla modularis

A. spinoletta - Syringophilopsis spinolettus

A. trivialis - Aulonastes anthus, Neopicobia anthi, Syringophilopsis blaszaki Motacilla alba - Aulonastus albus

M. flava - Aulonastus albus, Syringophiloidus motacillae

\section{Fam. Muscicapidae}

Cichladusa arquata - Picobia cichladusa

Cossypha heuglini - Picobia echo

Copsychus malabricus - Picobia echo

C. saularis - Picobia echo

Erithacus rubecula - Torotrogla rubeculi

Ficedula hypoleuca - Rafapicobia zirnitra, Syringophilopsis kazmierski

F. parva - Syringophilopsis kazmierski

Luscinia akahige - Rafapicobia zirnitra

L. cyanura - Rafapicobia zirnitra

L. megarhynchos - Torotrogla lusciniae

L. sibilans - Rafapicobia zirnitra

L. svecica - Torotrogla lusciniae

Muscicapa dauurica (= latirostris) - Syringophilopsis muscicapus

Myrmecocichla arnotti - Picobia myrmecocichla

Phoenicurus auroreus - Rafapicobia zirnitra

Ph. moussieri - Picobia phoenicuri

Ph. ochruros - Betasyringophyloidus phoenicurus

Ph. phoenicurus - Syringophilopsis kazmierski

Saxicola rubetra - Betasyringophyloidus saxicolus, Rafapicobia zirnitra

\section{Fam. Nectariniidae}

Anthreptes longuemarei - Aulobia afroanthreptes, Neoaulonastus cinnyris

A. malacensis - Aulobia anthreptes

A. neglectus - Aulobia afroanthreptes, Neoaulonastus cinnyris

A. reichenowi - Aulonastus nectariniphilus

Cinnyris mediocris - Neoaulonastes cinnyris

C. shelleyi - Aulobia nectariniae

C. chalybeus - Picobia oritis

C. erythrocercus - Picobia oritis

C. jugularis - Aulobia nectariniae

C. mariquensis - Aulobia nectariniae

C. oustaleti - Picobia oritis

C. regius - Aulobia nectariniae

C. shelleyi-Aulobia nectariniae 
C. talatala - Picobia oritis

C. venustus - Picobia oritis

Cyanomitra olivacea - Picobia oritis

C. oritis - Picobia oritis

C. verreauxi - Picobia oritis

Hedydipna collaris - Picobia hedydipna

Leptocoma sericea - Aulobia nectariniae

L. zeylonica - Neoaulonastus sidorchukae

Nectarinia takazze - Aulonastus nectariniphilus

\section{Fam. Oriolidae}

Oriolus chinensis - Syringophiloidus oriolus

O. oriolus - Syringophiloidus oriolus

\section{Fam. Panuridae}

Panurus biarmicus - Picobia biarmicus

\section{Fam. Paradisaeidae}

Cicinnurus regius - Neoperisterophila regiusi

Manucodia ater - Picobia lemi

M. chalybatus - Picobia lemi

\section{Fam. Paridae}

Parus major - Neopicobia pari

Periparus venustulus - Neopicobia pari

Poecile carolinensis - Syringophiloidus jackowiaki

P. palustris - Syringophilopsis palustris

P. varius - Neopicobia pari

\section{Fam. Parulidae}

Geothlypis philadelphia - Betasyringophiloidus seiuri

Helmitheros vermivorum - Betasyringophiloidus seiuri

Oreothlypis peregrina - Betasyringophiloidus seiuri

O. [Vermivora] rufiapilla - Betasyringophiloidus seiuri

Parkesia noveboracensis - Betasyringophiloidus seiuri

Phainopepla nitens - Torotrogla villosa

Protonotaria citrea - Syringophilopsis dendroicae

Seiurus aurocapillus - Betasyringophiloidus seiuri

Setophaga coronata - Aulobia dendroicae, Syringophilopsis dendroicae

S. carulescens - Syringophilopsis dendroicae

S. [Dendroica] graciae - Syringophilopsis dendroicae

S. [D.] nigrescens - Aulobia dendroicae

S. palmarum - Betasyringophiloidus seiuri

S. pensylvanica - Betasyringophiloidus seiuri

S. petechia - Syringophilopsis dendroicae 
Cardellina [Wilsonia] pusilla - Neopicobia troglodytes, Syringophilopsis dendroicae

\section{Fam. Passerellidae}

Melospiza georgiana - Betasyringophiloidus seiuri

M. lincolnii - Betasyringophiloidus seiuri

M. melodia - Betasyringophiloidus seiuri

Pipilo erythrophthalmus - Aulonastus pipili

Spizella breweri - Syringophiloidus auduboni, S. sialius

Zonotrichia albicolis - Betasyringophiloidus seiuri, Syringophiloidus zonotrichia

Z. atricapilla - Aulonastus emberizicus

\section{Fam. Passeridae}

Passer domesticus - Krantziaulonastus dubinini, Picobia passeri, Syringophiloidus minor, Syringophilopsis hunanensis, S. passericus

P. hispaniolensis - Syringophiloidus minor

P. montanus - Syringophilopsis fringillae, Syringophiloidus minor, S. montanus Petronia petronia - Syringophiloidus petronicus

\section{Fam. Pellorneidae}

Illadopsis rufipennis - Picobia illadopsae

\section{Fam. Phylloscopidae}

Phylloscopus collybita - Neosyringophilopsis phylloscopi

Ph. trochilus - Neosyringophilopsis phylloscopi

\section{Fam. Pipridae}

Corapipo altera - Syringophilopsis bochkovi

Manacus vitellinus - Syringophilopsis bochkovi

\section{Fam. Ploceidae}

Dinemellia dinemelli - Krantziaulonastus yoyomi, Picobia dinemellia

Euplectes axillaris - Neoaulonastus tanzanicus

Hyphanturgus nigricollis brachypterus - Syringophilopsis nitens

Malimbus nitens - Syringophilopsis nitens

Ploceus xanthops - Betasyringophiloidus seiuri

P. aurantius - Syringophiloidus plocei

P. cucullatus - Syringophiloidus plocei

P. nelicourvi - Neoaulonastus tanzanicus

P. nigerrimus - Syringophiloidus plocei

P. nigricollis - Syringophilopsis nitens

P. ocularis - Picobia ploceus

Ploceus baglafecht [Othyphantes stuhlmanni] - Betasyringophiloidus seiuri

Ploceus [Pachyphantes] superciliosus - Betasyringophiloidus seiuri

P. vitellinus - Syringophilopsis nitens

Pseudonigrita arnaudi - Syringophiloidus pseudonigritae 
Quelea erythrops - Betasyringophiloidus seiuri, Syringophilopsis nitens

Q. quelea - Neoaulonastus quelea

Fam. Polioptilidae

Polioptila caerulea - Syringophilopsis polioptilus

Fam. Prunellidae

Prunella atrogularis - Neopicobia modularis

P. modularis - Aulonastus prunellae, Neopicobia modularis, Torotrogla modularis

Fam. Ptilogonatidae

Phainopepla nitens - Torotrogla villosa

Fam. Pycnonotidae

Chlorocichla flaviventris - Syringophilopsis veselovsky

Eurillas latirostris - Syringophilopsis veselovsky

Pycnonotus barbatus - Picobia pycnonoti, Syringophilopsis veselovsky

P. goiavier - Picobia pycnonoti

P. xanthopygos - Picobia pycnonoti, Torotrogla pycnonotus

Fam. Remizidae

Remiz pendulinus - Neoaulonastus remizus

Fam. Rhinocriptidae

Pteroptochos tarnii - Syringophiloidus tarnii

Scytalopus magellanicus - Picobia magellani

Teledromas fuscus - Syringophiloidus teledromas

Fam. Sittidae

Sitta carolinensis - Syringophilopsis sittae

S. europaea - Syringophilopsis sittae

\section{Fam. Sturnidae}

Aplonis panayensis - Picobia indonesiana

Enodes erythrophris - Picobia indonesiana

Gracula religiosa - Syringophiloidus graculae

Lamprotornis superbus - Picobia lamprotornis

Mino dumontii - Picobia indonesiana

Sturnus cineraceus - Picobia sturni

S. unicolor - Picobia sturni

S. vulgaris - Krantziaulonastus buczekae Picobia sturni, Syringophiloidus presentalis, Syringophilopsis sturni

\section{Fam. Sylviidae}

Sylvia atricapilla - Aulobia sylviae, Neoaulonastus bisetatus

S. borin - Syringophilopsis borini

S. communis - Neoaulonastus bisetatus

S. curruca - Aulobia sylviae, Neoaulonastus bisetatus, Picobia currucae, Syringophilopsis faini

S. hortensis - Picobia currucae 
S. nisoria - Aulobia sylviae, Picobia currucae

\section{Fam. Thamnophilidae}

Hypocnemis cantator - Rafapicobia thamnophili

Myrmeciza ferruginea - Rafapicobia thamnophili

Myrmoborus leucophrys - Rafapicobia thamnophili

Myrmotherula longipennis - Rafapicobia thamnophili

Pygiptila stellaris - Phipicobia pygiptilae

Thamnophilus insignis - Rafapicobia thamnophili

\section{Fam. Thraupidae}

Dacnis cayana - Syringophiloidus stawarczyki

Emberizoides herbicola - Neopicobia herbicolae

Sicalis luteola - Syringophilopsis emberizae

Sporophila torqueola - Syringophiloidus sporophila

Thamnophilus rufus - Syringophiloidus stawarczyki

Fam. Timaliidae

Cyanoderma [Stachyris] pyrrhops - Syringophilopsis stachyris

\section{Fam. Troglodytidae}

Campylorhynchus brunneicapillum - Syringophiloidus sp.

Henicorhina leucosticta - Aulonastus henicorhina

H. leucophrys - Picobia troglodytidus

Microcerculus marginatus - Syringophiloidus microcerculus

Thryomanes bewickii - Neopicobia troglodytes

Thryothorus ludovicianus - Syringophiloidus thryothorus

Troglodytes aedon - Neopicobia troglodytes, Neosyringophilopsis troglodytis

T. troglodytes - Neosyringophilopsis troglodytis

\section{Fam. Turdidae}

Catharus [= Hylocichla] fuscescens - Syringophilopsis hylocichlae

Copsychus albospecularis - Rafapicobia copsychus

Hylocichla mustelina - Picobia hylocichlae

Sialia mexicana - Syringophiloidus sialius

Turdus albicollis - Syringophilopsis turdi

T. atrogularis - Syringophiloidus parapresentalis, Syringophilopsis turdi

T. iliacus - Syringophiloidus parapresentalis, Syringophilopsis turdi

T. merula - Torotrogla merulae, Syringophiloidus parapresentalis

T. migratorius - Syringophiloidus parapresenalis, S. presentalis, Syringophilopsis turdi, Torotrogla merulae

T. pilaris - Syringophiloidus parapresentalis, Syringophilopsis turdi

T. philomelos - Syringophiloidus philomelosus, Syringophilopsis turdi, Torotrogla merulae

T. torquatus - Torotrogla merulae

T. viscivorus - Torotrogla merulae 
Fam. Tyrranidae

Elaenia ruficeps - Picobia myiopagi

Empidonax hammondii - Syringophilopsis empidonax

E. wrightii - Syringophilopsis empidonax

Hemitriccus margariraceiventer - Picobia schmidti

Lophotriccus pileatus - Picobia schmidti, Syringophilopsis szeffleri

Myiozetetes similis - Aulonastus similis

Sayornis nigricans - Picobia sayornis

Tolmomyias sulphurescens - Picobia ochoi

Tyrranus tyrranus - Syringophilopsis tyranni

T. dominicensis - Syringophilopsis tyranni

T. forficatus - Syringophilopsis tyranni

T. verticalis - Syringophilopsis tyranni

Myiarchus cinerascens - Syringophilopsis tyranni

M. crinitus - Syringophilopsis tyranni

Myiopagis gaimardii - Picobia myiopagi

M. subplacens - Picobia myiopagi, Syringophilopsis tyranni

Satrapa icterophrys - Syringophilopsis tyranni

\section{Fam. Vangidae}

Prionops scopifrons - Neoaulonastus prionops, Picobia meru

\section{Fam. Vireonidae}

Vireo hypochryseus - Syringophilopsis catesbyi

V. olivaceus - Syringophilopsis catesbyi

\section{Fam. Zosteropidae}

Zosterops erythropleurus - Neoaulonastus zosterops

Z. senegalensis - Neoaulonastus zosterops

\section{Pelecaniformes}

Fam. Ardeidae

Ardea alba egretta - Trypetoptila casmerodia

Ixobrychus cinnamomeus - Ixobrychiphilus wallacei

\section{Fam. Threskiornithidae}

Geronticus calurus - Stibarokris geronticus

Phimosus infuscatus - Selenonycha inspirata

Platalea leucorodia - Stibarokris platalea

Plegadis falcinellus - Stibarokris brevisetosus

Theristicus caudatus - Stibarokris theristicus

\section{Phoenicopteriformes}

\section{Fam. Phoenicopteridae}

Phoeniconaias minor - Stibarokris phoeniconaias

Phoenicopterus ruber - Stibarokris phoeniconaias

Ph. chilensis - Stibarokris phoeniconaias 


\section{Piciformes}

Fam. Lybiidae

Pogoniulus bilineatus - Neosyrigophilopsis lybidus, Picineoaulonastus pogoniulus

P. pusillus - Picineoaulonastus pogoniulus

Tanopicobia trachyphoni - Trachyphonus erythrocephalus

\section{Fam. Picidae}

Campethera nubica - Picobia dryobatis

Celeus elegans - Neopicobia ea

C. flavus - Neopicobia ea

C. torquatus - Neopicobia ea

Chrysocolaptes lucidus - Neoaulonastes chrysocolaptes

Colaptes [Picumnus] rubiginosus - Neopicobia freya

Dendrocopos borealis - Picobia dryobatis

D. darjellensis - Picobia dryobatis

D. leucotos - Picobia dryobatis, Neoaulonastus picidus

D. macei - Picobia dryobatis

D. major - Picobia dryobatis, Neoaulonastus picidus

D. minor hortorum - Picobia dryobatis

Dendropicos fuscescens - Picobia dryobatis, Syringophiloidus picidus

D. goerthae - Neopicobia freya, Picobia dryobatis

D. griseocephalus - Neopicobia freya

D. namaquus - Picobia dryobatis

D. stierlingi - Picobia dryobatis

D. syriacus - Picobia dryobatis

Dryocopus galeatus - Neopicobia freya, Picobia dryobatis

Jynx torquilla - Picobia dryobatis

Melanerpes carolinus - Picobia dryobatis,

M. erythrocephalus - Picobia dryobatis

M. formicivorus - Syringophiloidus carolae

Picoides pubescens - Picobia dryobatis, Picisyringophilus kratos

P. mixtus - Picobia dryobatis

P. scalaris - Picobia dryobatis

P. tridactylus - Picobia dryobatis

Piculus rubiginosus - Neopicobia freya

Picumnus exilis - Neopicobia hepburni

P. olivaceus - Neopicobia hepburni

P. sclateri - Neopicobia hepburni

P. temminckii - Neopicobia hepburni

Picus awokera - Picobia heeri

P. canus - Picobia heeri, Neoaulonastus picidus 
P. chlorolophus - Picobia heeri

P. erythropygius - Picobia heeri

P. flavinucha-Picobia heeri

P. mentalis - Picobia mentalis

P. puniceus - Picobia heeri

P. squamatus - Picobia heeri

P. viridis - Picobia heeri

Veneliornis cassini - Picobia dryobatis

V. dignus - Picobia dryobatis

V. fumigatus - Picobia dryobatis

V. kirkii - Picobia dryobatis

V. maculifrons - Picobia dryobatis

V. passerines - Picobia dryobatis

V. sanguineus - Picobia dryobatis

V. spilogaster - Picobia dryobatis

Fam. Rhamphastidae

Ramphastos sulfuratus - Rafaicobia ramphastos

R. vietellinus - Rafapicobia ramphastos

Fam. Semnornithidae

Semnornis ramphastinus - Rafapicobia olszanowskii

\section{Procellariiformes}

\section{Fam. Procellariidae}

Ardenna pacifica - Procellariisyringophilus bulwerius

Bulwerius bulwerius - Procellariisyringophilus bulwerius

Calonectris diomedea - Procellariisyringophilus bulwerius

Puffinus pacificus - Stibarokris mariasi

Fam. Hydrobatidae

Psittaciformes

Hydrobates pelagicus - Stibarokris hydrobates

Fam. Psittacidae

Amazona aestiva - Psittaciphilus amazonae

A. amazonica - Psittaciphilus amazonae

A. finschi - Neoaulobia mironovi

Aratinga canicularis - Neoaulobia mexicana

A. pertinax-Megasyringophilus kethleyi, Neoaulobia mexicana

A. jandaya - Megasyringophilus kethleyi, Neoaulobia aratingae

Bolborhynchus aymara - Peristerophila mucuya

Brotogeris jugularis cyanoptera - Rafapicobia brotogeris

B. versicolurus - Megasyringophilus kethleyi, Peristerophila mucuya

Forpus cyanopygius - Peristerophila forpi

Pionus senilis - Terratosyringophilus pioni 
Poicephalus senegalus - Neoaulobia pulyerti, Lawrencicobia poicephali

\section{Fam. Psittaculidae}

Agapornis fischeri - Neoaulobia agapornis

A. nigrigenis - Neoaulobia agapornis

A. personatus - Neoaulobia agapornis

A. roseicollis - Neoaulobia agapornis

A. taranta - Neoaulobia agapornis

Eos bernea - Megasyringophilus eos

Geoffroyus geoffroyi - Megasyringophilus geoffroyus

Glossopsitta porphyrocephala - Neopicobia glossopsitta

Loriculus philippensis - Neoaulobia puylaerti

L. pusillus - Neoaulobia puylaerti

Lorius garrulus - Terratosyringophilus loricatus

L. lory - Terratosyringophilus reichholfi

Platycercus eximius - Megasyringophilus platycercus

Psittacula cyanocephala - Megasyringophilus cyanocephala, Neoaulobia psittaculae

P. eupatria - Megasyringophilus cyanocephala

P. krameri - Megasyringophilus cyanocephala

Trichoglossus chlorolepidotus - Megasyringophilus trichoglossus

T. euteles - Megasyringophilus trichoglossus

T. haematodus - Peristerophila mucuya, Terratosyringophilus loricinus

T. ornatus - Megasyringophilus dubinini

\section{Fam. Cacatuidae}

Cacatua galerita - Megasyringophilus cacatua

C. tenuirostris - Neoaulobia krafti

C. sulphurea - Lawrencipicobia sulphurea

Calyptorhynchus funereus - Neoaulobia cacatua

C. lathami - Lawrencipicobia calyptorhyncha

Probosciger aterrimus - Neoaulobia cacatua

Fam. Strigopidae

Nestor meridionalis - Peristerophila nestoriae

\section{Pterocliformes}

Fam. Pteroclidae

Pterocles coronatus - Columbiphilus pteroclesi

P. senegallus - Pteroclidisyringophus re, Columbiphilus pteroclesi

\section{Strigiformes}

\section{Fam. Strigidae}

Asio otus - Bubophilus aluconis, B. asiobius

Athene cunicularia - Neobubophilus atheneus

A. brama - Neobubophilus atheneus 
A. noctua - Neobubophilus atheneus

Bubo ascalaphus - Bubophilus ascalaphus

B. africanus - Bubophilus ascalaphus

B. bubo - Bubophilus ascalaphus

B. virginianus - Bubophilus ascalaphus

Megascops choliba - Megasyringophilus dalmas

Strix aluco - Bubophilus aluconis

S. woodfordii - Bubophilus aluconis

Fam. Tytonidae

Tyto alba affinis - Bubophilus ascalaphus, B. tytonis

\section{Suliformes}

Fam. Phalacrocoracidae

Phalacrocorax auritus - Stibarokris phalacrus

Ph. carbo - Stibarokris dastychi

\section{Tinamiformes}

Fam. Tinamidae

Eudromia elegans - Tinamiphilopsis elegans

Nothura boraquira - Tinamiphilopsis ariconte

N. minor - Tinamiphilopsis ariconte

Rhynchotus rufescens - Tinamiphilopsis ariconte

\section{Trogoniformes}

Fam. Trogonidae

Pharomachrus antisianus - Syringophiloidus quetzali

Ph. mocinno - Syringophiloidus quetzali

Trogon citreolus - Syringophilopsis trogoni

T. melanocephalus - Syringophilopsis trogoni

\section{Upupiformes}

Fam. Phoeniculidae

Phoeniculus purpureus - Picobia phoeniculi

Fam. Upupidae

Upupa epops africana - Picobia phoeniculi

Upupa epops epops - Peristerophila upupi, Picobia phoeniculi

\section{Bibliography of Syringophilidae}

[Amirhanov F.M.] Амирханов Ф. М. 1970. [Syringophilosis of chicken in Azerbaidzhan] Сирингофилез кур в Азербайджане. - Second Acarological Conference, Proc., 1, Ed. Naukova dumka, Kiev: 31-32 (in Russian).

[Amirhanov F.M.] Амирханов Ф. М. 1973. 1973. On study of fauna of acaroid mites (Acaroidea) in Azerbaijan. - Izv. Akad. Nauk Az SSR, Ser Biol. Nauk, 2: 93-94. 
Banks N. 1907. Catalogue of the Acarina, or Mites, of the United States. - Proc. U.S. National Museum, 32(1553): 595-625.

Berlese A. 1887. Acari, Myriopoda et Scorpiones hucusque in Italia reperta. - Padova, fasc. 37, No 9, 10.

Bilal D.I.K. 2012. The first record of Ornithocheyletia hallae Smiley, 1970 (Acariformes: Actinedida: Cheyletidae) and its prevalance on Pseudolynchia canariensis (Mcquart, 1840) (Diptera: Pupipara: Hippoboscidae) in Turkey. - Türkiye Parazitolojii Dergisi, 36(3): 169-173.

Bochkov A.V. 2000. Syringophilus numidae sp. n., a new species of quill mites (Acarina: Syringophilidae) ex Numida meleagris (Galliformes: Phasianidae) from South Africa. Acarina, 8(2): 103-104.

Bochkov A.V. 2001. On systematics of mites of the genus Syringophiloidus (Acara: Syringophilidae) from the European part of Russia. - Parazitologyia, 35(2): 149-154 (Russian, Summ. Engl.).

Bochkov A.V., D. Apanaskevich. 2001. Two new species of the family Syringophilidae (Acari: Cheyletoidea) from passeriform birds collected in the Altai. - Folia Parasitologica, 48: 321-325.

Bochkov A.V., D.V. Chistyakov. 2001. Description of Charadriphilus lyudmilae gen. et sp. n. (Acari: Syringophilidae) from Scolopax rustica (Aves: Scolopacidae) in NW Russia. Zoosystematica Rossica, 9(2): 2000: 295-297.

Bochkov A. V., A. Fain. 2003. New and little known species of the family Syringophilidae (Acari: Cheyletoidea) from parrots (Aves: Psittaciformes). - Acarina, 11(1): 1-8.

Bochkov A. V., A. Fain, M. Skoracki. 2004. New quill mites of the family Syringophilidae (Acari: Cheyletoidea). - Systematic Parasitology, 57: 135-150.

Bochkov A. V., M. Flannery, G. Spicer. 2009. Mites of the genus Torotrogla (Prostigmata: Syringophilidae) from North American Passerines. - J. Med. Entomol., 46(2): 183-197.

Bochkov A. V., T.D. Galloway. 2001. Parasitic cheyletoid mites (Acari: Cheyletoidea) associated with passeriform birds (Aves: Passeriformes) in Canada. - Canadian Journal of Zoology, 79: 2014-2028.

Bochkov A. V., T.D. Galloway. 2004. New species and records of cheyletoid mites (Acari: Cheyletoidea) from birds in Canada. - Journal of the Kansas Entomological Society, 77: 26-44.

Bochkov A. V., S.V. Mironov. 1998. Quill mites of the family Syringophilidae Lavoipierre, 1953 (Acariformes: Prostigmata) parasitic on birds (Aves) of the fauna of the former USSR. - Acarina, 6(1-2): 3 -16.

Bochkov A. V., S.V. Mironov. 1999. New quill mite species of the family Syringophilidae (Acari: Cheyletoidea) from the European part of Russia. - Acarina, 7(1): 35-45.

Bochkov A. V., S.V. Mironov, N.T. Kravtsova. 2000. Two new syringophilid mites from the Green finch Carduelis chloris (Passeriformes: Fringillidae) from Kirghizia. - Genus, 11:351-358.

Bochkov A. V., S.V. Mironov, M. Skoracki. 2002. Four new quill mite species of the genus Syringophilopsis Kethley, 1970 (Acariformes: Prostigmata). - Acarina, 2001, 9(2): 233-239.

Bochkov A.V., T. Perez. 2002. New quill mites of the family Syringophilidae (Acari: Cheyletoidea) parasitizing Mexican parrots. - Belgian J. of Entomology, 4: 145-159. 
Bochkov A.V., K. Skirnisson, K. 2011. Description of the life stages of quill mite Mironovialagopus sp. nov. (Acari: Syringophilidae) parasitizing the rock ptarmigan Lagopus muta (Phasianidae) from Iceland. - Parasitological Research, 108: 715-722.

Bochkov A.V., M. Skoracki, S. Hendricks, G.S. Spicer. 2011. Further investigations of mite genus Syringophiloidus (Acariformes: Syringophilidae) from North American Passerines. - Systematic Parasitology, 79(3): 201-211.

Bochkov A. V., G. Williams, H. Proctor. 2005. First record of Picobia zumpti (Acari: Syringophilidae) from quills of the rock pigeon in North America and description of the male. - Belgian J. of Entomology, 7: 121-127.

Broda L., M. Dabert, E. Glowska. 2016. Aulonastus similis n.sp., a new quill mite species (Syringophilidae) parasitizing passeriform birds (Tyrannidae and Cardinalidae) in Mexico. - Systematic Parasitology, 93(7): 715-719.

Brown N. Sandra. 1971. A survey of the Arthropod parasites of pigeons (Columba livia) in Boston. - J. Parasitol., 57(6): 1379-1380.

Brown N.S., G.I. Wilson. 1975. A comparison of the ectoparasites of the house sparrow (Passer domesticus) from North America and Europe. - The American Midland Naturalist, 94(1): 154-165.

Casto S.D. 1973. A method for collection of the quill mite, Syringophiloidus minor (Berlese) (Prostigmata) from living birds. - J. of Medical Entomology - Honolulu, 10: 524.

Casto S.D. 1974a. Quill wall thickness and feeding of Syringophiloidus minor (Berlese)(Acarina: Syringophilidae). - Annals of the Entomological Society of America, 67: 824.

Casto S.D. 1974b. A nocturnal dispersal rhythm in the quill mite, Syringophiloidus minor (Berlese)(Prostigmata: Syringophilidae). - J. Med. Entomol., 10: 113-114.

Casto S.D. 1974c. Entry and exit of syringophilid mites (Acarina: Syringophilidae) from the lumen of the quill. - Wilson Bull., 86(3): 272-278.

Casto S.D. 1974d. Observations on the karyotype and maturation of the F1 generation of Syringophiloidus minor (Acarina: Syringophilidae). - Ann. Ent. Soc. of America, 67: 136-137.

Casto S.D. 1975a. Generation time of male quill mites (Syringophiloidus minor) (Berlese)( Acarina: Syringophilidae). - Am. Midland Nat., 93: 495-498.

Casto S.D. 1975b. Mortality in the quill mite, Syringophiloidus minor (Acarina: Syringophilidae). - Ann. Entomol. Soc. Am., 68: 551-552.

Casto S.D. 1975c. The effect of the postjuvenal molt in the house sparrow on infestations of the quill mite, Syringophiloidus minor (Berlese)( Acarina: Syringophilidae). - J. Med. Entomol., 12: 23-27.

Casto S.D. 1976a. Host records and observations of quill mites (Acarina: Syringophilidae) from Texas birds. - Southw. Entomol., 1(4): 155-160.

Casto S.D. 1976b. Dispersal of the quill mite Syringophiloidus minor (Berlese)(Acarina: Syringophilidae). - J. Med. Entomol., 13: 357-360.

Casto S.D. 1977. Cuculiphilus lobatus gen. n., sp. n. representing a new subfamily of quill mites (Acarina: Syringophilidae) from the groove-billed ani, Crotophaga sulcirostris (Cuculiformes: Cuculidae). - Southwestern Naturalist, 22(2): 169-176. 
Casto S.D. 1978. A new name for Cuculiphilus (Acarina: Syringophilidae). - Southwestern Naturalist, 23: 158.

Casto S.D. 1979. A new syringophilid mite from the white-winged dove. - Tex. J. Sci., 31: 225-229.

Casto S.D. 1980a. A new genus of syringophilid mites from galliform birds in Texas. - Tex. J. Sci., 32(3): 233-240.

Casto S.D. 1980b. A new quill mite (Acarina: Syringophilidae) from the ground dove. - Southw. Entomol., 5(1): 1-5.

Chirov P.A., N.T. Kravtsova. 1995. [A new genus and new species of mites of the family Syringophilidae]. - Parazitologiya, 29(5): 370-379 (in Russian).

Clark G.M. 1964. The acarine genus Syringophilus in North American birds. - Acarologia, 6(1): 77-92.

Domrow R. 1991. Acari Prostigmata (excluding Trombiculidae) Parasitic on Australian Vertebrates: an Annotated Checklist, Keys and Bibliography. - Invertebr. Taxon., 4: 1283-1376.

Dubinin W.B. 1957. [A new classification of the mites of the superfamilies Cheyletoidea W. Dub. and Demodicoidea W. Dub. (Acariformes, Trombidiformes)]. - Parazitologicheskii sbornik, Zool. Inst. AN S.S.S.R., 17: 17-136 (in Russian).

Dubinina E.V. 1987. Family Syringophilidae: 300-301. In: Soboleva R.G.[Ed.] [Insects and Acari of the Far East of medical and veterinary importance], Nauka, Leningrad : 1-309.

Ewing H.E. 1911. Predaceous and parasitic Acarina. - Psyche, 18: 37-43.

Fain A., A.V. Bochkov, S.V. Mironov. 2000. New genera and species of quill mites of the family Syringophilidae (Acari: Prostigmata). - Bull. Inst. Royal des Sciences Naturelles de Belgique (Entomology), 70: 33-70.

Filimonova S.A. 2008. Peculiarities of the internal anatomy in the mite family Syringophilidae by the example of Syringophilopsis fringilla--a parasite of chaffinch. - Parazitologiia, 42(5): 395-404.

Filimonova S.A. 2009. The ultrastructural investigation of the midgut in the quill mite Syringophilopsis fringilla (Acari, Trombidiformes: Syringophilidae). - Arthropod structure \& development, 38(4): 303-313.

Filimonova S.A., S.V. Mironov. 2010. Functional morphology of the gnathosoma in the quill mite Syringophilopsis fringilla Fritsch (Acari : Prostigmata : Syringophilidae). - Zool. Anzeiger, 249: 165-180.

Fritsch W. 1958. Die Milbengattung Syringophilus Heller 1880 (Subordo Trombidiformes, Fam. Myobiidae Mégnin 1877). - Zool. Jb. Syst., 86(3): 227-244.

Glowska E. 2013. Neoaulonastus grewlingi sp.nov. (Prostigmata: Cheyletoidea: Syringophilidae) a new quill mite species parasitizing Falcunculus frontatus (Passeriformes: Falcunculidae) in Australia. - Acta Parasitologica, 58(1): 18-20.

Glowska E. 2014. New quill mites (Cheyletoidea : Syringophilidae) parasitizing tyrannid birds (Passeriformes: Tyrannidae) in Peru. - Zootaxa, 3814(1): 139-145. 
Glowska E. 2015a. Stibarokris annae n. sp. from Ciconia maguari (Gmelin) (Ciconiiformes: Ciconiidae), a new species of quill mites (Acariformes: Syringophilidae). Exhibiting dimorphism of females. - Systematic Parasitology, 92(1): 73-79.

Glowska E. 2015b. Stibarokris mariasi sp.nov. - a new quill mite species (Acariformes: Syringophilidae) parasitizing Puffinus pacificus (Gmelin) (Procellariidae) on Johnston Atoll. Acta Parasitologica, 60(4): 614-617. https://doi.org/10.1515/ap-2015-0086

Glowska E., L. Broda, C.A. Gebhard, J. Dabert. 2016. A new quill mite Syringophiloidus plocei sp. nov. (Prostigmata: Syringophilidae) parasitizing ploceid birds (Passeriformes) in Gabon - a combined description using morphology and DNA barcode data. - Acta Parasitologica, 61: 562-566.

Glowska E., L. Broda, J. Dabert. 2019. Insight into the species diversity of the quill mite genus Betasyringophiloidus Skoracki, 2011 (Prostigmata: Syringophilidae) on the basis of the DNA barcodes. - Folia Parasitologica, 66: 009.

Glowska E., M. Chrzanowski, K. Kaszewska. 2015. Checklist of the Quill mites (Acariformes: Syringophilidae) of the World. - Zootaxa, 3968(1): 1-81.

Glowska E., A. Dragun-Damian, J. Dabert. 2012. Picobia dziabaszewskii sp. nov. (Acari, Syringophilidae)—combined description (morphology with DNA barcode data) of a new quill mite species parasitizing Garrulax formosus (Passeriformes: Leiothrichidae). - Zootaxa, 3224: 57-61.

Glowska E., A. Dragun-Damian, J. Dabert. 2012. A new quill mite Syringophiloidus pseudonigritae sp. nov. (Prostigmata, Syringophilidae) parasitizing Pseudonigrita arnaudi (Passeriformes, Ploceidae) - a combined description using morphology and DNA barcode data. - Zootaxa, 3532: 64-68.

Glowska E., Z.K. Filutowska, M. Dabert, M. Gerth. 2020. Microbial composition of enigmatic bird parasites: Wolbachia and Spiroplasma are the most important bacterial associates of quill mites (Acariformes: Syringophilidae). - Microbiologyopen. 9(5): e964. doi: 10.1002/mbo3.964.

Glowska E., I. Laniecka. 2012. Syringophilopsis davidi sp. nov. (Prostigmata, Syringophilidae) a new quill mite species parasitizing Calandrella brachydactyla (Passeriformes, Alaudidae) in Egypt. - Acta Parasitologica, 57(4): 385-387.

Glowska E., I. Laniecka. 2013. Two new quill mite species (Prostigmata: Syringophilidae) parasitizing Australian birds. - Zootaxa, 3670(3): 385-390.

Glowska E., I. Laniecka. 2014. A new quill mite species Neopicobia hepburni sp. nov. (Cheyletoidea: Syringophilidae) parasitizing picid birds (Piciformes: Picidae) in Peru. - Acta Parasitologica, 59: 635-637.

Glowska E., I. Laniecka, Ch.M. Milensky. 2015. Two new picobiin mite species (Acari: Cheyletoidea: Syringophilidae) parasitizing passerine birds in Guyana. - Acta Parasitologica, 60(3): 488-493.

Glowska E., I. Laniecka, K. Romanowska. 2018. A new quill mite Torotrogla emberizae sp. nov. (Acariformes: Syringophilidae) from the Chestnut-eared Bunting (Passeriformes: Emberizidae) in Japan (morphology and DNA barcode data). - Acta Parasitologica, 63(4): 791-794. 
Glowska E., C. Milensky. 2014. New species of the genus Picobia (Cheyletoidea: Syringophilidae) parasitizing tyrannid birds (Passeriformes: Tyrannidae) in Gabon. - Zootaxa, 3821(3): 373-383..

Glowska E., K. Romanowska, B.K. Schmidt, M. Dabert. 2018. Combined description (morphology with DNA barcode data) of a new quill mite Torotrogla paenae n. sp. (Acariformes: Syringophilidae) parasitising the Kalahari scrub-robin Cercotrichas paena (Smith) (Passeriformes: Muscicapidae) in Namibia. - Systematic Parasitology, 95(8-9): 863-869.

Glowska E., B.K. Schmidt. 2014a. New taxa of the subfamily Picobiinae (Cheyletoidea: Syringophilidae) parasitizing antbirds and gnateaters (Passeriformes: Thamnophilidae, Conopophagidae) in Guyana. - Zootaxa, 3861(2): 193-200.

Glowska E., B.K. Schmidt. 2014b. New quill mites (Cheyletoidea: Syringophilidae) parasitizing the black-headed paradise-flycatcher Terpsiphone rufiventer (Passeriformes: Monarchidae) in Gabon. - Zootaxa, 3786(1): 57-64.

Glowska E., M. Skoracki. 2009. Anomalous in chaetotaxy of the quill mites Torotrogla merulae Skoracki et al., 2000 (Acari: Prostigmata: Syringophilidae). - Acta Parasitologica, 54: 364-367.

Glowska E., M. Skoracki. 2010. Meitingsunes, a new genus of quill mites (Acari: Cheyletoidea: Syringophilidae). - Zootaxa, 2514: 61-67.

Glowska E., M. Skoracki. 2011a. New species of quill mites (Acari, Cheyletoidea, Syringophilidae) and the first record of male for the genus Stibarokris. - Zootaxa, 2817: 63-68.

Glowska E., M. Skoracki. 2011b. Two new quill mite species (Acari, Cheyletoidea, Syringophilidae) parasitizing Dinemellia dinemelli (Rüppell)(Passeriformes, Ploceidae) . - Zootaxa, 3114: 63-68.

Glowska E., M. Skoracki, F. Khourly. 2007. A new species and new records of syringophilid mites (Acari: Prostigmata: Syringophilidae) from birds of Jordan. - Zootaxa, 1635: 63-68. Goff M. Lee. 1982. Syringophilidae mites. - Proc. Hawaii. Ent. Soc., 24(1), 1979: 13.

Grandjean F. 1944. Observations sur les acariens de la famille Stigmaeidae. - Archives des Sciences Physiques et Naturelles, 26: 103-131.

Gritsenko E.F. 1969. [Biology and ecology of the quill mite Syringophilus bipectinatus Heller, 1880]. - Avtoreferat Dissert. Doc. Biol. Nauk, Stavropol, 17 p. (in Russian).

Gritsenko E.F. 1973. The biology and ecology of the quill mite Syringophilus bipectinatus Heller, 1880. - Proc. $3^{\text {rd }}$ Intern. Congress of Acarology, Prague, 1971: 515-516.

Grossi A.A., H. C. Proctor. The Distribution of Quill Mites (Betasyringophiloidus seiuri) Among Flight Feathers of the Ovenbird (Seiurus aurocapilla). - J. Parasitol., 106(1): 82-89.

Haller G. 1878. Freyana und Picobia. - Zeit. wiss. Zool., Siebold und Kolliker, Leipzig, 30: 81-98.

Hancock J. L. 1895. A new Trombidian. - Amer. Nat., 29: 382-385.

Hancock J. L. 1898. Picobia villosa (Hancock). - Amer. Nat., 29: 866-867.

Heller A. 1880. Die Schmarotzer. - Münich und Leipzig: 186-188. 
Hirst S. 1920. Notes on Acari parasitic on birds with descriptions of two new species. - Ann. Mag. Nat. Hist., 6: 121-122.

Hirst S. 1922. Mites injurious to domestic animals. - Brit. Mus. Econ. Series, 13: 1-107.

Hood D.E., H.E. Welch. 1980. A seasonal study of the parasites of the red-winged blackbird 537. Hromada M., M. Klimovičova. 2015. From dusty collections to descriptions of new species birds in Sarisske Museum Bardejov as valuable source for investigating mite biodiversity. - Folia Oecologica, Prešov, 98-108.

Hromada M., M. Klimovičova, M. Unsold, M. Skoracki. 2016. Host-parasite relationships in the system composed by cuckoos and quill mites. - Syst. and Applied Acarology, 21(4): $528-536$.

Hwang J.C. 1959. Case reports of the quill mite, Syringophilus bipectinatus, in poultry. - Proc. Helminthol. Soc. Wash. D.C., 26: 47-50.

Jones J. 1968. Some parasites of the common crow, Corvus brachyrhynchos Brehm, from Ohio. - The Ohio J. of Science, 68(1): 25-31.

Johnson D.E., J.B. Kethley. 1973. A numerical phenetic study of the quill mites of the family Syringophilidae (Acari). - J. Parasitol., 59(3): 520-530.

Kaszewska K., K. Kavetska, M. Skoracki. 2014. Two new species of quill mites of the family Syringophilidae (Acariformes: Cheyletoidea) associated with treronine doves (Columbiformes: Treroninae). - Zootaxa, 3846: 293-300.

Kaszewska K., M. Skoracki. 2018. Two new quill mite species of the genus Psittaciphilus Fain, Bochkov \& Mironov, 2000 (Acariformes: Syringophilidae) associated with pigeons and doves (Columbiformes: Columbidae). - Systematic Parasitology, 95(8): 953-958.

Kaszewska K., M.Skoracki, M.Hromada. 2018. A review of the genus Gunabopicobia Skoracki and Hromada (Acariformes: Prostigmata: Syringophilidae) associated with birds of the order Columbiformes. - Intern. J. of Acarology, 44(7): 288-299.

Kaszewska K., M. Skoracki, J.Z Kosicki, M. Hromada. 2020. New species and records of the quill mites of the genus Peristerophila Kethley, 1970 (Acariformes: Syringophilidae) associated with pigeons and doves (Aves: Columbiformes). - Zootaxa, 4878(2): 349-361.

Kaszewska K., M. Skoracki, K. Kavetska. 2016. Two new Meitingsunes species (Acari: Syringophilidae) from Indonesian doves (Columbiformes: Columbidae). - Zootaxa, 4109(4): 479-486.

Kaszewska K., M. Skoracki, K. Kavetska. 2020. The mites of the genus Meitingsunes Glowska and Skoracki (Acariformes: Syringophilidae) associated with pigeons and doves (Aves: Columbiformes): taxonomic studies with description of two new species. $-\cdot \cdot$ International Journal of Acarology, 46(6):1-7.

Kethley J.B. 1970a. A revision of the family Syringophilidae (Prostigmata: Acarina). - Contrib. Amer. Entomol. Inst., 5(6): 1-76.

Kethley J.B. 1970b. Population regulation in quill mites (Acarina: Syringophilidae). - Ecology, 52: 1113-1118.

Kethley J. B. 1973a. Population regulation in quill mites (Acarina: Syringophilidae). - Proc. $3^{\text {rd }}$ Intern. Congress of Acarology, Prague, 1971: 781. 
Kethley J.B. 1973b. A new genus and species of quill mites (Acarina: Syringophilidae) from Colinus virginianus (Galliformes: Phasianidae) with notes on developmental chaetotaxy. - Fieldiana Zool., 65(1): 1-8.

Kivganov D.A., G.S. Sharafat. 1995. Review of the family Syringophilidae (Acari) with the description of new genera and species. - Zool. Zhurnal, 74(4): 82-91 (in Russian).

Klimovičová M., M. Hromada. 2014. New hosts and localities of quill mites (Acari: Syringophilidae) parasitising birds in Slovakia. - Folia Oecologica, Prešov, 11: 31-39.

Klimovičová M., P. Mikula, N. Kahure, M. Hromada. 2014a. A review of quill mites (Acari: Syringophilidae) parasitising Kenyan birds. - Zootaxa, 3857(4): 571-580.

Klimovičová M., R. Smolák, M. Hromada. 2014b. A new species and new host records of the quill mites (Acari: Syringophilidae) associated with sunbirds (Passeriformes: Nectariniidae). - Acta Parasitologica, 59(2): 255-258.

Klimovičová M., M. Skoracki, M. Hromada. 2016. Two new species of quill mites (Acari: Syringophilidae) associated with the Chestnut-fronted Helmetshrike Prionops scopifrons (Peters)(Passeriformes: Fangidae) in Kenya. - Zootaxa, 4137(4): 578-584.

Klimovičová M., M. Skoracki, P. Njoroge, M. Hromada. 2016. Two New Species of the Family Syringophilidae (Prostigmata: Syringophilidae) Parasitising Bushshrikes (Passeriformes: Malaconotidae). - Journal of Parasitology, 102(2):187-192.

Lavoipierre M.M. 1953. The undescribed male and female of the pigeon quill mite, Syringophilus columbae, Hirst 1920. - Trans. Roy. Soc. Trop. Med. Hygiene, 47: 7.

Lawrence R.F. 1959. New mites parasites of African birds (Myobiidae, Cheyletidae). - Parasitology, 49(3-4): 416-438.

Leonovich S.A., S.A. Filimonova. 2017. The quill mite Syringophilopsis fringilla (Fritsch) (Acari: Trombidiformes: Syringophilidae): the structure of sensory organs providing feeding of the parasite in the feather quill. - Entomol. Review, 97(3): 383-394.

Liu Bai-li. 1988. On three species of quill mites from China (Acari: Syringophilidae). - Acta Zootaxonomica Sinica, 13: 274-277.

Lukoschus F.S., H.A.P.M. Lombert. 1979. Parasites of Western Australia VII: Observation on Quill Wall Mites (Acarina: Astigmata). - Western Australian Museum, 7(3): 265-327.

Marciniak N., M. Skoracki, M. Hromada. 2019a. Quill mites of the family Syringophilidae (Acariformes: Prostigmata) associated with Cockatoos (Psittaciformes: Cacatuidae). Zootaxa, 4565(2): 190-200.

Marciniak N., M. Skoracki, M. Hromada. 2019b. Peristerophila nestoriae, a new species of quill mite of the family Syringophilidae (Acariformes: Prostigmata) parasitizing New Zealand Kaka Nestor meridionalis (Gmelin)(Psittaciformes: Strigopidae). - New Zealand Journal of Zoology. http://doi.org/10.1080/03014223.2019.1607406

Michalik J., M. Skoracki, B. Sikora. 2005. Quill mites (Acari: Syringophilidae); a new vector of bacterial zoonotic pathogens circulating in an enzootic avian cycle? In: Buczek A., Blaszak C. (eds) Arthropods, a variety of forms and interactions. Pp. 91-105.

Nattress B., M. Skoracki. 2007. Quill mites of the family Syringophilidae Lavoipierre, 1953 parasitic on birds in England (Acari: Cheyletoidea). - Genus, 18(1): 139-145. 
Nattress B., M. Skoracki. 2007. A new species and further records of quill mites (Acari: Cheyletoidea: Syringophilidae) parasitic on birds (Aves) in England. - Zootaxa, 2133: 49-54. Oudemans A.C. 1904a. Acarologische Aanteekeningen, XIII. - Entomol. Ber., 1(19): 171.

Oudemans A.C. 1904b. Acarologische Aanteekeningen, XIV. - Entomol. Ber., 1(20): 190-191. Oudemans A.C. 1906. Révision des Chélétinés. - Mém. Soc. Zool. France, 19: 36-144.

Philips J.R., R.A. Norton. 1978. Bubophilus ascalaphus gen. et sp. n. (Acarina: Syringophilidae) from the quills of a great horned owl (Bubo virginianus). - J. Parasitol., 64(5): 900-904.

Pidhorna S.Y., K.J. Chernychko, D.A. Kivganov, V.A. Trach, O.F. Deli. 2020. New and interesting records of quill mites (Acari: Prostigmata: Syringophilidae) of passerine birds of southwestern Ukraine. - Persian Journal of Acarology, 9(3): 213-223.

Pires E.O., E. Daemon. 2007. Biological and ecological aspects of quill mites, parasites of domestichen Gallus gallus domesticus (Aves, Phasianidae) from rusting breeding locations in the municipality of Juiz de Fora, Minas Gerais, Brasil. - Revista Brasileira de Zoociencias, 9: 95-102.

Poppe S.A. 1887. Über parasitische Milben. - Abhandl. Nat. wiss. Ver. Bremen, 10: 205.

Radford C. D. 1953. The mites (Acarina: Analgesidae) living on or in the feathers of birds. -Parasitology, 43: 199-230.

Rebrassier R.E., E. Martin. 1932. Syringophilus bipectinatus, a quill mite of poultry. - Science, 76: 128.

Schmäschke R., M. Sachse, K. Eulenberger, R. Schöne. 2003. Quill mites - little-known parasites of Birds. - Verh. Ber. Erkrg. Zootiere, Rom: 127-133.

Schmidt K., M. Skoracki. 2007. A new name for Syringonomus Kethley, 1970 (Acari: Prostigmata: Syringophilidae) not Hope et Murphy, 1969. - Genus, 18: 545.

Schwabe O. 1956. A quill mite of poultry - a case report. - J. of the American Veterinary \& Medical Association, 129: 481-482.

Sikora B., M. Fajfer, K. Kavetska, M. Skoracki. 2012. Three new species of quill mites (Acari: Syringophilidae) parasitizing the wrens (Aves: Troglodytidae). - Zootaxa, 3167: 57- 65.

Sikora B., M. Fajfer, M. Skoracki. 2011. Quill mites (Acari: Syringophilidae) from mimid birds (Aves: Mimidae). - Zootaxa, 3027: 29-38.

Sikora B., K. Kaszewska, M. Skoracki. 2014. Two new quill mites of the family Syringophilidae (Acari: Prostigmata) parasitising the tapaculos (Passeriformes: Rhinocriptidae) in South America. - Zootaxa, 3895(3): 419-426.

Sikora B., M. Unsoeld, M. Skoracki. 2016. Aulonastus paridus spec. nov.-a parasite of two bird species of the genus Melaniparus in Kenya and Tanzania. - Spixiana, 39 (2): 149-152.

Skoracki M. 1999a. New genus and species of Syringophilidae from Eurasian reed-warbler, Acrocephalus scirpaceus (Sylviidae: Passeriformes)(Acari: Prostigmata). - Genus (Wroclaw), 10(1): 155-162.

Skoracki M. 1999b. Syringophilus bipectinatus Heller, 1880 i Syringophilopsis troglodytis (Fritsch, 1958); (Acari: Prostigmata: Syringophilidae), gatunki roztoczy nowe dla fauny Polski. - Przeglad Zoologiczny, 43(3-4): 183 -184.

Skoracki M. 2002a. Three new species of quill mites of the genus Aulonastus Kethley, 1970 (Acari: Prostigmata: Syringophilidae) from passerine birds. - Acta Parasitologica, 47(4): 300-305. 
Skoracki M. 2002b. Three new species of the ectoparasitic mites of the genus Syringophiloidus Kethley, 1970 (Acari: Syringophilidae) from passeriform birds from Slovakia. - Folia Parasitologica, 49: 305-313.

Skoracki M. 2004a. A new name for Dissonus Skoracki, 1999 not Wilson, 1906 (Acari: Prostigmata: Syringophilidae). - Genus, 15(3): 445-446.

Skoracki M. 2004b. A review of quill mites of the genus Syringophiloidus Kethley, 1970 parasitizing quills of passeriform birds, with descriptions of four new species (Acari: Prostigmata: Syringophilidae). - Genus, Wroclaw, 15(2): 281-300.

Skoracki M. 2004c. Quill mites of the genus Syringophilopsis (Acari, Syringophilidae) from passeriform birds of Poland with descriptions of five new species. - Acta Parasitologica, 49(1): 45-62.

Skoracki M. 2004d. New data on systematics of the quill mites of the genus Torotrogla Kethley, 1970 (Acari, Syringophilidae). - Belg. J. Entomol., 6: 303-314.

Skoracki M. 2005a. A new genus of ectoparasitic mites of the family Syringophilidae (Acari, Cheyletoidea) from the treeswifts (Apodiformes, Hemiprocnidae). - Acta Parasitologica, 50(4): 336-343.

Skoracki M. 2005b. A review of the quill mites (Acari: Syringophilidae) parasitizing parrots (Aves: Psittaciformes) with description of three new species. - Acarina, 13(2): 127-136.

Skoracki M. 2005c. A new genus and species of quill mites (Acari: Syringophilidae) from a king bird-of-paradise Cicinnurus regius (L.). - Systematic Parasitology, 60: 155 -158.

Skoracki M. 2008a. Crotophagisyringophilus n.g. (Acari: Syringophilidae), a new genus of parasitic quill mites. - Syst. Parasitol., 71(1): 75-80.

Skoracki M. 2008b. A new genus of syringophilid mites (Acari: Cheyletoidea: Syringophilidae) from cuculiform birds (Aves: Cuculiformes). - Folia parasitologica, 55: 155-158.

Skoracki M. 2008c. Description of two new quill mite species (Acari: Syringophilidae). Acarina, 16(1): 45-50.

Skoracki M. 2011. Quill mites (Acari: Syringophilidae) of the Palaearctic region. - Zootaxa, 2840: 1-414.

Skoracki M. 2017. Quill mites (Acariformes: Syringophilidae) associated with birds of Mexico. - Zootaxa, 4282(1):179. .

Skoracki M., M. Antczak, J. Riegert, D. Fainová, V. Mikeš. 2009. New species and new records of quill mites (Acari: Syringophilidae) inhabiting African passerins (Aves: Passeriformes). - Acta Zool. Acad. Sci. Hungaricae, 55(2): 123-137.

Skoracki M., C. Blaszak, R. Ehrnsberger. 2002. Die Milben in der Zoologischen Staatssammlung München. Teil 1. Familie Syringophilidae (Acari: Prostigmata: Cheyletoidea). - Spixiana, 25: 97-99.

Skoracki M., A.V. Bochkov. 2002. A new quill mite species Bubophilus asiobius sp. n. (Acari: Syringophilidae) from the Long-eared Owl Asio otus (Strigiformes: Strigidae). - Genus, 13(1): 141-144.

Skoracki M., A.V. Bochkov. 2010. Syringophilid mites (Acari: Syringophilidae) of Kazakhstan. - Zootaxa, 2549: 52-68. 
Skoracki M., A.V. Bochkov, B.M. OConnor. 2011. Notes on syringophilid mites (Acariformes: Syringophilidae) from aquatic birds in North America. - Acta Parasitologica, 56(3): 325-330.

Skoracki M., A.V. Bochkov, G. Wauthy. 2004. Revision of the quill mites of the genus Picobia Haller, 1878 (Acari: Syringophilidae) with notes on their host-parasites relationships. Insect Syst. Evol., 35: 155-176.

Skoracki M., J. Dabert. 1999a. New species of Syringophilidae from African birds (Acari: Prostigmata). - Genus, 10(3): 523-527.

Skoracki M., J. Dabert. 1999b. A new species of the genus Syringophilopsis Kethley, 1970 (Acari: Prostigmata: Syringophilidae) from the Tree Pipit Anthus trivialis (Passeriformes: Motacillidae). - Acarina, 7(2): 89-92.

Skoracki M., J. Dabert. 2000. Syringophilopsis albicollisi, a new species of the quill mite of the family Syringophilidae Lavoipierre, 1953 (Acari: Prostigmata). - Acarina, 8(1): 59-63.

Skoracki M., J. Dabert. 2001a. Two new species of the parasitic mites of the genus Aulobia Kethley, 1970 (Acari: Syringophilidae) from African birds. - Acta Parasitologica, 46(3) 208-215.

Skoracki M., J. Dabert. 2001b. The quill mites of the genus Syringophilopsis (Kethley, 1970) from African birds. - Acarina, 9(1): 105-112.

Skoracki M., J. Dabert. 2002. A review of parasitic mites of the family Syringophilidae (Acari: Prostigmata) from African birds, with description of four new species. - Acta Parasitologica, 47(2): 137 - 146.

Skoracki M., J. Dabert, R. Ehrnsberger. 2000. A new quill mite (Acari: Syringophilidae) from the Blackbird. - Osnabr. Naturwissensch. Mitt., 26: 191-198.

Skoracki M., J. Dabert, R. Schmäschke. 2006. Observations on the quill mites (Acari: Syringophilidae) from charadriiform birds. - Zootaxa, 1156: 51-64.

Skoracki M., M. Flannery, G. Spicer. 2008. Quill mites of the genus Syringophilopsis Kethley, 1970 (Acari: Syringophilidae) from North American birds. - Folia parasitologica, 55: 291-300.

Skoracki M., M. Flannery, G. Spicer. 2009. New data on systematics of the quill mites of the genus Syringophiloidus Kethley, 1970 (Acari, Syringophilidae) from North American birds. - Acta Parasitologica, 54(1) 64-72.

Skoracki M., E. Glowska. 2008a. Two new species of the quill mite genus Aulobia Kethley, 1970 (Acari, Syringophilidae) associated with sunbirds (Passeriformes, Nectariniidae). - Acta Parasitologica, 53(2): 179-185.

Skoracki M., E. Glowska. 2008b. Quill mites (Acari: Syringophilidae) associated with columbiform birds. - Genus, 19(1): 151-160.

Skoracki M., E. Glowska. 2008c. Two new species of the genus Picobia Haller (Acari: Syringophilidae) from Australian and Indonesian passeriform birds. - New Zealand J. of Zoology, 35: 281-286.

Skoracki M., E. Glowska, A.V. Bochkov. 2013. Phylogeny of quill mites of the family Syringophilidae (Acari: Prostigmata) based on their external morphology. - European J. of Entomology, 110: 663-675. 
Skoracki M., E. Glowska, J. Lontkowski, T. Stawarczyk. 2010. Picobia ictericus sp. n., an ectoparasite of two icterid bird species from Brazil (Acari: Prostigmata: Syringophilidae). - Genus, Wroclaw, 21(1): 143-148.

Skoracki M., E. Glowska, B. Sikora. 2008. Four new species of the quill mite genus Picobia Heller, 1889 (Acari: Syringophilidae) parasitizing birds in the Australian Region. - Zootaxa, 1961: 58-68.

Skoracki M., G. Hebda. 2004. Quill mites (Acari: Syringophilidae) from Aegithalos caudatus (Passeriformes: Aegithalidae). - Zootaxa, 691: 1-6.

Skoracki M., S.A. Hendricks, G.S. Spicer. 2010a. Systematics of the ectoparasitic quill mites of the genus Aulobia Kethley, 1970 (Acari: Syringophilidae) with the description of a new species. - Zootaxa, 2399: 31-41.

Skoracki M., S.A. Hendricks, G.S. Spicer. 2010b. New species of parasitic quill mites of the genus Picobia (Acari: Syringophilidae: Picobiinae) from North American birds. - J. of Medical Entomology, 47(5): 727-742.

Skoracki M., S.A. Hendricks, G.S. Spicer. 2010c. Four new species of Aulonastus Kethley, 1970 (Acari: Syringophilidae) from North American passerines. - Systematic Parasitology, 76: $131-144$.

Skoracki M., S.A. Hendricks, G.S. Spicer. 2011. Systematics of the genus Syringophilopsis Kethley, 1970 (Acari: Prostigmata: Syringophilidae) with description of three new species from North American passerines. - Zootaxa, 2793: 1-22.

Skoracki M., M. Hromada. 2013. A review of picobiine mites (Acari: Syringophilidae: Picobiinae) parasiting African birds. - Folia Parasitologica, 60: 192-212.

Skoracki M., M. Hromada, K. Kaszewska, B. Sikora. 2020. Females of the quill mite genera Peristerophila and Castosyringophilus (Acariformes: Syringophilidae) are two morphological forms: ontogenetic and population evidences. - Systematic \& Applied Acarology, 25(10):1803-1820.

Skoracki M., M. Hromada, K. Kaszewska, M. Unsoeld. 2018. Peristerophila falcophila sp. nov., a new species and first record of quill mites (Acariformes: Syringophilidae) parasitizing birds of the order Falconiformes. - Acta Parasitologica, 63(4): 744-749.

Skoracki M., M. Hromada, L. Kuczyński. 2001. Torotrogla lullulae, a new species of the family Syringophilidae Lavoipierre 1953 (Acari: Prostigmata: Cheyletoidea). - Genus, 12(1): 87-92.

Skoracki M., M. Hromada, P. Pervuznakova, W. Wamiti. 2019. Mites of the family Syringophilidae (Acariformes: Cheyletoidea) parasitizing waxbills of the genus Estrilda (Passeriformes: Estrildidae). - Systematic and applied acarology, 24(9): 1799-1808.

Skoracki M., M. Hromada, B. Sikora. 2017. Castosyringophilus meropis sp. n. (Acariformes: Syringophilidae) - A new quill mite species parasitising the world population of Merops apiaster Linnaeus (Coraciiformes: Meropidae). - Folia parasitologica, 64: 024.

Skoracki M., M. Hromada, K. Kaszewska, B. Sikora. 2020. Females of the quill mite genera Peristerophila and Castosyringophilus (Acariformes: Syringophilidae) are two morphological forms: ontogenetic and population evidences. - Syst. \& Applied Acarology, 25(10): 1803-1820. 
Skoracki M., M. Hromada, B. Sikora. 2020. Quill mites of the family Syringophilidae (Acariformes: Prostigmata) parasitizing coraciiform birds (Aves: Coraciiformes) - Zootaxa, 4802(1): 169-181.

Skoracki M., M. Hromada, P. Tryjanowski. 2001. Description of a new species of quill mite Syringophiloidus weiszii sp.n. (Acari: Prostigmata: Syringophilidae) from Great Grey Shrike Lanius excubitor. - Acta Parasitologica, 46(1): 30-34.

Skoracki M., M. Hromada, M. Unsoeld. 2013. Three new quill mite species of the genus Neoaulonastus Skoracki (Acari: Syringophilidae) parasitizing passerines in Tanzania. Zootaxa, 3616(4): 367-377.

Skoracki M., M. Hromada, W. Wamiti. 2011. A new species and new host records of syringophilid mites (Acari: Syringophilidae) from passerines from Kenya. - Zootaxa, 2922: 34-40.

Skoracki M., M. Hromada, M.Zmudzinski, M. Unsoeld, B.Sikora. 2018. Parasitic quill mites of the Family Syringophilidae (Acariformes: Prostigmata) associated with Sub-Saharan Sunbirds (Passeriformes: Nectariniidae): species composition and host-parasite relationships. - J. of Medical Entomology, 55(6): 1464-1477.

Skoracki M., K. Kaszewska, K. Kavetska. 2015. Two new species of the syringophilid quill mites (Acari: Prostigmata: Syringophilidae) parasitizing apodiform birds (Aves: Apodiformes). - Zootaxa, 4052(5): 583-588.

Skoracki M., K. Kaszewska, M. Unsoeld, M. Skorupski. 2015. First record of parasitic quill mites of the family Syringophilidae (Acari: Prostigmata: Cheyletoidea) on an avian representative of the order Caprimulgiformes. - International Journal of Acarology, 41(2): 128-131.

Skoracki M., K. Kavetska, M. Ozminski, B. Sikora. 2012. The morphology of juvenile stages of Bubophilus aluconis Nattress and Skoracki, 2009 (Acari: Syringophilidae) with a description of a male. - Annals of Parasitology, 58: 37- 48.

Skoracki M., G. Kiljan. 2002. Picobia paludicola sp. n. a new species of quill mite (Acari: Prostigmata: Syringophilidae) from the Aquatic Warbler Acrocephalus paludicola (Passeriformes: Sylviidae). - Zootaxa, 91: 1-6.

Skoracki M., M. Klimovičová, M. Muchai, M. Hromada. 2014. New taxa of the family Syringophilidae (Acari: Prostigmata) from African barbets and woodpeckers (Piciformes: Lybiidae, Picidae). - Zootaxa, 3768(2): 178-188.

Skoracki M., J. Lontkowski, T. Stawarczyk. 2010. New taxa of the parasitic quill mites associated with accipitrid birds indicating close relationship of falcond birds to Psittaci-Columbi clade. - J. of Nat. History, 44: 1203-1214.

Skoracki M., W. Magowski. 2001. Two new species of quill mites of the genus Picobia (Acari: Prostigmata: Syringophilidae) from passerine birds of Poland. - Acarina, 9(1) 113 -119.

Skoracki M., W. Magowski, J. Dabert. 2001. Picobia polonica sp. n. (Acari: Prostigmata: Syringophilidae), a new species of quill mite from the domestic hen, Gallus gallus domesticus (Aves: Phasianidae). - Folia Parasitologica, 48: 154 -158. 
Skoracki M., J. Michalik, B. Sikora. 2005. Habitat selection among the quill mites (Acari: Syringophilidae). In: Buczek A., Blaszak C. (eds) Arthropods, a variety of forms and interactions. Pp. 91-105.

Skoracki M., J. Michalik, B. Sikora. 2010. Prevalence and habitat preference of quill mites (Acari: Syringophilidae) parasitizing forest passerine birds in Poland. - Acta Parasitologica, 55(2): 188-193.

Skoracki M., J. Michalik, B. Skotarczak, A. Rymaszewska, B. Sikora, T. Hofman, B. Wodecka, M. Sawczuk. 2006. First detection of Anaplasma phagocytophilum in quill mites (Acari: Syringophilidae) parasitizing passerine birds. - Microbes and Infection, 8: 303-307.

Skoracki M., S.V. Mironov. 2013. New species and records of quill mites of the family Syringophilidae (Acariformes: Prostigmata) from the passerines (Aves: Passeriformes) from the Russian Far East. - Zootaxa, 3641: 554-564.

Skoracki M., S.V. Mironov, S. Bermúdez. 2019. A new syringophilid mite (Acariformes: Syringophilidae) from manakins (Passeriformes: Pipridae) in Panama. - Acarina, 27(2): 229-232.

Skoracki M., S.V. Mironov, M. Unsoeld. 2013. The first records of quill mites of the family Syringophilidae (Acariformes: Prostigmata: Cheyletoidea) from trogoniform birds (Aves: Trogoniformes). - Zootaxa, 3701: 291-297.

Skoracki M., P. Møller, P. Tryjanowski. 2003. A new species of parasitic mites of the genus Syringophiloidus Kethley 1970 (Acari: Syringophylidae) from the barn swallow Hirundo rustica Linnaeus, 1758. - Parasite, 10: 17- 20.

Skoracki M., B. OConnor. 2010. New taxa of quill mites (Acari: Cheyletoidea: Syringophilidae). - Zootaxa, 2341: 1-32.

Skoracki M., B. OConnor, S.M. Goodman, N. Marciniak-Musial. 2016. New species and records of syringophilid mites (Acariformes: Syringophilidae) associated with Malagasy birds. - Systematic and Applied Acarology, 21(10): 1534

Skoracki M., K. Scibek, B. Sikora. 2012. New genus and three new species of quill mites (Acari: Syringophilidae: Picobiinae) parasitizing puffbirds (Aves: Piciformes). - Folia Parasitologica, 59: 229-236.

Skoracki M., B. Sikora. 2002. New ectoparasitic mites of the family Syringophilidae (Acari: Prostigmata: Cheyletoidea) associated with birds from Argentina. - Zootaxa, 27: 1-8.

Skoracki M., B. Sikora. 2003. Quill mites (Acari: Prostigmata: Syringophilidae) from African passeriform birds. - Zootaxa, 129: 1-10.

Skoracki M., B. Sikora. 2004a. Tinamiphilopsis elegans gen. nov. et sp. nov., a first record of the quill mites (Acari, Syringophilidae) from tinamou birds (Tinamiformes, Tinamidae). Acta Parasitologica, 49(4): 348-352.

Skoracki M., B. Sikora. 2004b. A new genus and four new species of quill mites (Acari: Prostigmata: Syringophilidae) from phasianid birds (Galliformes: Phasianidae). - Parasite, 11: 379-386. Skoracki M., B. Sikora. 2005. Neosyringophilopsis, a new genus of the subfamily Syringophilinae (Acari: Syringophilidae). - Zootaxa, 1052: 21-28. 
Skoracki M., B. Sikora. 2008a. Blaszakia, a new genus of quill mites (Acari: Syringophilidae) parasitizing turacos (Aves: Musophagiformes). - Annales Zoologici (Warszawa), 58(2): 327-332.

Skoracki M., B. Sikora. 2008b. Terratosyringophilus reichholfi, a new species of quill mites parasitizing the black-capped lory Lorius lory (L.) in New Guinea. - Spixiana, 31(2): 195-198.

Skoracki M., B. Sikora. 2011. Quill mites (Acari: Syringophilidae) associated with galliform birds (Aves: Galliformes). - Zootaxa, 2954: 13-30.

Skoracki M., B. Sikora. 2014. Two new quill mite species of the family Syringophilidae (Acari: Prostigmata) parasitizing the house sparrow Passer domesticus (L.)(Aves: Passeriformes). - Zootaxa, 3765(2): 194-200.

Skoracki M., B. Sikora, L. Jerzak, M. Hromada. 2020. Tanopicobia gen. nov., a new genus of quill mites, its phylogenetic placement in the subfamily Picobiinae (Acariformes: Syringophilidae) and picobiine relationships with avian hosts. - PLoS One, 15[1 (e0225982)].

Skoracki M., B. Sikora, M. Hromada. 2019. First Record of Quill Mites (Acariformes: Syringophilidae: Picobiinae) Living in the Quill Walls of Parrots. - J. Med. Entomol., 56(6): $1610-1613$.

Skoracki M., B. Sikora, M. Marciniak, M. Zmudzinski. 2016. Syringophiloidus bucerotidus sp. nov. (Acari: Syringophilidae), a new quill mite species parasitizing hornbills (Aves: Bucerotidae) in the Sub-Saharan Africa. - International Journal of Acarology, 43(1):

Skoracki M., B. Sikora, J. Michalik. 2004. Budowa morfologiczna ektopasożytniczych roztoczy z rodziny Syringophilidae (Acari: Prostigmata). In: Blaszak C., Buczek A. (eds) Arthropods, Parasite-Host relationships. Pp.19-22 (in Polish).

Skoracki M., B. Sikora, M. Ozminski. 2012. A new quill mite species (Acari: Syringophilidae) parasitising tinamous (Aves: Tinamiformes). - Systematic Parasitology, 81: 109-113.

Skoracki M., B. Sikora, G.S. Spicer. 2016. A review of the subfamily Picobiinae Johnston and Kethley, 1973 (Acariformes: Prostigmata: Syringophilidae). - Zootaxa, 4113(1): 1-95.

Skoracki M., A. Skoracka. 1999. Syringophilopsis turdi (Fritsch, 1958) i Torotrogla gaudi Bochkov et Mironov, 1998 dwa gatunki roztoczy z rodziny Syringophilidae nowe dla fauny Polski. - Przegl. Zool., 43 (3-4): 185 -186.

Skoracki M., Solarczyk P. 2012. New picobiin mites (Acari: Syringophilidae: Picobiinae) associated with woodcreeper birds (Passeriformes: Dendrocolaptidae). - Zootaxa, 3406: 59-66.

Skoracki M., P. Solarczyk, B. Sikora. 2012. Three new species of picobiine mites (Acari: Syringophilidae) parasitising African Flycatchers (Aves: Muscicapidae). - Systematic Parasitology, 83: 123-135.

Skoracki M., G.S. Spicer, B.M. OConnor. 2014. A review of mites of the subfamily Picobiinae Johnston \& Kethley, 1973 (Prostigmata: Syringophilidae) from North American birds. - Systematic Parasitology, 87: 99-110.

Skoracki M., G.S. Spicer, B. M. OConnor. 2016. A systematic review of the subfamily Syringophilinae (Acari: Syringophilidae) of the Nearctic region. Part 1: quill mites associated with passerines (Aves: Passeriformes). - Zootaxa, 4084(4): 451-494.

Skoracki M., P. Tryjanowski, M. Hromada. 2002. Two new parasitic mite species of the genus Syringophilopsis from true Shrikes (Aves: Laniidae). - Parasite, 9: 11-16. 
Skoracki M., M. Unsoeld, K. Kavetska. 2014. Syringophilid mites (Acari: Syringophilidae) associated with the rails (Aves: Rallidae) and a key to the species of the genus Rafapicobia Skoracki, 2011. - Systematic Parasitology, 88: 227-232.

Skoracki M., M. Unsoeld, K. Kavetska, K. Kaszewska. 2014. Quill mites of the subfamily Picobiinae (Acari: Syringophilidae) associated with woodpeckers (Aves: Piciformes: Picidae). - Systematic Parasitology, 85: 213-217.

Skoracki M., M. Unsoeld, N. Marciniak, B. Sikora. 2016. Diversity of Quill Mites of the Family Syringophilidae (Acari: Prostigmata) Parasitizing Owls (Aves: Strigiformes) With Remarks on the Host-Parasite Relationships. - Journal of Medical Entomology, 53: 4: 815-826.

Skoracki M., M. Unsoeld, M. Ozminski. 2013. A new genus of the quill mites of the family Syringophilidae (Acari: Cheyletoidea) associated with mousebirds (Aves: Coliiformes). - Systematic Parasitology, 85: 213-217.

Skoracki M., M. Unsoeld, M. Skorupski. 2014. Otidiformes, a new avian host order for quill mites of the family Syringophilidae (Acari: Prostigmata). - Intern. Journal of Acarology, 40: 349-352.

Skoracki M., M. Unsoeld, M. Skorupski, K. Kavetska. 2014. Syringophilid mites (Acari: Syringophilidae) associated with the rails (Aves: Rallidae) and a key to the species of the genus Rafapicobia Skoracki, 2011. - Systematic Parasitology, 88(3): 227-232.

Skoracki M., S.V. Zabludovskaya, A.V. Bochkov. 2012. A review of Prostigmata (Acariformes: Trombidiformes) permanently associated with birds. - Acarina, 20: 67-107.

Skoracki, M., K. Zawierucha. 2016. Chenophila nanseni sp. n. (Acari: Syringophilidae) parasitizing the barnacle goose in Svalbard. - Pol. Polar Res., 37(1): 121-130.

Skoracki M., M. Zmudzinski, P. Solarczyk. 2017. Ixobrychiphilus, a new genus of the family Syringophilidae (Acariformes: Prostigmata). - Acarologia, 57(2): 269-273.

Skoracki M., M. Zmudzinski, M. Unsoeld, B. Sikora. 2016. First Records of the Syringophilid Mites (Acariformes: Prostigmata) Parasitizing Ibises and Spoonbills (Pelecaniformes: Threskiornithidae), With Description of Four New Species. - J. of Medical Entomology, 53(6): 1312-1321.

Skoracki M., M. Zmudzinski, B. Sikora. 2020. Rafapicobia olszanowskii, a New Species of Syringophilid Mite (Acariformes: Syringophilidae) from Semnornis ramphastinus (Piciformes: Semnornithidae). - Annales Zoologici, 70(3): 449-452.

Sun X. (undated). Other medical mites. 105-106. In: Chen X., Ma E. (eds). Researches of acarology in China. - Chongqing Publishing House. Chongqing: 1-170.

Turk F.A. 1953. I. - A synonymic catalogue of British Acari: Part I. - Ann. Mag. Nat. Hist., $12^{\text {th }}$ Ser., 61: 1-26.

Tymcio K. K. Kavetska, M. J. Czyz, M. Skoracki. 2013. Onthogeny of Chenophila platyrhynchos sp.nov. (Acari: Syringophilidae), an ectoparasite of the Mallard Anas platyrhynchos (Anseriformes: Anatidae). - Turkish J. of Zoology, 37: 659-667.

Womersley H. 1941. Notes on the Cheyletidae (Acarina, Trombidoidea) of Australia and New Zealand with descriptions of new species. - Rec. South Australian Mus., 7 (1): 51-64. 
Zmudzinski M., M. Skoracki. 2016. An updated check-list of the family Syringophilidae (Acariformes:Prostigmata). https://sites.google.com/site/syringophilidae/(accessed 7 April 2019).

Zmudzinski M., M. Skoracki. 2017. A new species Selenonycha inspirata n.sp. (Acariformes: Syringophilidae) from the bare-faced ibis Phimosus infuscatus (Lichtenstein)(Pelecaniformes: Threskiornithidae) as an exemple of host- switching event. - Systematic Parasitology, 94(5):593-598

Zmudzinski M., M. Skoracki, M. Hromada. 2019. Neoaulonastus sidorchukae, a new species of quill mites (Acariformes: Syringophilidae) associated with the purple-rumped sunbird Leptocoma zeylonica (Linnaeus) (Passeriformes: Nectariniidae) from Sri Lanka. - Zootaxa, 4647(1): 83-87.

Zmudzinski M., M. Skoracki, M. Hromada, M. Unsoeld. 2018. Niglarobia vanelli, a new species of syringophilid quill mite associated with lapwings (Charadriiformes: Charadriidae). Acta Parasitologica, 63(4): 704-708.

Zmudzinski M., P. Solarczik, M. Skoracki. 2016. A new species and new records of syringophilid mites (Acariformes: Prostigmata: Cheyletoidea) associated with aquatic birds. - Annales Zoologici (Warsaw), 66(3): 431-435.

Zmudzinski M., M. Unsoeld. 2016. New distribution of Picobia caudati Skoracki and Hebda, 2004 (Acariformes: Syringophilidae): an ectoparasite of Long-tailed Tit Aegithalos caudatus (L.) (Passeriformes: Aegithalidae). - Ann. Parasitol., 62(4): 355-357.

Zmudzinski M., M. Unsoeld. 2017. A new species of the quill mite genus Chenophila (Acariformes: Syringophilidae) from the Marbled Teal Marmaronetta angustirostris (Menetries) (Anseriformes: Anatidae) in Turkey. - Acta Parasitologica, 62(2): 477-481.

Zmudzinski M., M. Unsoeld. 2019. Quill mites (Acariformes: Syringophilidae) parasitizing birds in Germany: new host records and descriptions of two new species from Limosa lapponica (L.)(Aves: Scolopacidae). - Syst. and Applied Acarology, 24(3): 362-376.

Zmudzinski M., M. Unsoeld, W. Knee, M. Skoracki. 2015. New host records for parasitic mites of the family Syringophilidae from accipitriform birds (Aves: Accipitriformes). - Annals of Parasitology, 61(4): 291-293. 


\title{
Superfamily Cloacaroidea Bochkov et OConnor
}

\author{
Cloacaroidea Bochkov \& O’Connor, 2008: 335
}

The small family Epimyodicidae has been initially considered subfamily of Cloacaridae, but the analysis of Bochkov (2002) and Bochkov \& O'Connor (2008) has shown the need to give it familial status under the new superfamily Cloacaroidea. Although small (19 sp.), the superfamily embraces mites with peculiar localisation and high value in reconstructiing the ways of formation of parasitic mites. 


\title{
The Mite Family Cloacaridae
}

\author{
Cloacaridae Camin, Moss, Oliver \& Singer, 1967: 263
}

This small ( $15 \mathrm{sp}$.) family has been established almost simultaneously by Camin, Moss, Oliver \& Singer (1967) and by Fain (1968). The genera Cloacarus Camin et al., Emyduracarus Fain and Caminacarus Fain contain species living in the rectum of turtles and tortoises in Israel, USA, China, Africa, and Australia. They are attached to the mucose or live more or less submucose. Theodoracarus testudinis Fain live in the muscle tissue of tortoises. Chelonacarus elongatus Pence et Wright, 1998 had been discovered as parasite of sea turtles (Chelonia mydas).

Besides the typical subfamily Cloacarinae, latter has been described the only representative of Pneumophaginae (Pneumophagus bubonis Fain et Smiley, 1989) from the lungs of Bubo virginianus.

\section{Composition of the family Cloacaridae:}

Fam. Cloacaridae Camin, Moss, Oliver et Singer, 1967 - 6 gen., 15 sp.

Subfam. Cloacarinae Camin, Moss, Oliver et Singer, 1967 - 14 sp.

Caminacarus Fain, 1968 - 9 sp.

Chelonacarus Pence et Wright, 1998 - 1 sp.

Cloacarus Camin, Moss, Oliver et Singer, 1967 - 2 sp.

Emyduracarus Fain, 1968 - 1 sp.

Theodoracarus Fain, 1968 - 1 sp.

Subfam. Pneumophaginae Fain et Smiley, 1989 - 1 sp.

Pneumophagus Fain et Smiley, 1989 - 1 sp.

\section{Catalogue of Cloacaridae}

Family Cloacaridae Camin, Moss, Oliver et Singer

Cloacaridae Camin, Moss, Oliver \& Singer, 1967: 263

Type genus: Cloacarus Camin, Moss, Oliver et Singer, 1967

Subfamily Cloacarinae Camin, Moss, Oliver et Singer

Cloacarinae Camin, Moss, Oliver \& Singer, 1967: 263

Type genus: Cloacarus Camin, Moss, Oliver et Singer, 1967 
Genus Caminacarus Fain

Caminacarus Fain, 1968: 6

Type species: Caminacarus theodori Fain, 1968

Caminacarus chrysemys Pence et Casto

Caminacarus chrysemys Pence \& Casto, 1975: 133

Type locality: Laplace, Louisiana

Distribution: USA (Louisiana)

Host: Chrysemys scripta elegans (Testudines: Emydidae)

\section{Caminacarus costai Fain}

Caminacarus costai Fain, 1968: 10

Type locality: Benjamina

Distribution: Israel

Host: Mauremys caspica (Testudines: Emydidae)

Caminacarus dawsoni Bochkov et OConnor

Caminacarus dawsoni Bochkov \& OConnor, 2008: 340

Type locality: USA

Distribution: USA

Host: Graptemys pseudogeographica (Testudines: Emydidae)

Caminacarus deirochelys Fain

Caminacarus deirochelys Fain, 1968: 18

Type locality: Englewoord

Distribution: USA (Florida)

Host: Deirochelys reticularia (Testudines: Emydidae)

Caminacarus pelomedusae Fain

Caminacarus pelomedusae Fain, 1968: 13

Locality: Niarembe, Ituri (DR Congo), Tabora

Distribution: DR Congo, Tanzania

Hosts: Pelomedusa galeata, P. subrufa (Testudines: Pelomedusidae)

\section{Caminacarus pelusios Fain}

Caminacarus pelusios Fain, 1968: 13

Type locality: Lake Mohasi

Distribution: Rwanda

Host: Pelusios castaneus (Testudines: Pelomedusidae) 
Caminacarus sinensis Fain

Caminacarus sinensis Fain, 1968: 16

Type locality: China

Distribution: China

Host: Trionyx sinensis (Testudines: Trionychidae)

Caminacarus terrapenae Pence et Casto

Caminacarus terrapenae Pence \& Casto, 1975: 13.

Type locality: Louisiana

Distribution: USA (Louisiana)

Host: Terrapene carolina (Testudines: Emydidae)

Caminacarus theodori Fain

Caminacarus theodori Fain, 1968: 7

Type locality: Benjamina

Distribution: Israel

Host: Mauremys caspica (Testudines: Emydidae)

Genus Chelonacarus Pence et Wright

Chelonacarus Pence \& Wright, 1998: 835

Type species: Chelonacarus elongatus Pence et Wright, 1998

Chelonacarus elongatus Pence et Wright

Chelonacarus elongatus Pence \& Wright, 1998: 836

Type locality: Bocas del Toro, Isla de Colon

Distribution: Panama

Host: Chelonia mydas (Testudines: Cheloniidae)

Genus Cloacarus Camin, Moss, Oliver et Singer

Cloacarus Camin, Moss, Oliver \& Singer, 1967: 264

Type species: Cloacarus faini Camin et Singer, 1967

Cloacarus beeri Camin et Oliver

Cloacarus beeri Camin \& Oliver, in Camin et al., 1967: 269

Type locality: Douglas Lake, Michigan

Distribution: USA (Michigan)

Host: Chrysemys picta (Chelonia: Emydidae)

Cloacarus faini Camin et Singer

Cloacarus faini Camin \& Singer, in Camin et al., 1967: 265 
Type locality: stream near U.S. Route 59 at county line between Douglas Co. and Franklin Co., Kansas

Distribution: USA (Kansas)

Host: Chelydra serpentina (Chelonia: Chelydridae)

Genus Emyduracarus Fain

Emyduracarus Fain, 1968: 20

Type species: Emydurarus australis Fain, 1968

\section{Emydurarus australis Fain}

Emydurarus australis Fain, 1968: 20; Domrow, 1991: 1347

Type locality: St. Lawrence (Queensland)

Distribution: Australia (Coastal central Queensland)

Host: Elseya [Emydura] latisternum (Testudines: Chelidae)

Genus Theodoracarus Fain

Theodoracarus Fain, 1968: 23

Type species: Theodoracarus testudinis Fain, 1968

\section{Theodoracarus testudinis Fain}

Theodoracarus testudinis Fain, 1968: 23

Type locality: Jerusalem

Distribution: Israel

Host: Testudo graeca ibera (Testudines: Testudinidae)

Subfamily Pneumophaginae Fain et Smiley

Pneumophaginae Fain \& Smiley, 1989: 112

Type genus: Pneumophagus Fain et Smiley, 1989

Genus Pneumophagus Fain et Smiley

Pneumophagus Fain \& Smiley, 1989: 112

Type species: Pneumophagus bubonis Fain et Smiley, 1989

Pneumophagus bubonis Fain et Smiley

Pneumophagus bubonis Fain \& Smiley, 1989: 112

Type locality: East Lansing, Michigan

Distribution: USA (Michigan)

Host: Bubo virginianus (Strigiformes: Strigidae) 


\section{Index of Cloacaridae}

(Names in bold are accepted as valid)

australis, Emyduracarus 458

beeri, Cloacarus 457

bubonis, Pneumophagus 458

Caminacarus 456

Chelonacarus 457

chrysemys, Caminacarus 456

Cloacaridae 455

Cloacarinae 455

Cloacarus 457

costai, Caminacarus 456

dawsoni, Caminacarus 456

deirochelys, Caminacarus 456 elongatus, Chelonacarus 457

Emyduracarus 458

faini, Cloacarus 457

pelomedusae, Caminacarus 456

pelusios, Caminacarus 456

Pneumophaginae 458

Pneumophagus 458

sinensis, Caminacarus 457

terrapenae, Caminacarus 457

testudinis, Theodoracarus 458

Theodoracarus 458

theodori, Caminacarus 457

\section{Distribution of Cloacaridae}

\section{AFRICA}

Congo DR (Fain, 1968) - Caminacarus pelomedusae

Rwanda (Fain, 1968) - Caminacarus pelusios

Tanzania (Fain, 1968) - Caminacarus pelomedusae

ASIA

China (Fain, 1968) - Caminacarus sinensis

Israel (Fain, 1968) - Caminacarus costai, C. theodori, Theodoracarus testudinis

\section{NORTH AMERICA}

USA (Fain \& Smiley, 1989; Pence \& Casto, 1975; Bochkov \& OConnor, 2008) - Caminacarus chrysemys, C. deirochelys, C. dawsoni, C. terrapenae, Cloacarus beeri, C. faini, Pneumophagus bubonis

\section{SOUTH AMERICA}

Panama (Pence \& Wright, 1998) - Chelonacarus elongatus

\section{OCEANIA}

Australia (Fain, 1968; Domrow, 1991) - Emyduracarus australis 


\section{Hosts of Cloacaricae}

\section{Reptilia}

\section{Testudines}

Fam. Chelidae

Emydura latisternum - Emydurarus australis

Fam. Cheloniidae

Chelonia mydas - Chelonacarus elongatus

Fam. Chelydridae

Chelydra serpentina - Cloacarus faini

Fam. Emydidae

Chrysemys scripta elegans - Caminacarus chrysemys

Deirochelys reticularia - Caminacarus deirochelys

Graptemys pseudogeographica - Caminacarus dawsoni

Mauremys caspica - Caminacarus costai

Terrapene carolina - Caminacarus terrapenae

Fam. Pelomedusidae

Pelomedusa galeata - Caminacarus pelomedusae

Pelomedusa subrufa - Caminacarus pelomedusae

Pelusios castaneus - Caminacarus pelusios

Fam. Testudinidae

Testudo graeca ibera - Theodoracarus testudinis

Fam. Trionychidae

Trionyx sinensis - Caminacarus sinensis

Aves

\section{Strigiformes}

Fam. Strigidae

Bubo virginianus - Pneumophagus bubonis

\section{Bibliography of Cloacaridae}

Bochkov A.V. 2008. Origin and evolution of parasitism of mites of the Infraorder Eleutherengona (Acari: Prostigmata). Report I. Lower Raphignathae. - Parazitologiya, 42(5): $337-359$.

Bochkov A.V., B.M. OConnor. 2008. A new mite superfamily Cloacaridae and its position within the Prostigmata (Acariformes). - J. Parasitology, 94(2): 335-344.

Camin J. H., W. W. Moss, J. H. Oliver Jr., G. Singer. 1967. Cloacaridae, a new family of Cheyletoid mites from the cloaca of aquatic turtles (Acari: Acariformes: Eleutherengona). - J. Med. Ent., 4(3): 261-272. 
Domrow R. 1991. Acari Prostigmata (excluding Trombiculidae) Parasitic on Australian Vertebrates: an Annotated Checklist, Keys and Bibliography. - Invertebr. Taxon., 4: 1283-1376.

Fain A. 1968. Notes sur les Acariens de la famille Cloacaridae Camin et al. Parasites du Cloaque et des tissus profonds des tortues (Cheyletoidea: Trombidiformes). - Bull. Inst. r. Sci. nat. Belgique, 44(15): 1-33.

Fain A., R. L. Smiley. 1989. A new Cloacarid mite (Acari: Cloacaridae) from the lungs of the Great horned owl, Bubo virginianus, from the U. S. A. - Intern. J. Acarol., 15(2): 111-115.

Pence D. B., S.D. Casto. 1975. Two new species of the genus Caminacarus (Acarina: Cloacaridae) from Turtles in Louisiana. - J. Parasitol., 61(1): 133-138.

Pence D., S.D. Wright. 1998. Chelonacarus elongatus n.gen., n. sp. (Acari: Cloacaridae) from the cloaca of the green turtle Chelonia mydas (Cheloniidae). - J. of Parasitology, 84(4): 835-839. 


\title{
The Mite Family Epimyodicidae
}

\author{
Epimyodicinae Fain, Lukoschus \& Rosmalen, 1982: 4 \\ Epimyodicidae: Bochkov, 2002: 651
}

The representatives of Epimyodicinae have been discovered in loose deep connective subcutaneous tissues of Soricomorpha and Rodentia, so far only in Europe and North America.

So far only four species are known from subcutaneous tissues of Soricomorpha (Sorex, Crocidura, Talpa) and Rodentia (Microtus, Peromyscus, Sylvaemus).

\section{Catalogue of Epimyodicidae}

Family Epimyodicidae Fain, Lukoschus et Rosmalen

Epimyodicinae Fain, Lukoschus \& Rosmalen, 1982: 4

Epimyodicidae: Bochkov, 2002: 651

Type genus: Epimyodex Fain et Orts, 1969

Genus Epimyodex Fain et Orts

Epimyodex Fain \& Orts, 1969: 66

Type species: Epimyodex talpae Fain et Orts, 1969

Epimyodex crocidurae Fain, Lukoschus et Rosmalen

Epimyodex crocidurae Fain, Lukoschus \& Rosmalen, 1982: 5

Type locality: Nijmegen

Distribution: Holland

Host: Crocidura russula (Soricomorpha: Soricidae)

Epimyodex microti Fain, Lukoschus et Rosmalen

Epimyodex microti Fain, Lukoschus \& Rosmalen, 1982: 7; Fain \& Bochkov, 2001: 223

Type locality: Nijmegen (Holland)

Distribution: Holland, Italy, USA

Hosts: Microtus arvalis, M. savii (Rodentia: Arvicolidae), Peromyscus leucopus, P. maniculatus (Rodentia: Cricetidae); Sylvaemus sylvaticus (Muridae)

Epimyodex soricis Fain et Bochkov

Epimyodex soricis Fain \& Bochkov, 2001: 221- 223.

Type locality: Lincoln Co., Oregon

Distribution: USA (Oregon)

Hosts: Sorex trowbridgii, S. vagrans (Soricomorpha: Soricidae) 
Epimyodex talpae Fain et Orts

Epimyodex talpae Fain \& Orts, 1969: 66

Type locality: Lillois, Belgium

Distribution: Belgium

Hosts: Talpa europaea, T. romana (Soricomorpha: Talpidae, Talpinae)

\section{Index of Epimyodicidae}

(Names in bold are accepted as valid)

soricis, Epimyodex 462

Epimyodex 462

Epimyodicidae 462

Epimyodicinae 462 crocidurae, Epimyodex 462

talpae, Epimyodex 463

microti, Epimyodex 462

soricis, Epimyodex 462

\section{Distribution of Epimyodicidae}

\section{EUROPE}

Belgium (Fain \& Orts, 1969) - Epimyodex talpae

Holland (Fain, Lukoschus \& Rosmalen, 1982) - Epimyodex crocidurae, E. microti

Italy (Fain, Lukoschus \& Rosmalen, 1982) - Epimyodex microti, E. talpae

\section{NORTH AMERICA}

USA (Fain, Lukoschus \& Rosmalen, 1982; Fain \& Bochkov, 2001) - Epimyodex microti, E. soricis

\section{Hosts of Epimyodicidae}

\section{Mammalia}

\section{Soricomorpha}

Fam. Soricidae

Crocidura russula - Epimyodex crocidurae

Sorex trowbridgii - Epimyodex soricis

S. vagrans - Epimyodex soricis

Fam. Talpidae

Talpa europaea - Epimyodex talpae

T. romana - Epimyodex talpae 


\section{Rodentia}

Fam. Arvicolidae

Microtus arvalis - Epimyodex microti

M. savii - Epimyodex microti

\section{Fam. Cricetidae}

Peromyscus leucopus - Epimyodex microti

P. maniculatus - Epimyodex microti

\section{Fam. Muridae}

Sylvaemus sylvaticus - Epimyodex microti

\section{Bibliography of Epimyodicidae}

Bochkov A. V. 2002. Classification and phylogeny of mites of the superfamily Cheyletoidea (Acari: Prostigmata). - Entomologicheskoe Obozrenie [In Russian but also translated into English in Entomol. Rev.]. 81: 488-513.

Bochkov A.V., B.M. OConnor. 2008. A new mite superfamily Cloacaridae and its position within the Prostigmata (Acariformes). - J. Parasitology, 94(2): 335-344.

Fain A. 1959. Deux nouveaux genres d'Acariens vivant dans l'épaisseur des muqueuses nasale et buccale chez un lémurien (Trombidiformes: Demodicidae). - Bull. Ann. Soc. r. Ent. Belgium, 95(9 -10): 263-273.

Fain A., A.V. Bochkov. 2001. A new species of the genus Epimyodex Fain and Orts, 1969 (Acari: Cloacaridae: Epimyodecinae) parasitizing Sorex trowbridgii (Soricidae) from the U.S.A. - Internat. J. Acarol., 27(3): 221-223.

Fain A., F. S. Lukoschus, P.G. Rosmalen. 1982. Observations on the genus Epimyodex Fain \& Orts, 1969, with description of two new species. Transfer of this genus to the Cloacaridae (Acarina, Prostigmata). - Bull. Inst. r. Sci. nat. Belg., 54(3): 1-10.

Fain A., S. Orts. 1969. Epimyodex talpae n. g., n. sp. parasite sous-cutané de la taupe en Belgium (Demodicidae: Trombidiformes). - Acarologia, 11(1): 65-68. 


\section{Acknowledgements}

Many prominent Acarologists provided literature and advice to the present author in the complicated task to put together and to update the huge information on the seven families of mites treated in this volume. Some will certainly be ommited, but cordial gratitude is first of all due to A. Fain, A. Bochkov, U. Gerson, I. Literák, F. Dusbábek, J. B. Kethley, R. F. Lawrence, B. OConnor, R. V. Southcott, J. de la Cruz, K.M. Jack, H. Rak, F. M. Summers, M. Shiba, R. Domrow, C.H.W. Flechtmann, I. M. Newell, K. Kawashima, A. Hoffmann, D. B. Pence, M. Skoracki, F.S. Lukoschus, Ch. D. Radford, V. Černy, W.W. Moss, W. B. Nutting, J.N. Izdebska, K.M.T. Giesen, M. Nadchatram, S.E. Thewke, K. Uchikawa, V. Bukva, J. O. Whitaker, A. Baker, V. Amaral, M.K.P. Meyer, E. Glowska, M. Hromada, A. Khaustov, A. Saboori, M. Klimovičová, K. Kaszewska, G. Spicer, M. Mironov, M. Zmudzinski, L. Corpuz-Raros, V.I. Volgin, J.L. Izdebska, R.L. Smiley, H. Klompen, M. Dabert, E. Ueckermann. 